\title{
cells
}

\section{The Molecular and Cellular Basis of Retinal Diseases}

Edited by

Steven J. Pittler and Steven J. Fliesler Printed Edition of the Special Issue Published in Cells 


\section{The Molecular and Cellular Basis of Retinal Diseases}





\section{The Molecular and Cellular Basis of Retinal Diseases}

Editors

Steven J. Pittler

Steven J. Fliesler 


\section{Editors}

Steven J. Pittler

Department of Optometry and Vision Science, Vision Science

Research Center, University of Alabama at Birmingham USA
Steven J. Fliesler

Departments of Ophthalmology and Biochemistry and the

Neuroscience Graduate Program, The State University of New York (SUNY)—University at Buffalo

USA

Editorial Office

MDPI

St. Alban-Anlage 66

4052 Basel, Switzerland

This is a reprint of articles from the Special Issue published online in the open access journal Cells (ISSN 2073-4409) (available at: https://www.mdpi.com/journal/cells/special_issues/basis_retinal_ diseases).

For citation purposes, cite each article independently as indicated on the article page online and as indicated below:

LastName, A.A.; LastName, B.B.; LastName, C.C. Article Title. Journal Name Year, Article Number, Page Range.

ISBN 978-3-03936-654-5 (Hbk)

ISBN 978-3-03936-655-2 (PDF)

Cover image courtesy of Steven Pittler.

(c) 2020 by the authors. Articles in this book are Open Access and distributed under the Creative Commons Attribution (CC BY) license, which allows users to download, copy and build upon published articles, as long as the author and publisher are properly credited, which ensures maximum dissemination and a wider impact of our publications.

The book as a whole is distributed by MDPI under the terms and conditions of the Creative Commons license CC BY-NC-ND. 


\section{Contents}

About the Editors $\ldots \ldots \ldots \ldots \ldots \ldots \ldots \ldots \ldots \ldots \ldots \ldots$

Preface to "The Molecular and Cellular Basis of Retinal Diseases" $\ldots \ldots \ldots \ldots \ldots$ ix

Bright Asare-Bediako, Sunil K. Noothi, Sergio Li Calzi, Baskaran Athmanathan, Cristiano P. Vieira, Yvonne Adu-Agyeiwaah, Mariana Dupont, Bryce A. Jones, Xiaoxin X. Wang, Dibyendu Chakraborty, Moshe Levi, Prabhakara R. Nagareddy and Maria B. Grant

Characterizing the Retinal Phenotype in the High-Fat Diet and Western Diet Mouse Models of Prediabetes

Reprinted from: Cells 2020, 9, 464, doi:10.3390/cells9020464 . . . . . . . . . . . . . . . .

Sriganesh Ramachandra Rao, Steven J. Fliesler, Mai N. Nguyen, Pravallika Kotla and Steven J. Pittler

Lack of Overt Retinal Degeneration in a K42E Dhdds Knock-In Mouse Model of RP59

Reprinted from: Cells 2020, 9, 896, doi:10.3390/cells9040896 . . . . . . . . . . . . . . . .

Marci L. DeRamus, Stephanie J. Davis, Sriganesh Ramachandra Rao, Cyril Nyankerh, Delores Stacks, Timothy W. Kraft, Steven J. Fliesler and Steven J. Pittler

Selective Ablation of Dehydrodolichyl Diphosphate Synthase in Murine Retinal Pigment Epithelium (RPE) Causes RPE Atrophy and Retinal Degeneration

Reprinted from: Cells 2020, 9, 771, doi:10.3390/cells9030771 . . . . . . . . . . . . . . . .

Xiaoping Qi, Sayak K. Mitter, Yuanqing Yan, Julia V. Busik, Maria B. Grant and Michael E. Boulton

Diurnal Rhythmicity of Autophagy Is Impaired in the Diabetic Retina

Reprinted from: Cells 2020, 9, 905, doi:10.3390/cells9040905 . . . . . . . . . . . . . . . .

T. J. Hollingsworth and Alecia K. Gross

Innate and Autoimmunity in the Pathogenesis of Inherited Retinal Dystrophy

Reprinted from: Cells 2020, 9, 630, doi:10.3390/cells9030630 . . . . . . . . . . . . . . .

Andrew P. Voigt, Elaine Binkley, Miles J. Flamme-Wiese, Shemin Zeng, Adam P. DeLuca, Todd E. Scheetz, Budd A. Tucker, Robert F. Mullins and Edwin M. Stone

Single-Cell RNA Sequencing in Human Retinal Degeneration Reveals Distinct Glial Cell Populations

Reprinted from: Cells 2020, 9, 438, doi:10.3390/cells9020438 . . . . . . . . . . . . . . . .

Alyson Wolk, Dilara Hatipoglu, Alecia Cutler, Mariya Ali, Lestella Bell, Jian Hua Qi, Rupesh Singh, Julia Batoki, Laura Karle, Vera L. Bonilha, Oliver Wessely, Heidi Stoehr, Vincent Hascall and Bela Anand-Apte

Role of FGF and Hyaluronan in Choroidal Neovascularization in Sorsby Fundus Dystrophy Reprinted from: Cells 2020, 9, 608, doi:10.3390/cells9030608 . . . . . . . . . . . . . . . .

Gayle B. Collin, Navdeep Gogna, Bo Chang, Nattaya Damkham, Jai Pinkney, Lillian F. Hyde, Lisa Stone, Jürgen K. Naggert, Patsy M. Nishina and Mark P. Krebs

Mouse Models of Inherited Retinal Degeneration with Photoreceptor Cell Loss

Reprinted from: Cells 2020, 9, 931, doi:10.3390/cells9040931 . . . . . . . . . . . . . . . . . 105

Paige A. Winkler, Laurence M. Occelli and Simon M. Petersen-Jones

Large Animal Models of Inherited Retinal Degenerations: A Review

Reprinted from: Cells 2020, 9, 882, doi:10.3390/cells9040882 . . . . . . . . . . . . . . . 173 
Lars Tebbe, Mashal Kakakhel, Mustafa S. Makia, Muayyad R. Al-Ubaidi and Muna I. Naash The Interplay between Peripherin 2 Complex Formation and Degenerative Retinal Diseases Reprinted from: Cells 2020, 9, 784, doi:10.3390/cells9030784 . . . . . . . . . . . . . . . . . . 201

\section{Theodore G. Wensel}

Phosphoinositides in Retinal Function and Disease

Reprinted from: Cells 2020, 9, 866, doi:10.3390/cells9040866 . . . . . . . . . . . . . . . . . . . . 227

Emilie Picard, Alejandra Daruich, Jenny Youale, Yves Courtois and Francine Behar-Cohen From Rust to Quantum Biology: The Role of Iron in Retina Physiopathology

Reprinted from: Cells 2020, 9, 705, doi:10.3390/cells9030705 . . . . . . . . . . . . . . . . . 245

Valeria De Matteis and Loris Rizzello

Noble Metals and Soft Bio-Inspired Nanoparticles in Retinal Diseases Treatment: A Perspective Reprinted from: Cells 2020, 9,679, doi:10.3390/cells9030679 . . . . . . . . . . . . . . . . . . . . . 275

Tirthankar Sinha, Larissa Ikelle, Muna I. Naash and Muayyad R. Al-Ubaidi

The Intersection of Serine Metabolism and Cellular Dysfunction in Retinal Degeneration Reprinted from: Cells 2020, 9,674, doi:10.3390/cells9030674 . . . . . . . . . . . . . . . . . . . . 299

Sonali Nashine and M. Cristina Kenney

Effects of Mitochondrial-Derived Peptides (MDPs) on Mitochondrial and Cellular Health in AMD

Reprinted from: Cells 2020, 9, 1102, doi:10.3390/cells9051102 . . . . . . . . . . . . . . . . . 313 


\section{About the Editors}

Steven J. Pittler is a professor at the Department of Optometry and Vision Science, a senior scientist and director of the Vision Science Research Center, with secondary appointments in the Departments of Ophthalmology and Biochemistry and Molecular Genetics. He received his undergraduate training in biochemistry from Michigan State University (1983). He completed his doctoral studies in Biochemistry at Michigan State University (1989), after which he was an NRSA Neurobiology Postdoctoral Fellow (Ocular Molecular Genetics) at Cullen Eye Institute, Baylor College of Medicine. In 1991, he was an assistant professor of Biochemistry and Molecular Biology at the University of South Alabama. In 1995, he became Director of the Center for Eye Research, at the College of Medicine, University of South Alabama. Moreover, in 1995, Dr. Pittler was the recipient of the Cogan Award for excellence in vision research from the Association for Research in Vision and Ophthalmology. He came to the University of Alabama in Birmingham in 1999, and was promoted to the rank of professor in 2000. In 2013, he received the National Eye Institute Audacious Idea Award.

Steven J. Fliesler is a SUNY Distinguished Professor, UB Distinguished Professor, the Meyer H. Riwchun Endowed Chair Professor of Ophthalmology, and Vice-Chair/Director of Research in the Department of Ophthalmology, State University of New York (SUNY)—University at Buffalo (UB). He also holds concurrent appointments as a professor in the Department of Biochemistry and in the Neuroscience Graduate Program at UB, as well as being a Department of Veterans Affairs Research Career Scientist at the Buffalo VA Medical Center, VA Western NY Healthcare System. Dr. Fliesler obtained a PhD in biochemistry from Rice University, completed a postdoctoral fellowship at the Cullen Eye Institute/Baylor College of Medicine, and was previously on the faculties of Baylor College of Medicine, Bascom Palmer Eye Institute/University of the Miami School of Medicine, and the Saint Louis University School of Medicine, prior to joining the faculty of SUNY-University at Buffalo in 2008. His research is focused on inborn errors of cholesterol and isoprenoid metabolism and their impact on the development, structure and function of the retina, as well as on blast injury to the eye, using animal models. He has published more than 150 peer-reviewed journal articles, book chapters and review articles, and is the editor of two books. His research program has been funded continuously for more than 35 years by multiple grants from the NEI/NIH and private foundations, as well as, more recently, MERIT Awards from the U.S. Department of Veterans Affairs. Dr. Fliesler recently served on the Board of Trustees of the Association for Research in Vision and Ophthalmology (ARVO), representing the Retinal Cell Biology (RC) Section, as well as being President and immediate past President of ARVO. In 2009, he was inducted as a Silver-tier Fellow of ARVO (FARVO) and, in 2014, became a Gold-tier FARVO. In addition, he is a past councilor for North America, and Treasurer and President of the International Society for Eye Research (ISER). Dr. Fliesler is the Editor-in-Chief of Experimental Eye Research and serves on six other journal editorial boards, including Molecular Vision and the Journal of Lipid Research. 



\section{Preface to "The Molecular and Cellular Basis of Retinal Diseases"}

Our goal with this series of articles was to bring together many prominent vision scientists to report on various aspects of ocular disease, with a focus on the use of animal models to elucidate the underlying mechanisms of pathobiology involved. Rather than limit the focus to only certain aspects of retinal research, we contacted a broad representation of vision scientists studying the retina, in the hopes of compiling a more comprehensive overview of the field. We believe that you will find, herein, a breadth of studies touching on many of the key areas of current retina research.

Steven J. Pittler, Steven J. Fliesler

Editors 



\title{
Characterizing the Retinal Phenotype in the High-Fat Diet and Western Diet Mouse Models of Prediabetes
}

\author{
Bright Asare-Bediako ${ }^{1}$, Sunil K. Noothi ${ }^{2}$, Sergio Li Calzi ${ }^{2}$, Baskaran Athmanathan ${ }^{3}$, \\ Cristiano P. Vieira ${ }^{2}$, Yvonne Adu-Agyeiwaah ${ }^{1}$, Mariana Dupont ${ }^{1}$, Bryce A. Jones ${ }^{4}$, \\ Xiaoxin X. Wang ${ }^{5}$, Dibyendu Chakraborty ${ }^{2}$, Moshe Levi ${ }^{5}$, Prabhakara R. Nagareddy ${ }^{3}$ \\ and Maria B. Grant ${ }^{2, *}$ \\ 1 Vision Science Graduate Program, School of Optometry, University of Alabama at Birmingham, \\ Birmingham, AL 35233, USA; basareb@uab.edu (B.A.-B.); yvonnad@uab.edu (Y.A.-A.); \\ mdupont@uab.edu (M.D.) \\ 2 Department of Ophthalmology and Visual Sciences, School of Medicine, The University of Alabama \\ at Birmingham, Birmingham, AL 35294, USA; sunilnooti@uabmc.edu (S.K.N.); scalzi@uabmc.edu (S.L.C.); \\ cvieira@uabmc.edu (C.P.V.); dchakraborty@uabmc.edu (D.C.) \\ 3 Division of Cardiac Surgery, Department of Surgery, Ohio State University Wexner Medical Center, \\ Columbus, OH 43210, USA; baskaran.athmanathan@osumc.edu (B.A.); \\ prabhakara.nagareddy@osumc.edu (P.R.N.) \\ 4 Department of Pharmacology and Physiology, Georgetown University, Washington, DC 20057, USA; \\ baj46@georgetown.edu \\ 5 Department of Biochemistry and Molecular \& Cellular Biology, Georgetown University, \\ Washington, DC 20057, USA; xiaoxin.wang@georgetown.edu (X.X.W.); moshe.levi@georgetown.edu (M.L.) \\ * Correspondence: mariagrant@uabmc.edu
}

Received: 9 January 2020; Accepted: 13 February 2020; Published: 18 February 2020

\begin{abstract}
We sought to delineate the retinal features associated with the high-fat diet (HFD) mouse, a widely used model of obesity. C57BL/6 mice were fed either a high-fat (60\% fat; HFD) or low-fat (10\% fat; LFD) diet for up to 12 months. The effect of HFD on body weight and insulin resistance were measured. The retina was assessed by electroretinogram (ERG), fundus photography, permeability studies, and trypsin digests for enumeration of acellular capillaries. The HFD cohort experienced hypercholesterolemia when compared to the LFD cohort, but not hyperglycemia. HFD mice developed a higher body weight $(60.33 \mathrm{~g}$ vs. $30.17 \mathrm{~g}, p<0.0001)$ as well as a reduced insulin sensitivity index (9.418 vs. 62.01, $p=0.0002$ ) compared to LFD controls. At 6 months, retinal functional testing demonstrated a reduction in a-wave and b-wave amplitudes. At 12 months, mice on HFD showed evidence of increased retinal nerve infarcts and vascular leakage, reduced vascular density, but no increase in number of acellular capillaries compared to LFD mice. In conclusion, the HFD mouse is a useful model for examining the effect of prediabetes and hypercholesterolemia on the retina. The HFD-induced changes appear to occur slower than those observed in type 2 diabetes (T2D) models but are consistent with other retinopathy models, showing neural damage prior to vascular changes.
\end{abstract}

Keywords: retinal phenotype; neural infarcts; vascular leakage

\section{Introduction}

Diabetes is now considered a worldwide epidemic [1,2]. Recent reports indicate that over $90 \%$ of diabetic individuals have type 2 diabetes (T2D) [3,4]. The most common microvascular complication of diabetes is diabetic retinopathy (DR) [2]. Despite a growing number of different approaches to arrest $\mathrm{DR}$, the incidence and prevalence of DR continues to rise [5]. The understanding of the pathogenesis of DR remains incomplete [4], and this is, in part, due to the lack of readily available models that completely recapitulate the metabolic phenotype [6]. The high-fat diet (HFD) mouse model has 
been described as a robust model for investigating obesity-associated T2D and its related metabolic complications [7]. Studies have shown that HFD-fed mice develop obesity, impaired glucose tolerance, and reduced insulin sensitivity [8,9] with systemic manifestations involving adipose tissue [10], liver [8], and kidneys [11]. However, the ocular changes associated with the HFD model have not been fully investigated. Moreover, the typical Western diet (WD; $40 \%$ fat) has also been given to rodents to recapitulate obesity-driven pathology. However, to mimic the features of T2D, the administration of low-dose Streptozotocin (STZ) is also given to the WD mice [12-14].

The retinal response to high fat exposure would likely involve local changes in the expression of lipid transport proteins, such as the liver $\mathrm{X}$ receptors (LXRs). The LXRs are the key transcription factors that regulate lipid and cholesterol metabolism [15]. While liver $X$ receptor alpha (LXR $\alpha)$ is expressed only in some tissues, the expression of liver $X$ receptor beta (LXR $\beta$ ) is ubiquitous [12]. Previously we showed that whole body $L X R \alpha / \beta$ deficiency resulted in the generation of increased numbers of acellular capillaries, while LXR agonists improved DR in Streptozotocin (STZ)-induced diabetes [12] and in diabetic Lepr ${ }^{\mathrm{db} / \mathrm{db}}(\mathrm{db} / \mathrm{db})$ mice [16]; however, it is not known if the WD modulates the expression of LXR in the retina.

Retinopathy is typically characterized by macroglia activation and gliosis identified by glial fibrillary acidic protein (GFAP) overexpression, which can be considered as a marker for retinal damage $[17,18]$. In the healthy mammalian retina, GFAP is expressed only in astrocytes and not in Muller cells. Following inherited or acquired retinal pathology, GFAP is expressed also in Muller cells $[19,20]$. GFAP expression in Muller cells has been widely used as a cellular marker for retinal pathology [21-25]. Hypoxia-inducible factor 1 alpha (HIF-1 $\alpha$ ) is known to be a key regulator of a tissue's response to hypoxia [26] and plays a role in obesity-induced metabolic syndrome. It has been shown that HFD leads to gradual increase in HIF- $1 \alpha$ and associated pathological changes in the liver $[27,28]$. However, the role of HIF- $1 \alpha$ in the retina of WD-fed mice is not known.

A better understanding of DR in obesity-driven models is needed and may facilitate the optimal choice of disease models for future investigations. Thus, in the present study, we hypothesized that HFD and WD feeding would result in a distinct retinal phenotype and a time course slower than that observed in models of T2D, such as the $\mathrm{db} / \mathrm{db}$ mouse [29] or the high fructose and high fat fed mouse [30]. For this purpose, we characterized not only systemic endpoints of glucose and lipid metabolism but also the function of the retina and development of retinal pathology, including retinal vascular changes and changes in expression of the critical proteins LXR $\beta$, HIF- $1 \alpha$, and GFAP.

\section{Materials and Methods}

\subsection{Animals}

All animal experiments were approved by the University of Alabama at Birmingham (IACUC-20467, approved on 06/16/2016) and Georgetown University (animal project \#2017-0059, approved on 10/27/2017), and followed the Association for Research in Vision and Ophthalmology Statement for the Use of Animals. Six to eight-week-old C57BL/6J mice were fed either a low-fat diet (LFD) (10\%kcal fat, $70 \% \mathrm{kcal}$ carbohydrate, $20 \%$ protein), a Western diet ( $40 \% \mathrm{kcal}$ fat, $43 \% \mathrm{kcal}$ carbohydrate, $17 \% \mathrm{kcal}$ protein), or a HFD (60\% kcal fat, $20 \% \mathrm{kcal}$ carbohydrate, $20 \% \mathrm{kcal}$ protein) for up to 12 months. Diets were purchased from Research Diets, Inc, New Brunswick, NJ, USA. Full details of the composition of each diet is given in Supplementary Table S1.

\subsection{Body Composition, Glucose Tolerance, and Insulin Sensitivity Testing}

The fat mass, lean mass, and water content of the animals were measured by magnetic resonance imaging using EchoMRI (Echo Medical Systems, LLC, Houston, TX, USA). For glucose and insulin tolerance tests, mice were fasted for 5-6 h, injected intraperitoneally with D-glucose at $1.5 \mathrm{~g} / \mathrm{kg}$ of lean mass and tail bled for glucose and insulin measurements. Blood glucose and insulin levels were 
measured $0,15,30,45,60$, and 120 min after glucose administration. The insulin sensitivity index (ISI) was estimated using the Matsuda-Defronzo method [31].

\subsection{Electroretinogram (ERG)}

ERGs were performed using a LKC Bigshot ERG system. Briefly, mice were dark-adapted overnight. The animals were anesthetized with ketamine $(80 \mathrm{mg} / \mathrm{kg}$ total body mass) and xylazine $(15 \mathrm{mg} / \mathrm{kg}$ total body mass), then dilated with atropine/phenylephrine under dim red light. Once dilated, animals were exposed to 5 full-field white light flashes at 0.25 and $2.5 \mathrm{~cd} . \mathrm{s} / \mathrm{m}^{2}$ under scotopic conditions. The animals were then light-adapted for $5 \mathrm{~min}$ and exposed to 10-15 full-field white light flashes at 10 and $25 \mathrm{~cd} . \mathrm{s} / \mathrm{m}^{2}$ under photopic conditions. Responses were averaged and analyzed using the LKC EM software.

\subsection{Fundus Photography and Fluorescein Angiography}

Fundus photography and fluorescein angiography were performed using the Phoenix Micron IV retinal imaging microscope (Phoenix Technology Group, Pleasanton, CA, USA). Briefly, mice were anesthetized with ketamine and xylazine, then dilated with atropine/phenlylephrine, as described above. Once dilated, the animals were placed on the instrument and fundus photographs were taken. Animals were then given intraperitoneal injection of fluorescein (AK-FLUOR $10 \%$, Sigma Pharmaceuticals, North Liberty, IA, USA) and the retinal vasculature was imaged with blue light illumination after 5-8 $\mathrm{min}$ when all the vessels were filled.

\subsection{Acellular Capillaries Quantification}

Trypsin digestion of the retina was performed according to a previously published protocol $[32,33]$. Briefly, eyeballs were enucleated and incubated in $4 \%$ paraformaldehyde overnight. Retinas were isolated, washed, and digested in elastase solution (40 Units elastase/mL; Sigma-Aldrich, St. Louis, MO, USA) to remove the non-vascular tissue. The vascular beds were mounted on glass slides followed by staining with periodic acid-Schiff's base and hematoxylin. About 5-6 fields from the central to mid-periphery were imaged and the number of acellular capillaries per square millimeter were quantified.

\subsection{Immunohistochemistry}

Immunohistochemical staining of mouse retinas was performed according to a previously published protocol [34]. Briefly, mice were euthanized and eyes were immediately enucleated and fixed in $4 \%$ paraformaldehyde (PFA) solution for $15 \mathrm{~min}$. Cornea and lenses were carefully removed and posterior cups were incubated in $15 \%$ sucrose solution in phosphate-buffered saline (PBS) overnight at $4{ }^{\circ} \mathrm{C}$ after washing briefly in PBS. Posterior cups were transferred to $30 \%$ sucrose in PBS for $3-4 \mathrm{~h}$, then embedded in optimal cutting temperature (O.C.T) medium and immediately frozen on dry ice. The frozen samples were stored at $-80^{\circ} \mathrm{C}$ until further processing. The sections were thawed at room temperature for $4 \mathrm{~h}$, washed in PBS for $5 \mathrm{~min}$, and permeabilized with $0.25 \%$ Triton-X in PBS for $5 \mathrm{~min}$ at room temperature. Sections were blocked with $10 \%$ horse serum in $1 \%$ bovine serum albumin (BSA) for $2 \mathrm{~h}$ then incubated with primary antibody diluted in blocking solution (1:100 dilution) overnight at $4{ }^{\circ} \mathrm{C}$. The antibodies used were rabbit anti-GFAP (Abcam, MA, USA), mouse mAB HIF- $1 \alpha$ antibody (Novus Biologicals, CO, USA), LXR- $\beta$ polyclonal antibody (Invitrogen, IL, USA), rabbit anti-Vimentin (Cell Signaling Technology, MA, USA), and isolectin GS-IB4 Alexa Fluor 568 (Life Technologies, OR, USA). Sections were then washed and incubated in fluorescent-labeled secondary antibodies (goat anti-rabbit IgG Alexa Fluor 488, Life Technologies, OR, USA) for $1 \mathrm{~h}$ at room temperature, followed by washing and incubation with 4',6-diamidino-2-phenylindole, dihydrochloride (DAPI) solution (Life Technologies, OR, USA) for $5 \mathrm{~min}$ at room temperature. Finally, sections were washed and mounted with anti-fade mounting medium (Vector Laboratories, CA, USA) for imaging. Image analysis was completed in a masked fashion using four images taken at defined positions and quantified using ImageJ software. 
The analysis was performed in a masked fashion by three separate observers, then averaged. To achieve unbiased results, positive and negative controls were included alongside experimental test and control groups. Fluorescent microscopy was performed by trained masked operators. To address selection bias in immunofluorescence, the entire areas of retinal cross-sections were imaged.

\subsection{Statistics}

All experiments were repeated at least 3 times. All data were assessed using one-way ANOVA. When the results were significant, we determined which means differed from each other using Tukey's multiple-comparisons test. Results are expressed as mean \pm standard error of the mean (SEM). Statistical analysis was performed using GraphPad Prism, with $p<0.05$ considered statistically significant. Only significant comparisons are shown in the figures. All the examiners were blinded to the identities of the samples they were analyzing.

\section{Results}

\subsection{HFD Mice Have Normal Glucose Levels but Are Insulin-Resistant}

We first sought to validate our model by confirming in our cohort that HFD feeding led to similar degrees of body weight gain as reported in the literature [10,35]. Mice on HFD showed increased body weights by 4 weeks of feeding $(p=0.0020)$. This increase was sustained throughout the 12-month observation period (Figure 1A). At 12 months, HFD mice had moderately higher lean mass (difference of $5.580 \pm 1.003 \mathrm{~g}, p<0.0001$ ) and water content (difference of $4.517 \pm 0.876, p=0.0001$ ) but a markedly increased fat mass (difference of $21.15 \pm 2.362, p<0.0001$ ) compared to LFD controls (Figure 1B). Unexpectedly, chronic high-fat feeding did not cause hyperglycemia. Despite feeding mice with a HFD for 12 months, the HbA1c levels were not different between HFD and LFD mice (Figure 1C). At 12 months, there was no difference in fasting blood glucose levels (Figure 1D, basal) and intraperitoneal glucose tolerance test (IP-GTT) did not show any significant differences between LFD and HFD mice (Figure 1D). However, due to the very high levels of insulin in HFD mice $(0.6 \mathrm{ng} / \mathrm{mL}$ for LFD and $3.5 \mathrm{ng} / \mathrm{mL}$ for HFD; Figure 1E, basal), the insulin sensitivity index demonstrated that HFD mice had much lower insulin sensitivity compared to LFD mice (Figure 1E,F). Also, plasma total cholesterol levels were higher in HFD mice compared to LFD (174.4 vs. 114.9, $p=0.0008$ ) (Figure 1G)
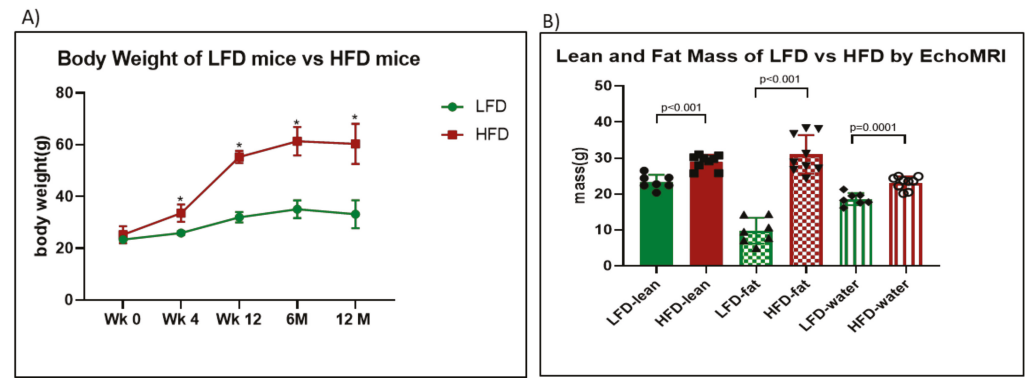

Figure 1. Cont. 

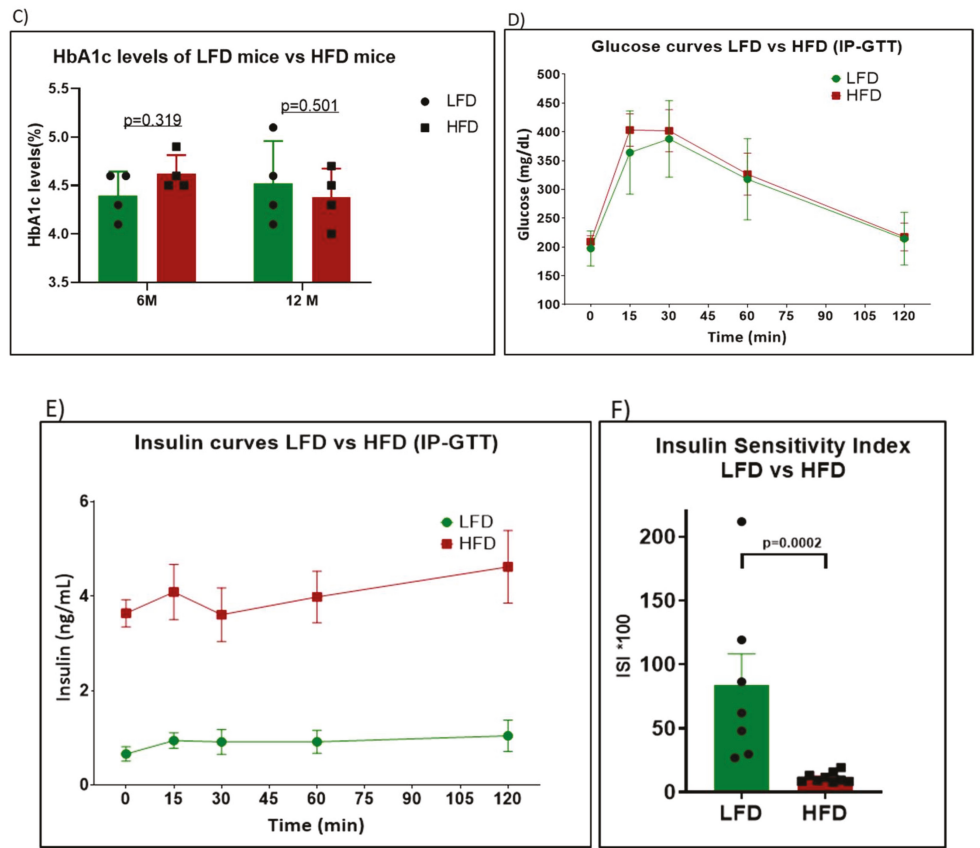

G)

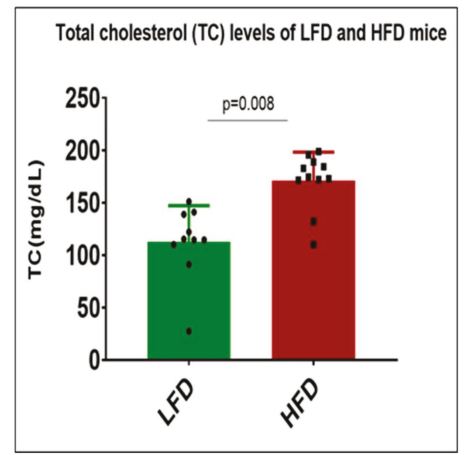

Figure 1. Body weight, glucose levels, and insulin sensitivity of high-fat diet (HFD) mice vs. low-fat diet (LFD) mice. (A) Body weights as measured for mice on LFD (green) and HFD (red) for 12 months. $\left({ }^{*} p<0.000001 ; n=6\right)$. (B) Lean mass, fat mass and water content of LFD mice vs. HFD mice. (C) Glycated hemoglobin $(\mathrm{HbA1c})$ levels measured for the mice after 6 months and 12 months. (D-G) Glucose curves ( $p>0.46$ for all time points), insulin curves ( $p<0.0018$ for all time points), insulin sensitivity index, and total cholesterol levels for LFD mice vs. HFD mice, respectively, following intraperitoneal glucose tolerance test (IP-GTT) after 12 months of feeding.

\subsection{HFD Mice Have Functional Deficits in Their Retinas}

Full-field ERG under both scotopic and photopic conditions was performed at 6 months and 12 months of HFD feeding (Figure 2A-D). HFD mice at 6 months showed significantly reduced aand b-wave amplitudes under scotopic conditions $\left(p=0.00125\right.$ and $p=0.000002$ for $0.25 \mathrm{~cd} . \mathrm{s} / \mathrm{m}^{2}$ and $2.5 \mathrm{~cd} . \mathrm{s} / \mathrm{m}^{2}$ stimulus luminance, respectively) but not photopic conditions when compared to LFD mice. After 12 months of feeding of the respective diets, the difference was not significant $(p=0.183$ and 
$p=0.154$ for $0.25 \mathrm{~cd} . \mathrm{s} / \mathrm{m}^{2}$ and $2.5 \mathrm{~cd} . \mathrm{s} / \mathrm{m}^{2}$ stimulus luminance respectively) (Figure 2C,D). Interestingly, when comparing 6 and 12 months of LFD feeding, the mice experienced marked reductions in both the a- and b- waves under both photopic and scotopic conditions at 12 months (Figure 2E,F), but no significant difference was noted in the HFD-fed mice (Figure 2G,H).
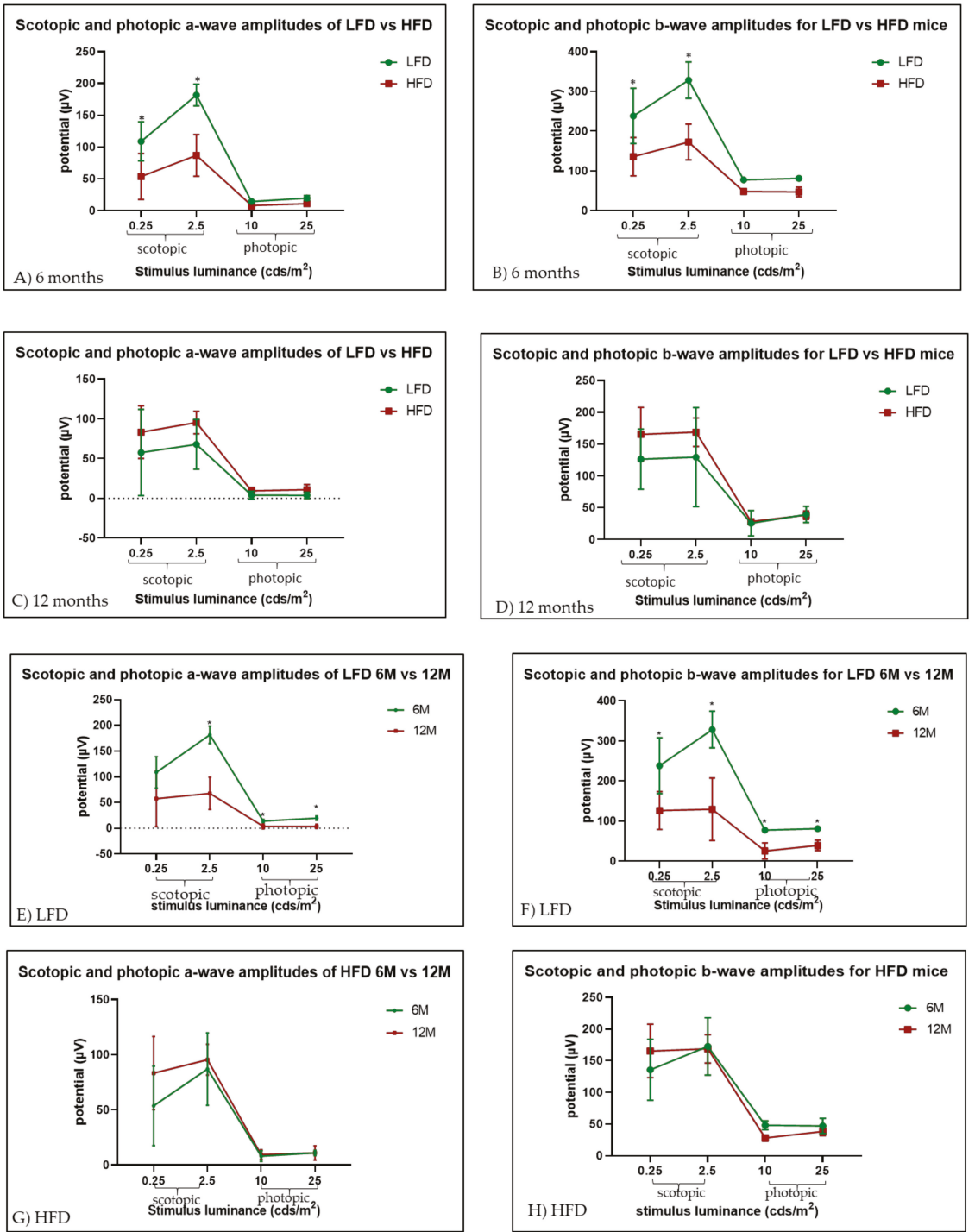

Figure 2. Assessment of retinal function of LFD mice versus HFD mice by electroretinogram (ERG). The amplitudes of a-waves and b-waves were assessed under both scotopic and photopic conditions for LFD mice and HFD mice after 6 months (A,B) and 12 months (C,D). LFD mice showed a significant reduction in retinal response between 6 months and 12 months of feeding (E,F), but HFD mice did $\operatorname{not}(\mathbf{G}, \mathbf{H}) ;(n=4$ for both groups). 


\subsection{Fundus Photography shows Neural Retinal Lesions in HFD Mice}

In humans, DR is associated with retinal lesions such as hemorrhages, microaneurysms, exudates, and "cotton wool spots" [36]. Fundus photography using Micron IV demonstrated retinal pathology in the HFD mice. Though not statistically significant, HFD mice showed a trend of increased numbers of "lipid-laden-like" lesions (Figure 3A) after 6 months ( $p=0.057)$. However, with 12 months of feeding, HFD mice showed significantly higher number of lesions in the retina (Figure 3B).

\section{A) 6 months}
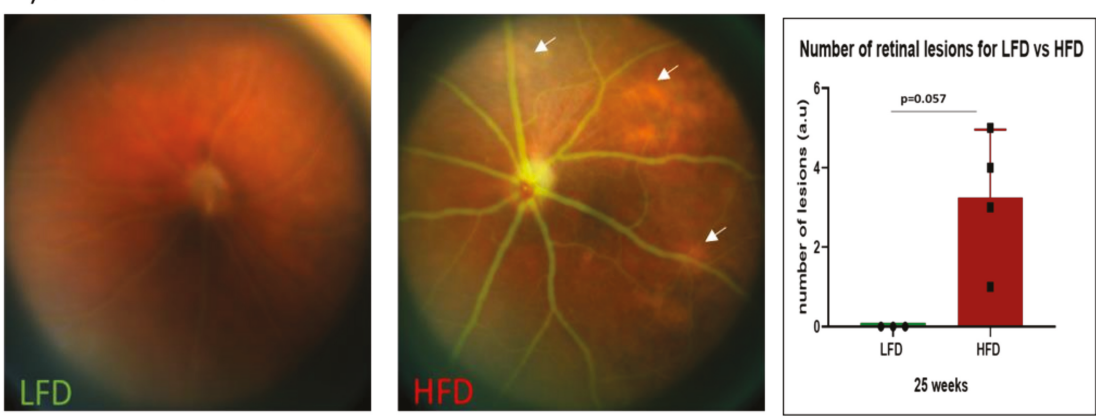

\section{B) 12 months}
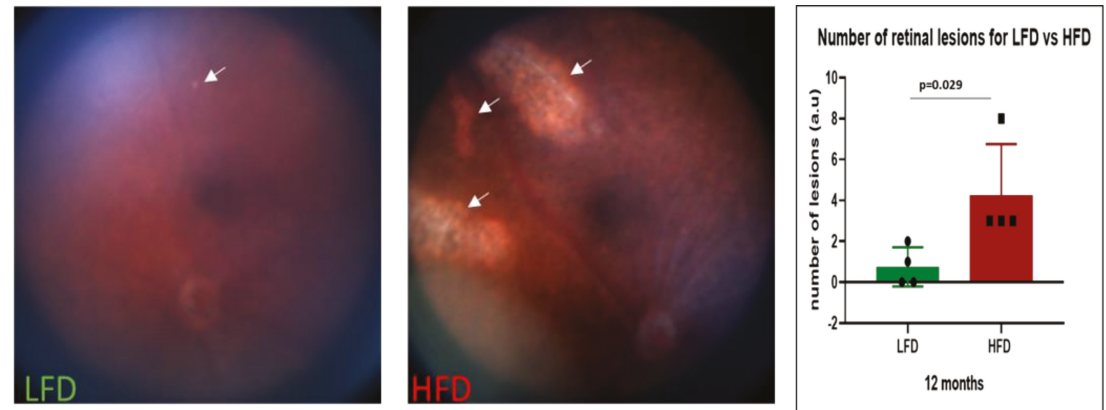

\section{C) 6 months}

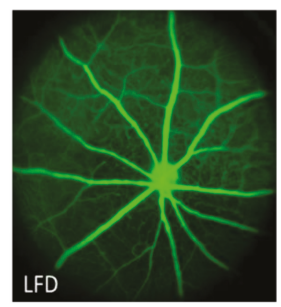

\section{D) 12 months}

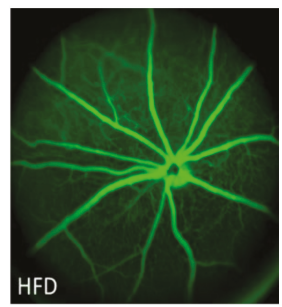

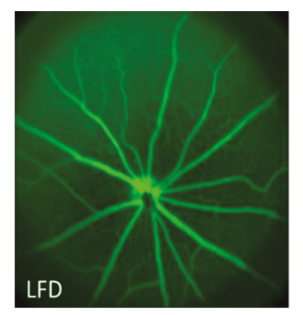

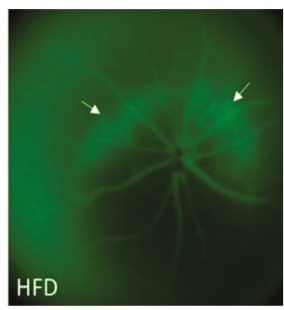

Figure 3. Assessment of retinal lesions by fundus photography $(\mathbf{A}, \mathbf{B})$ and vascular leakage by fluorescein angiography (C,D). HFD mice developed more neural infarcts ((A,B), white arrows) than LFD mice. No infarct was observed for LFD after 6 months (A). However, vascular leakage was observed in HFD mice after 12 months of feeding ((D), white arrows). 


\subsection{Vascular Permeability Changes in HFD Mice}

A hallmark of DR in humans is increased vascular permeability, ultimately leading to diabetic macular edema in humans. To determine if HFD mice developed a breakdown in the blood-retinal barrier, we assessed vascular leakage by fluorescein angiography (FA). At 6 months of HFD feeding, FA did not show any evidence of retinal vascular leakage and were similar to FAs in LFD controls (Figure 3C). However, after 12 months of HFD feeding, increased leakage of fluorescein was observed in the retina compared to LFD control retinas (Figure 3D).

\subsection{Acellular Capillary Formation in HFD Mice}

A well-established feature of diabetic microvascular dysfunction is an increase in the number of acellular capillaries in the retina, defined as basal membrane tubes lacking endothelial cells and pericyte nuclei. At 12 months of HFD feeding, there was no significant increase in acellular capillary numbers in the HFD mice (Figure 4B,C) compared to the LFD mice (Figure 4A,C). However, the HFD retinas showed lower vascular densities compared to LFD retinas (Figure 4D).

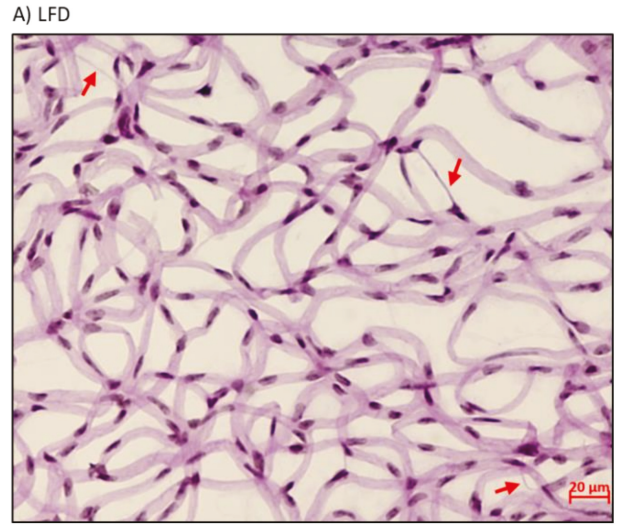

\section{B) HFD}

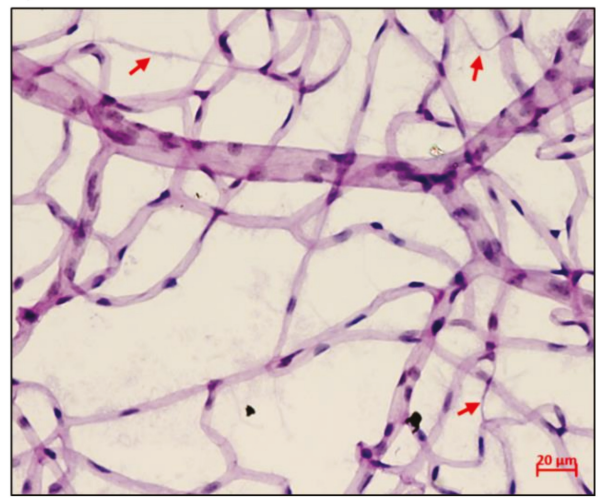

C)

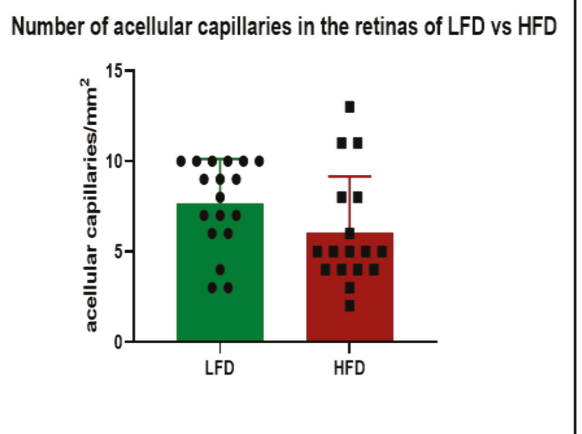

D)

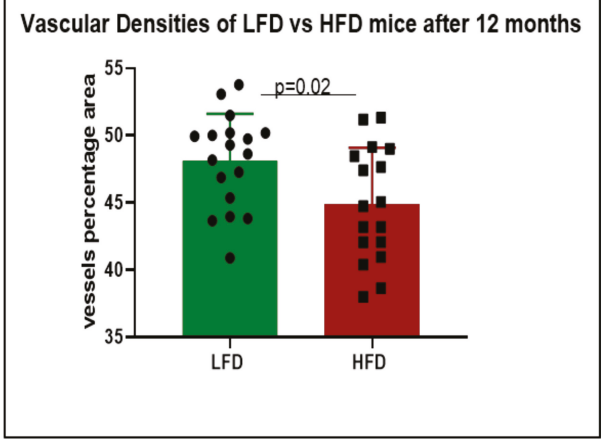

Figure 4. Enumeration of acellular capillaries in LFD and HFD mice after 12 months of feeding. Red arrows indicate acellular capillaries in the retinas of LFD (A) and HFD (B) mice. There was no significant difference in the number of acellular capillaries between both groups $(\mathbf{C})(p=0.086)$. However, HFD retinas showed lesser vascular densities compared to LFD retinas (D). 


\subsection{Retinal Damage, Hypoxia, and Lipid Transport in WD Mice}

While the HFD represents a diet with $60 \%$ fat content that is used as a model of obesity and T2D, the WD with $40 \%$ fat content has garnered popularity as it represents a regimen closer to that actually ingested by humans. Since the WD diet has lower fat content and is not associated with hyperglycemia, we hypothesized that if retinal changes were present they would be subtle compared to those we observed with HFD feeding. To test the validity of our hypothesis, we performed IHC studies and first examined whether there was evidence of glial activation by examining expression of the glial marker GFAP after 6 months of WD feeding. Although there was no statistically significant difference $(p=0.88)$ in the total expression of GFAP between retinas of WD and LFD mice (Figure 5A-C), increased expression of GFAP was observed in selected Vimentin-positive Muller cells in the WD mice (Figure 5G-I) compared to LFD (Figure 5D-F). Increased expression of GFAP in Muller cells is supportive of increased oxidative stress and inflammation in these cells, and suggests that the impact of WD is not experienced uniformly across all Muller cells [37,38].

To assess whether WD feeding induced retinal hypoxia, changes in HIF-1 $\alpha$ expression were examined by IHC. After 6 months of WD feeding, a significant increase $(p=0.025)$ in expression of HIF-1 $\alpha$ was seen in WD mice (Figure 6D) compared to LFD mice (Figure 6C). This was not observed after 3 months of WD feeding (Figure 6A,B). Quantitation of HIF-1 $\alpha$ expression is shown in Figure 6E, demonstrating that WD-fed mice exhibit higher levels than LFD-fed mice. Co-localization with isolectin, a known vascular endothelial cell marker, showed increased expression of HIF- $1 \alpha$ in some endothelial cells in WD mice (I-K) but not in LFD mice (F-H). Higher magnification images from two different WD samples are shown in Figure 6L,M.

Retinal lipid content is regulated in part by liver $X$ receptor beta (LXR $\beta$ ) expression. We next examined changes in LXR $\beta$ expression in the two experimental cohorts. In control mice, LXR $\beta$ localized predominantly in the ganglion cell layer, as well as the inner nuclear layer (Figure 7A), which is the location of the bipolar cells, horizontal cells, and amacrine cells. There was a significant reduction in expression of LXR $\beta$ in WD only in the ganglion cell layer $(p=0.0079)$ after 3 months of feeding (Figure 7B). However, after 6 months of WD feeding, WD mice (Figure 7E) showed significantly reduced expression of LXR $\beta$ in the ganglion cell layer $(p=0.0374)$, inner nuclear layer $(p<0.0001)$, and outer nuclear layer, as well as in the photoreceptors of the outer nuclear layer $(p=0.0020)$. The expression of LXR $\beta$ was reduced after 6 months compared to 3 months of feeding in both LFD $(p<0.0001)$ and WD $(p<0.0001)$ in the nuclear and ganglion cell layers, suggesting an age-related loss in LXR $\beta$.
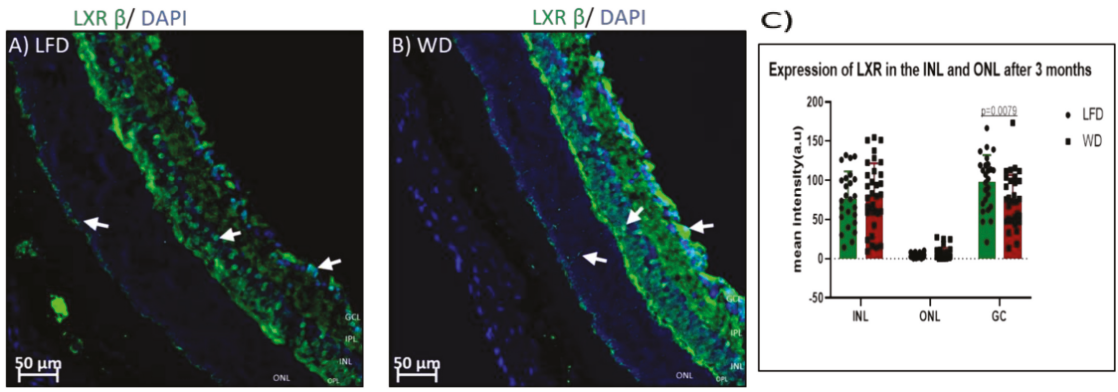

Figure 5. Cont. 

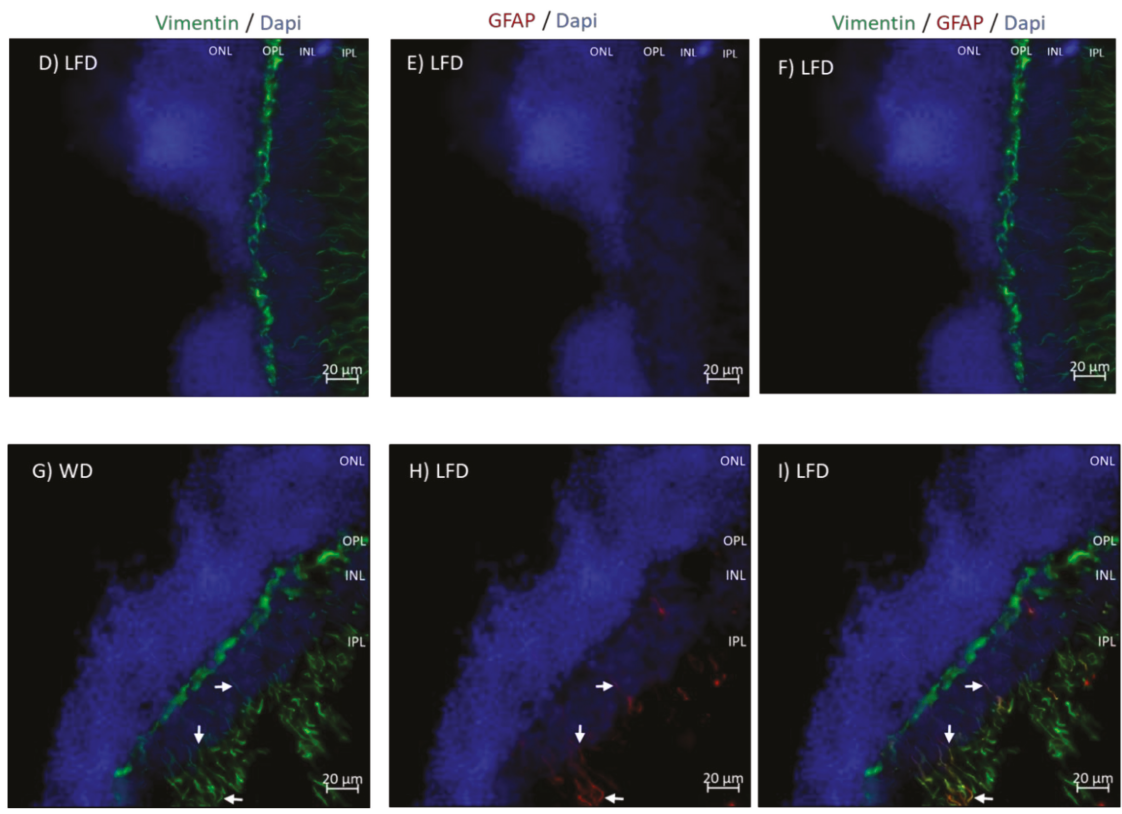

Figure 5. Retinal glial fibrillary acidic protein (GFAP) expression after 6 months of feeding. Some Muller cells in Western diet (WD) retinas express GFAP (A,C, white arrows), but not in LFD (A,B), indicating that the impact of WD is not uniform across all Muller cells. Co-localization with Vimentin, a known Mueller cell marker, showed increased expression of GFAP in some Mueller cells in WD mice (G-I) but not in LFD mice (D-F).

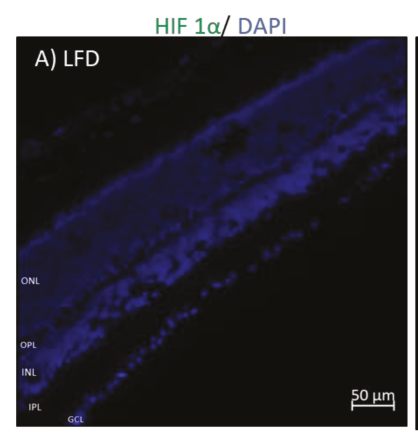

HIF $1 \alpha /$ DAPI

Figure 6. Cont. 

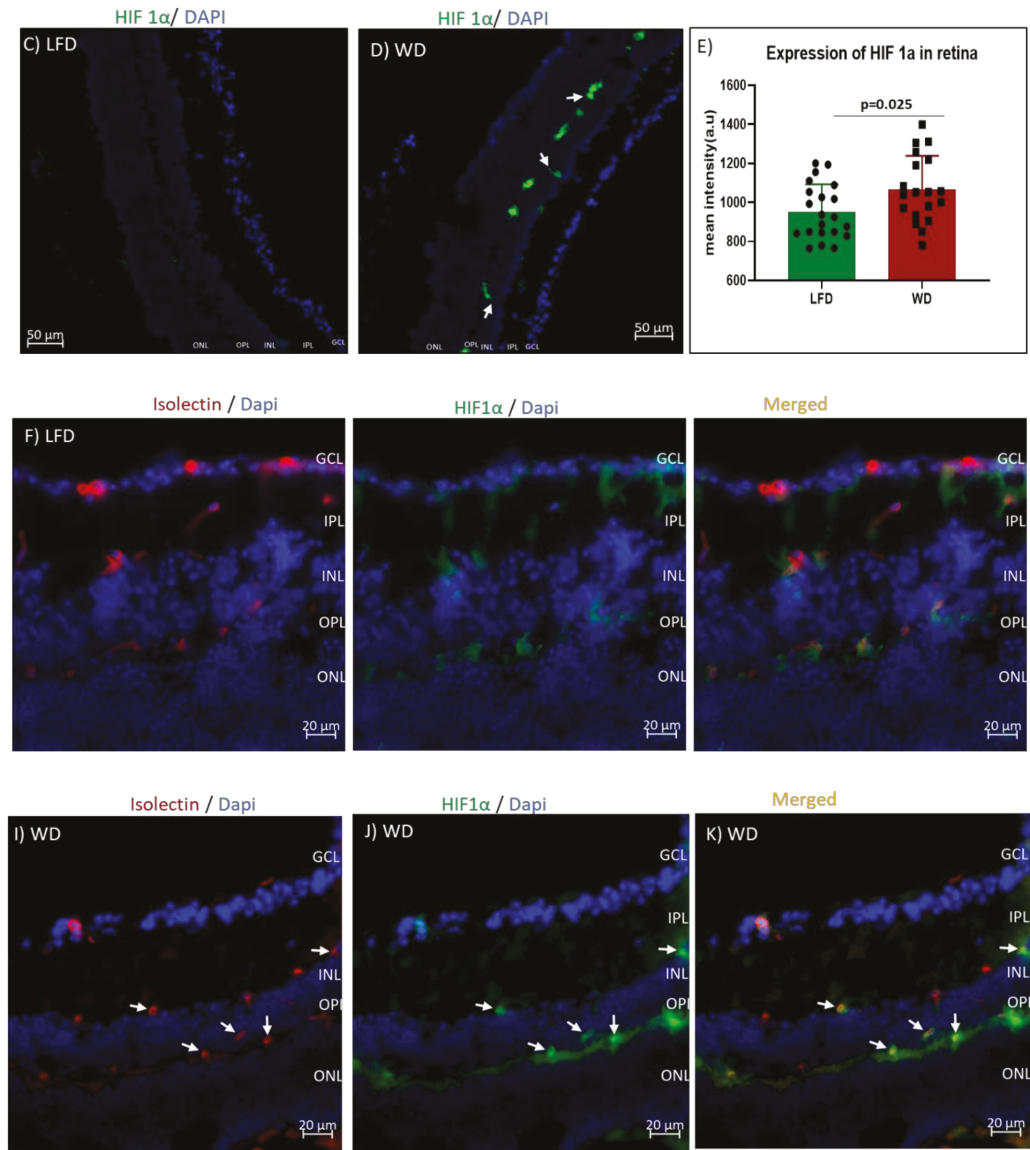

Isolectin / HIF1 $\alpha$ / Dapi

Isolectin / HIF1 $\alpha$ / Dapi
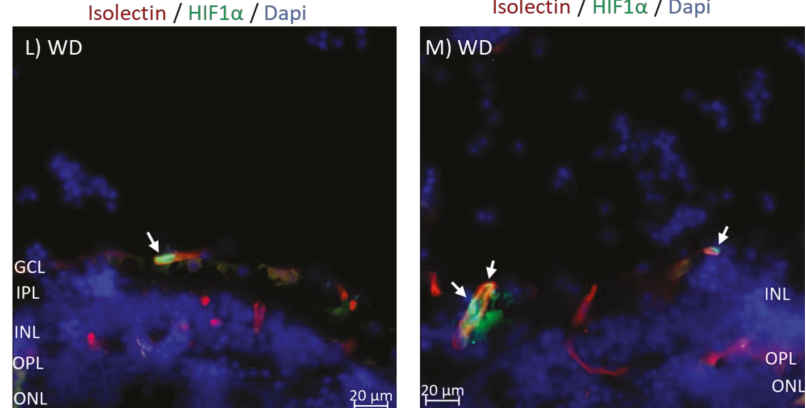

Figure 6. Retinal hypoxia-inducible factor 1 alpha (HIF-1 $\alpha$ ) expression after 3 and 6 months of WD feeding. There was increased expression of HIF- $1 \alpha$ in WD retinas (D, white arrows) compared to LFD retinas $(\mathbf{C})$, as shown by quantification $(\mathrm{E})$. Also, there was no significant difference in expression of HIF- $1 \alpha$ after 3 months of feeding $(\mathbf{A}, \mathbf{B})$. Co-localization with isolectin, a known vascular endothelial cell marker, showed increased expression of HIF-1 $\alpha$ in some endothelial cells in WD mice (I-K) but not in LFD mice (F-H). (L,M) Magnified merged images from two different WD samples. 

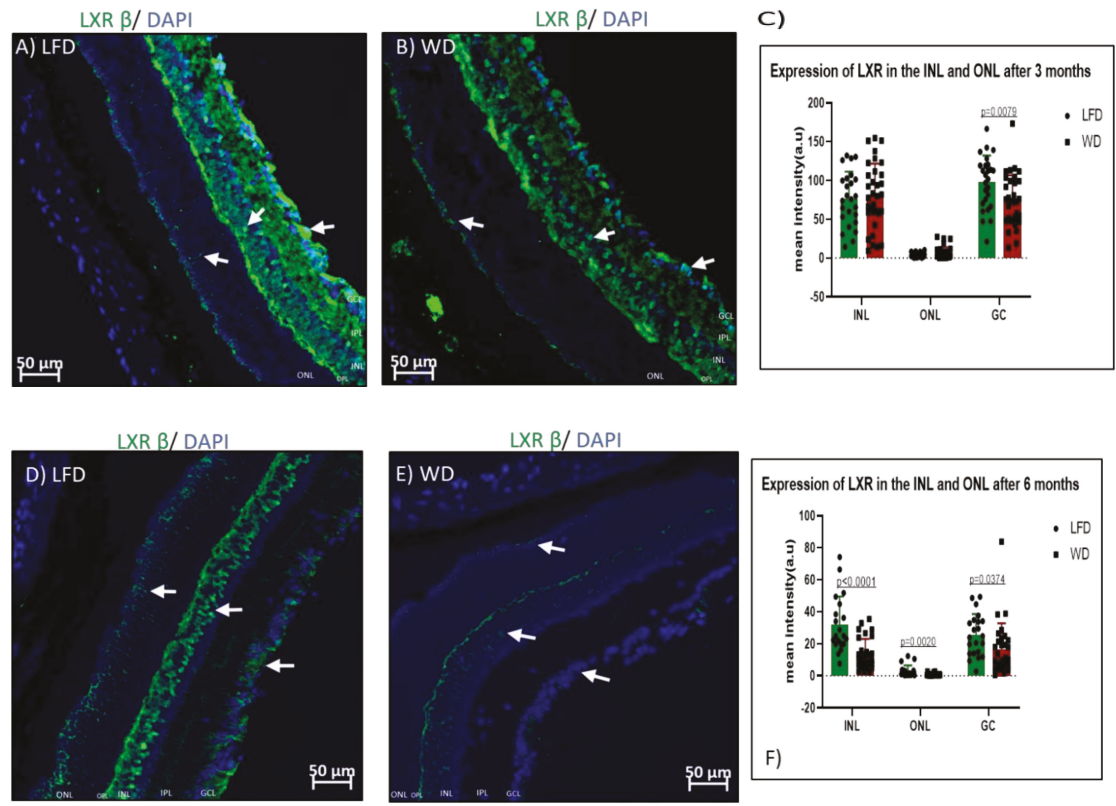

Figure 7. Retinal liver $X$ receptor beta (LXR $\beta$ ) expression after 3 and 6 months of feeding. After 3 months of either WD or LFD feeding, there was significant reduction in the expression of LXR $\beta$ in only the ganglion cell layer of WD mice (B) compared to LFD mice (A). However, after 6 months of feeding, there was reduced expression of LXR $\beta$ in the ganglion cell layer as well as inner and outer nuclear layers of WD mice (E, white arrows) compared to LFD mice (D). Quantification of LXR in the inner nuclear layer (INL) and outer nuclear layer (ONL) at 3 months shows reductions in the ganglion cell (GC) layer (C). At 6 months, reductions are seen in the INL, ONL, and ganglion cell (GC) layer of the WD-fed mice when compared to LFD mice.

\section{Discussion}

Diabetic retinopathy causes both neural and vascular defects, with neural deficits preceding vascular changes [6,39-42]. Even before the onset of clinically detectable retinopathy, diabetic patients have a reduced ERG implicit time [43] and high-frequency flicker amplitude [44]. Later, they experience decreased vascular density [45]. In this study, we have shown that HFD feeding results in a suitable model of prediabetes, with the HFD cohort exhibiting insulin resistance and hypercholesterolemia without hyperglycemia. The retinopathy that is exhibited occurs over a slower time course than in T2D models, where both hyperglycemia and hyperinsulinemia exist.

The HFD mouse has previously been described as a model for T2D [7,46], as C57BL/6J mice fed HFD develop obesity and insulin resistance [47,48], but as we show in this study, this model has a distinct timeline and different characteristics than those seen in T2D. We show that HFD mice have hypercholesterolemia and insulin resistance but the absence of hyperglycemia, which is typical of T2D models.

In agreement with the literature, our study shows that mice fed a HFD have a sustained increase in body weight $[6,49,50]$. As confirmed by EchoMRI, the increase in body weight is primarily due to elevated body fat mass. After 12 weeks of feeding, HFD mice showed a two-fold increase in body fat mass over control LFD mice. Despite the marked increase in fat mass, HFD mice did not develop overt hyperglycemia. Glycated hemoglobin levels measured at 6 months and 12 months showed that both groups had normal glycated hemoglobin, thus indicating a key difference between the HFD model and other T2D rodent models, many of which are genetic. However, HFD mice develop hyperinsulinemia (Figure 1E,F), and their insulin production is sufficient to maintain euglycemia, 
as indicated by their glycated hemoglobin levels. The marked hyperinsulinemia we observed is supported by the literature [6,51-54]. In contrast, T2D in humans is characterized by not only insulin resistance but also the presence of sustained hyperglycemia and elevated $\mathrm{HbA} 1 \mathrm{c}$ levels. When only insulin resistance is present, individuals are described as prediabetics $[55,56]$.

Insulin resistance is believed to play a key role in diabetic neuropathy by increasing oxidative stress and mitochondrial dysfunction [57,58], and may also drive the early neural retinal dysfunction that we observed in our HFD mice. Thus, the HFD mice secrete sufficiently elevated insulin to maintain a normal glucose level, and as such the HFD model may be better characterized as a prediabetes model. Importantly, the incidence of prediabetes is often higher than that of diabetes [59]. The prevalence of prediabetes is also increasing; it is estimated that more than 470 million people worldwide will be suffering from prediabetes by 2030 [60]. Most importantly, the three classical microvascular complications, retinopathy, neuropathy, and nephropathy, have all been documented in individuals with prediabetes [61].

While classifications of diabetes remain "glucose-centric", our study draws attention to the importance of earlier events, when glucose levels are still normal. Thus, in our model, hyperinsulinemia with hypercholesterolemia will likely lead to the retinal pathology observed. Not surprisingly, these pathologies take a longer time to develop than those typically seen when hyperglycemia is also present.

Systemic and retinal lipid abnormalities have been shown to promote retinal damage $[16,62,63]$. Previously, we demonstrated that diabetes-induced disruption of the LXR axis results in abnormal lipid metabolism, inadequate vascular repair, and localized and systemic inflammation [16,64]. The LXRs (LXR $\alpha$ and LXR $\beta$ ) play important roles in cholesterol homeostasis [65]. They regulate the expression of reverse cholesterol transporters [12]. Activation of LXRs using pharmacological agents repress inflammatory genes such as TNF- $\alpha$ and IL-1 $\beta$ [66], inhibit the expression of pro-apoptotic factors [67], and prevent the development of DR [12]. We showed that use of GW3965, an LXR agonist, resulted in normalization of cholesterol homeostasis and repression of inflammatory genes, such as iNOS, IL-1 $\beta$, ICAM-1, and CCL2 in the retina [16]. We found that inadequate cholesterol removal due to deficiency in LXR and reduced oxysterol production in the retina due to loss of cytochromes p450 27A1 and 46A1 resulted in widespread retinal pathology [68]. In the current study, we showed that concentrations of $40 \%$ fat in the diet were sufficient to reduce expression of LXR in the inner and outer nuclear layers.

Our study showed that HFD mice develop neural retinal deficits after 6 months of feeding, as both $\mathrm{a}$-waves and b- waves were reduced under scotopic conditions. Unexpectedly, the a- and b- wave responses for LFD mice was significantly less after 12 months compared to the response after 6 months of feeding ( $p<0.01$ for both scotopic and photopic conditions), which suggests that the LFD may have detrimental effects on the neural retina. Because the composition of the diets must be isocaloric, when the amount of fat is reduced, some other dietary component needs to be increased to compensate. Inn the LFD, the amount of sucrose increases from $72 \mathrm{~g}$ to $354 \mathrm{~g}$ and $315 \mathrm{~g}$ of corn starch is also added so that the LFD can be isocaloric with the HFD. However, this largely occurs at the expense of making the diet high in carbohydrates. The literature supports that LFD may be detrimental [69-71]. While we were unable to find literature supporting the impact of LFD specifically on ERGs, the systemic consequences of LFD may indirectly affect the retina, for example by reduced availability of fat-soluble vitamins or changing retinal cholesterol metabolism. Moreover, the increased sucrose and cornstarch in the LFD may have direct deleterious effects [72,73]. LFDs promote insulin resistance, and while most of the research has been performed in humans, these findings may have relevance to murine studies. LFD, typically considered a high carbohydrate diet, is known to promote inflammation [74-76]. A recent study compared ERGs in HFD fed rats, Streptozotocin (STZ) rats and type 2 diabetes (T2D) rats at 6 months to controls. Kowluru found differences between the diabetic ERGs and controls, but no differences between the ERGs of the HFD rats compared to controls; however, Kowluru did not look at 12 month tests and the study was performed in rats, not in mice [77]. Thus, it is difficult to compare these findings with our results.

While neural damage was detected at 6 months, the vascular damage was not observed until much later. This is in agreement with Rajagopal et al. [6], who demonstrated that vascular damage was not 
observed at 6 months of HFD feeding. However, despite the absence of vascular damage after 6 months of HFD, we observed the presence of "lipid-laden like" lesions, and also neural infarcts similar to what is described in humans as "cotton-wool" spots. These lesions, which appeared to increase as the retinopathy progressed in the HFD mice, could become a useful measure of retinal damage and may be sensitive enough to use as a novel endpoint for the preclinical investigation of therapeutic agents.

GFAP is normally expressed in retinal astrocytes in rodents; however, during stress and inflammation, Muller cells [37] respond by increasing GFAP expression. In this study, we show that WD induces GFAP expression in selective Muller cells, supporting the presence of increased stress and inflammation in the retina of these mice. Kim et al. have reported increased inflammation in other tissues such as adipose tissue and intestines [78]. Lee et al. showed increased numbers of activated macrophages in the retina of HFD mice [79]. In both humans and rodents, obesity-induced diabetes is associated with hypoxia in tissues such adipose tissue, and suppression of HIF- $1 \propto$ mitigates tissue-specific pathological changes associated with HFD [80]. The liver, brain, kidney, and heart display tissue-specific regulation of HIF-1 $\alpha$ under systemic hypoxia [81]. After 6 months, but not after 3 months, we observed that HIF- $1 \alpha$ expression is increased in the WD retinas compared to LFD controls. Similar to our observation in the retina of 3-month-old mice on WD, Prasad et al. showed the absence of pimonidazole staining in the kidneys of 10-11-week old $\mathrm{db} / \mathrm{db}$ mice [82], also indicating the absence of hypoxia response in the kidneys at this time point.

\section{Conclusions}

Our study demonstrates that HFD feeding generates a useful prediabetes model. Specifically, the combination of hypercholesterolemia and insulin resistance are sufficient to induce retinal dysfunction with a slower time course of development compared to T2D models such as the $\mathrm{db} / \mathrm{db}$ mouse. In agreement with reports describing diabetes models, we show that neural functional deficits are the earliest indicator of damage in the retina of this prediabetes model before vascular changes. Key molecular targets such as HIF- $1 \alpha$ and the LXRs provide insights into the retinal pathobiology observed in this hypercholesterolemic, hyperinsulinemic model. The appearance and frequency of neural infarcts or "lipid-laden lesions" in the retina of HFD mice could represent a novel endpoint for evaluation of therapeutic interventions.

Supplementary Materials: The following are available online at http://www.mdpi.com/2073-4409/9/2/464/s1, Table S1: Detailed Composition of high-fat diet (HFD), Western diet (WD), and low-fat diet (LFD).

Author Contributions: Conceptualization, M.L., P.R.N., and M.B.G.; data curation, B.A.-B. and S.K.N.; formal analysis, B.A.-B., S.K.N., B.A.J., and M.B.G.; funding acquisition, M.B.G.; investigation, B.A.-B., S.K.N., S.L.C., B.A., C.P.V., Y.A.-A., M.D., B.A.J., X.X.W., D.C., and M.B.G.; methodology, B.A.-B., S.K.N., P.R.N., and M.B.G.; project administration, P.R.N. and M.B.G.; resources, M.L., P.R.N., and M.B.G.; supervision, M.L. and P.R.N.; validation, B.A.-B. and S.L.C.; visualization, B.A.-B.; writing-original draft, B.A.-B., S.K.N., P.R.N., and M.B.G.; writing-review and editing, B.A.-B., S.K.N., S.L.C., B.A., C.P.V., Y.A.-A., B.A.J., M.L., P.R.N., and M.B.G. All authors have read and agreed to the published version of the manuscript.

Funding: M.G is supported by NIH funding (EY012601, EY028037, and EY028858). P.R.N is supported by NIH funding (HL122505, HL137799); M.L is supported by NIH funding (DK116567). BAJ is supported by the National Center for Advancing Translational Sciences of the NIH under award number TL1TR001431.

Conflicts of Interest: The authors declare no conflict of interest.

\section{References}

1. Cho, N.; Shaw, J.; Karuranga, S.; Huang, Y.; Fernandes, J.D.R.; Ohlrogge, A.; Malanda, B. IDF Diabetes Atlas: Global estimates of diabetes prevalence for 2017 and projections for 2045. Diabetes Res. Clin. Pr. 2018, 138, 271-281. [CrossRef] [PubMed]

2. Chatterjee, S.; Khunti, K.; Davies, M.J. Type 2 diabetes. Lancet 2017, 389, 2239-2251. [CrossRef]

3. Busik, J.V.; Tikhonenko, M.; Bhatwadekar, A.; Opreanu, M.; Yakubova, N.; Caballero, S.; Player, D.; Nakagawa, T.; Afzal, A.; Kielczewski, J.; et al. Diabetic retinopathy is associated with bone marrow neuropathy and a depressed peripheral clock. J. Exp. Med. 2009, 206, 2897-2906. [CrossRef] [PubMed] 
4. Turner, R.C.; Cull, C.A.; Frighi, V.; Holman, R.R.; UK Prospective Diabetes Study (UKPDS) Group. Glycemic Control With Diet Sulfonylurea, Metformin, or Insulin in Patients with Type 2 Diabetes MellitusProgressive Requirement for Multiple Therapies (UKPDS 49). JAMA 1999, 281, 2005. [CrossRef] [PubMed]

5. Yao, Z.; Gu, Y.; Zhang, Q.; Liu, L.; Meng, G.; Wu, H.; Xia, Y.; Bao, X.; Shi, H.; Sun, S. Estimated daily quercetin intake and association with the prevalence of type 2 diabetes mellitus in chinese adults. Eur. J. Nutr. 2019, 58, 819-830. [CrossRef]

6. Rajagopal, R.; Bligard, G.W.; Zhang, S.; Yin, L.; Lukasiewicz, P.; Semenkovich, C.F. Functional Deficits Precede Structural Lesions in Mice With High-Fat Diet-Induced Diabetic Retinopathy. Diabetes 2016, 65, 1072-1084. [CrossRef]

7. Winzell, M.S.; Ahrén, B. The high-fat diet-fed mouse: A model for studying mechanisms and treatment of impaired glucose tolerance and type 2 diabetes. Diabetes 2004, 53, S215-S219. [CrossRef]

8. Liou, C.-J.; Lee, Y.-K.; Ting, N.-C.; Chen, Y.-L.; Shen, S.-C.; Wu, S.-J.; Huang, W.-C. Protective Effects of Licochalcone A Ameliorates Obesity and Non-Alcoholic Fatty Liver Disease Via Promotion of the Sirt-1/AMPK Pathway in Mice Fed a High-Fat Diet. Cells 2019, 8, 447. [CrossRef]

9. Collins, S.; Martin, T.L.; Surwit, R.S.; Robidoux, J. Genetic vulnerability to diet-induced obesity in the C57BL/6J mouse: Physiological and molecular characteristics. Physiol. Behav. 2004, 81, 243-248. [CrossRef]

10. Illesca, P.; Valenzuela, R.; Espinosa, A.; Echeverría, F.; Soto-Alarcon, S.; Ortiz, M.; Videla, L.A. Hydroxytyrosol supplementation ameliorates the metabolic disturbances in white adipose tissue from mice fed a high-fat diet through recovery of transcription factors nrf2, srebp-1c, ppar- $\gamma$ and nf-kb. Biomed. Pharmacother. 2019, 109, 2472-2481. [CrossRef]

11. Declèves, A.-E.; Mathew, A.V.; Armando, A.M.; Han, X.; Dennis, E.A.; Quehenberger, O.; Sharma, K.; Decl\&egraveves, A.-E. AMP-activated protein kinase activation ameliorates eicosanoid dysregulation in high-fat-induced kidney disease in mice. J. Lipid Res. 2019, 60, 937-952. [CrossRef] [PubMed]

12. Hazra, S.; Rasheed, A.; Bhatwadekar, A.; Wang, X.; Shaw, L.C.; Patel, M.; Caballero, S.; Magomedova, L.; Solis, N.; Yan, Y.; et al. Liver X Receptor Modulates Diabetic Retinopathy Outcome in a Mouse Model of Streptozotocin-Induced Diabetes. Diabetes 2012, 61, 3270-3279. [CrossRef]

13. Wang, X.X.; Jiang, T.; Shen, Y.; Caldas, Y.; Miyazaki-Anzai, S.; Santamaria, H.; Urbanek, C.; Solis, N.; Scherzer, P.; Lewis, L.; et al. Diabetic Nephropathy Is Accelerated by Farnesoid X Receptor Deficiency and Inhibited by Farnesoid X Receptor Activation in a Type 1 Diabetes Model. Diabetes 2010, 59, $2916-2927$. [CrossRef] [PubMed]

14. Chen, X.; Yuan, H.; Shi, F.; Zhu, Y. Effect of garden cress in reducing blood glucose, improving blood lipids and reducing oxidative stress in a mouse model of diabetes induced by a high fat diet and streptozotocin. J. Sci. Food Agric. 2019. [CrossRef] [PubMed]

15. Zheng, W.; Mast, N.; Saadane, A.; Pikuleva, I.A. Pathways of cholesterol homeostasis in mouse retina responsive to dietary and pharmacologic treatments. J. Lipid Res. 2015, 56, 81-97. [CrossRef]

16. Hammer, S.S.; Beli, E.; Kady, N.; Wang, Q.; Wood, K.; Lydic, T.A.; Malek, G.; Saban, D.R.; Wang, X.X.; Hazra, S.; et al. The Mechanism of Diabetic Retinopathy Pathogenesis Unifying Key Lipid Regulators, Sirtuin 1 and Liver X Receptor. EBioMedicine 2017, 22, 181-190. [CrossRef]

17. Li, Q.; Zemel, E.; Miller, B.; Perlman, I. Early Retinal Damage in Experimental Diabetes: Electroretinographical and Morphological Observations. Exp. Eye Res. 2002, 74, 615-625. [CrossRef]

18. Krady, J.K.; Basu, A.; Allen, C.M.; Xu, Y.; LaNoue, K.F.; Gardner, T.W.; Levison, S.W. Minocycline reduces proinflammatory cytokine expression, microglial activation, and caspase-3 activation in a rodent model of diabetic retinopathy. Diabetes 2005, 54, 1559-1565. [CrossRef]

19. Dahl, D. The radial glia of Müller in the rat retina and their response to injury. An immunofluorescence study with antibodies to the glial fibrillary acidic (GFA) protein. Exp. Eye Res. 1979, 28, 63-69. [CrossRef]

20. Osborne, N.N.; Block, F.; Sontag, K.-H. Reduction of ocular blood flow results in glial fibrillary acidic protein (GFAP) expression in rat retinal Müller cells. Vis. Neurosci. 1991, 7, 637-639. [CrossRef]

21. Penn, J.S.; Thum, A.L.; Rhem, M.N.; Dell, S.J. Effects of oxygen rearing on the electroretinogram and GFA-protein in the rat. Investig. Ophthalmol. Vis. Sci. 1988, 29, 1623-1630.

22. Tanaka, Y.; Takagi, R.; Ohta, T.; Sasase, T.; Kobayashi, M.; Toyoda, F.; Shimmura, M.; Kinoshita, N.; Takano, H.; Kakehashi, A. Pathological Features of Diabetic Retinopathy in Spontaneously Diabetic Torii Fatty Rats. J. Diabetes Res. 2019, 2019, 8724818. [CrossRef] [PubMed] 
23. Fan, Y.; Lai, J.; Yuan, Y.; Wang, L.; Wang, Q.; Yuan, F. Taurine protects retinal cells and improves synaptic connections in early diabetic rats. Curr. Eye Res. 2020, 45, 52-63. [CrossRef]

24. Bahr, H.I.; Abdelghany, A.A.; Galhom, R.A.; Barakat, B.M.; Arafa, E.-S.A.; Fawzy, M.S. Duloxetine protects against experimental diabetic retinopathy in mice through retinal GFAP downregulation and modulation of neurotrophic factors. Exp. Eye Res. 2019, 186, 107742. [CrossRef]

25. Gu, L.; Xu, H.; Zhang, C.; Yang, Q.; Zhang, L.; Zhang, J. Time-dependent changes in hypoxia-and gliosis-related factors in experimental diabetic retinopathy. Eye 2019, 33, 600. [CrossRef]

26. Chen, J.; Chen, J.; Fu, H.; Li, Y.; Wang, L.; Luo, S.; Lu, H. Hypoxia exacerbates nonalcoholic fatty liver disease via the HIF-2 $\alpha /$ PPAR $\alpha$ pathway. Am. J. Physiol. Metab. 2019, 317, E710-E722. [CrossRef]

27. Han, J.; He, Y.; Zhao, H.; Xu, X. Hypoxia inducible factor-1 promotes liver fibrosis in nonalcoholic fatty liver disease by activating PTEN/p65 signaling pathway. J. Cell. Biochem. 2019, 120, 14735-14744. [CrossRef]

28. Carabelli, J.; Burgueño, A.L.; Rosselli, M.S.; Gianotti, T.F.; Lago, N.R.; Pirola, C.J.; Sookoian, S. High fat diet-induced liver steatosis promotes an increase in liver mitochondrial biogenesis in response to hypoxia. J. Cell. Mol. Med. 2011, 15, 1329-1338. [CrossRef]

29. Beli, E.; Yan, Y.; Moldovan, L.; Vieira, C.P.; Gao, R.; Duan, Y.; Prasad, R.; Bhatwadekar, A.; White, F.A.; Townsend, S.D.; et al. Restructuring of the Gut Microbiome by Intermittent Fasting Prevents Retinopathy and Prolongs Survival in db/db Mice. Diabetes 2018, 67, 1867-1879. [CrossRef]

30. Li, M.; Reynolds, C.M.; Gray, C.; Patel, R.; Sloboda, D.M.; Vickers, M.H. Long-term effects of a maternal high-fat: High-fructose diet on offspring growth and metabolism and impact of maternal taurine supplementation. J. Dev. Orig. Heal. Dis. 2019, 1-8. [CrossRef]

31. Matsuda, M.; DeFronzo, R.A. Insulin sensitivity indices obtained from oral glucose tolerance testing: Comparison with the euglycemic insulin clamp. Diabetes Care 1999, 22, 1462-1470. [CrossRef]

32. Veenstra, A.; Liu, H.; Lee, C.A.; Du, Y.; Tang, J.; Kern, T.S. Diabetic Retinopathy: Retina-Specific Methods for Maintenance of Diabetic Rodents and Evaluation of Vascular Histopathology and Molecular Abnormalities. Curr. Protoc. Mouse Boil. 2015, 5, 247-270. [CrossRef]

33. Bhatwadekar, A.D.; Duan, Y.; Chakravarthy, H.; Korah, M.; Caballero, S.; Busik, J.V.; Grant, M.B. Ataxia telangiectasia mutated dysregulation results in diabetic retinopathy. Stem Cells 2016, 34, 405-417. [CrossRef]

34. Léger, H.; Santana, E.; Beltran, A.W.; Luca, F.C. Preparation of Mouse Retinal Cryo-sections for Immunohistochemistry. J. Vis. Exp. 2019, e59683. [CrossRef]

35. Fan, S.; Zhang, Y.; Hu, N.; Sun, Q.; Ding, X.; Li, G.; Zheng, B.; Gu, M.; Huang, F.; Sun, Y.-Q.; et al. Extract of Kuding Tea Prevents High-Fat Diet-Induced Metabolic Disorders in C57BL/6 Mice via Liver X Receptor (LXR) $\beta$ Antagonism. PLoS ONE 2012, 7, e51007. [CrossRef]

36. Engerman, R.L. Pathogenesis of diabetic retinopathy. Diabetes 1989, 38, 1203-1206. [CrossRef]

37. Kumar, B.; Gupta, S.K.; Nag, T.C.; Srivastava, S.; Saxena, R.; Jha, K.A.; Srinivasan, B.P. Retinal neuroprotective effects of quercetin in streptozotocin-induced diabetic rats. Exp. Eye Res. 2014, 125, 193-202. [CrossRef]

38. Eisenfeld, A.J.; Bunt-Milam, A.H.; Sarthy, P.V. Müller cell expression of glial fibrillary acidic protein after genetic and experimental photoreceptor degeneration in the rat retina. Investig. Ophthalmol. Vis. Sci. 1984, $25,1321-1328$

39. Lieth, E.; Gardner, T.W.; Barber, A.J.; Antonetti, D.A. Retinal neurodegeneration: Early pathology in diabetes. Clin. Exp. Ophthalmol. Viewopoint 2000, 28,3-8. [CrossRef]

40. Barber, A.J. A new view of diabetic retinopathy: A neurodegenerative disease of the eye. Prog. Neuro-Psychopharmacol. Biol. Psychiatry 2003, 27, 283-290. [CrossRef]

41. Stratton, I.M.; Kohner, E.M.; Aldington, S.J.; Turner, R.C.; Holman, R.R.; Manley, S.E.; Matthews, D.R.; UKPDS Group UKPDS 50. Risk factors for incidence and progression of retinopathy in Type II diabetes over 6 years from diagnosis. Diabetologia 2001, 44, 156-163. [CrossRef]

42. Tang, J.; Kern, T.S. Inflammation in diabetic retinopathy. Prog. Retin. Eye Res. 2011, 30, 343-358. [CrossRef]

43. Fortune, B.; Schneck, E.M.; Adams, A.J. Multifocal electroretinogram delays reveal local retinal dysfunction in early diabetic retinopathy. Investig. Ophthalmol. Vis. Sci. 1999, 40, 2638-2651.

44. McAnany, J.J.; Park, J.C.; Chau, F.Y.; Leiderman, Y.I.; Lim, J.I.; Blair, N.P. Amplitude loss of the high-frequency flicker electroretinogram in early diabetic retinopathy. Retin. 2019, 39, 2032-2039. [CrossRef] [PubMed]

45. Zeng, Y.; Cao, D.; Yu, H.; Yang, D.; Zhuang, X.; Hu, Y.; Li, J.; Yang, J.; Wu, Q.; Liu, B.; et al. Early retinal neurovascular impairment in patients with diabetes without clinically detectable retinopathy. Br. J. Ophthalmol. 2019, 103, 1747-1752. [PubMed] 
46. Sone, H.; Kagawa, Y. Pancreatic beta cell senescence contributes to the pathogenesis of type 2 diabetes in high-fat diet-induced diabetic mice. Diabetologia 2005, 48, 58-67. [CrossRef]

47. Surwit, R.S.; Kuhn, C.M.; Cochrane, C.; McCubbin, A.J.; Feinglos, M.N. Diet-induced type II diabetes in C57BL/6J mice. Diabetes 1988, 37, 1163-1167. [CrossRef]

48. Surwit, R.S.; Feinglos, M.N.; Rodin, J.; Sutherland, A.; Petro, E.A.; Opara, E.C.; Kuhn, C.M.; Rebuffé-Scrive, M. Differential effects of fat and sucrose on the development of obesity and diabetes in C57BL/6J and A/J mice. Metabolism 1995, 44, 645-651. [CrossRef]

49. Cani, P.D.; Neyrinck, A.M.; Fava, F.; Knauf, C.; Burcelin, R.G.; Tuohy, K.M.; Gibson, G.R.; Delzenne, N.M. Selective increases of bifidobacteria in gut microflora improve high-fat-diet-induced diabetes in mice through a mechanism associated with endotoxaemia. Diabetologia 2007, 50, 2374-2383. [CrossRef]

50. Lin, S.; Thomas, T.; Storlien, L.; Huang, X. Development of high fat diet-induced obesity and leptin resistance in C57Bl/6J mice. Int. J. Obes. 2000, 24, 639-646. [CrossRef]

51. Membrez, M.; Blancher, F.; Jaquet, M.; Bibiloni, R.; Cani, P.D.; Burcelin, R.G.; Corthesy, I.; Chou, C.J.; Macé, K. Gut microbiota modulation with norfloxacin and ampicillin enhances glucose tolerance in mice. FASEB J. 2008, 22, 2416-2426. [CrossRef] [PubMed]

52. Rabot, S.; Membrez, M.; Bruneau, A.; Gerard, P.; Harach, T.; Moser, M.; Raymond, F.; Mansourian, R.; Chou, C.J. Germ-free C57BL/6J mice are resistant to high-fat-diet-induced insulin resistance and have altered cholesterol metabolism. FASEB J. 2010, 24, 4948-4959. [CrossRef] [PubMed]

53. Uysal, K.T.; Wiesbrock, S.M.; Marino, M.W.; Hotamisligil, G.S. Protection from obesity-induced insulin resistance in mice lacking TNF- $\alpha$ function. Nature 1997, 389, 610-614. [CrossRef] [PubMed]

54. Elchebly, M. Increased Insulin Sensitivity and Obesity Resistance in Mice Lacking the Protein Tyrosine Phosphatase-1B Gene. Sci. 1999, 283, 1544-1548. [CrossRef]

55. Nathan, D.M.; Buse, J.B.; Davidson, M.B.; Ferrannini, E.; Holman, R.R.; Sherwin, R.; Zinman, B. Medical management of hyperglycemia in type 2 diabetes: A consensus algorithm for the initiation and adjustment of therapy: A consensus statement of the American Diabetes Association and the European Association for the Study of Diabetes. Diabetes Care 2009, 32, 193-203. [CrossRef] [PubMed]

56. Kim, K.; Kim, E.S.; Yu, S.-Y. Longitudinal Relationship Between Retinal Diabetic Neurodegeneration and Progression of Diabetic Retinopathy in Patients With Type 2 Diabetes. Am. J. Ophthalmol. 2018, 196, 165-172. [CrossRef]

57. Kim, B.; McLean, L.L.; Philip, S.S.; Feldman, E.L. Hyperinsulinemia induces insulin resistance in dorsal root ganglion neurons. Endocrinology 2011, 152, 3638-3647. [CrossRef]

58. Kim, B.; Feldman, E.L. Insulin resistance in the nervous system. Trends Endocrinol. Metab. 2012, 23, $133-141$. [CrossRef]

59. Das, A.K.; Kalra, S.; Tiwaskar, M.; Bajaj, S.; Seshadri, K.; Chowdhury, S.; Sahay, R.; Indurkar, S.; Unnikrishnan, A.G.; Phadke, U.; et al. Expert Group Consensus Opinion: Role of Anti-inflammatory Agents in the Management of Type-2 Diabetes (T2D). J. Assoc. Physicians India 2019, 67, 65-74.

60. Tabák, A.G.; Herder, C.; Rathmann, W.; Brunner, E.J.; Kivimäki, M. Prediabetes: A high-risk state for diabetes development. Lancet 2012, 379, 2279-2290. [CrossRef]

61. Lamparter, J.; Raum, P.; Pfeiffer, N.; Peto, T.; Höhn, R.; Elflein, H.; Wild, P.; Schulz, A.; Schneider, A.; Mirshahi, A. Prevalence and associations of diabetic retinopathy in a large cohort of prediabetic subjects: The Gutenberg Health Study. J. Diabetes its Complicat. 2014, 28, 482-487. [CrossRef]

62. Tikhonenko, M.; Lydic, T.A.; Opreanu, M.; Calzi, S.L.; Bozack, S.; McSorley, K.M.; Sochacki, A.L.; Faber, M.S.; Hazra, S.; Duclos, S.; et al. N-3 Polyunsaturated Fatty Acids Prevent Diabetic Retinopathy by Inhibition of Retinal Vascular Damage and Enhanced Endothelial Progenitor Cell Reparative Function. PLoS ONE 2013, 8, e55177. [CrossRef] [PubMed]

63. Busik, J.V.; Esselman, W.J.; Reid, E.G. Examining the role of lipid mediators in diabetic retinopathy. Clin. Lipidol. 2012, 7, 661-675. [CrossRef] [PubMed]

64. Hammer, S.S.; Busik, J.V. The role of dyslipidemia in diabetic retinopathy. Vis. Res. 2017, 139, $228-236$. [CrossRef] [PubMed]

65. Calkin, A.C.; Tontonoz, P. Liver x receptor signaling pathways and atherosclerosis. Arter. Thromb. Vasc. Boil. 2010, 30, 1513-1518. [CrossRef] [PubMed]

66. Zelcer, N.; Tontonoz, P. Liver $X$ receptors as integrators of metabolic and inflammatory signaling. J. Clin. Investig. 2006, 116, 607-614. [CrossRef] 
67. Steffensen, K.R.; Jakobsson, T.; Gustafsson, J.-Å. Targeting liver X receptors in inflammation. Expert Opin. Ther. Targets 2013, 17, 977-990. [CrossRef]

68. Saadane, A.; Mast, N.; Trichonas, G.; Chakraborty, D.; Hammer, S.; Busik, J.V.; Grant, M.B.; Pikuleva, I.A. Retinal Vascular Abnormalities and Microglia Activation in Mice with Deficiency in Cytochrome P450 46A1-Mediated Cholesterol Removal. Am. J. Pathol. 2019, 189, 405-425. [CrossRef]

69. Sacks, F.M.; Lichtenstein, A.H.; Wu, J.H.; Appel, L.J.; Creager, M.A.; Kris-Etherton, P.M.; Miller, M.; Rimm, E.B.; Rudel, L.L.; Robinson, J.G.; et al. Dietary Fats and Cardiovascular Disease: A Presidential Advisory from the American Heart Association. Circulation 2017, 136, e1-e23. [CrossRef]

70. Andraski, A.B.; Singh, S.A.; Lee, L.H.; Higashi, H.; Smith, N.; Zhang, B.; Aikawa, M.; Sacks, F.M. Effects of Replacing Dietary Monounsaturated Fat With Carbohydrate on HDL (High-Density Lipoprotein) Protein Metabolism and Proteome Composition in Humans. Arter. Thromb. Vasc. Boil. 2019, 39, 2411-2430. [CrossRef]

71. Bolla, A.M.; Caretto, A.; Laurenzi, A.; Scavini, M.; Piemonti, L. Low-Carb and Ketogenic Diets in Type 1 and Type 2 Diabetes. Nutrients 2019, 11, 962. [CrossRef] [PubMed]

72. Gomes, J.A.; Silva, J.F.; Silva, G.C.; Gomes, G.F.; de Oliveira, A.C.; Soares, V.L.; Oliveira, M.C.; Ferreira, A.V.; Aguiar, D.C. High-refined carbohydrate diet consumption induces neuroinflammation and anxiety-like behavior in mice. J. Nutr. Biochem. 2019, 77, 108317. [CrossRef] [PubMed]

73. Tobias, D.K.; Chen, M.; Manson, J.E.; Ludwig, D.S.; Willett, W.; Hu, F.B. Effect of low-fat diet interventions versus other diet interventions on long-term weight change in adults: A systematic review and meta-analysis. Lancet Diabetes Endocrinol. 2015, 3, 968-979. [CrossRef]

74. Vega-López, S.; Venn, B.J.; Slavin, J.L. Relevance of the Glycemic Index and Glycemic Load for Body Weight, Diabetes, and Cardiovascular Disease. Nutrients 2018, 10, 1361. [CrossRef] [PubMed]

75. Livesey, G.; Taylor, R.; Livesey, H.F.; Buyken, A.E.; Jenkins, D.J.A.; Augustin, L.S.A.; Sievenpiper, J.L.; Barclay, A.W.; Liu, S.; Wolever, T.M.S.; et al. Dietary Glycemic Index and Load and the Risk of Type 2 Diabetes: Assessment of Causal Relations. Nutrients 2019, 11, 1436. [CrossRef]

76. Myette-Côté, É.; Durrer, C.; Neudorf, H.; Bammert, T.D.; Botezelli, J.D.; Johnson, J.D.; DeSouza, C.A.; Little, J.P. The effect of a short-term low-carbohydrate, high-fat diet with or without postmeal walks on glycemic control and inflammation in type 2 diabetes: A randomized trial. Am. J. Physiol. Integr. Comp. Physiol. 2018, 315, R1210-R1219.

77. Kowluru, R.A. Retinopathy in a Diet-Induced Type 2 Diabetic Rat Model, and Role of Epigenetic Modifications. Diabetes 2020, db191009. [CrossRef]

78. Kim, K.-A.; Gu, W.; Lee, I.-A.; Joh, E.-H.; Kim, N.-H. High Fat Diet-Induced Gut Microbiota Exacerbates Inflammation and Obesity in Mice via the TLR4 Signaling Pathway. PLoS ONE 2012, 7, e47713. [CrossRef]

79. Lee, J.-J.; Wang, P.-W.; Yang, I.-H.; Huang, H.-M.; Chang, C.-S.; Wu, C.-L.; Chuang, J.-H. High-Fat Diet Induces Toll-Like Receptor 4-Dependent Macrophage/Microglial Cell Activation and Retinal Impairment. Investig. Opthalmol. Vis. Sci. 2015, 56, 3041. [CrossRef]

80. Krishnan, J.; Danzer, C.; Simka, T.; Ukropec, J.; Walter, K.M.; Kumpf, S.; Mirtschink, P.; Ukropcova, B.; Gasperikova, D.; Pedrazzini, T. Dietary obesity-associated hif1 $\alpha$ activation in adipocytes restricts fatty acid oxidation and energy expenditure via suppression of the sirt2-nad+ system. Genes Dev. 2012, 26, 259-270. [CrossRef]

81. Stroka, D.M.; Burkhardt, T.; Desbaillets, I.; Wenger, R.H.; Neil, D.A.; Bauer, C.; Gassmann, M.; Candinas, D. Hif-1 is expressed in normoxic tissue and displays an organ-specific regulation under systemic hypoxia. FASEB J. 2001, 15, 2445-2453. [CrossRef] [PubMed]

82. Prasad, P.; Li, L.-P.; Halter, S.; Cabray, J.; Ye, M.; Batlle, D. Evaluation of renal hypoxia in diabetic mice by BOLD MRI. Investig. Radiol. 2010, 45, 819-822. [CrossRef] [PubMed] 


\title{
Lack of Overt Retinal Degeneration in a K42E Dhdds Knock-In Mouse Model of RP59
}

\author{
Sriganesh Ramachandra Rao ${ }^{1,2, \dagger}$, Steven J. Fliesler ${ }^{1,2, \dagger}$, Pravallika Kotla ${ }^{3}$, Mai N. Nguyen ${ }^{3}$ and \\ Steven J. Pittler ${ }^{3, *}$ \\ 1 Research Service, VA Western NY Healthcare System, Buffalo, NY 14215, USA; \\ sramacha@buffalo.edu (S.R.R.); fliesler@buffalo.edu (S.J.F.) \\ 2 Departments of Ophthalmology and Biochemistry and Neuroscience Graduate Program, \\ The State University of New York- University at Buffalo, Buffalo, NY 14209, USA \\ 3 Department of Optometry and Vision Science, Vision Science Research Center, University of Alabama at \\ Birmingham, School of Optometry, Birmingham, AL 35294, USA; pkotla@uab.edu (P.K.); \\ mnnguyen@uab.edu (M.N.N.) \\ * Correspondence: pittler@uab.edu; Tel.: +1-205-934-6744 \\ + These authors contributed equally to this work.
}

Received: 17 February 2020; Accepted: 4 April 2020; Published: 7 April 2020

\begin{abstract}
Dehydrodolichyl diphosphate synthase (DHDDS) is required for protein $N$-glycosylation in eukaryotic cells. A K42E point mutation in the DHDDS gene causes an autosomal recessive form of retinitis pigmentosa (RP59), which has been classified as a congenital disease of glycosylation (CDG). We generated K42E Dhdds knock-in mice as a potential model for RP59. Mice heterozygous for the Dhdds K42E mutation were generated using CRISPR/Cas9 technology and crossed to generate Dhdds ${ }^{\mathrm{K} 42 \mathrm{E} / \mathrm{K} 42 \mathrm{E}}$ homozygous mice. Spectral domain-optical coherence tomography (SD-OCT) was performed to assess retinal structure, relative to age-matched wild type (WT) controls. Immunohistochemistry against glial fibrillary acidic protein (GFAP) and opsin (1D4 epitope) was performed on retinal frozen sections to monitor gliosis and opsin localization, respectively, while lectin cytochemistry, plus and minus PNGase-F treatment, was performed to assess protein glycosylation status. Retinas of $D h d d_{s}{ }^{\mathrm{K} 42 \mathrm{E} / \mathrm{K} 42 \mathrm{E}}$ mice exhibited grossly normal histological organization from 1 to 12 months of age. Anti-GFAP immunoreactivity was markedly increased in Dhdds ${ }^{\mathrm{K} 42 \mathrm{E} / \mathrm{K} 42 \mathrm{E}}$ mice, relative to controls. However, opsin immunolocalization, ConA labeling and PNGase-F sensitivity were comparable in mutant and control retinas. Hence, retinas of $D h d d s^{\mathrm{K} 42 \mathrm{E} / \mathrm{K} 42 \mathrm{E}}$ mice exhibited no overt signs of degeneration, yet were markedly gliotic, but without evidence of compromised protein $\mathrm{N}$-glycosylation. These results challenge the notion of RP59 as a DHDDS loss-of-function CDG and highlight the need to investigate unexplored RP59 disease mechanisms.
\end{abstract}

Keywords: retinitis pigmentosa; knock-in mouse model; congenital disorder of glycosylation; retina

\section{Introduction}

Retinitis pigmentosa (RP) represents a group of hereditary retinal degenerative disorders of diverse genetic origins that have as their common trait the progressive, irreversible dysfunction, degeneration, and demise of retinal photoreceptor cells, with rods initially undergoing these pathological changes followed eventually by cones [1,2]. Relatively recently, a K42E point mutation in the dehydrodolichyl diphosphate synthase (DHDDS) gene was shown to cause a rare, recessive form of RP (RP59; OMIM \#613861) [3-5]. DHDDS catalyzes cis-prenyl chain elongation in the synthesis of dolichyl diphosphate (Dol-PP), which is required for protein $N$-glycosylation [6,7]. DHDDS catalyzes the condensation of multiple units of isopentenyl pyrophosphate (IPP, also called isopentenyl diphosphate) to farnesyl pyrophosphate (FPP, also called farnesyl diphosphate) to produce Dol-PP [8,9]. This is used 
as the "lipid carrier" onto which oligosaccharide chains are built that are ultimately transferred to specific asparagine $(N)$ residues on nascent polypeptide chains in the lumen of the endoplasmic reticulum (ER) to form $N$-linked glycoproteins [10]. The monophosphate (Dol-P) is used as a sugar carrier, transferring sugars from their corresponding sugar-nucleotide adducts (e.g., UDP-glucose, GDP-mannose, etc.) to the growing Dol-PP-linked oligosaccharide chains in the ER. Mutations in rhodopsin that block its glycosylation have been shown to cause retinal degeneration in vertebrate animals [11,12]. In addition, pharmacological inhibition of protein $N$-glycosylation with tunicamycin has been shown to disrupt retinal photoreceptor outer segment (OS) disc membrane morphogenesis in vitro [13], as well as to cause retinal degeneration with progressive shortening and loss of photoreceptor OSs in vivo [14].

In the present study, we created a DHDDS K42E homozygous knock-in mouse model (hereafter called $D h d d s^{\mathrm{K} 42 \mathrm{E} / \mathrm{K} 42 \mathrm{E}}$ ) of RP59-since K42E is the most prevalent point mutation in the RP59 patient population [3-5] - to study its underlying pathological mechanism, with the working hypothesis that defective protein $\mathrm{N}$-glycosylation underlies the retinal dysfunction and degeneration observed in human RP59. Herein, we present a description of the generation and initial characterization of the phenotypic features of the $D h d d s^{\mathrm{K} 42 \mathrm{E} / \mathrm{K} 42 \mathrm{E}}$ mouse model. Surprisingly, although we expected to observe an early onset, progressive, and potentially severe retinal degeneration, this was not the case. The retina appeared histologically intact and normal according to spectral domain optical coherence tomography (SD-OCT) analysis for up to at least one year of age. However, there was evidence of gliotic reactivity (glial fibrillary acidic protein (GFAP) immunostaining), despite the lack of obvious neuronal degeneration or cell death/loss. Also, despite the homozygous mutation in Dhdds, we found no evidence of compromised protein $\mathrm{N}$-glycosylation in mutant mouse retinas.

\section{Materials and Methods}

\subsection{Animals}

Heterozygous (K42E/+) Dhdds knock-in (KI) mice were generated on a C57Bl/6J background by Applied StemCell (Milipitas, CA, USA). Briefly, CRISPR guide RNA (5'-TCGCTATGCCAAGAAGTGTC-3' with PAM site AGG) was generated using in vitro transcription and was used to create a double strand break in the murine Dhdds locus to promote introduction of a single-stranded oligodeoxynucleotide (SSO) carrying the K42E mutation and a second silent DNA polymorphism to eliminate the PAM recognition site required for cleavage by CAS9 (5'-ATTATCTGTTCTCTTCTACAGGCTGGCCCAGTACCCAAACATATCGCGTTCATAATGGACGGC AACCGTCGCTATGCCAAGGAGTGTCAAGTGGAGCGCCAGGAGGGCCACACACAGGGCTTCA ATAAGCTTGCTGAGGTGGGTGCGGGTGACAGAGCCTAGA-3'). Mouse zygotes were injected with 100, 100, and $250 \mathrm{ng} / \mu \mathrm{L}$ of Cas9 enzyme, guide RNA, and SSO, respectively, which were then transferred into pseudo pregnant CD-1 females. Three potential founder (F0) pups were identified out of 13 mice tested, and an F0 founder was verified by DNA sequence analysis. Sequence-validated heterozygous (Dhdds ${ }^{\mathrm{K} 42 \mathrm{E} /+}$ ) mice were crossed to generate homozygous (Dhdds ${ }^{\mathrm{K} 42 \mathrm{E} / \mathrm{K} 42 \mathrm{E}}$ ) mice, as confirmed by PCR and DNA sequencing (see below). C57Bl/6J wild type (WT) mice, age- and sex-matched, were used as controls. All procedures conformed to the ARVO Statement for the Use of Animals in Ophthalmic and Vision Research, and were approved by the Institutional Animal Care and Use Committee (IACUC) of the University of Alabama at Birmingham. All animals were maintained on a standard 12/12 h light/dark cycle (20-40 lux ambient room illumination), fed standard rodent chow, provided water ad libitum, and housed in plastic cages with standard rodent bedding.

\subsection{PCR Genotyping and DNA Analysis}

PCR primers were designed that spanned the targeted region (forward primer, 5'-TCTAGGCTCTGTCACCCGCA-3' and reverse primer 5'-TCTAGGCTCTGTCACCCGCA- ${ }^{\prime}$ ) amplifying a $292 \mathrm{bp}$ segment of DNA in both WT and $D h d d_{s}{ }^{\mathrm{K} 42 \mathrm{E} / \mathrm{K} 42 \mathrm{E}}$ mice. For initial verification of the knock-in, PCR products were sequenced in the UAB Heflin Center for Genomic Sciences. The presence 
of the knock-in sequence was confirmed in subsequent generations by restriction enzyme digestion with StyI, which cleaves the knock-in allele only (data not shown). Knock-in alleles were independently verified by Transnetyx, Inc. (Cordova, TN, USA) using proprietary technology. While the analysis was set up to recognize and differentiate the knock-in mutation and the PAM site polymorphism, only the knock-in mutation was maintained in all subsequent breeding.

\subsection{Spectral Domain Optical Coherence Tomography (SD-OCT)}

In vivo retinal imaging was performed as previously described in detail by DeRamus et al. [15], using a Bioptigen Model 840 Envisu Class-R high-resolution SD-OCT instrument (Bioptigen/Leica, Inc.; Durham, NC, USA). Data were collected from Dhdds ${ }^{\mathrm{K} 42 \mathrm{E} / \mathrm{K} 42 \mathrm{E}}$ and WT mice at postnatal day (PN) 1 (KI, $\mathrm{n}=5 ; \mathrm{WT}, \mathrm{n}=9), 2(\mathrm{KI}, \mathrm{n}=4 ; \mathrm{WT}, \mathrm{n}=8), 3(\mathrm{KI}, \mathrm{n}=5$; WT $\mathrm{n}=3), 8(\mathrm{KI}, \mathrm{n}=4$; WT $\mathrm{n}=5)$, and 12 months (mos) $(\mathrm{KI}, \mathrm{n}=3$; WT $\mathrm{n}=3$ ) to assess retinal structure. Layer thicknesses were determined manually using Bioptigen InVivoVue ${ }^{\circledR}$ and Bioptigen Diver ${ }^{\circledR}$ V. 3.4.4 software and the data were analyzed and graphed using Microsoft Excel software.

\subsection{Immunohistochemistry (IHC)}

Procedures utilized for fixation, O.C.T. embedment, and sectioning of mouse eyes were as described in detail previously by Ramachandra Rao et al. [16]. In brief, eyes were immersion fixed overnight in phosphate-buffered saline (PBS) containing freshly prepared paraformaldehyde $(4 \% \mathrm{v} / \mathrm{v})$, appropriately cryopreserved, embedded in O.C.T., and cryosectioning was performed on a Leica Model CM3050 S Cryostat (Leica Biosystems, Wetzlar, Germany). Retinal sections were first "blocked" with $0.1 \%$ BSA, $0.5 \%$ serum (species corresponding to secondary antibody host) in Tris-buffered saline containing $0.1 \%$ Tween-20 (TBST), then incubated for $1 \mathrm{~h}$ at room temperature with a rabbit polyclonal antibody against glial fibrillary acidic protein (GFAP; DAKO/Agilent, Santa Clara, CA, USA; 1:500 dilution in TBST) and a mouse monoclonal antibody against the C-terminal epitope of opsin (1D4; Novus Biologicals, Littleton, CO, USA; 1:500 dilution in TBST), followed by incubation with fluor-conjugated secondary antibodies (AlexaFluor ${ }^{\circledR}-488$ conjugated anti-mouse IgG, AlexaFluor ${ }^{\circledR}-568$ conjugated anti-rabbit IgG; Thermo Fisher Scientific, Waltham, MA, USA; 1:500 dilution in TBST). Sections were then counterstained with DAPI and cover slipped with anti-fade mounting medium (Vectashield ${ }^{\circledR}$; Vector Laboratories, Burlingame, CA, USA) and viewed with a Leica TCS SPEII DMI4000 scanning laser confocal microscope (Leica Biosystems). Images were captured using a 40X oil immersion (RI 1.518 ) objective under normal laser intensity ( $10 \%$ of laser power source), arbitrary gain ( $850 \mathrm{~V})$ and offset (-0.5) values, to optimize signal-to-noise ratio. Digital images were captured and stored as TIFF files on a PC computer.

\subsection{Lectin Cytochemistry}

Paraformaldehyde-fixed eyes (as described above) were processed for paraffin embedment. Paraffin sections of mouse eyes were then incubated (45 $\mathrm{min}$ at room temperature) with biotinylated Concanavalin-A (ConA, B-1005; Vector Laboratories; 1:200 dilution in PBS), followed by incubation with AlexaFluor ${ }^{\circledR}-488$ conjugated streptavidin (Thermo Fisher Scientific; 1:500 dilution in PBS) and AlexaFluor ${ }^{\circledR}$-647-conjugated peanut agglutinin (PNA, L32460; Thermo Fisher Scientific; 1:250 dilution in PBS), with or without pre-treatment $\left(37^{\circ} \mathrm{C}\right.$, overnight) with peptide:N-glycosidase $\mathrm{F}$ (PNGase-F, 200 U, P0704S; New England Biolabs, Inc., Ipswich, MA, USA). Sections were DAPI-stained and mounted using Vectashield mounting media, and digital images obtained using scanning laser confocal microscopy as described above [16]. 


\section{Results}

\subsection{Generation and Validation of K42E DHDDS Knock-In Mutation}

K42E knock-in mice were generated commercially using CRISPR-Cas9 technology. The K42E knock-in mutations in both heterozygous and homozygous mice were confirmed by DNA sequence for one of the heterozygous F0 founder mice, which is shown in Figure 1. Both the A-to-G and G-to-A transitions that lead to the K42E mutation and the Q44Q silent polymorphism, respectively, are heterozygous (arrows). Intra-litter mating was done to establish at least fourth generation homozygous mice that were used for all subsequent analyses. Heterozygous mice were initially characterized by SD-OCT and histology and found not to differ from WT (not shown).

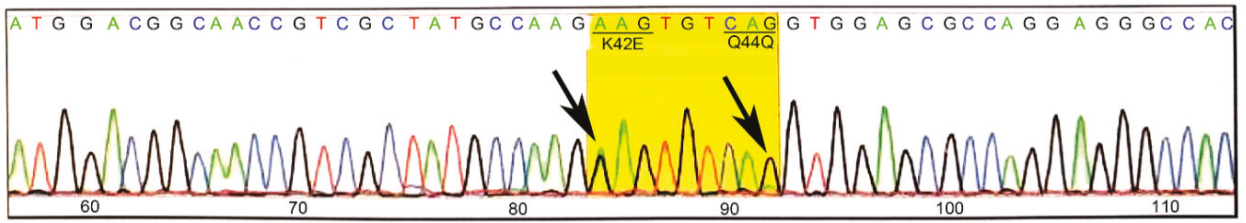

Figure 1. DNA sequence analysis of a tail DNA from a K42E/+ founder mouse. Tail DNA was amplified with primers that cover a $292 \mathrm{bp}$ segment spanning the target region. The sequence analysis confirmed the presence (arrows) of the K42E (A-to-G) mutation and the Q44Q (G-to-A) polymorphism that was included to eliminate the CRISPR-related PAM site.

\subsection{SD-OCT Analysis Reveals No Evidence for Retinal Degeneration in Dhdds ${ }^{K 42 E / K 42 E}$ Mice}

SD-OCT provides a non-invasive means of assessing retinal morphology in vivo. Qualitative SD-OCT images obtained from wild type (WT) and Dhdds ${ }^{\mathrm{K} 42 \mathrm{E} / \mathrm{K} 42 \mathrm{E}}$ mice are presented in Figure 2. From these images, it is clear that the gross morphology of the retina in the homozygous knock-in animals, from PN 1 to 12 months of age, are comparable to that observed in fully mature, age-matched WT control mice. All retinal histological layers were intact and of normal appearance. Hence, there was no evidence of retinal degeneration, even up to one year of age.

We used SD-OCT to perform quantitative analysis of retinal morphology to compare ocular tissue layer thicknesses in WT and knock-in mice. Figure 3 compares data obtained at PN 1, 2, 3, 8, and 12 mos for Dhdds ${ }^{\mathrm{K} 42 \mathrm{E} / \mathrm{K} 42 \mathrm{E}}$ mice, compared to age-matched WT control littermates. The data are shown both with respect to outer nuclear layer (ONL) thickness (yellow and gray lines) as well as total neural retina thickness (blue and orange lines) as a function of distance from the optic nerve head $(\mathrm{ONH}$, point 4 in each graph) along the vertical meridian, for both the inferior and superior hemispheres. No differences in these quantitative metrics of retinal morphology were observed with respect to genotype, consistent with the representative OCT images shown in Figure 2. 

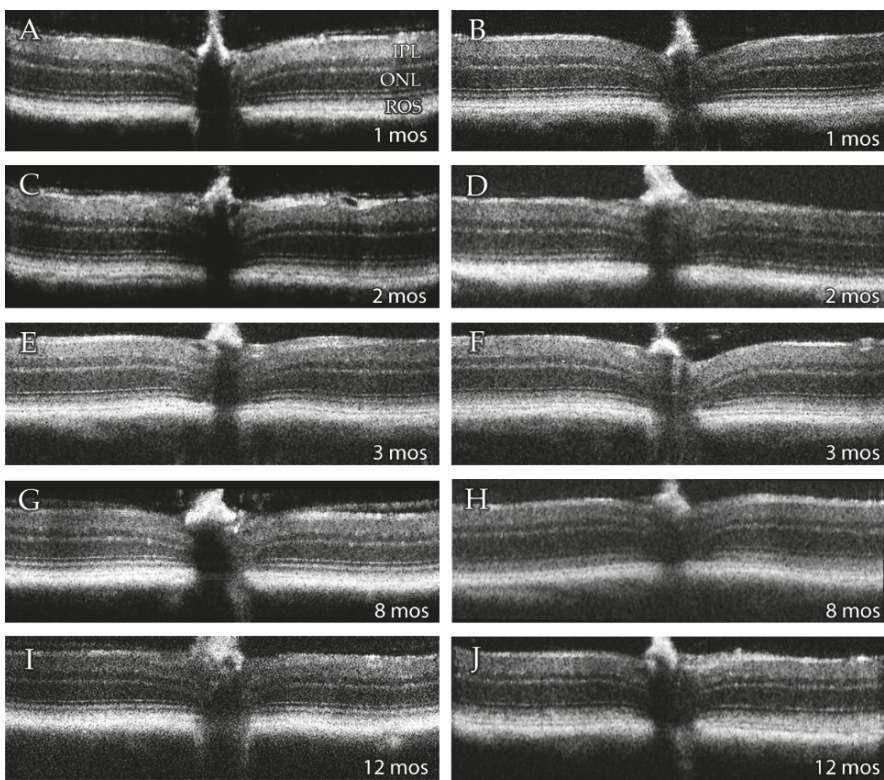

Figure 2. Representative averaged SD-OCT images of retinas from (left panels: A,C,E,G,I) 1-, 2-, 3-, 8and 12-months (mos) old wild type (WT), and (right panels: B,D,F,H,J) 1-, 2-, 3-, 8-, and 12-months old Dhdds ${ }^{\mathrm{K} 42 \mathrm{E} / \mathrm{K} 42 \mathrm{E}}$ mice. Abbreviations: IPL, inner plexiform layer; ONL, outer nuclear layer; ROS, rod outer segment layer. No changes were observed at any age in retinas of $D h d d s^{\mathrm{K} 42 \mathrm{E} / \mathrm{K} 42 \mathrm{E}}$ mice compared to WT mice.

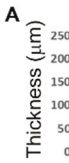

B

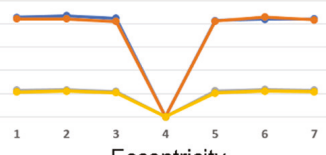
Eccentricity

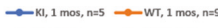

D

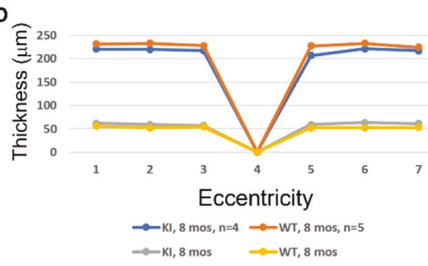

Figure 3. Analysis of the ONL thickness (yellow and gray lines) and total retinal thickness (blue and orange lines) in WT and Dhdds ${ }^{\mathrm{K} 42 \mathrm{E} / \mathrm{K} 42 \mathrm{E}}$ mice ranging in age from PN 1 to 12 months. (A) 1 month, (B) 2 months, (C) 3 months, (D) 8 months, (E) 12 months. Outer nuclear layer (ONL) thickness and total retina thickness measurements (in microns), as a function of genotype and distance from the optic nerve head $(\mathrm{ONH})$ along the vertical meridian in both the inferior and superior hemispheres. Genotypes: WT and Dhdds ${ }^{\mathrm{K} 42 \mathrm{E} / \mathrm{K} 42 \mathrm{E}}$ mice. No significant differences were observed between the groups.

\subsection{Gliotic Reactivity, Despite Lack of Overt Neural Retina Degeneration, in Dhdds ${ }^{K 42 E / K 42 E}$ Mice}

We performed immunohistochemical analysis on frozen sections of fixed, O.C.T.-embedded WT and Dhdds ${ }^{\mathrm{K} 42 \mathrm{E} / \mathrm{K} 42 \mathrm{E}}$ mouse eyes at PN 2 months of age, probing with antibodies against GFAP 
(polyclonal) and a C-terminal epitope of rod opsin (1D4 monoclonal). As shown in Figure 4, whereas WT control retinas only exhibited GFAP immunoreactivity (pseudocolored red) along the vitreoretinal interface, corresponding to astrocytes and Müller glia "end feet", retinas from Dhdds ${ }^{\mathrm{K} 42 \mathrm{E} / \mathrm{K} 42 \mathrm{E}}$ mice exhibited extensive, robust anti-GFAP labeling in a radial pattern. This extended throughout the inner retinal layers to the outer plexiform layer (OPL), in addition to intense labeling along the vitreoretinal interface. The latter results are indicative of massive gliotic activation, which is remarkable considering the lack of overt retinal degeneration or loss of retinal neurons (per the SD-OCT data; see Figures 2 and 3). Gliosis in Dhdds ${ }^{\mathrm{K} 42 \mathrm{E} / \mathrm{K} 42 \mathrm{E}}$ mouse retinas was also detected at PN one month and persisted even at PN six months of age (data not shown). Anti-opsin immunolabeling (pseudocolored green) was comparable in both WT and Dhdds ${ }^{\mathrm{K} 42 \mathrm{E} / \mathrm{K} 42 \mathrm{E}}$ retinas. Notably, the label was confined to the OS layer; there was no mislocalization of opsin to the plasma membrane of the cell in the IS or ONL layer-unlike what is often observed in degenerating photoreceptor cells in various animal models-suggesting normal trafficking of opsin to the outer segment, and consistent with a lack of overt photoreceptor degeneration. It is worth noting that the green labeling in a few cells in the inner retina in Figure 4 is due to mouse-on-mouse binding of the monoclonal antibody to endogenous IgG in blood vessels. It does not represent true anti-opsin immunolabeling.
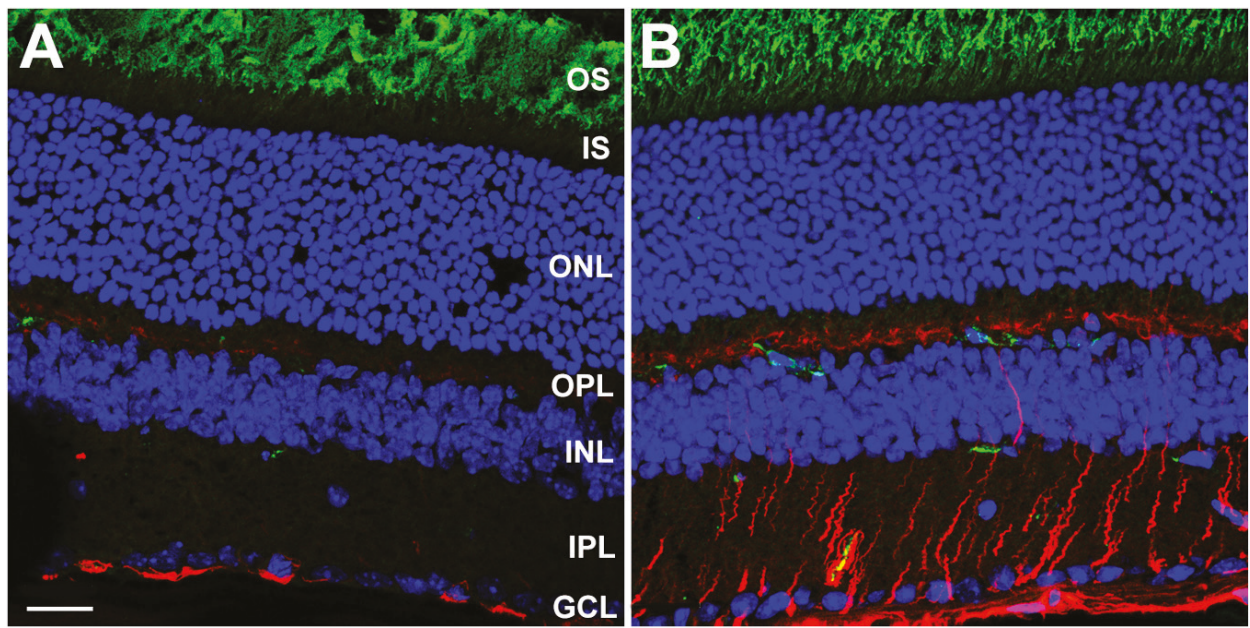

Figure 4. Laser confocal microscopy images of (A) WT control and (B) Dhdds ${ }^{\mathrm{K} 42 \mathrm{E} / \mathrm{K} 42 \mathrm{E}}$ mouse retina frozen sections at PN 2 months of age, stained with antibodies to GFAP (pseudocolor: red) and rod opsin (pseudocolor: green), and counterstained with DAPI (blue). Scale bar (both panels) is $20 \mu \mathrm{m}$. Abbreviations: OS, outer segment layer; IS, inner segment layer; ONL, outer nuclear layer; OPL, outer plexiform layer; INL, inner nuclear layer; IPL, inner plexiform layer; GCL, ganglion cell layer. Scale bar (both panels) is $20 \mu \mathrm{m}$.

\subsection{Lack of Defective Protein Glycosylation in Dhdds ${ }^{K 42 E / K 42 E}$ Mouse Retinas}

The $N$-linked oligo-saccharides of glycoproteins contain alpha-linked mannose residues as constituents, which are cognate ligands for the lectin concanavalin A (Con A) [17]. Hence, ConA lectin cytochemistry offers a reliable means of detecting the presence (or absence) of N-linked oligo-saccharides in tissue sections of Dhdds ${ }^{\mathrm{K} 42 \mathrm{E} / \mathrm{K} 42 \mathrm{E}}$ mice, and a way to directly test the current hypothesis that RP59 is driven by lack of glycosylation. This is because the synthesis of oligosaccharide chains in cells and tissues obligatorily depends upon the presence of Dol-PP and Dol-P (which requires upstream DHDDS activity). Furthermore, N-linked oligosaccharide chains are selectively susceptible to hydrolysis by peptide:N-glycosidase F (PNGase-F) [18]; hence, tissue sections treated with PNGase-F should exhibit a marked loss of Con A binding (serving as a true negative control), thereby mimicking the scenario 
where upstream DHDDS activity may be lacking. We performed ConA lectin cytochemical analysis on retinal sections from WT control and $D h d d s^{\mathrm{K} 42 \mathrm{E} / \mathrm{K} 42 \mathrm{E}}$ mice at PN six months of age, with and without pre-treatment with PNGase-F. The results are shown in Figure 5.
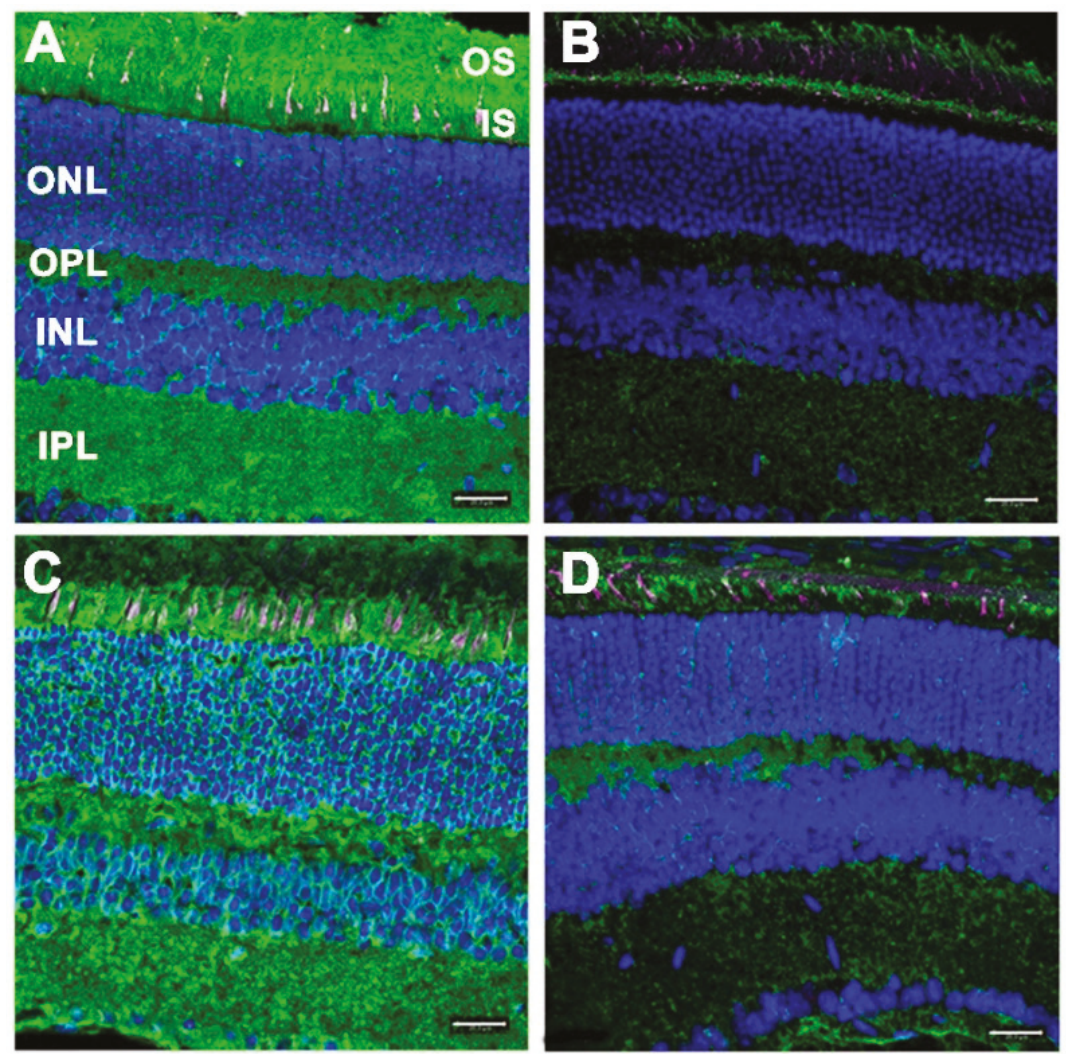

Figure 5. ConA lectin cytochemical analysis of retinas from $(\mathbf{A}, \mathbf{B}) \mathrm{WT}$ control and (C,D) Dhdds $\mathrm{K}^{\mathrm{K} 2 \mathrm{E} / \mathrm{K} 42 \mathrm{E}}$ mice at PN 6 months of age, with $(\mathbf{B}, \mathbf{D})$ or without $(\mathbf{A}, \mathbf{C})$ pretreatment with PNGase-F. ConA binding (green); PNA binding (magenta); DAPI counterstain (blue). Abbreviations are the same as in the Figure 4 legend. Scale bar (all panels): $20 \mu \mathrm{m}$.

Normally, $N$-linked glycoproteins are present throughout the retina, being notably enriched in photoreceptor cells and the synaptic endings of neurons (IPL, OPL). Hence, the inner and outer segment layers (IS and OS, respectively), including the glycoconjugate-rich interphotoreceptor matrix (IPM), as well as the inner and outer plexiform layers (IPL and OPL, respectively) were robustly labeled with fluor-tagged ConA in untreated WT retinal sections (Figure 5 A). As expected, treatment of WT retinal tissue sections with PNGase-F dramatically reduced the level of ConA binding throughout the retina (Figure 5B). Notably, retinal sections from Dhdds ${ }^{\mathrm{K} 42 \mathrm{E} / \mathrm{K} 42 \mathrm{E}}$ mice also exhibited robust, pan-retinal ConA binding (Figure 5C), comparable to that of WT controls. Upon treatment with PNGase-F, most of the ConA staining was lost (Figure 5D). These results obviate any significant DHDDS loss-of-function in Dhdds ${ }^{\mathrm{K} 42 \mathrm{E} / \mathrm{K} 42 \mathrm{E}}$ mice.

Peanut agglutinin (PNA) binds to the disaccharide Gal- $\beta(1-3)$-GalNAc in glycoproteins and glycolipids [17]. Oligosaccharides containing this disaccharide are highly enriched in the extracellular matrix surrounding cone photoreceptor outer segments (the "cone matrix sheath") $[19,20]$. Thus, PNA binding can be used to selectively label cone photoreceptors in retinal tissue sections, since rod 
photoreceptors and their associated "rod matrix sheath" lack such glycan chains [21]. Furthermore, oligosaccharides containing Gal- $\beta(1-3)$-GalNAc are generally O-linked (e.g., through Ser or Thr residues), rather than $N$-linked, and their synthesis is not dolichol-dependent in mammalian cells [22]. Furthermore, the PNA-binding disaccharide epitope is not susceptible to PNGase-F hydrolysis [17]. Hence, we expected to observe no appreciable differences in the binding of PNA to retinal tissue sections from Dhdds ${ }^{\mathrm{K} 2 \mathrm{E} / \mathrm{K} 42 \mathrm{E}}$ retinas vs. WT controls, nor effects of PNGase-F treatment on PNA binding, either with regard to labeling intensity or distribution. These expectations were realized, as illustrated in Figure 5 (magenta staining, all four panels). Both $D h d d s^{\mathrm{K} 42 \mathrm{E} / \mathrm{K} 42 \mathrm{E}}$ and control retinas exhibited comparable distribution of PNA-positive cone matrix sheaths, suggesting persistence of viable cone photoreceptors in the K42E mutants.

\section{Discussion}

Here, we have presented the generation and initial characterization of a novel mouse model of RP59, where we have achieved global homozygous knock-in of the K42E Dhdds mutation specifically associated with RP59 [3-5]. Based upon the clinical presentation of RP59 in human patients [3-5], as well as the demonstrable importance of dolichol-dependent protein glycosylation in maintaining the normal structure and function of the vertebrate retina [11-14], we expected to observe retinal degeneration and retinal thinning in $D h d d s^{\mathrm{K}} 42 \mathrm{E} / \mathrm{K} 42 \mathrm{E}$ mice, particularly in mice homozygous for the K42E mutation. This expectation was also predicated on a preliminary report [23], using a similar K42E mouse knock-in model, that claimed nearly $50 \%$ loss of OS length and reduction in ONL thickness by about two-thirds at PN 3 months of age, compared to WT mouse retinas. However, we observed no evidence of retinal degeneration in $D h d d s^{\mathrm{K} 42 \mathrm{E} / \mathrm{K} 42 \mathrm{E}}$ mice up to one year of age. Furthermore, despite the confirmed mutation of Dhdds, we found no evidence for defective protein $N$-glycosylation in the retinas of these mice. The retinas were labeled robustly with fluor-tagged ConA lectin, irrespective of genotype. These findings are in good agreement with observations made by Sabry et al. [24] who found normal mannose incorporation into $N$-linked oligosaccharides using either siRNA silencing of DHDDS in a HepG2 cell line or in RP59 (severe mutation) patient fibroblasts. The ConA binding observed in our study is further consistent with observations made by Wen et al., who observed that rather than any loss in dolichols, there was an alteration in dolichol chain lengths (increased D17:D18 ratio) in RP59 patients compared to normal human subjects, but without obvious hypoglycosylation of serum transferrin [25]. These findings collectively suggest hypoglycosylation-independent retinal degeneration in RP59, the mechanism of which still remains to be elucidated.

Understanding the pathophysiological and biochemical mechanisms underlying RP59 remains limited due to the lack of a validated vertebrate animal model that faithfully mimics the key hallmarks of the disease. Heretofore, only a zebrafish model of RP59 has been documented, using global knock-down of DHDDS expression by injection of morpholino oligonucleotides at the one-cell embryo stage [26]. In that case, the fish exhibited defective photoresponses and their cone outer segments (as assessed indirectly by PNA staining) were dramatically shortened, if not nearly absent. It should be noted that zebrafish have a highly cone-rich retina, unlike humans or mice (which have highly rod-dominant retinas). Also, the reduction and loss of PNA binding in the zebrafish knock-down retinas most likely reflects degeneration and death of cone photoreceptors, with concomitant degeneration and loss of their outer segments, due to their requirement for dolichol. Unlike the zebrafish Dhdds knock-down model, the murine RP59 model generated in the present study exhibits robust PNA staining in the outer retina, suggesting persistence of viable cone photoreceptors. In a parallel study (Ramachandra Rao et al., unpublished), we have observed Dhdds transcript distribution in all retinal nuclear layers by in situ hybridization, consistent with the fact that all cells require dolichol derivatives to support protein $\mathrm{N}$-glycosylation. Taken together, these findings suggest that the K42E Dhdds mutation does not affect cone photoreceptor viability. Recently (Ramachandra Rao et al., manuscript submitted for publication), we also generated a conditional Dhdds knockout mouse model, with targeted ablation of Dhdds in retinal rod photoreceptors, using a Cre-lox approach; however, unlike the K42E knock-in 
model, the rod-specific Dhdds knockout model exhibits profound, rapid retinal degeneration, with almost complete loss of photoreceptors by PN 6 weeks. Yet, there was no evidence of compromised protein $\mathrm{N}$-glycosylation prior to the onset of photoreceptor degeneration. In addition, as reported in a companion article in this Special Issue of Cells [27], targeted ablation of Dhdds in retinal pigment epithelium, (RPE) cells in mice also results in a progressive, but somewhat slower, retinal degeneration.

As pointed out by Zelinger et al. [4], the phenotype of RP59 only involves the retina; there is no observable dysfunction or pathology in other tissues and organs in RP59 patients. Hence, those authors speculated that the K42E mutation, "alters, rather than abolishes, enzymatic function, perhaps either by reducing the level of DHDDS protein or by preventing requisite interactions between DHDDS and a photoreceptor-specific protein" [4]. They also suggested, alternatively, that mutation of DHDDS might result in, "a toxic accumulation of isoprenoid compounds," such as occurs in various forms of neuronal ceroid lipofuscinosis (e.g., Batten disease). While such speculations may turn out to be true, there is no direct empirical evidence extant to support this hypothesis. It is also entirely possible, however, that mutations (whether K42E or others) in DHDDS may affect its interactions with its enzymatic partner, Nogo-B receptor (NgBR, encoded by the Nus1 gene) [8,9], with concomitant alterations in dolichol synthesis and protein $\mathrm{N}$-glycosylation [28,29]. At present, nothing is known about the expression of Nogo-B receptor or its interactions with DHDDS, specifically in the retina. Our Dhdds ${ }^{\mathrm{K} 42 \mathrm{E} / \mathrm{K} 42 \mathrm{E}}$ mouse line and retinal cell type-specific conditional DHDDS knockout mice offer potentially valuable model systems in which to pursue further investigations along these lines. In addition, we are currently pursuing studies employing dual, targeted ablation of DHDDS and NgBR in the retina. (See also the article by DeRamus et al., in this Special Issue of Cells, regarding an RPE-specific DHDDS knockout mouse model [27].)

Our findings bring into question the current concept that RP59 is a member of a large and diverse class of diseases known as "congenital disorders of glycosylation" (CDGs) [30,31]. While, in principle, it would be reasonable to consider RP59 as a CDG, due to the associated mutation(s) in DHDDS, there is no direct evidence to demonstrate a glycosylation defect in the human retinal disease or in any animal model of RP59 generated to date. The mechanism underlying the DHDDS-dependent retinal degeneration in human arRP patients remains to be elucidated, but is more complex than simply loss-of-function of DHDDS.

Author Contributions: Conceptualization, S.J.P. and S.J.F.; methodology, S.J.P., S.J.F., S.R.R., P.K.; M.N.N.; validation, S.R.R., M.N.N., P.K., S.J.P. and S.J.F.; formal analysis, M.N.N., S.J.P., S.J.F. and S.R.R.; investigation, S.J.P., S.R.R., P.K.; resources, S.J.P. and S.J.F.; data curation, S.J.P. and S.J.F.; writing-original draft preparation, S.J.F.; writing-review and editing, S.J.F., S.J.P., P.K., M.N.N. and S.R.R.; visualization, S.J.F., S.R.R. and S.J.P.; supervision, S.J.P. and S.J.F.; project administration, S.J.P. and S.J.F.; funding acquisition, S.J.P. and S.J.F. All authors have read and agreed to the published version of the manuscript.

Funding: This research was supported by U.S. Department of Health and Human Services (National Institutes of Health (NIH)/National Eye Institute (NEI)) grant R01 EY029341 to S.J.P. and S.J.F., and NIH/NEI core grant P30 003039 to S.J.P.; a Fight for Sight Summer Student Fellowship to S.R.R.; a Career-Starter Research Grant from the Knights Templar Eye Foundation to S.R.R.; as well as support from the UAB Vision Science Research Center (S.J.P., M.N.N., P.K.) and facilities and resources provided by the VA Western NY Healthcare System (S.J.F., S.R.R.).

Acknowledgments: We thank Isaac Cobb for technical assistance with OCT and genotyping. The opinions expressed herein do not reflect those of the Department of Veteran Affairs or the U.S. Government.

Conflicts of Interest: The authors declare no conflict of interest. The funders had no role in the design of the study; in the collection, analyses, or interpretation of data; in the writing of the manuscript, or in the decision to publish the results.

\section{References}

1. Hartong, D.T.; Berson, E.L.; Dryja, T.P. Retinitis pigmentosa. Lancet 2006, 368, 1795-1809. [CrossRef]

2. Zhang, Q. Retinitis Pigmentosa: Progress and Perspective. Asia Pac. J. Ophthalmol. (Phila) 2016, 5, $265-271$. [CrossRef] [PubMed] 
3. Zuchner, S.; Dallman, J.; Wen, R.; Beecham, G.; Naj, A.; Farooq, A.; Kohli, M.A.; Whitehead, P.L.; Hulme, W.; Konidari, I.; et al. Whole-exome sequencing links a variant in DHDDS to retinitis pigmentosa. Am. J. Hum. Genet. 2011, 88, 201-206. [CrossRef]

4. Zelinger, L.; Banin, E.; Obolensky, A.; Mizrahi-Meissonnier, L.; Beryozkin, A.; Bandah-Rozenfeld, D.; Frenkel, S.; Ben-Yosef, T.; Merin, S.; Schwartz, S.B.; et al. A missense mutation in DHDDS, encoding dehydrodolichyl diphosphate synthase, is associated with autosomal-recessive retinitis pigmentosa in Ashkenazi Jews. Am. J. Hum. Genet. 2011, 88, 207-215. [CrossRef] [PubMed]

5. Lam, B.L.; Zuchner, S.L.; Dallman, J.; Wen, R.; Alfonso, E.C.; Vance, J.M.; Pericak-Vance, M.A. Mutation K42E in dehydrodolichol diphosphate synthase (DHDDS) causes recessive retinitis pigmentosa. Adv. Exp Med. Biol. 2014, 801, 165-170. [CrossRef] [PubMed]

6. Parodi, A.J.; Leloir, L.F. The role of lipid intermediates in the glycosylation of proteins in the eucaryotic cell. Biochim. Biophys. Acta 1979, 559, 1-37. [CrossRef]

7. Hemming, F.W. Control and manipulation of the phosphodolichol pathway of protein N-glycosylation. Biosci. Rep. 1982, 2, 203-221. [CrossRef]

8. Giladi, M.; Edri, I.; Goldenberg, M.; Newman, H.; Strulovich, R.; Khananshvili, D.; Haitin, Y.; Loewenstein, A. Purification and characterization of human dehydrodolychil diphosphate synthase (DHDDS) overexpressed in E. coli. Protein Expr. Purif. 2017, 132, 138-142. [CrossRef]

9. Lisnyansky Bar-El, M.; Lee, S.Y.; Ki, A.Y.; Kapelushnik, N.; Loewenstein, A.; Chung, K.Y.; Schneidman-Duhovny, D.; Giladi, M.; Newman, H.; Haitin, Y. Structural Characterization of Full-Length Human Dehydrodolichyl Diphosphate Synthase Using an Integrative Computational and Experimental Approach. Biomolecules 2019, 9. [CrossRef]

10. Behrens, N.H.; Leloir, L.F. Dolichol monophosphate glucose: An intermediate in glucose transfer in liver. Proc. Natl. Acad. Sci. USA 1970, 66, 153-159. [CrossRef]

11. Tam, B.M.; Moritz, O.L. The role of rhodopsin glycosylation in protein folding, trafficking, and light-sensitive retinal degeneration. J. Neurosci. 2009, 29, 15145-15154. [CrossRef] [PubMed]

12. Murray, A.R.; Vuong, L.; Brobst, D.; Fliesler, S.J.; Peachey, N.S.; Gorbatyuk, M.S.; Naash, M.I.; Al-Ubaidi, M.R. Glycosylation of rhodopsin is necessary for its stability and incorporation into photoreceptor outer segment discs. Hum. Mol. Genet. 2015, 24, 2709-2723. [CrossRef] [PubMed]

13. Fliesler, S.J.; Rayborn, M.E.; Hollyfield, J.G. Membrane morphogenesis in retinal rod outer segments: Inhibition by tunicamycin. J. Cell Biol. 1985, 100, 574-587. [CrossRef] [PubMed]

14. Fliesler, S.J.; Rapp, L.M.; Hollyfield, J.G. Photoreceptor-specific degeneration caused by tunicamycin. Nature 1984, 311, 575-577. [CrossRef]

15. DeRamus, M.L.; Stacks, D.A.; Zhang, Y.; Huisingh, C.E.; McGwin, G.; Pittler, S.J. GARP2 accelerates retinal degeneration in rod cGMP-gated cation channel beta-subunit knockout mice. Sci. Rep. 2017, 7, 42545. [CrossRef]

16. Ramachandra Rao, S.; Pfeffer, B.A.; Mas Gomez, N.; Skelton, L.A.; Keiko, U.; Sparrow, J.R.; Rowsam, A.M.; Mitchell, C.H.; Fliesler, S.J. Compromised phagosome maturation underlies RPE pathology in cell culture and whole animal models of Smith-Lemli-Opitz Syndrome. Autophagy 2018, 1-22. [CrossRef]

17. Goldstein, I.J.; Hayes, C.E. The lectins: Carbohydrate-binding proteins of plants and animals. Adv. Carbohydr. Chem. Biochem. 1978, 35, 127-340. [CrossRef]

18. Wang, T.; Voglmeir, J. PNGases as valuable tools in glycoprotein analysis. Protein Pept. Lett. 2014, 21, 976-985. [CrossRef]

19. Blanks, J.C.; Johnson, L.V. Specific binding of peanut lectin to a class of retinal photoreceptor cells. A species comparison. Invest. Ophthalmol. Vis. Sci. 1984, 25, 546-557.

20. Johnson, L.V.; Hageman, G.S.; Blanks, J.C. Interphotoreceptor matrix domains ensheath vertebrate cone photoreceptor cells. Invest. Ophthalmol. Vis. Sci. 1986, 27, 129-135.

21. Fariss, R.N.; Anderson, D.H.; Fisher, S.K. Comparison of photoreceptor-specific matrix domains in the cat and monkey retinas. Exp. Eye Res. 1990, 51, 473-485. [CrossRef]

22. Gemmill, T.R.; Trimble, R.B. Overview of N- and O-linked oligosaccharide structures found in various yeast species. Biochim. Biophys. Acta 1999, 1426, 227-237. [CrossRef]

23. Li, Y.; Lam, B.L.; Guan, Z.; Wang, Z.; Wang, N.; Chen, Y.; Wen, R. Photoreceptor degeneration in the DHDDSK42E/K42E mouse. Invest. Ophthalmol. Vis. Sci. 2014, 55, 4371. 
24. Sabry, S.; Vuillaumier-Barrot, S.; Mintet, E.; Fasseu, M.; Valayannopoulos, V.; Heron, D.; Dorison, N.; Mignot, C.; Seta, N.; Chantret, I.; et al. A case of fatal Type I congenital disorders of glycosylation (CDG I) associated with low dehydrodolichol diphosphate synthase (DHDDS) activity. Orphanet. J. Rare Dis. 2016, 11, 84. [CrossRef]

25. Wen, R.; Lam, B.L.; Guan, Z. Aberrant dolichol chain lengths as biomarkers for retinitis pigmentosa caused by impaired dolichol biosynthesis. J. Lipid Res. 2013, 54, 3516-3522. [CrossRef]

26. Wen, R.; Dallman, J.E.; Li, Y.; Zuchner, S.L.; Vance, J.M.; Pericak-Vance, M.A.; Lam, B.L. Knock-down DHDDS expression induces photoreceptor degeneration in zebrafish. Adv. Exp. Med. Biol. 2014, 801, 543-550. [CrossRef]

27. DeRamus, M.L.; Davis, S.J.; Rao, S.R.; Nyankerh, C.; Stacks, D.; Kraft, T.W.; Fliesler, S.J.; Pittler, S.J. Selective Ablation of Dehydrodolichyl Diphosphate Synthase in Murine Retinal Pigment Epithelium (RPE) Causes RPE Atrophy and Retinal Degeneration. Cells 2020, 9, 771. [CrossRef]

28. Harrison, K.D.; Park, E.J.; Gao, N.; Kuo, A.; Rush, J.S.; Waechter, C.J.; Lehrman, M.A.; Sessa, W.C. Nogo-B receptor is necessary for cellular dolichol biosynthesis and protein N-glycosylation. EMBO J. 2011, 30, 2490-2500. [CrossRef]

29. Park, E.J.; Grabinska, K.A.; Guan, Z.; Stranecky, V.; Hartmannova, H.; Hodanova, K.; Baresova, V.; Sovova, J.; Jozsef, L.; Ondruskova, N.; et al. Mutation of Nogo-B receptor, a subunit of cis-prenyltransferase, causes a congenital disorder of glycosylation. Cell Metab. 2014, 20, 448-457. [CrossRef]

30. Haeuptle, M.A.; Hennet, T. Congenital disorders of glycosylation: An update on defects affecting the biosynthesis of dolichol-linked oligosaccharides. Hum. Mutat. 2009, 30, 1628-1641. [CrossRef]

31. Ng, B.G.; Freeze, H.H. Perspectives on Glycosylation and Its Congenital Disorders. Trends Genet. 2018, 34, 466-476. [CrossRef] [PubMed]

(C) 2020 by the authors. Licensee MDPI, Basel, Switzerland. This article is an open access article distributed under the terms and conditions of the Creative Commons Attribution (CC BY) license (http://creativecommons.org/licenses/by/4.0/). 



\title{
Article \\ Selective Ablation of Dehydrodolichyl Diphosphate Synthase in Murine Retinal Pigment Epithelium (RPE) Causes RPE Atrophy and Retinal Degeneration
}

\author{
Marci L. DeRamus ${ }^{1}$, Stephanie J. Davis ${ }^{1}$, Sriganesh Ramachandra Rao ${ }^{2}$, Cyril Nyankerh ${ }^{1}$, \\ Delores Stacks ${ }^{1}$, Timothy W. Kraft ${ }^{1}$, Steven J. Fliesler ${ }^{2}$ and Steven J. Pittler ${ }^{1, *}$ \\ 1 Department of Optometry and Vision Science, Vision Science Research Center, University of Alabama at \\ Birmingham, Birmingham, AL 35294, USA; marcismith1952@gmail.com (M.L.D.); \\ stephanie.davis@bison.howard.edu (S.J.D.); cyrilnya@uab.edu (C.N.); delorez@uab.edu (D.S.); \\ twkraft@uab.edu (T.W.K.) \\ 2 Departments of Ophthalmology and Biochemistry, State University of New York-University at Buffalo, \\ Buffalo, NY 14209, USA; and Research Service, VA Western NY Healthcare System, Buffalo, NY 14215, USA; \\ sramacha@buffalo.edu (S.R.R.); fliesler@buffalo.edu (S.J.F.) \\ * Correspondence: pittler@uab.edu; Tel.: +1-205-934-6744
}

Received: 12 February 2020; Accepted: 17 March 2020; Published: 21 March 2020

\begin{abstract}
Patients with certain defects in the dehydrodolichyl diphosphate synthase (DHDDS) gene (RP59; OMIM \#613861) exhibit classic symptoms of retinitis pigmentosa, as well as macular changes, suggestive of retinal pigment epithelium (RPE) involvement. The DHDDS enzyme is ubiquitously required for several pathways of protein glycosylation. We wish to understand the basis for selective ocular pathology associated with certain DHDDS mutations and the contribution of specific ocular cell types to the pathology of mutant Dhdds-mediated retinal degeneration. To circumvent embryonic lethality associated with Dhdds knockout, we generated a Cre-dependent knockout allele of murine Dhdds (Dhdds ${ }^{f l x f f l x}$ ). We used targeted Cre expression to study the importance of the enzyme in the RPE. Structural alterations of the RPE and retina including reduction in outer retinal thickness, cell layer disruption, and increased RPE hyper-reflectivity were apparent at one postnatal month. At three months, RPE and photoreceptor disruption was observed non-uniformly across the retina as well as RPE transmigration into the photoreceptor layer, external limiting membrane descent towards the RPE, and patchy loss of photoreceptors. Functional loss measured by electroretinography was consistent with structural loss showing scotopic a- and b-wave reductions of $83 \%$ and $77 \%$, respectively, at three months. These results indicate that RPE dysfunction contributes to DHDDS mutation-mediated pathology and suggests a more complicated disease mechanism than simply disruption of glycosylation.
\end{abstract}

Keywords: retinal degeneration; retinitis pigmentosa; retinal pigment epithelium dystrophy; RPE transmigration; Cre-Lox technology; mouse models

\section{Introduction}

Retinitis pigmentosa (RP) and related disorders are characterized by degeneration and loss of photoreceptors, attenuation of retinal blood vessels, pigment deposits, and a waxy pallor of the optic disc that result in impaired night vision and peripheral and central vision loss [1]. Defective protein glycosylation in the retinal pigment epithelium (RPE) has been associated with retinal degeneration elicited by photoreceptor abnormalities and impaired phagocytosis of aging photoreceptor outer segment (OS) membranes [2-5]. One such example, dehydrodolichyl diphosphate synthase (DHDDS; OMIM \#608172), is an essential enzyme in the mevalonate pathway where it functions ubiquitously in 
isoprenoid chain elongation to form dolichols that comprise 17-20 isoprene units. The phosphorylated form of dolichol, dolichol pyrophosphate is necessary for $\mathrm{N}$-glycosylation at specific residues in many membrane proteins [6]. There is no known unique function for DHDDS in the retina, or in any other ocular tissue. There are numerous $\mathrm{N}$-glycosylated proteins in the retina and photoreceptors, such as rhodopsin [7] and the cGMP-gated cation channel in rods. Additionally, in the RPE, there are several glycosylated structural proteins, ion channels and transport proteins that contribute to the transepithelial potential of the polarized RPE monolayer [8-10].

Mutations in the gene encoding DHDDS lead to a recessive form of RP called RP59 (OMIM \#613861, DHDDS, K42E), which was first identified in several families of Ashkenazi Jewish origin [11-13]. RP59 is considered to belong to the family of human genetic diseases known as "congenital disorders of glycosylation" (CDGs) [14]. Two other mutations in the DHDDS gene (T206A and R98W), which occur heterozygously with the K42E mutation, were also reported, but have not been studied in detail $[15,16]$. Patients with the K42E mutation in DHDDS exhibit all the cardinal features of RP as well as macular changes [12], suggesting possible RPE involvement. While the K42E, T206A, and R98W mutations are only associated with known RP symptoms, another mutation in the DHDDS gene has led to infant morbidity at seven months of age [17].

As a first step to assess the role of DHDDS in specific retinal cell types and to understand the molecular mechanism of RP59, we created a Cre-lox dependent line of mice that allows targeted, cell type-specific deletion of Dhdds in cells of interest. Using a conditional knockout was necessary to circumvent embryonic lethality associated with global knockout of $D h d d s[18,19]$. Here, we describe the generation and characterization of a $D h d d s^{f l x} f f l x$ CreRPE mouse line (i.e., RPE-specific Dhdds knockout) and its validation as a model of RPE atrophy and retinal degeneration. We show that RPE-specific deletion of Dhdds induces structural and functional deficits in the RPE and the photoreceptors, which suggests that RPE pathology may be a significant contributor to the retinal degeneration observed in patients with RP59 mutations.

\section{Materials and Methods}

\subsection{Generation of Dhdds flxfflx CreRPE Mice}

A construct containing lacZ flanked by FLP-FRT and Dhdds exon 3 flanked by loxP sites from the Knockout Mouse Project (KOMP, UC Davis, Davis, CA, USA) was linearized and introduced into mouse ES cells (C57Bl/6J background) at the Roswell Park Cancer Institute (RPCI) Gene Targeting and Transgenic Facility (Buffalo, NY, USA) using standard technology. To confirm the correctly targeted cells, polymerase chain reaction (PCR) was performed with the primers listed below (see PCR Genotyping, below). The lacZ cassette was excised with FLP-FRT recombinase, and excision was confirmed by PCR. Mouse lines that carried the Dhdds loxP conditional knockout allele were crossed to generate homozygotes and the latter were also crossed to a mouse line (on a C57Bl/6J background) carrying a homozygous transgene expressing Cre recombinase under the control of the RPE-specific VMD2 (vitelliform macular degeneration 2) promoter [20,21]. RPE-specific expression of Cre in the VMD2 promoter-driven mouse line was confirmed by crossing those mice with a ZsGreen reporter mouse

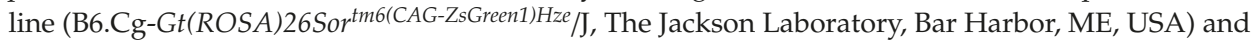
examining the retinas by confocal fluorescence microscopy. Genotypes of offspring were confirmed by PCR with Dhdds- and Cre RPE-specific primer pairs. All mice used in this study were treated following the ARVO Statement on the Use of Animals in Ophthalmic and Vision Research and the policies of the University of Alabama at Birmingham (UAB) Institutional Animal Care and Use Committee (IACUC). This project was approved for animal use on April 2019 by the UAB IACUC and requires updated approvals each year (protocol number IACUC-21270). All animals were maintained on a standard 12/12-h light/dark cycle, fed standard rodent chow, provided water ad libitum, and housed in plastic cages with standard rodent bedding. 


\subsection{PCR Genotyping}

Mouse genomic DNA samples obtained from tail snips were verified by PCR using primers Dhdds-FWD: 5'-GTGTCATCCCCTGCTGCAGAT-3' and Dhdds-REV: 5'-TGGGTGTAGTGGCTCAGGTC-3' for genotype identification of floxed Dhdds alleles designed in a region which was conserved in both wild type (WT) and floxed alleles and also in the region flanking the loxP sites. The expected PCR product sizes for the WT and floxed alleles are 393 and $517 \mathrm{bp}$, respectively, thus differentiating WT, heterozygous floxed, and homozygous floxed alleles. PCR verification of Cre transgene modification was carried out using the following forward and reverse primer sets for Cre RPE 5'-AGGTGTAGAGAAGGCACTTAGC-3' and 5'-CTAATCGCCATCT-TCCAGCAGG-3', respectively, yielding a $411 \mathrm{bp}$ product. RPE specific expression and activity of Cre-recombinase in VMD2-RPE Cre was verified by breeding these mouse lines against an ZsGreen reporter mouse strain (B6.Cg-Gt(ROSA)26Sortm6(CAG-ZsGreen1)Hze/J, Stock\# 007906; The Jackson Laboratory, Bar Harbor, ME, USA) and monitoring ZsGreen expression in the retina.

\subsection{Spectral-Domain Optical Coherence Tomography}

Spectral-domain optical coherence tomography (SD-OCT) $(840$ nm; Bioptigen, Inc./Leica, Durham, NC, USA) was used to obtain in vivo images of the retina at one, two, and three months of age. OCT images were collected with Bioptigen InVivoVue ${ }^{\circledR} 1.4$ software and Bioptigen Diver ${ }^{\circledR} 2.0$ software was used to analyze outer nuclear layer (ONL) thickness and full retinal thickness (F.R.T.) across all retinal layers at five eccentricities spanning two-thirds of the retina centered at the optic nerve head. A detailed description of this procedure has been reported previously [22]. F.R.T. was calculated from the difference of markers 1 and 10 and ONL thickness was calculated from the difference between 5 and 6 .

\subsection{Fundus Examination and Fluorescein Angiography}

Fundus examination was performed with a Micron IV digital fundus microscope (Phoenix Technology Group, Pleasanton, CA, USA), using the mouse objective and hydroxypropylmethyl cellulose $2.5 \%$ on the surface of the cornea. Digital images of the fundus were captured with dedicated StreamPix ${ }^{\circledR} 6$ digital software (NorPix, Inc., Montreal, Quebec, Canada) and processed using Adobe ${ }^{\circledR}$ Photoshop ${ }^{\circledR} 6$ (Adobe Systems, Inc., San Jose, CA, USA).

\subsection{Visual Function Testing}

Full-field scotopic and photopic electroretinograms (ERGs) were obtained as described in detail previously using an OcuScience ${ }^{\circledR}$ HMsERG instrument (OcuScience, Henderson, NV, USA) [22]. Response amplitudes and implicit times of a-waves and b-waves representing the activity of photoreceptors and bipolar cells, respectively, were quantified. In brief, mice were dark-adapted overnight, anesthetized, and then placed on a heating pad to maintain body temperature. Following pupil dilation with $2.5 \%$ phenylephrine and $1 \%$ tropicamide ophthalmic solutions, a thin silver wire electrode was placed on the cornea (interfaced with methylcellulose and covered with a specially designed contact lens) and referenced to a needle ground electrode in the cheek. The responses to Ganzfeld flash stimuli, spanning a range of five log units, were measured and recorded; following light adaptation to background illumination, cone-driven responses also were recorded.

\subsection{Histology/Immunohistochemistry}

The methodologies used in this study have been described in detail previously [23]. Briefly, for conventional histology, eyes ( $n \geq 4$ per condition) were fixed by immersion in freshly prepared $4 \%$ paraformaldehyde in $0.125 \mathrm{M} \mathrm{Na}$-phosphate buffer, $\mathrm{pH} 7.4$, at $4{ }^{\circ} \mathrm{C}$ overnight, embedded in paraffin, and tissue sections (toluidine blue-stained) were viewed with an Olympus BH2 photomicroscope equipped with a Nikon digital camera. Digitized images were collected and further analyzed with ImagePro 
Plus ${ }^{\circledR}$ software, Version 4.1 (Media Cybernetics; Rockville, MD, USA). For immunohistochemistry, frozen sections of retinal tissue (embedded in Tissue-Plus ${ }^{\mathrm{TM}}$ Optimal Cutting Temperature (O.C.T.) compound; Thermo Fisher Scientific, Waltham, MA, USA), obtained with a cryostat and collected on glass microscope slides were incubated with suitable primary antibodies, with detection by application of species-specific, fluor-conjugated secondary antibodies, counterstaining nuclei with DAPI, followed by laser confocal immunofluorescence microscopy (Leica TCS SPE scanning confocal microscope; Leica Microsystems Inc., Buffalo Grove, IL, USA), as previously reported [24].

\subsection{Fluorescein Angiography}

Fluorescein angiography was performed following intraperitoneal injection (i.p., $10 \mu \mathrm{L} / \mathrm{gram}$ body weight) of a $10 \mathrm{mg} / \mathrm{mL}$ solution of AK-Fluor 10\% (Sigma Pharmaceuticals; Liberty, IA, USA) in PBS. Uptake of fluorescein and fundus imaging was monitored with a Micron IV Retinal Imaging Microscope (Phoenix Technology Group, Inc., Pleasanton, CA, USA).

\subsection{Electron Microscopy}

Mouse eyes were processed for plastic embedment, ultramicrotomy, and EM analysis essentially as described in detail previously [23]. Immediately after sacrifice, eyes were orientated by marking the superior hemisphere along the vertical meridian at the limbus with a hot needle, before starting the dissection. A cut was made in the superior cornea and the eyes were fixed for $2 \mathrm{~h}$ at $4{ }^{\circ} \mathrm{C}$ in fresh $0.1 \mathrm{M}$ sodium phosphate buffer ( $\mathrm{pH} 7.4)$, containing $2.5 \%(\mathrm{v} / \mathrm{v})$ glutaraldehyde, $2.0 \%$ formaldehyde and $0.025 \% \mathrm{CaCl}_{2}$. After a $20-30 \mathrm{~min}$ primary fixation, the superior cornea and lens were removed, and fixation was continued overnight. The fixed eyes were then rinsed with $0.1 \mathrm{M}$ sodium cacodylate buffer ( $\mathrm{pH} 7.4$ ) containing $0.025 \% \mathrm{CaCl}_{2}$, and then post-fixed for $1 \mathrm{~h}$ in $1 \%$ osmium tetroxide in $0.1 \mathrm{M}$ sodium cacodylate buffer. After post-fixation, the eyes were rinsed twice in $0.1 \mathrm{M}$ sodium cacodylate buffer and once in distilled water, then dehydrated in graded ethanol series followed by propylene oxide and infiltration overnight in Spurr's resin. The eyes were then embedded in resin-filled BEEM ${ }^{\circledR}$ capsules (Polysciences, Warrington, PA, USA) and allowed to polymerize in a $70{ }^{\circ} \mathrm{C}$ oven for $48 \mathrm{~h}$. Tissue sections were obtained with a Reichert-Jung Ultracut $\mathrm{E}^{\circledR}$ microtome using a diamond knife. Thin (60-80 nm thickness) sections were collected on copper 75/300 mesh grids and stained with $2 \%$ (v/v) uranyl acetate and Reynolds' lead citrate. Sections were viewed with a JEOL 100CX electron microscope at an accelerating voltage of $60 \mathrm{keV}$.

\subsection{Serial Block-Face Scanning Electron Microscopy (SBF-SEM)}

Samples prepared for TEM as described above were further processed by Thermo Fisher Scientific (Waltham, MA, USA) using an Apreo VolumeScope ${ }^{\mathrm{TM}}$ serial block-face scanning electron microscope (SBF-SEM). Excess resin was removed from the tissue using a Leica ultramicrotome. The trimmed blocks were then glued to a SEM stub (Agar Scientific, AGG1092450) using a two-component silver conductive epoxy, H20E EPO-TEK (Ted Pella, Inc.; Redding, CA, USA). To minimize charging of the block by the electron beam, the bottom and sides of the block were sputter-coated with a $30 \mathrm{~nm}$ thick gold film layer. The samples were then imaged on the VolumeScope ${ }^{\mathrm{TM}}$ operating in low vacuum mode at $50 \mathrm{~Pa}$ and using a lens mounted backscattered detector. All of the data sets were imaged with an accelerating voltage of $2.2 \mathrm{kV}$ and a beam current of $100 \mathrm{pA}$ using 1- $\mu$ s dwell time combined with two-line integration. Two regions of interest (ROIs) were acquired on the knock-out sample (KO-148). For ROI1, 738 sections were collected with the internal microtome set to a $40 \mathrm{~nm}$ cutting thickness (z resolution) with an area of $92.9 \mu \mathrm{m} \times 89.1 \mu \mathrm{m}$ at $10 \mathrm{~nm} /$ pixel. For ROI2, 745 sections were collected with an area of $97.6 \mu \mathrm{m} \times 96.3 \mu \mathrm{m}$ using the same imaging condition as ROI1. For the wild type sample (WT-146), an area of $92.9 \mu \mathrm{m} \times 89.1 \mu \mathrm{m}$ was imaged at $10 \mathrm{~nm} /$ pixel using a cutting thickness (z resolution) of $40 \mathrm{~nm}$. The acquired data sets were finally aligned and visualized using 3D volume rendering to highlight the RPE anomalies using Amira software (Thermo Fisher Scientific). 


\subsection{Statistical Analysis}

For ERG analyses, we evaluated differences between genetically modified vs. WT control mice across flash intensities by performing repeated measures two-way ANOVA with Holm-Sidak post-hoc analysis at each time point. To evaluate functional (visual acuity, a-wave and b-wave amplitudes and implicit times) and structural (SD-OCT retinal layer thickness) parameters across time, we used a two-way repeated measures ANOVA (time $X$ treatment conditions) with Holm-Sidak post-hoc analysis. For biochemical and quantitative immunohistochemical data, binary statistical comparisons between specific genetically modified vs. WT control group data were analyzed using an unpaired Student's $t$-test.

\section{Results}

\subsection{Generation of a Floxed Dhdds Mouse Line}

The scheme used for the generation of a Dhdds conditional allele, employing a validated Dhdds construct (from KOMP) and mouse embryonic stem cells (ESCs), is shown in Figure 1 (see Materials and Methods, above). Clones from confirmed flippase recognition target (FRT)-excised alleles were used to generate Dhdds heterozygous and homozygous mouse lines on a C57Bl/6J background.
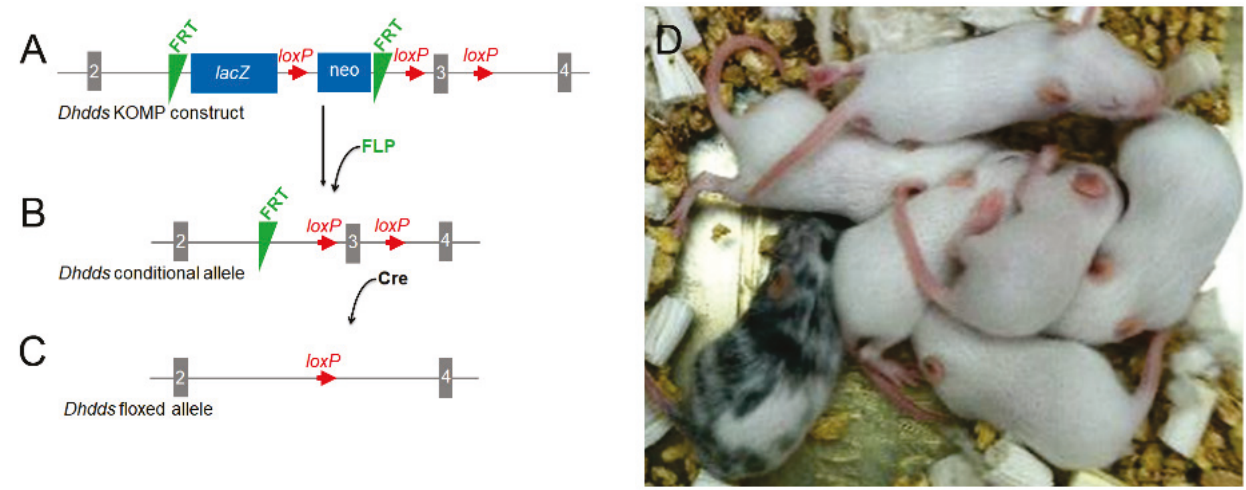

Figure 1. Generation of Cre-dependent Dhdds conditional knockout (KO) mice. (A) A validated Dhdds construct from the Knockout Mouse Project (KOMP) (U.C. Davis) was linearized and introduced into mouse ESCs. (B) Transformed cells were treated with FLP-FRT recombinase and PCR was used to verify lacZ cassette excision. (C) Clones from confirmed FRT-excised alleles were used to generate Dhdds ${ }^{f l x f f l x-}$ mice. (D) Pups carrying the Dhdds floxed allele were identified by coat appearance.

\subsection{Validation of Retinal Cell Type-Specific Cre-Expressing Mouse Lines}

To assess the specificity and efficiency of the Cre recombinase in the RPE, we cross-bred transgenic mice expressing Cre recombinase (bred to homozygosity) with a ZsGreen Ai6 reporter mouse line, and then evaluated ZsGreen expression in the retina by confocal fluorescence microscopy. As expected, WT mouse retinas (a negative control) did not exhibit ZsGreen expression (Figure 2A). However, ZsGreen fluorescence was detected in Cre recombinase-positive mice specifically in the RPE layer by 1 postnatal (PN) month (Figure 2B), with $>90 \%$ of the RPE cells being labeled. We subsequently confirmed that Cre recombinase continued to be expressed robustly and specifically in the RPE for $>3$ months (data not shown). 


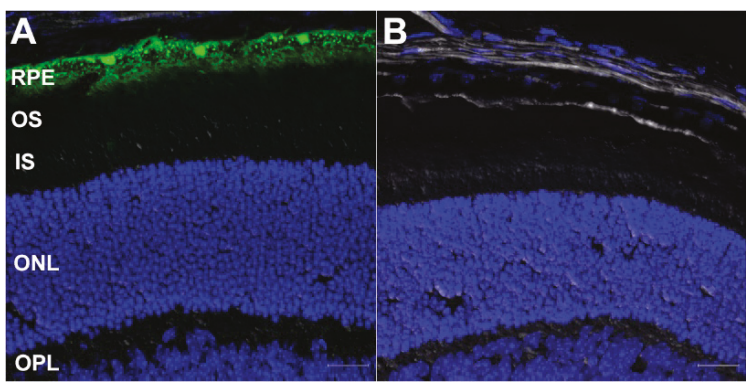

Figure 2. Cre recombinase-dependent ZsGreen expression (green) in mouse retina. (A) Retina from a postnatal $(\mathrm{PN})$ one-month old CreRPE $\times$ ZsGreen reporter mouse, demonstrating ZsGreen expression specifically in retinal pigment epithelium (RPE) cells. (B) Retina from an age-matched, wild type (WT) mouse retina, demonstrating lack of ZsGreen expression. Nuclei counterstained with DAPI (blue). Abbreviations: IS, photoreceptor inner segment layer; OS, photoreceptor outer segment layer; ONL, outer nuclear layer; OPL, outer plexiform layer. Scale bars (both panels): $20 \mu \mathrm{m}$.

3.3. RPE-Specific Ablation of Dhdds Causes a Geographic Atrophy-Like Phenotype and Retinal Degeneration, Involving Photoreceptors

In vivo retinal imaging using SD-OCT (Figure 3) showed comparable normal layer stratification in WT, CreRPE and Dhdds ${ }^{+f f x}$ CreRPE age-matched mice in each group. However, Dhdds $s^{f l x f l x}$ CreRPE mice showed altered hyper-reflectivity at all ages (indicated by red arrows, Figure 3), indicative of pathologic changes and a reduction in outer retinal layer thickness.

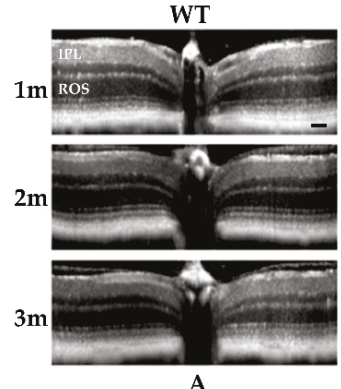

A

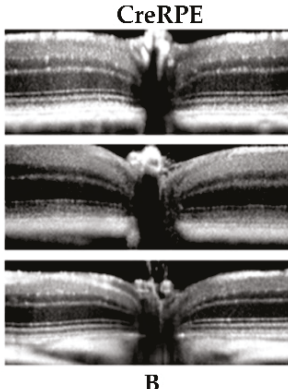

B

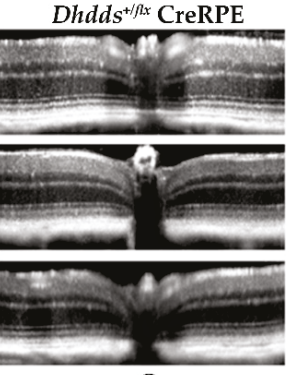

C

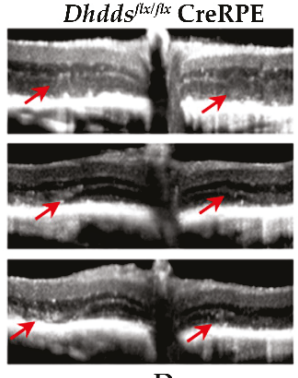

D

Figure 3. SD-OCT revealed structural changes in Dhdds $f x f f l x$ CreRPE mice at all ages. OCT scans were performed at 1, 2, and 3 months (m) postnatal for each genotype. (A) WT, (B) CreRPE, (C) Dhdds ${ }^{+/ f l x}$ CreRPE all showed normal layer stratification. (D) Dhdds flxfflx CreRPE showed alterations of hyper-reflectivity at all ages, indicative of pathologic changes (red arrows) and reduction in layer thickness, particularly in the outer retina. IPL, inner plexiform layer, ROS, rod outer segment. Scale bar (shown in WT $1 \mathrm{~m}$ panel): $50 \mu \mathrm{m}$, applies to all panels.

"Spidergram" plots of average thickness values (in $\mathrm{mm}$ ) vs. retinal eccentricity (distance from the optic nerve head $(\mathrm{ONH})$ along with vertical meridian) for the outer nuclear layer (ONL) and FRT are shown in Figure 4. No significant differences were observed when comparing WT, heterozygous, or Cre-only mice for both ONL and FRT measurements. However, Dhddfflxfflx CreRPE mice showed a significant reduction (vs. WT) in ONL and F.R.T. values at all ages analyzed ( $\mathrm{n} \geq 4$, all $p<0.001)$.

Histologically, at PN 3 months, light micrograph images revealed that all retina layers in mice lacking Cre expression appeared normal (Figure 5A and Supplementary Materials, Figure S1A). In contrast, the age-matched Dhdds ${ }^{\mathrm{fl} x / f \mathrm{fl}}$ Cre RPE mice displayed a geographic atrophy-like RPE appearance with the most degeneration observed mid-centrally throughout the retina (Supplementary Materials, Figure S1B). There were regions of well-preserved Dhdds ${ }^{\mathrm{fl} / \mathrm{flx}}$ Cre RPE retina that 
showed severe RPE pathology (Figure 5B). Notably, the descent of the external limiting membrane (ELM) [25] towards Bruch's membrane was also observed (Figure 5C,E). There was a near-total loss of photoreceptors and RPE within the most affected regions (Figure 5D). Very well-preserved regions were also observed in the periphery (Figure 5F).
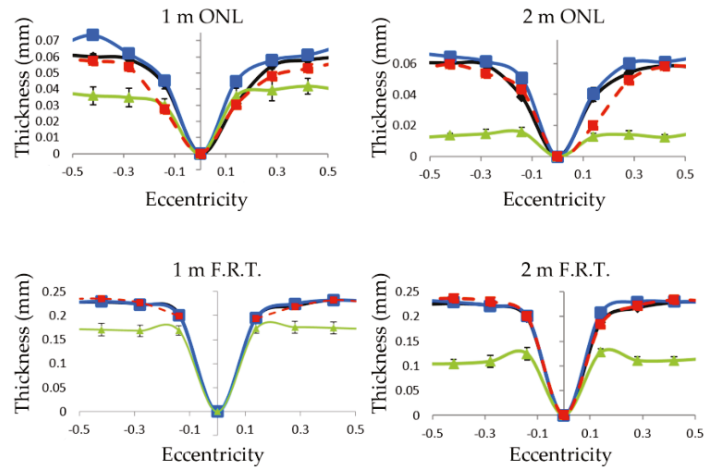

$-\mathrm{WT}$

- Dhdds $^{+/ f x}$ CreRPE

$\rightarrow$ CreRPE

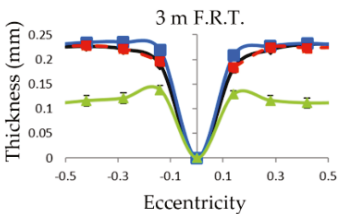

Figure 4. Quantitative morphometric analysis of WT, CreRPE, Dhdds ${ }^{+/ f l x}$ CreRPE, and Dhdds $f l x f f l x$ CreRPE mice SD-OCT data. Average OCT measurements at each eccentricity revealed no significant differences when comparing WT, CreRPE and Dhdds ${ }^{+/ f l x}$ CreRPE mice for both outer nuclear layer (ONL) and F.R.T. thickness measurements ( $\mathrm{n}=4$ for all genotypes, except for WT at one month ( $1 \mathrm{~m}$; $\mathrm{n}=8$ ) and two months ( $2 \mathrm{~m} ; \mathrm{n}=5)$. However, significant changes were observed when comparing WT and Dhdds ${ }^{f l x} f f x$ CreRPE mice at any age with respect to both ONL and F.R.T. values.
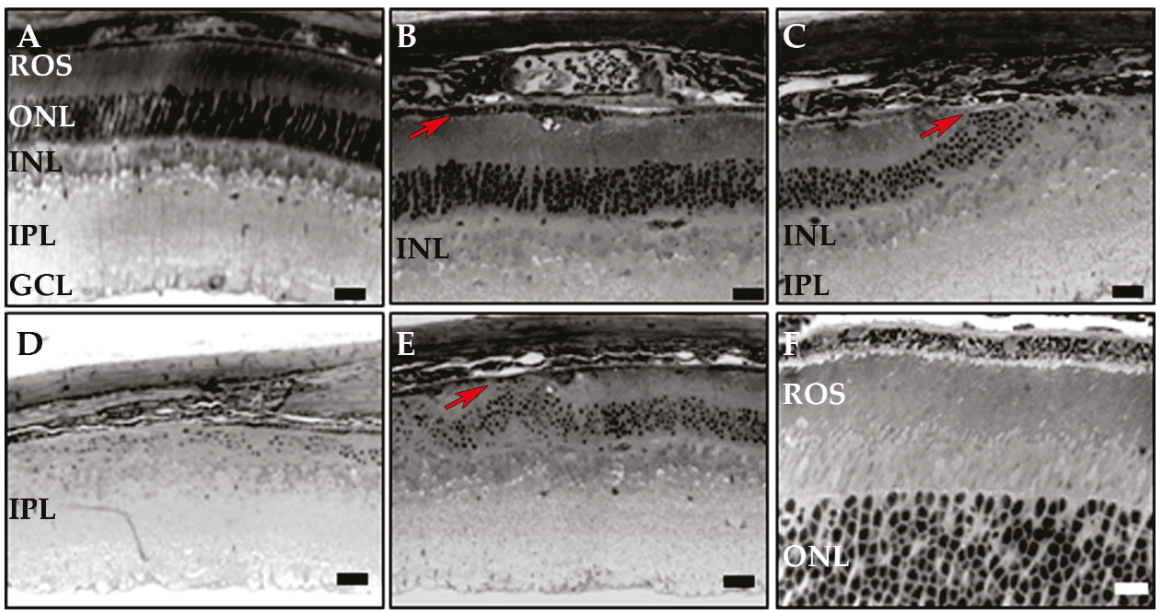

Figure 5. Pathology observed in Dhddf $f^{f l x f l x}$ Cre RPE mouse retina. (A) Dhdds $s^{f l x f f l x}$ retina without Cre expression appears normal. (B-F) Five regions of a retina from a Dhdds ${ }^{f l x f f l x}$ CreRPE mouse expressing Cre in RPE are shown. (B,F) Relatively well-preserved peripheral retina with some photoreceptor loss, outer segment (OS) shortening, and differing severity of RPE pathology are shown (arrow in panel B points to severely compromised RPE. (C,E) Transition zones of severe to mild retinal pathology showing loss of photoreceptors, severe compromise of RPE and external limiting membrane (ELM) descent (arrows). (D) A more central region of the retina showing severe cell loss in both the retina and RPE. Scale bars (all panels, except F): $20 \mu \mathrm{m}$; scale bar, panel F: $10 \mu \mathrm{m}$. ROS, rod outer segments; ONL, outer nuclear layer, INL, inner nuclear layer; IPL, inner plexiform layer, GCL, ganglion cell layer. 
Additional pathology was revealed by higher magnification EM analysis (Figure 6, panels $\mathrm{C}-\mathrm{O})$. In contrast, Dhdds ${ }^{f l x f f l x}$ retinas without Cre expression were indistinguishable from WT retinas (Figure 6A,B). The two areas most affected showed severe RPE dystrophy with concomitant degeneration and loss of photoreceptor cells (Figure 6C,D,F,G,J,M-O). ELM descent was apparent in areas where there was a transition from milder to more severe pathology (* in panels D-F,J). RPE cell transmigration was apparent in the outer retina (arrows, panels G,J,O,L). Thus, compared to WT neural retina and RPE, the observed RPE anomalies including migration of nucleus and RPE melanosomes, displacement of the ELM, and shortened misshaped outer segments throughout the retina were seen in the Dhddfflxfflx CreRPE mice.
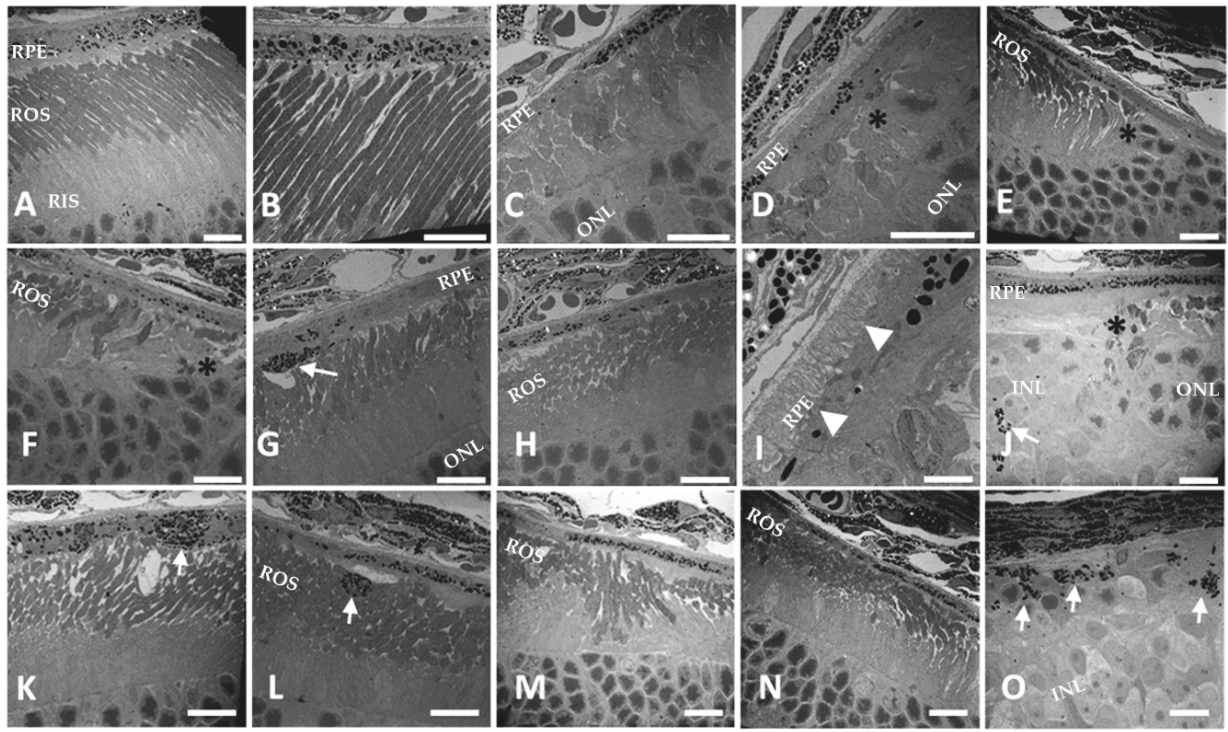

Figure 6. High magnification observation of Dhdds ${ }^{f l x f f l x}$ CreRPE retina. EM images of $3 \mathrm{~m}(\mathbf{A}, \mathbf{B})$ $D h d d s^{f l x f f l x}$ and (C-O) Dhdds ${ }^{f l x f f l x}$ CreRPE mice. Mice homozygous for the floxed Dhdds allele (Dhdds $\left.{ }^{f l x} / f l x\right)$ are not distinguishable from WT. (A) Rod outer segments are properly aligned and all retinal layers are intact (only rod inner and outer segments and the outer nuclear layer are shown). (B) The RPE shows normal thickness and melanin distribution. (C-O) Examples of mildly and severely compromised RPE and retina in Dhdds ${ }^{f l x f l x}$ CreRPE mice. Severe RPE and outer retina degeneration is concentrated mid-centrally on both sides of the optic nerve head (see histology, Figure 5, and Supplementary Materials, Figure S1). Severe RPE/PR atrophy is observed in the most affected central regions (C-F,I,J,O). External limiting membrane (ELM) descent $\left(^{*}\right)$ towards the RPE is apparent in the transition regions from severely affected to more intact regions $(\mathbf{D}-\mathbf{F}, \mathbf{J})$. Transmigration of RPE cellular material into the ROS space (arrows) is also seen $(\mathbf{G}, \mathbf{J}, \mathbf{L}, \mathbf{O})$. Extended RPE basal fenestrations (arrowheads, $\mathbf{I}$ ) are apparent in the most affected regions $(\mathbf{D}, \mathbf{I})$. Outside of the central region of severe degeneration are regions with compromised but still apparent retinal and RPE layers (K-N). Scale bars (all panels, except I): $10 \mu \mathrm{m}$; panel I, $2.5 \mu \mathrm{m}$.

To further examine the effects of Dhdds deletion in the RPE, serial block face-scanning electron microscopy was used to examine a $\sim 100 \mu^{3}$ region of the $D h d d s^{f l x f f l x}$ retina across an area of transition from milder to more severe pathology (Supplementary Materials, Videos S1-3). In WT mice, the retina appeared normal in all layers across the entire block. Examination of two regions of the Dhdds ${ }^{f l x f f l x}$ Cre RPE retina showed areas of severe compromise as well as areas where retinal histology was more well-preserved. Transmigration of RPE nuclei and melanosomes into the photoreceptor 
region (subretinal space and photoreceptor outer segment layer, and even deeper, into the ONL) was also apparent.

\subsection{Altered Scotopic and Photopic ERG Amplitudes in Dhdds flx/flx CreRPE Mice}

Analysis by ERG (Figure 7) showed that both homozygous and heterozygous Dhdds ${ }^{f l x f l x}$ CreRPE mice exhibited reduced scotopic ERG a- and b-wave responses. At PN 1 month, scotopic a-wave responses $(289 \pm 42 \mu \mathrm{V} ; \mathrm{n}=5)$ were significantly reduced in Dhdds ${ }^{\text {flx/flx }}$ CreRPE mice compared to WT mice $(395 \pm 14 \mu \mathrm{V} ; \mathrm{n}=8 ; p<0.05)$. Scotopic b-wave responses also were reduced for both $D h d d s^{+/ f l x}$ CreRPE $(772 \pm 42 \mu \mathrm{V} ; \mathrm{n}=20)$ and $D^{2} d d s^{f l x f f l x}$ CreRPE $(479 \pm 69 \mu \mathrm{V} ; \mathrm{n}=5)$ mice, compared to WT mice $(934 \pm 38 \mu \mathrm{V} ; \mathrm{n}=8 ; p<0.01)$. At PN 2 months of age, Dhdds flxfflx CreRPE scotopic ERG a-wave $(223 \pm$ $48 \mu \mathrm{V} ; \mathrm{n}=10)$ and b-wave $(477 \pm 88 \mu \mathrm{V} ; \mathrm{n}=10)$ responses and Dhdds ${ }^{+/ f l x}$ CreRPE a-wave $(305 \pm 19 \mu \mathrm{V}$; $\mathrm{n}=10)$ and $\mathrm{b}$-wave $(557 \pm 64 \mu \mathrm{V} ; \mathrm{n}=10)$ responses were significantly reduced compared to the WT a-wave $(366 \pm 10 \mu \mathrm{V} ; \mathrm{n}=15)$ and $\mathrm{b}$-wave $(935 \pm 27 \mu \mathrm{V} ; \mathrm{n}=15)$ responses $(p<0.005$ for all comparisons). At PN 3 months, scotopic a-wave amplitudes were reduced only in Dhdds ${ }^{\text {flx/flx }}$ CreRPE $(60 \pm 48 \mu \mathrm{V}$; $\mathrm{n}=6)$ compared to WT mice $(354 \pm 16 \mu \mathrm{V} ; \mathrm{n}=18 ; p<0.001)$; b-wave responses were reduced in both Dhdds ${ }^{+/ f l x}$ CreRPE $(662 \pm 88 \mu \mathrm{V} ; \mathrm{n}=6)$ and Dhdds ${ }^{f l x / f l x}$ CreRPE $(150 \pm 105 \mu \mathrm{V} ; \mathrm{n}=6)$ mice compared to WT mice $(914 \pm 35 \mu \mathrm{V} ; \mathrm{n}=18) p<0.05$ and $p<0.01$, respectively).
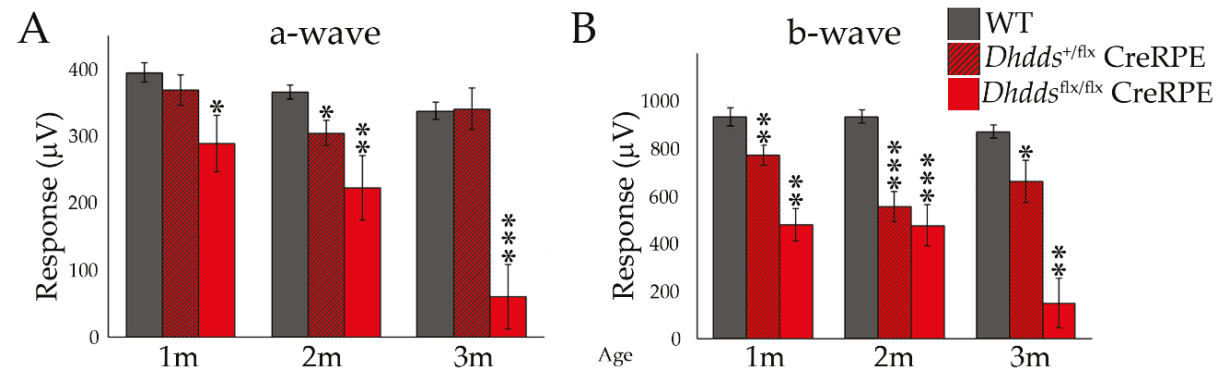

Figure 7. Scotopic ERG analysis of WT, Dhdds $+f f x$ CreRPE, Dhdds ${ }^{f l x f f l x}$ CreRPE mice. Maximum responses to saturating light stimuli showed significant decreases in (A) a-wave and (B) b-wave amplitudes for Dhdds ${ }^{+/ f l x}$ CreRPE and Dhdds flxfflx CreRPE when compared to WT mice at 1, 2 and 3 postnatal months. Statistical significance: ${ }^{*} p<0.05,{ }^{* *} p<0.01,{ }^{* * *} p<0.001$.

The photopic a-wave and b-wave responses (Figure 8) at PN 1 month were not different when comparing WT $(\mathrm{n}=5)$ and Dhdds $/ f l x$ CreRPE $(\mathrm{n}=13)$ mice, however the photopic responses of $D h d d s f l x f l x$ CreRPE mice $(n=4)$ were significantly lower. Photopic ERG responses were significantly different only for $D h d d s^{+/ f l x}$ CreRPE a-wave $(29 \pm 2 \mu \mathrm{V} ; \mathrm{n}=4 ; p<0.01)$, but not b-wave, responses at PN 2 months of age. In Dhdds ${ }^{f l x f f l x}$ CreRPE mice, b-wave $(86 \pm 19 \mu \mathrm{V} ; \mathrm{n}=6)$ amplitudes were significantly reduced $(p<0.01)$, compared to WT mice $(n=13)$, but not the a-wave responses. At PN 3 months, Dhdds ${ }^{f l x} / f l x$ CreRPE mice exhibited significant functional impairment in the photopic a-wave $(2 \pm 1 \mu \mathrm{V}$; $\mathrm{n}=4)$ and b-wave $(7 \pm 1 \mu \mathrm{V} ; \mathrm{n}=4)$ responses compared to WT a-wave $(14 \pm 1 \mu \mathrm{V} ; \mathrm{n}=9)$ and b-wave responses $(144 \pm 8 \mu \mathrm{V} ; \mathrm{n}=9 ; p<0.001$ for all comparisons).

Optokinetic reflex (OKR) analysis (Supplementary Materials, Figure S3), a measure of retina-tobrain transmission (i.e., visual capacity), showed reductions in photopic (4-31\%) and scotopic (8-29\%) contrast sensitivity over the range of 0.031 to $0.272 \mathrm{c} / \mathrm{d}$ in PN 3-month old Dhdds ${ }^{f l x / f l x}$ CreRPE mice $(p<0.05)$, compared to WT controls of the same age. However, no differences in spatial frequency (a measure of visual acuity) were observed between the different mouse lines. This is partly evident in the OKR scotopic and photopic plots, which show a similar high-frequency cut-off for all three mouse lines examined. 
A

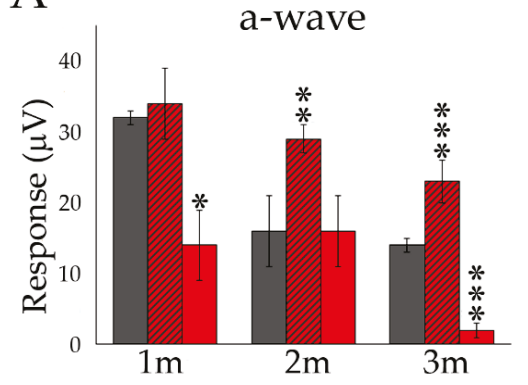

B

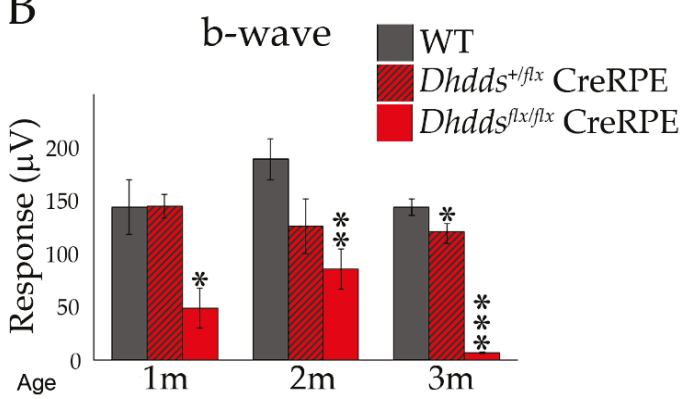

Figure 8. Photopic ERG analysis of WT, CreRPE Dhdds ${ }^{+/ f l x}$, CreRPE Dhdds $f$ flftx mice. (A) a-wave responses were significantly lower in CreRPE Dhdds ${ }^{--}$at $1(\mathrm{n}=4)$ and $3(\mathrm{n}=4)$ months postnatal compared to WT (one month $(1 \mathrm{~m}), \mathrm{n}=5$; three months $(3 \mathrm{~m}), \mathrm{n}=9$ ) mice. (B) Photopic b-wave amplitudes for Dhdds ${ }^{+f f l x}$ CreRPE mice showed no statistically significant differences when compared to WT mice at $1(n=13)$ and $2(n=4)$ postnatal months, but were reduced by 3 months $(n=4)$. In contrast, the responses of Dhdds ${ }^{f l x f l x}$ CreRPE mice (two months $(2 \mathrm{~m}), \mathrm{n}=6$ ) were significantly lower when compared to WT mice at all postnatal ages examined. Statistical significance: ${ }^{*} p<0.05,{ }^{* *} p<0.01$, $* * * p<0.001$.

\section{Discussion}

Studies involving genetic screening of families with autosomal recessive retinitis pigmentosa have implicated a founder missense mutation (K42E) in the gene encoding DHDDS [12,26]. DHDDS is required for $\mathrm{N}$-glycosylation of proteins by adding multiple copies of isopentenyl pyrophosphate (IPP) to farnesyl pyrophosphate (FPP) to produce dehydrodolichyl diphosphate (Dedol-PP), a precursor of dolichol, which is utilized as a sugar carrier in protein glycosylation in the endoplasmic reticulum [6]. Even though the generation of isoprenoid chains is complex, involving multiple enzymes and enzyme complexes [27], only DHDDS and Nogo-B receptor are required for long-chain isoprenoid synthesis $\left(C_{70}-C_{120}\right)$ [28]. Previous studies have reported that mutations in the opsin gene that abolish N-linked glycosylation cause retinal degeneration [28-30]. To understand the basis for the ocular pathology associated with DHDDS mutation, we utilized a Cre recombinase conditional knockout (Dhddf $f^{f x / f l x}$ CreRPE) driven under the control of the RPE-specific VMD2 promoter to achieve RPE-targeted excision of the loxP-modified Dhdds gene, which renders the enzyme non-functional.

The VMD2 construct was originally generated to be conditional on the presence of doxycycline; however, we found that the mice displayed a phenotype in the absence of doxycycline induction. While we cannot rule out the presence of some level of doxycycline in the standard chow mouse diet, it is more likely that Cre expression was due to "leaky" expression, which bypassed doxycycline control, as confirmed in Figure 2. Since the promoters used for cell-specific targeting of Cre expression turn on after development is complete, developmental changes that would otherwise preempt retina/RPE development were not observed.

The expression of Cre recombinase in the Cre lines was confirmed using a ZsGreen reporter strategy. The Dhdds flxfflx CreRPE mice exhibited about $90 \%$ coverage of Cre expression by PN 1 month (Figure 2) which persisted up to 3 months at least. We crossed the conditional Dhdds $s^{f l x f l x}$ lines with heterozygous VMD2 Cre lines to generate homozygous Dhdds flxfflx mice with RPE-specific knockout of DHDDS expression as the model for our study.

In vivo imaging suggested that ONL thickness and F.R.T. were comparable between age-matched WT and Dhdds ${ }^{+f f x}$ CreRPE mice, but significantly reduced in Dhdds stxfllx CreRPE mice. While the specific pathology is observed in much greater detail in the histological (light microscopy; Figure 5) and ultrastructural (electron microscopy; Figure 6) images provided, it is clear that the pathology indicated in the SD-OCT tomograms (Figure 3D) is consistent with the histological observations. This altered structural integrity of $D h d d s s^{f l x} / f l x$ CreRPE retinas also corresponded to a significant decrease 
in a-wave and b-wave compared to age-matched WT at all flash intensities. Unexpectedly, the scotopic a- and b-waves also were reduced in Dhdds ${ }^{+f f x}$ CreRPE mice, which may suggest a functional change that occurs prior to any obvious retinal structural changes and suggests that 50\% DHDDS activity is insufficient in the RPE to maintain the required enzymatic activity level. Thus, carriers of Dhdds mutations also may develop visual defects, depending on the nature of the mutation, and other factors, such as genetic background and environment. This agrees with the rod-cone dystrophy reported in patients with autosomal recessive RP [16]. However, we cannot rule out the expression of a truncated protein that leads to a gain of function. This could explain the significant attenuation of the photoresponse in mice heterozygous for the floxed allele (Figures 7 and 8). It could also possibly explain the manifestation of the disease only in ocular tissues, if the gain of function is due to specific targeting of retina-specific protein complexes. Further experimentation will be required to sort this out.

We carried out further experiments to examine the fundus of older Dhdds ${ }^{f l x f f l x}$ CreRPE mice at 6, 8 and 10 months PN. These fundus images obtained with fluorescein angiography showed the classical signs of RP including abnormal pigmentation in 8-month old Dhdds ${ }^{f l x f f l x}$ CreRPE mice; vascular changes, including microaneurysms, increased vessel tortuosity and attenuation, were also observed by 6 months of age (Supplementary Materials, Figure S2).

Originally it was proposed that the cause of the pathology in DHDDS-related RP patients (RP59) is defective glycosylation of rod opsin because of reduced DHDDS enzyme activity [12,26]. While this assumption may explain the classic RP symptoms observed, it fails to explain the AMD-like macular involvement. Interestingly, Lam and colleagues [11] found no N-glycosylation deficiency in RP59 patients, based upon isoelectric focusing gel analysis of plasma transferrin (a systemic glycoprotein). In addition, in a related study (Rao et al., manuscript submitted for publication), utilizing a rod photoreceptor-specific knockout of Dhdds in mice, we found no evidence for a resulting lack of protein glycosylation in the retina; yet, there was a rapidly progressing photoreceptor degeneration, resulting in complete loss of photoreceptors by PN 6 weeks of age. Also, in a companion article in this Special Issue [31], we generated a mouse model harboring the global K42E homozygous Dhdds mutation associated with RP59 patients, but observed no retinal degeneration, even out to 9 postnatal months of age. Clearly, mutations that only partially diminish enzymatic activity would be far less severe than a complete ablation of the gene encoding the enzyme.

\section{Conclusions}

From these observations, we conclude that targeted ablation of Dhdds selectively in RPE cells results in perturbation of DHDDS-dependent processes, resulting in structural and functional deficits in both the RPE and photoreceptors, in a manner resembling geographic atrophy. The degeneration progresses relatively slowly (compared to the rod-specific Dhdds ablation model) over the course of a few months, rather than weeks.

Supplementary Materials: The following are available online at http://www.mdpi.com/2073-4409/9/3/771/s1, Figure S1: Stitched images of $D h d d s f l x f l x$ and $D h d d s f l x f l x$ Cre RPE mouse retina, Figure S2: Fundus imaging and fluorescein angiography (FA), Figure S3: Contrast sensitivity and spatial frequency assessment in WT, Dhdds $f(x) f l x$, and Dhddf $f^{f l f f l x}$ Cre RPE mice, Video S1: Serial Block-Face Scanning Electron Microscopy of WT Dhdds $f^{f l x f f l x}$, Video S2: Serial Block-Face Scanning Electron Microscopy of region 1 of Dhdds ${ }^{f l x f f l x}$ Cre RPE, Video S3: Serial Block-Face Scanning Electron Microscopy of region 2 of Dhdds $s^{f l x f l x}$ Cre RPE.

Author Contributions: Conceptualization, S.J.P., S.R.R., S.J.F. and M.L.D.; methodology, S.J.D., M.L.D., S.J.F., and S.J.P.; software, S.J.P., M.L.D., and T.W.K; validation, S.J.D., M.L.D., D.S., S.R.R., and S.J.P.; data curation, M.L.D., C.N., S.R.R., S.J.F. and S.J.P.; writing—original draft preparation, M.L.D., C.N., and S.J.P.; writing-review and editing, S.J.F., S.J.P., S.R.R., M.L.D., and D.S., visualization, S.J.P., S.R.R., and S.J.F.; supervision, T.W.K., S.J.F., and S.J.P.; project administration, S.J.F. and S.J.P.; funding acquisition, S.J.P. and S.J.F. All authors have read and agreed to the published version of the manuscript.

Funding: This research was supported by U.S.P.H.S. (National Institutes of Health/National Eye Institute) grant R01 EY029341 to S.J.P. and S.J.F., support from the UAB Vision Science Research Center, and a core grant P30 EY003039 to SJP, as well as facilities and resources provided by the VA Western NY Healthcare System (S.J.F., S.R.R.). 
Acknowledgments: We thank Jeffrey Messinger for expert technical assistance with light level histology and TEM, Isaac Cobb for technical assistance with some of the experiments, Amy Stablewski (Roswell Park Cancer Institute (RPCI) Gene Targeting and Transgenic Facility) for the generation of Dhdds flxfflx mice, and Yun Le (University of Oklahoma Health Sciences Center) for generously providing the CreRPE mouse line. The opinions expressed herein do not reflect those of the Department of Veteran Affairs or the U.S. Government.

Conflicts of Interest: The authors declare no conflict of interest. The funders of this study had no role in the design; in the collection, analyses, or interpretation of data; in the writing of the manuscript; or in the decision to publish the results.

\section{References}

1. Dryja, T.P.; McGee, T.L.; Reichel, E.; Hahn, L.B.; Cowley, G.S.; Yandell, D.W.; Sandberg, M.A.; Berson, E.L. A point mutation of the rhodopsin gene in one form of retinitis pigmentosa. Nature 1990, 343, 364-366. [CrossRef] [PubMed]

2. Bok, D.; Hall, M.O. The role of the pigment epithelium in the etiology of inherited retinal dystrophy in the rat. J. Cell Biol. 1971, 49, 664-682. [CrossRef] [PubMed]

3. Clark, V.M.; Hall, M.O. RPE cell surface proteins in normal and dystrophic rats. Investig. Ophthalmol. Vis. Sci. 1986, 27, 136-144.

4. Clark, V.M.; Zhou, X.Y.; Pfeffer, B.A. Partial characterization of fucosylated cell surface glycoproteins of cultured RPE. Curr. Eye Res. 1990, 9, 977-986. [CrossRef] [PubMed]

5. Edwards, R.B.; Szamier, R.B. Defective phagocytosis of isolated rod outer segments by RCS rat retinal pigment epithelium in culture. Science 1977, 197, 1001-1003. [CrossRef]

6. Buczkowska, A.; Swiezewska, E.; Lefeber, D.J. Genetic defects in dolichol metabolism. J. Inherit. Metab. Dis. 2015, 38, 157-169. [CrossRef]

7. Giladi, M.; Edri, I.; Goldenberg, M.; Newman, H.; Strulovich, R.; Khananshvili, D.; Haitin, Y.; Loewenstein, A. Purification and characterization of human dehydrodolychil diphosphate synthase (DHDDS) overexpressed in E. coli. Protein Expr. Purif. 2017, 132, 138-142. [CrossRef]

8. Kean, E.L. The dolichol pathway in the retina and its involvement in the glycosylation of rhodopsin. Biochim. Biophys. Acta 1999, 1473, 272-285. [CrossRef]

9. Nowycky, M.C.; Wu, G.; Ledeen, R.W. Glycobiology of ion transport in the nervous system. Adv. Neurobiol. 2014, 9, 321-342. [CrossRef]

10. Wimmers, S.; Karl, M.O.; Strauss, O. Ion channels in the RPE. Prog. Retin. Eye Res. 2007, 26, 263-301. [CrossRef]

11. Lam, B.L.; Zuchner, S.L.; Dallman, J.; Wen, R.; Alfonso, E.C.; Vance, J.M.; Pericak-Vance, M.A. Mutation K42E in dehydrodolichol diphosphate synthase (DHDDS) causes recessive retinitis pigmentosa. Adv. Exp. Med. Biol. 2014, 801, 165-170. [CrossRef] [PubMed]

12. Zelinger, L.; Banin, E.; Obolensky, A.; Mizrahi-Meissonnier, L.; Beryozkin, A.; Bandah-Rozenfeld, D.; Frenkel, S.; Ben-Yosef, T.; Merin, S.; Schwartz, S.B.; et al. A missense mutation in DHDDS, encoding dehydrodolichyl diphosphate synthase, is associated with autosomal-recessive retinitis pigmentosa in Ashkenazi Jews. Am. J. Hum. Genet. 2011, 88, 207-215. [CrossRef] [PubMed]

13. Venturini, G.; Koskiniemi-Kuendig, H.; Harper, S.; Berson, E.L.; Rivolta, C. Two specific mutations are prevalent causes of recessive retinitis pigmentosa in North American patients of Jewish ancestry. Genet. Med. 2015, 17, 285-290. [CrossRef] [PubMed]

14. Ng, B.G.; Freeze, H.H. Perspectives on Glycosylation and Its Congenital Disorders. Trends Genet. 2018, 34, 466-476. [CrossRef]

15. Biswas, P.; Duncan, J.L.; Maranhao, B.; Kozak, I.; Branham, K.; Gabriel, L.; Lin, J.H.; Barteselli, G.; Navani, M.; Suk, J.J.; et al. Genetic analysis of ten pedigrees with inherited retinal degeneration (IRD) by exome sequencing and phenotype-genotype association. Physiol. Genom. 2017, 49, 216-229. [CrossRef]

16. Kimchi, A.; Khateb, S.; Wen, R.; Guan, Z.; Obolensky, A.; Beryozkin, A.; Kurtzman, S.; Blumenfeld, A.; Pras, E.; Jacobson, S.G.; et al. Nonsyndromic Retinitis Pigmentosa in the Ashkenazi Jewish Population: Genetic and Clinical Aspects. Ophthalmology 2018, 125, 725-734. [CrossRef]

17. Sabry, S.; Vuillaumier-Barrot, S.; Mintet, E.; Fasseu, M.; Valayannopoulos, V.; Heron, D.; Dorison, N.; Mignot, C.; Seta, N.; Chantret, I.; et al. A case of fatal Type I congenital disorders of glycosylation (CDG I) associated with low dehydrodolichol diphosphate synthase (DHDDS) activity. Orphanet J. Rare Dis. 2016, 11, 84. [CrossRef] 
18. Ferrara, N.; Carver-Moore, K.; Chen, H.; Dowd, M.; Lu, L.; O'Shea, K.S.; Powell-Braxton, L.; Hillan, K.J.; Moore, M.W. Heterozygous embryonic lethality induced by targeted inactivation of the VEGF gene. Nature 1996, 380, 439-442. [CrossRef]

19. Rucker, E.B., 3rd; Dierisseau, P.; Wagner, K.U.; Garrett, L.; Wynshaw-Boris, A.; Flaws, J.A.; Hennighausen, L. $\mathrm{Bcl}-\mathrm{x}$ and Bax regulate mouse primordial germ cell survival and apoptosis during embryogenesis. Mol. Endocrinol. 2000, 14, 1038-1052. [CrossRef]

20. Le, Y.Z.; Zheng, W.; Rao, P.C.; Zheng, L.; Anderson, R.E.; Esumi, N.; Zack, D.J.; Zhu, M. Inducible expression of cre recombinase in the retinal pigmented epithelium. Investig. Ophthalmol. Vis. Sci. 2008, 49, 1248-1253. [CrossRef] [PubMed]

21. Le, Y.Z.; Zhu, M.; Anderson, R.E. Cre Recombinase: You Can't Live with It, and You Can't Live Without It. Adv. Exp. Med. Biol. 2016, 854, 725-730. [CrossRef] [PubMed]

22. DeRamus, M.L.; Stacks, D.A.; Zhang, Y.; Huisingh, C.E.; McGwin, G.; Pittler, S.J. GARP2 accelerates retinal degeneration in rod cGMP-gated cation channel beta-subunit knockout mice. Sci. Rep. 2017, 7, 42545. [CrossRef] [PubMed]

23. Stricker, H.M.; Ding, X.Q.; Quiambao, A.; Fliesler, S.J.; Naash, M.I. The Cys214->Ser mutation in peripherin/rds causes a loss-of-function phenotype in transgenic mice. Biochem. J. 2005, 388, 605-613. [CrossRef] [PubMed]

24. Tu, C.; Li, J.; Jiang, X.; Sheflin, L.G.; Pfeffer, B.A.; Behringer, M.; Fliesler, S.J.; Qu, J. Ion-current-based proteomic profiling of the retina in a rat model of Smith-Lemli-Opitz syndrome. Mol. Cell. Proteom. 2013, 12, 3583-3598. [CrossRef] [PubMed]

25. Zanzottera, E.C.; Ach, T.; Huisingh, C.; Messinger, J.D.; Spaide, R.F.; Curcio, C.A. Visualizing retinal pigment epithelium phenotypes in the transition to geographic atrophy in age-related macular degeneration. Retina 2016, 36, S12-S25. [CrossRef]

26. Zuchner, S.; Dallman, J.; Wen, R.; Beecham, G.; Naj, A.; Farooq, A.; Kohli, M.A.; Whitehead, P.L.; Hulme, W.; Konidari, I.; et al. Whole-exome sequencing links a variant in DHDDS to retinitis pigmentosa. Am. J. Hum. Genet. 2011, 88, 201-206. [CrossRef]

27. Grabińska, K.A.; Park, E.J.; Sessa, W.C. cis-Prenyltransferase: New insights into protein glycosylation, rubber synthesis, and human diseases. J. Biol. Chem. 2016, 291, 18582-18590. [CrossRef]

28. Park, E.J.; Grabinska, K.A.; Guan, Z.; Stranecky, V.; Hartmannova, H.; Hodanova, K.; Baresova, V.; Sovova, J.; Jozsef, L.; Ondruskova, N.; et al. Mutation of Nogo-B receptor, a subunit of cis-prenyltransferase, causes a congenital disorder of glycosylation. Cell Metab. 2014, 20, 448-457. [CrossRef]

29. Murray, A.R.; Fliesler, S.J.; Al-Ubaidi, M.R. Rhodopsin: The functional significance of asn-linked glycosylation and other post-translational modifications. Ophthalmic Genet. 2009, 30, 109-120. [CrossRef]

30. White, D.A.; Fritz, J.J.; Hauswirth, W.W.; Kaushal, S.; Lewin, A.S. Increased sensitivity to light-induced damage in a mouse model of autosomal dominant retinal disease. Investig. Ophthalmol. Vis. Sci. 2007, 48, 1942-1951. [CrossRef]

31. Ramachanra Rao, S.; Fliesler, S.J.; Nguyen, M.N.; Kotla, P.; Pittler, S.J. Lack of overt retinal degeneration in K42E DHDDS knock-in mouse model of RP59. Cells 2020. submitted.

(C) 2020 by the authors. Licensee MDPI, Basel, Switzerland. This article is an open access article distributed under the terms and conditions of the Creative Commons Attribution (CC BY) license (http://creativecommons.org/licenses/by/4.0/). 

Article

\title{
Diurnal Rhythmicity of Autophagy Is Impaired in the Diabetic Retina
}

\author{
Xiaoping Qi ${ }^{1}$, Sayak K. Mitter ${ }^{1}$, Yuanqing Yan ${ }^{2}$, Julia V. Busik ${ }^{3}$, Maria B. Grant ${ }^{1}$ \\ and Michael E. Boulton ${ }^{1, *}$ \\ 1 Department of Ophthalmology and Visual Sciences, University of Alabama at Birmingham, AL 35294, USA; \\ xqi@uabmc.edu (X.Q.); sayakmitter@gmail.com (S.K.M.); mariagrant@uabmc.edu (M.B.G.) \\ 2 Department of Neurosurgery, The University of Texas Health Science Center, Houston, TX 77030, USA; \\ Yuanqing.Yan@uth.tmc.edu \\ 3 Department of Physiology, Michigan State University, East Lansing, MI 48824, USA; busik@msu.edu \\ * Correspondence: meboulton@uabmc.edu
}

Received: 1 March 2020; Accepted: 2 April 2020; Published: 7 April 2020

\begin{abstract}
Retinal homeostasis is under both diurnal and circadian regulation. We sought to investigate the diurnal expression of autophagy proteins in normal rodent retina and to determine if this is impaired in diabetic retinopathy. C57BL/6J mice and Bio-Breeding Zucker (BBZ) rats were maintained under a $12 \mathrm{~h} / 12 \mathrm{~h}$ light/dark cycle and eyes, enucleated over a $24 \mathrm{~h}$ period. Eyes were also collected from diabetic mice with two or nine-months duration of type 1 diabetes (T1D) and Bio-Breeding Zucker diabetic rat (BBZDR/wor rats with 4-months duration of type 2 diabetes (T2D). Immunohistochemistry was performed for the autophagy proteins Atg7, Atg9, LC3 and Beclin1. These autophagy proteins (Atgs) were abundantly expressed in neural retina and endothelial cells in both mice and rats. A differential staining pattern was observed across the retinas which demonstrated a distinctive diurnal rhythmicity. All Atgs showed localization to retinal blood vessels with Atg7 being the most highly expressed. Analysis of the immunostaining demonstrated distinctive diurnal rhythmicity, of which Atg9 and LC3 shared a biphasic expression cycle with the highest level at 8:15 am and 8:15 pm. In contrast, Beclin1 revealed a 24-h cycle with the highest level observed at midnight. Atg7 was also on a 24-h cycle with peak expression at 8:15 am, coinciding with the first peak expression of Atg9 and LC3. In diabetic animals, there was a dramatic reduction in all four Atgs and the distinctive diurnal rhythmicity of these autophagy proteins was significantly impaired and phase shifted in both T1D and T2D animals. Restoration of diurnal rhythmicity and facilitation of autophagy protein expression may provide new treatment strategies for diabetic retinopathy.
\end{abstract}

Keywords: diurnal rhythm; autophagy; retina; diabetes; diabetic retinopathy

\section{Introduction}

Macroautophagy (hereafter referred to as autophagy) is an evolutionarily conserved cellular catabolic mechanism that facilitates the degradation of damaged cellular organelles and proteins, by targeting them to the lysosomes and recycling the macromolecules for the rebuilding of cellular machinery [? ? ? . Autophagy undergoes rhythmic variation in accordance with circadian patterns of rest/activity and feeding in adult mammals [? ]. Dysregulated autophagy has been implicated in several neurodegenerative disorders, hepatitis, cancer, aging associated diseases and in the general aging process [? ? ? ? ? ]. Recently, a growing body of evidence indicates that dysregulated autophagy is also linked to diabetes [? ? ? ].

The disruption of circadian rhythm has a profound negative impact on health and is associated with elevated risk for several diseases [? ]. The physiological relevance of an altered circadian rhythm in diabetes is evidenced by the observation of a high incidence of myocardial dysfunction, acute 
coronary syndrome, sudden cardiac death, and ischemic stroke in diabetics during the night, compared to a higher frequency during the day in non-diabetics [? ? ? ? ]. In diabetic conditions, Bmal1 and Clock are inactivated, causing deregulated glucose homeostasis and suppressed diurnal variation in glucose and triglycerides, along with reduced gluconeogenesis [? ]. Streptozotocin (STZ)-induced type 1 diabetes (T1D) in mice exhibits altered phase of the circadian clock in the heart [? ] and a significant reduction of circadian sensitivity to low-intensity light in the retina [? ]. Furthermore, STZ-mice develop a deficiency in their ability to re-entrain the circadian rhythm when subjected to a phase advance of the 12L/12D cycle [? ]. Bio-Breeding Zucker diabetic rat (BBZDR/Wor) and Goto-Kakizaki rat type 2 diabetes (T2D) models also show impairment of the molecular clock, suggesting that the disruption of the circadian clock is a common phenomenon in both T1D and T2D [? ? ].

Several hallmarks of diabetic retinopathy can be recapitulated in rodent retina deficient of clock genes [? ? ]. Reduced tube formation and increased senescence of endothelial cells coupled with impaired progenitor-mediated repair is observed in Per2 mutant mice, emphasizing the importance of the circadian clock in retinal homeostasis [? ]. Recent studies on autophagy in the retina have shed light on the association of key molecules of the autophagic pathway with phagocytosis of photoreceptor outer segments (POS) by the retinal pigmented epithelium (RPE) [? ? ]. Photoreceptor disk shedding has been widely reported to exhibit diurnal rhythmicity in the retina [? ? ] and the tight coupling of phagocytic ingestion and autophagic degradation of the POS to this diurnal rhythm is a critical aspect of retinal homeostasis [? ? ].

The role of the peripheral clock and diurnal variation on the regulation of autophagy in the normal and diabetic rodent retinas and the fate of autophagy in the diabetic retina remain unexplored. Understanding these control mechanisms may help find effective treatments for diabetic retinopathy. In this study, we demonstrate that the spatial distribution and temporal expression of autophagy proteins show a diurnal rhythm and that this is depressed and phase shifted in the diabetic retina.

\section{Materials and Methods}

\subsection{Experimental Animals}

All animal procedures were performed in accordance with a) protocols approved by the Institutional Animal Care and Use Committees at University of Florida, Gainesville, FL, USA (\#CR-201106001), Michigan State University, Lansing, MI, USA (\#Busik09/14-160-00, and Indiana University, Indianapolis, IN, USA (\#10574 MD/R), b, the National Institutes of Health Guide for Care and Use of Laboratory Animals and c) the ARVO Statement for the Use of Animals in Ophthalmic and Vision Research. Male C57BL/6J mice were purchased from Jackson Labs at 6 weeks of age and male BBZDR/wor T2D rats and lean heterozygote nondiabetic control littermates were obtained from biomedical research models (BRM, Inc.) Worcester, Massachusetts at 5 months of age. C57BL/6J mice $(n=240)$ and BBZ rats $(n=75)$ were maintained on a standard $12 / 12 \mathrm{~h}$ light/dark cycle $(6.00$ am lights on/6.00 pm lights off). T1D was induced in eight-week-old C57BL/6 mice ( $\mathrm{n}=120)$ by five consecutive intraperitoneal injection of freshly prepared streptozotocin (STZ) solution with the concentration of $40 \mathrm{mg} / \mathrm{kg}$ body weight in $0.1 \mathrm{M} /$ citrate buffer, $\mathrm{pH} 4.5$ as previously described [? ? ]. The control group received sodium citrate buffer (vehicle) alone. Diabetes was confirmed by two consecutive blood glucose levels $>240 \mathrm{mg} / \mathrm{dl}$, using the AlphaTrak ${ }^{\circledR}$ blood glucose monitor and test strip system (Abbot laboratories, Irvine, CA, USA), according to the manufacturer's instruction. No animal used in this study required insulin injections. Eyes from one group were collected at 2 months following establishment of diabetes and the second cohort 9 months after diabetes induction (the age of the animals at these time points being 4 and 11 months, respectively). We also investigated inbred Bio-Breeding Zucker (BBZDR)/wor T2D rats $(n=37)$ and age-matched controls $(n=38)$ [? ]. BBZDR/wor rats spontaneously became diabetic at $\sim 10$ weeks of age and were maintained for 4 months after the onset of diabetes. A second group of C57BL/6J mice $(n=24)$ were dark adapted for $48 \mathrm{~h}$ before sacrifice and referred to as being on the dark/dark cycle. In all groups, animals were euthanized by 
deep anesthesia with isoflurane followed by cervical dislocation and eyes were immediately enucleated every 2 or $3 \mathrm{~h}$ over a $24 \mathrm{~h}$ period and prepared for immunofluorescence staining. For animals in the dark cycle, euthanasia and enucleation were performed in a dark room under a red safe light. For each set of experiments, all animals were started at the same time point to clearly identify any phase shift in peak or trough of ATG expression. The number of biological replicates of the enucleated eyes at each time point is $n=10$ for mice and $n=3$ for rats. Only one eye from each animal was used in the assessment.

\subsection{Immunofluorescence Microscopy}

Eyes were processed for standard paraffin embedding and $4 \mu \mathrm{m}$ sections were prepared. Rodent Decloaker (Biocare Medical LLC, Concord, CA, USA Catalog\# RD913L) was used to unmask antigens and non-specific binding was blocked with 10\% normal goat sera and 5\% BSA for $1 \mathrm{~h}$ at room temperature. Sections received either mouse monoclonal anti-Beclin1 (BD Transduction Lab, San Jose, CA, USA, Cat\#612112), or rabbit polyclonal antibodies against Atg7, Atg9 and LC3 (provided by Dr. Dunn, Department of Anatomy and Cell Biology, University of Florida, Gainesville, FL, USA), diluted in phosphate buffered saline (PBS) with 1\% normal goat sera plus $1 \%$ bovine serum albumin (BSA) (Beclin1 1:20; Atg7-1:300; Atg9 and LC3 - 1:100). After washing, sections were incubated with an appropriate FITC-conjugated secondary antibody for $1 \mathrm{~h}$. In some sections, colocalization of autophagy proteins within the retinal vasculature was confirmed by dual staining with the endothelial cell marker, TRITC-agglutinin (Vector Labs, Burlingame, CA, USA, Cat\#RCA120). Sections were covered with Vectashield mounting medium/DAPI (Vector Labs, Inc.). Sections were viewed using a Zeiss Axioplan 2 Upright Fluorescence Microscope with Qimage/QCapture software Version 8 (QImaging Corporate, Surrey, British Columbia, Canada). Omission of the primary antibody was the baseline control and all the fluorescence photographs were obtained under the same scaled conditions.

\subsection{Grading of Immunostaining}

Three independent masked observers, using a previously described grading system [? ? ], graded the intensity of the immunoreactivity for each antibody in transverse retinal sections. The intensity of labeling was graded qualitatively as: 10 , strong bright intense immunoreactivity, 9 , bright intense; 8 , uniformly intense; 7 , patchy and intense; 6 , uniform and moderate; 5 , patchy and moderate; 4 , uniform and weak; 3 , patchy and weak; 2 , uniform and very weak; 1 , patchy and very weak; and 0 , none. A mean score \pm SEM for each group was determined from the scores of all graders for each retina structure.

\subsection{Trypsin Digestion and the Detection of Superoxide}

The retinal vasculature was prepared as previously described [? ]. Briefly, mouse eyes were fixed overnight in $4 \%$ paraformaldehyde, freshly made in PBS. The retinas were dissected from the eye cups, kept in water overnight, and digested in 3\% trypsin (Invitrogen-Gibco, Grand Island, NY, USA) for $3 \mathrm{~h}$ at $37^{\circ}$ Celcius. The tissue was mounted carefully on a glass slide under a dissection microscope. The tissue was stained with PAS-H\&E (periodic acid Schiff-hematoxylin and eosin; Gill No.3; Sigma-Aldrich), according to the instruction manual. The images were taken using a Zeiss light microscope equipped with a digital camera (AxioCam MRC5, Axiovert 200; Carl Zeiss Meditec, Inc., Dublin, CA, USA), using 20X objective lenses. Eight to ten representative fields from each quadrant of the retina were imaged and the number of acellular capillaries per square millimeter of retina were quantified.

For detecting the reactive oxygen species (ROS), the superoxide indicator, hydroethidine, was used to detect the production of superoxide radicals, as previously described [? ]. Superoxide oxidizes hydroethidine to yield a red fluorescent signal at approximately $600 \mathrm{~nm}$. Mice received two intraperitoneal injections, $15 \mathrm{~min}$ apart, of freshly prepared hydroethidine $(20 \mathrm{mg} / \mathrm{kg}$; Invitrogen $)$ and were euthanized $18 \mathrm{~h}$ after injection. The fluorescence intensity was measured in the neural retina 
using a fluorescent plate reader (BioTek, Winooski, VT, USA) and a spectrofluorometer (FLUOstar Optima; BMG Labtechnologies, Cary, NC, USA). The relative fluorescence intensity was calculated by normalizing to protein concentration.

\subsection{Statistical Analysis}

The diurnal data are time series data, and single cosine analysis was employed to fit the data. The data was considered as diurnal oscillation by a zero-amplitude test, with a $p$-value less than 0.05. All experiments were assessed by comparing two groups mean scores from control animals and diabetic animals using a Student's $t$-test, plus ANOVA for multiple comparisons by using Prism ${ }^{\circledR}$ statistical software ver. 5 (GraphPad, La Jolla, CA, USA) with differences of $p$ value $<0.05$ were considered statistically significant. Results are expressed as mean $\pm \mathrm{SEM}$.

\section{Results}

\subsection{Autophagy Proteins Exhibit Diurnal Expression/Localization in Normal Mouse Neural Retina}

To determine if autophagic protein expression in the neural retina had an intrinsic diurnal rhythm, we assessed the expression and localization of four autophagy proteins, Atg7, LC3, Atg9 and Beclin1 in the normal retinas of young mice (age $=3$ months) that were maintained under standard $12 \mathrm{~h} \mathrm{light} / 12 \mathrm{~h}$ dark conditions. When we examined the tissue at two-hour intervals over a $24 \mathrm{~h}$ period, these proteins exhibited not only a distinct diurnal rhythm, but remarkably showed distinct staining patterns across the layers of the neural retina in mice (????). Atg9 and LC3 exhibited a biphasic 12/12 $\mathrm{h}$ circadian cycle with zenith at 8:15 AM and 8:15 PM and nadir at 2:15 AM and 2:15 PM $(p<0.05)$ (Figure ??A-D). By contrast, Atg7 and Beclin1 expression showed a monophasic rhythm and the overall expression of these proteins was much lower than either Atg9 or LC3 (Figure ??E-H). The expression of Atg7 started to rise at 4:15 AM, peaked at 8:15 AM and gradually decreased until 10:15 $\mathrm{AM}(p<0.05)$ (Figure ??E-G). Beclin1 expression was highest at around midnight and reached lowest levels at midday $(p<0.05)$ (Figure ??F-H). Omission of the primary antibody showed no staining in mouse retina (Figure ??D).

An assessment of the staining pattern of the proteins at zenith revealed that Atg9 and LC3 were localized throughout the retina, predominantly within the retinal ganglion cell (RGC) layer, inner (INL) and outer nuclear layer (ONL) (Figure ??A,B). Atg7 was only weakly localized to the neural retina in both the inner and outer nuclear layers and showed stronger localization in the photoreceptor outer segment. Beclin1 staining was detected throughout the retina, with highest intensity in the inner and outer plexiform layers and in the photoreceptors. Closer observation of the retina revealed that ATG7 and 9 showed remarkably strong association within the inner retinal vasculature (Figure ??C).

Using dual staining for endothelial cells and autophagy proteins, we confirmed that Atg9, LC3, Atg7 and Beclin1 were all expressed in both the inner and outer retinal plexus of the retinal vasculature (Figure ??C). 

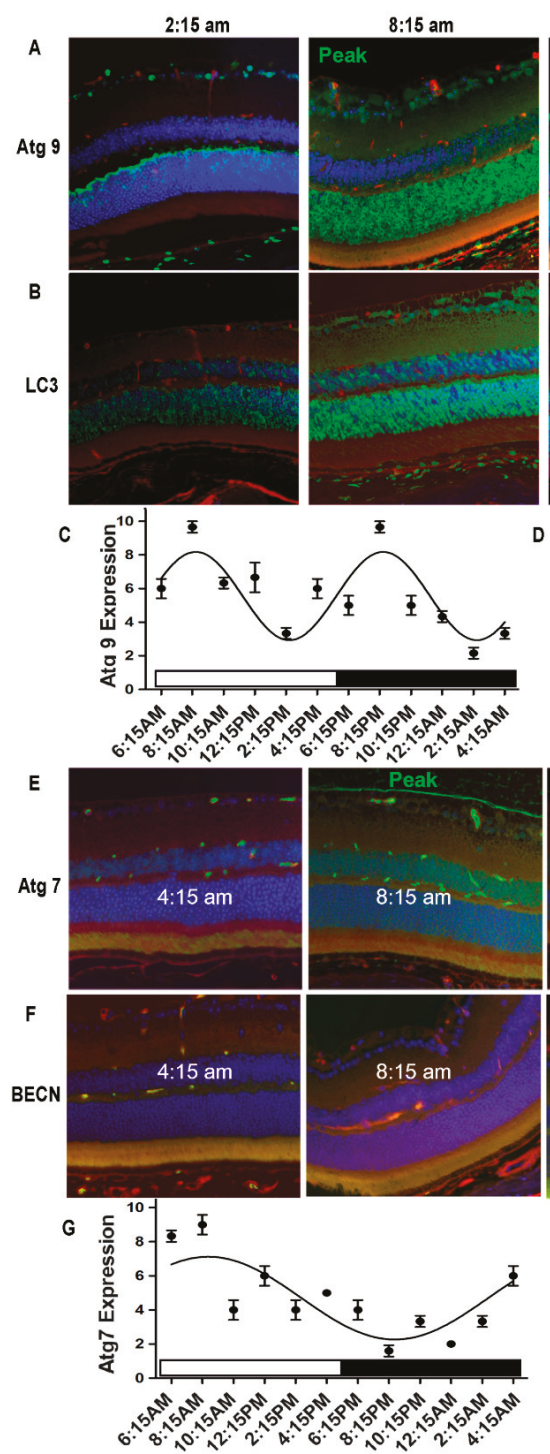

$8: 15$ am

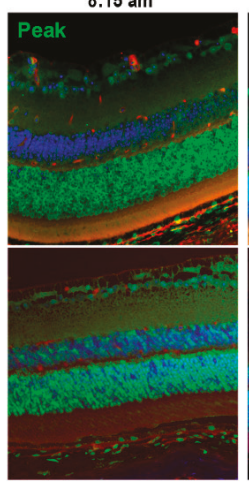

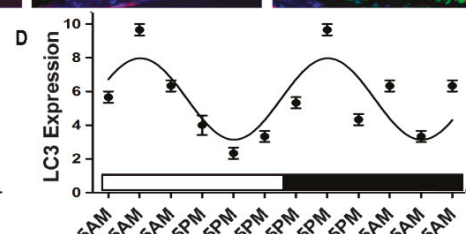

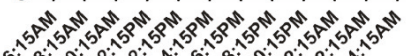
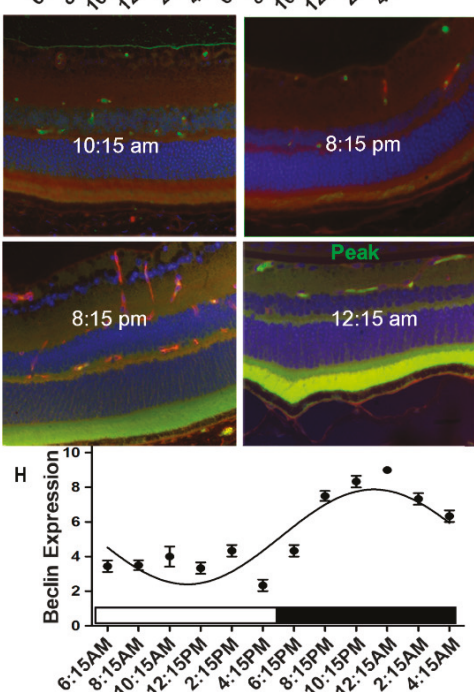

Figure 1. Autophagy proteins exhibited diurnal expression/localization in normal mouse neural retina. Retina were harvested every $2 \mathrm{~h}$ over a $24 \mathrm{~h}$ time period. Antibodies for autophagy proteins (ATGs) ATG9, Microtubule-associated protein 1A/1B-light chain 3 (LC3), ATG7 and Beclin1 (BECN) were used to detect respective protein expression (green) in the retina, Tetramethylrhodamine (TRITC) agglutinin for vessels (red) and DAPI (blue) shows nuclear staining. Distinct diurnal rhythmic patterns of the autophagic proteins Atg9 and LC3 expressions (A,B) revealed a biphasic diurnal cycle (12/12 h), with peaks at 8:15 AM and 8:15 PM and lowest levels at 2:15 AM and 2:15 PM (C,D). Atg7 and Beclin1 (BECN) expressions (E,F) were on a monophasic $24 \mathrm{~h}$ cycle. Atg7 expression peaked at 8:15 AM and lowest levels were at 10:15 AM. The peak of Beclin1 expression was at midnight and lowest levels were observed during the late morning $(\mathbf{H})$. All animals were maintained in a standard 12/12 h light/dark phase with lights ON at 6:00 AM and lights OFF at 6:00 PM. The error bars in the diurnal plots represent the mean+SEM and diurnal oscillation had a $p$-value less than 0.05 . 


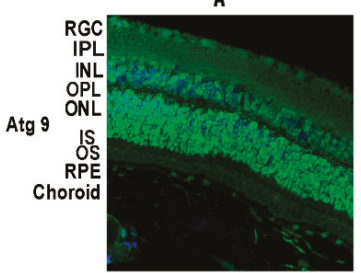

LC3
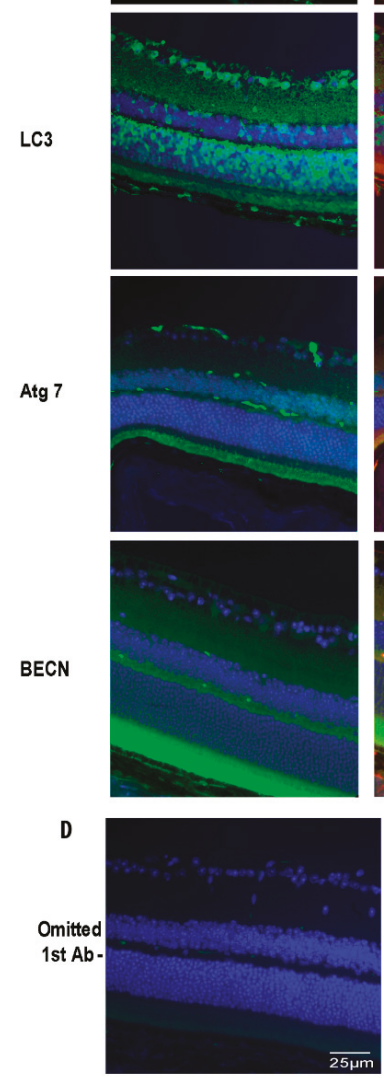

B
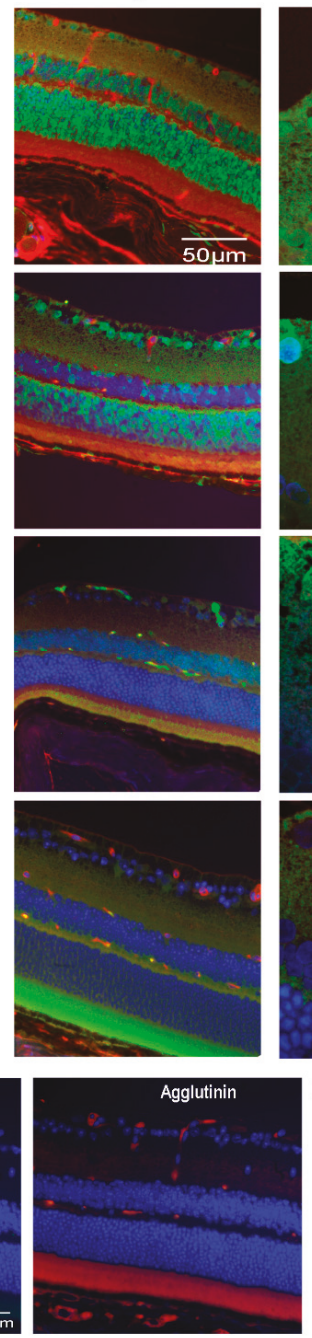

c
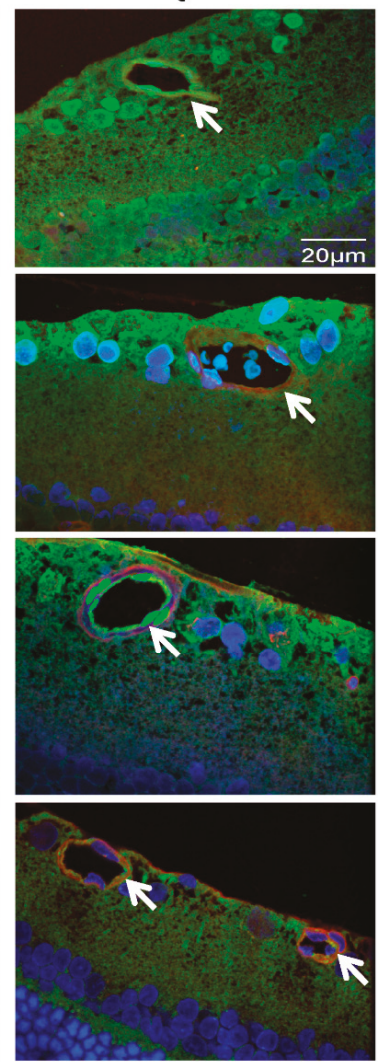

E

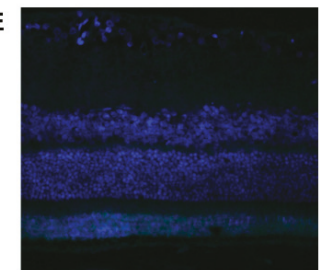

Figure 2. Localization of autophagy proteins in normal mouse retina and vasculature. Animals were kept in tight 12/12-h light/dark cycle before the experiment. Antibodies for ATG9, LC3, ATG7 and Beclin1 (BECN) were used to detect respective protein expression (green) in the retinas with different staining patterns and DAPI was used to stain nuclei (blue) (A). Agglutinin, an endothelial cell marker, conjugated with TRITC (red) was used to co-localize with antibodies specific to individual autophagy proteins (FITC) in the retinal vasculature (B). High magnification images demonstrated autophagy protein localization to the endothelial cells and pericytes of the retinal vasculature (C). No fluorescence was observed with omission of the primary antibody in the mouse retina. Using agglutinin staining, the section displayed retinal vascular patterns with normal architecture (D). Omitted autophagy primary antibody in rat retina also was negative (E). The arrows indicate autophagy protein localization to retinal vessels. RGC, retina ganglion cell; IPL, inner plexiform layer; INL, inner nuclear layer; OPL, outer plexiform layer; ONL, outer nuclear layer; IS, inner segment; OS, outer segment; RPE, retinal pigment epithelium. 


\subsection{Autophagic Activity in the Retina Is Tightly Entrained by Light}

We repeated immunohistochemistry of ATG9, ATG7, LC3 and Beclin1 in the retina (and the retinal vasculature) of $48 \mathrm{~h}$ dark adapted mice and compared the results to those of 12/12 h light/dark adapted mice. The immunohistochemistry data indicated that the periodic oscillations, as well as overall levels of the autophagic proteins ATG9 and LC3, were attenuated in the $48 \mathrm{~h}$ dark adapted mice retina when compared to the 12/12 h dark/dark controls, both in the retina and the vasculature (Figure ??A). Similarly, peaks in Atg7 and Beclin1 were phase shifted compared to the light/dark controls and overall expression levels were reduced (Figure ??B). We concluded that diurnal oscillations in autophagic activity in the murine retina depend, at least in part, on light-effected entrainment.
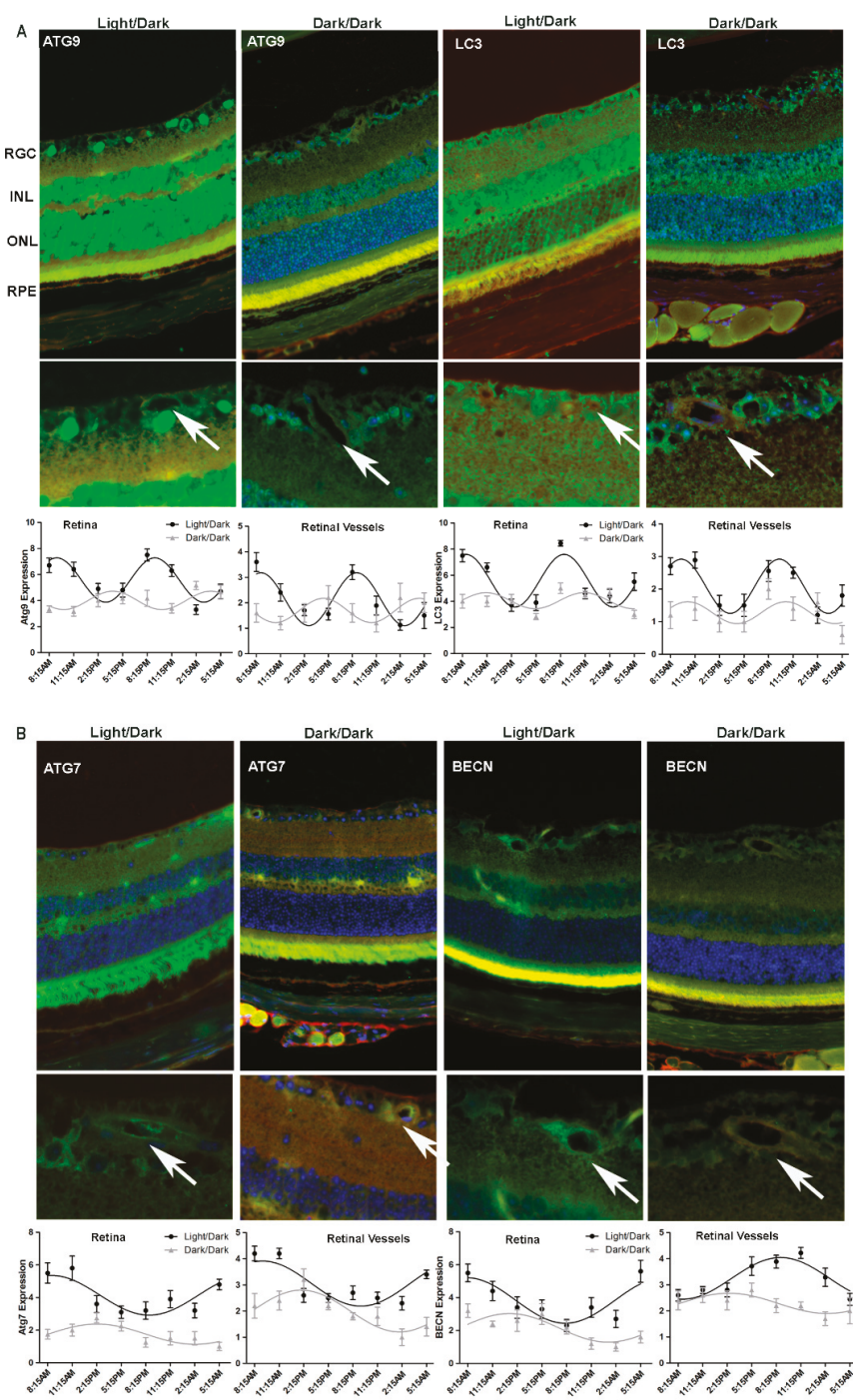

Figure 3. Immunohistochemistry confirmed alterations in diurnal patterns of autophagic gene expression upon light cycle disruption. 
Mice were separated into two groups. One group was kept under 12/12-h light/dark cycle and the other group was kept in the dark for 48-h (dark/dark), before being euthanized. Samples were collected every $3 \mathrm{~h}$ for $24 \mathrm{~h}$ and were analyzed by immunohistochemistry for autophagic markers (A) ATG9 (B) LC3 (C) ATG7 and (D) Beclin1 (BECN). Autophagy protein expression is green, TRITC agglutinin for vessels red and DAPI nuclear staining blue. The arrows indicate autophagy protein localization to retinal vessels. RGC, retina ganglion cell; INL, inner nuclear layer; OPL, outer plexiform layer; ONL, outer nuclear layer; RPE, retinal pigment epithelium. The error bars in the circadian plots represent the mean + SEM and diurnal oscillation had a $p$-value less than 0.05 .

\subsection{Diurnal Rhythmicity of Expression of Autophagic Proteins Is Dampened in T1D}

Having established that autophagy in the retina possesses a diurnal rhythm, we next examined the rhythmicity of autophagy proteins in T1D. We chose mice from 2- and 9-months duration of diabetes to assess the expression of autophagic proteins. To confirm that our T1D mice exhibited the expected retinopathy features [? ? ], we performed trypsin-digests of the retina from nondiabetic C57BL/6J (Figure ??A, left) and T1D (Figure ??A, right). T1D mice exhibited an increase in the number of acellular capillaries per unit area. Quantitative measurements of acellular capillaries suggested a dramatic 4-fold increase, in contrast to the age-matched nondiabetic mouse of retinas that showed a normal vascular pattern (Figure ??B). These eyes showed significantly higher levels of superoxide anions in the neural retina, typical of retinopathy in this model (Figure ??C).
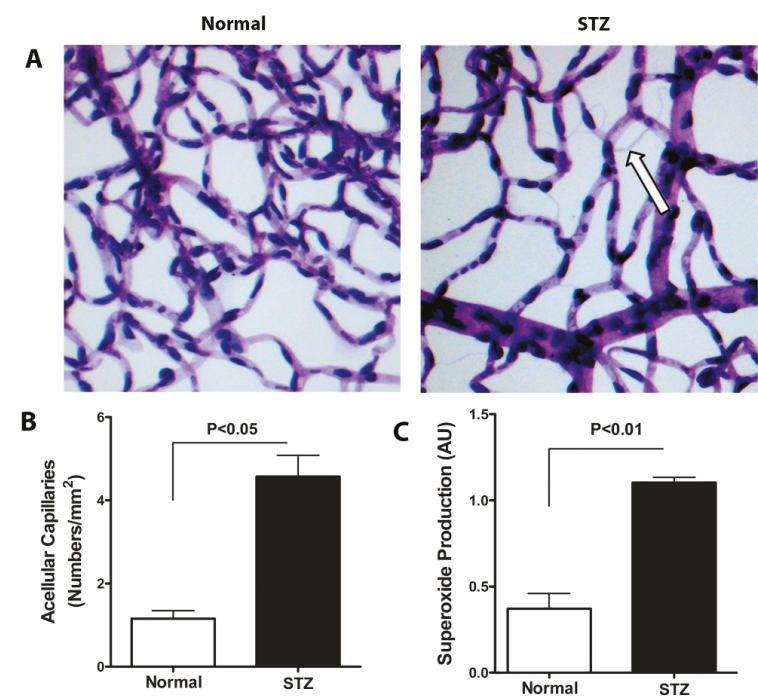

Figure 4. Evaluation of acellular capillary formation and superoxide anion generation in T1D mice. Representative images of trypsin-digested retinal vascular preparations from 4-month old control mice and 4-month old mice with 2-month duration of T1D (A) The arrow indicates an acellular capillary. Quantitative measurement of acellular capillaries was significantly increased in diabetic eyes $(\mathrm{n}=6)$. (B) STZ-induced diabetic retina showed $>1.5$ fold increase in superoxide anion levels, $p<0.01$ (C) The errors bars represent SEM.

While immunohistochemistry results from 4-month old normal control mice corresponded with the respective results shown in ????, we observed a dramatic attenuation of diurnal rhythmicity (amplitudes), as well as overall levels of the autophagic proteins in the T1D mice retina (Figure ??). This loss of amplitude was noted for ATG9, LC3 and Beclin1 expression in the diabetic retina, in either duration of diabetes group when compared to the respective age-matched controls (????). ATG7 exhibited a shift in 
phase in both the retina and the vasculature of mice with 2-month duration of diabetes while in mice with 9-months duration of diabetes the phase-shift was prominent in the retina and there was no significant variation in expression between time-points in the retinal vasculature (Figures ??B and ??B).
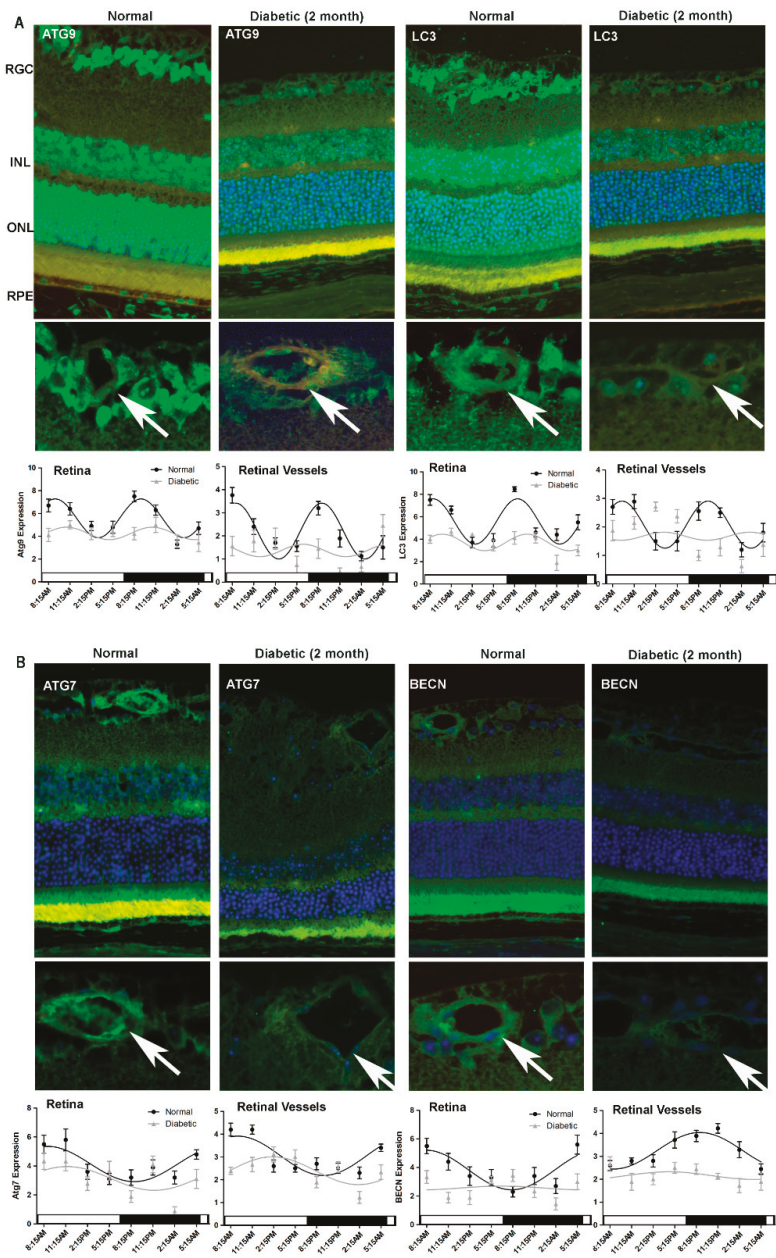

Figure 5. Impairment of diurnal rhythmicity of autophagy in T1D mice with two-months duration of diabetes. Retinas were collected from C57Bl/6J mice with two-months duration of T1D and age-matched control mice. Immunostaining and intensity analyses of retina and retinal vasculature demonstrated a dramatic loss of oscillatory amplitude of autophagic protein expression in the diabetic animals compared to normal mice (A,B). Autophagy protein expression is green, TRITC agglutinin for vessels red and DAPI nuclear staining blue. The arrows indicate autophagy protein localization to retinal vessels. RGC, retina ganglion cell; INL, inner nuclear layer; OPL, outer plexiform layer; ONL, outer nuclear layer; RPE, retinal pigment epithelium. Loss and phase-shifting of diurnal rhythmicity in diabetic retinopathy is demonstrated in single cosine plots, with ordinary least square fitted $(p<0.01$, $\mathrm{n}=10$ ). All animals were maintained in a standard 12/12-h light/dark phase with lights ON at 6:00 AM and lights OFF at 6:00 PM. The error bars in the circadian plots represent the mean+SEM and diurnal oscillation had a $p$-value less than 0.05 . 

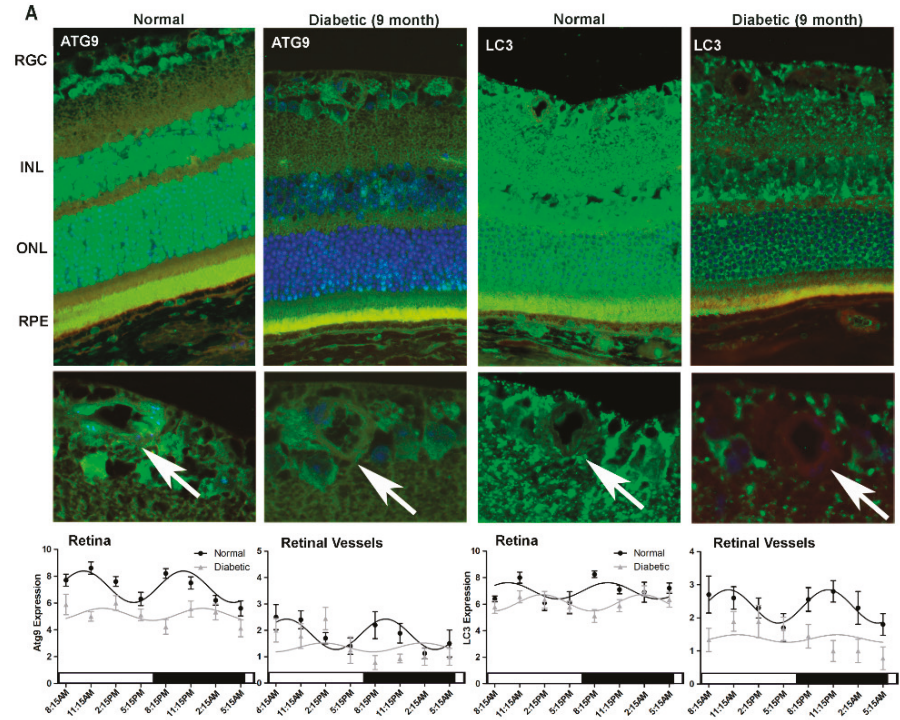

Retinal Vessels

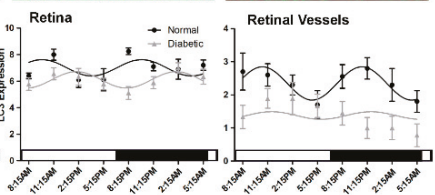

B
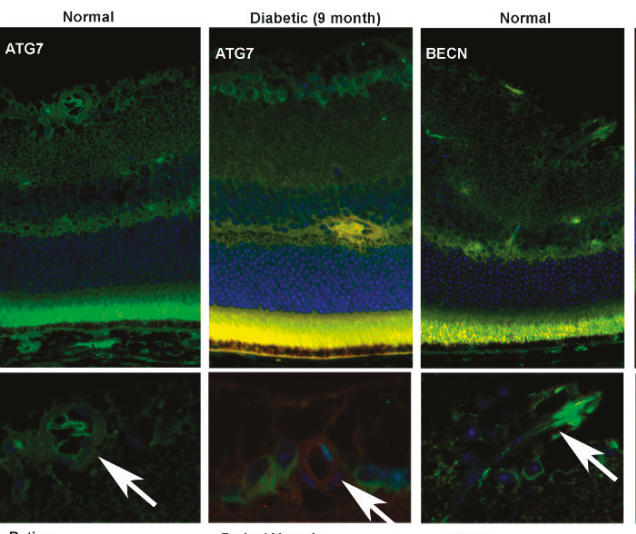

Diabetic (9 month)
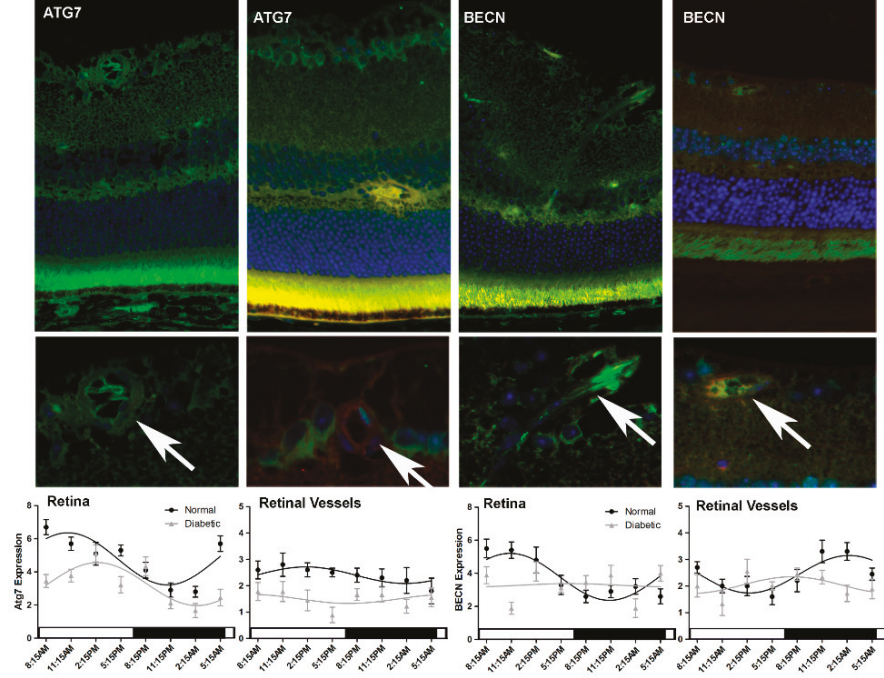

Figure 6. Impairment of diurnal rhythmicity of autophagy in T1D mice with nine-months duration of diabetes. Retinas were collected from C57Bl/6J mice with nine-months duration of T1D and age-matched control mice. Immunostaining and intensity analyses of retina and retinal vasculature demonstrated a dramatic loss of oscillatory amplitude of autophagic protein expression in the diabetic animals compared to normal mice (A.B). Autophagy protein expression is green, TRITC agglutinin for vessels red and DAPI nuclear staining blue. The arrows indicate autophagy protein localization to retinal vessels. RGC, retina ganglion cell; INL, inner nuclear layer; OPL, outer plexiform layer; ONL, outer nuclear layer; RPE, retinal pigment epithelium. Loss and phase-shifting of diurnal rhythmicity in diabetic retinopathy is demonstrated in single cosine plot with ordinary least square fitted $(p<0.01$, $\mathrm{n}=10$ ). All animals were maintained in a standard 12/12-h light/dark phase with lights ON at 6:00 AM and lights OFF at 6:00 PM. The error bars in the circadian plots represent the mean+SEM and diurnal oscillation had a $p$-value less than 0.05 . 


\subsection{Autophagy Proteins Were Suppressed Severely in T2D Rats}

We next examined autophagy in the retina of T2D rats following 4-months duration of diabetes (Figure ??). The normal age-matched rats selected as controls demonstrated a similar staining pattern of immunohistochemistry across the retina as were observed in normal mice (Figure ??A-D). Atg9 (Figure ??A) and LC3 (Figure ??B) were mainly present in the ganglion cell layer, inner and outer nuclear layers in the normal rat retina, similar to what was observed in normal mice (Figure ??) but their expression was dramatically decreased in the retinas of the diabetic animals by $49 \%$ to $58 \%$, respectively. For better demonstration of changes in autophagic protein levels in the vasculature of diabetic retinas, we showed higher magnification images. Both Atg9 and LC3 clearly distributed within the retinal endothelia of normal rats but were completely absent from the endothelia of the diabetic retinas (Figure ??E,F). Atg7 was strongly expressed in normal retinal vessels near the ganglion cells layer, meanwhile the small vessels in inner and outer plexus layers were also stained (Figure ??C). Semi-quantitative analysis revealed that the level of Atg7 was deceased by $52 \%, p<0.05$ in diabetic retinas. Diabetic retina also displayed pathologic changes in retina vessels, which appeared to be abnormally protruded toward the intravitreous cavity. Beclin1 staining was detected across the retina, including retinal vessels in the plexus and photoreceptors, however, Beclin1 staining was diminished in diabetic retinas by 53\%, $p<0.01$ (Figure ??D). Quantitative analyses of autophagic proteins in the retina of control and diabetic mice demonstrated, not only suppressed levels, but also revealed impairment in diurnal rhythmicity in T2D rats. Expression of Atg9 and LC3 were severely suppressed with insignificant biphasic oscillatory pattern and ATG7 and Beclin1 were phase-shifted by approximately for 4-6 h. We concluded that disruption in autophagy is a characteristic phenomenon of both T1D and T2D. 


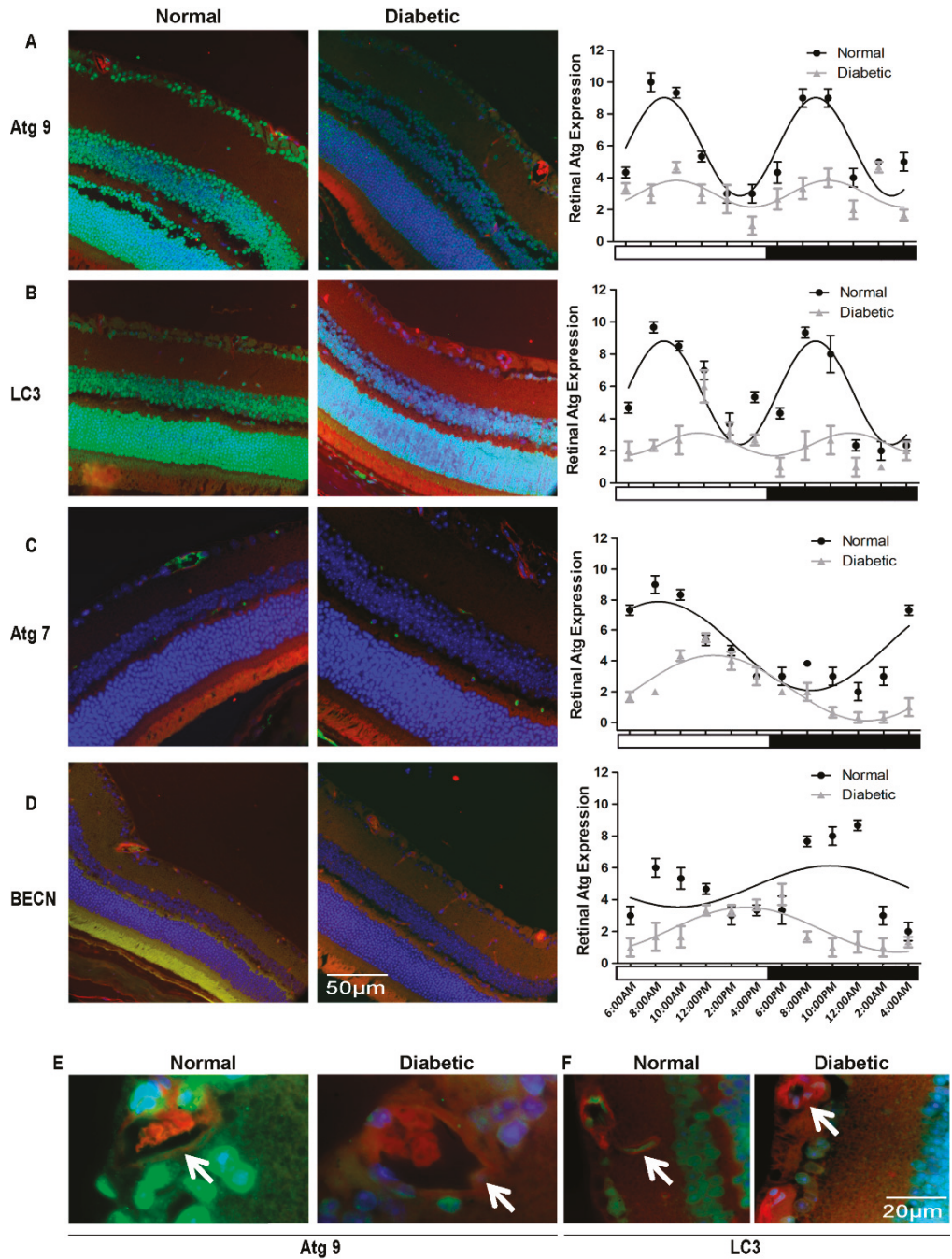

Figure 7. Impairment of diurnal rhythmicity of autophagy in T2D rats. Eyes were collected from 6.5-month old BBZDR/wor type 2 diabetic rats with 4-month duration of diabetes and the normal age-matched rats selected as controls. Immunostaining demonstrated a dramatic decrease in amplitude of diurnal rhythmicity of autophagic protein expression in the retina of the diabetic animals compared to normal rats. Representative micrographs of immunostained sections at 8:15 am are shown for (A) ATG9, (B) LC3 (C) ATG7 (D) Beclin1 (BECN). Autophagy protein expression is green, TRITC agglutinin for vessels red and DAPI nuclear staining blue. The arrows indicate autophagy protein localization to retinal vessels. RGC, retina ganglion cell; INL, inner nuclear layer; OPL, outer plexiform layer; ONL, outer nuclear layer; RPE, retinal pigment epithelium. ATG7 and Beclin1 displayed phase-shifting in the diurnal oscillation (C) and (D) respectively. Loss and phase-shifting of diurnal rhythmicity in diabetic retinopathy is demonstrated in single cosine plot with ordinary least square fitted $(p<0.01, \mathrm{n}=10)$. High magnification images confirmed the loss of autophagy proteins ATG9 and LC3 in the retinal vasculature (E). All animals were maintained in a standard 12/12-h light/dark phase with lights ON at 6:00 AM and lights OFF at 6:00 PM. The error bars in the circadian plots represent the mean + SEM and diurnal oscillation had a $p$-value less than 0.05 . 


\section{Discussion}

Autophagy is a critical and indispensable housekeeping process in the cells of the retina. Numerous reports support the functional relevance of autophagy in specific cells of the retina and how dysregulated autophagy contributes to retinal malfunction and degeneration [? ? ? ? ? ]. While acute short-term noxious stimuli (e.g., nutrient deprivation; hypoxia and endoplasmic reticulum (ER) stress; oxidative stress arising from light, lipofuscin, POS phagocytosis by RPE or mitochondrial ROS generated in retinal cells (which have a generally high metabolic rate)) can stimulate autophagy as a generally cytoprotective mechanism, it must be acknowledged that diurnal basal autophagic modulation is a critical factor in abrogating the unavoidable regular cellular damage that occurs during the basic functioning of retinal cells. An efficient regulatory molecular circuit is required in the retina, that would modulate both the intensity and duration of basal autophagic activity in specific cell types and thus meet their regular housekeeping demands [? ]. Previous studies have suggested the existence of autophagic rhythm in rodent heart muscles and liver, as well as kidney proximal tubules [? ? ? ]. In this study, we establish the existence of diurnal regulation of autophagic activity, in both the neural and vascular cells of the retina. We show evidence that certain autophagic markers like Beclin1, ATG9 and LC3 are highly expressed across the retina, while ATG7 shows preferential staining patterns and is enriched only in certain layers (????). The first report on diurnal rhythmicity in autophagy in the retina recorded increased autophagosome formation following outer disk shedding in rat photoreceptors [? ]. Our results regarding the biphasic oscillation of diurnal rhythmicity of LC3 expression agree with the findings in a report by Yao et al. 2014, who also observed two peaks of elevated autophagic activity [? ]. Our results in Figure ?? make a strong argument in favor of the influence of external stimuli such as light for the entrainment and maintenance of healthy amplitudes of oscillation of autophagic protein expression in the retina. However, we observed that even in the absence of light entrainment, the retina continued to display rhythmicity in ATG7, ATG9, LC3 and BECN, but were lower in amplitude and out of phase, suggesting that there may be an intrinsic diurnal rhythm of autophagy in the retina not dependent on light entrainment.

Our study encompasses autophagy in aging and diabetic conditions. We successfully demonstrate that perturbed autophagy is a characteristic feature of aging. It has been suggested that macroautophagy suffers a decline with aging and is replaced partly by other forms of autophagy [? ]. It remains to be determined if other forms of autophagy i.e., the chaperone mediated autophagy and microautophagy are also under regulation by the circadian system and, if so, whether their rhythmicity is affected in aging and disease. In addition to the above, our study reveals that the level of disruption in T1D and T2D is significant, not only in younger mice, but also in older animals (????). Compared to age-matched control mouse retinas, the dramatic deviations in the oscillatory patterns or the overall levels of one or more Atgs in both two- and nine-month old diabetic mouse retina could be an indication of faulty housekeeping and pathological outcomes, such as the accumulation of damaged mitochondria and ROS production [? ? ? ?].

A limitation of this study is that it relies on immunohistochemistry to determine changes in the expression of autophagy proteins throughout the neural retina. We did not attempt to confirm our findings by assessing gene expression or protein levels using Western blot. Neither did we assess autophagic flux by determining the LC3II:LC3I ratio. The reasons for this were a combination of the large number of animals needed; regional/cell-specific changes would be masked in whole retinal preps and the non-canonical role of LC3 in retinal and immune cell phagocytosis [? ] could complicate the analysis of the autophagic flux. Furthermore, based on our data, there is a need to determine the mechanistic link between diurnal changes and autophagy in the neural retina. There is considerable evidence that diurnal/circadian rhythm is associated with the induction of autophagy [? ? ], a key regulator of autophagy; the mechanistic target of rapamycin (mTOR) is regulated by the circadian clock [? ] and the circadian regulation of metabolism is mediated through reciprocal signaling between the clock and metabolic regulatory networks such as autophagy [? ]. Recently, Ryzhikov and colleagues reported that diurnal rhythms spatially and temporarily organize autophagy [? ]. They reported that 
basal autophagy rhythms could be resolved into two antiphase clusters that were distinguished by the subcellular location of targeted proteins. Daytime autophagy was directed towards cytosolic proteins and proteosomal degradation, while nighttime autophagy was directed towards ER and mitochondrial removal. There is now a need to better understand the mechanistic control of these processes.

As mentioned above, autophagy in diabetes and related diseases has become an area of intense research, with focus on how this pathway may be targeted in synergy, with other therapeutic approaches to encourage a better clinical outcome. Our study, along with other recent reports, adds a novel extension to the mammalian diurnal rhythmicity in its relevance in regulation of biological processes. It provides a novel link between dysregulated autophagy and disrupted diurnal rhythm in the aging and diabetic retina and suggests that our analyses of myriad biological processes in the retina should be reconsidered from a diurnal perspective, in order to better comprehend age-related vision loss and disease pathology.

Author Contributions: Conceptualization, M.E.B., M.B.G. and J.V.B; Data curation, X.Q., S.K.M; Formal analysis, X.Q., Y.Y.; Investigation, X.Q., S.K.M., M.E.B. Resources, M.E.B., J.V.B., M.B.G.; Project administration: M.E.B., J.V.B., M.B.G. Writing—original draft preparation, X.Q., M.E.B.; Writing—review and editing, X.Q., S.K.M., Y.Y., J.V.B., M.B.G., M.E.B. All authors have read and agreed to the published version of the manuscript.

Funding: M.E.B. is supported by NIH funding (EY019688, EY021626) and an unrestricted grant from Research to Prevent Blindness. J.V.B. is supported by NIH funding (EY01EY028049, R01EY016077; R01EY025383).

Acknowledgments: The animal experiments were performed over a period of time when MEB, MBG, XQ and SKM were at University of Florida and then Indiana University, before moving to UAB. All data were analyzed at UAB.

Conflicts of Interest: The authors declare no conflict of interest.

\section{References}

1. Choi, A.M.; Ryter, S.W.; Levine, B. Autophagy in human health and disease. N. Engl. J. Med. 2013, 368, 651-662. [CrossRef]

2. Mitter, S.K.; Boulton, M.E. Autophagy in ocular physiology. In Autophagy in Current Trends in Cellular Physiology and Pathology; Gorbunov, N.V., Schneider, M., Eds.; InTech: Open Access: London, UK, 2016; pp. 287-327.

3. Sridhar, S.; Botbol, Y.; Macian, F.; Cuervo, A.M. Autophagy and disease: Always two sides to a problem. J. Pathol. 2012, 226, 255-273. [CrossRef]

4. Sachdeva, U.M.; Thompson, C.B. Diurnal rhythms of autophagy: Implications for cell biology and human disease. Autophagy 2008, 4, 581-589. [CrossRef]

5. Boya, P.; Esteban-Martinez, L.; Serrano-Puebla, A.; Gomez-Sintes, R.; Villarejo-Zori, B. Autophagy in the eye: Development, degeneration, and aging. Prog. Retin. Eye Res. 2016, 55, 206-245. [CrossRef]

6. Czaja, M.J.; Ding, W.X.; Donohue, T.M., Jr.; Friedman, S.L.; Kim, J.S.; Komatsu, M.; Lemasters, J.J.; Lemoine, A.; Lin, J.D.; Ou, J.H.; et al. Functions of autophagy in normal and diseased liver. Autophagy 2013, 9, 1131-1158. [CrossRef]

7. Kiriyama, Y.; Nochi, H. The Function of Autophagy in Neurodegenerative Diseases. Int. J. Mol. Sci. 2015, 16, 26797-26812. [CrossRef]

8. Mancias, J.D.; Kimmelman, A.C. Mechanisms of Selective Autophagy in Normal Physiology and Cancer. J. Mol. Biol. 2016, 428, 1659-1680. [CrossRef]

9. Yin, Y.; Sun, G.; Li, E.; Kiselyov, K.; Sun, D. ER stress and impaired autophagy flux in neuronal degeneration and brain injury. Ageing Res. Rev. 2016. [CrossRef]

10. Ebato, C.; Uchida, T.; Arakawa, M.; Komatsu, M.; Ueno, T.; Komiya, K.; Azuma, K.; Hirose, T.; Tanaka, K.; Kominami, E.; et al. Autophagy is important in islet homeostasis and compensatory increase of beta cell mass in response to high-fat diet. Cell Metab. 2008, 8, 325-332. [CrossRef]

11. Fujitani, Y.; Kawamori, R.; Watada, H. The role of autophagy in pancreatic beta-cell and diabetes. Autophagy 2009, 5, 280-282. [CrossRef]

12. Goldman, S.; Zhang, Y.; Jin, S. Autophagy and adipogenesis: Implications in obesity and type II diabetes. Autophagy 2010, 6, 179-181. [CrossRef] [PubMed] 
13. Graham, T.E.; Abel, E.D. Autophagy in diabetes and the metabolic syndrome. In Autophagy in Health and Disease; Gottlieb, R.A., Ed.; Academic Press: London, UK, 2013; pp. 117-140.

14. Shi, S.Q.; Ansari, T.S.; McGuinness, O.P.; Wasserman, D.H.; Johnson, C.H. Circadian disruption leads to insulin resistance and obesity. Curr. Biol. Cb 2013, 23, 372-381. [CrossRef] [PubMed]

15. Busik, J.V.; Tikhonenko, M.; Bhatwadekar, A.; Opreanu, M.; Yakubova, N.; Caballero, S.; Player, D.; Nakagawa, T.; Afzal, A.; Kielczewski, J.; et al. Diabetic retinopathy is associated with bone marrow neuropathy and a depressed peripheral clock. J. Exp. Med. 2009, 206, 2897-2906. [CrossRef] [PubMed]

16. Ikeda, H.; Yong, Q.; Kurose, T.; Todo, T.; Mizunoya, W.; Fushiki, T.; Seino, Y.; Yamada, Y. Clock gene defect disrupts light-dependency of autonomic nerve activity. Biochem. Biophys. Res. Commun. 2007, 364, 457-463. [CrossRef] [PubMed]

17. Ruiter, M.; Buijs, R.M.; Kalsbeek, A. Hormones and the autonomic nervous system are involved in suprachiasmatic nucleus modulation of glucose homeostasis. Curr. Diabetes Rev. 2006, 2, 213-226. [CrossRef] [PubMed]

18. Thomas, H.E.; Redgrave, R.; Cunnington, M.S.; Avery, P.; Keavney, B.D.; Arthur, H.M. Circulating endothelial progenitor cells exhibit diurnal variation. Arterioscler. Thromb. Vasc. Biol. 2008, 28, e21-e22. [CrossRef]

19. Kohsaka, A.; Laposky, A.D.; Ramsey, K.M.; Estrada, C.; Joshu, C.; Kobayashi, Y.; Turek, F.W.; Bass, J. High-fat diet disrupts behavioral and molecular circadian rhythms in mice. Cell Metab. 2007, 6, 414-421. [CrossRef]

20. Young, M.E.; Razeghi, P.; Taegtmeyer, H. Clock genes in the heart: Characterization and attenuation with hypertrophy. Circ. Res. 2001, 88, 1142-1150. [CrossRef]

21. Lahouaoui, H.; Coutanson, C.; Cooper, H.M.; Bennis, M.; Dkhissi-Benyahya, O. Clock genes and behavioral responses to light are altered in a mouse model of diabetic retinopathy. PLoS ONE 2014, 9, e101584. [CrossRef]

22. Ando, H.; Ushijima, K.; Yanagihara, H.; Hayashi, Y.; Takamura, T.; Kaneko, S.; Fujimura, A. Clock gene expression in the liver and adipose tissues of non-obese type 2 diabetic Goto-Kakizaki rats. Clin. Exp. Hypertens. 2009, 31, 201-207. [CrossRef]

23. Bhatwadekar, A.D.; Yan, Y.; Qi, X.; Thinschmidt, J.S.; Neu, M.B.; Li Calzi, S.; Shaw, L.C.; Dominiguez, J.M.; Busik, J.V.; Lee, C.; et al. Per2 mutation recapitulates the vascular phenotype of diabetes in the retina and bone marrow. Diabetes 2013, 62, 273-282. [CrossRef]

24. Frost, L.S.; Lopes, V.S.; Bragin, A.; Reyes-Reveles, J.; Brancato, J.; Cohen, A.; Mitchell, C.H.; Williams, D.S.; Boesze-Battaglia, K. The Contribution of Melanoregulin to Microtubule-Associated Protein 1 Light Chain 3 (LC3) Associated Phagocytosis in Retinal Pigment Epithelium. Mol. Neurobiol. 2015, 52, 1135-1151. [CrossRef] [PubMed]

25. Kim, J.Y.; Zhao, H.; Martinez, J.; Doggett, T.A.; Kolesnikov, A.V.; Tang, P.H.; Ablonczy, Z.; Chan, C.C.; Zhou, Z.; Green, D.R.; et al. Noncanonical autophagy promotes the visual cycle. Cell 2013, 154, 365-376. [CrossRef] [PubMed]

26. Bosch, E.; Horwitz, J.; Bok, D. Phagocytosis of outer segments by retinal pigment epithelium: Phagosome-lysosome interaction. J. Histochem. Cytochem. Off. J. Histochem. Soc. 1993, 41, 253-263. [CrossRef] [PubMed]

27. Young, R.W. The daily rhythm of shedding and degradation of cone outer segment membranes in the lizard retina. J. Ultrastruct. Res. 1977, 61, 172-185. [CrossRef]

28. Rodriguez-Muela, N.; Koga, H.; Garcia-Ledo, L.; de la Villa, P.; de la Rosa, E.J.; Cuervo, A.M.; Boya, P. Balance between autophagic pathways preserves retinal homeostasis. Aging Cell 2013, 12, 478-488. [CrossRef]

29. Yao, J.; Jia, L.; Shelby, S.J.; Ganios, A.M.; Feathers, K.; Thompson, D.A.; Zacks, D.N. Circadian and noncircadian modulation of autophagy in photoreceptors and retinal pigment epithelium. Investig. Ophthalmol. Vis. Sci. 2014, 55, 3237-3246. [CrossRef]

30. Feit-Leichman, R.A.; Kinouchi, R.; Takeda, M.; Fan, Z.; Mohr, S.; Kern, T.S.; Chen, D.F. Vascular damage in a mouse model of diabetic retinopathy: Relation to neuronal and glial changes. Investig. Ophthalmol. Vis. Sci. 2005, 46, 4281-4287. [CrossRef]

31. Hazra, S.; Rasheed, A.; Bhatwadekar, A.; Wang, X.; Shaw, L.C.; Patel, M.; Caballero, S.; Magomedova, L.; Solis, N.; Yan, Y.; et al. Liver $\mathrm{X}$ receptor modulates diabetic retinopathy outcome in a mouse model of streptozotocin-induced diabetes. Diabetes 2012, 61, 3270-3279. [CrossRef]

32. Bhutto, I.A.; McLeod, D.S.; Hasegawa, T.; Kim, S.Y.; Merges, C.; Tong, P.; Lutty, G.A. Pigment epithelium-derived factor (PEDF) and vascular endothelial growth factor (VEGF) in aged human choroid and eyes with age-related macular degeneration. Exp. Eye Res. 2006, 82, 99-110. [CrossRef] 
33. McLeod, D.S.; Lefer, D.J.; Merges, C.; Lutty, G.A. Enhanced expression of intracellular adhesion molecule-1 and P-selectin in the diabetic human retina and choroid. Am. J. Pathol. 1995, 147, 642-653. [PubMed]

34. Kuwabara, T.; Cogan, D.G. Studies of retinal vascular patterns. I. Normal architecture. Arch. Ophthalmol. 1960, 64, 904-911. [CrossRef] [PubMed]

35. Komeina, K.; Usui, S.; Shen, J.; Rogers, B.S.; Campochiaro, P.A. Blockade of neuronal nitric oxide synthase reduces cone cell death in a model of retinitis pigmentosa. Free Radic. Biol. Med. 2008, 45, 905-912. [CrossRef] [PubMed]

36. Hammes, H.P.; Lin, J.; Renner, O.; Shani, M.; Lundqvist, A.; Betsholtz, C.; Brownlee, M.; Deutsch, U. Pericytes and the pathogenesis of diabetic retinopathy. Diabetes 2002, 51, 3107-3112. [CrossRef] [PubMed]

37. Toda, N.; Nakanishi-Toda, M. Nitric oxide: Ocular blood flow, glaucoma, and diabetic retinopathy. Prog. Retin. Eye Res. 2007, 26, 205-238. [CrossRef] [PubMed]

38. Krohne, T.U.; Kaemmerer, E.; Holz, F.G.; Kopitz, J. Lipid peroxidation products reduce lysosomal protease activities in human retinal pigment epithelial cells via two different mechanisms of action. Exp. Eye Res. 2010, 90, 261-266. [CrossRef]

39. Viiri, J.; Amadio, M.; Marchesi, N.; Hyttinen, J.M.; Kivinen, N.; Sironen, R.; Rilla, K.; Akhtar, S.; Provenzani, A.; D'Agostino, V.G.; et al. Autophagy activation clears ELAVL1/HuR-mediated accumulation of SQSTM1/p62 during proteasomal inhibition in human retinal pigment epithelial cells. PLoS ONE 2013, 8, e69563. [CrossRef]

40. Chu, Y.K.; Lee, S.C.; Byeon, S.H. VEGF rescues cigarette smoking-induced human RPE cell death by increasing autophagic flux: Implications of the role of autophagy in advanced age-related macular degeneration. Investig. Ophthalmol. Vis. Sci. 2013, 54, 7329-7337. [CrossRef]

41. Mohlin, C.; Taylor, L.; Ghosh, F.; Johansson, K. Autophagy and ER-stress contribute to photoreceptor degeneration in cultured adult porcine retina. Brain Res. 2014, 1585, 167-183. [CrossRef]

42. Rodriguez-Muela, N.; Hernandez-Pinto, A.M.; Serrano-Puebla, A.; Garcia-Ledo, L.; Latorre, S.H.; de la Rosa, E.J.; Boya, P. Lysosomal membrane permeabilization and autophagy blockade contribute to photoreceptor cell death in a mouse model of retinitis pigmentosa. Cell Death Differ. 2015, 22, $476-487$. [CrossRef]

43. Mitter, S.K.; Song, C.; Qi, X.; Mao, H.; Rao, H.; Akin, D.; Lewin, A.; Grant, M.; Dunn, W., Jr.; Ding, J.; et al. Dysregulated autophagy in the RPE is associated with increased susceptibility to oxidative stress and AMD. Autophagy 2014, 10, 1989-2005. [CrossRef] [PubMed]

44. Ma, D.; Li, S.; Molusky, M.M.; Lin, J.D. Circadian autophagy rhythm: A link between clock and metabolism? Trends Endocrinol. Metab. Tem. 2012, 23, 319-325. [CrossRef]

45. Ma, D.; Panda, S.; Lin, J.D. Temporal orchestration of circadian autophagy rhythm by C/EBPbeta. Embo J. 2011, 30, 4642-4651. [CrossRef] [PubMed]

46. Pfeifer, U.; Scheller, H.; Ormanns, W. Diurnal rhythm of lysosomal organelle decomposition in liver, kidney and pancreas. Acta Histochemica Suppl. 1976, 16, 205-210.

47. Pfeifer, U.; Strauss, P. Autophagic vacuoles in heart muscle and liver. A comparative morphometric study including circadian variations in meal-fed rats. J. Mol. Cell. Cardiol. 1981, 13, 37-49.

48. Reme, C.E.; Sulser, M. Diurnal variation of autophagy in rod visual cells in the rat. Graefes Arch. Clin. Exp. Ophthalmol. 1977, 203, 261-270. [CrossRef]

49. Kanwar, M.; Chan, P.S.; Kern, T.S.; Kowluru, R.A. Oxidative damage in the retinal mitochondria of diabetic mice: Possible protection by superoxide dismutase. Investig. Ophthalmol. Vis. Sci. 2007, 48, 3805-3811. [CrossRef]

50. Kowluru, R.A.; Chan, P.S. Oxidative stress and diabetic retinopathy. Exp. Diabetes Res. 2007, 2007, 43603. [CrossRef]

51. Wu, Y.; Tang, L.; Chen, B. Oxidative stress: Implications for the development of diabetic retinopathy and antioxidant therapeutic perspectives. Oxid. Med. Cell. Longev. 2014, 2014, 752387. [CrossRef]

52. Zhong, Q.; Kowluru, R.A. Diabetic retinopathy and damage to mitochondrial structure and transport machinery. Investig. Ophthalmol. Vis. Sci. 2011, 52, 8739-8746. [CrossRef]

53. Maiese, K. Moving to the Rhythm with Clock (Circadian) Genes, Autophagy, mTOR, and SIRT1 in Degenerative Disease and Cancer. Curr. Neurovasc. Res. 2017, 14, 299-304. [CrossRef] 
54. Cao, R. mTOR Signaling, Translational Control, and the Circadian Clock. Front. Genet. 2018, 9, 367. [CrossRef] 55. Ryzhikov, M.; Ehlers, A.; Steinberg, D.; Xie, W.; Oberlander, E.; Brown, S.; Gilmore, P.E.; Townsend, R.R.; Lane, W.S.; Dolinay, T.; et al. Diurnal Rhythms Spatially and Temporally Organize Autophagy. Cell Rep. 2019, 26, 1880-1892.e6. [CrossRef] [PubMed]

(C) 2020 by the authors. Licensee MDPI, Basel, Switzerland. This article is an open access article distributed under the terms and conditions of the Creative Commons Attribution (CC BY) license (http://creativecommons.org/licenses/by/4.0/). 

Article

\title{
Innate and Autoimmunity in the Pathogenesis of Inherited Retinal Dystrophy
}

\author{
T. J. Hollingsworth ${ }^{1,2}$ and Alecia K. Gross ${ }^{2,3, *}$ \\ 1 Hamilton Eye Institute, Department of Ophthalmology, University of Tennessee Health Science Center, \\ Memphis, TN 38163, USA; thollin1@uthsc.edu \\ 2 Department of Optometry and Vision Science, University of Alabama at Birmingham, \\ Birmingham, AL 35294, USA \\ 3 Department of Neurobiology, University of Alabama at Birmingham, Birmingham, AL 35294, USA \\ * Correspondence: agross@uab.edu; Tel.: +1-205-975-8396
}

Received: 28 January 2020; Accepted: 3 March 2020; Published: 5 March 2020

\begin{abstract}
Inherited retinal dystrophies (RDs) are heterogenous in many aspects including genes involved, age of onset, rate of progression, and treatments. While RDs are caused by a plethora of different mutations, all result in the same outcome of blindness. While treatments, both gene therapy-based and drug-based, have been developed to slow or halt disease progression and prevent further blindness, only a small handful of the forms of RDs have treatments available, which are primarily for recessively inherited forms. Using immunohistochemical methods coupled with electroretinography, optical coherence tomography, and fluorescein angiography, we show that in rhodopsin mutant mice, the involvement of both the innate and the autoimmune systems could be a strong contributing factor in disease progression and pathogenesis. Herein, we show that monocytic phagocytosis and inflammatory cytokine release along with protein citrullination, a major player in forms of autoimmunity, work to enhance the progression of RD associated with a rhodopsin mutation.
\end{abstract}

Keywords: retinal degeneration; immunity; autoimmunity; rhodopsin; citrullination; retinitis pigmentosa

\section{Introduction}

Inherited retinal dystrophies (RDs) are the result of mutations in genes associated with cells of the outer retina, primarily rod and cone photoreceptors and retinal pigment epithelium (RPE) [1]. RDs are often highly heterogenous in every aspect of the disease, from the age of onset to the rate of progression, to the very mechanisms underlying the pathogenesis [2]. In order to study the molecular mechanisms of pathogenesis underlying retinal disease, numerous animal models, mostly in mouse, have been generated, carrying genetic defects in several genes causing the disease. A major cause of $\mathrm{RDs}$, specifically, autosomal dominant retinitis pigmentosa (RP), is the presence of dominantly inherited mutations in the gene for the rod photoreceptor protein rhodopsin [2]. While most rhodopsin mutations cause protein misfolding and subsequent apoptosis, the more severe mutations result in improper rhodopsin trafficking and can lead to a subsequent loss of outer segment formation [2]. These mutations result in improper localization of both normal and mutant rhodopsin to the cellular membrane of the inner segment, nuclear, and axonal regions of the rod photoreceptor cells [1]. Two rhodopsin mutations resulting in the earliest onset of retinal degeneration, rhodopsin Ter349Glu and Gln344Ter, behave in this manner [2]. In recent years, many studies have shown the involvement of the immune system in the pathogenesis of many retinal diseases including glaucoma, age-related macular degeneration (AMD), RP, and others [3,4]. Also brought to light is the role of autoimmunity in RDs including AMD and RP [3,5-8]. While the eye is immune-privileged, the homeostatic disruptions caused by the progression of RDs can allow for a loss of this immune-privileged status, mainly due to the breakdown 
of the blood retinal barrier and choroidal neovascularization. In this study, we show the involvement of pro-inflammatory pathways in the progression of rhodopsin-mediated RD using the Ter349Glu rhodopsin knock-in mouse model of the disease, including monocytic phagocytosis and activation of the Janus Kinase/Signal Transducer and Activator of Transcription (JAK/STAT) pathway. We also show the presence of known autoimmunity proteins and protein modifications in the rhodopsin Ter349Glu mutant mouse.

\section{Materials and Methods}

\subsection{Measuring Electrical Function in the Ter349Glu Rhodopsin Knock-in Mouse by Electroretinogram (ERG)}

Wild-type $(+/+$, WT), Ter349Glu rhodopsin heterozygous and homozygous mice at one month of age were dark-adapted overnight $(\mathrm{O} / \mathrm{N})$. The following day, the mice were anesthetized using ketamine/xylazine ( $14.3 \mathrm{mg} / \mathrm{mL}$ ketamine $/ 2.8 \mathrm{mg} / \mathrm{mL}$ xylazine in PBS, $\mathrm{pH} 7.4)$, and ERGs were performed. Dark-adapted flashes (505 nm stimulus) of varying intensities were achieved using neutral density (ND) filters of nominal optical densities (OD) 4.8, 1.4, 3.6, 2.4, 1.8, 1.2, 0.6, and 0.0. A dark-adapted green camera flash performed with no attenuation. Data were analyzed using Labview and IgorPro software.

\subsection{Monitoring the Ter349Glu Rhodopsin Knock-in Mouse Retina for Vascular and Laminar Abnormalities}

WT and Ter349Glu homozygous mice at 4 weeks of age were anesthetized using ketamine/xylazine $(14.3 \mathrm{mg} / \mathrm{mL}$ ketamine $/ 2.8 \mathrm{mg} / \mathrm{mL}$ xylazine in PBS, pH7.4). The mice were subsequently examined by optical coherence tomography (OCT) (Bioptigen $840 \mathrm{~nm}$ Spectral Domain-OCT, Durham, NC, USA) to measure retinal thickness and assess for structural anomalies. After OCT, the mice were injected intraperitoneally with $100 \mu \mathrm{L}$ of $4 \%$ fluorescein, and the retinas were imaged approximately 1 to 2 minutes post-fluorescein injection by fluorescein angiography (FA), using a $488 \mathrm{~nm}$ lamp co-equipped with a Micron III digital microscope (Phoenix Laboratories, Mukilteo, WA, USA) to assess the retinal vasculature for abnormalities including neovascularization, retinal hemorrhage, and vessel alterations.

\subsection{Immunohistochemical Survey for Inflammatory Markers in the C-Terminal Mutant Rhodopsin Knock-in Mouse Retina}

Whole eyes from WT, Ter349Glu rhodopsin heterozygous and homozygous mice 4 , 8 , and/or 12 weeks of age were fixed in $4 \%$ paraformaldehyde (PFA) in PBS, $\mathrm{pH} 7.4$, overnight at $4{ }^{\circ} \mathrm{C}$, cryoprotected in 30\% sucrose in PBS, pH 7.4, frozen in optimal cutting temperature medium, and cryosectioned into $10 \mu \mathrm{m}$-thick sections. After washing away the medium, sections were prepped for immunolabeling using heat-mediated antigen retrieval in $10 \mathrm{mM}$ sodium citrate, $\mathrm{pH}$ 6.0, with $0.05 \%$ Tween- 20 for $1 \mathrm{~h}$. After cooling, the slides were washed in PBS and subsequently blocked in $10 \%$ goat serum $/ 5 \% \mathrm{BSA} / 0.5 \%$ Triton X-100 in PBS for $1 \mathrm{~h}$ at RT. Following blocking, labeling for markers of retinal inflammation including activated macrophages (F4/80), phosphorylated STAT3 (pSTAT3), and suppressor of cytokine signaling 3 (SOCS3) was performed using fluorescent immunohistochemistry. For both pSTAT3 (Cell Signaling Technology, Danvers, MA, USA) and SOCS3 (abcam, Cambridge, United Kingdom) labeling, a goat anti-rabbit IgG secondary antibody conjugated to horseradish peroxidase (HRP) was allowed to bind the primary antibodies, and Cy3-Tyramide Signal Amplification (TSA, Perkin Elmer, Waltham, MA, USA) was used to visualize the proteins. Briefly, TSA uses the peroxidase activity of HRP to generate reactive oxygen species from peroxide. These reactive oxygen species then oxidize and covalently bind the Cy3-conjugated tyramide molecule to the nearby proteins including the antigen/antibody complex, thus amplifying the signal. Macrophage labeling was performed using an antibody to F4/80-antigen (Bio-Rad, Hercules, CA, USA) followed by a goat anti-rat IgG secondary antibody conjugated to AlexaFluor488 (Invitrogen, Carlsbad, CA, USA). All sections were co-labeled for rhodopsin using B6-30N or K62-82 (provided by W. Clay Smith, University of Florida, Gainesville, FL, USA) and goat anti-mouse $\operatorname{IgG}_{1}$ or $\operatorname{IgG}_{3}$ conjugated to AlexaFluor488 (Invitrogen). Labeling for Müller cells was performed using an antibody against glial fibrillary acidic protein (GFAP, EMD Millipore, 
Burlington, MA, USA) followed by goat anti-mouse secondary $\operatorname{IgG}_{1}$ conjugated to AlexaFluor647 (Invitrogen). All images were captured as Z-stacks and expressed as maximum intensity projections.

\subsection{Analysis of Retinal Citrullination in the Ter349Glu/Ter349Glu Rhodopsin Mouse Retina by Fluorescent Immunohistochemistry}

Whole eyes from 10- to 12-week-old mice were fixed in 4\% PFA in PBS, pH 7.4, overnight at $4{ }^{\circ} \mathrm{C}$, cryoprotected in $30 \%$ sucrose in PBS, $\mathrm{pH}$ 7.4, frozen in optimal cutting temperature medium, and cryosectioned into $10 \mu \mathrm{m}$-thick sections. After washing away the medium, the sections were treated using heat-mediated antigen retrieval by boiling in $10 \mathrm{mM}$ sodium citrate, $\mathrm{pH} 6.0$, with $0.05 \%$ Tween-20 for $1 \mathrm{~h}$. After cooling, the slides were washed in PBS and subsequently blocked in $10 \%$ goat serum $/ 5 \%$ BSA/0.5\% Triton X-100 in PBS for $1 \mathrm{~h}$ at RT. Following blocking, the sections were incubated with primary antibodies against peptidyl arginine deiminase 4 (PAD4, ProteinTech, Rosemont, IL, USA) and citrullinated peptides (Clone F95, EMD Millipore, Burlington, MA, USA) O/N at $4{ }^{\circ} \mathrm{C}$. After washing in PBS, the slides were probed using goat anti-rabbit IgG conjugated to AlexaFluor 488 (Invitrogen) and goat anti-mouse IgM conjugated to AlexaFluor555 (Invitrogen), and the nuclei were labeled with DAPI (Invitrogen). After washing in PBS, the slides were mounted using Prolong Diamond Anti-Fade Mountant (Invitrogen) and imaged using a Zeiss 710 Laser Scanning Confocal Microscope (Zeiss, Oberkochen, Germany). All images were captured as Z-stacks and expressed as maximum intensity projections.

\section{Results}

\subsection{Loss of Functional ERG in Early-Onset RD}

Patients expressing the Ter349Glu mutant rhodopsin experience a loss of photoreceptor function earlier in life, resulting in early and rapid central vision loss, when compared to other mutants of rhodopsin [9]. We tested if the Ter349Glu knock-in mouse displayed a similar early-onset (4 weeks of age) loss of visual capacity by ERG (Figure 1). Compared to +/+ mice $(n=5)$, the Ter349Glu/Ter349Glu mice $(n=5)$ showed an increase in the threshold of the dark-adapted b-wave by three orders of magnitude, with a maximum amplitude about $25 \%$ that of $+/+$ mice, while the maximum a-wave amplitude was only about $6 \%$ that of $+/+$ mice. This indicated a drastic loss of rod photoreceptor function. Interestingly, when compared to $+/+$ and Ter349Glu/Ter349Glu mice, Ter349Glu/+ mice $(n=4)$ appeared to have a gain of function with an increase in sensitivity and a decrease in response latency without significant changes in amplitudes.

\subsection{Effects of RD-Associated Photoreceptor Loss on Retinal Vasculature and Laminar Architecture}

In cases of $\mathrm{RP}$, retinal degeneration exerts effects on both retinal vasculature and laminar architecture in the forms of attenuated vessels and outer nuclear layer (ONL) thinning, respectively [10]. To examine the Ter349Glu/Ter349Glu retina for such abnormalities, FA and OCT were performed on 4-week-old +/+ and Ter349Glu/Ter349Glu animals in triplicate (Figure 2). Using FA, when compared to +/+ mice, Ter349Glu/Ter349Glu mice exhibited heterogeneous types and degrees of vascular abnormalities. The most common anomalies included attenuated vessels, tortuous vessels indicating hyperoxia, and reduced retinal venous and arterial vessel numbers. Using OCT, Ter349Glu/Ter349Glu mice exhibited the expected thinning of the ONL; however, at the interfaces between choroid, RPE, and rod outer segments (ROS)/ rod inner segments (RIS) layers the mice also exhibited a possible edema, likely due to loss of contacts between the RPE and the photoreceptors, associated with the lack of ROS. This edema was observed extending to varying degrees both inferiorly and superiorly to the optic nerve. Representative images were taken from the retina inferior to the optic nerve. 

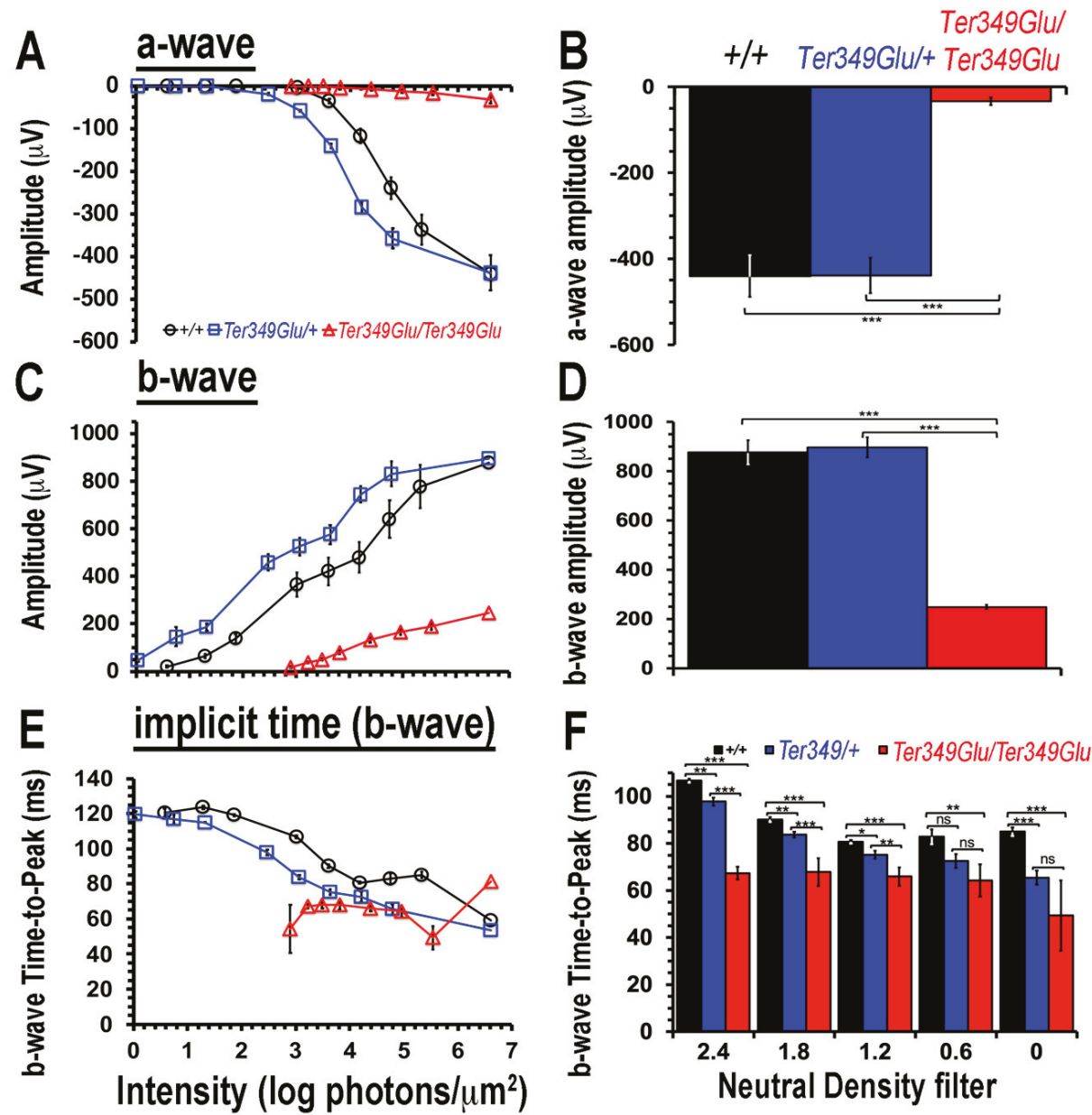

Figure 1. Electroretinogram (ERG) responses decline in mice expressing Ter349Glu rhodopsin. Using ERG to record extracellular potential differences across the retina, the electrophysiological function of +/+, Ter349Glu/+, and Ter349Glu/Ter349Glu mice was monitored by measuring a-, b-waves, and response latencies (Time-to-Peak, TTP) under increasing stimulus intensities (A,C,E). Graphs compare maximum average wave amplitudes and TTP (B,D,F) under dark-adapted conditions. Data analyzed using two-tailed T-test and expressed as the mean \pm S.E.M. ${ }^{*}, p<0.05 ;{ }^{* *}, p<0.01$; ***, $p<0.001$; ns, not significant. 


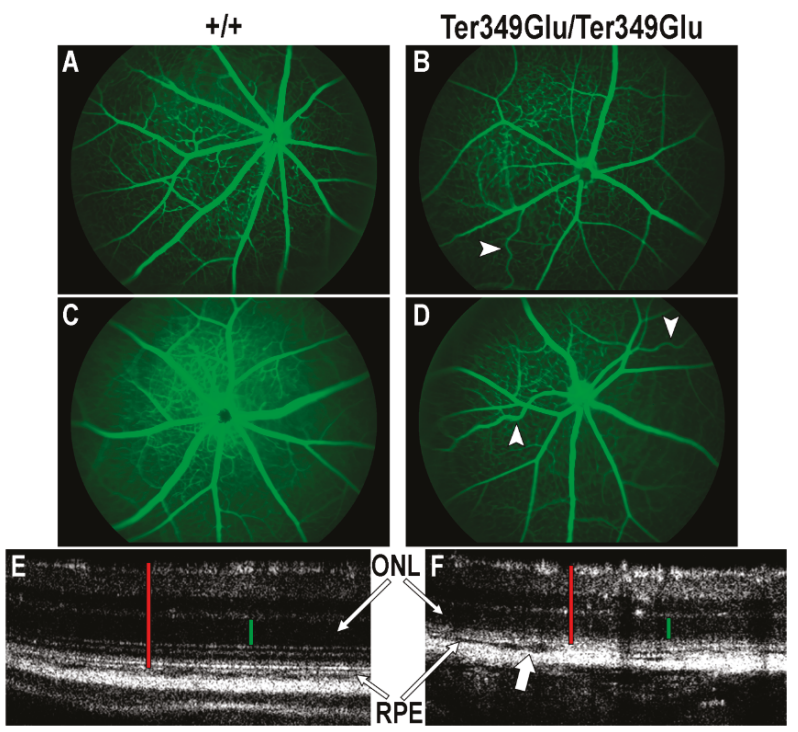

Figure 2. Ter349Glu rhodopsin knock-in mouse retina exhibits both vascular and laminar abnormalities. (A-D) Utilizing fluorescein angiography (FA), the state of the retinal vasculature of 4-week-old +/+ (A,B) and Ter349Glu/Ter349Glu (C,D) mice was examined. Abnormal phenotypes varied in severity among mice, with overall attenuated retinal vessels and tortuous retinal vessels (arrowheads) being commonplace amongst all mice examined. (E,F) Optical coherence tomography (OCT) was used to examine the retinas of 4-week-old +/+ (E) and Ter349Glu/Ter349Glu mice (F) for architectural abnormalities. Ter349Glu/Ter349Glu mice exhibited thinning of the outer nuclear layer (ONL) and patches of varying degrees of separation among the choroid, retinal pigment epithelium (RPE), and photoreceptors (block arrow), indicative of edema. Retinal thickness (red calipers) $=240 \mu \mathrm{m}(\mathrm{E})$ and $180 \mu \mathrm{m}(\mathbf{F}) ;$ ONL (green calipers) $=60 \mu \mathrm{m}(\mathbf{E})$ and $50 \mu \mathrm{m}(\mathbf{F})$.

\subsection{Activated Monocytes Are Present in RD Retinas from Rhodopsin Mutant Knock-in Mice}

The retina contains resident macrophages similarly to the cortex, known as microglia, and these cells remain in the inner retinal layers under normal physiological conditions. Here, they remain in an inactivated state unless triggered by cytokine signaling or apoptotic signals [11]. Other types of leukocytes are typically not resident in the retina, and evidence of these cells in ocular tissues is indicative of retinal inflammation. Damage to retinal and choroidal vessels can allow leakage of not only blood-borne macrophages into the retina, but also cytokines, antibodies, and a plethora of other inflammatory factors [12]. Unfortunately, no method exists to differentiate between microglia and blood-borne macrophages; however, due to the observation of abnormal vasculature and retinal edemas, we chose to monitor the $+/+$ and Ter349Glu/Ter349Glu retinas for activated macrophages as a whole, using an antibody against F4/80 antigen, a cell surface glycoprotein expressed upon macrophage maturation (Figure 3). F4/80-positive macrophages were found in multiple animals from the Ter349Glu/Ter349Glu cohort at 12 weeks of age, with the most labeling observed in sections from Ter349Glu/Ter349Glu animals where nearly the whole retina was degenerated. Macrophages remained in the outer retina after almost total rod cell death (12 weeks). These macrophages were not observed in $+/+$ sections from any animal ( $n=3$ at all ages). 


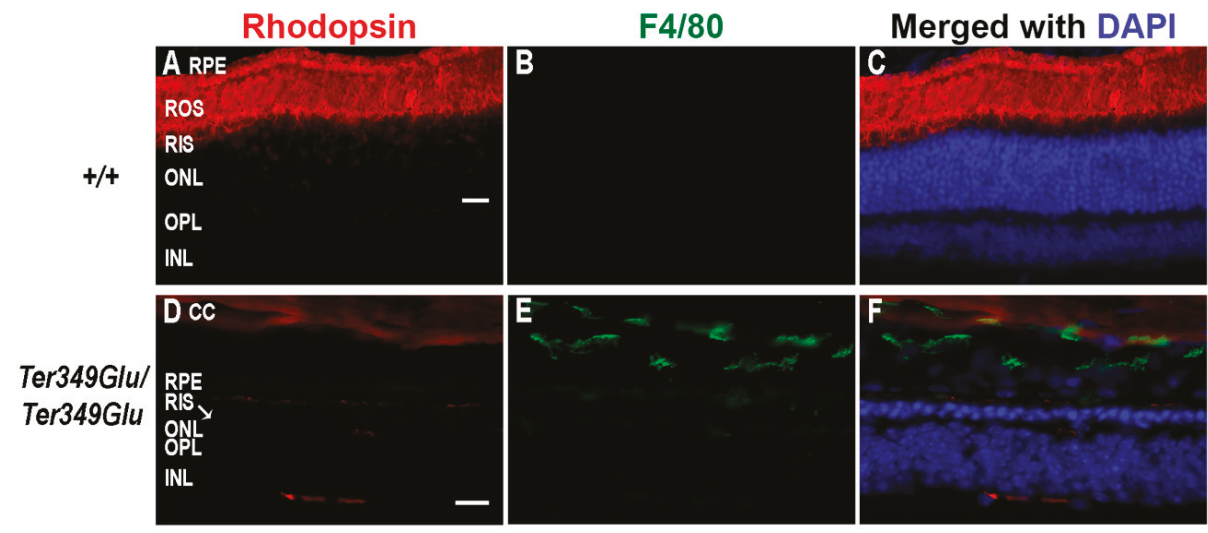

Figure 3. Retinas from retinal dystrophy (RD) mice exhibit monocyte activation. Using fluorescent immunohistochemistry, the presence of activated macrophages was examined in $+/+(\mathbf{A}-\mathbf{C})$ and Ter349Glu/Ter349Glu (D-F) mice at 12 weeks of age. Retinal sections were labeled with anti-F4/80 antigen (green) and K62-82 (rhodopsin, red) antibodies. Nuclei were labeled with DAPI (blue). Autofluorescence in the choroid was observed in the red channel. CC, choriocapillaris; ROS, rod outer segments; RIS, rod inner segments; OPL, outer plexiform layer. Scale bars $=20 \mu \mathrm{m}$.

\subsection{Activation of the Pro-Inflammatory JAK/STAT Pathway and Its Inhibitor SOCS3 in RD}

STAT3 is a downstream signaling partner of JAK. In inflammatory conditions, STAT3 is activated when cytokines such as IL-6, ciliary neurotrophic factor, leukemia inhibitory factor, and others bind to and activate the glycoprotein 130 (gp130) receptor [13]. Gp130 activates JAK, which in turn phosphorylates STAT proteins (pSTAT). Upon phosphorylation, pSTATs form both homo- and heterodimers, allowing nuclear entry and activation of gene transcription. The protein SOCS3 works as a negative regulator of the JAK/STAT pathway by binding the gp130 receptor and JAK together and blocking active sites involved in phosphorylation of STAT3, thus prohibiting further signal transduction [14]. In instances where inflammatory signaling needs to be slowed or stopped, SOCS3 works to perform this task. To examine the Ter349Glu knock-in mouse retina for inflammatory cytokine signaling, retinas from both +/+, Ter349Glu/+, and Ter349Glu/Ter349Glu mice were labeled with an antibody against pSTAT3 (Figure 4 ). We found that $+/+$ retinas showed no STAT3 activation across all ages, while the Ter349Glu/+ and Ter349Glu/Ter349Glu mice exhibited increasing activation with age, beginning at 8 weeks and 4 weeks, respectively ( $n=3$ for all ages).

Early pSTAT3 activation began in the inner nuclear layer (INL) and, by 12 weeks, extended to the RPE. The location of the activated nuclei in the INL suggested the nuclei belonged to Müller cells. Indeed, co-labeling for GFAP, a marker of gliosis in Müller cells, showed Müller cells to be pSTAT3-positive (Figure 5). 


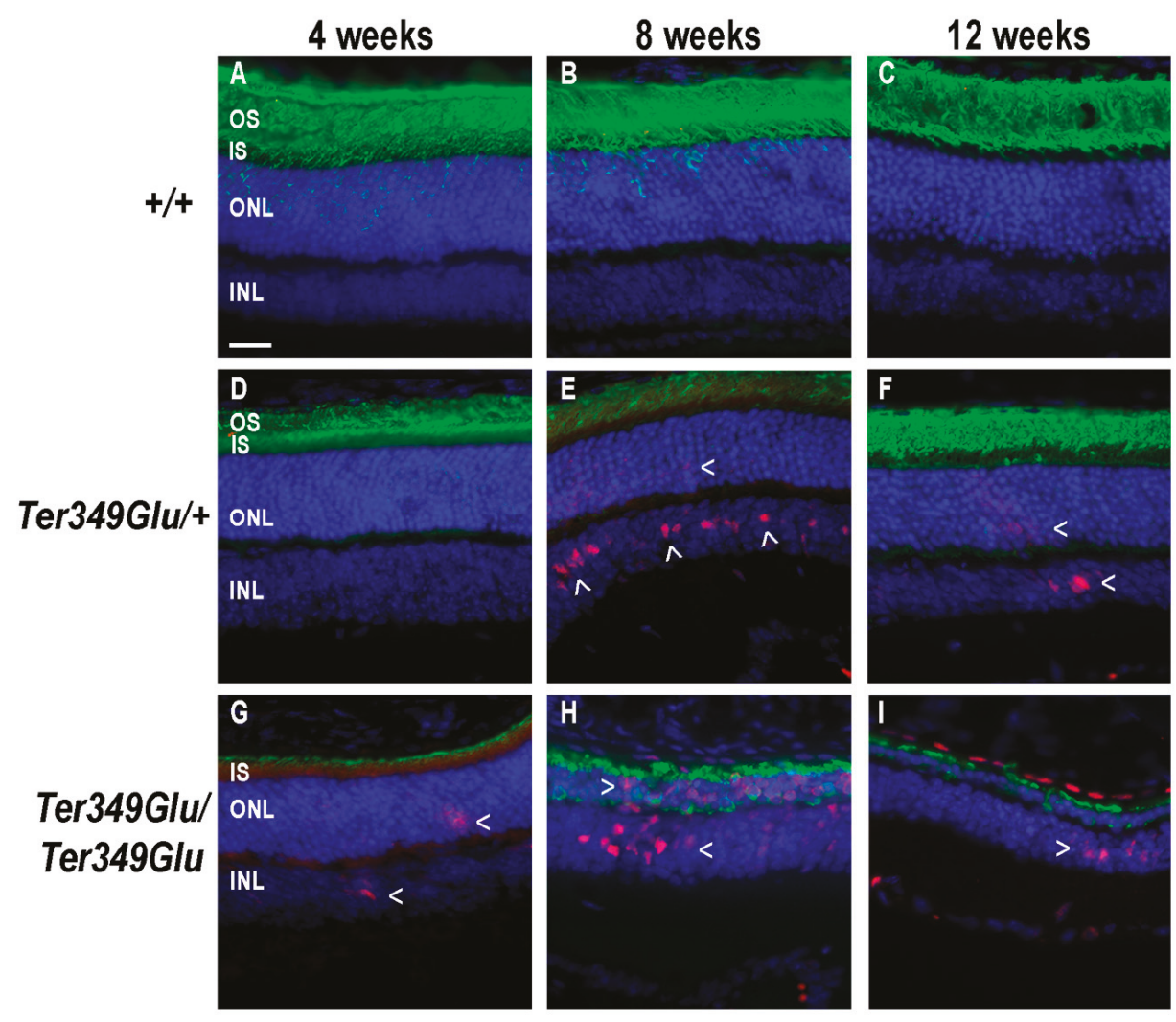

Figure 4. JAK/STAT pathway is activated in Ter349Glu rhodopsin knock-in mouse retina. Activation of the JAK/STAT pathway was examined using fluorescent immunohistochemistry on retinas from WT (+/+, A-C), Ter349Glu heterozygous (Ter349Glu/+, D-F), and Ter349Glu homozygous (Ter349Glu/Ter349Glu, G-H) mice. Retinal sections were treated for antigen retrieval and labeled for phosphorylated STAT3 (pSTAT3, red) and rhodopsin (green). Nuclei were labeled with DAPI (blue). Arrowheads (>), areas of JAK/STAT activation; OS, outer segments; IS, inner segments; INL, inner nuclear layer. Scale bar = $20 \mu \mathrm{m}$.

Since Müller cells maintain retinal homeostasis, this finding suggests they may act to respond to inflammatory cytokines, as STAT3 activation indicates the presence of pro-inflammatory cytokines in the neural retina, likely being released from activated macrophages within the retina. It should be noted that in these images, labeling for rhodopsin can be seen in the ONL even though rhodopsin is not normally localized in large amounts in this region. This is likely an artifact due to a heightened number of anti-rhodopsin epitopes following the antigen retrieval process. Labeling for SOCS3 revealed minimal expression in +/+ retinas while, similarly to pSTAT3, Ter349Glu/+ and Ter349Glu/Ter349Glu mice began expressing SOCS3 in the neural retina and RPE at 4 weeks of age; with time, the expression increased in Ter349Glu/Ter349Glu mice and decreased in Ter349Glu/+ mice (Figure 6, $n=3$ at all ages). 


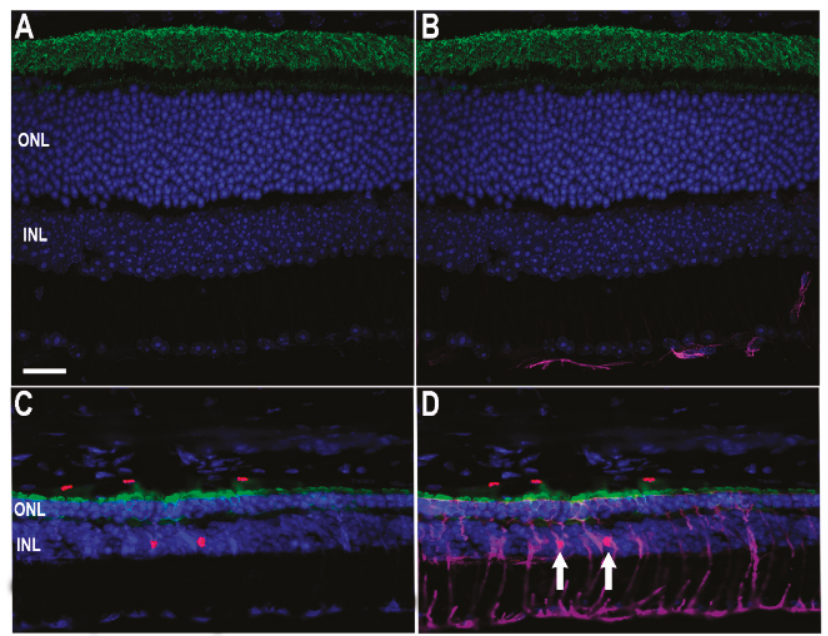

Figure 5. Identification of Müller cell nuclei as INL centers for STAT3 activation. To assess which retinal cells exhibited STAT3 phosphorylation, labeling was performed on +/+ (A,B) and Ter349Glu/Ter349Glu (C,D) animals for glial fibrillary acidic protein (purple), a marker of astrocytes and gliotic Müller cells, rhodopsin (green), and pSTAT3 (red). Nuclei were labeled with DAPI (blue). Arrows show red nuclei surrounded by purple. Scale bar $=20 \mu \mathrm{m}$.

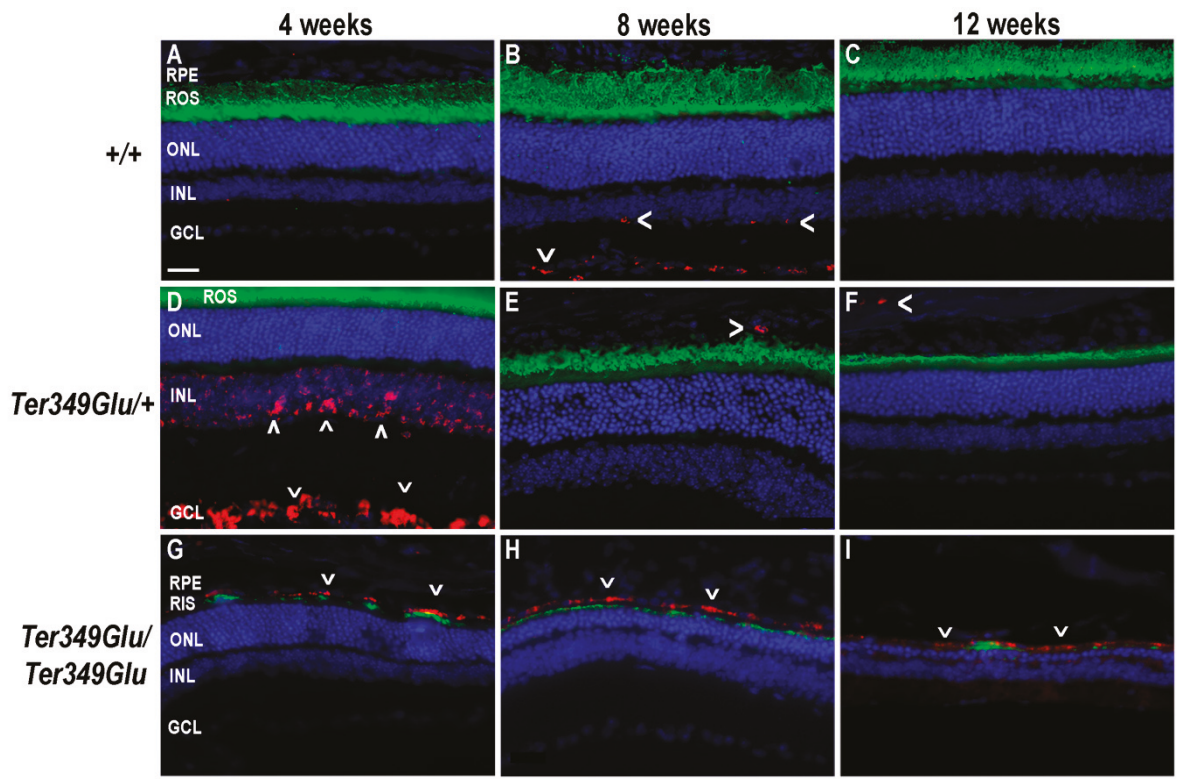

Figure 6. The JAK/STAT antagonist SOCS3 is expressed in Ter349Glu rhodopsin knock-in mouse retina. Using fluorescent immunohistochemistry, the expression of SOCS3, an antagonist to the JAK/STAT pathway, was examined in retinas from wild-type (WT, +/+, A-C), Ter349Glu heterozygous (Ter349Glu/+, D-F), and Ter349Glu homozygous (Ter349Glu/Ter349Glu, G-I) mice. Retinal sections were treated for antigen retrieval and labeled for SOCS3 (red) and rhodopsin (green). Nuclei were labeled with DAPI (blue). GCL, ganglion cell layer; arrowheads $(<)$, SOCS3 labeling. Scale bar $=20 \mu \mathrm{m}$. 


\subsection{Cell-Specific Expression of PAD4 and Heightened Citrullination in Early-Onset RD}

Recent studies have shown an increase in the expression of the deiminating enzyme PAD4 and increased citrullination in the event of ocular insult of the anterior segment [15]. Due to the inherent ability of citrullinated proteins to cause autoimmunity [16-22] and our previous finding that PAD4 is the primary retinal PAD in mouse [23], we tested the Ter349Glu retina for changes in PAD4 expression and citrullination compared with WT retina at 10 to 12 weeks of age (Figure 7). WT retina exhibited expression of PAD4 and exhibited INL nuclear citrullination, as shown previously [23]; however, the Ter349Glu retina exhibited higher levels of citrullination, much of it spanning the entire retina. This was observed in parallel with PAD4 expression increases, especially in the photoreceptors.

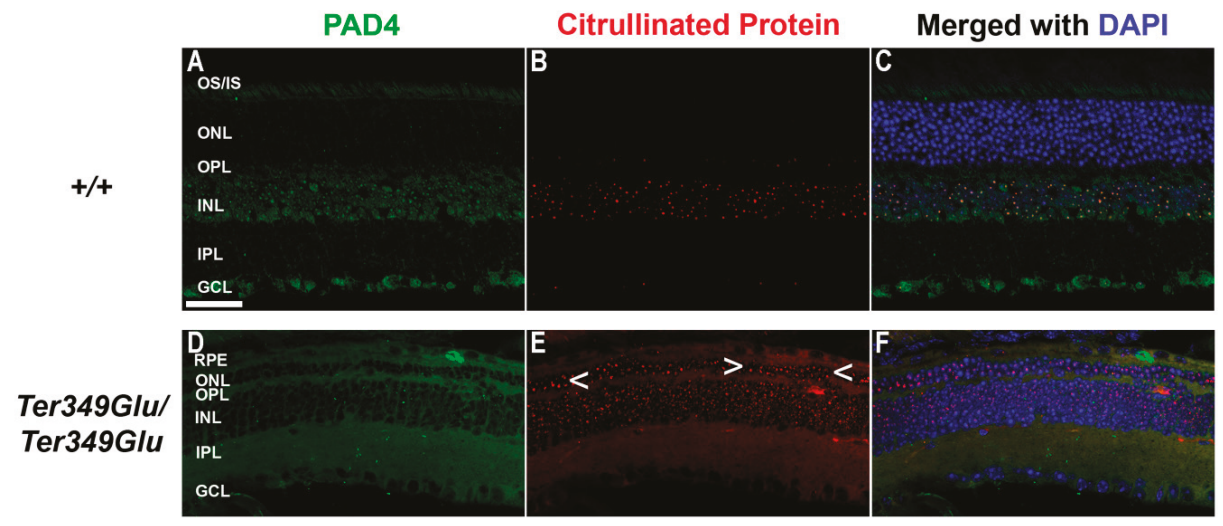

Figure 7. Expression of PAD4 and citrullination of retinal proteins in normal and degenerated states. WT (+/+, A-C) and Ter349Glu/Ter349Glu (D-F) mice at 10 to 12 weeks of age were labeled for PAD4 (green) and citrullinated peptides (red). Nuclei were labeled with DAPI (blue). Arrowheads $(<)$, areas of increased citrullination in ONL; OS/IS, photoreceptor outer and inner segments; IPL, inner plexiform layer; Scale bar $=20 \mu \mathrm{m}$.

\section{Discussion}

Increasing amounts of evidence linking the immune system to ocular disease has emerged in the last decade. Studies focusing on glaucoma, AMD, RP, and other RDs have continued to show increased presence of many immune components including monocytes (blood-borne and resident), pro- and anti-inflammatory cytokines, and autoantibodies in many models of these diseases [24-26]. While all of these diseases present an initial insult of genetic and/or environmental origins, these findings overwhelmingly implicate the immune system in the pathogenesis of RDs. For example, the prevalence of single-nucleotide polymorphisms in the genes coding for complement factor $\mathrm{H}$, complement factor I, as well as many other components of the innate immune system bolster the chances of developing AMD in otherwise normal patients [27,28]. In addition, models of glaucoma have shown the presence of macrophages and T-lymphocytes in the eye along with autoantibodies to proteins of the retina $[3,4,29,30]$. Models of RP have also shown similar features, including monocytic phagocytosis of both diseased and healthy retinal cells as well as numerous cytokines such as interleukin-1, interleuken-6, vascular endothelial growth factor, and others [4,31,32]. While the molecular mechanisms of the primary genetic assault have been teased out by copious amounts of work using cell culture and animal models for many RDs (i.e., loss of outer segment formation, disrupted visual cycle, etc.) $[1,2,33]$, the full mechanisms of pathogenesis and progression of RDs still need thorough investigation before we fully understand them. In our findings, the Ter349Glu/Ter349Glu mice exhibited an almost complete loss of photoreceptor function by ERG; however, the Ter349Glu/+ mice had heightened a and $b$ waves while also showing a decrease in $b$ wave latency compared to the $+/+$ 
animals. This phenomenon might be explained by the expression levels of mutant rhodopsin compared to WT rhodopsin, as Ter349Glu rhodopsin expression levels are less than half of the WT levels [2]. This lower level of expression while still having 50\% WT rhodopsin could result in a somewhat thinner outer segment with less rhodopsin protein in the discs, thus allowing for faster rates of diffusion of the phototransduction cascade components, decreasing the response latency, while normal WT rhodopsin activates the cascade, enhancing the signal. We also showed that animals with more advanced RD had significant numbers of macrophages, a feature found in many RDs, as well as activation of a known pro-inflammatory pathway (JAK/STAT). Interestingly, the Ter349Glu/+ mice showed SOCS3 expression in the inner retinal layers early in life (4 weeks of age), with a dramatic decrease in expression with age, while the $+/+$ mice had minimal SOCS3 expression, which peaked at 8 weeks of age, and the Ter349Glu/Ter349Glu mice exhibited a somewhat constant SOCS3 expression in the outer retina and RPE. While the relatively stable SOCS3 expression in the Ter349Glu/Ter349Glu mice is explainable when compared to the levels of STAT3 phosphorylation which was present throughout the first 3 months of the animals' life, the stark difference in SOCS 3 expression the Ter349Glu/+ mice remains to be elucidated. More experiments examining the activation and deactivation of the proinflammatory JAK/STAT pathway are needed to better understand these expression differences. Further work will aim at pinning down the cytokines responsible for pathway activation; experiments inhibiting the pathway activation using antagonists to the JAK/STAT pathway and/or those inhibiting specific cytokine(s) will better underpin this pathway's role in RD. Due to the nature of STAT3 role in numerous developmental pathways [13], a more targeted approach to delivering inhibitory compounds directly to the eye would be necessary. Future experiments will also work to decrease the number of activated macrophages in RD models to attempt to rescue some photoreceptor degeneration due to excessive phagocytosis and/or macrophage-derived cytokines. Work is already underway testing the inhibition of PAD4 in RD models to rescue retinal cells, retinal function, or both [34]. This work will be achieved using PAD4-deficient mice as well as inhibitors of the enzyme. Due to the excessive citrullination observed in Ter349Glu/Ter349Glu mice, it is not far-fetched to think that lowering or preventing this post-translation modification from occurring could lead to a slower rate of disease progression, allowing for not only longer lasting vision in patients affected but also an extended opportunity to correct the genetic insult, thus preventing further retinal degeneration. In all, our work further contributes to the increasing volume of studies indicating a role of the immune system in RDs as well as provides possible targets for the treatment of these debilitating blinding diseases.

Author Contributions: Conceptualization, A.K.G. and T.J.H.; methodology, A.K.G. and T.J.H.; investigation, A.K.G. and T.J.H.; resources, A.K.G. and T.J.H.; data curation, A.K.G. and T.J.H.; writing-original draft preparation, T.J.H.; writing-review and editing, A.K.G. and T.J.H.; visualization, A.K.G. and T.J.H.; project administration, A.K.G.; funding acquisition, A.K.G. All authors have read and agreed to the published version of the manuscript.

Funding: This research was funded by the NIH R01EY019311 and the E. Matilda Ziegler Foundation. Center support for the project was provided by NIH P30EY003039.

Acknowledgments: We thank Brian Simms and Meredith Hubbard for technical assistance and W. Clay Smith for the K62-82 rhodopsin antibody.

Conflicts of Interest: The authors declare no conflicts of interest.

\section{References}

1. Hollingsworth, T.J.; Gross, A.K. Defective trafficking of rhodopsin and its role in retinal degenerations. In International Review of Cell and Molecular Biology; Academic Press: Cambridge, MA, USA, 2012; Volume 293. [CrossRef]

2. Hollingsworth, T.J.; Gross, A.K. The severe autosomal dominant retinitis pigmentosa rhodopsin mutant Ter349Glu mislocalizes and induces rapid rod cell death. J. Biol. Chem. 2013, 288, 29047-29055. [CrossRef]

3. Bhattacharya, S.K.; Crabb, J.S.; Bonilha, V.L.; Gu, X.; Takahara, H.; Crabb, J.W. Proteomics implicates peptidyl arginine deiminase 2 and optic nerve citrullination in glaucoma pathogenesis. Investig. Ophthalmol. Vis. Sci. 2006, 47, 2508-2514. [CrossRef] [PubMed] 
4. Wooff, Y.; Man, S.M.; Aggio-Bruce, R.; Natoli, R.; Fernando, N. IL-1 family members mediate cell death, inflammation and angiogenesis in retinal degenerative diseases. Front. Immunol. 2019, 10, 1618. [CrossRef] [PubMed]

5. Iannaccone, A.; Giorgianni, F.; New, D.D.; Hollingsworth, T.J.; Umfress, A.; Alhatem, A.H.; Neeli, I.; Lenchik, N.I.; Jennings, B.J.; Calzada, J.I.; et al. Circulating autoantibodies in age-related macular degeneration recognize human macular tissue antigens implicated in autophagy, immunomodulation, and protection from oxidative stress and apoptosis. PLoS ONE 2015, 10, e0145323. [CrossRef] [PubMed]

6. Bhattacharya, S.K. Retinal deimination in aging and disease. IUBMB Life 2009, 61, 504-509. [CrossRef] [PubMed]

7. Bonilha, V.L.; Shadrach, K.G.; Rayborn, M.E.; Li, Y.; Pauer, G.J.; Hagstrom, S.A.; Bhattacharya, S.K.; Hollyfield, J.G. Retinal deimination and PAD2 levels in retinas from donors with age-related macular degeneration (AMD). Exp. Eye Res. 2013, 111, 71-78. [CrossRef]

8. Iannaccone, A.; Hollingsworth, T.J.; Koirala, D.; New, D.D.; Lenchik, N.I.; Beranova-Giorgianni, S.; Gerling, I.C.; Radic, M.Z.; Giorgianni, F. Retinal pigment epithelium and microglia express the CD5 antigen-like protein, a novel autoantigen in age-related macular degeneration. Exp. Eye Res. 2017, 155, 64-74. [CrossRef] [PubMed]

9. Bessant, D.A.; Khaliq, S.; Hameed, A.; Anwar, K.; Payne, A.M.; Mehdi, S.Q.; Bhattacharya, S.S. Severe autosomal dominant retinitis pigmentosa caused by a novel rhodopsin mutation (Ter349Glu). Mutations in brief no. 208. Online. Hum. Mutat. 1999, 13, 83. [CrossRef]

10. Rezaei, K.A.; Zhang, Q.; Chen, C.L.; Chao, J.; Wang, R.K. Retinal and choroidal vascular features in patients with retinitis pigmentosa imaged by OCT based microangiography. Graefes Arch. Clin. Exp. Ophthalmol. 2017, 255, 1287-1295. [CrossRef]

11. Fontainhas, A.M.; Wang, M.; Liang, K.J.; Chen, S.; Mettu, P.; Damani, M.; Fariss, R.N.; Li, W.; Wong, W.T. Microglial morphology and dynamic behavior is regulated by ionotropic glutamatergic and GABAergic neurotransmission. PLoS ONE 2011, 6, e15973. [CrossRef]

12. Zhao, L.; Ma, W.; Fariss, R.N.; Wong, W.T. Minocycline attenuates photoreceptor degeneration in a mouse model of subretinal hemorrhage microglial: inhibition as a potential therapeutic strategy. Am. J. Pathol. 2011, 179, 1265-1277. [CrossRef] [PubMed]

13. Levy, D.E.; Lee, C.K. What does Stat3 do? J. Clin. Investig. 2002, 109, 1143-1148. [CrossRef] [PubMed]

14. Kershaw, N.J.; Murphy, J.M.; Liau, N.P.; Varghese, L.N.; Laktyushin, A.; Whitlock, E.L.; Lucet, I.S.; Nicola, N.A.; Babon, J.J. SOCS3 binds specific receptor-JAK complexes to control cytokine signaling by direct kinase inhibition. Nat. Struct. Mol. Biol. 2013, 20, 469-476. [CrossRef] [PubMed]

15. Wizeman, J.W.; Nicholas, A.P.; Ishigami, A.; Mohan, R. Citrullination of glial intermediate filaments is an early response in retinal injury. Mol. Vis. 2016, 22, 1137-1155.

16. Acharya, N.K.; Nagele, E.P.; Han, M.; Coretti, N.J.; DeMarshall, C.; Kosciuk, M.C.; Boulos, P.A.; Nagele, R.G. Neuronal PAD4 expression and protein citrullination: possible role in production of autoantibodies associated with neurodegenerative disease. J. Autoimmun. 2012, 38, 369-380. [CrossRef]

17. Bicker, K.L.; Thompson, P.R. The protein arginine deiminases: Structure, function, inhibition, and disease. Biopolymers 2013, 99, 155-163. [CrossRef]

18. Dwivedi, N.; Neeli, I.; Schall, N.; Wan, H.; Desiderio, D.M.; Csernok, E.; Thompson, P.R.; Dali, H.; Briand, J.P.; Muller, S.; et al. Deimination of linker histones links neutrophil extracellular trap release with autoantibodies in systemic autoimmunity. FASEB J. 2014, 28, 2840-2851. [CrossRef]

19. Dwivedi, N.; Radic, M. Citrullination of autoantigens implicates NETosis in the induction of autoimmunity. Ann. Rheum. Dis. 2014, 73, 483-491. [CrossRef]

20. Lundberg, K.; Nijenhuis, S.; Vossenaar, E.R.; Palmblad, K.; van Venrooij, W.J.; Klareskog, L.; Zendman, A.J.; Harris, H.E. Citrullinated proteins have increased immunogenicity and arthritogenicity and their presence in arthritic joints correlates with disease severity. Arthritis Res. Ther. 2005, 7, R458-R467. [CrossRef]

21. Nicholas, A.P.; Sambandam, T.; Echols, J.D.; Barnum, S.R. Expression of citrullinated proteins in murine experimental autoimmune encephalomyelitis. J. Comp. Neurol. 2005, 486, 254-266. [CrossRef]

22. Turunen, S.; Huhtakangas, J.; Nousiainen, T.; Valkealahti, M.; Melkko, J.; Risteli, J.; Lehenkari, P. Rheumatoid arthritis antigens homocitrulline and citrulline are generated by local myeloperoxidase and peptidyl arginine deiminases 2, 3 and 4 in rheumatoid nodule and synovial tissue. Arthritis Res. Ther. 2016, 18, 239. [CrossRef] [PubMed] 
23. Hollingsworth, T.J.; Radic, M.Z.; Beranova-Giorgianni, S.; Giorgianni, F.; Wang, Y.; Iannaccone, A. Murine Retinal Citrullination Declines With Age and is Mainly Dependent on Peptidyl Arginine Deiminase 4 (PAD4). Investig. Ophthalmol. Vis. Sci. 2018, 59, 3808-3815. [CrossRef] [PubMed]

24. Lu, Z.; Lin, V.; May, A.; Che, B.; Xiao, X.; Shaw, D.H.; Su, F.; Wang, Z.; Du, H.; Shaw, P.X. HTRA1 synergizes with oxidized phospholipids in promoting inflammation and macrophage infiltration essential for ocular VEGF expression. PLoS ONE 2019, 14, e0216808. [CrossRef]

25. Murakami, Y.; Ishikawa, K.; Nakao, S.; Sonoda, K.H. Innate immune response in retinal homeostasis and inflammatory disorders. Prog. Retin. Eye Res. 2019, 74, 100778. [CrossRef] [PubMed]

26. Ronning, K.E.; Karlen, S.J.; Miller, E.B.; Burns, M.E. Molecular profiling of resident and infiltrating mononuclear phagocytes during rapid adult retinal degeneration using single-cell RNA sequencing. Sci. Rep. 2019, 9, 4858. [CrossRef] [PubMed]

27. Mansoor, N.; Wahid, F.; Azam, M.; Shah, K.; den Hollander, A.I.; Qamar, R.; Ayub, H. Molecular mechanisms of complement system proteins and matrix metalloproteinases in the pathogenesis of age-related macular degeneration. Curr. Mol. Med. 2019, 19, 705-718. [CrossRef]

28. Landowski, M.; Kelly, U.; Klingeborn, M.; Groelle, M.; Ding, J.D.; Grigsby, D.; Bowes Rickman, C. Human complement factor $\mathrm{H} Y 402 \mathrm{H}$ polymorphism causes an age-related macular degeneration phenotype and lipoprotein dysregulation in mice. Proc. Natl. Acad. Sci. USA 2019, 116, 3703-3711. [CrossRef]

29. Reinehr, S.; Kuehn, S.; Casola, C.; Koch, D.; Stute, G.; Grotegut, P.; Dick, H.B.; Joachim, S.C. HSP27 immunization reinforces AII amacrine cell and synapse damage induced by S100 in an autoimmune glaucoma model. Cell Tissue Res. 2018, 371, 237-249. [CrossRef]

30. Tsai, T.; Grotegut, P.; Reinehr, S.; Joachim, S.C. Role of heat shock proteins in glaucoma. Int. J. Mol. Sci. 2019, 20, 5160. [CrossRef]

31. Yi, Q.Y.; Wang, Y.Y.; Chen, L.S.; Li, W.D.; Shen, Y.; Jin, Y.; Yang, J.; Wang, Y.; Yuan, J.; Cheng, L. Implication of inflammatory cytokines in the aqueous humour for management of macular diseases. Acta Ophthalmol. 2019. [CrossRef]

32. Zhao, L.; Zabel, M.K.; Wang, X.; Ma, W.; Shah, P.; Fariss, R.N.; Qian, H.; Parkhurst, C.N.; Gan, W.B.; Wong, W.T. Microglial phagocytosis of living photoreceptors contributes to inherited retinal degeneration. EMBO Mol. Med. 2015, 7, 1179-1197. [CrossRef] [PubMed]

33. den Hollander, A.I.; Roepman, R.; Koenekoop, R.K.; Cremers, F.P. Leber congenital amaurosis: genes, proteins and disease mechanisms. Prog. Retin. Eye Res. 2008, 27, 391-419. [CrossRef] [PubMed]

34. Iannaccone, A.; Radic, M.Z. Increased protein citrullination as a trigger for resident immune system activation, intraretinal inflammation, and promotion of anti-retinal autoimmunity: intersecting paths in retinal degenerations of potential therapeutic relevance. Adv. Exp. Med Biol. 2019, 1185, 175-179. [CrossRef] [PubMed]

(C) 2020 by the authors. Licensee MDPI, Basel, Switzerland. This article is an open access article distributed under the terms and conditions of the Creative Commons Attribution (CC BY) license (http://creativecommons.org/licenses/by/4.0/). 
Article

\title{
Single-Cell RNA Sequencing in Human Retinal Degeneration Reveals Distinct Glial Cell Populations
}

\author{
Andrew P. Voigt ${ }^{1,2}$, Elaine Binkley ${ }^{1,2}$, Miles J. Flamme-Wiese ${ }^{1,2}$, Shemin Zeng ${ }^{1,2}$, \\ Adam P. DeLuca ${ }^{1,2}$, Todd E. Scheetz ${ }^{1,2}$, Budd A. Tucker ${ }^{1,2}$, Robert F. Mullins ${ }^{1,2}$ and \\ Edwin M. Stone ${ }^{1,2, *}$ \\ 1 Department of Ophthalmology and Visual Sciences, The University of Iowa Carver College of Medicine, \\ Iowa City, IA 52242, USA; andrew-voigt@uiowa.edu (A.P.V.); elaine-binkley@uiowa.edu (E.B.); \\ miles-flamme-wiese@uiowa.edu (M.J.F.-W.); shemin-zeng@uiowa.edu (S.Z.); \\ adam-deluca@uiowa.edu (A.P.D.); scheetzt@ivr.uiowa.edu (T.E.S.); budd-tucker@uiowa.edu (B.A.T.); \\ robert-mullins@uiowa.edu (R.F.M.) \\ 2 Institute for Vision Research, The University of Iowa, Iowa City, IA 52242, USA \\ * Correspondence: edwin-stone@uiowa.edu
}

Received: 19 December 2019; Accepted: 10 February 2020; Published: 13 February 2020

\begin{abstract}
Degenerative diseases affecting retinal photoreceptor cells have numerous etiologies and clinical presentations. We clinically and molecularly studied the retina of a 70-year-old patient with retinal degeneration attributed to autoimmune retinopathy. The patient was followed for 19 years for progressive peripheral visual field loss and pigmentary changes. Single-cell RNA sequencing was performed on foveal and peripheral retina from this patient and four control patients, and cell-specific gene expression differences were identified between healthy and degenerating retina. Distinct populations of glial cells, including astrocytes and Müller cells, were identified in the tissue from the retinal degeneration patient. The glial cell populations demonstrated an expression profile consistent with reactive gliosis. This report provides evidence that glial cells have a distinct transcriptome in the setting of human retinal degeneration and represents a complementary clinical and molecular investigation of a case of progressive retinal disease.
\end{abstract}

Keywords: autoimmune retinopathy; retinal degeneration; Müller cell; single-cell

\section{Introduction}

Photoreceptor cells are highly specialized, terminally differentiated neurons that detect photons of light and transmit this information to bipolar cells in the retina. Unfortunately, their exacting structural and metabolic requirements make them very susceptible to a large number of acquired and genetic sources of injury, leading to irreversible vision loss [1]. Degenerative diseases affecting photoreceptor cells have multiple etiologies. For example, genetic variants in over 100 genes have been shown to cause heritable photoreceptor degeneration [2]. However, photoreceptor degeneration can also be immune mediated, as in the case of autoimmune retinopathy (AIR), where circulating retinal autoantibodies lead to inflammation and downstream photoreceptor destruction [3]. Photoreceptor loss can also occur secondary to damage or dysfunction of adjacent cells and extracellular structures; for example, diseases affecting the retinal pigment epithelium (RPE), Bruch's membrane, or choroid can lead to increased oxidative stress and decreased metabolic support to the outer retina [4].

One approach for studying retinal degeneration is to characterize transcriptomic changes within diseased retina using microarrays or, more recently, next-generation sequencing of cDNA libraries (RNA sequencing, or RNA-Seq). Conventional gene expression studies with RNA-Seq have analyzed pools of retinal RNA from numerous cell types [5,6]. However, the high degree of cellular complexity and diversity in the human retina can prevent detection of even large gene expression changes that are 
restricted to specific classes of cells that are relatively unrepresented in the pool [7]. This concern has been largely obviated by the development of single-cell RNA sequencing, which has recently been employed to characterize the transcriptome of individual retinal cell populations. The neural retina is well suited for dissociation into single-cells, and protocols for recovery of viable, singlet cells are well established [8,9]. Such protocols facilitated the exploration of the murine retina transcriptome in the first report of Drop-Seq single-cell RNA sequencing [10]. Since this initial investigation, several additional studies have described the transcriptome of murine retina [10-12] and more recently, human retina [13-15] at the single-cell level.

In this report, we describe the clinical course of a 70-year-old patient with progressive photoreceptor degeneration attributed to AIR. We perform single-cell RNA sequencing on paired foveal and peripheral retinal samples from this patient and four unaffected control patients to investigate how different populations of retinal cells respond to photoreceptor degeneration. A total of 23,429 cells were recovered in this experiment, including 7189 cells from the AIR patient. This study provides insight into the responses of the retina to a blinding inflammatory condition at the cellular and transcriptional levels.

\section{Materials and Methods}

Human Donor Eyes: Eyes from the human donors utilized for this study were acquired from the Iowa Lions Eye Bank in accordance with the Declaration of Helsinki and following full consent of the donors' next of kin. The Institutional Review Board at the University of Iowa has judged that experiments performed on the donated eyes of deceased individuals does not fall under human subjects rules. All of the experiments in present paper were on the eyes of deceased individuals donated to science by the donors' next of kin. The work we performed in this paper was not human subjects research. Donor information is presented in Table 1. All tissue was received in the laboratory within $7 \mathrm{~h}$ post-mortem and processed immediately. A $2 \mathrm{~mm}$ foveal centered punch and an $8 \mathrm{~mm}$ peripheral retinal punch from the inferotemporal region centered on the equator were acquired with a disposable trephine from each donor. For the AIR donor, the OS was used for single-cell RNA sequencing and the OD was preserved in freshly generated $4 \%$ paraformaldehyde in phosphatidylcholine buffer solution. Frozen sections from the macula and peripheral retina were prepared as described previously [16]. Sections were stained with hematoxylin-eosin stain.

Table 1. Sample information from the donor eyes utilized in this study. Note that donor eyes 1-3 serve as controls for the current study and have been previously published [13].

\begin{tabular}{|c|c|c|c|c|c|c|}
\hline Donor & Age & Sex & $\begin{array}{c}\text { Time } \\
\text { Postmortem }\end{array}$ & Eye & Cause of Death & Ophthalmologic History \\
\hline Donor 1 & 89 & Male & $5: 21$ & OD & Cancer & $\begin{array}{l}\text { Early stage glaucoma documented; } \\
\text { histologically normal }\end{array}$ \\
\hline Donor 2 & 54 & Male & $5: 29$ & OD & Cardiac arrest & $\begin{array}{c}\text { No records received; histologically } \\
\text { normal }\end{array}$ \\
\hline Donor 3 & 82 & Female & $4: 18$ & OD & $\begin{array}{l}\text { Cardiopulmonary } \\
\text { arrest }\end{array}$ & $\begin{array}{c}\text { No ophthalmic records; histologically } \\
\text { normal }\end{array}$ \\
\hline Donor 4 & 76 & Male & $5: 14$ & OS & $\begin{array}{l}\text { Respiratory } \\
\text { failure/cancer }\end{array}$ & $\begin{array}{l}\text { posterior vitreous detachment OU, } \\
\text { nuclear sclerosis OU; histologically } \\
\text { normal }\end{array}$ \\
\hline Donor 5 & 70 & Male & $6: 36$ & OS & $\begin{array}{l}\text { Chronic obstructive } \\
\text { pulmonary disease }\end{array}$ & AIR, see results 3.1 \\
\hline
\end{tabular}

Dissociation for single-cell analysis: The overlying retinal tissue was peeled off of the retinal pigment epithelium and choroid. Retinal tissue was subsequently dissociated in 20 units $/ \mathrm{mL}$ of papain with $0.005 \%$ DNase I (Worthington Biochemical Corporation, Lakewood NJ) for $1.25 \mathrm{~h}$ on a shaker at $37^{\circ} \mathrm{C}$. Dissociated cell suspensions were frozen in DMSO-based Recovery Cell Culture Freezing Media (Life Technologies Corporation, Grand Island NY) in a Cryo-Safe cooler (CryoSafe, Summerville SC) to cool at $1{ }^{\circ} \mathrm{C} / \mathrm{min}$ at $-80^{\circ} \mathrm{C}$ for $3-8 \mathrm{~h}$ before storage in liquid nitrogen. 
Sample Preparation: Cryopreserved retinal samples were rapidly thawed and resuspended in phosphatidylcholine buffer solution with $0.04 \%$ non-acetylated bovine serum albumin (New England Biolabs, Ipswich, MA, USA) at a concentration of 1000 cells $/ \mu \mathrm{L}$. Viability analysis was performed with the Annexin V/Dead Cell Apoptosis Kit (Life Technologies Corporation, Eugene, OR, USA), with viability $>90 \%$ using the Countess II FL Automated Cell Counter (ThermoFisher Scientific, Waltham, MA, USA). Next, single cells were captured and barcoded using the Chromium system v3.0 chemistry kit (10X Genomics, Pleasanton, CA, USA). Barcoded libraries were pooled before sequencing on the HiSeq 4000 platform (Illumina, San Diego, CA, USA), generating 150 base pair paired-end reads.

Immunohistochemistry: Immunohistochemical experiments were performed on frozen tissue sections from donor eyes fixed in $4 \%$ paraformaldehyde. Sections were blocked with $1 \mathrm{mg} / \mathrm{mL}$ of bovine serum albumin before one-hour incubation with anti-ANXA1 (1:1.7, Developmental Studies Hybridoma Bank, Iowa City, IA) or Blue Cone Opsin (1:200, Millipore, AB5407), Red/Green Cone Opsin (1:200, Millipore, AB5405), and RetP1 (1:1000, Thermo Scientific). Sections were subsequently washed and incubated with Alexa-546-conjugated anti-mouse IgG (1:200, Invitrogen) or Alexa-488-conjugated anti-mouse IgG (1:200, Invitrogen) and Alexa-546-conjugated anti-rabbit IgG (1:200, Invitrogen). Each secondary antibody was supplemented with $100 \mu \mathrm{g} / \mathrm{mL}$ diamidino-phenyl-indole (DAPI, Sigma). Sections were incubated for $30 \mathrm{~min}$ before washing and cover slipping. Negative controls were included by omitting each primary antibody. Sections were photographed with an epifluorescent microscope (Olympus BX41) equipped with a digital camera (SPOT-RT; Diagnostic Instruments).

Computational Analysis: In addition to the two new donors sequenced for this study, we recently reported single-cell RNA sequencing on paired foveal $(2 \mathrm{~mm})$ and peripheral neural retina isolated from three human donors [13] with identical sample processing. FASTQ files from the previous experiment ( $\mathrm{n}=3$ paired samples, donors 1-3; GSE130636) and the current experiment ( $\mathrm{n}=2$ paired samples, donors 4-5) were utilized for downstream analysis. Briefly, FASTQ files were generated from basecalls with the bcl2fastq software (Illumina, San Diego, CA, USA) by the University of Iowa Institute of Human Genetics. Next, FASTQ files were mapped to the hg19 genome with CellRanger (v3.0.1) [17]. Cells with unique gene counts fewer than 200 were filtered, and cells with greater than 7000 unique genes per cell were removed to eliminate potential doublets. Libraries were aggregated to the same effective sequencing depth, and log-normalization of aggregated reads was performed with Seurat (v2.3.4) using a scale factor of 10,000 [18]. All raw and processed data have been deposited in NCBI's Gene Expression Omnibus (GSE142449).

\section{Results}

\subsection{Patient Description}

The patient initially presented to the neuro-ophthalmology service at the age of 51 for evaluation of decreased peripheral visual fields and photopsias in both eyes. He had no family history of inherited retinal degeneration. At presentation, his visual acuity was 20/20 in each eye, but he was found to have peripheral visual field loss. Ophthalmoscopic examination early in his disease course showed granular juxtapapillary pigmentary changes and mild vascular attenuation in both eyes. Electroretinogaphy (ERG) at presentation was consistent with widespread retinal dysfunction affecting both rods and cones. He experienced relatively rapid progression of his visual field loss and was seen by the inherited retinal degeneration service with concern for retinitis pigmentosa versus autoimmune retinopathy (AIR). Cancer-associated retinopathy was also considered, but his workup for malignancy was negative and his ERG was felt to be inconsistent with a paraneoplastic process at that time.

He ultimately developed peripheral bone-spicule-like pigmentary changes in both eyes (Figure 1A,B, 7 years after initial presentation) and progressive visual field constriction (Figure 1C,D, 8 years after initial presentation). Molecular evaluation for an inherited retinal degeneration, including whole exome sequencing, was performed but failed to identify a genetic etiology for his condition (for methods see [2]). He developed colon cancer several years after presentation, but this was thought to 
be unrelated to his ocular disease. He was given a presumed diagnosis of autoimmune retinopathy and ultimately required treatment for cystoid macular edema with intravitreal steroids.
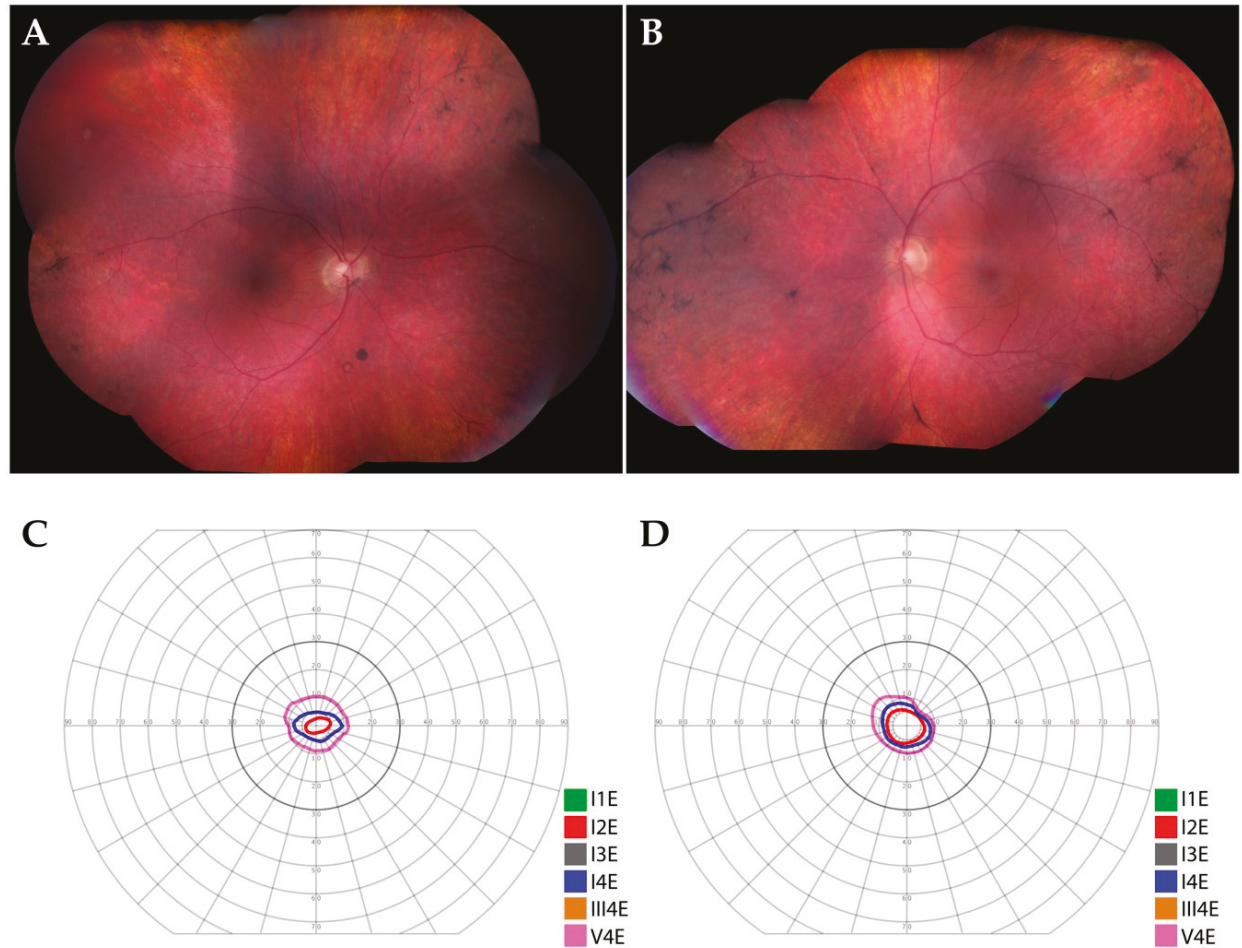

Figure 1. Clinical findings in a patient with retinal degeneration. (A,B): Montage color fundus photographs of the right (A) and left (B) eyes. There was granular, retinal pigment epithelial atrophy in the mid-periphery of both eyes, in addition to peripheral bone-spicule-like pigmentary changes and pigment clumps in both eyes. Arteriolar attenuation was notable in both eyes. (C,D): Goldman visual fields of the right (C) and left (D) eyes. There was severe constriction of the peripheral visual field in both eyes.

At the time of his last follow up with the retina service at the age of 69 , his visual acuity was $20 / 40+1$ in the right eye and 20/100 in the left eye with stable peripheral pigmentary changes in both eyes, and no cystoid macular edema. At age 70, the patient expired and donated his eyes for ophthalmic research. Evaluation of the patient's serum with Western blotting revealed the presence of antibodies that reacted with a 23 kilodalton protein in human retina.

\subsection{Histological Findings}

Sections from an eye with normal ocular history (Figure 2A), from the macula of the AIR donor (in the OD, the eye with better visual acuity) (Figure 2B), and from the periphery of the AIR donor (Figure 2C,D) were acquired. The macula of the AIR donor showed a loss of rod photoreceptors with only a single layer of attenuated cone cells remaining. In spite of the photoreceptor cell loss, the RPE was confluent and the inner retina appeared intact, with discrete inner nuclear layers and ganglion cell layers. In the periphery, the AIR donor showed complete loss of inner and outer segments and of the outer nuclear layer (Figure 2C). Considerable pigment migration into the inner retina was also observed in the periphery of the AIR donor (Figure 2D). 

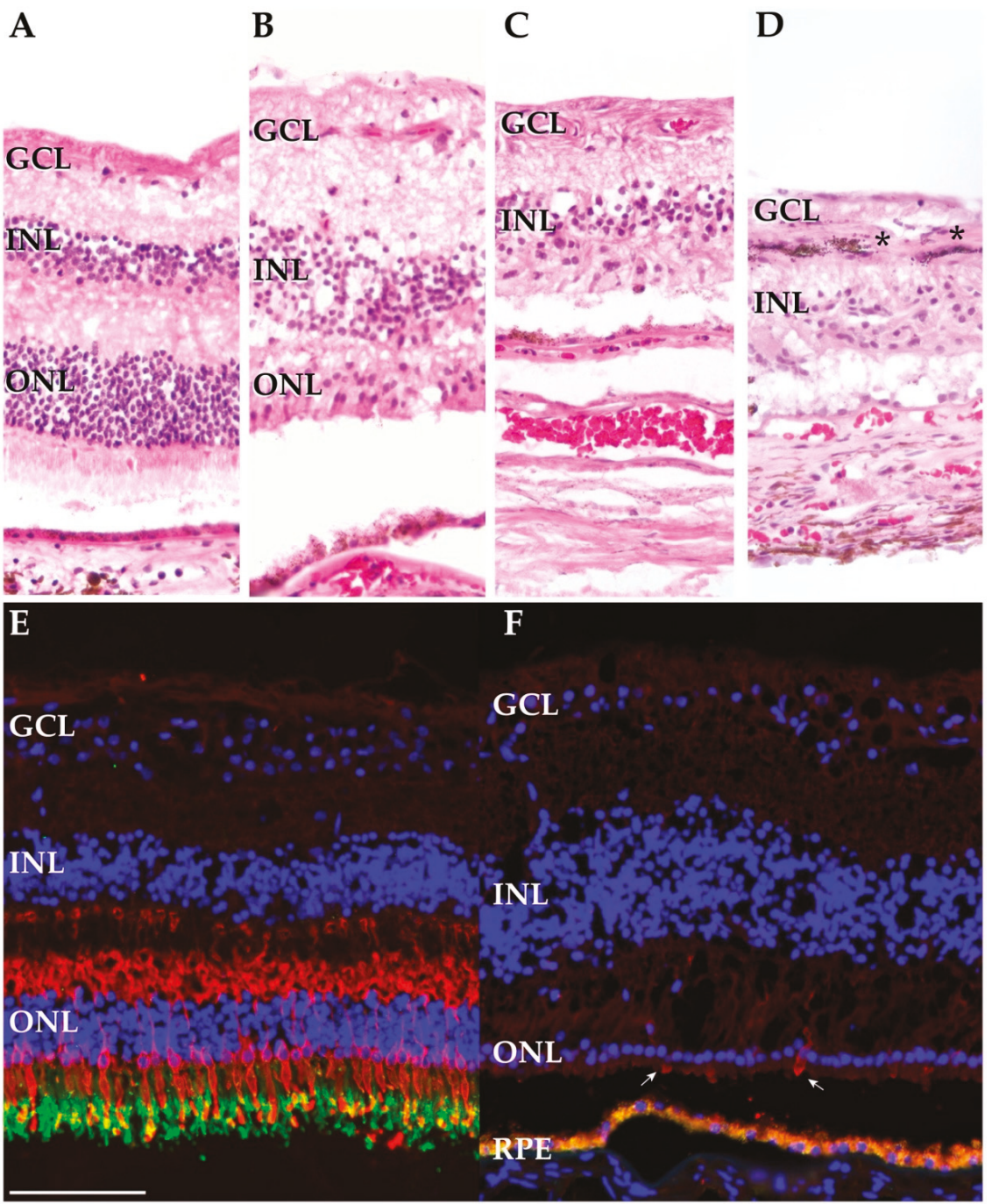

Figure 2. Histological and immunohistochemical investigation of the autoimmune retinopathy (AIR) and control donors. (A-D). Hematoxylin and eosin staining of the AIR and control donors. Sections from the periphery of a control donor (donor 2) (A), the macula (OD) of the AIR donor (B), and the periphery of the AIR donor (C,D). The AIR macula demonstrates intact ganglion cell and inner nuclear layers with attenuated cone photoreceptor outer segments. In contrast, in the periphery of the AIR donor complete loss of the outer nuclear layer (C) and retinal pigment epithelium (RPE) pigment migration into the inner retina (D) is observed $\left({ }^{*}\right)$. (E,F): Cone opsins (blue cone opsin and red/green cone opsins) are labeled in red while RetP1 is labeled in green. (E): A macula from a donor with normal ocular history demonstrates abundant labeling of cone opsins and rhodopsin. Of note, the RPE below the photoreceptors is out of frame. (F) The macula from the AIR donor demonstrates a complete lack of rod photoreceptors with rare, extremely attenuated cone photoreceptors (arrows). Autofluorescent lipofuscin from the RPE appears below the photoreceptor cells. Scalebar (100 microns) for all subpanels is provided in (E). 
Cone and rod photoreceptor cells were also visualized with fluorescent immunohistochemistry (IHC). Within the macula of a donor with normal ocular history, abundant cone opsin and rhodopsin labeling was observed (Figure 2E). In contrast, the AIR donor demonstrates complete loss of the rod specific opsin rhodopsin as well as extreme attenuation of cone photoreceptors (Figure 2F).

\subsection{Single-Cell Gene Profiling of Diseased Cell Populations}

Paired foveal and peripheral retinal punches were acquired from each of the five donors. While the four control donors had grossly normal retinas upon examination in the laboratory (Figure 3A), the donor with AIR had abundant peripheral pigmentation with a mostly unaffected macula (Figure 3B). After gentle dissociation, single-cell RNA sequencing was performed on each foveal and peripheral sample, and a total of 23,429 cells were recovered after filtering (Figure 3C). A total of 23 clusters were identified, and expression profiles were used to assign each cluster to its corresponding retinal cell type (Figure 3D). All major populations of retinal neurons, as well as supporting retinal endothelial cells, pericytes, glial cells, and microglia, were identified.

Next, the distribution of recovered cell types was compared between the AIR donor and the four control donors (Table S1). As the cellular composition of the retina varies between the fovea and periphery, comparisons were stratified by region. Within the fovea, cone photoreceptor cells are more abundant than rod photoreceptor cells, and cone photoreceptor cells synapse one-to-one with bipolar cells and upstream retinal ganglion cells (Figure 4A). The fovea centralis comprises the central $0.65-0.70 \mathrm{~mm}$ of the retina, consisting exclusively of cone photoreceptor cells and excluding vascular elements [19]. Our use of $2 \mathrm{~mm}$ foveal centered punches completely captures the fovea centralis but also includes some central rod photoreceptors and retinal endothelial cells. In the four control donors, all major populations of inner retinal neurons were recovered from the fovea (Figure 4B). No RPE cells were detected, suggesting that the foveal retinal punch was well separated from the underlying RPE and choroid. Unlike the control donors, no rod photoreceptor cells were detected in the foveal punch from the donor with AIR. However, a similar proportion of foveal cone photoreceptor cells were recovered in the AIR donor and the control donors. In addition, the AIR donor demonstrated a moderate increase in the proportion of recovered foveal bipolar and Müller cells.

In the periphery, rod photoreceptor cells were predominant, and peripheral bipolar cells receive input from multiple rod photoreceptor cells (Figure 4D). In the four control donors, peripheral rod photoreceptor cells were much more abundant than cone photoreceptor cells, and relatively few microglia or astrocytes were detected (Figure 4E). In contrast, only a single rod photoreceptor cell was recovered from the periphery of the AIR donor while microglia and astrocyte cells were recovered in much higher frequency. In addition, a small proportion of RPE cells were recovered from the periphery of the AIR donor, consistent with the histological observation of peripheral RPE migration into the retina (Figure 2D).

Next, the transcriptomic consequences of photoreceptor degeneration in the AIR donor were investigated. For each cell type, gene expression was compared between cells originating from the AIR donor and the control donors, and the proportion of significantly differentially expressed genes (adjusted $p$-value $<0.05$ ) that exhibited an absolute log fold-change in expression greater than 0.5 was calculated. As gene expression within a single cell type can vary between the fovea and the periphery [13], this analysis was again stratified by region. Within the fovea, Müller cells and horizontal cells demonstrated modest expression differences, with a total of $1.1 \%$ and $1.2 \%$ of assayed genes significantly enriched in the AIR Müller cell and horizontal cell populations, respectively (Figure 4C). A greater proportion of differentially expressed genes between the AIR and control donors were identified in the periphery (Figure 4F). Müller and astrocyte glial cells both demonstrated a modest proportion of genes significantly enriched in the periphery of the AIR donor $(1.3 \%$ and $2.1 \%$, respectively), as did microglia and horizontal cells ( $2.2 \%$ and $2.6 \%$, respectively). Differential expression results for each comparison are shown in detail in Table S2. 

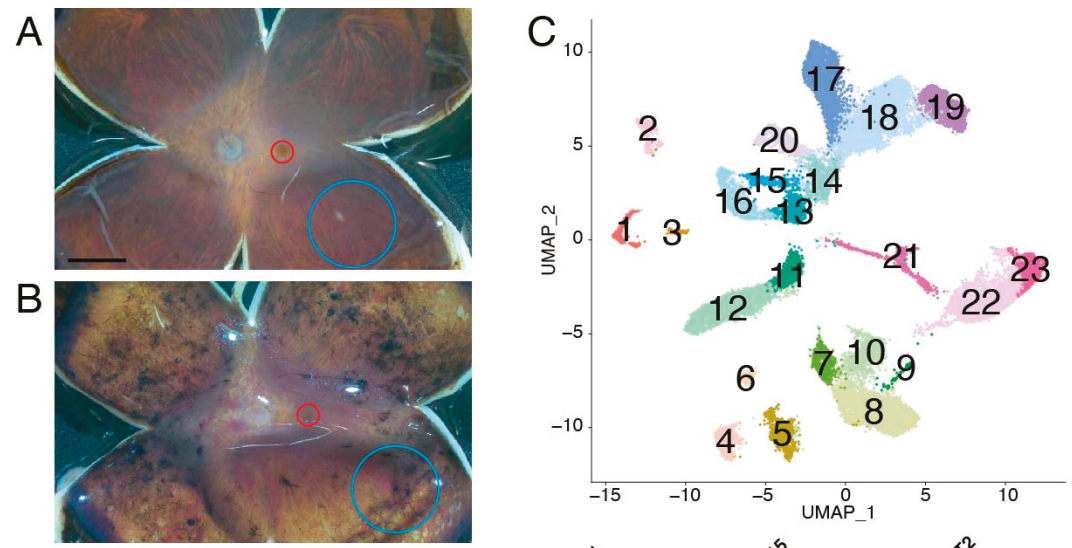

D
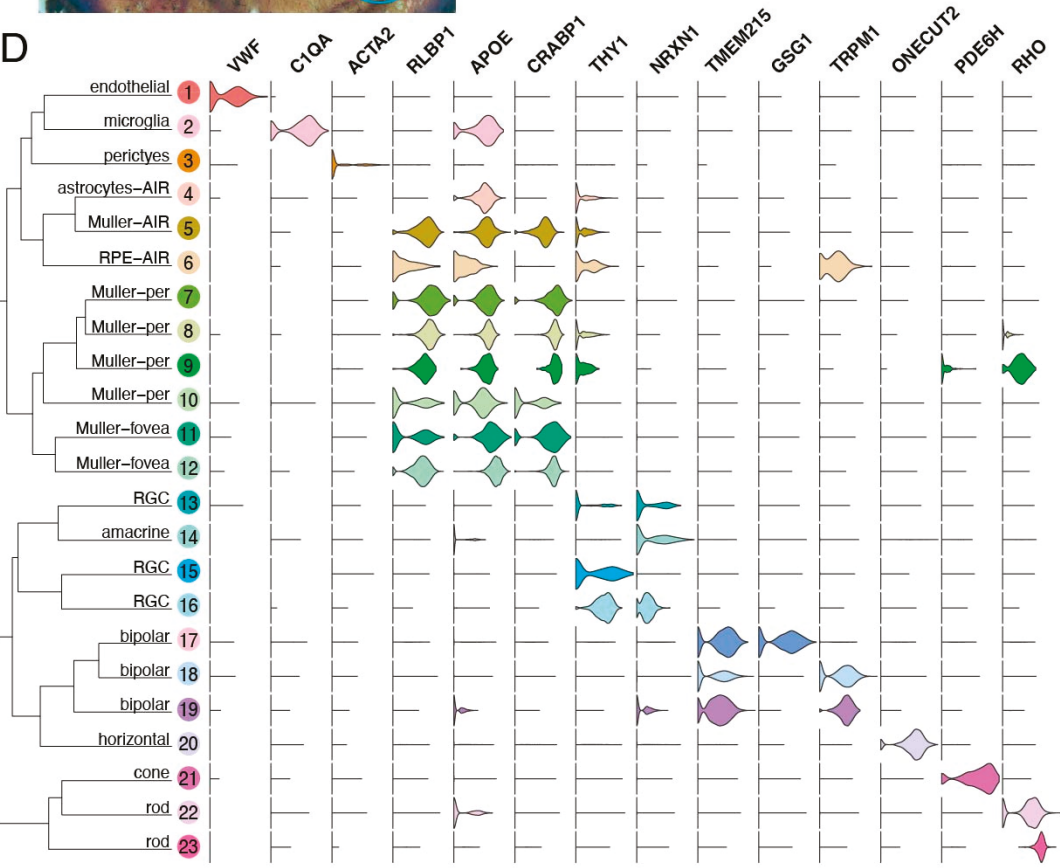

Figure 3. Single-cell RNA sequencing of the AIR donor. (A,B): Five human donor eyes were used for this study. A gross image of a control eye (donor 4) (A) and the AIR eye (donor 5) (B) are included. From each eye, a $2 \mathrm{~mm}$ foveal centered punch (red) and an $8 \mathrm{~mm}$ peripheral punch isolated from the inferotemporal region (blue) were acquired and gently dissociated. Scalebar (A) is $5 \mathrm{~mm}$. (C): Single-cell RNA sequencing of retinal cells from the AIR donor and four control patients. A total of 23,429 cells were recovered after filtering. Unsupervised clustering of cells resulted in 23 clusters, which are visualized with uniform manifold approximation and projection (UMAP) dimensionality reduction, where each point represents the multidimensional transcriptome of a single-cell and each cluster of cells is depicted in a different color. (D): Violin plots depict the expression of cell-type specific genes across the 23 identified clusters. Per $=$ peripheral retina. AIR = autoimmune retinopathy. RPE = retinal pigment epithelium. RGC = retinal ganglion cell. 
A
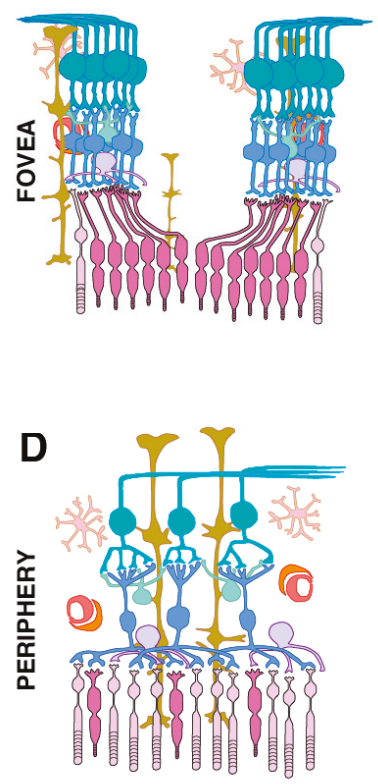
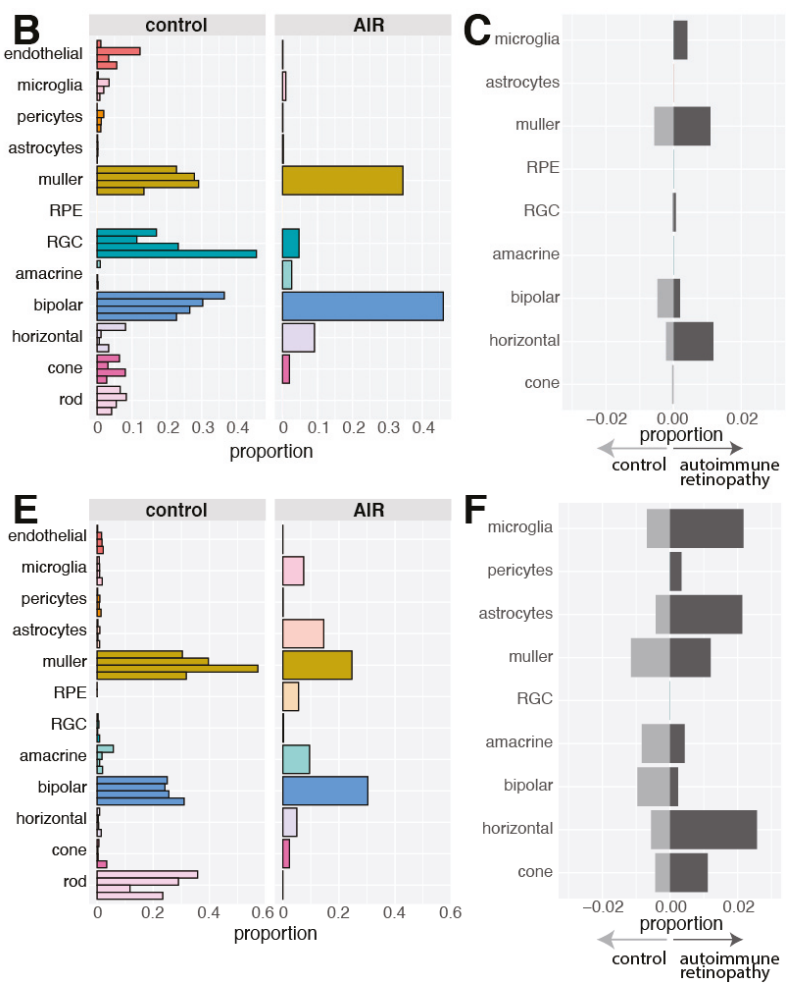

Figure 4. Library composition of recovered cells. (A): In the fovea, cone photoreceptor cells synapse with one bipolar cell, which synapse with one retinal ganglion cell. (B): The proportion of each cell type recovered from the fovea of the four control donors and the autoimmune retinopathy donor. No foveal rods were recovered from the AIR donor. (C): In order to visualize the degree of gene expression differences within each population of cells between the AIR and control donors, differential expression analysis was performed. In each cell type, the number of differentially expressed genes that were enriched in the AIR donor and the control donors were enumerated and divided by the total number of expressed genes (in at least 10\% of cells). For example, $1.09 \%$ of foveal Müller cell genes were significantly enriched in the AIR donor (dark grey), while $0.57 \%$ of foveal Müller cell genes were significantly enriched in the control donors (light grey). (D): In the periphery, multiple rod photoreceptor cells synapse with a single bipolar cell. (E): The proportion of each cell type recovered from the periphery of the four control donors and the periphery of the AIR donor. (F): As in (C), the proportion of differentially expressed genes between the AIR and control donors was performed in each cell type. More genes were differentially expressed in the periphery compared to the fovea (C). As no RPE cells originated from control donors, differential expression could not be performed in the periphery for this cell type.

While most clusters contained cells from each of the five donors, Clusters 4-6 were comprised predominantly of cells from the periphery of the AIR patient (each cluster possessing $>85 \%$ of cells from the AIR donor) (Figure 5A). Therefore, gene expression patterns from these clusters were further investigated. Cluster 4 was classified as astrocytes (Figure 5C,D). Cells in this cluster demonstrated high expression of the glial fibrillary acid protein (GFAP), which is widely expressed in astrocytes responding to neuronal injury [20], and the astrocyte-specific inflammatory cytokine IFITM3 [21]. A total of 624 cells were recovered in Cluster 4 and 551 of them $(88 \%)$ originated from the periphery of the AIR donor. Differential expression analysis was performed to investigate if astrocytes from the AIR 
donor demonstrated a reactive gene expression profile (Figure S1). Astrocytes from the AIR donor were enriched for SOCS3 [22], SLPI [23], and CH25H [24], genes that have all been previously found to be expressed in astrocytes responding to CNS injury. In addition, reactive glial cells are involved in inflammatory responses, and have been shown to increase the production of pro-inflammatory chemokines [25]. The chemokine CXCL2 was highly enriched $(\log F C=1.47)$ in peripheral astrocytes from the AIR donor.

Cluster 5, with $98 \%$ of cells originating from the periphery of the AIR donor, was interpreted as Müller glial cells. Cells in this cluster highly expressed the Müller cell genes RLBP1 and CRALBP1 (Figure 5E). Six additional clusters of Müller glia were identified, which largely separated Müller cells of peripheral (Clusters 6-10) and foveal (Clusters 11-2) origin (Figure 5A). As previously shown in monkey [26] and human [13] retina, foveal and peripheral Müller cells have distinct gene expression profiles (Figure 5G). Interestingly, foveal Müller cells from the AIR donor clustered with foveal Müller cells from the other four donors, and differential expression analysis yielded relatively few expression differences (log fold-change greater than 1.25) (Figure 5H). NFKBAI, which has been previously associated with glial cell degeneration, [27] was the most upregulated gene in the AIR donor's foveal Müller cells.

In contrast, the majority of peripheral Müller cells from the AIR donor formed their own cluster (Cluster 5). Differential expression revealed numerous expression differences between peripheral Müller cells from the AIR donor versus peripheral Müller cells from other donors (Figure 5I). Among the first hallmarks of reactive gliosis is the increased expression of intermediate filament proteins [28]. The intermediate filament gene GFAP $(\operatorname{logFC}=2.54)$ was the most enriched gene in Müller cells from the AIR donor, which was also observed to be more abundant in the AIR donor at the protein level (Figure S2). In addition, peripheral Müller cells from the AIR donor were enriched for ANXA1 and ANXA2, which have been shown to be upregulated in reactive glial populations in the brain [29]. Immunofluorescent IHC also demonstrates increased ANXA1 labeling in cells of the AIR donor (Figure 5B), which co-localizes with GFAP expression (Figure S3).

Cluster 6, with $99 \%$ of cells originating from the periphery of the AIR donor, was interpreted as retinal pigment epithelium (RPE) cells (Figure 5F). Cells in this cluster demonstrated high expression of SERPINF1 (the gene encoding PEDF) and RLBP1 (the gene encoding cellular retinaldehyde binding protein). Although retinal samples were dissected away from the underlying RPE and choroid, the recovery of RPE cells suggests that either some RPE cells migrated into the inner retina or remained adhered to the outer retina after dissection, consistent with both the clinical observation of bone spicule like pigmentation in the patient's neurosensory retina (Figure 1A-B) and the morphological finding of pigment migration into the inner retina (Figure 2D). 

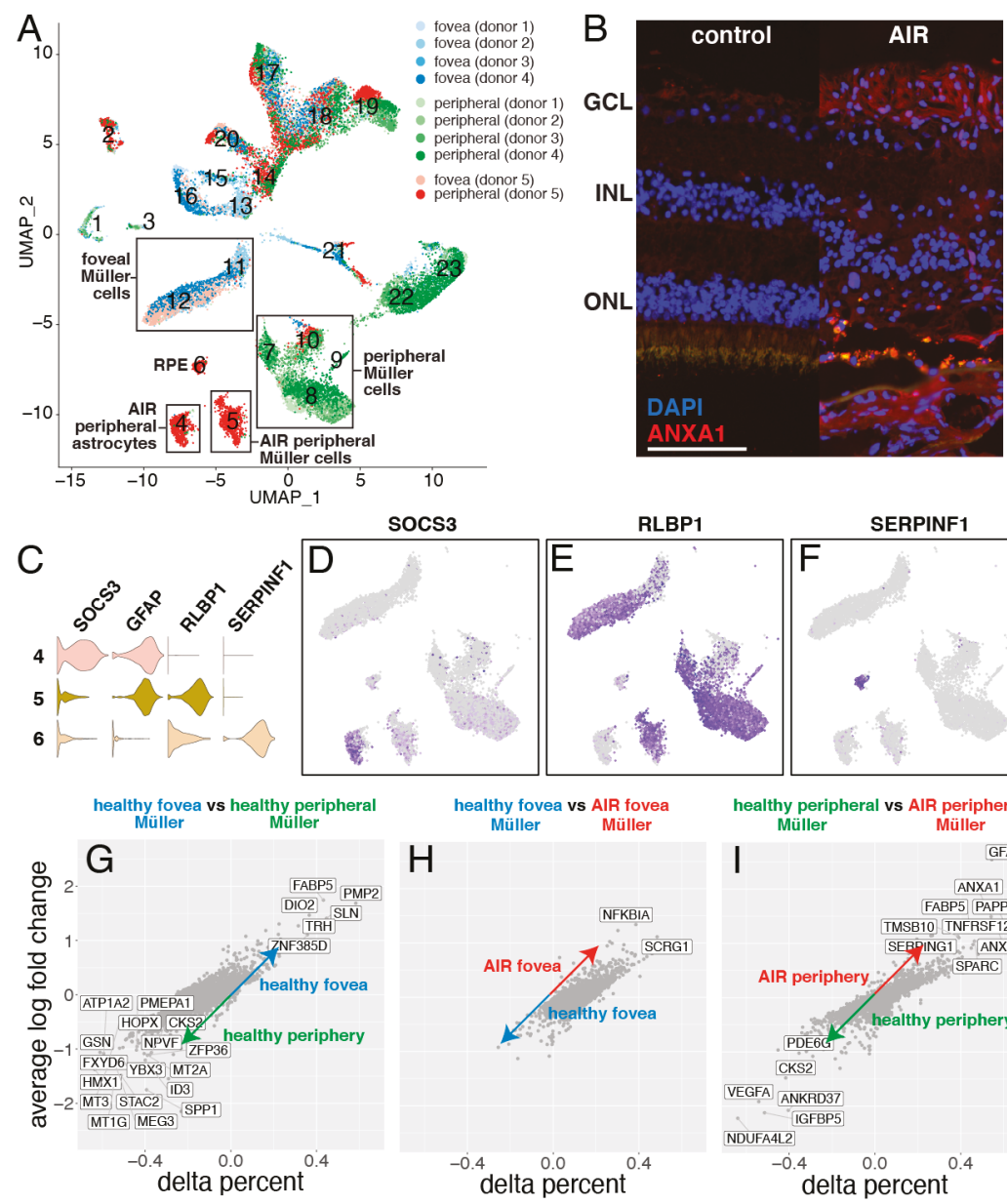

SOCS3
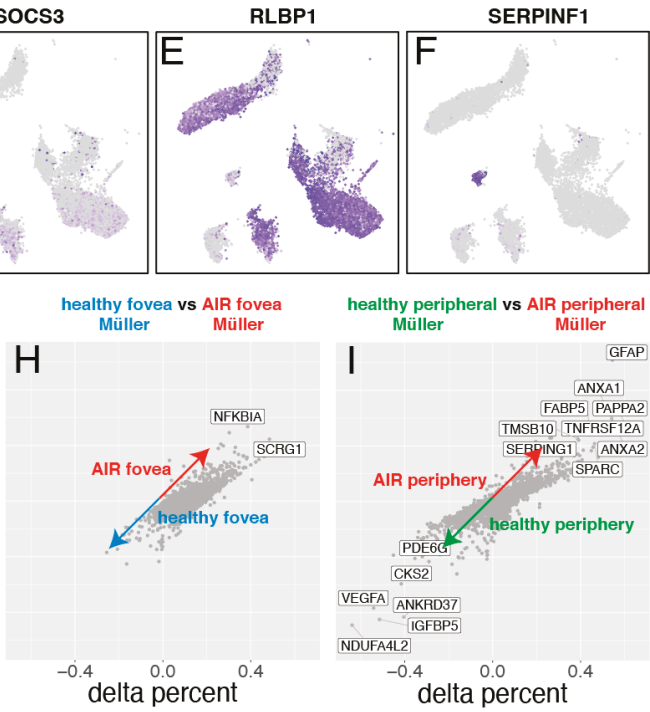

Figure 5. Exploration of autoimmune retinopathy dominant clusters. (A): The library composition of each cluster is displayed, with cells originating from the control foveas represented in shades of blue while cells originating from the control peripheries are in shades of green. Cells originating from the AIR donor are colored light red (fovea) or dark red (periphery). Three clusters (Cluster 4-6) consist predominantly of cells from the periphery of the AIR donor. (B): Immunofluorescent labeling of ANXA1 in the retina of a control donor (left) and the AIR donor (right). The AIR donor demonstrates increased ANXA1-labeling of the inner retina. Scale bar $=100$ microns. (C): Violin plots of SOCS3, GFAP, RLBP1, and SERPINF1 expression are used to classify the cell types of clusters 4-6. (D): Cluster 4 specifically expressed SOCS3, which is enriched in reactive astrocytes. (E): Cluster 5 and Clusters 7-12 express the Müller cell specific gene RLBP1. (F): Cluster 6 expresses the RPE-specific gene SERPINF1. (G): Healthy foveal and peripheral Müller cells have distinct gene expression profiles. The variable delta percent along the $\mathrm{x}$-axis represents the proportion of foveal Müller cells that express the gene of interest minus the proportion of peripheral Müller cells that express that gene. $(\mathbf{H})$ : Foveal Müller cells originating from the control donors have similar gene expression profiles to foveal Müller cells originating from the AIR donor. (I): In contrast, peripheral Müller cells from control versus the AIR donor demonstrated more transcriptomic differences. Genes with a log fold-change greater than 1.0 and a delta percent greater than 0.35 are labeled in $(\mathbf{G}-\mathbf{I})$. 


\section{Discussion}

Autoimmune retinopathy (AIR) is a blinding, immune-mediated inflammatory condition in which anti-retinal antibodies result in retinal cell destruction. In most cases, photoreceptor cells are the primarily targeted cell type, although antibodies against bipolar cells have also been reported [30]. The clinical presentation of the patient in this report follows the classical trajectory of sudden, bilateral loss of peripheral vision, consistent with rod photoreceptor cell dysfunction [31]. The etiology of AIR is broadly subdivided into paraneoplastic and non-paraneoplastic disease. Identifying anti-retinal antibodies can support a diagnosis of AIR, however unaffected individuals may also have circulating anti-retinal antibodies, limiting the specificity of this diagnostic assay [32,33].

Consistent with the clinical history of peripheral visual deterioration, histological examination of the AIR patient revealed a profound loss of both cone and rod photoreceptor cells and destruction of the inner and outer photoreceptor segments in the periphery (Figure 2C). However, in the macula, rare attenuated cone photoreceptor cells were still present (Figure 2F). Single-cell RNA sequencing supported these clinical and histological findings. Only a single rod photoreceptor cell was recovered from the periphery of the AIR donor while numerous foveal cones were recovered. In addition, single-cell RNA sequencing identified the presence of RPE cells in the periphery of the AIR donor, as was observed on histological examination (Figure 2D). Gene expression comparisons between the AIR donor and the four control donors were remarkably similar for most cell types. However, peripheral astrocytes and Müller glial cells were more abundant and demonstrated unique expression signatures in the AIR patient. Collectively, these expression data corroborate the clinical and histologic findings and provide evidence that single-cell RNA sequencing can be a complementary tool for investigating the molecular features of a human retinal disease.

The retina contains two major classes of glial cells: Müller cells and astrocytes. Müller cells are elongated cells that extend from the external limiting membrane (apical end) to the internal limiting membrane (basal feet). Müller cells provide metabolic and structural support to retinal neurons, ensheathing neural somas and comprising an important part of the blood retina barrier. Astrocytes also metabolically support the retina, however astrocytes do not originate from the embryonic retinal neuroepithelium but rather enter the retina by migrating along the developing optic nerve [34]. As opposed to Müller cells, astrocytes are star-shaped cells with radiating processes located in the nerve fiber and ganglion cell layers. Both astrocytes and Müller glial cells are capable of responding to retinal injury and exerting neuroprotective effects on the retina in a process known as reactive gliosis [35]. In this wound response process, glial cells proliferate and undergo changes in gene expression for improved neuronal protection and repair [36,37].

In the donor with AIR, the transcriptional response of the glial cells can likely be attributed to their interactions with degenerating retina. Within the fovea, where the retina clinically and histologically was most intact, Müller cells from the AIR donor were transcriptionally similar to foveal Müller cells from the control patients. Yet in the peripheral retina, where the AIR donor experienced progressive visual field loss and a complete loss of the outer nuclear layer, peripheral Müller cells segregated into a distinct cluster and demonstrated a reactive gliotic phenotype (Figure 5I). Likewise, many astrocytes were recovered from the periphery of the AIR donor that expressed genes implicated in reactive gliosis (Figure S1). Reactive astrogliosis is marked by astrocyte proliferation and migration, which may have led to an increased number of peripherally localized astrocytes available for recovery in the AIR donor, consistent with recent single-cell RNA sequencing studies characterizing microglial proliferation in response to retinal damage in mice [38]. Collectively, the gliotic injury response induced by Müller cells and astrocytes has many neuroprotective benefits, yet chronic gliotic activation can further injure retinal neurons and disrupt the blood-retinal barrier, leading to worsening vision $[39,40]$. In the setting of chronic retinal injury, interventions that modulate gliotic activation may optimize preservation of remaining retinal function [41].

While glial cells from the AIR donor demonstrated reactive transcriptional changes, most inner retinal cell populations from this donor had remarkably similar gene expression profiles to the control 
donors (Figure 4C,F). Likewise, histological examination revealed preserved inner retinal morphology with discrete inner nuclear and ganglion cell layers (Figure 2B,C). Collectively, these findings suggest that even in the setting of photoreceptor cell degeneration, the inner retinal wiring remains largely undamaged. The presence of morphologically and transcriptomically normal inner retinal cells is promising for prospective photoreceptor degeneration treatments, including autologous retinal cellular replacement strategies [42].

There are several limitations to this study. First, AIR is a rare retinal disease, preventing us from including multiple patients with this condition in this investigation. As a result, gene expression differences between the AIR donor and the four control donors are valuable for hypothesis generation but should be interpreted with caution. Second, while all samples had identical sample processing, certain cell types might have a selective advantage in cellular recovery for single-cell RNA sequencing. Recovered proportions of cells at the single-cell level (Figure 4B,E) should not be interpreted as the true cellularity of the retina.

This study provides a complementary investigation of the clinical and molecular response of the retina in AIR. Clinical, histologic, and transcriptomic evidence identify the loss of cone and rod photoreceptor cells with relative preservation of inner retinal cell types. The gliotic transcriptional profile of astrocyte and Müller glial populations observed in this case provides some new insight into the retina's response to photoreceptor degeneration.

Supplementary Materials: The following are available online at http://www.mdpi.com/2073-4409/9/2/438/s1, Figure S1: AIR astrocytes demonstrate a reactive gene expression profile. Figure S2: Increased GFAP in the AIR donor versus a healthy control donor. Figure S3 ANXA1 and GFAP co-localization in the AIR donor. Table S1: Library Composition, Table S2: Differential expression between the AIR donor versus four control donors.

Author Contributions: Conceptualization, R.F.M. and E.M.S.; methodology, A.P.V., E.B., R.F.M., E.M.S.; software, A.P.V., A.P.D., T.E.S.; validation, E.B., M.J.F.-W., S.Z.; formal analysis, A.P.V., A.P.D., T.E.S.; investigation, A.P.V., E.B., M.J.F.-W., S.Z., A.P.D., T.E.S., B.A.T., R.F.M., E.M.S.; resources, T.E.S., B.A.T., R.F.M., E.M.S. data curation, A.P.V., E.B., T.E.S., R.F.M.; writing-original draft preparation, A.P.V., E.B., B.A.T., R.F.M., E.M.S.; writing-review and editing, M.J.F.-W., S.Z., A.P.D., T.E.S.; visualization, A.P.V., E.B., R.F.M.; supervision, T.E.S., B.A.T., R.F.M., E.M.S.; project administration, E.M.S.; funding acquisition, T.E.S., B.A.T., R.F.M., E.M.S. All authors have read and agreed to the published version of the manuscript.

Funding: This research was funded by NIH grants T32 GM007337, R21 EY027038, and P30 EY025580 with support from Research to Prevent Blindness and the Elmer and Sylvia Sramek Charitable Foundation.

Acknowledgments: We wish to thank the Iowa Lions Eye Bank, the donors, and their families for the generous role in this research. The ANXA1 monoclonal antibody developed by the Clinical Proteomics Technologies for Cancer and was obtained from the Developmental Studies Hybridoma Bank, created by the National Institute of Child Health and Human Development of the NIH and maintained at the Department of Biology, The University of Iowa (Iowa City, IA).

Conflicts of Interest: The authors declare no conflict of interest.

\section{References}

1. Wright, A.F.; Chakarova, C.F.; Abd El-Aziz, M.M.; Bhattacharya, S.S. Photoreceptor degeneration: Genetic and mechanistic dissection of a complex trait. Nat. Rev. Genet. 2010, 11, 273-284. [CrossRef]

2. Stone, E.M.; Andorf, J.L.; Whitmore, S.S.; DeLuca, A.P.; Giacalone, J.C.; Streb, L.M.; Braun, T.A.; Mullins, R.F.; Scheetz, T.E.; Sheffield, V.C.; et al. Clinically Focused Molecular Investigation of 1000 Consecutive Families with Inherited Retinal Disease. Ophthalmology 2017, 124, 1314-1331. [CrossRef] [PubMed]

3. Adamus, G.; Ren, G.; Weleber, R.G. Autoantibodies against retinal proteins in paraneoplastic and autoimmune retinopathy. BMC Ophthalmol. 2004, 4, 5. [CrossRef] [PubMed]

4. Kannan, R.; Hinton, D.R. Sodium iodate induced retinal degeneration: New insights from an old model. Neural Regen. Res. 2014, 9, 2044-2045. [PubMed]

5. Li, M.; Jia, C.; Kazmierkiewicz, K.L.; Bowman, A.S.; Tian, L.; Liu, Y.; Gupta, N.A.; Gudiseva, H.V.; Yee, S.S.; Kim, M.; et al. Comprehensive analysis of gene expression in human retina and supporting tissues. Hum. Mol. Genet. 2014, 23, 4001-4014. [CrossRef] [PubMed] 
6. Whitmore, S.S.; Wagner, A.H.; DeLuca, A.P.; Drack, A.V.; Stone, E.M.; Tucker, B.A.; Zeng, S.; Braun, T.A.; Mullins, R.F.; Scheetz, T.E. Transcriptomic analysis across nasal, temporal, and macular regions of human neural retina and RPE/choroid by RNA-Seq. Exp. Eye Res. 2014, 129, 93-106. [CrossRef]

7. Hwang, B.; Lee, J.H.; Bang, D. Single-cell RNA sequencing technologies and bioinformatics pipelines. Exp. Mol. Med. 2018, 50, 96. [CrossRef]

8. Sarthy, P.V.; Lam, D.M. Isolated cells from a mammalian retina. Brain Res. 1979, 176, 208-212. [CrossRef]

9. Feodorova, Y.; Koch, M.; Bultman, S.; Michalakis, S.; Solovei, I. Quick and reliable method for retina dissociation and separation of rod photoreceptor perikarya from adult mice. MethodsX 2015, 2, 39-46. [CrossRef]

10. Macosko, E.Z.; Basu, A.; Satija, R.; Nemesh, J.; Shekhar, K.; Goldman, M.; Tirosh, I.; Bialas, A.R.; Kamitaki, N.; Martersteck, E.M.; et al. Highly Parallel Genome-wide Expression Profiling of Individual Cells Using Nanoliter Droplets. Cell 2015, 161, 1202-1214. [CrossRef]

11. Rheaume, B.A.; Jereen, A.; Bolisetty, M.; Sajid, M.S.; Yang, Y.; Renna, K.; Sun, L.; Robson, P.; Trakhtenberg, E.F. Single cell transcriptome profiling of retinal ganglion cells identifies cellular subtypes. Nat. Commun. 2018, 9, 2759. [CrossRef] [PubMed]

12. Clark, B.S.; Stein-O'Brien, G.L.; Shiau, F.; Cannon, G.H.; Davis-Marcisak, E.; Sherman, T.; Santiago, C.P.; Hoang, T.V.; Rajaii, F.; James-Esposito, R.E.; et al. Single-Cell RNA-Seq Analysis of Retinal Development Identifies NFI Factors as Regulating Mitotic Exit and Late-Born Cell Specification. Neuron 2019, 102, 1111.e1115-1126.e1115. [CrossRef] [PubMed]

13. Voigt, A.P.; Whitmore, S.S.; Flamme-Wiese, M.J.; Riker, M.J.; Wiley, L.A.; Tucker, B.A.; Stone, E.M.; Mullins, R.F.; Scheetz, T.E. Molecular characterization of foveal versus peripheral human retina by single-cell RNA sequencing. Exp. Eye Res. 2019, 184, 234-242. [CrossRef]

14. Hu, Y.; Wang, X.; Hu, B.; Mao, Y.; Chen, Y.; Yan, L.; Yong, J.; Dong, J.; Wei, Y.; Wang, W.; et al. Dissecting the transcriptome landscape of the human fetal neural retina and retinal pigment epithelium by single-cell RNA-seq analysis. PLoS Biol 2019, 17, e3000365. [CrossRef]

15. Lukowski, S.W.; Lo, C.Y.; Sharov, A.A.; Nguyen, Q.; Fang, L.; Hung, S.S.; Zhu, L.; Zhang, T.; Grunert, U.; Nguyen, T.; et al. A single-cell transcriptome atlas of the adult human retina. EMBO J. 2019, 38, e100811. [CrossRef] [PubMed]

16. Barthel, L.K.; Raymond, P.A. Improved method for obtaining 3-microns cryosections for immunocytochemistry. J. Histochem. Cytochem. 1990, 38, 1383-1388. [CrossRef]

17. Zheng, G.X.Y.; Terry, J.M.; Belgrader, P.; Ryvkin, P.; Bent, Z.W.; Wilson, R.; Ziraldo, S.B.; Wheeler, T.D.; McDermott, G.P.; Zhu, J.; et al. Massively parallel digital transcriptional profiling of single cells. Nat. Commun. 2017, 8, 14049. [CrossRef]

18. Butler, A.; Hoffman, P.; Smibert, P.; Papalexi, E.; Satija, R. Integrating single-cell transcriptomic data across different conditions, technologies, and species. Nat. Biotechnol. 2018, 36, 411-420. [CrossRef]

19. Yuodelis, C.; Hendrickson, A. A qualitative and quantitative analysis of the human fovea during development. Vision Res. 1986, 26, 847-855. [CrossRef]

20. Sofroniew, M.V.; Vinters, H.V. Astrocytes: Biology and pathology. Acta Neuropathol. 2010, 119, 7-35. [CrossRef]

21. Ibi, D.; Nagai, T.; Nakajima, A.; Mizoguchi, H.; Kawase, T.; Tsuboi, D.; Kano, S.; Sato, Y.; Hayakawa, M.; Lange, U.C.; et al. Astroglial IFITM3 mediates neuronal impairments following neonatal immune challenge in mice. Glia 2013, 61, 679-693. [CrossRef] [PubMed]

22. Okada, S.; Nakamura, M.; Katoh, H.; Miyao, T.; Shimazaki, T.; Ishii, K.; Yamane, J.; Yoshimura, A.; Iwamoto, Y.; Toyama, Y.; et al. Conditional ablation of Stat 3 or Socs 3 discloses a dual role for reactive astrocytes after spinal cord injury. Nat. Med. 2006, 12, 829-834. [CrossRef] [PubMed]

23. Ghasemlou, N.; Bouhy, D.; Yang, J.; Lopez-Vales, R.; Haber, M.; Thuraisingam, T.; He, G.; Radzioch, D.; Ding, A.; David, S. Beneficial effects of secretory leukocyte protease inhibitor after spinal cord injury. Brain 2010, 133, 126-138. [CrossRef] [PubMed]

24. Zhu, Z.; Hu, Y.; Zhou, Y.; Zhang, Y.; Yu, L.; Tao, L.; Guo, A.; Fang, Q. Macrophage Migration Inhibitory Factor Promotes Chemotaxis of Astrocytes through Regulation of Cholesterol 25-Hydroxylase Following Rat Spinal Cord Injury. Neuroscience 2019, 408, 349-360. [CrossRef]

25. Farina, C.; Aloisi, F.; Meinl, E. Astrocytes are active players in cerebral innate immunity. Trends Immunol. 2007, 28, 138-145. [CrossRef] 
26. Peng, Y.R.; Shekhar, K.; Yan, W.; Herrmann, D.; Sappington, A.; Bryman, G.S.; van Zyl, T.; Do, M.T.H.; Regev, A.; Sanes, J.R. Molecular Classification and Comparative Taxonomics of Foveal and Peripheral Cells in Primate Retina. Cell 2019, 176, 1222.e1222-1237.e1222. [CrossRef]

27. Haenold, R.; Weih, F.; Herrmann, K.H.; Schmidt, K.F.; Krempler, K.; Engelmann, C.; Nave, K.A.; Reichenbach, J.R.; Lowel, S.; Witte, O.W.; et al. NF-kappaB controls axonal regeneration and degeneration through cell-specific balance of RelA and p50 in the adult CNS. J. Cell Sci. 2014, 127, 3052-3065. [CrossRef]

28. Lu, Y.B.; Iandiev, I.; Hollborn, M.; Korber, N.; Ulbricht, E.; Hirrlinger, P.G.; Pannicke, T.; Wei, E.Q.; Bringmann, A.; Wolburg, H.; et al. Reactive glial cells: Increased stiffness correlates with increased intermediate filament expression. FASEB J. 2011, 25, 624-631. [CrossRef]

29. Eberhard, D.A.; Brown, M.D.; VandenBerg, S.R. Alterations of annexin expression in pathological neuronal and glial reactions. Immunohistochemical localization of annexins I, II (p36 and p11 subunits), IV, and VI in the human hippocampus. Am. J. Pathol. 1994, 145, 640-649.

30. Choi, E.Y.; Kim, M.; Adamus, G.; Koh, H.J.; Lee, S.C. Non-Paraneoplastic Autoimmune Retinopathy: The First Case Report in Korea. Yonsei Med. J. 2016, 57, 527-531. [CrossRef]

31. Rahimy, E.; Sarraf, D. Paraneoplastic and non-paraneoplastic retinopathy and optic neuropathy: Evaluation and management. Surv. Ophthalmol. 2013, 58, 430-458. [CrossRef] [PubMed]

32. Braithwaite, T.; Vugler, A.; Tufail, A. Autoimmune retinopathy. Ophthalmologica 2012, 228, 131-142. [CrossRef] [PubMed]

33. Adamus, G.; Brown, L.; Schiffman, J.; Iannaccone, A. Diversity in autoimmunity against retinal, neuronal, and axonal antigens in acquired neuro-retinopathy. J. Ophthalmic Inflamm. Infect. 2011, 1, 111-121. [CrossRef] [PubMed]

34. Stone, J.; Dreher, Z. Relationship between astrocytes, ganglion cells and vasculature of the retina. J. Comp. Neurol. 1987, 255, 35-49. [CrossRef] [PubMed]

35. de Hoz, R.; Rojas, B.; Ramirez, A.I.; Salazar, J.J.; Gallego, B.I.; Trivino, A.; Ramirez, J.M. Retinal Macroglial Responses in Health and Disease. Biomed. Res. Int. 2016, 2016, 2954721. [CrossRef]

36. Barres, B.A. The mystery and magic of glia: A perspective on their roles in health and disease. Neuron 2008, 60, 430-440. [CrossRef]

37. Sarthy, V.P.; Sawkar, H.; Dudley, V.J. Endothelin2 Induces Expression of Genes Associated with Reactive Gliosis in Retinal Muller Cells. Curr. Eye Res. 2015, 40, 1181-1184. [CrossRef]

38. Todd, L.; Palazzo, I.; Suarez, L.; Liu, X.; Volkov, L.; Hoang, T.V.; Campbell, W.A.; Blackshaw, S.; Quan, N.; Fischer, A.J. Reactive microglia and IL1beta/IL-1R1-signaling mediate neuroprotection in excitotoxin-damaged mouse retina. J. Neuroinflammation 2019, 16, 118. [CrossRef]

39. Coorey, N.J.; Shen, W.; Chung, S.H.; Zhu, L.; Gillies, M.C. The role of glia in retinal vascular disease. Clin. Exp. Optom. 2012, 95, 266-281. [CrossRef]

40. Baumann, B.; Sterling, J.; Song, Y.; Song, D.; Fruttiger, M.; Gillies, M.; Shen, W.; Dunaief, J.L. Conditional Muller Cell Ablation Leads to Retinal Iron Accumulation. Invest. Ophthalmol. Vis. Sci. 2017, 58, 4223-4234. [CrossRef]

41. Peng, L.; Parpura, V.; Verkhratsky, A. EDITORIAL Neuroglia as a Central Element of Neurological Diseases: An Underappreciated Target for Therapeutic Intervention. Curr. Neuropharmacol. 2014, 12, 303-307. [CrossRef] [PubMed]

42. Burnight, E.R.; Giacalone, J.C.; Cooke, J.A.; Thompson, J.R.; Bohrer, L.R.; Chirco, K.R.; Drack, A.V.; Fingert, J.H.; Worthington, K.S.; Wiley, L.A.; et al. CRISPR-Cas9 genome engineering: Treating inherited retinal degeneration. Prog. Retin. Eye Res. 2018, 65, 28-49. [CrossRef] [PubMed]

(C) 2020 by the authors. Licensee MDPI, Basel, Switzerland. This article is an open access article distributed under the terms and conditions of the Creative Commons Attribution (CC BY) license (http://creativecommons.org/licenses/by/4.0/). 


\title{
Role of FGF and Hyaluronan in Choroidal Neovascularization in Sorsby Fundus Dystrophy
}

\author{
Alyson Wolk ${ }^{1,2}$, Dilara Hatipoglu ${ }^{1}$, Alecia Cutler ${ }^{1}$, Mariya Ali ${ }^{1}$, Lestella Bell ${ }^{1,3}$, Jian Hua Qi ${ }^{1}$, \\ Rupesh Singh ${ }^{1}$, Julia Batoki ${ }^{1}$, Laura Karle ${ }^{1}$, Vera L. Bonilha ${ }^{1,2,3}$, Oliver Wessely ${ }^{2,4}$, \\ Heidi Stoehr ${ }^{5}$, Vincent Hascall ${ }^{6}$ and Bela Anand-Apte ${ }^{1,2,3, *}$ \\ 1 Cole Eye Institute \& Lerner Research Institute, Cleveland Clinic Foundation, Cleveland, OH 44195, USA; \\ wolka@ccf.org (A.W.); Hatipod@ccf.org (D.H.); cutlera@ccf.org (A.C.); alim2@ccf.org (M.A.); \\ belll3@ccf.org (L.B.); qij@ccf.org (J.H.Q.); Singhr4@ccf.org (R.S.); Batokij@ccf.org (J.B.); \\ lauraikarle@gmail.com (L.K.); bonilhav@ccf.org (V.L.B.) \\ 2 Cleveland Clinic Lerner College of Medicine, Department of Molecular Medicine, Case Western Reserve \\ University, Cleveland, OH 44195, USA; Wesselo@ccf.org \\ 3 Cleveland Clinic Lerner College of Medicine at Case Western Reserve University, Department of \\ Ophthalmology, Cleveland, OH 44195, USA \\ 4 Department of Cardiovascular and Metabolic Sciences, Lerner Research Institute, Cleveland Clinic \\ Foundation, Cleveland, $\mathrm{OH} 44195$, USA \\ 5 Institute of Human Genetics, University of Regensburg, 93053 Regensburg, Germany; \\ Heidi.Stoehr@klinik.uni-regensburg.de \\ 6 Department of Biomedical Engineering, Lerner Research Institute, Cleveland Clinic Foundation, Cleveland, \\ OH 44195, USA; Hascalv@ccf.org \\ * Correspondence: anandab@ccf.org
}

Received: 11 January 2020; Accepted: 28 February 2020; Published: 4 March 2020

\begin{abstract}
Sorsby's fundus dystrophy (SFD) is an inherited blinding disorder caused by mutations in the tissue inhibitor of metalloproteinase-3 (TIMP3) gene. The SFD pathology of macular degeneration with subretinal deposits and choroidal neovascularization (CNV) closely resembles that of the more common age-related macular degeneration (AMD). The objective of this study was to gain further insight into the molecular mechanism(s) by which mutant TIMP3 induces CNV. In this study we demonstrate that hyaluronan (HA), a large glycosaminoglycan, is elevated in the plasma and retinal pigment epithelium (RPE)/choroid of patients with AMD. Mice carrying the S179C-TIMP3 mutation also showed increased plasma levels of HA as well as accumulation of HA around the RPE in the retina. Human RPE cells expressing the S179C-TIMP3 mutation accumulated HA apically, intracellularly and basally when cultured long-term compared with cells expressing wildtype TIMP3. We recently reported that RPE cells carrying the S179C-TIMP3 mutation have the propensity to induce angiogenesis via basic fibroblast growth factor (FGF-2). We now demonstrate that FGF-2 induces accumulation of HA in RPE cells. These results suggest that the TIMP3-MMP-FGF-2-HA axis may have an important role in the pathogenesis of CNV in SFD and possibly AMD.
\end{abstract}

Keywords: sorsby's fundus dystrophy; hyaluronan; neovascularization; retina

\section{Introduction}

Sorsby's fundus dystrophy (SFD) is a dominantly inherited, degenerative disease of the macula that is characterized by bilateral loss of central vision as a consequence of choroidal neovascularization (CNV) [1-6]. Specific mutations in the tissue inhibitor of metalloproteinase 3 (TIMP3) gene involving exon 5 , exon 1 or the intron 4-exon 5 boundary have been shown to be causative [7-14]. In comparative studies using TIMP3 deficient mice, S179C-TIMP3 transgenic mice and in vitro culture experiments we have determined that TIMP3 partially inhibits angiogenesis by blocking the binding of vascular 
endothelial growth factor (VEGF) to VEGF Receptor 2 (VEGFR2). We have also demonstrated that the S179C-TIMP3 mutant protein induces angiogenesis via VEGF and fibroblast growth factor 2 (FGF-2) [15-21].

TIMP3 is produced constitutively by the retinal pigment epithelium (RPE) and choroidal endothelial cells [2,20]. It is a normal component of Bruch's membrane [22] and binds to sulfated glycosaminoglycans of the extracellular matrix (ECM) $[23,24]$. Hyaluronan (HA) is a large glycosaminoglycan that is a significant component of peri-cellular and extracellular matrices. HA is essential for numerous physiological functions that are dependent on its chain size and its interactions with various effector proteins and receptors [25]. HA has been implicated in the regulation of neovascularization and endothelial barrier function [26]. While studies have demonstrated that signaling via $\mathrm{HA}$ and its cell surface receptor CD44 accentuates $\mathrm{CNV}$ in mice using a laser-induced model [27], the exact molecular mechanism by which HA regulates tissue remodeling and neovascularization is unknown.

We have recently reported that RPE cells expressing mutant TIMP3 secrete increased amounts of FGF-2 [28] and that this contributes to increased angiogenesis. FGF-2 has been shown to be important in tumor angiogenesis, but its role in CNV has been less well studied. The most direct evidence for a role of FGF-2 in CNV comes from studies in which Flk1-Cre or Tie2-Cre mediated deletions of FGF receptor 1 (FGFR1) and FGF receptor 2 (FGFR2) in endothelial cells resulted in reduced laser-induced CNV in mice [29]. Extracellular matrix components such as heparan sulfate proteoglycans (HSPGs) bind and regulate the activity of growth factors such as FGF-2 [30] and have a critical role in the regulation of neovascularization [31]. In addition, the observation that activation of the FGFR-STAT3 pathway can induce a hyaluronan-rich microenvironment that can affect tumor growth [32] led us to test the hypothesis that in addition to VEGF, FGF-2 and hyaluronan also have critical roles in the increased neovascularization induced by mutant TIMP3 in Sorsby's fundus dystrophy.

\section{Materials and Methods}

\subsection{Human Samples}

Patients with AMD and controls (without AMD or any other retinal disease) were recruited from the eye clinics at Cole Eye Institute under Cleveland Clinic Foundation approved IRB protocols. Plasma samples were prepared and stored at $-80{ }^{\circ} \mathrm{C}$. Samples from patients $(\mathrm{n}=49$, with 26 males and 23 females) given a clinical diagnosis of geographic atrophy or $\mathrm{CNV}$ and age-matched controls $(\mathrm{n}=59$ with 28 males and 31 females) were included in this pilot study to evaluate HA in the plasma. Normal and/or AMD post-mortem eyes were obtained from the Cleveland Eye Bank, the National Disease Research Interchange (Philadelphia, PA, USA) or from the Cole Eye Institute Eye Tissue Repository through the Foundation Fighting Blindness (FFB) Eye Donor Program (Columbia, MD, USA). All post-mortem tissue were obtained in accordance with the policies of the Eye Bank Association of America and the Institutional Review Board of the Cleveland Clinic Foundation (IRB\#14-057). Eye bank records accompanying the donor eyes indicated whether the donor had AMD or no known eye diseases. The analyzed tissue included FFB donations \#714 (82 y.o.), \#781 (80 y.o.), \#711 (83 y.o.), \#722 (90 y.o.), \#716 (80 y.o.) and \#739 (90 y.o.), identified as AMD. Postmortem eyes from a 95 (\#784), 92 (\#979) and a 91 year-old donor without a history of retinal disease were used as controls. Eyes were enucleated 4 to $22 \mathrm{~h}$ postmortem and fixed in $4 \%$ paraformaldehyde and $0.5 \%$ glutaraldehyde in phosphate buffer. The globes were stored in $2 \%$ paraformaldehyde in D-PBS.

\subsection{Mice}

All mice utilized in this study were housed in the Cole Eye Institute vivarium under approved Institutional Animal Care and Use Committee (IACUC) protocols. All procedures on the mice were in accordance with ARVO statement for the Use of Animals in Ophthalmic and Vision Research and conformed to the National Institutes of Health Guide for the Care and Use of Animals in Research 
and to the ARVO statement for the use of animals in ophthalmic and vision research. Timp3 ${ }^{+/ S 179 C}$ mice were generated in the laboratory of Dr. Bernhard Weber using site-directed mutagenesis and homologous recombination in embryonic stem (ES) cells to generate mutant ES cells carrying the Timp3 $3^{\text {S179C }}$ allele. Heterozygous breeding of Timp3 ${ }^{+/ S 179 C}$ [33] produced homozygous Timp3 $3^{\text {S179C/S179C }}$ mice and age-matched littermate controls in a C57BL6 background. Similarly, heterozygous Timp3 $3^{+/-}$ mice [34] were bred to generate Timp3 ${ }^{-/-}$knockouts and TIMP3 $^{+/+}$littermate controls. Eyes were enucleated following euthanasia and fresh frozen in tissue-plus optical cutting temperature embedding medium (Scigen, \#4583) for sectioning and histology. Blood samples were collected via cardiac puncture and plasma prepared via standard protocols.

\subsection{Hyaluronan Enzyme-Linked Immunosorbent Assay (ELISA)}

Plasma from human patients with and without AMD and from SFD mouse models were measured for HA contents by solid-phase sandwich ELISA in 96-well plates (Costar, \#9018) using the Hyaluronan Duo-Set ELISA kit (R\&D Systems, \#DY3614-05).

\subsection{Immunofluorescence}

Retina sections and flat-mounted ARPE-19 cells grown on polyester trans-wells were fixed for $5 \mathrm{~min}$ in $4 \%$ paraformaldehyde and blocked in $1 \%$ bovine serum albumin with $0.1 \%$ Triton X-100 in phosphate-buffered saline. Human sections were processed with melanin bleaching kit to remove autofluorescence (Polysciences, Inc., Warrington, PA, USA, \#24883A-B). Samples were incubated overnight with biotinylated HA binding protein, (Millipore Sigma, \#385911) or primary antibodies (anti-ezrin, clone 3C12, Invitrogen, Carlsbad, CA, USA \#MA5-13862) in humidified chambers at 4 ${ }^{\circ} \mathrm{C}$. Subsequently, secondary antibodies (anti-mouse AlexaFluor 594, streptavidin-AlexaFluor 488, streptavidin-AlexaFluor 647, all from ThermoFisher Scientific, Waltham, MA, USA) were incubated with samples at room temperature for one hour in the dark. Rhodamine-phalloidin (Thermo Fisher Scientific, R415) was incubated together with secondary antibodies. Then, 4' ,6-diamidino-2-phenylindole (DAPI) was used to stain nuclei of murine sections and cell culture mounts and SYTOX green (ThermoFisher Scientific, \#S7020) was used to stain nuclei in human sections. Imaging by confocal microscopy was performed (Leica TCS-SP8, Exton, PA, USA). The localization of Bruch's membrane was determined by its autofluorescence at $405 \mathrm{~nm}$.

\subsection{Hyaluronidase Treatment of Retina Sections}

Hyaluronidase from Streptomyces hyalurolyticus (Millipore Sigma, Burlington, MA USA, \#H1136) was used to treat retina sections as described previously [35]. Streptomyces hyaluronidase was resuspended in $0.1 \mathrm{M}$ sodium acetate buffer, $\mathrm{pH}$ 5.0, at $100 \mathrm{U} / \mathrm{mL}$. To prevent any nonspecific digestion, the following protease inhibitors were added to the sodium acetate buffer: $1 \mathrm{mM}$ iodoacetic acid, $1 \mathrm{mM}$ phenylmethyl sulfonylfluoride, $1 \mathrm{mM}$ EDTA, $1 \mu \mathrm{g} / \mathrm{mL}$ pepstatin $\mathrm{A}, 250 \mu \mathrm{g} / \mathrm{mL}$ ovomucoid. Hyaluronidase solution $\left(100 \mathrm{mU} / \mathrm{mL}\right.$ of hyaluronidase in $\mathrm{PBS}$ with $\mathrm{CaCl}_{2}(0.1 \mathrm{~g} / \mathrm{L})$ and $\left.\mathrm{MgCl}_{2}(0.1 \mathrm{~g} / \mathrm{L})\right)$ was applied onto the sections for $3 \mathrm{~h}$ at $37^{\circ} \mathrm{C}$. Slides were subsequently fixed in $4 \%$ paraformaldehyde and examined by fluorescence microscopy.

\subsection{Cells and Reagents}

ARPE-19 cells stably expressing S179C-TIMP3, wild-type-TIMP3 (WT), or vector alone were reported previously [19]. Cells were expanded in DMEM-F12 with 10\% FBS before transfer to polyester inserts coated with mouse laminin (Corning Inc., Corning, NY, USA, \#23017). 720,000 cells and 100,000 cells were plated per well in each well of a 12-well plate or 24-well plate, respectively using a previously published protocol [36]. Essentially, ARPE-19 cells were cultured for at least 2 weeks in nicotinamide-supplemented media with $1 \%$ FBS. Media were replaced twice per week. Cells were serum-starved for $24 \mathrm{~h}$ before treatment with the FGF Receptor inhibitor BGJ-398 (Selleckchem, Houston, TX, USA, \#S2183) for 48 h. Similarly, cells were treated with FGF-2 (Gibco from Thermo 
Fisher Scientific, \#13256-029) with the required cofactor heparin sodium salt (1 $\mu \mathrm{g} / \mathrm{mL}$, Sigma Aldrich, \#H3149) for $48 \mathrm{~h}$ after serum starving for $24 \mathrm{~h}$.

\subsection{Quantitation of Immunofluorescence by Integrated Density Analysis}

Fluorescence intensity of HABP staining was quantified using integrated density analysis as previously described $[37,38]$. For all the RPE cell culture confocal microscopy images, fluorescence was quantitated using a standard measure of integrated density, which is the product of area and mean gray value. A custom written automated image analysis code was developed using Matlab (MATLAB 2019a, The MathWorks, Inc., Natick, MA, USA) for separating the desired color channel from the image, thereby obtaining the total area (in pixels), the mean gray value, and the integrated density.

\subsection{In Vivo Imaging and Laser Injury Model}

Laser mediated CNV was induced as described previously [28]. Briefly, mice were anesthetized with $65-68 \mathrm{mg} / \mathrm{kg}$ sodium pentobarbital delivered intra-peritoneally. Topical $0.5 \%$ procaine solution was applied for cornea anesthesia. Following anesthesia, pupils were dilated with $0.5 \%$ topical tropicamide/phenylephrine combination drops (Santen Pharmaceuticals, Osaka, Japan).

Four laser spots were placed in the superior, superior-temporal, or superior-nasal quadrants of the fundus using a green solid-state laser (Oculight by Iridex Corp., Mountain View, CA, USA) (532 nm; $2500 \mathrm{~mW}$; $0.50 \mathrm{~s}$ pulse duration; $50 \mu \mathrm{m}$ spot size) using a slit lamp delivery system and a microscope coverslip placed and affixed to the cornea with a drop of Systane Ultra artificial tears (Alcon, Ft Worth, TX, USA). All animals were scanned immediately after laser injury with optical coherence tomography (Envisu R2210 UHR Leica Microsystems Inc., Wetzlar, Germany) to confirm successful RPE-Bruch's membrane rupture, an endpoint in laser-induced CNV models.

\subsection{Statistical Analysis}

All parameters in the study were distributed normally. Data are expressed as mean \pm SEM. Differences were tested by unpaired t-test (Figure 1, Figure 2 and Figure 3) or by using multiple t-tests employing two-stage linear step-up procedure of Benjamini, Krieger and Yekutieli, with a false discovery rate set to $1 \%$ (Figure 4 and Figure 5). Each group was analyzed separately without the assumption of consistent standard deviation. $p<0.05$ values were considered statistically significant. Statistical analysis was performed with GraphPad Prism 7.03 (GraphPad Software, Inc., San Diego, CA, USA).

\section{Results}

\subsection{Hyaluronan is Elevated in Plasma and RPE/Choroid of Patients with AMD}

Age-related macular degeneration (AMD) is usually seen as two main types. "Dry" AMD where deposits called drusen develop in the macular region that ultimately progress to a late stage in which there is atrophy of the macula (geographic atrophy). "Wet" AMD describes AMD in which patients develop abnormal growth and leakage of the choroid vessels beneath and into the retina, termed choroidal neovascularization (CNV). HA contents were measured in plasma from patients with late stage AMD (geographic atrophy or choroidal neovascularization) and from age-matched controls without the disease. ELISA analysis (Figure 1A) indicates that HA contents were significantly increased in the plasma of patients with late-stage AMD (mean \pm SEM: $111.8 \pm 5.78 \mathrm{ng} / \mathrm{mL}$ ) compared with plasma of controls without AMD (32.91 \pm 5.75$)$. 
A

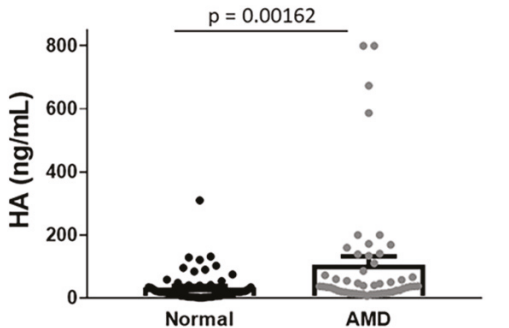

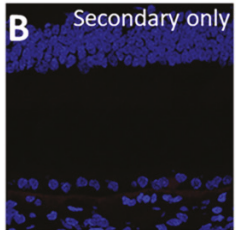
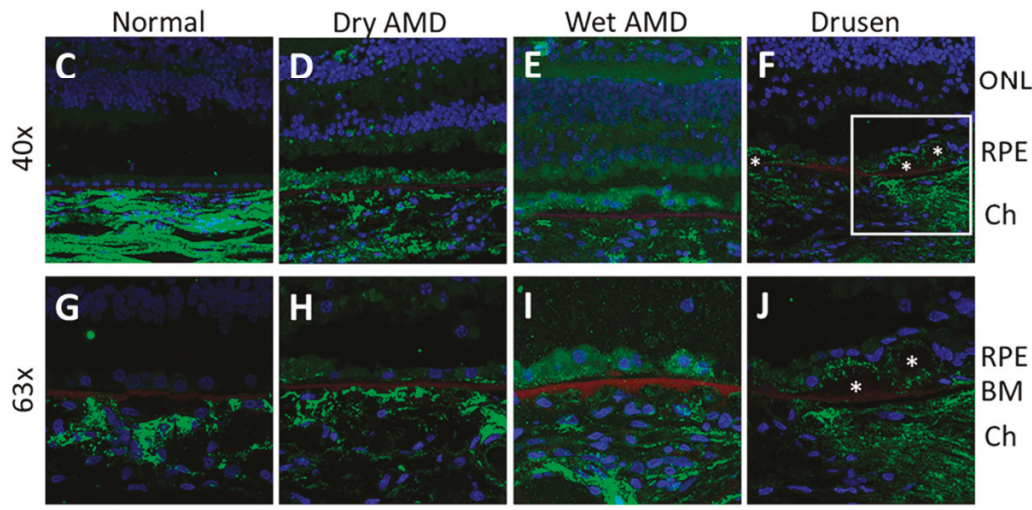

Figure 1. Hyaluronan HA is increased in circulation and in the RPE of age-related macular degeneration (AMD) patients. (A) HA was increased in plasma from patients with late-stage AMD (GA or CNV) compared to age-matched, normal controls. Data are presented as mean \pm SEM (B-J) Representative human retina sections stained with HA binding protein (HABP) (B) Human retina section stained with streptavidin-AlexaFluor 647 in the absence of HA binding protein serves as a specificity control. (C-J) Human retina sections stained with streptavidin-AlexaFluor 647 in the presence of HA binding protein. HA is increased in the RPE in patients with dry $\operatorname{AMD}(\mathbf{D}, \mathbf{H})$, wet $\operatorname{AMD}(\mathbf{E}, \mathbf{I})$, and around drusen $(\mathbf{F}, \mathbf{J})$ compared to the RPE from an aged-match normal control (C,G). 40× images (B-F), 63× images (G-J). Green: HA; red: Bruch's membrane determined by its autofluorescence at $405 \mathrm{~nm}$; blue: DAPI. Asterisks indicate drusen $(\mathrm{F}, \mathrm{J})$. GA—geographic atrophy; $\mathrm{CNV}$ - choroidal neovascularization; ONL—outer nuclear layer; RPE—retinal pigment epithelium; Ch—choroid; DAPI-4',6-diamidino-2-phenylindole.

To evaluate the distribution of HA in the retina under physiological and pathological conditions, sections from post-mortem human donor eyes from 3 controls and 6 AMD (4 dry and 2 wet AMD) patients were stained for HA using biotinylated HA binding protein (HABP). HA was found to be localized predominantly in the choroid of normal eyes (Figure 1C) as described previously [39,40]. Increased deposition of HA was seen around the RPE in AMD eyes (both in the dry (Figure 1D,H) and wet AMD specimens (Figure 1E,I)). HA was particularly enhanced in drusen and in areas of atrophy (Figure $1 F, J)$ in AMD specimens. The sections stained with secondary antibody alone serves as a specificity control and shows minimal staining compared with sections stained with HABP (Figure 1B).

\subsection{Increased Plasma HA and Accumulation of HA in the RPE of SFD Mice}

We utilized two mouse models to study the potential role of TIMP3 in the regulation of HA in the retina: mice lacking TIMP3 [34] and mice carrying the S179C-TIMP3 SFD mutation [33]. Plasma from S179C-TIMP3 and TIMP3-KO mice at 4-6 weeks of age was collected and HA contents were analyzed by ELISA. HA content of plasma was significantly increased in mice lacking TIMP3 as well 
as in mice carrying the S179C-TIMP3 mutation (Figure 2A), suggesting that TIMP3 may be important in regulating $\mathrm{HA}$.
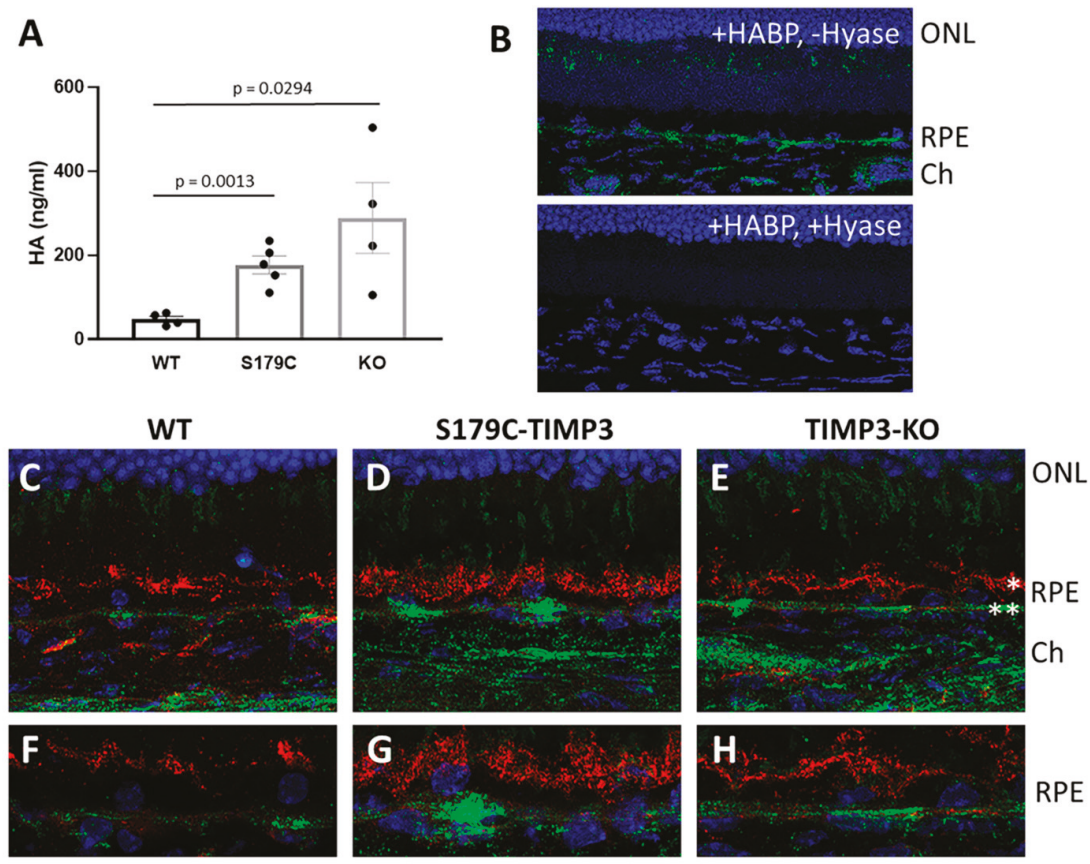

Figure 2. HA is increased in circulation and in the RPE and choroid in mouse models of Sorsby's fundus

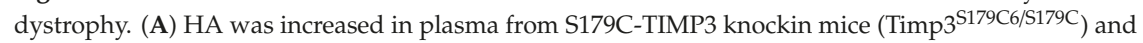
TIMP3-KO $\left(\right.$ Timp3 $\left.^{-/-}\right)$mice compared to wild-type $(\mathrm{WT})$ littermates. $(\mathrm{n} \geq 5)$. Data are presented as mean \pm SEM. (B) Mouse retina sections stained with biotinylated HA binding protein (HABP) in the absence (upper panel) or presence (lower panel) of hyaluronidase to detect HA. HABP staining is specific for HA as shown by the absence of staining in sections treated with hyaluronidase (lower panel). Green: HA; blue: DAPI. (C-H) Representative images of HA staining of mouse sections from wild-type (WT) mice (C,F), S179C-TIMP3 mutant mice (D,G) and TIMP3-KO mice (E,H). HA is increased in the RPE and choroid of S179C-TIMP3 (D) and TIMP3-KO (E) mice compared to wild-type (WT) littermate controls (C). HA (green) is predominantly localized to the basal surface of the RPE (**) (F-H) and not to the apical surface $\left(^{*}\right)$ as shown by co-staining with ezrin, a marker for the apical microvilli of RPE (red). $40 \times$ images (C-E); $63 \times$ images (F-H). $\mathrm{n} \geq 3$ for all immunohistochemistry data.

To determine if there was a similar correlation between the plasma HA levels and the accumulation of HA in the RPE as observed in human sections with AMD, we evaluated accrual of HA in the retinas of mice ( 8 weeks of age) lacking TIMP3 or carrying the SFD mutation. Cryosections of retina from mice of each specific genotype and wild-type littermates were stained for HA content with HABP. To ascertain that HABP binds HA specifically, sections were treated with hyaluronidase prior to staining with HABP. Indeed, pre-treatment with hyaluronidase resulted in absence of staining with HABP (Figure 2B, lower panel). S179C-TIMP3 mice (Figure 2D) and TIMP3-KO mice (Figure 2E) show increased accumulation of HA beneath the RPE and in the choroid compared to that seen in wildtype littermates (Figure 2C). Staining with antibodies to ezrin served as a marker for RPE apical microvilli (Figure 2C-H), and the higher magnification images (Figure 2F-H) confirmed the RPE localization of HA to the basal surface of the cells. 
To identify the potential mechanism by which S179C-TIMP3 regulates HA we utilized stable human RPE lines (ARPE-19) expressing S179C-TIMP3 [20]. ARPE-19 cells (expressing S179C-TIMP3, wildtype TIMP3 (WT-TIMP3) and empty vector (Vector)) were cultured for 2-6 weeks in 1\% serum on trans-well inserts and stained for HA. Increased accumulation of HA was observed in RPE cells expressing S179C-TIMP3 (Figure 3C,G) compared with cells transfected with empty vector (Figure 3A,G) or expressing wildtype TIMP3 (Figure 3B,G). The accumulation was predominantly intracellular and apical in the RPE (Figure 3D-F). There appear to be multiple layers of S179C-TIMP3 RPE cells on the transwell compared with a single monolayer for WT-TIMP3 and vector cells, which might suggest epithelial-mesenchymal transition.
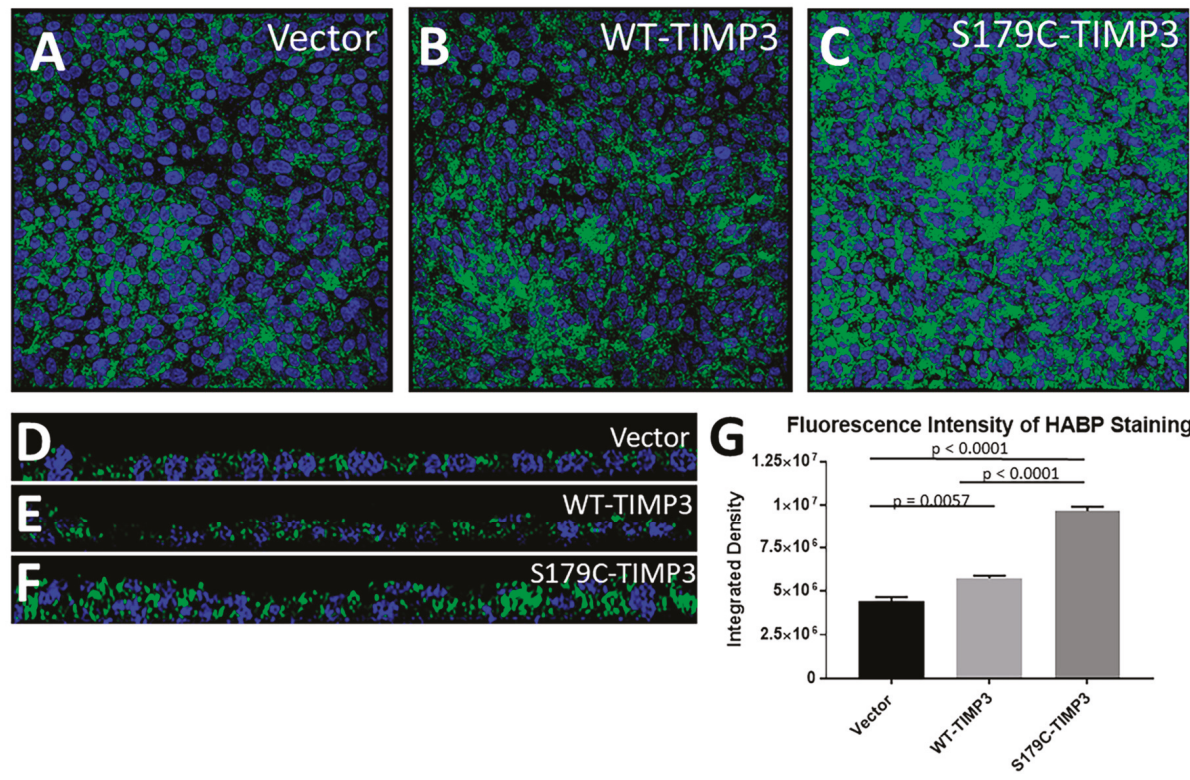

Figure 3. HA is increased in S179C-TIMP3 RPE cells in culture. (A-C) ARPE-19 cells expressing S179CTIMP3 grown in culture for at least 2 weeks on trans-well inserts have increased HA (C) compared to WT-TIMP3 expressing cells (B) or vector only controls (A).(D-F) Z-plane images of HA in RPE monolayers grown on trans-well inserts show increased intracellular HA in S179C-TIMP3 cells. Green: HA; blue: DAPI. (G) Fluorescence intensity was quantitated by integrated density measurement $(\mathrm{n} \geq 4$, for each cell line). Data are presented as mean \pm SEM.

\subsection{FGF-2 Contributes to HA Accumulation in the RPE}

We have recently reported that RPE cells expressing S179C-TIMP3 secrete higher amounts of FGF-2 compared with control cells [28]. Previous studies have suggested that FGF signaling has the propensity to increase HA accumulation [32]. To experimentally test this hypothesis in RPE cells, we evaluated the ability of FGF-2 to induce HA accumulation in primary porcine RPE cells. Cells cultured for 3 weeks on trans-well inserts in 1\% serum were treated with $0 \mathrm{ng} / \mathrm{mL}, 10 \mathrm{ng} / \mathrm{mL}, 25 \mathrm{ng} / \mathrm{mL}$, or $100 \mathrm{ng} / \mathrm{mL}$ of FGF-2 in the presence of $1 \mu \mathrm{g} / \mathrm{mL}$ heparin, a cofactor for FGF receptor signaling. FGF-2 induced HA accumulation in a dose-dependent manner (Figure 4A-I) with maximum HA deposits being observed with a dose of 25 and $100 \mathrm{ng} / \mathrm{mL}$ (Figure $4 \mathrm{D}, \mathrm{H}$ ). This was confirmed by quantitation of fluorescence by integrated density measurements (Figure 4I). The FGF-2 induced accumulation of HA was seen predominantly on the apical surface of the RPE with increased basal and peri-cellular accumulation at higher doses (Figure 4E-H). 

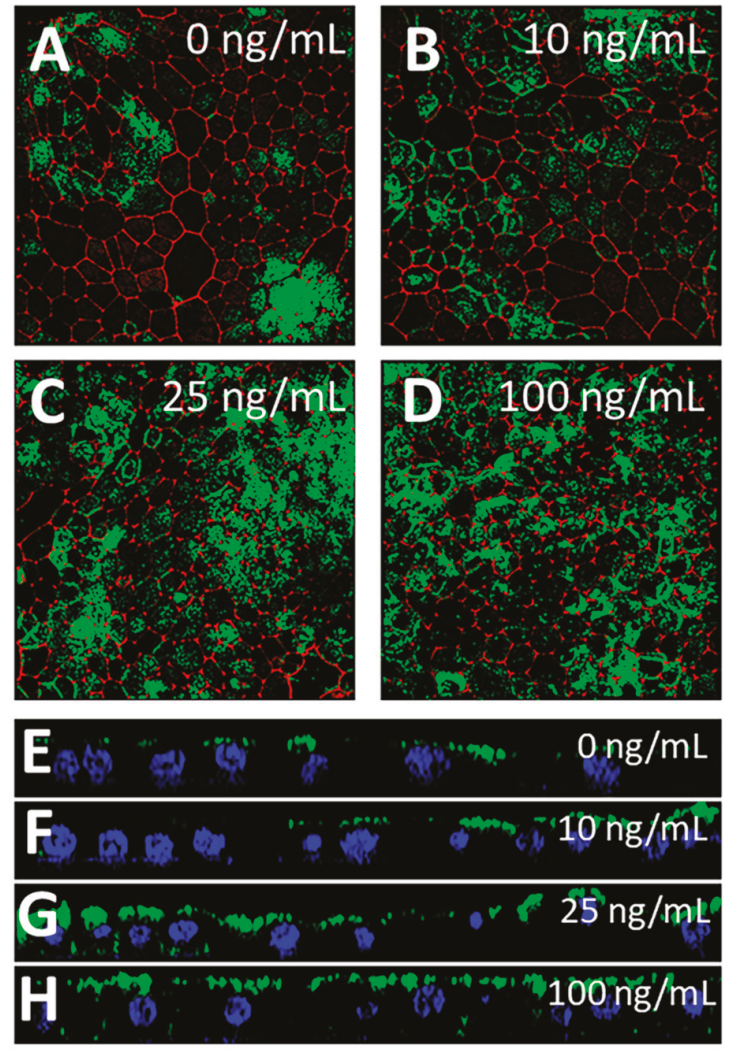

\section{Fluorescence Intensity of HABP Staining}

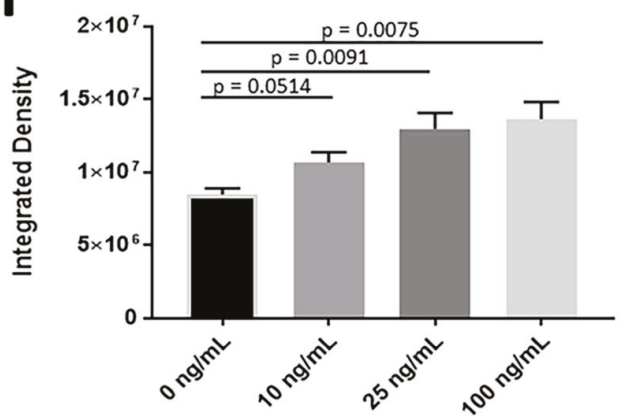

Figure 4. FGF-2 induces HA accumulation in primary RPE cells. (A-D) FGF-2 induced HA accumulation in primary porcine RPE cells in a dose-dependent manner (A) $0 \mathrm{ng} / \mathrm{mL},($ B) $10 \mathrm{ng} / \mathrm{mL},($ C) $25 \mathrm{ng} / \mathrm{mL}$, (D) $100 \mathrm{ng} / \mathrm{mL}$. Fluorescence intensity was quantitated by integrated density measurement $(\mathrm{I})(\mathrm{n} \geq$ 4, for each cell line). Data are presented as mean \pm SEM (E-H) Z-plane images show that increased concentrations of FGF-2 induce increased apical accumulation of HA in addition to some peri-cellular and basal deposits of HA at high doses of FGF-2. Green: HA, red: phalloidin; blue: DAPI.

To determine if the accumulation of HA seen in RPE cells expressing S179C-TIMP3 was a consequence of increased FGF signaling, cells (RPE cells expressing S179C-TIMP3, wildtype TIMP3 or empty vector) were treated with BGJ-398, an FGF receptor inhibitor. $10 \mu \mathrm{M}$ BGJ-398 decreased HA 
content in RPE cells expressing S179C-TIMP3 (Figure 5C,F,H,I) and WT-TIMP3 (Figure 5B,E,I) but not vector only transfected cells (Figure 5A,D,I) when compared with their respective untreated cells that served as controls (Figure 5A: vector, 5B: WT-TIMP3 and 5C,G: S179C-TIMP3). Quantitation of fluorescence by integrated density analysis revealed that $10 \mu \mathrm{M}$ BGJ-398 decreased HA accumulation in S179C-TIMP3 cells $63.1 \%(S D=0.241)$ and only $43.4 \%(S D=0.291)$ in wildtype cells (Figure $5 \mathrm{I})$. Quantitation confirmed that BGJ-398 had no significant effect on vector only cells (Figure 5I), suggesting an FGF-specific mechanism for HA accumulation in S179C-TIMP3 cells.
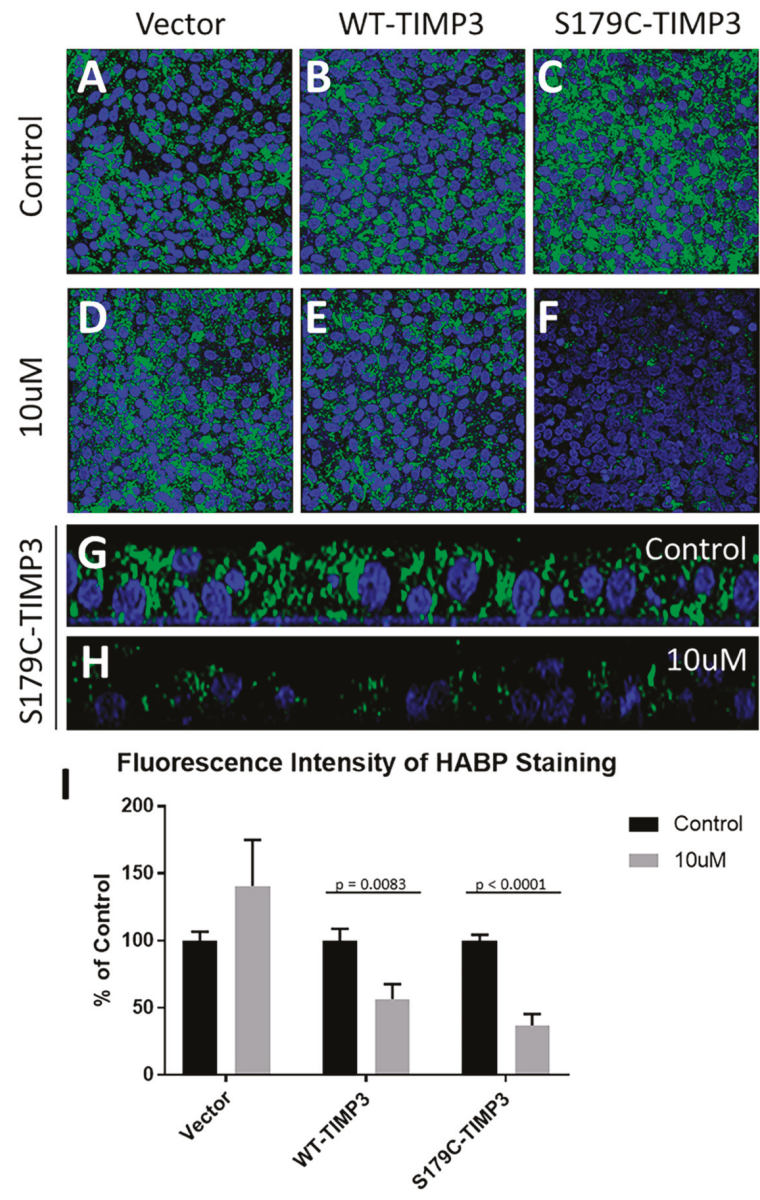

Figure 5. Inhibition of FGF signaling decreases HA accumulation in S179C-TIMP3 RPE cells. Vector only (A), WT-TIMP3 (B), and S179C-TIMP3 (C) expressing ARPE-19 cells were grown on trans-well inserts for 4 weeks before treatment with FGF receptor inhibitor BGJ-398. Treatment with BGJ-398 decreased HA accumulation in S179C-TIMP3 expressing RPE cells $(\mathbf{F}, \mathbf{H}, \mathbf{I})$ and to a lesser extent in WT-TIMP3 expressing cells (E,I) but has no effect on control vector RPE cells (A,D,I). (I) Control (Black Bar) indicates respective untreated cells compared with BGJ-398 treated cells (Grey bar). (G,H) Z-plane images of S179C-TIMP3 cells in the absence $(\mathbf{G})$ and presence $(\mathbf{H})$ of $10 \mu \mathrm{M}$ BGJ-398 S179C-TIMP3 cells show an overall reduction in HA accumulation, including intracellular HA, after treatment with BGJ-398. Green: HA; blue: DAPI. (I) data are presented as mean $\pm \operatorname{SEM}(n \geq 6)$. 


\subsection{Increased HA is Associated with CNV in AMD and SFD}

Sections of post-mortem eyes from a patient with CNV showed significant HA deposition around the RPE (Figure 6C,D) when compared with control eyes (Figure 6A,B). While S179C-TIMP3 mice do not demonstrate a florid SFD phenotype as seen in humans, they do have increased susceptibility to experimental laser-induced CNV [28] as do the TIMP3-KO mice [41]. We have previously shown that FGF-2 from S179C-TIMP3 RPE cells can stimulate angiogenesis [28]. Since FGF-2 contributes to HA accumulation in the RPE and to angiogenesis, we evaluated if HA content and distribution was altered in laser-induced CNV lesions in S179C-TIMP3 mice. As described previously lesions in S179C-TIMP3 mice were larger and leakier compared to controls [28]. Increased HA accumulation was observed in CNV in S179C-TIMP3 mice (Figure 6H-J) when compared to lesions in control mice (Figure 6E-G) which appears to be a consequence of altered distribution to the CNV lesions in S179C-TIMP3 mice.
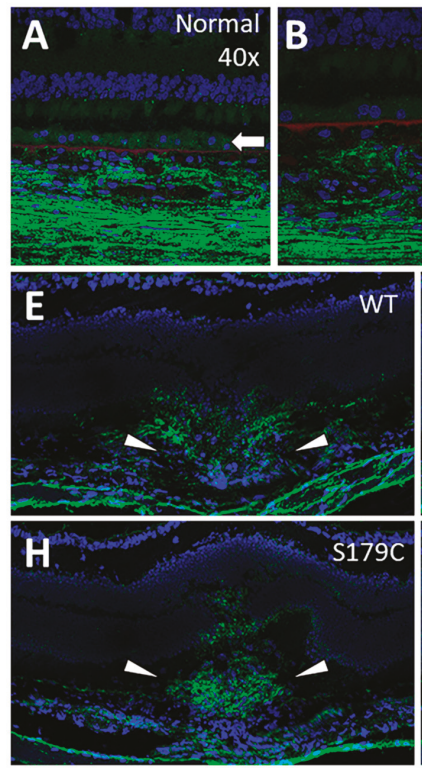
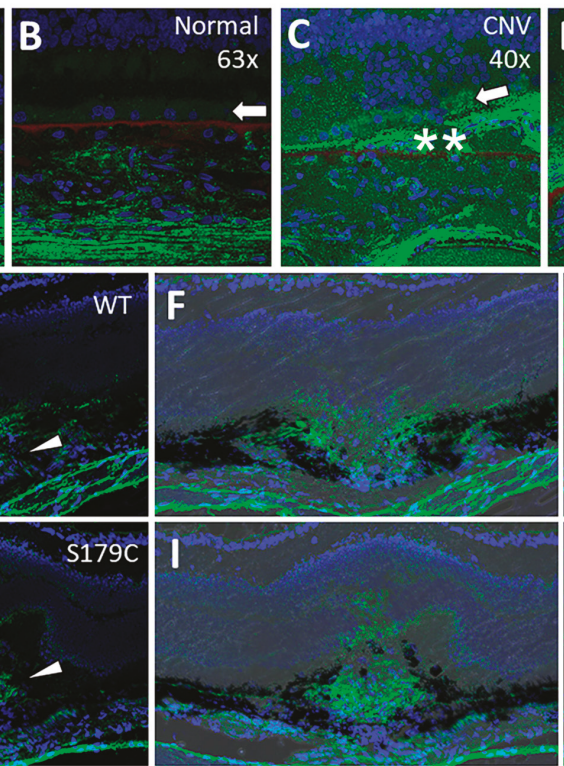
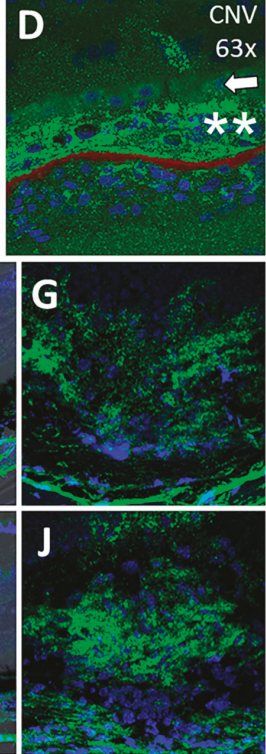

Figure 6. HA is increased in choroidal neovascular lesions in AMD patients and in S179C-TIMP3 mice. (A-D) HA is increased in the RPE in a patient with CNV $(\mathbf{C}, \mathbf{D})$ and in the CNV lesion compared to the RPE of a normal patient (A,B). Green: HA; blue: nuclei; red: Bruch's membrane. Arrows indicate $\mathrm{RPE}$, double asterisks indicate CNV lesion. (E-G) HA is increased in laser-induced CNV lesions in S179C-TIMP3 mice compared to wild-type (WT) littermates 5 days post injury. (E,H) Arrows indicate CNV lesion. (F,I) Brightfield image overlaid on fluorescent image shows disruption of RPE and Bruch's membrane. (G,J) HA accumulation is diffuse and appears predominantly in the borders of the lesion in WT (E-G) compared to dense mass within the lesions of S179C-TIMP3 mice (H-J). Green: HA; blue: nuclei.

\section{Discussion}

Sorsby's fundus dystrophy (SFD) is a rare macular dystrophy characterized by vision loss due to persistent choroidal neovascularization [1-6]. SFD is an autosomal dominant, fully penetrant degenerative disease of the macula and is notable for its similarity in histopathological features to AMD [3-6]. The majority of SFD patients develop CNV, as well as confluent, 20-30 $\mu \mathrm{m}$ thick, amorphous deposits between the basement membrane of the RPE and Bruch's membrane. TIMP3 and/or its downstream substrates have been postulated to have a role in the pathogenesis of both SFD and AMD, because accumulation of TIMP3 has been observed in subretinal deposits in SFD [42] as 
well as in AMD drusen [43-45]. In this study, we show that hyaluronan accumulates around the RPE in AMD as well as in CNV lesions of mice expressing S179C-TIMP3.

One interesting observation from our studies was the increase in HA in the plasma of patients with AMD as well as in mice lacking TIMP3 or carrying the S179C-TIMP3 mutation. Although we observe significant differences in the plasma levels of HA in patients with advanced AMD (GA and CNV), the number of patients analyzed ( $n=49$ controls) and $n=59$ (AMD) is not sufficient to determine if this is of prognostic value. In addition, we did not have access to samples from patients at different degrees of severity to be able to draw conclusions with this or the rate of progression of the disease. Future studies are warranted to address this question. While patients with SFD generally demonstrate disease localized to the retina, our results may be a consequence of ubiquitous expression of TIMP3 in a variety of tissues in the body [46] which could potentially explain the systemic increase in HA. Whether there is accumulation of HA in other tissues in S179C TIMP3 mice has not been evaluated.

Our studies with RPE cells suggest that the increase in HA in these cells is likely a consequence of increased FGF. A recent study [47] suggests that TIMP proteins can control FGF-2 bioavailability in skeletal tissue and the same might be true of multiple tissues leading to systemic increase in HA in the plasma. The exact mechanism by which TIMP3 regulates FGF bioavailability in the RPE is currently unknown, but it is highly likely that the TIMP-metalloproteinase axis likely has a key role. The extracellular matrix (ECM) serves as a high capacity reservoir for FGF-2 and early studies have demonstrated that matrix metalloproteases (MMPs) have the ability to mobilize FGF-2 to a soluble phase that results in receptor activation [48]. Additional studies identifying the molecular mechanisms by which TIMP3 regulates FGF-2 bioavailability will provide insight into the pathophysiology of the disease.

FGF-2 is sufficient to increase HA accumulation and distribution in the RPE, and blocking FGF signaling in S179C-TIMP3 RPE cells brings HA levels back to normal. The mechanism by which FGF-2 increases HA accumulation is not understood. HA is endogenously synthesized by a family of membrane-integrated glycosyltransferases, called hyaluronan synthases (HAS 1-3) and is exported directly into the ECM [49,50]. Hyaluronidases (HYAL1-2) are a class of enzymes that degrade HA [51]. A balance between HA synthesizing and degrading activity keeps HA at physiological levels. In order to determine the mechanism of accumulation of HA in the RPE in SFD, we performed quantitative PCR analysis of HAS1-3 and HYAL1-2 from RPE isolated from S179C-TIMP3, TIMP3-KO mice and wildtype littermate controls. Interestingly, we observed no changes in gene expression of any of these enzymes in the mutant mice (Supplementary Figure S1). Therefore, at least in the RPE in SFD mice, the differences in HA content are not due to increased expression of the synthases nor decreased expression of canonical degradation enzymes. However, there are other possibilities that need to be explored in the future. HA production could be modulated by decreasing enzyme recycling from endosomes back to the cell surface as seen in keratocytes [52]. Additionally, there is a possibility that other non-canonical hyaluronidases such as KIAA1199 [53] and Tmem2 [54] could be involved. Alternatively, as previously reported degradation might be prevented by increased binding proteins on HA and leading to net accumulation [55].

HA has been shown to exhibit a diverse array of biological functions including a role in the response to tissue damage and inflammation [56]. Our studies demonstrating accumulation of HA in laser-induced CNV lesions corroborates previous studies [27]. This study also reported an increase in CD44 and HAS2 mRNA following laser injury [27]. It is possible that the increased accumulation of HA in laser-induced CNV lesions in S179C-TIMP3 mice might result from similar increases in mRNA transcription.

Chronic low-grade inflammation has been suggested to contribute to age-related macular degeneration [57]. In the laser-induced mouse model of $\mathrm{CNV}$, inflammatory processes have been shown to play a role in the development and regression of the lesions. A number of reports link HA remodeling to the modulation of neuroinflammation with low-molecular weight HA being pro-inflammatory and high molecular weight HA being anti-inflammatory [58-60]. While we see increased deposition of HA 
in and around the RPE, we have not determined its physical properties such as size and molecular weight distribution in the tissue.

The receptor engagement of HA in the retina or it's downstream signaling under physiological or pathological conditions has not yet been identified and will be important as we determine its exact role in the pathology of macular degenerative disease. In our study we demonstrate that primary porcine RPE cells deposited HA predominantly on the apical surface under physiological conditions similar to what had been previously reported for human RPE cells [61]. Our data revealed that FGF-2 induced HA accumulation apically as well as between cells and on the basal surface, suggesting that in addition to increased total HA content, the distribution of HA may be important for disease pathogenesis and warrants further investigation. We have recently reported that the secretion of FGF-2 by RPE cells expressing S179C-TIMP3 led to increased angiogenesis [28]. Whether HA is modified in the endothelial glycocalyx as a consequence of FGF-2 has not been studied and might provide further insight into the pathogenesis of CNV in AMD and SFD leading to the identification of novel therapeutic approaches.

Supplementary Materials: The following are available online at http:/www.mdpi.com/2073-4409/9/3/608/s1, Figure S1: RNA was isolated from mouse RPE using the Simultaneous RPE cell Isolation and RNA Stabilization method (SRIRS method) using the RNA Plus Mini Kit (Qiagen). Quantitative PCR was performed following reverse transcription using TaqMan probes for the mouse genes Has1 (A), Has2 (B), Has3 (No signal), Hyal1 (C), Hyal2 (D), and 18S ribosomal RNA (rRNA) (Applied Biosystems). 18S rRNA was used as endogenous control for each gene tested. mRNA expression was calculated using 2- $\Delta \Delta \mathrm{Ct}$ method and shown relative to expression in wildtype littermate mice.

Author Contributions: Conceptualization, A.W., O.W., V.H. and B.A.-A.; data curation, A.W.; formal analysis, B.A.-A.; funding acquisition, B.A.-A.; methodology, A.W., D.H., A.C., M.A., L.B., J.H.Q., R.S., J.B. and L.K.; project administration, B.A.-A.; resources, V.L.B., O.W. and H.S.; software, R.S.; supervision, B.A.-A.; validation, A.W.; writing—original draft, A.W.; writing—review and editing, A.W., H.S., V.H. and B.A.-A. All authors have read and agreed to the published version of the manuscript.

Funding: This work was supported in part by US National Institute of Health EY027083 (BA-A), EY026181 (BA-A), P30EY025585(BA-A), T32EY024236 (AW), EY022768 (JHQ), EY027750 (VLB), Research to Prevent Blindness (RPB) Challenge Grant and RPB Lew Wasserman award to BA-A, Cleveland Eye Bank Foundation Grant and funds from Cleveland Clinic Foundation.

Acknowledgments: The authors thank the retina specialists at Cole Eye Institute from whose clinics patients with or without AMD were recruited. The authors are grateful for the generous gift of TIMP-3 null mice from Rama Khokha at the Princess Margaret Cancer Centre-Ontario Cancer Institute, Toronto, Canada and to Emma Lessieur who served as the research coordinator at the time the blood samples were collected from patients The authors also acknowledge the support of Foundation Fighting Blindness in setting up the Eye Tissue Repository. We wish to extend a sincere apology to colleagues whose work was not cited due to space limitations.

Conflicts of Interest: The authors declare no conflict of interest. The funders had no role in the design of the study; in the collection, analyses, or interpretation of data; in the writing of the manuscript, or in the decision to publish the results.

\section{References}

1. Sorsby, A.; Mason, M.E.; Gardener, N. A fundus dystrophy with unusual features (late onset and dominant inheritance of a central retinal lesion showing oedema, haemorrhage and exudates developing into generalised choroidal atrophy with massive pigment proliferation). Br. J. Ophthalmol. 1949, 33, 67-97. [CrossRef] [PubMed]

2. Della, N.G.; Campochiaro, P.A.; Zack, D.J. Localization of timp-3 mRNA expression to the retinal pigment epithelium. Investig. Ophthalmol. Vis. Sci. 1996, 37, 1921-1924.

3. Holz, F.G.; Haimovici, R.; Wagner, D.G.; Bird, A.C. Recurrent choroidal neovascularization after laser photocoagulation in sorsby's fundus dystrophy. Retina 1994, 14, 329-334. [CrossRef] [PubMed]

4. Jacobson, S.G.; Cideciyan, A.V.; Regunath, G.; Rodriguez, F.J.; Vandenburgh, K.; Sheffield, V.C.; Stone, E.M. Night blindness in sorsby's fundus dystrophy reversed by vitamin a. Nat. Genet. 1995, 11, 27-32. [CrossRef]

5. Kalmus, H.; Seedburgh, D. Probable common origin of a hereditary fundus dystrophy (sorsby's familial pseudoinflammatory macular dystrophy) in an english and australian family. J. Med. Genet. 1976, 13, 271-276. [CrossRef] 
6. Polkinghorne, P.J.; Capon, M.R.; Berninger, T.; Lyness, A.L.; Sehmi, K.; Bird, A.C. Sorsby's fundus dystrophy. A clinical study. Ophthalmology 1989, 96, 1763-1768. [CrossRef]

7. Barbazetto, I.A.; Hayashi, M.; Klais, C.M.; Yannuzzi, L.A.; Allikmets, R. A novel timp3 mutation associated with sorsby fundus dystrophy. Arch. Ophthalmol. 2005, 123, 542-543. [CrossRef]

8. Felbor, U.; Benkwitz, C.; Klein, M.L.; Greenberg, J.; Gregory, C.Y.; Weber, B.H. Sorsby fundus dystrophy: Reevaluation of variable expressivity in patients carrying a timp3 founder mutation. Arch. Ophthalmol. 1997, 115, 1569-1571. [CrossRef]

9. Felbor, U.; Stohr, H.; Amann, T.; Schonherr, U.; Weber, B.H. A novel ser156cys mutation in the tissue inhibitor of metalloproteinases-3 (timp3) in sorsby's fundus dystrophy with unusual clinical features. Hum. Mol. Genet. 1995, 4, 2415-2416. [CrossRef]

10. Langton, K.P.; Barker, M.D.; McKie, N. Localization of the functional domains of human tissue inhibitor of metalloproteinases-3 and the effects of a sorsby's fundus dystrophy mutation. J. Biol. Chem. 1998, 273, 16778-16781. [CrossRef]

11. Langton, K.P.; McKie, N.; Curtis, A.; Goodship, J.A.; Bond, P.M.; Barker, M.D.; Clarke, M. A novel tissue inhibitor of metalloproteinases-3 mutation reveals a common molecular phenotype in sorsby's fundus dystrophy. J. Biol. Chem. 2000, 275, 27027-27031. [PubMed]

12. Lin, R.J.; Blumenkranz, M.S.; Binkley, J.; Wu, K.; Vollrath, D. A novel his158arg mutation in timp3 causes a late-onset form of sorsby fundus dystrophy. Am. J. Ophthalmol. 2006, 142, 839-848. [CrossRef] [PubMed]

13. Tabata, Y.; Isashiki, Y.; Kamimura, K.; Nakao, K.; Ohba, N. A novel splice site mutation in the tissue inhibitor of the metalloproteinases-3 gene in sorsby's fundus dystrophy with unusual clinical features. Hum. Genet. 1998, 103, 179-182. [PubMed]

14. Weber, B.H.; Vogt, G.; Pruett, R.C.; Stohr, H.; Felbor, U. Mutations in the tissue inhibitor of metalloproteinase-3 (timp-3) in patients with sorsby's fundus dystrophy. Nat. Genet. 1994, 8, 352-356. [CrossRef]

15. Qi, J.H.; Anand-Apte, B. Tissue inhibitor of metalloproteinase-3 (timp3) promotes endothelial apoptosis via a caspase-independent mechanism. Apoptosis 2015, 20, 523-534. [CrossRef]

16. Qi, J.H.; Dai, G.; Luthert, P.; Chaurasia, S.; Hollyfield, J.; Weber, B.H.; Stohr, H.; Anand-Apte, B. S156c mutation in tissue inhibitor of metalloproteinases-3 induces increased angiogenesis. J. Biol. Chem. 2009, 284, 19927-19936. [CrossRef]

17. Qi, J.H.; Ebrahem, Q.; Ali, M.; Cutler, A.; Bell, B.; Prayson, N.; Sears, J.; Knauper, V.; Murphy, G.; Anand-Apte, B. Tissue inhibitor of metalloproteinases-3 peptides inhibit angiogenesis and choroidal neovascularization in mice. PLoS ONE 2013, 8, e55667. [CrossRef]

18. Qi, J.H.; Ebrahem, Q.; Anand-Apte, B. Tissue inhibitor of metalloproteinases-3 and sorsby fundus dystrophy. Adv. Exp. Med. Biol. 2003, 533, 97-105.

19. Qi, J.H.; Ebrahem, Q.; Moore, N.; Murphy, G.; Claesson-Welsh, L.; Bond, M.; Baker, A.; Anand-Apte, B. A novel function for tissue inhibitor of metalloproteinases-3 (timp3): Inhibition of angiogenesis by blockage of VEGF binding to vegf receptor-2. Nat. Med. 2003, 9, 407-415. [CrossRef]

20. Qi, J.H.; Ebrahem, Q.; Yeow, K.; Edwards, D.R.; Fox, P.L.; Anand-Apte, B. Expression of sorsby's fundus dystrophy mutations in human retinal pigment epithelial cells reduces matrix metalloproteinase inhibition and may promote angiogenesis. J. Biol. Chem. 2002, 30, 30. [CrossRef]

21. Stohr, H.; Anand-Apte, B. A review and update on the molecular basis of pathogenesis of sorsby fundus dystrophy. Adv. Exp. Med. Biol. 2012, 723, 261-267. [PubMed]

22. Fariss, R.N.; Apte, S.S.; Olsen, B.R.; Iwata, K.; Milam, A.H. Tissue inhibitor of metalloproteinases-3 is a component of bruch's membrane of the eye. Am. J. Pathol. 1997, 150, 323-328. [PubMed]

23. Troeberg, L.; Lazenbatt, C.; Anower, E.K.M.F.; Freeman, C.; Federov, O.; Habuchi, H.; Habuchi, O.; Kimata, K.; Nagase, H. Sulfated glycosaminoglycans control the extracellular trafficking and the activity of the metalloprotease inhibitor timp-3. Chem. Biol. 2014, 21, 1300-1309. [CrossRef] [PubMed]

24. Yu, W.H.; Yu, S.; Meng, Q.; Brew, K.; Woessner, J.F., Jr. Timp-3 binds to sulfated glycosaminoglycans of the extracellular matrix. J. Biol. Chem. 2000, 275, 31226-31232. [CrossRef] [PubMed]

25. Itano, N.; Kimata, K. Altered hyaluronan biosynthesis in cancer progression. Semin. Cancer Biol. 2008, 18, 268-274. [CrossRef] [PubMed]

26. Singleton, P.A. Hyaluronan regulation of endothelial barrier function in cancer. Adv. Cancer Res. 2014, 123, 191-209. 
27. Mochimaru, H.; Takahashi, E.; Tsukamoto, N.; Miyazaki, J.; Yaguchi, T.; Koto, T.; Kurihara, T.; Noda, K.; Ozawa, Y.; Ishimoto, T.; et al. Involvement of hyaluronan and its receptor cd44 with choroidal neovascularization. Investig. Ophthalmol. Vis. Sci. 2009, 50, 4410-4415. [CrossRef]

28. Qi, J.H.; Bell, B.; Singh, R.; Batoki, J.; Wolk, A.; Cutler, A.; Prayson, N.; Ali, M.; Stoehr, H.; Anand-Apte, B. Sorsby fundus dystrophy mutation in tissue inhibitor of metalloproteinase 3 (timp3) promotes choroidal neovascularization via a fibroblast growth factor-dependent mechanism. Sci. Rep. 2019, 9, 17429. [CrossRef]

29. Oladipupo, S.S.; Smith, C.; Santeford, A.; Park, C.; Sene, A.; Wiley, L.A.; Osei-Owusu, P.; Hsu, J.; Zapata, N.; Liu, F.; et al. Endothelial cell fgf signaling is required for injury response but not for vascular homeostasis. Proc. Natl. Acad. Sci. USA 2014, 111, 13379-13384. [CrossRef]

30. Sarrazin, S.; Lamanna, W.C.; Esko, J.D. Heparan sulfate proteoglycans. Cold Spring Harb. Perspect. Biol. 2011, 3, a004952. [CrossRef]

31. Weis, S.M.; Cheresh, D.A. Tumor angiogenesis: Molecular pathways and therapeutic targets. Nat. Med. 2011, 17, 1359-1370. [CrossRef] [PubMed]

32. Bohrer, L.R.; Chuntova, P.; Bade, L.K.; Beadnell, T.C.; Leon, R.P.; Brady, N.J.; Ryu, Y.; Goldberg, J.E.; Schmechel, S.C.; Koopmeiners, J.S.; et al. Activation of the fgfr-stat3 pathway in breast cancer cells induces a hyaluronan-rich microenvironment that licenses tumor formation. Cancer Res. 2014, 74, 374-386. [CrossRef] [PubMed]

33. Weber, B.H.; Lin, B.; White, K.; Kohler, K.; Soboleva, G.; Herterich, S.; Seeliger, M.W.; Jaissle, G.B.; Grimm, C.; Reme, C.; et al. A mouse model for sorsby fundus dystrophy. Investig. Ophthalmol. Vis. Sci. 2002, 43, 2732-2740.

34. Leco, K.J.; Waterhouse, P.; Sanchez, O.H.; Gowing, K.L.; Poole, A.R.; Wakeham, A.; Mak, T.W.; Khokha, R. Spontaneous air space enlargement in the lungs of mice lacking tissue inhibitor of metalloproteinases-3 (timp-3). J. Clin. Investig. 2001, 108, 817-829. [CrossRef]

35. Tammi, R.; Ripellino, J.A.; Margolis, R.U.; Maibach, H.I.; Tammi, M. Hyaluronate accumulation in human epidermis treated with retinoic acid in skin organ culture. J. Investig. Dermatol. 1989, 92, 326-332. [CrossRef]

36. Hazim, R.A.; Volland, S.; Yen, A.; Burgess, B.L.; Williams, D.S. Rapid differentiation of the human rpe cell line, arpe-19, induced by nicotinamide. Exp. Eye Res. 2019, 179, 18-24. [CrossRef]

37. Venkatareddy, M.; Verma, R.; Kalinowski, A.; Patel, S.R.; Shisheva, A.; Garg, P. Distinct requirements for vacuolar protein sorting 34 downstream effector phosphatidylinositol 3-phosphate 5-kinase in podocytes versus proximal tubular cells. J. Am. Soc. Nephrol. 2016, 27, 2702-2719. [CrossRef]

38. Scheuring, D.; Lofke, C.; Kruger, F.; Kittelmann, M.; Eisa, A.; Hughes, L.; Smith, R.S.; Hawes, C.; Schumacher, K.; Kleine-Vehn, J. Actin-dependent vacuolar occupancy of the cell determines auxin-induced growth repression. Proc. Natl. Acad. Sci. USA 2016, 113, 452-457. [CrossRef]

39. Hollyfield, J.G. Hyaluronan and the functional organization of the interphotoreceptor matrix. Investig. Ophthalmol. Vis. Sci. 1999, 40, 2767-2769.

40. Hollyfield, J.G.; Rayborn, M.E.; Tammi, R. Hyaluronan localization in tissues of the mouse posterior eye wall: Absence in the interphotoreceptor matrix. Exp. Eye Res. 1997, 65, 603-608. [CrossRef]

41. Ebrahem, Q.; Qi, J.H.; Sugimoto, M.; Ali, M.; Sears, J.E.; Cutler, A.; Khokha, R.; Vasanji, A.; Anand-Apte, B. Increased neovascularization in mice lacking tissue inhibitor of metalloproteinases-3. Investig. Ophthalmol. Vis. Sci. 2011, 52, 6117-6123. [CrossRef] [PubMed]

42. Fariss, R.N.; Apte, S.S.; Luthert, P.J.; Bird, A.C.; Milam, A.H. Accumulation of tissue inhibitor of metalloproteinases-3 in human eyes with sorsby's fundus dystrophy or retinitis pigmentosa. Br. J. Ophthalmol. 1998, 82, 1329-1334. [CrossRef] [PubMed]

43. Crabb, J.W.; Miyagi, M.; Gu, X.; Shadrach, K.; West, K.A.; Sakaguchi, H.; Kamei, M.; Hasan, A.; Yan, L.; Rayborn, M.E.; et al. Drusen proteome analysis: An approach to the etiology of age-related macular degeneration. Proc. Natl. Acad. Sci. USA 2002, 99, 14682-14687. [CrossRef] [PubMed]

44. Kamei, M.; Apte, S.S.; Rayborn, M.E.; Lewis, H.; Hollyfield, J.G. Timp-3 accumulation in drusen and bruch's membrane in eyes from donors with age-related macular degeneration (1997). In Degenerative Diseases of the Retina; Luvail, M.M., Anderson, R.E., Hollyfield, J.G., Eds.; Plenum Press: New York, NY, USA, 1997; pp. 11-15.

45. Kamei, M.; Hollyfield, J. Timp-3 in bruch's membrane: Changes during aging and in age-related macular degeneration. Investig. Ophthalmol. Vis. Sci. 1999, 40, p2367-p2375. 
46. Apte, S.S.; Mattei, M.-G.; Olsen, B.R. Cloning of the cdna encoding human tissue inhibitor of metalloproteinase-3 (timp-3) and mapping of the timp-3 gene to chromosome 22. Genomics 1994, 19, 86-90. [CrossRef]

47. Saw, S.; Aiken, A.; Fang, H.; McKee, T.D.; Bregant, S.; Sanchez, O.; Chen, Y.; Weiss, A.; Dickson, B.C.; Czarny, B.; et al. Metalloprotease inhibitor timp proteins control fgf-2 bioavailability and regulate skeletal growth. J. Cell Biol. 2019, 218, 3134-3152. [CrossRef]

48. Rifkin, D.B.; Moscatelli, D.; Bizik, J.; Quarto, N.; Blei, F.; Dennis, P.; Flaumenhaft, R.; Mignatti, P. Growth factor control of extracellular proteolysis. Cell Differ. Dev. 1990, 32, 313-318. [CrossRef]

49. DeAngelis, P.L. Hyaluronan synthases: Fascinating glycosyltransferases from vertebrates, bacterial pathogens, and algal viruses. Cell Mol. Life Sci. 1999, 56, 670-682. [CrossRef]

50. Tien, J.Y.; Spicer, A.P. Three vertebrate hyaluronan synthases are expressed during mouse development in distinct spatial and temporal patterns. Dev. Dyn. 2005, 233, 130-141. [CrossRef]

51. Stern, R.; Kogan, G.; Jedrzejas, M.J.; Soltes, L. The many ways to cleave hyaluronan. Biotechnol. Adv. 2007, 25, 537-557. [CrossRef]

52. Tammi, R.; Rilla, K.; Pienimaki, J.P.; MacCallum, D.K.; Hogg, M.; Luukkonen, M.; Hascall, V.C.; Tammi, M. Hyaluronan enters keratinocytes by a novel endocytic route for catabolism. J. Biol. Chem. 2001, 276, 35111-35122. [CrossRef] [PubMed]

53. Marella, M.; Jadin, L.; Keller, G.A.; Sugarman, B.J.; Frost, G.I.; Shepard, H.M. Kiaa1199 expression and hyaluronan degradation colocalize in multiple sclerosis lesions. Glycobiology 2018, 28, 958-967. [CrossRef] [PubMed]

54. De Angelis, J.E.; Lagendijk, A.K.; Chen, H.; Tromp, A.; Bower, N.I.; Tunny, K.A.; Brooks, A.J.; Bakkers, J.; Francois, M.; Yap, A.S.; et al. Tmem2 regulates embryonic VEGF signaling by controlling hyaluronic acid turnover. Dev. Cell 2017, 40, 123-136. [CrossRef] [PubMed]

55. Nagaoka, A.; Yoshida, H.; Nakamura, S.; Morikawa, T.; Kawabata, K.; Kobayashi, M.; Sakai, S.; Takahashi, Y.; Okada, Y.; Inoue, S. Regulation of hyaluronan (ha) metabolism mediated by hybid (hyaluronan-binding protein involved in ha depolymerization, kiaa1199) and ha synthases in growth factor-stimulated fibroblasts. J. Biol. Chem. 2015, 290, 30910-30923. [CrossRef]

56. Garantziotis, S.; Savani, R.C. Hyaluronan biology: A complex balancing act of structure, function, location and context. Matrix Biol. 2019, 78-79, 1-10. [CrossRef]

57. Nita, M.; Grzybowski, A.; Ascaso, F.J.; Huerva, V. Age-related macular degeneration in the aspect of chronic low-grade inflammation (pathophysiological parainflammation). Mediators Inflamm. 2014, 2014, 930671. [CrossRef]

58. Avenoso, A.; Bruschetta, G.; D’Ascola, A.; Scuruchi, M.; Mandraffino, G.; Saitta, A.; Campo, S.; Campo, G.M. Hyaluronan fragmentation during inflammatory pathologies: A signal that empowers tissue damage. Mini Rev. Med. Chem. 2020, 20, 54-65. [CrossRef]

59. Mueller, A.M.; Yoon, B.H.; Sadiq, S.A. Inhibition of hyaluronan synthesis protects against central nervous system (cns) autoimmunity and increases cxcl12 expression in the inflamed cns. J. Biol. Chem. 2014, 289, 22888-22899. [CrossRef]

60. Scuruchi, M.; D’Ascola, A.; Avenoso, A.; Campana, S.; Abusamra, Y.A.; Spina, E.; Calatroni, A.; Campo, G.M.; Campo, S. 6-mer hyaluronan oligosaccharides modulate neuroinflammation and alpha-synuclein expression in neuron-like sh-sy5y cells. J. Cell Biochem. 2016, 117, 2835-2843. [CrossRef]

61. deS Senanayake, P.; Calabro, A.; Nishiyama, K.; Hu, J.G.; Bok, D.; Hollyfield, J.G. Glycosaminoglycan synthesis and secretion by the retinal pigment epithelium: Polarized delivery of hyaluronan from the apical surface. J. Cell Sci. 2001, 114, 199-205. 



\title{
Mouse Models of Inherited Retinal Degeneration with Photoreceptor Cell Loss
}

\author{
Gayle B. Collin ${ }^{1,+}$, Navdeep Gogna ${ }^{1,+}$, Bo Chang ${ }^{1}$, Nattaya Damkham ${ }^{1,2,3}$, Jai Pinkney ${ }^{1}$, \\ Lillian F. Hyde ${ }^{1}$, Lisa Stone ${ }^{1}$, Jürgen K. Naggert ${ }^{1}$, Patsy M. Nishina ${ }^{1, *}$ and Mark P. Krebs ${ }^{1, *}$ \\ 1 The Jackson Laboratory, Bar Harbor, Maine, ME 04609, USA; gayle.collin@jax.org (G.B.C.); \\ navdeep.gogna@jax.org (N.G.); bo.chang@jax.org (B.C.); nattaya.damkham@jax.org (N.D.); \\ jai.pinkney@jax.org (J.P.); Lillian.Hyde@jax.org (L.F.H.); lisa.stone@jax.org (L.S.); \\ juergen.naggert@jax.org (J.K.N.) \\ 2 Department of Immunology, Faculty of Medicine Siriraj Hospital, Mahidol University, \\ Bangkok 10700, Thailand \\ 3 Siriraj Center of Excellence for Stem Cell Research, Faculty of Medicine Siriraj Hospital, Mahidol University, \\ Bangkok 10700, Thailand \\ * Correspondence: patsy.nishina@jax.org (P.M.N.); mark.krebs@jax.org (M.P.K.); \\ Tel.: +1-207-2886-383 (P.M.N.); +1-207-2886-000 (M.P.K.) \\ + These authors contributed equally to this work.
}

Received: 29 February 2020; Accepted: 7 April 2020; Published: 10 April 2020

\begin{abstract}
Inherited retinal degeneration (RD) leads to the impairment or loss of vision in millions of individuals worldwide, most frequently due to the loss of photoreceptor (PR) cells. Animal models, particularly the laboratory mouse, have been used to understand the pathogenic mechanisms that underlie PR cell loss and to explore therapies that may prevent, delay, or reverse RD. Here, we reviewed entries in the Mouse Genome Informatics and PubMed databases to compile a comprehensive list of monogenic mouse models in which PR cell loss is demonstrated. The progression of PR cell loss with postnatal age was documented in mutant alleles of genes grouped by biological function. As anticipated, a wide range in the onset and rate of cell loss was observed among the reported models. The analysis underscored relationships between RD genes and ciliary function, transcription-coupled DNA damage repair, and cellular chloride homeostasis. Comparing the mouse gene list to human $\mathrm{RD}$ genes identified in the RetNet database revealed that mouse models are available for $40 \%$ of the known human diseases, suggesting opportunities for future research. This work may provide insight into the molecular players and pathways through which PR degenerative disease occurs and may be useful for planning translational studies.
\end{abstract}

Keywords: visual photoreceptor cell loss; mouse genetic models; retinitis pigmentosa; Leber congenital amaurosis; ciliopathies

\section{Introduction}

Inherited forms of retinal degeneration (RD) encompass a genetically and clinically heterogeneous group of disorders estimated to cause vision impairment and loss in more than 5.5 million individuals worldwide [1,2], with 282 mapped and identified retinal degenerative disease genes documented in the RetNet human database [3]. Animal models, such as non-human primates [4], dogs [5], mice [6,7], zebrafish [8], and fruit flies [9], have been used to identify candidates for human retinal disease genes, to elucidate pathological mechanisms, and to serve as a resource for exploring therapeutic approaches. As potential therapies for retinal diseases are investigated, the need for animal models increases. Information about the disease onset and rate of progression, the pathogenic pathways involved, and the genetic background in which the disrupted genes are situated are all factors that 
must be considered when selecting appropriate models for testing therapeutics. These factors will also play a role in interpreting the outcome of treatment studies.

The purpose of this review is to compile a searchable list of mouse models of inherited retinal diseases caused by single gene mutations that specifically lead to the post-developmental rod and/or cone photoreceptor (PR) cell loss. To identify these models, we reviewed mouse-specific data available in the Mouse Genome Informatics (MGI) and National Center for Biotechnology Information (NCBI) databases at The Jackson Laboratory (JAX) and National Institutes of Health (NIH), respectively. We recorded, when available, PR cell loss data from publications describing mutant alleles of the genes identified. We also included representative fundus photographs and optical coherence tomography (OCT) images of selected mouse models from the Eye Mutant Resource (EMR) and the Translational Vision Research Models (TVRM) programs at JAX as examples of the retinal phenotypes found among mouse models that fit our criteria. We attempted to cluster genes based on the function and then compared the progression of PR cell loss among these clusters to provide potential insights into disease mechanisms. We also compared our list of mouse genes associated with PR cell loss with the RetNet gene list, to highlight mouse models for specific retinal diseases, to reveal opportunities to create novel models, and to identify candidate genes within human loci for which a causative gene is currently unknown. Finally, we coordinated with the MGI team to incorporate our annotations into the MGI database, which will allow future analyses using tools available through that platform. It is hoped that our work will be useful as a resource for investigators to assist in the selection of appropriate mouse models within and across functional clusters in new studies to understand and develop treatments for human retinal degenerative disease.

In the three decades since the genes linked to PR loss phenotype were first identified in the mouse and human [10-12], rapid progress in understanding the genetic basis of inherited RD has been summarized in many excellent reviews. Many of the topics presented in the current article have been discussed previously in reviews of mouse RD models $[6,7,13,14]$ and in summaries of our work at JAX [15-21]. Although we have made every effort to acknowledge the many contributions to this field, we note that there is a large body of relevant literature and apologize in advance to authors whose reviews or articles we may have inadvertently overlooked.

\section{Background}

\subsection{Photoreceptor (PR) Cell Structure}

PR cells are sensory neurons within the retina that detect light and signal this event to other cells. Since PR cells are essential for vision, their loss can dramatically and negatively affect the quality of life. PR cells include rod and cone cells (Figure 1a,b) that occupy the outermost layers of the neurosensory retina. Although intrinsically photosensitive retinal ganglion cells have also been described as photoreceptors [22], we did not include them in this review, as their contribution to RD is unknown. Rod and cone photoreceptors possess unique structures that serve to compartmentalize processes that are critical for cell function and maintenance.

- The outer segment (OS), which is cylindrical in rod PR cells and tapered in cones, contains phototransduction proteins that sense light and amplify the ensuing signal, culminating in PR cell hyperpolarization (Figure 1c). Much of the phototransduction apparatus is localized to double-bilayer discs formed by evagination of the plasma membrane at the base of the OS. These discs are largely internalized in rods except at the base of the OS, but remain contiguous with the plasma membrane in cones to yield a highly convoluted OS surface [23].

- The OS is stabilized by a ciliary axoneme, which runs through much of its length (Figure 1d; Ax). At the proximal end of the axoneme, the connecting cilium, analogous to the transition zone in other cilia (Figure 1d; CC-TZ), serves as a conduit through which all membrane and protein components destined for the OS are thought to pass. At the base of the connecting cilium lies the basal body (Figure 1d; BB), a cylindrical organelle derived from the mother centriole. Altogether 
these structures represent a modified primary cilium that encompasses an extensive network of protein complexes that transport proteins and lipids and shares characteristics with primary cilia in many other cell types. The ciliary networks also function to prevent the flow of OS components to other parts of the cell and may associate with the intracellular trafficking apparatus to ensure the directed movement of needed components to the OS.

a

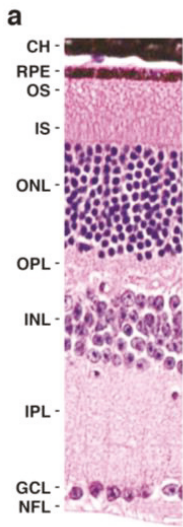

b

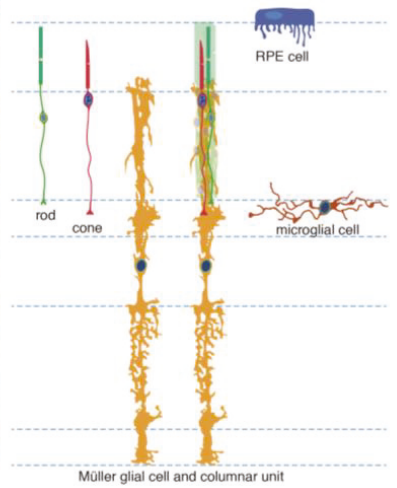

c

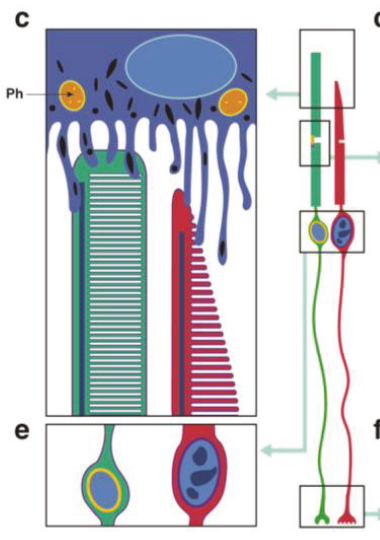

d

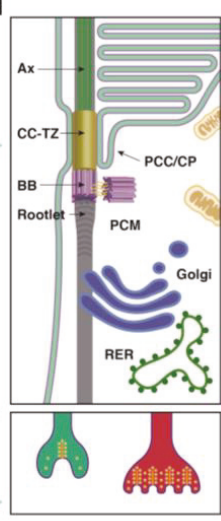

Figure 1. Retinal tissue organization emphasizing cell types and subcellular structures that may be sites of pathological processes in mouse models of photoreceptor (PR) cell loss. (a) A radial section of the posterior eye stained with hematoxylin and eosin shows the layered structure of the retina. $\mathrm{CH}$, choroid; RPE, retinal pigment epithelium; IS, inner segment; OS, outer segment; ONL, outer nuclear layer; OPL, outer plexiform layer; INL, inner nuclear layer; IPL, inner plexiform layer; GCL, ganglion cell layer; NFL, nerve fiber layer. (b) Two PR cell types (rods and cones) and additional cell types that may be the target of processes implicated in PR cell loss. Dashed lines indicate alignment with retinal layers in (a). A columnar unit consisting of one cone and one Müller cell and roughly 20 rod cells is shown. (c-f) Details of PR and RPE cells. (c) PR cell OSs contain flattened discs (rod, left) or incomplete discs (cone, right) where the light sensing apparatus is located. OS tips engulfed by RPE cell apical processes are digested in phagolysosomes $(\mathrm{Ph})$. (d) The base of the OS, the connecting cilium, and the apical portion of a rod cell IS (adapted with permission from [24]). The axoneme (Ax) and rootlet provide physical stability to the cilium. BB, basal bodies; CC-TZ, connecting cilium-transition zone; $\mathrm{PCM}$, pericentriolar matrix; $\mathrm{PCC} / \mathrm{CP}$, periciliary complex/ciliary pocket; $\mathrm{RER}$, rough endoplasmic reticulum. (e) The PR cell soma is largely occupied by the nucleus. (f) Rod and cone synaptic termini include presynaptic ribbons and associated neurotransmitter vesicles.

- $\quad$ The inner segment (IS) contains the biosynthetic machinery and energy sources needed to produce and assemble newly synthesized phototransduction proteins and their associated membranes (Figure 1d). The capacity of this cellular factory is impressive, as up to $10 \%$ of the OS is shed daily and removed via phagocytosis by the retinal pigment epithelium (see below) and must be renewed. Most protein and lipid components are synthesized de novo, but the IS also has an extensive recycling machinery that can reassemble components provided from outside the cell.

- $\quad$ The cell body or soma includes the nucleus, which is highly condensed in rod PR cells, but is larger in cones and includes patches of heterochromatin (Figure 1e). To increase the density of rod and cone OSs in the retina, the somas are stacked in columns within the outer nuclear layer (ONL). This arrangement necessitates thin cell extensions reaching from the soma to the IS or to the synapse. PR cell loss is measured by counting ONL nuclei, which are prominently stained in retinal sections (Figure 1a), or in the case of rods, which are more abundant than cones, by measuring ONL thickness from micrographs or by OCT. 
- The PR cell terminus contains ribbon synapses close to the presynaptic membrane loaded with vesicles containing the excitatory neurotransmitter glutamate (Figure 1f). In the dark, a steady-state level of glutamate is released at the synapse, which is reduced when the cells are hyperpolarized in the light. Changes in glutamate levels at the synapse signal postsynaptic secondary neurons in the inner nuclear layer, which communicate with ganglion cells on the vitreal surface of the retina that connect through long axons to the visual cortex of the brain.

\subsection{Neighboring Cells}

Müller glia are radial cells that span much of the neurosensory retina, reaching from the internal limiting membrane at the vitreal surface of the retina to the external limiting membrane on the scleral edge of the ONL [25]. Within the ONL, fine Müller cell extensions appear to ensheath the PR cell soma. As they also interact with vascular layers within the retina, Müller cells may provide essential nutrients to PR cells, which do not directly contact the circulation. They also regulate extracellular volume, ion and water homeostasis, serve to modify neuronal activity through release of neuroactive compounds, and modulate immune and inflammatory responses [26]. At the external limiting membrane, Müller cell endfeet engage rod and cone cell ISs in intercellular adhesion interactions, including tight junctions, which create a diffusion barrier. Notably, the arrangement of an Müller cell, rods, and a cone cell has been proposed to form a columnar unit (Figure 1b), which may result in physiological and functional coordination of these cell types.

RPE cells (Figure 1b,c) constitute an epithelial monolayer that lies between the retina and a capillary bed, the choriocapillaris. The flow of water, ions, small molecules, and metabolites from the blood to the outer retina is thus regulated by RPE cells. Their apical surface features microvilli and microplicae (Figure 1b,c) that contact roughly the outermost third of OSs and play important roles in recycling molecules needed for PR renewal. These apical processes also mediate initial steps in the daily phagocytosis of OS tips. As an epithelium with high-resistance intercellular junctions [27], the RPE performs an important barrier function, disruption of which may cause PR degeneration.

Microglial cells form ramified networks within the same retinal layers as the retinal vasculature, which includes the superficial, intermediate and deep vascular beds. Microglia at the level of the outer plexiform layer in healthy retinas extend dendritic arms into the ONL (Figure 1b), where they contact PR soma as part of a dynamic survey process. During development and in rare events that occur in healthy retinas, these cells engulf and phagocytose PR soma, presumably in response to a defect in PR function.

PRs form synaptic connections in the outer plexiform layer with secondary neurons, including bipolar and horizontal cells. Although these connections are critical for signal transmission, they are not as extensive as the contacts made between PRs and Müller glia, RPE cell apical processes, or the dynamic extensions on microglial cells, and were therefore omitted from the summary diagram in Figure 1. Nevertheless, perturbation of the interactions among these cells may lead to PR degeneration, conceivably due to an alteration of signal transmission.

\subsection{Inherited Diseases that Cause PR Cell Loss}

Major monogenic inherited RDs in which PR cells are lost include: retinitis pigmentosa [28], Leber congenital amaurosis [29], and syndromic disorders that manifest disease in multiple organs, including the eye, particularly ciliopathies, such as Joubert [30], Bardet-Biedl [31], or Usher [32] syndrome. The remarkable success of gene augmentation therapy for a form of Leber congenital amaurosis has invigorated research efforts to treat these diseases [33]. 


\section{Methods}

\subsection{Public Database and Literature Searches}

The search strategy employed in this review is summarized in Figure 2. Initially, the MGI database [34] was queried to identify mutant protein-coding genes associated with a mammalian disease phenotype indicating a loss of PR cells. MGI is a curated database that includes expert annotation based on full-text searching of 148 selected journals, which is limited compared to literature databases, such as PubMed. Typically, only the first paper describing a new allele is fully curated for phenotype data in the database due to resource constraints. Although our analysis yielded 159 mutant genes that were associated with PR cell loss, a number of mutant genes known to cause this phenotype were absent or not annotated, possibly due to these aforementioned limitations.

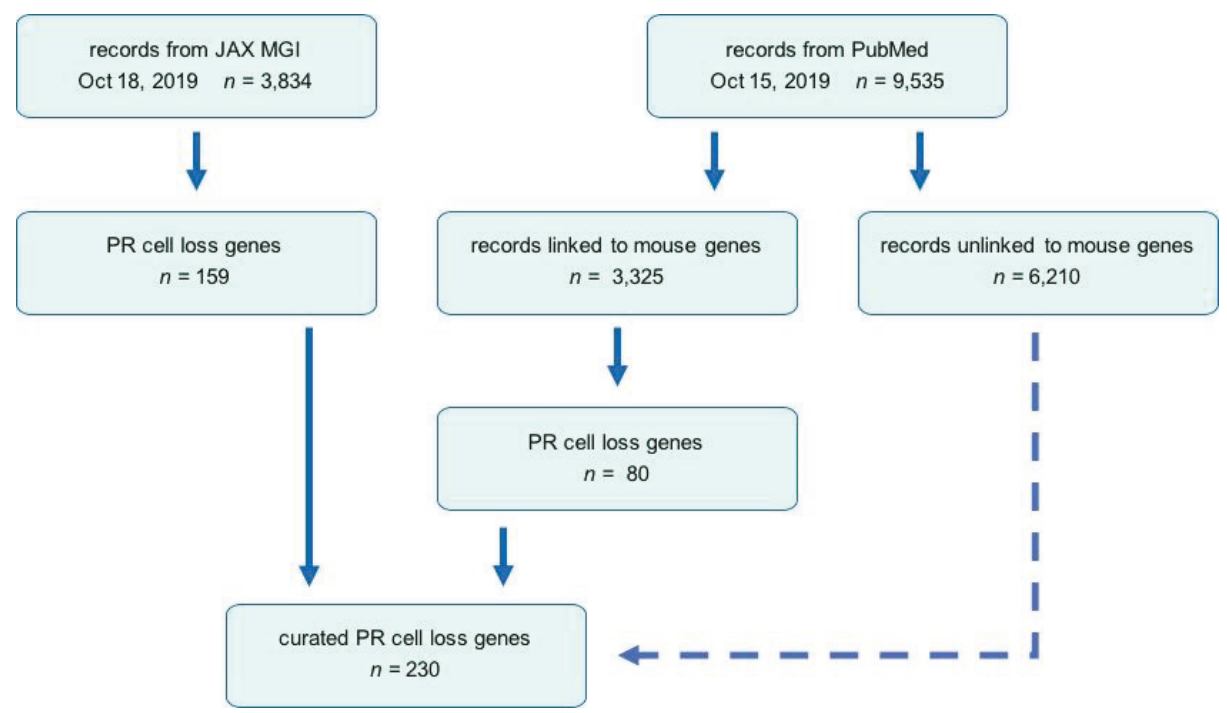

Figure 2. Flow chart depicting the progression of the search strategy utilized in this review. The number of records or genes identified from them is indicated at each stage of the process. The dashed line indicates that some genes were identified from records that remain to be systematically screened.

To expand the search, we used NCBI databases, including PubMed [35] and Gene. We refined a PubMed query by searching with keyword phrases to generate article lists, using the Gene option of Find Related Data to yield mouse genes linked to the articles, and then assessing whether these genes were on our MGI list. The goal was to develop a broad query that included as many genes from the MGI list as possible but also included additional hits. The most successful was: (ONL OR "outer nuclear layer" OR retina* OR PR OR rod OR rods OR cone*) AND (degener* OR loss OR thin* OR thick*) AND (mouse OR mice OR murine), which captured $>97 \%$ of the MGI list. Restricting this query to entries posted to PubMed on or before October 15, 2019, yielded 9535 articles. To review these articles efficiently we tried two approaches, the first generating a spreadsheet containing hyperlinks in which each linked gene symbol was combined with the Boolean query, and the second using mouse gene identification numbers corresponding to the linked genes and applying an Entrez script that accessed the Gene and PubMed databases to find all articles satisfying the Boolean query for each linked gene. 


\subsection{Search Strategy}

Each MGI database-derived entry was curated manually or automatically to identify candidate models that reported PR degeneration as a phenotype, as described above. In the case of PubMed entries, although the automated approaches were useful for quickly identifying genes that satisfied our criteria, neither was comprehensive, and additional candidate models were identified by review of the title and abstract from some of the remaining articles in the full collection of 9535 articles. Subsequently, an independent coauthor identified an original publication for each candidate gene and determined if PR cell loss was reported. If sufficient evidence for PR cell loss was obtained, the gene and mouse model was assigned to one of 11 categories. Genes within each category were curated further by coauthors who identified alternate alleles and extracted information regarding the disease phenotype induced by the disruption of a gene. Each entry in Table S1 is the result of the examination of an original article (indicated by PubMed ID numbers, PMIDs) and data from MGI to capture information such as mutation type, associated human diseases, and disease onset and progression.

\subsection{Comparative Analysis and Updating the MGI Database}

Once our final list was completed, we used tools in Excel to compare it to a list constructed from online tables downloaded on 8 December 2019, from RetNet, a public compilation of human genes linked to inherited RD. We also provided our data to the MGI team at JAX, who assigned allele nomenclature, added strain information for newly described mutants, and updated phenotype data for alleles that were present in the MGI database but not yet annotated with respect to PR cell loss. This review has been referenced at MGI so that the alleles documented in the article can be examined using MGI tools or downloaded in tabular format for analysis with other software. The collaborative approach between mouse phenotyping experts and the MGI team may be attractive for ensuring that this useful resource remains current in the face of limited funding, personnel, and time.

\subsection{Inclusion/Exclusion Criteria}

Monogenic models generated from a variety of sources were included in Table S1. However, in the case of conditional models, only those for which a germline null allele was reported in the MGI database that resulted in embryonic, prenatal, or postnatal lethality were included. We excluded the following models from Table S1: those for which a causative gene had yet to be identified and for which complementation tests were unavailable; those requiring multiple genes for the presentation of the disease phenotype; those based on overexpressing transgenes; and those in which PR degeneration depended on experimental interventions, such as an altered diet, drug treatment, or exposure to bright illumination. Environmental influences on retinal diseases are very important and may affect the progression of PR cell loss, but models that depend on environmental conditions are challenging to compare because of the significant variation among the types of environmental perturbations and the methods used to apply them. We also excluded models that exhibited a reduction in the PR cell number during development but not a progressive loss with age, and those where IS and/or OS dysmorphology or reduction in length was observed without a loss of PR cells, as indicated by a reduction in nuclei number or ONL thickness within the time frame reported in the papers. Although these models were excluded from Table S1, examples are included in the Results.

\subsection{Heterogeneity of Data}

The type and frequency of data gathered varied greatly among the studies reviewed. In some papers, only one figure with one retinal section was offered as evidence for PR degeneration, while other papers showed extensive quantitation of their data. To document potential sources of variability in the data, we indicate the method by which the degree of degeneration was determined, either by measuring ONL thickness or by count of nuclei in the ONL, typically the number of rows of nuclei spanning the ONL but sometimes a total count of ONL nuclei in a fixed area of a retinal micrograph. 
In some instances, when data was quantified in spider plots or bar graphs, mean values obtained from the central retina were used in estimating the PR loss. We normalized the data among studies by recording the percent degeneration as determined by dividing the mutant values by the corresponding values from age-matched controls as reported in each publication.

\subsection{Comparison of Progressive PR Cell Loss}

To compare progressive PR cell loss among models, we fit normalized data from each article to an exponential decay that includes a delay, or offset [36]. Ranges of either age or photoreceptor numbers, if reported, were averaged. Fitting was performed in Excel Visual Basic using a piecewise equation that modeled the delay with a straight line at $100 \%$ and the remaining points with a monoexponential decay to $0 \%$. Two adjustable parameters, the delay and the decay rate constant, were optimized. We calculated the age at which PR cell numbers reached $50 \%$ of control values $\left(D_{50}\right)$ as a measure of progression. Roughly one third of the datasets contained only a single point within the exponential regime, which was insufficient to calculate $\mathrm{D}_{50}$. In these cases, $\mathrm{D}_{50}$ was calculated at the extremes of zero delay and infinite rate, and the mean of these values was used as a $\mathrm{D}_{50}$ estimate.

\subsection{Generation of Primary Data Using Fundus Imaging and OCT Scans}

Fundus photographs of EMR mutants were taken in unanesthetized mice treated with $1 \%$ cyclopentolate to dilate or enlarge the pupil with an in vivo bright field retinal imaging microscope equipped with image-guided OCT capabilities (Micron III; Phoenix Laboratories, Inc., Pleasanton, CA, USA) as previously described [20]. This system allows for the visualization of the location of the OCT scan using the real-time Micron III bright-field image. A superimposed line placed directly on the image over the retinal feature being examined delivers precise cross-sectional information, allowing for the assessment of changes in layer thickness and morphological alterations.

Fundus photodocumentation for TVRM mutants and C57BL/6J control mice was performed using a Micron III or IV retinal camera (Phoenix Laboratories, Inc., Pleasanton, CA, USA) as described [37], except that $1 \%$ cyclopentolate or $1 \%$ atropine was used as a dilating agent, and in some cases, mice were anesthetized with isoflurane. OCT imaging to assess retinal layer thickness in Nmnat ${ }^{\text {tvrm113, }}$,

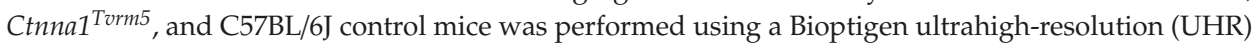
Envisu R2210 spectral domain OCT (SDOCT) imaging system for volume scanning as described [37,38] with ketamine/xylazine (1.6 mL ketamine $(100 \mathrm{mg} / \mathrm{mL}), 1.6 \mathrm{~mL}$ xylazine $(20 \mathrm{mg} / \mathrm{mL})$, and $6.8 \mathrm{~mL}$ sodium chloride $(0.9 \% w / v))$ as an anesthetic. A representative B-scan through the optic nerve head was derived from the OCT volume dataset. Rpgrip $1^{\text {nmf247 }}$ and Alms $1^{\text {Gt(XH152)Byg }}$ were assessed on the same OCT system by obtaining a linear B-scan with the following parameters: length, $1.9 \mathrm{~mm}$; width, $1.9 \mathrm{~mm}$; angle, 0 degrees; horizontal offset, $0 \mathrm{~mm}$; vertical offset, $0 \mathrm{~mm}$; A-scans/B-scan, 1000 lines; B-scans, 1 line; frames/B-scan, 20 frames; and inactive A-scans/B-scan, 80 lines. Linear scans were registered and averaged in the InVivoVue program to merge the 20 frames into a single image.

\section{Results}

\subsection{Summary of Studies that Report PR Cell Loss}

The combined searches of MGI and PubMed databases yielded a total of 230 genes associated with PR cell loss. Ultimately, 3834 reports at MGI and 3325 at PubMed, which most typically characterized one mutant gene but on rare occasions described more than one, were used in the present review. The distribution of retrieved publications sorted by functional categories is summarized in Table S1. The genes identified in these models are summarized in Figure 3. Descriptions of gene and protein symbols used in the text, figures, and Table S1 are provided in Table S2. 


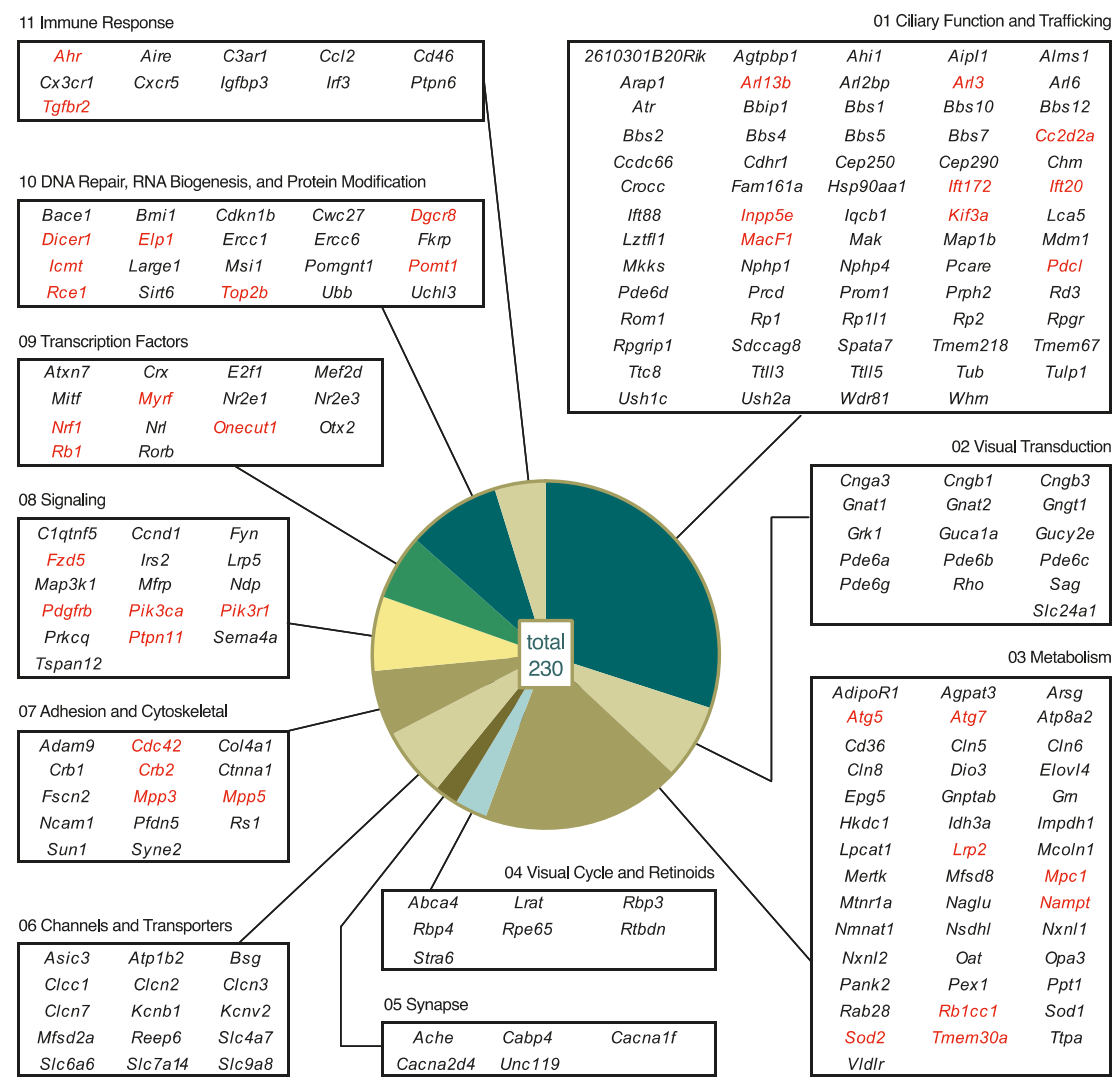

Figure 3. Genes associated with PR cell loss in monogenic mouse models of retinal degeneration (RD). Genes identified by combined review of the Mouse Genome Informatics (MGI) database and articles from a PubMed query were assigned to the indicated functional categories as described in the text. Genes for which mutant alleles are available only in the conditional form are displayed in red. Conditional alleles were included only in instances where germline null alleles resulted in embryonic, prenatal or postnatal lethality. For additional details on inclusion/exclusion criteria, see Section 3.4.

\subsubsection{PR Cell Loss Models}

The mouse models described in Table S1 were either spontaneous (12\%) or chemically induced mutants $(11 \%)$, or those produced through genetic engineering approaches $(77 \%)$. This latter group, which was by far the largest, utilized standard homologous recombination, gene-traps, nuclease mediated approaches such as CRISPR/Cas9, and conditionals to mediate genomic changes. Additionally, four models of inadvertent transgene insertion into a unique gene, whose disruption led to PR degeneration, were included within this group. Interesting examples of differences in the disease onset or rate of progression were demonstrated in different models of the same gene (e.g., Aipl1) that may be related to allelic differences, null versus missense mutations, or genetic background effects [21,39,40]. Most of the genetically engineered models in Table S1 tended to be in mixed, segregating genetic backgrounds that might impact phenotypic expression (discussed below).

Within the genetically engineered category, a relatively large group of models, $16 \%$, were conditional models, representing 39 genes (Figure 3; red). Generation of conditional mutants is based on the Cre-Lox recombination approach, which requires a floxed gene and a cre-driver to excise the 
targeted genomic region in a spatial (e.g., cell/tissue specific) or temporal manner (e.g., induction by chemicals such as doxycycline). Since $30 \%$ of all null mutations lead to embryonic lethality, as they represent genes that are essential during development, conditionals are often used to examine the adult function of genes [41]. This was the case in $92 \%$ of the conditional models described here, as standard organism-wide removal of the genes was reported to be embryonic, perinatal, or postnatal lethal. Thus, conditionals allow us to learn the function of a gene post-developmentally. Conditionals are also sometimes used to determine the cellular contributions to a disease phenotype. If a gene is expressed in multiple retinal cell types, by removing them systematically and examining the consequent phenotype, one can learn how the loss of function of the gene within particular cell types affects the disease phenotype. For example, removal of Arl3 from rod PRs using a Rho-icre driver shows a later and slower rate of degeneration than that found with Six3-cre, a Cre driver that expresses in early retinal development. This suggests that Arl3 in rods is necessary for PR survival but that Arl3 function in other retinal cell types also affects PR survival [42]. The most widely used Cre models include: for targeting retinal progenitor cells, $\mathrm{Tg}(\mathrm{rx} 3$-icre)1Mjam, $\mathrm{Tg}$ (Six3-cre)69Frty, $\mathrm{Tg}$ (Chx10-EGFP/cre,-ALPP)2Clc, $\mathrm{Tg}$ (Crx-cre)1Tfur, and $\mathrm{Tg}$ (Pax6-cre,GFP)2Pgr; for targeting rods, $\mathrm{Tg}$ (Rho-icre)1Ck, $\mathrm{Tg}$ (RHO-cre)8Eap, and Pde6g tm1(cre/ERT2)Eye; for targeting M-cone PRs, Tg(OPN1LW-cre)4Yzl (also known as HRGP-cre); for targeting PRs, $\mathrm{Tg}$ (Rbp3-cre)528Jxm (also known as IRBP-cre); for targeting RPE, $\mathrm{Tg}$ (BEST1-cre)1Jdun,

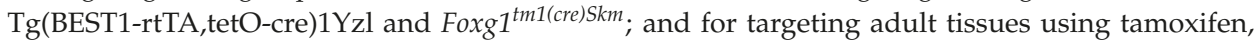
$\operatorname{Tg}\left(\right.$ CAG-cre/Esr $\left.{ }^{*}\right) 5$ Amc.

\subsubsection{Mouse Models from Phenotyping Programs}

The models listed in Table S1 come from many sources. In addition to individual investigator-initiated efforts, currently the largest contributor to ocular models is the International Mouse Phenotyping consortium, in which 19 phenotyping centers from 11 countries participate to systematically characterize knockout mice generated in a standardized manner [41,43]. All centers do some eye phenotyping, thus providing a window into potential models. Although only a few models from this program are included in Table S1, as most are not yet fully characterized, it is anticipated that this consortium will provide a wealth of models for individual laboratories to study. For example, in the MGI database, 39 IMPC models were identified with "reduced retinal thickness" that with further characterization may reveal PR degeneration.

At The Jackson Laboratory, the Eye Mutant Resource (EMR) and the Translational Vision Research Models (TVRM) programs are dedicated to screen for or generate mouse models with ocular diseases. The EMR has been screening retired breeders by slit lamp biomicroscopy, indirect ophthalmoscopy, and electroretinography since 1988. Retired breeders from the production and genetic resources colonies are screened. Heritable mutants are phenotypically and genetically characterized and the spontaneous mutants are distributed worldwide. The TVRM program arose from the JAX Neuromutagenesis Facility. Mice for this program are generated by chemical mutagenesis or genetic engineering. Carefully characterized mutants are also distributed. Examples of mutants from the EMR and TVRM programs are shown in Figures 4 and 5, respectively. 


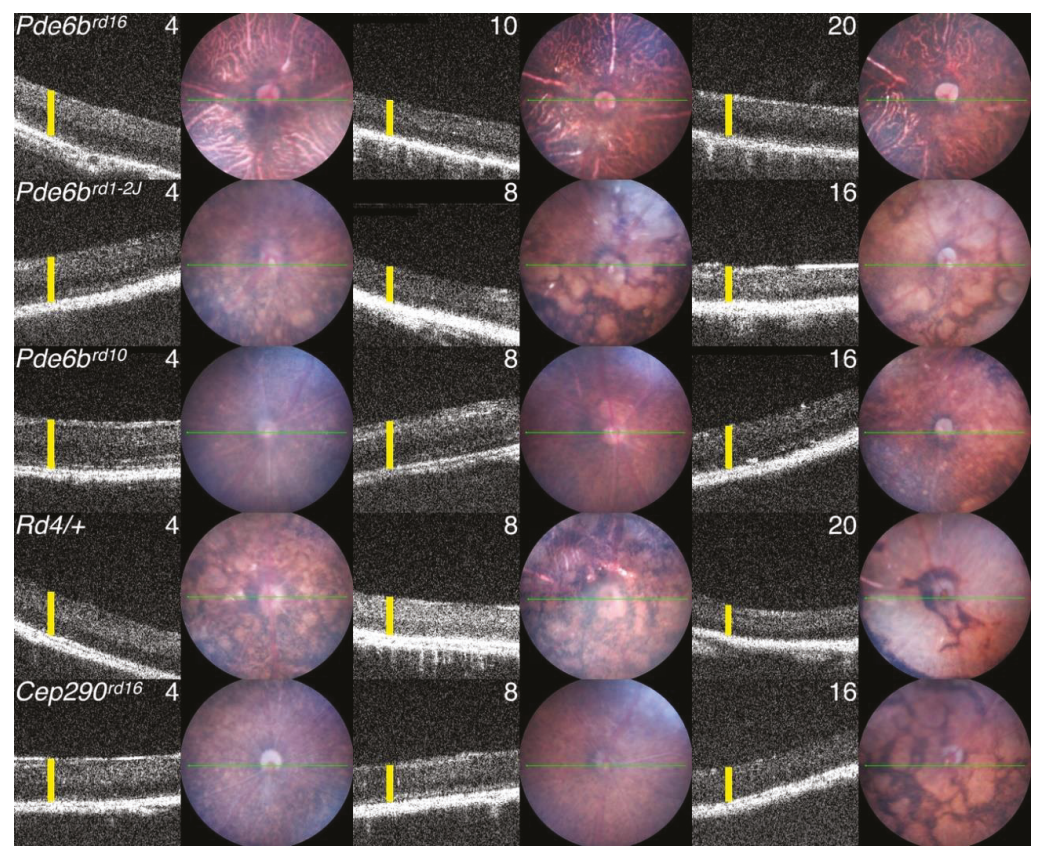

(a)

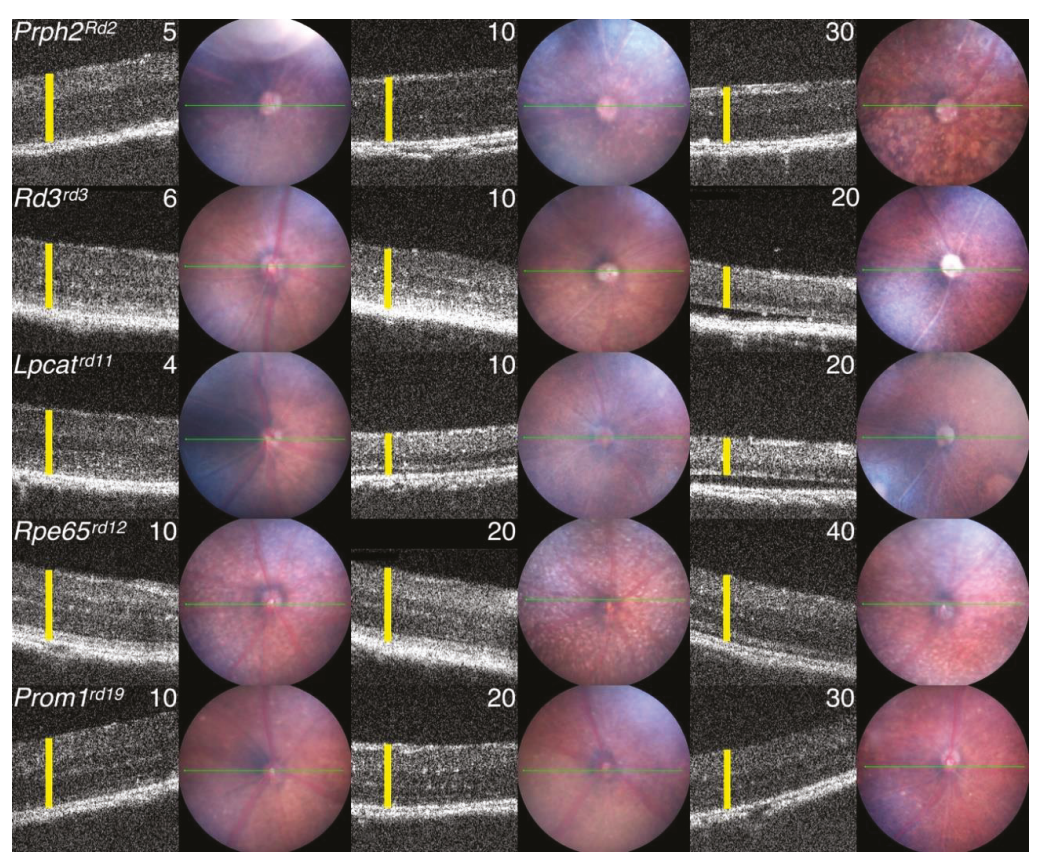

(b)

Figure 4. Cont. 


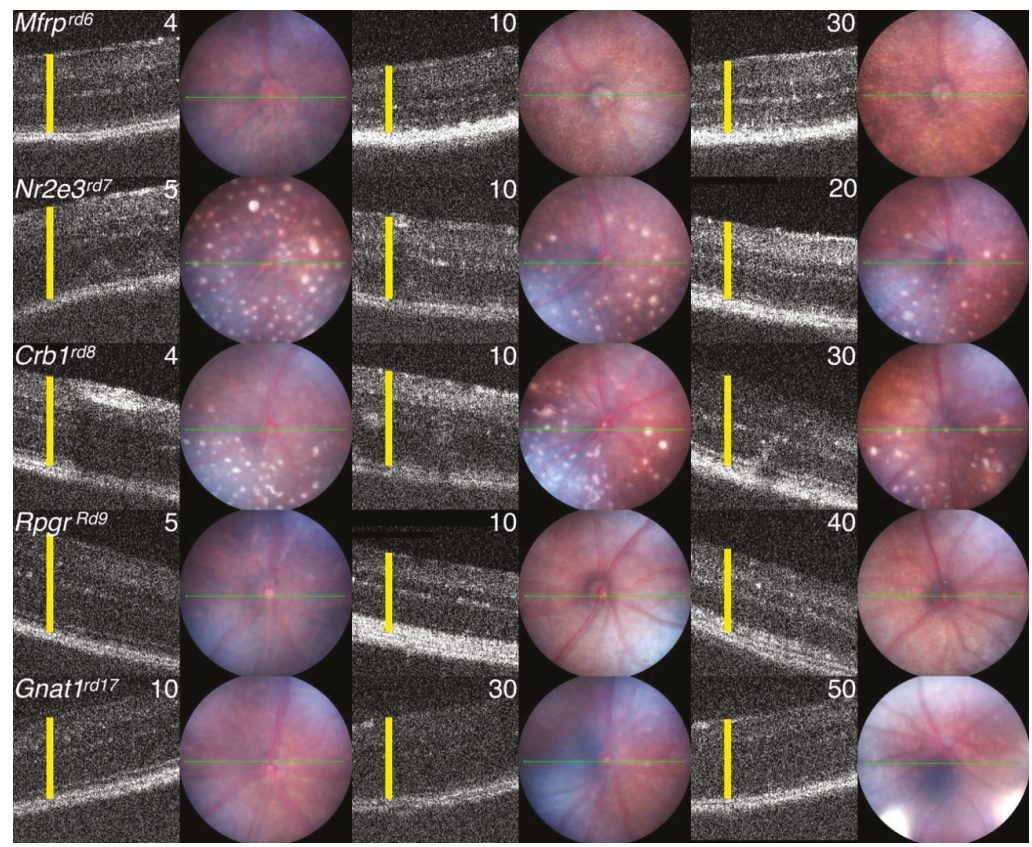

(c)

Figure 4. (a) Characterization of mouse models from the Eye Mutant Resource (EMR) program at JAX. Example optical coherence tomography (OCT) and fundus images were taken from rapid RD models: Pde6b $b^{r d 1}$ (B6.C3-Pde6b $b^{r d 1} H p s 4^{\text {le }} / \mathrm{J}$, Stock No: 000002), Pde6b ${ }^{\text {rd1-2J }}$ (C57BL/6J-Pde6 $b^{r d 1-2 J} / \mathrm{J}$, Stock No: 004766), Pde6b ${ }^{r d 10}$ (B6.CXB1-Pde6b ${ }^{r d 10} / \mathrm{J}$, Stock No: 004297), Rd4/+ (STOCK In(4)56Rk/J, Stock No: 001379) and Cep290 ${ }^{r d 16}$ (B6.Cg-Cep290 ${ }^{r d 16} /$ Boc, Stock No: 012283) (b) Example images from

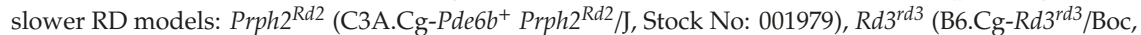

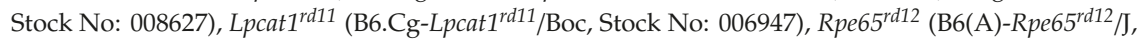
Stock No: 005379) and Prom $1^{\text {rd19 }}$ (B6.BXD83-Prom1 ${ }^{\text {rd19} / B o c, ~ S t o c k ~ N o: ~ 026803) . ~(c) ~ E x a m p l e s ~ f r o m ~ s l o w ~}$ and very slow RD models: Mfrp ${ }^{r d 6}$ (B6.C3Ga-Mfrp $p^{r d 6} / \mathrm{J}$, Stock No: 003684), Nr2e $3^{r d 7}\left(\mathrm{~B} 6 . \mathrm{Cg}-\mathrm{Nr} 2 e 3^{r d 7} / \mathrm{J}\right.$, Stock No: 004643), Crb1 ${ }^{r d 8}$ (STOCK Crb1 $1^{r d 8} / \mathrm{J}$, Stock No: 003392), Rpgr ${ }^{R d 9}$ (C57BL/6J-Rpgr ${ }^{R d 9} / \mathrm{Boc}$,

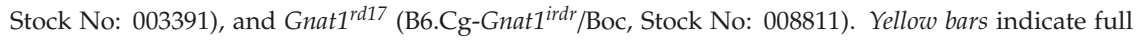
retinal thickness. Values correspond to the mouse age at the time of imaging (weeks).

\section{Analysis}

\subsection{Progression of PR Cell Loss}

While a host of effects can occur as a result of disruptions in genes expressed in PRs and ancillary cell types that functionally impair vision, such as night blindness, or color vision defects, the focus of the models described here are those that bear single gene mutations that lead to actual PR cell loss. From a review of the models in Table S1, significant PR cell loss is reported as early as postnatal day 7 (P7) and can extend throughout the lifetime of animals examined. The progression of PR cells loss is also highly variable and includes models that progress rapidly with complete ablation within several weeks to models with extremely slow progression where only $<10 \%$ PR cell loss is noted over the span of time in which animals were examined. Generally, while rapid to moderate progression led to almost complete PR ablation, slow and very slow progression, or degeneration in models that primarily affect cone PRs left a substantial number of PR cells intact. 


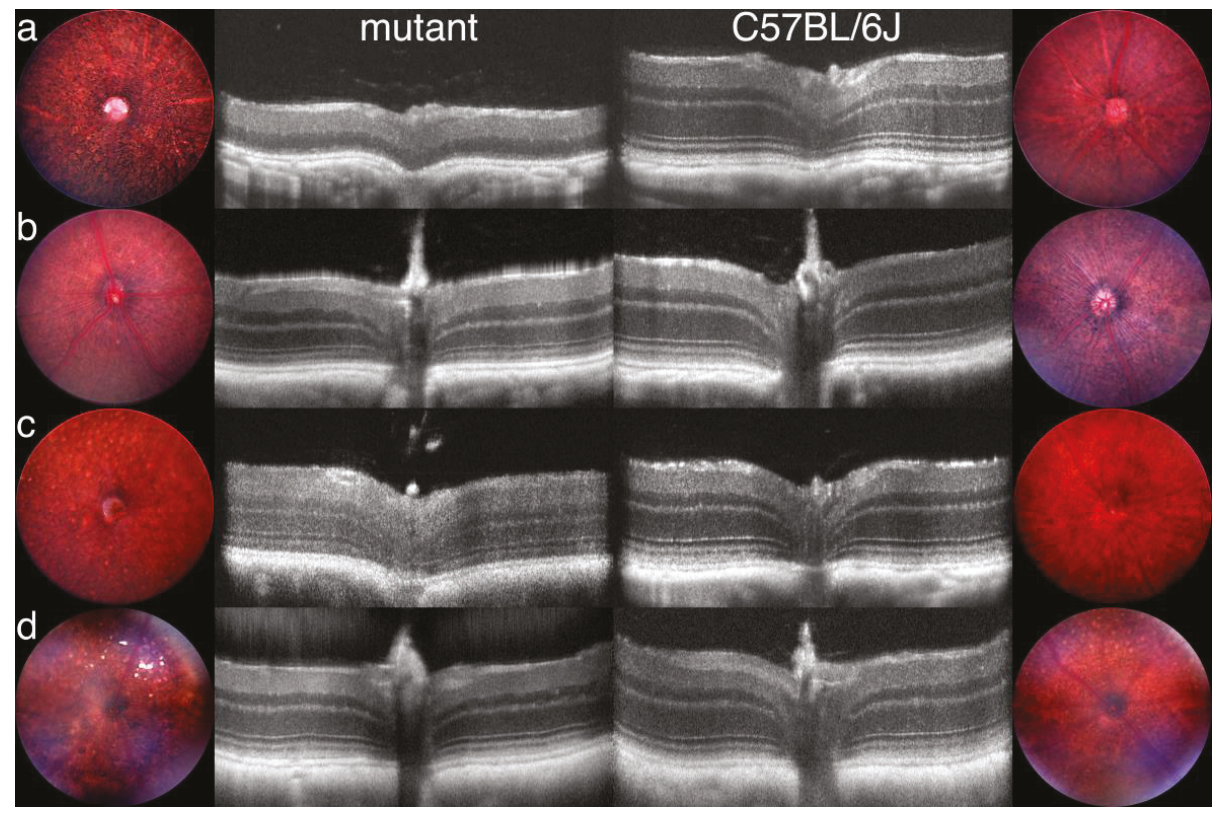

Figure 5. Characterization of mouse models from the Translational Vision Research Models (TVRM) program at JAX. A fundus image (circular panels) and corresponding OCT B-scan are shown for homozygous (a) Rpgrip $1^{\text {nmf247 }}$, at one month of age; (b) Nmnat $1^{\text {tvrm113 }}$, at two months;

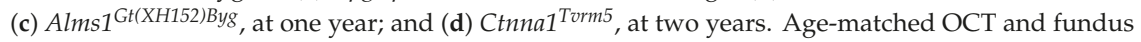
images for C57BL6/J control mice are shown to the right of the mutant images. PR cell loss is indicated by a decreased ONL thickness. Fundus images were acquired using a Micron III or IV retinal camera. The vertical dimension of OCT images was doubled to emphasize changes in retinal layer thicknesses.

To compare cell loss among functionally similar genetic models, models in each category of Table $\mathrm{S} 1$ were sorted based on the estimated age at which the PR cell population had degenerated by $50 \%$ compared to control values, defined as $D_{50}$ (Figure 6). This quantity represents neither the rate of PR cell loss nor the delay before loss commences, although both parameters are used to calculate it. Rather, $\mathrm{D}_{50}$ provides a common measure of progression that allows both complete and sparse datasets to be evaluated. With sufficient data, delay, and exponential decay constants were calculated and are reflected by the shaded bars in Figure 6. Many datasets with fewer measurements, some containing a single point, allowed only an estimate of $\mathrm{D}_{50}$ (Figure 6; filled circles, range lines indicate estimated limits). Figure 6 may be used to identify models in which overall PR cell loss progresses at an earlier age (lower $\mathrm{D}_{50}$ ), proceeds at a higher rate once initiated (shorter bar), or is accompanied by a substantial delay (bar starts farther to the right), which may aid in experimental design. Values for $\mathrm{D}_{50}$, the exponential decay constant $\mathrm{k}$, and the delay, when available, are also included in Table S1. We relate qualitative descriptions of progression to $\mathrm{D}_{50}$ as follows: rapid, $<2$ months; moderate, 2 to $<6$ months; slow, 6 to $<12$ months; and very slow, $\geq 12$ months. 

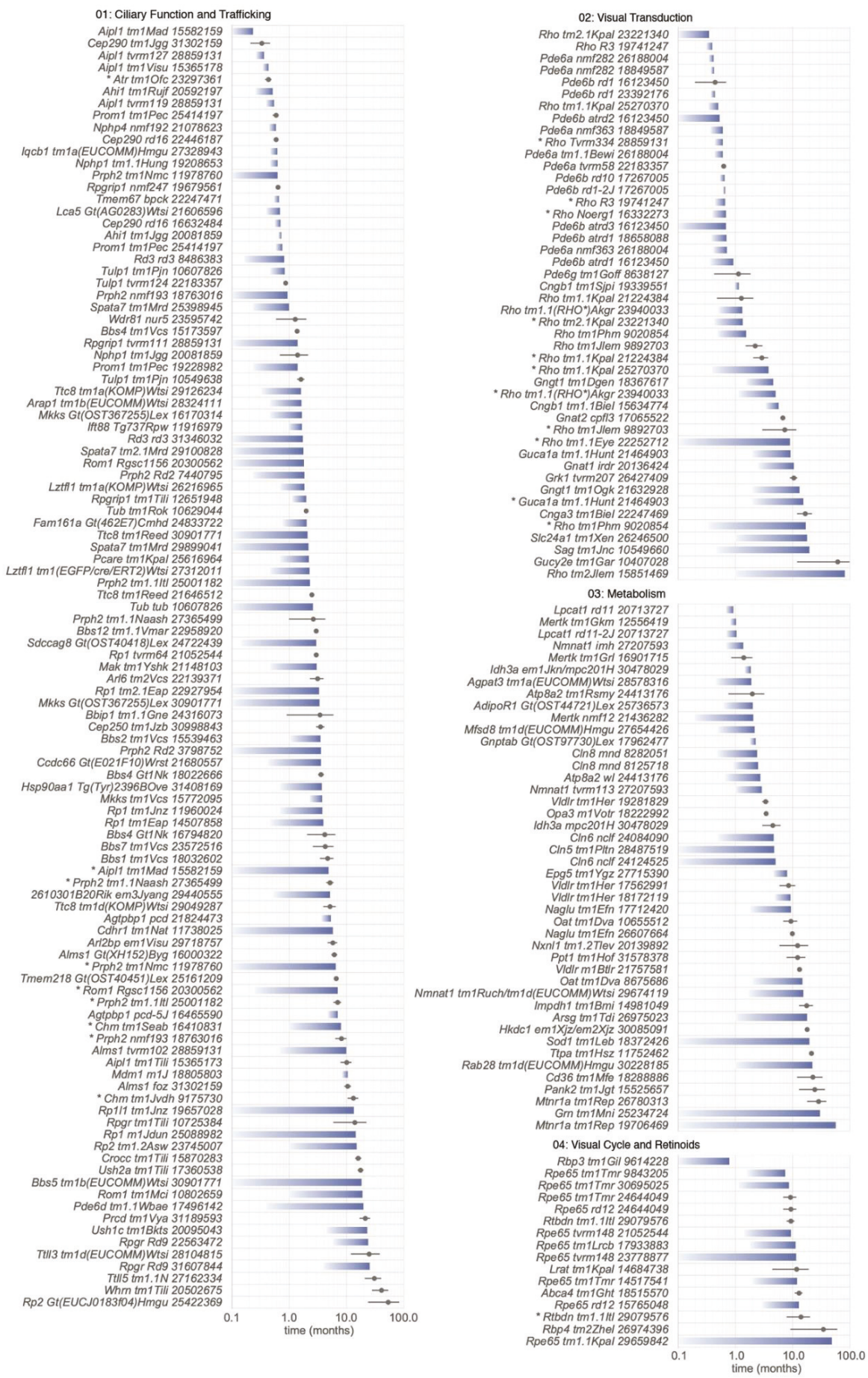

Figure 6. Cont. 

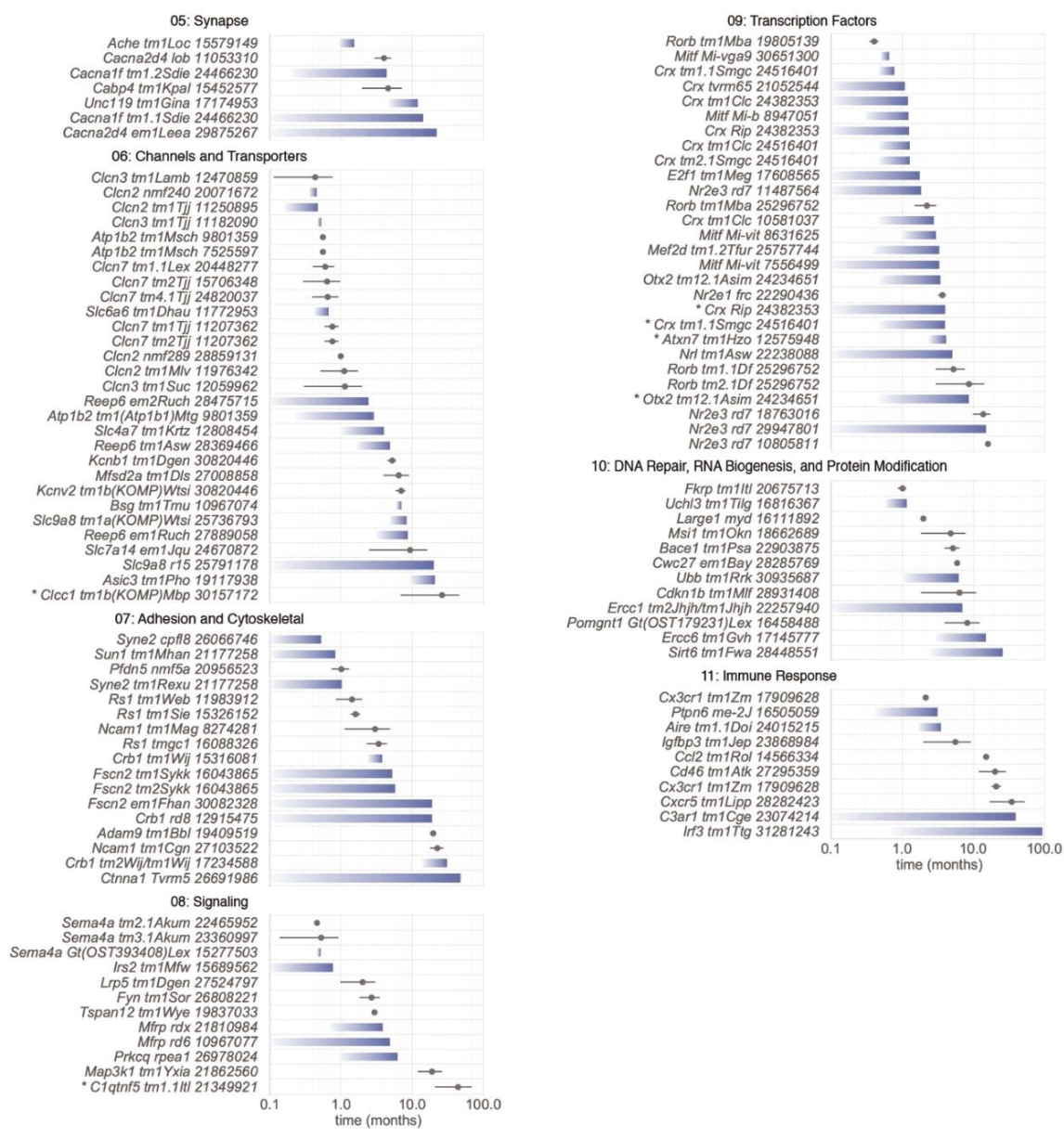

Figure 6. Progression of PR cell loss in mouse models by functional category. Models in each category were sorted by the age at which the estimated number of PR cells reached $50 \%$ of wild-type $\left(\mathrm{D}_{50}\right)$ as determined by fitting reported data to an exponential decay function combined with a delay. Models are identified by gene symbol, MGI allele symbol, and PMID. Models are homozygous except as indicated by * (heterozygous) or by the presence of two allele symbols (compound heterozygous). Left- and right-hand margins of shaded bars represent the delay and $D_{50}$ values, respectively. Bars with a delay value of $\leq 0.1$ month derive from datasets in which no values at $100 \%$ of wild type were reported. The midpoint and range of estimated $D_{50}$ values for datasets with only one point in the $5-95 \%$ range is indicated by closed circles and range lines, respectively. In cases where the dataset consisted of a single point at $50 \%$, no estimate was needed.

Figure 6 does not include models in which the age of the animal at the time of measurements was not provided, or was reported as "adult", although these models were included in Table S1. We also omitted models where only cone PR cell degeneration was reported, as limited data and a lack of cone nuclei counts made mathematical modeling of cell loss unreliable. Finally, we omitted all conditional alleles from Figure 6, as the efficiency and specificity of inactivation of genes, as well as the temporal expression of different transgenic Cre drivers utilized varies, making comparison of these alleles difficult. 


\subsection{Biological Processes Affected by Mutations}

Categorization of PR cell loss genes (Figure 3) relied on current functional knowledge, similarity to other genes, and localization, if known. Genes are often expressed in multiple cell types and serve different functions within the retina. We placed genes in categories that were most pertinent to their roles in the PR or effect on PR survival. The data in Table S1 can be explored after reassigning models to different categories if desired. In the descriptions below, we provide an overview for the first three categories and then a detailed description of the effect of gene disruptions on biological functions included in these categories. Subsequent categories are described without overview.

\subsubsection{Category 01: Ciliary Function and Trafficking}

Overview. Disruptions in a vast number of genes associated with PR sensory cilia result in $\mathrm{RD}[24,44]$. The onset and rate of PR cell loss may vary depending on the role of a given gene in maintaining ciliary structural integrity and function during early PR development and maintenance in adulthood. Retinal defects within any one of the key ciliary structures and/or processes such as ciliogenesis, OS morphogenesis, or PR homeostasis may result in the loss of rod and cone PR cells. Understanding the pathophysiological mechanisms involved in PR cell loss requires a detailed examination of the components and functionalities of the PR sensory cilium.

The mouse PR sensory cilium is a specialized structure comprised of a tubulin-rich axoneme with a symmetrical " $9+0$ " arrangement of microtubular doublets [45]. The connecting cilium (CC) is akin to the transition zone (TZ) of primary cilia [46,47] and serves as a passageway for the movement of proteins from the organelle-rich IS to the photosensory OS. The OS is composed of an elongated distal axoneme with adjacent stacked membranous discs decorated with proteins necessary for phototransduction. It has been hypothesized that extension of the PR plasma membrane towards the apical RPE provides a convenient sink in the OS for the storage of a large number of membrane proteins [48]. In the CC-TZ, axonemal microtubule doublets interconnect the distal axoneme and basal body, and also connect to the periciliary membrane via Y-linkers. The CC-TZ harbors membrane-associated and soluble proteins that coordinate as gatekeepers to regulate the entry, retention, and exit of proteins in and out of the OS $[47,49,50]$. At the axonemal base, the ciliary membrane is anchored by the nine microtubular triplets of the basal body and its associated appendages.

In developing murine PRs, the connecting cilium and OSs assemble through a series of coordinated events. Shortly after cell cycle exit of retinal progenitor cells, the mother centriole docks to a primary ciliary vesicle initiating its expansion and fusion with the plasma membrane. Concurrently, the microtubular axoneme elongates towards the apical end of the neuroblastic layer [51]. In rod PRs, ciliogenesis typically begins shortly after birth with the appearance of a mature centriolar-bound ciliary vesicle at around P4 [52]. Subsequently, OS biogenesis occurs asynchronously between P8 and P14 [52-54] while axoneme and PM extension continue until OS maturation around P19-25 [52,53]. During this process, intraflagellar transport (IFT) provides an efficient mechanism for the movement and delivery of crucial proteins to the developing OS [55,56], as discussed in greater detail below.

Over the past several decades, two disparate mechanisms of rod disc morphogenesis have been debated, a vesicular fusion model $[57,58]$, which postulates that membrane discs originate from rhodopsin bearing vesicles that undergo intracellular membrane fusion, and the classic evagination model [59], which proposes that new discs result from the evagination of the plasma membrane at the base of the OS. Recent ultrastructural studies in mouse rod PRs have provided compelling evidence that support the classic evagination model. By high resolution microscopy, several groups demonstrated the plasma membrane origin of the evaginated rod disc membranes [60], which subsequently flatten, elongate, and become enclosed [52,60,61].

OS membranous discs undergo a rapid and continuous turnover with approximately 75 rod discs being shed daily, corresponding to $10 \%$ of the OS [53,62]. At the ciliary tip, aged discs are removed by the adjacent RPE through phagocytosis. Consequently, OS renewal requires a continuous flow of new proteins from IS to the OS through the connecting cilium, a process that requires careful regulation. 
For the CC-TZ gate to function properly, efficient mechanisms are needed to prevent the entry of undesired proteins and to remove the non-OS proteins improperly targeted to the OS. At the base of the CC-TZ lie transition fibers, which act as a barrier in conjunction with other CC-TZ components, such as the membrane-associated Meckel syndrome (MKS) complex. The BBSome, an octameric coat complex, is thought to coordinate the delivery and removal of proteins from the OS during ciliary formation and maturation $[63,64]$.

Finally, protein trafficking through the CC-TZ is important for PR development and maintenance $[51,65,66]$. The movement of protein cargo from the IS to the OS requires a highly regulated passage of vesicles along microtubules that dock and fuse with the periciliary membrane to deliver their cargo at the CC base. Movement of targeted proteins through the cilia to the OS may be facilitated by IFT transport machinery [65] or by a lipidated protein trafficking system [67,68]. During IFT, protein cargo associate with IFT particles that attach to kinesin and dynein motors and move along the axonemal microtubules in both anterograde and retrograde directions, respectively. The BBSome is known to associate with IFT particles and may provide a mechanism for the removal of non-targeted protein accumulation in the OS [69].

Ciliogenesis. As the ciliary axoneme serves as an important conduit for the IFT movement of signaling molecules, it comes as no surprise that the disruption of ciliogenesis genes may result in significant developmental abnormalities causing early lethality and/or rapid PR degeneration. Genes essential for the elongation of the proximal axoneme (A and B tubules) include Kif3a [70], which encodes a subunit of kinesin 2, Iqcb1 [71], Arl3 [42], and Arl13b [72]. While patients with missense mutations in ARL13B present with Joubert-associated features [73], a null mutation, Arl13b ${ }^{\text {hnn }}$, results in embryonic lethality in mice [74]. Axonemal disturbances and a failure to form OS discs are observed in developing retinas with conditional Arl13b disruption [72].

Axonemal and ciliary membrane extension. Disruptions in genes that affect ciliary extension include Rp1/Rp1l1/Spata7 (distal axoneme), Mak, and Pcare (C2orf71; ciliary membrane). Mice with knockout alleles of Rp1 (Rp $1^{t m 1 E a p}$ and $R p 1^{t m 1 J n z}$ ) and Spata7 (Spata $\left.T^{t m 1 M r d}\right)$ show a progressive, moderate loss in PR cells through the first year of life. $R p 1^{m 1 J d u n}$ mice homozygous for a Leu66Pro missense mutation experience a much slower degeneration with $30 \%$ of PRs left at 26 months of age. Conditional ablation studies of Spata7 in PRs and in the RPE have shown that the disruption of SPATA7 in rod and cone PRs, but not in the RPE, is the molecular basis of the retinal degenerative phenotype [75].

Ciliary Gate and the CC-TZ. Sensory/primary cilia and their gatekeepers (CC-TZ) are found abundantly in most cell types [76]. Thus, the disease spectrum of ciliary proteins is extensive given their roles in ciliary trafficking, signaling, and development. Disruptions in CC-TZ genes may result in isolated cases of inherited retinal dystrophies such as Leber congenital amaurosis or in multisystemic, ciliopathies such as Joubert, Meckel, or Senior-Løken Syndrome. Such syndromic ciliopathies may include a multitude of disease phenotypes such as brain malformations, renal cysts, nephronophthisis, and retinal dystrophy.

Within the CC-TZ reside MKS and NPHP modules that closely interact and form multiple distinct protein complexes $[47,50,77-79]$. The MKS complex includes membrane-associated proteins, such as MKS1 and TMEM67, while NPHP complex proteins, such as NPHP1 and NPHP4, associate in closer proximity to the ciliary axoneme. Mice harboring mutations in genes coding for these complex-associated proteins form normal cilia, however, display early abnormalities in OS morphogenesis. After ciliary biogenesis, retinas in these mutant mice quickly degenerate, eliminating most PRs by 3-4 weeks of age. Genes whose disruptions affect the ciliary gate functions of the CC-TZ and cause rapid degeneration include Nphp1, Nphp4, Ahi1, Iqcb1, Tmem67, and Cep290. In humans, mutations in CEP290 can lead to primarily single-organ diseases such, as retinitis pigmentosa and nephronophthisis, or pleiotropic diseases, such as the Joubert, Meckel, and Bardet-Biedl syndromes. The most studied allele is $r d 16$, which harbors a 297 basepair in-frame deletion in Cep290. Compared to Cep290 ${ }^{\text {tm1.11gg }}$ knockout mice, which show a rapid $78 \%$ loss at P14, Cep $290^{\text {rd16 }}$ homozygotes have a longer disease progression with a $60 \%$ ONL loss at three weeks of age. 
Basal bodies and associated pericentriolar material (PCM). The basal body is a structure derived from the mother centriole and resides at the base of the cilium along with the daughter centriole, neighboring centriolar satellites and other related PCM. Proteins positioned at the ciliary base can also be seen in centrosomes of dividing cells (ALMS1, CEP250, and C8ORF37). Specifically, ALMS1 and CEP250 (CNAP1) localize in close proximity to each other at the proximal ends of centrioles [80]. ALMS1 encodes a 460kDa protein that when disrupted results in the Alström syndrome (ALMS) [81,82]. Mice with a gene trap, frameshift, and nonsense mutations recapitulate human ALMS disease features such as obesity, diabetes, and neurosensory deficits [21,83,84]. The proper formation of the connecting cilium and the slow progression of PR cell loss in Alms $1^{\text {Gt(XH152)Byg }}$ [83], Alms $1^{\text {foz }}$ [84],

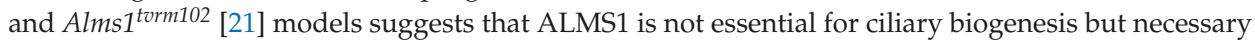
for overall PR homeostasis.

The ciliary base contains supportive structures necessary for the proper docking of cargo to the ciliary membrane. Targeted Macf1 null mutants fail to develop the ciliary vesicle needed for basal body docking while conditional ablation of Macf1 in the developing retina disrupts retinal lamination and maturation [85]. Mutations in Cdcc66, which encodes a component of centriolar satellites and Sdccag8, which encodes a recruiter of PCM, result in an early onset but slow-moderately progressive disease $[86,87]$. The slower RD makes these alleles attractive models for therapeutic investigations.

Genetic mutations in CC2D2A, which encodes a component of the subdistal appendages of mother centrioles and basal bodies [88], have been observed in patients with Meckel Syndrome [89], Joubert Syndrome [90], and non-syndromic rod-cone dystrophy [91]. Mice with null mutations in C $c 2 d 2 a$ experience embryonic lethality due to the absence of subdistal appendages and nodal cilia [88]. The retinas of adult mice with tamoxifen-induced deletion of $C c 2 d 2 a$ in PRs have a significantly diminished ONL (2-3 layers) 12 weeks post-injection [92], suggesting that CC2D2A is necessary for ciliary homeostasis.

Periciliary membrane complex. At the periciliary membrane complex of PRs lies an Usher protein interactome complex that provides a scaffold for the anchoring of fibers to the periciliary membrane [93]. Mutations in genes encoding members of this complex, Ush1c, Whrn, and Ush2a, result in Usher syndrome, a disease that results in progressive hearing and vision loss. Multiple forms of Usher syndrome exist resulting in different degrees of the onset and severity of disease symptoms. In the mouse, targeted mutations in Usher genes results in a late-onset and very slow progression of PR degeneration. Homozygous Ush $2 a^{\text {tm } 1 \text { Tili }}$ mice have normal retinas at 10 months of age and lose $70 \%$ of their PRs by 20 months of age [94]. PR degeneration in Whrn ${ }^{\text {tm1Tili }}$ retinas is protracted with only $30 \%$ loss observed at 28 months of age [95].

Disc morphogenesis. Although the molecular mechanisms involved in OS disc morphogenesis are not completely understood, there has been considerable progress within the past decade with the emergence of refined ultrastructural methods. The OS protein, peripherin-2 (PRPH2), localizes to the rims of rod and cone discs and functions to establish and maintain the membrane rim curvature during disc formation and maintenance $[96,97]$. Recent investigations using the $P r p h 2^{R d 2}(r d s)$ mouse model [10] have suggested another role for PRPH2 during disc morphogenesis [52]. Using transmission electron microscopy, Salinas et al. [52] demonstrated that like other forms of cilia [98,99], PR sensory cilia have an innate ability to spew off ectosomes at the OS base. During normal development of the OS, ectosome release is inhibited and the retained membrane at the CC-TZ is transformed into discs upon membrane evagination. In the homozygous $\operatorname{Prph} 2^{R d 2}$ mice, discs fail to form resulting in the accumulation of ectosomes at the OS base. This finding led the authors to propose that PRPH2 may play a role in inhibiting ectosome release during normal rim formation [52].

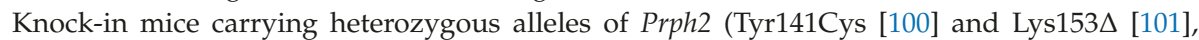
mimic the dominant RP disease observed in human patients. It is interesting that PR degeneration rates vary among $\operatorname{Prph} 2$ mutant alleles. While homozygous $\operatorname{Prph} 2^{R d 2}$ mice gradually lose their PRs within the first year [102,103], mice harboring a homozygous null mutation, $\operatorname{Prph}^{\mathrm{tm} 1 \mathrm{Nmc}}$ undergo a faster degeneration with most PRs lost by 4 months of age [104]. Heterozygous $\operatorname{Prph} 2^{t m 1 N m c}$ mice also 
experience PR loss, however the rate of decline is much slower [104]. Comparative studies of $\operatorname{Prph} 2^{R d 2}$ with rhodopsin double-knockout mice have suggested that abnormal accumulation of mislocalized rhodopsin may contribute to PR degeneration in $P r p h 2^{R d 2}$ [105]. Hence, the zygosity differences in degeneration rates may be a result of varying rhodopsin: PRPH2 ratios. In addition, the onset and severity of the disease may be influenced by the location of the mutation, as different PRPH2 domains have been implicated in dual roles during disc morphogenesis, the tetraspanin core in rim membrane curvature, and the $C$-terminal domain in ectosome release suppression [52].

ROM1 is thought to be involved in the regulation of OS disc formation. PRPH2 and ROM1 are closely associated at the disc rims in the OS. In humans, a double heterozygous disruption in both ROM1 (a PRPH2-interacting protein) and PRPH2 results in digenic RP [106]. While it is not clear whether defects in ROM1 alone causes RP in humans, mice with a monogenic Rom1 disruption show signs of dominant RP. At one month of age, Rom 1 knockout OS discs are visible but appear enlarged and slightly disorganized [107]. PRs slowly degenerated, reducing the ONL by $34 \%$ at 1 year of age. In contrast, Rom $1^{R g s c 1156}$ mice with a heterozygous missense mutation, p.W182R, show a 55\% loss of PRs at 35 weeks of age [108]. Furthermore, RD was more pronounced in homozygous mice. The degeneration in Rom $1^{R g s c 1156}$ mice may be a consequence of an early reduction in endogenous PRPH2 and ROM1 levels, which may interfere with PRPH2-mediated stabilization of disc outer rims.

PRCD, progressive rod-cone degeneration, is a rhodopsin-binding protein [109] that localizes to the OS disc rims [110]. Patients and canines with $P R C D^{C 2 Y}$ mutations have a slowly progressive form of rod-cone degeneration [111]. The Cys2Tyr mutation results in mislocalization of PRCD from the OS to the ONL where it is actively degraded [109]. In the PRs of mice with homozygous Prcd ${ }^{\text {tm1Vya }}$ mutations, loss of PRCD results in the formation of bulging discs that do not properly flatten and in the accumulation of extracellular vesicles that originate at the OS base [112]. Interestingly, mutant PRs are able to form membrane discs and the distribution of OS proteins and light response do not appear to be perturbed. While activated microglia infiltrate the interphotoreceptor space to remove extracellular vesicles and debris, removal is insufficient and PRs undergo a very slow degeneration. Homozygous $\operatorname{Prcd}^{\text {tm1Vya }}$ mice show only $36 \%$ ONL loss at 17 months [112] while in $\operatorname{Prcd}^{\text {tm1(KOMP)Mbp }}$ homozygotes a similar loss is observed at 30 weeks of age [110]. Both models are knockout alleles that target the $5^{\prime}$ end of Prcd but are on different genetic backgrounds. Further investigations are necessary to determine whether gene modifiers affect progressive PR cell loss in these two models.

IFT trafficking. IFT is essential for ciliogenesis in mammals [113] and disruption of this process often leads to abnormalities in embryonic development. In the mouse, null mutations in genes encoding subunits of the IFT-A (Ift122 [114], Ift88 [115], and Ttc21b [116]) and IFT-B (Ift172 [117], Ift80 [118], and Traf3ip1 [119]) complexes result in embryonic lethalities, many of which are attributable to ciliary-related disturbances in hedgehog signaling [120,121]. These findings further highlight the integral role of cilia and IFT machinery during embryogenesis.

Hypomorphic and conditional alleles have been useful for elucidating the roles of IFT components in retinal disease. The hypomorphic allele, Ift8 $88^{T} \mathrm{~g}^{737 R p w}$, contains a transgenic insertion resulting in a $2.7 \mathrm{~kb}$ intronic deletion. Homozygous Ift $88^{\mathrm{Tg}} 737 \mathrm{Rpw}$ mice exhibit disorganized OSs as early as P10 and a progressive degeneration of PRs that reduces the ONL to one layer at P77 [66]. Rod-specific ablation of Ift172 [122] leads to mislocalization of rhodopsin, RP1, and TTC21B (IFT139) and rapid degeneration of PRs. Conditional depletion of Ift20 in M cones and mature rods both results in opsin mislocalization suggesting that proper opsin trafficking hinges on functional IFT components [123]. To gain a clearer understanding of the roles that IFT molecules play in both rod and cone PRs, additional studies using conditional models are warranted to elucidate the contributions of impaired IFT components to PR cell loss.

Lipidated protein trafficking. Lipid modification of proteins, such as prenylation or acylation, helps direct intracellular protein targeting and regulates protein activity [124]. These hydrophobic modifications help tether their protein partners to the surface of specific membranes throughout the cell, such as the ER, Golgi, transport vesicles, or plasma membranes. Improper trafficking of lipidated 
proteins can result in RD. RP2 is a GTPase activating protein that interacts with ARL3 to regulate assembly and movement of membrane-associated protein complexes [125]. Homozygous mice with mutations in the gene encoding RP2 exhibit a slowly progressive rod-cone degeneration [126,127]. ARL3, a small GTPase, traffics lipidated membrane-associated proteins to the rod OS [128]. Although Arl3 knockout mice exhibit early postnatal lethality and Joubert-like features [42], mice with hypomorphic mutations survive to post-wean and display OS abnormalities as early as P9 [129]. Conditional ablation of Arl3 in the developing retina results in the absence of cilia, and therefore PR cells are rapidly lost [42]. In contrast, depletion of Arl 3 in mature rods leads to mislocalization of lipidated OS proteins, shortened OS, and a moderate progressive PR loss. These results are consistent with roles for ARL3 in ciliogenesis during development and cargo displacement during lipidated protein trafficking.

The ciliary TZ-associated protein, RPGR, binds and directs the ciliary targeting of INPP5E [130], a phosphoinositide phosphatase that is important for ciliogenesis [131]. Ciliary localization of RPGR itself requires modification with a prenyl group, which interacts with PDE6D [130], a prenyl-binding protein first discovered as a copurifying component of cGMP phosphodiesterase 6 (PDE6) [132]. Like Rpgr ${ }^{r d 9}$ [133] and Rpgrtm1Tili knockout mice [134], mice with Pde6d ${ }^{\text {tm1.1Wbae }}$ null mutations [135] undergo a very slow degeneration with at least $50 \%$ of PR cells remaining at 20 months of age. Rao et al. have demonstrated reduction of INPP5E in RPGR-deficient axonemal OSs [130]. Altogether, these observations validate RPGRs role in ciliary trafficking and homeostasis and suggest that other players may be involved in ciliary targeting of INPP5E.

Mutations in Aipl1 result in early and rapid loss of PR cells (Table S1). $\mathrm{D}_{50}<0.55$ months for the four germline alleles shown in Figure 6, Aipl1 ${ }^{\text {tm1Mad }}$, Aipl1 ${ }^{\text {tvrm127 }}$, Aipl1 ${ }^{\text {tm1Visu }}$, and Aipl1 ${ }^{\text {torm } 119}[21,39,136]$. AIPL1 is a protein chaperone that mediates the folding of phosphodiesterase 6 (PDE6), a key component of the visual transduction pathway that regulates cGMP levels (see Section 5.2.2. below) [137]. AIPL1 binding is promoted by prenylation of PDE6 subunits [137]. In Aipl1 mutants, PDE6 subunits are greatly diminished [136], providing further evidence for the importance of lipid modification in PR viability and vision.

BBSome assembly and regulation. Disruptions in the octameric BBSome complex or associated chaperonins may cause syndromic ciliopathies such as the Bardet-Biedl syndrome and McKusickKaufman syndrome. In mice, most gene disruptions that affect the BBSome [138] (BBS1, BBS2, BBS4, BBS7, BBIP1, TTC8, and ARL6), and its regulators (LZTFL1, MKKS, BBS10, and BBS12) result in a moderate degeneration of PRs. For instance, PRs in homozygous mice harboring gene trap or null mutations of $B b s 4, B b s 4^{G t 1 N k}[139]$, and Bbs $4^{t m 1 V c s}$ [140] appear to progressively decline after maturation with $>90 \%$ loss at 7 months of age. The delay and lack of ciliogenesis defects suggests that there may be some functional redundancy amongst components of the BBSome.

\subsubsection{Category 02: Visual Transduction}

Overview. Mutant alleles of genes encoding proteins responsible for light detection comprise a second category of models (Category 02: Visual Transduction; Figure 1c, Table S1). The multistep phototransduction process that detects light and amplifies this signal is similar in rod and cone cells, but the specific proteins that catalyze many of the steps are often unique to each cell type [141]. Phototransduction is initiated by the response of opsin-based light-sensitive $G$ protein coupled receptors that are covalently linked to vitamin A retinal as a cofactor. The receptor rhodopsin (RHO) is expressed exclusively in rod cells and is optimized to detect dim green light. Cone pigments that detect short or medium wavelength visible light (OPN1SW and OPN1MW, respectively) are exclusively expressed in cone cells, in some retinal regions coordinately within the same cell. These receptors constitute $>90 \%$ of OS protein and are localized to the disc membranes.

Light activation of RHO or cone pigments causes the bound retinal to isomerize from an 11-cis to an all-trans configuration, ultimately leading to its release from the receptor by hydrolysis. Isomerization results in a conformational change in the protein that alters its interaction with a bound heterotrimeric G protein, transducin, activating the exchange of GTP for GDP bound to the $\alpha$ subunit of this protein. 
In turn, activated $\alpha$ transducin-GTP binds the inhibitory $\gamma$ subunits of phosphodiesterase 6 , releasing it from the $\alpha$ and $\beta$ subunits of this complex, which are thereby activated to catalyze the conversion of cGMP to GMP. The ensuing reduction in cGMP levels in the OS closes the cGMP-gated cation channel, slowing the influx of $\mathrm{Na}^{+}$and $\mathrm{Ca}^{2+}$ ions, which hyperpolarizes the plasma membrane of the OS and, ultimately, the entire cell. Hyperpolarization causes $\mathrm{Ca}^{2+}$ channels to close at the cell synapse, which leads to a decrease in the calcium-dependent release of glutamate-containing vesicles into the synapse and activates postsynaptic bipolar neurons.

The process is regulated to ensure the highest sensitivity to illumination. Following its activation, rhodopsin is quenched by the action of arrestin, which binds to bleached opsin molecules that are phosphorylated by rhodopsin kinase. Resetting of the cell following the light flash requires the formation of cGMP from GTP, catalyzed by a membrane-bound guanylate cyclase, the subsequent closing of the cGMP-gated cation channel, and the restoration of electrolyte distribution across the plasma membrane as achieved by ion pumps and transporters. Hydrolyzed retinal is passed from the OS to the RPE as part of the visual cycle (see below), where it is re-isomerized and returned to the PR cell to regenerate bleached opsin. An additional visual cycle involving Müller cells contributes to the regeneration of cone pigments.

Visual pigments. Profound effects on PR viability are observed due to mutations that affect rod cells, which represent $97 \%$ of the PR population. Mouse models bearing Rho alleles exhibit semidominant and recessive rod cell loss phenotypes that vary greatly in the onset and rate, consistent with the variety of possible disease mechanisms that have been proposed for RHO mutations over decades of study. For example, some missense alleles in Table S1, such as those that encode the Pro23His, Cys110Tyr, Tyr178Cys, and Cys185Arg variants [21,142-146] may support a hypothesis that excessive RHO misfolding in the endoplasmic reticulum induces cellular stress pathways that lead to PR cell loss [147]. Although the pathways linking misfolded RHO to cell death are not fully resolved, recent studies of the Pro23His variant in cultured cells and in rats [148] or mice [145,146] suggest that stress pathways induced by the unfolded protein response are protective, and raise the possibility that increased intracellular calcium due to ER stress may cause cell death [146]. Misfolding may also explain the partial mislocalization of RHO Glu150Lys to the IS [149]. However, in this mutant, much of the protein appears to be correctly exported to the OS, where it leads to irregularly shaped and disorganized discs, possibly due to a defect in higher-order RHO organization [149]. Pro23His $\mathrm{RHO}$ also disrupts the orientation of discs during their morphogenesis, possibly through similar effects on higher-order structure [150].

By contrast, the effect of the Gln344Ter variant (Table S1), which is correctly folded but includes sequence extensions at the C-terminus that interfere with export to the OS [151], as well as the graded effect of heterozygous or homozygous knockout alleles Rho $o^{\text {tm1Jlem }}$ and Rho ${ }^{\text {tm1Phm }}[152,153]$ or the premature truncation mutant Arg107Ter (Table S1), provides evidence that a steady flow of RHO to the OS is essential for PR cell viability. These observations fit an emerging view that a proteostasis network, incorporating not only cellular stress pathways but also protein trafficking and degradation, regulates the cellular protein balance to ensure viability $[147,154]$. According to this view, a failure to sort vesicles bearing RHO from the Golgi to the periciliary membrane, or a partial or complete loss of the protein, leads to protein imbalance in the IS. This imbalance may induce cellular stress responses and also affect the trafficking of other molecules destined for the OS, such as other phototransduction proteins, lipids, and vitamin A, resulting in cellular toxicity. Finally, the RHO Asp190Asn variant (Table S1) appears to traffic properly to the OSs but may have structural defects that lead to constitutive signaling [155], which has been linked to PR degeneration [156]. The same mechanism may account for the effect of Rho mutants that result in rapid degeneration upon bright illumination [157] but were not included in Table S1 due to the dependence of the mutant phenotype on an environmental perturbation (see Discussion). Future studies of these and other models may resolve or converge the many proposed hypotheses to explain RHO-associated RD. 
Based on the often profound effect of Rho variants on rod cell viability, it might be expected that cone pigment variants would similarly cause cone PR cell loss. However, cones remain viable for more than 1.5 years in homozygous Opn1sw $w^{\text {tm1Pugh }}$ mice, which show a 1000-fold decrease in transcript and produce no detectable OPN1SW by immunoblotting, histochemistry, or single-cell recording of light responses [158]. Likewise, cones are viable for at least 10 months in homozygous Opn1mw $w^{t m 1 a(E U C O M M) W t s i}$ knockout mice, despite an absence of OPN1MW in immunoblotting and immunohistochemical studies [159]. These studies suggest fundamental differences in the cellular sensitivity of rod and cone cells to visual pigment deficiency. They also highlight the concern that reactivity to antibodies against cone opsins or other cone cell markers may be abolished even though the cells remain viable, and therefore may not be as reliable as counting cone nuclei [160] to assess cell loss.

Transducins. Rod transducin subunits $\alpha, \beta$, and $\gamma$ (encoded by Gnat1, Gnb1, and Gngt1, respectively) form the heterotrimeric $\mathrm{G}$ protein complex that is essential for propagating the signal from light-activated rhodopsin. Gnat1 knockout mice have attenuated rod responses and model congenital stationary night blindness (CSNB) [161]. Although slow PR loss was reported for this model, our measurement of ONL thickness at four weeks of age based on reported images yielded a value of $90 \%$ of wild type, matching the author's value at 13 weeks [161] and suggesting an early developmental difference rather than progressive cell loss. In support of this finding, others using the same strain reported ONL thickness was $85 \%$ of wild type at eight weeks of age with no evidence of significant cell loss up to 52 weeks of age [162]. By contrast, IRD2 mice, which are homozygous for a Gnat $1^{\text {irdr }}$ allele predicted to yield a prematurely truncated polypeptide, exhibit significant rod PR cell loss (Table S1) accompanied by late cone cell loss and reduced rod-specific ERG responses [163]. Homozygous Gnat ${ }^{i r d r}$ mice may recapitulate recessive rod-cone dystrophy, which has recently been linked to human GNAT1 variants predicted to encode prematurely truncated proteins [164-166]. The Gnat ${ }^{\text {irdr }}$ allele was discovered independently in $r d 17$ mice at JAX, suggesting a founder effect $[167,168]$.

Gnb1 knockout mice have not been studied due to embryonic and perinatal lethality. However knockout alleles of the gene encoding rod $\gamma$ transducin, Gngt $1^{\text {tm1Dgen }}$ and Gngt $1^{\text {tm1Ogk }}$, result in PR loss that is more rapid than in Gnat1 mutants $[169,170]$. In these strains, GNGT1 deficiency is accompanied by a 6- to 50-fold post-translational reduction of GNAT1 and GNB1, indicating a key role of the transducin $\gamma$ subunit in complex assembly. Gngt ${ }^{\text {tm1Dgen }}$-associated degeneration is rescued by heterozygous Gnb1 $1^{\text {Gt(proSStrap)4B8Yiw }}$ mice [171], which express retinal GNB1 at 50\% of wild type levels. This result suggests that the toxicity of GNGT1-deficiency is due to an excess of improperly assembled GNB1, which is targeted for degradation but exceeds the capacity of the proteasome [171]. This observation supports the proteostasis network model of PR degeneration [154].

Among genes encoding cone transducin subunits $\alpha, \beta$, and $\gamma$ (Gnat2, Gnb3, and Gngt2), only Gnat2 alleles have been reported to cause PR loss. A progressive reduction of cone cell ERG responses and a 27\% decrease in PNA-positive cells at 12 months of age in homozygous Gnat2 $2^{\text {tm1Erica }}$ mice (Table S1) is consistent with cone PR loss [172]. However, cone nuclei were not counted directly, so it is possible that cone cell loss is less pronounced than reported. The predicted GNAT2 Asp173Gly substitution in this model may alter guanine nucleotide binding [172], although how this change might cause cell loss is unresolved. Interestingly, mislocalized cone opsin OPN1MW in this model suggests endoplasmic reticulum stress, which is often associated with PR degeneration. Gnat2 $2^{\text {cpfl3 }}$ mice (Table S1) show no cone cell loss for at least 14 weeks but exhibit a slow loss of rod cells [173]. In contrast to these models, a recently developed Gnat2 knockout strain abolishes GNAT2 function without PR loss or dysmorphology in the oldest mice examined at 9 months of age [174]. Although human GNAT1-variants are a rare cause of achromatopsia [175], a stationary congenital colorblindness, the clinical presentation is variable and some cases are associated with a reduction in visual acuity with age [176] that may suggest progressive cone cell loss. The available mouse alleles may help to identify disease mechanisms that contribute to this phenotypic variability. 
Phosphodiesterase 6. Rod phosphodiesterase 6 consists of a catalytic $\alpha \beta$ complex encoded by $P d e 6 a$ and Pde6b and two inhibitory $\gamma$ subunits encoded by Pde6g. The control of cGMP levels by this enzyme is expected to affect both PR function and viability, as cGMP has a central role in the phototransduction cascade and PR cell metabolism [177], and elevated cGMP levels have been linked to PR cell loss [178]. Indeed, Pde6 $a$ and Pde6b mutants show depressed ERG responses at an early age and rapid PR loss with $\mathrm{D}_{50}$ values of 11-30 days (Figure 6, Table S1). A study of Pde6a mutations on the same strain background made use of an allelic series that varied in disease severity [179]. The order of disease progression due to the alleles reported in this study, nmf282 (Val685Met; fastest) > tm1.1Bewi (Arg562Trp) > nmf363 (Asp670Gly; slowest), is the same as assessed by $\mathrm{D}_{50}$ (Figure 6). This allelic series led to a correlation of more rapid PR degeneration with an increased number of cGMP-positive PR cells [179]. The same trend in the progression of disease in Pde6a $a^{n m f 282}$ and Pde6a ${ }^{n m f 363}$ mice was found earlier [180], but an opposite cGMP result was obtained, possibly due to the assessment of total retinal cGMP rather than a count of cGMP-positive PR cells [179] (a 0.1-month difference in the $\mathrm{D}_{50}$ of $P d e 6 a^{n m f 363}$ mice measured in the two studies may reflect strain differences that might also contribute to the difference in findings). The later study also combined two alleles that matched human PDE6A variants to create a compound heterozygote [179], mirroring the more typical situation in human genetic disease. Further, the allelic series highlighted a non-apoptotic cell death mechanism involving calpain rather than the expected caspase-mediated apoptotic process [179]. Both elevated cGMP and calpain activation have been observed in other mouse RD models [181]. Thus, allelic series as used in these studies are informative for assessing disease mechanisms and identifying potential differences in treatment efficacy that may reflect disease severity.

Of the Pde6b alleles described, $P d e 6 b^{r d 1}$ and $P d e 6 b^{r d 10}$ have been used most extensively as PR degeneration models. Pde6 $b^{r d 10}$ disease develops later, providing a longer window of opportunity to test therapeutic efficacy (Figure 6). The Pde6b $b^{\text {atrd1 }}$ model has an even slower progression $\left(\mathrm{D}_{50}=0.71\right)$ than Pde6b $b^{r d 10}$ mice $\left(D_{50}=0.65\right)$, which may make it more attractive for assessing the variation in treatment with disease severity (Figure 6, Table S1). Finally, loss of the inhibitory subunit in homozygous Pde6g ${ }^{\text {tm1Goff }}$ mice did not lead to an expected increase in catalytic activity; instead PDE6G was found to be essential for activation and possibly stable assembly of the holoenzyme [182].

Cone phosphodiesterase 6 includes two catalytic $\alpha$ subunits encoded by Pde6c and two inhibitory $\gamma$ subunits encoded by Pde6h. The Pde6c cpfl1 mutation leads to severely reduced cone ERG response at three weeks and progressive cone PR loss with age [15] as determined by counting cone nuclei (Bo Chang, unpublished data, presented in Table S1). This model mimics achromatopsia in humans, which is sometimes accompanied by cone PR cell loss [183]. Surprisingly, Pde6h knockout mice show no detectable functional cone loss or degeneration, likely due to the expression of the Pde6g subunit in mouse cones, which may compensate for PDE6H loss [184]. Variants in human PDE6H cause achromatopsia $[185,186]$ but cone cell loss has not been reported.

Cyclic nucleotide gated channels and cation exchanger. The decrease in cGMP levels resulting from PDE6 activation leads to the closing of cyclic nucleotide cation channels in the OS plasma membrane of both rods and cones. Channel closing diminishes the inward flux of $\mathrm{Na}^{+}$and $\mathrm{Ca}^{2+}$ ions that maintain the PR cell in a hyperpolarized state. The rod protein encoded by Cnga1 and Cngb1 is an $\alpha_{3} \beta_{1}$ heterotetramer, in which the $\beta$ subunit is a long isoform, CNGB1a $[187,188]$. Cnga1 mutations have not yet been described. Rod OSs of homozygous $\mathrm{Cngb1}^{\text {tm1.1Biel }}$ mice yield no detectable CNGB1a or CNGA1, and rapid PR loss is observed [189]. Together with evidence that CNGA1, but not CNGB1a, is capable of self-oligomerizing in heterologous expression systems, this result suggests that CNGB1 plays a critical role in stabilizing CNGA1 for channel assembly during synthesis in the secretory pathway and/or subsequent transport to the OS. Although the mechanisms leading to PR cell loss are unknown, low intracellular $\mathrm{Ca}^{2+}$ may overactivate guanylyl cyclase and cause toxicity due to elevated cGMP [189].

The cone channel encoded by Cnga3 and Cngb3 functions as an $\alpha_{2} \beta_{2}$ tetramer. Due to the absence of downstream synaptic signaling associated with channel defects, mutations in both genes result in 
a loss of cone ERG responses modeling achromatopsia. In addition, the alleles included in Table S1, $\mathrm{Cnga3}^{\text {cpfl5 }}, \mathrm{Cnga3}^{\text {tm1Biel }}, \mathrm{Cngb3}{ }^{\text {cpfl10 }}$, and $\mathrm{Cngb3}^{\text {tm1Dgen }}$ result in cone PR degeneration as assessed by marker analysis, although confirmation of cell loss by a direct nuclear count was lacking in some studies. The mechanism of cell death is unknown in these models, but by analogy may involve elevated cGMP as hypothesized in rods.

A critical component of phototransduction is SLC24A1 (also called NCKX1), which exports sodium and calcium ions in exchange for potassium. This activity is responsible for the decrease in intracellular $\mathrm{Ca}^{2+}$ upon closing of the cGMP-gated channels. Homozygous Slc24a1 ${ }^{\text {tm } 1 \mathrm{Xen}}$ mice exhibit slow degeneration, possible due to malformation of OS discs [190].

Guanylyl cyclase and activating proteins. Photoreceptor guanylyl cyclases function as homodimers encoded by two genes in mice, Gucy2e, and Gucy2f. In the homozygous Gucy2e $e^{\text {tm1Gar }}$ model, $\mathrm{D}_{50}$ was $>12$ months (Figure 6), indicating very slow rod PR cell loss, while cone cell numbers decreased rapidly to $33 \%$ of controls in 5 weeks [191]. Cone loss with rod preservation has been observed in Leber congenital amaurosis cases linked to variants of the human Gucy2e ortholog, GUCY2D [192]. However, Gucy $2 e^{\text {tm } 1 \text { Gar }}$ mice are not considered to model this disease because rod ERG function, though diminished, is still detectable [191]. Although Gucy2f knockout did not cause PR cell loss, double knockout of both guanylyl cyclase genes resulted in moderate degeneration [193]. Rod and cone ERG responses were abolished in this model, suggesting that the residual function in Gucy2 $e^{\text {tm1Gar }}$ mice was due to compensatory activity expressed from Gucy2f. The mechanism of PR cell loss in these models is unlikely to involve elevated cGMP as the enzymes needed for its production are ablated. The post-translational downregulation of other phototransduction proteins in double-knockout mice [193] may indicate a disruption of the proteostasis network that could explain PR cell loss.

Guanylyl cyclase activator proteins provide a feedback loop to restore cGMP levels. When intracellular $\mathrm{Ca}^{2+}$ is high, these proteins inhibit guanylyl cyclase; when $\mathrm{Ca}^{2+}$ levels are low, they switch to an activating $\mathrm{Mg}^{2+}$-bound conformation that promotes cGMP synthesis. This $\mathrm{Ca}^{2+}$-sensitive regulation permits PR cells to reestablish cGMP levels following light exposure due to lowered intracellular $\mathrm{Ca}^{2+}$, thereby resetting the cell for another stimulus. Double knockout of Guca1a and Guca1b, which encode the activator proteins in both rods and cones, had no detectable effect on retinal morphology up to eight months of age [194]. However, homozygous Guca1 $a^{\text {tm1.1Hunt }}$ mice, which have a Glu155Gly missense substitution identical to one found associated with a severe dominant cone dystrophy [195], result in rapid loss of cones and subsequently rods (Figure 6, Table S1). This mutation, like others associated with the human disease, may constitutively activate guanylyl cyclase due to a defect in calcium sensing [196], leading to cytotoxic accumulation of cGMP.

Recovery from light stimuli. Mechanisms to terminate the phototransduction cascade and recover the PR cell for additional stimuli include the phosphorylation of activated RHO by a Grk1-encoded kinase and the binding of Sag-encoded arrestin to the phosphorylated RHO. The binding of SAG limits transducin access to RHO and thereby prevents further activation of transducin and downstream processes. Significantly, defects in either gene induce photoreceptor cell loss, likely due to the accumulation of excess cGMP arising from unregulated active RHO. Early studies aimed at elaborating the role of the SAG or GRK1 proteins used mice raised in the dark [197,198], as typical vivarium cyclic light-dark rearing conditions were described as leading to rapid degeneration. Subsequent studies of homozygous Sag tm1Jnc [199] or homozygous Grk1 ${ }^{\text {tvrm } 207}$ mice [200] reveal slow PR cell loss with $\mathrm{D}_{50}>10$ months under normal rearing conditions.

\subsubsection{Category 03: Metabolism}

Overview. Inborn errors of metabolism constitute a heterogeneous group of disorders that affect metabolic pathways due to underlying genetic defects [201] and result in abnormalities in the synthesis or catabolism of biomolecules [201,202]. Many such inborn errors of metabolism are known to be associated with PR cell loss, manifested either as a primary ocular defect or as part of a systemic disease [201]. PR cells, with their high metabolic activity, are particularly vulnerable to defects in 
metabolism of biomolecules such as lipids, carbohydrates, nucleotides, and proteins, which provide energy and serve many other functions described below. Additionally, since organelles such as mitochondria and lysosomes are the major sites for cellular energy production and homeostasis, defects in organellar metabolism and function are also known to cause PR degeneration. The PR cell loss associated with different metabolic diseases varies in the age of onset, severity, and rate of progression (Figure 6, Table S1) and the underlying genetic defects can be categorized based on the type of biomolecular metabolism or the subcellular location of the pathways affected.

Biomolecular metabolism: lipids. PRs are extremely rich in lipids, which make up to $15 \%$ of their cellular wet weight as compared to $1 \%$ in most other cell types [203,204]. Phospholipids and cholesterol represent $90-95 \%$ and $4-6 \%(w / w)$ of total lipids, respectively [205]. The major phospholipids in rod outer segments include phosphatidylethanolamine, phosphatidylcholine, large amounts of phosphatidylserine, along with small amounts of sphingomyelin, phosphatidylinositol, and phosphatidic acid [205]. It has been suggested that the phospholipids in OS membranes are metabolically active and involved in generation of physiological mediators, and changes in metabolism of glycerolipids have been associated with transduction of visual stimuli [205]. Cholesterol has been reported to modulate the function of rhodopsin, a major protein of the OS membranes, by influencing membrane lipid properties [206]. Low-density lipoproteins (LDLs) are reported to be significant suppliers of PR lipids, especially cholesteryl esters $[207,208]$. The OSs of PRs are particularly rich in very-long-chain polyunsaturated fatty acids (PUFA), such as docasohexaenoic acid (DHA), which is considered to be essential for visual function [209], and phospholipid-containing DHA is suggested to help in isomerization of 11-cis-retinal to the all-trans form, which is further reduced for its entry into the visual cycle [210]. Recently, DHA has also been implicated in the maintenance of OS homeostasis [211] and mediating PR cell survival [212,213].

Thus, it is not surprising that disorders of lipid metabolism cause inherited PR degeneration. For example, mouse models for mutations in the elongation of very-long-chain fatty acids-like 4 (Elovl4) gene are reported to show features resembling Stargardt-like macular dystrophy in humans with cone degeneration preceding that of rods [214,215]. Mutations in genes involved in phospholipid metabolism such as Lpcat1 cause rapid PR degeneration (90\% and 75\% degeneration in Lpcat1 ${ }^{\text {rd11 }}$ and Lpcat $1^{\text {rd11-2J }}$ alleles, respectively by 47 days) [216]. Similarly, mutations in genes involved in cholesterol biosynthesis such as Nsdhl [217] or in the biosynthesis and regulation of DHA-containing phospholipids, such as Agpat3 and Adipor1, respectively [210], also cause PR degeneration, confirming the importance of lipids in preserving PR integrity. Since membrane phospholipid asymmetry is critical to performing various biological functions, mutations in genes important for its generation and maintenance, also lead to PR degeneration. For example, mutations in Atp8a2, a type of P4-ATPase that translocates and maintains phospholipid asymmetry show a 30-40\% PR degeneration by two months of age [218]. Similarly, conditional inactivation of Tmem30a, known to be required for folding and transport of several P4-ATPases to their plasma membrane destination $[219,220]$, also results in severe PR degeneration [221]. Tmem30a knockout mice exhibit a more severe phenotype compared to Atp8a2 knockout mice, possibly because Tmem30a binds multiple P4-ATPases [221].

Biomolecular metabolism: carbohydrate and nucleotide energy metabolism. The retina, and in particular PRs, have a high metabolic rate $[222,223]$ to support functions that are energetically demanding, such as phototransduction during constant illumination, maintenance of ion gradients in darkness, and performing anabolic metabolism to replace the approximately $10 \%$ of OSs that are lost every day to phagocytosis by RPE cells [223]. RPE cells also perform many energy demanding functions, such as maintenance of appropriate ionic and fluid composition in the subretinal space, uptake and conversion of all-trans-retinol to 11-cis-retinal and its transport back to photoreceptor cells, and OS phagocytosis. This high energy requirement makes the retina and RPE particularly vulnerable to functional deficits induced by deficits in energy metabolism [222]. The retina relies on blood-derived glucose and oxygen for its energy requirements. Additionally, PR cells use excess lactate obtained from Müller glial cells and convert it to pyruvate to provide energy via oxidative 
phosphorylation [222]. In addition to carbohydrates, the retina uses fatty acids [224] and nucleotides for its energy requirements [223].

Thus, neuronal activity and energy metabolism are tightly coupled and any mutations at the level of glucose, fatty acid or nucleotide biosynthesis can lead to PR degeneration. For example, mice lacking $H k d c 1$, which encodes a kinase found in the IS that phosphorylates glucose to glucose-6-phosphate, show $40 \%$ PR degeneration by 17 months [225]. Mice mutant for Vldlr, which encodes the receptor facilitating the uptake of triglyceride-derived fatty acids, show reduced cellular uptake and availability of fatty acids for energy production [224]. For some alleles of Vldlr (Vldlr ${ }^{m 1 B t r}$ and Vldlitm1Her), more than $50 \%$ of PRs are lost by $12-14$ months [226,227], with cones being affected more significantly than rods [228]. The decrease in net available energy may lead to greater cone loss, as cones have been reported to require three times more energy than rods [222]. Similarly, while in some cases, mutations in genes involved in nucleotide metabolism such as Nampt, show embryonic lethality [229], others such as mutation in Nmnat1, show severe PR degeneration by 4-6 months [230].

Biomolecular metabolism: hormones. The physiology of eye is also dependent on the action of several hormones [231]. Mouse models mutant for thyroid hormone metabolizing genes, such as Dio3, which is important for local amplification of triiodothyronine (T3), show selectively detrimental effects on cone cells [232]. This confirms the proposed role of thyroid hormone signaling in regulating cone viability and cone opsin expression [232,233]. Melatonin, a hormone that plays a role in sleep patterns, is known to have protective role against oxidative stress and apoptosis, and regulates retinal circadian rhythms [234]. A mouse model, mutant for the melatonin hormone receptor Mtnr1a, shows very slow PR degeneration (25\% in 18 months) [235].

Biomolecular metabolism: oxidative stress. The eye is constantly subjected to oxidative stress due to daily exposure to light, atmospheric oxygen, and high metabolic activities [236]. Reactive oxygen species (ROS) are derived from diatomic oxygen and processes such as mitochondrial respiration that form superoxide anion radicals, toxic bis-retinoids that undergo photo-oxidation, and lipids, such as PUFAs, that undergo peroxidation [237]. Having unpaired electrons confers a great degree of ROS reactivity that can damage biomolecules such as DNA, lipids and proteins, and organelles including mitochondria and lysosomes [238,239], thereby impairing their biological functions $[203,236]$. Compared to other cells, non-proliferative postmitotic cells such as PRs and RPE cells are particularly sensitive to oxidative damage due to the apparent absence of a DNA damage detection system [240-242].

Under physiological conditions, cellular redox homeostasis is maintained by a balance between ROS generation and antioxidant systems [236]. Antioxidant enzymes such as Sod1, Sod2, and Gpx4 are known to play a major role in ROS scavenging and changes in their expression or activity or both are reported to cause increased oxidative stress and are associated with diseases such as age-related macular degeneration (AMD) [243]. For example, mutations in the Sod1 gene, encoding a cytosolic $\mathrm{Cu}-\mathrm{Zn}$ superoxide dismutase that catalyzes the conversion of superoxide to hydrogen peroxide, are known to cause PR degeneration [244]. Sod2, which encodes a mitochondrial Mn superoxide dismutase, is required for survival and mutations in this gene lead to embryonic lethality $[244,245]$. Genes such as Nxnl1 and Nxnl2, known as rod-derived cone viability factors are also suggested to have antioxidant function and show cone degeneration when mutated [246,247], with Nxnl1 also showing a progressive rod cell loss [246]. Similarly, a mouse model for loss of Ttpa, coding for a protein that transports vitamin $\mathrm{E}$, which is known to have antioxidant function, also shows $40 \%$ PR degeneration by 20 months [248].

Organellar metabolism: lysosomes. The lysosome, a subcellular organelle is critical for performing several vital functions such as degradation of extracellular and intracellular material, nutrient sensing, energy metabolism, and maintaining cellular homeostasis [249]. Lysosomes contain a wide variety of hydrolytic enzymes that enzymatically degrade biomolecules such as polysaccharides, lipids, etc. [250]. Defects in lysosomal function results in lysosomal storage disorders, a group of inherited metabolic disorders sharing a common biochemical feature of accumulating incompletely degraded metabolites within the lysosomes. Lysosomal storage disorders are generally classified by the composition of the 
material accumulated within them and often differ depending on the lysosomal proteins affected, which reflect different cell biological processes that are affected but terminating in a similar pathology of reduced clearance of metabolic aggregates.

$\mathrm{RD}$ is an early consequence of lysosomal storage diseases, especially in neuronal ceroid lipofuscinoses (NCL) [251], also called Batten disease, an early-onset neurodegenerative disease with other systemic features such as dementia and epilepsy [252]. NCL may be caused by disruption of genes encoding lysosomal enzymes (Ppt1 and $C l n 5)$ and membrane proteins $(M f s d 8)$ as well as ER membrane ( $\mathrm{Cln} 6$ and $\mathrm{Cln} 8$ ) and secretory pathway ( $\mathrm{Gr}$ ) proteins, and is characterized by a common lysosomal accumulation of ceroid. Similar to the early retinal phenotype reported for most human NCLs, most mouse models for NCL disease show an early onset of PR degeneration, beginning at 1 month of age and showing greater than $60 \%$ degeneration by 6-9 months [253-256]. Additionally, similar to the adult-onset reported for mutations in human GRN, the mouse model for loss of Grn also shows a late onset PR degeneration by 12 months [257].

Mouse models for other lysosomal disorders, namely, mucopolysaccharidosis and mucolipidosis due to mutations in lysosomal proteins required for the breakdown of glycosaminoglycans and enzymes required for phosphorylation of glycoproteins, respectively, also develop PR degeneration. For example, mouse models for mucopolysaccharidosis with a mutation in Naglu present with a slowly progressive rod-cone degeneration [258], and for mucolipidosis with a mutation in Gnptab develop a severe PR degeneration with complete PR loss by 10 months [259].

The lysosome receives materials for degradation via two major pathways, autophagy and phagocytosis. Phagocytosis has an important function in maintaining retinal health since $10 \%$ of the OSs are phagocytosed daily by the RPE cells to dispose of waste such as photo-oxidative products while retaining and recycling useful contents back to the PR cells [260]. Phagocytosis by RPE requires its own machinery for processes such as recognition (e.g., Cd36), engulfment (e.g., Mertk), and degradation (lysosomal enzymes) of the extracellular material. Disruption of the phagocytic machinery due to absence/mutations in proteins involved in the phagocytic pathway, therefore, have severe consequences for PRs and can lead to PR cell death. Mouse models for mutations in genes involved in phagocytosis such as Mertk, Cd36, and Rab28 show PR degeneration with the loss of Mertk showing a more severe phenotype (>80\% degeneration by 60 days for Mertk $k^{t m 1 G r l}$ and Mertk $\left.{ }^{t m 1 G k m}\right)[261,262]$ than loss of Cd36 (17\% degeneration at 12 month) [263], and the model for Rab28 loss showing a more cone-specific response [264].

Autophagy is another lysosome-mediated degradation process essential for maintaining cellular homeostasis [265]. Autophagic flux, the complete dynamic process of autophagy, includes multiple steps involving the formation of phagosomes and autophagosomes, autophagosome fusion with lysosomes, the degradation of the intra-autophagosomal contents, and recycling [266]. Thus, both lysosomal function and autophagy are interconnected wherein disruption of the hydrolytic functions of lysosomes impairs autophagic flux and, conversely, lysosomal function requires normal flux through autophagy $[267,268]$. In the retina, autophagy plays a dual role: promoting cell survival against harmful stress, and cell death. High basal autophagic levels are maintained in RPE and PR cells. RPE cells being post-mitotic phagocytes are not self-renewing; the autophagy of intracellular components is therefore essential for a normal cellular function of the RPE [265]. In PR cells, autophagy occurs during various cellular activities such as OS degeneration [269], rhodopsin protein expression [270], visual cycle function, and PR apoptosis [271]. Mouse models of conditional inactivation of autophagy genes such as Atg5, Atg7, and Rb1cc1 in RPE cells show that these genes are indeed important for survival of the animal and show PR degeneration.

Organellar metabolism: mitochondria. Mitochondria, often referred to as "the powerhouse of the cell", are the major site for cellular energy production in the form of ATP via oxidative phosphorylation. They also perform other important functions such as ROS generation and scavenging, calcium regulation, steroid, and nucleotide metabolism, regulation of intermediary metabolism, and initiation of apoptosis [272]. Oxidative phosphorylation is carried out by the mitochondrial respiratory chain, 
which consists of five complexes located along the inner mitochondrial membrane. These complexes, in an intricately organized series of biochemical events, synthesize ATP from ADP in response to cellular energy demands. A large number of mitochondria are present in the rod and cone IS and in RPE cells. The total surface area of the inner mitochondrial membrane in cones is 3-fold greater than in rods, presumably accommodating more respiratory chain enzymes to generate more ATP. Cones require more ATP than rods as they do not saturate in bright light and use more ATP/sec for light transduction and phosphorylation [222].

Defective cellular energy production due to abnormal oxidative phosphorylation in mitochondria can therefore lead to PR degeneration. A mouse model for the Leu122Pro mutation of OPA3, a protein hypothesized to be important for maintaining the inner mitochondrial membrane, is reported to cause a multisystemic disease characterized by severely reduced vision, loss of ganglion cells and PR degeneration (by 50\%) at 3-4 months of age, a much more severe progression than observed in humans [273]. Similarly, a mouse model for a mutation in the gene for NAD-specific mitochondrial enzyme isocitrate dehydrogenase 3 (Idh $3 a$ ), catalyzing the rate limiting step of TCA cycle, also causes an early and severe PR degeneration (more than $90 \%$ ) by 90 days [274].

Extra-mitochondrial components of the tricarboxylic acid cycle and oxidative phosphorylation machinery have been localized to the rod OS [275]. It has been hypothesized that perturbation of this machinery results in excess ROS production, leading to PR cell death due to oxidative stress [275-277]. Mutations in a subset of mouse RD models in Table S1 alter genes (Mpc1, Opa3, Idh3a, Impdh1, and Oat) that encode mouse homologs of mitochondria-associated proteins identified in bovine rod OS [275]. Of these, only IDH3A is directly involved in cellular energy production [274]; the others may influence oxidative phosphorylation or the TCA cycle indirectly, possibly altering the generation of ROS. It may be of interest to determine whether PR cell loss in these mouse models correlates with an altered distribution of extra-mitochondrial oxidative phosphorylation proteins in the rod OS [278], or an increased ROS production, which can be measured in retinal explants [279].

Organellar metabolism: peroxisomes. Peroxisomes are subcellular organelles with various catabolic and anabolic functions such as catabolism of long chain fatty acids and biosynthesis of DHA and bile acids [280]. Several childhood multisystem disorders with prominent ophthalmological manifestations have been ascribed to the malfunction of the peroxisomes, either at the level of peroxisomal biogenesis (PBD) or single enzyme deficiencies [281]. While little is known about the metabolic role of these organelles in retina, studies have shown the presence of peroxisomes in nearly all layers of retina and RPE, albeit with differential expression of lipid metabolizing enzymes, suggesting different functions in different cell types [282]. For example, Zellweger spectrum disorder (ZSD) is a disease continuum known to result from inherited defects in Pex genes essential for normal peroxisome assembly. Mice homozygous for the G844D point mutation in Pex1 show a decreased ERG response and loss of cone PRs (up to $80 \%$ ) by 22 weeks, recapitulating the abnormal retinal function phenotype in ZSD patients with mild disease [283]. The retinal pathology in such disorders suggests the importance of peroxisomes in maintaining retinal homeostasis and function.

\subsubsection{Category 04: Visual Cycle and Retinoids}

The visual cycle reisomerizes vitamin A retinal that has been released from visual pigments in PR cells, allowing regeneration of the bleached pigments and the subsequent detection of additional light stimuli. The process is catalyzed by enzymes located in PR and RPE cells, so the retinoid intermediates in the process must be transported between them. Mutation of genes involved in the visual cycle pathway cause PR degeneration, in most instances with a moderate to slow progression depending on the allele and the genetic background. Most Rpe65 mutant alleles show moderately slow PR cell loss ( $\mathrm{D}_{50}=7-11$ months) [284-288]. Allelic effects are observed in models bearing missense mutations, Rpe6 $5^{\text {tm1 Lrcb }}$ [289] or Rpe65 ${ }^{\text {tm1.1Kpal }}$ [290], which cause slower progression than observed in Rpe65 $5^{\text {tm1Tmr }}$ knockout mice [285-288]. Abca4 ${ }^{\text {tm1Ght }}$ on the BALB/c strain, which also carries a homozygous Rpe65 Leu450Met mutation, show a late-onset PR degeneration with $40 \%$ loss by 11 months of age [291]. 
By contrast, the same Abca4 $4^{\text {tm } 1 \text { Ght }}$ mutation on a 129S4/SvJae background results in abnormal thickening of Bruch's membrane but normal ONL nuclei count and thickness [292]. Several visual cycle mutant alleles have other retinal abnormalities but normal ONL nuclei/thickness. For example, Abca4 ${ }^{\text {tm1.1Rsmy }}$ causes only autofluorescence and A2E accumulation [293] and Abca4 $4^{\text {tm2.1Kpal }}$ on C57BL/6*129Sv leads to a RPE defect but normal ONL nuclei count and thickness [294]. In addition, PR degeneration in Abca4 mutants can be induced by light exposure [295] or through interaction with other genes such as Rdh8 [296-298]. The Lrat ${ }^{\text {tm } 1 \text { Kpal }}$ mutation on a 129S6/SvEvTac ${ }^{*}$ C57BL/6J background results in mild PR degeneration, with $<10 \%$ loss at $4-5$ months [299]. However, a 35\% decrease in rod OS length was also reported in this model, indicating the importance of the visual cycle for OS maintenance. Another allele, Lrat ${ }^{\text {tm1.1Bok }}$, showed a similar loss of rod OS length and 18\% PR degeneration at 6 months of age [300]. The Rbp $3^{t m G i l}$ mutation results in the most rapid PR cell loss in this category $\left(D_{50}=0.79\right.$ months), possibly attributable to an early developmental role of the protein [301]. The Rbp $4^{\text {tm2Zhel }}$ congenic mutation on C57BL/6J showed 20\% PR cell loss in some peripheral areas and $10 \%$ in the central retina an age of 40 weeks [302]. Mutations in two genes that play a role in retinoid uptake in the eye also result in PR cell loss. The Rtbdn ${ }^{\text {tm1.11tl }}$ allele causes a slow degeneration with a $20 \%$ and $37 \%$ loss of PR nuclei at 240 days of age in heterozygotes and homozygotes, respectively. Stra $6^{\text {tm } 1 \mathrm{Nbg}}$ mice exhibit a normal number of rod PR nuclei but significant cone PR cell loss as detected by the

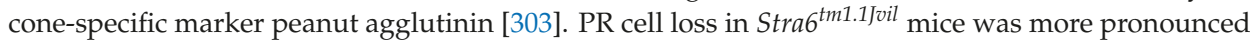
with vitamin A restriction [304].

\subsubsection{Category 05: Synapse}

PRs absorb light that passes through the anterior portion of the eye and convert the light to electrochemical signals that are transmitted through the neuroretina via synaptic connections to the optic nerve and visual cortex [305]. Thus, synapses, necessary for proper cell-to-cell communication, are critical for vision. Discussion of the complexity of PR synaptic development and function is reviewed in [306-308], and is beyond the scope of this review. Suffice to say that mutations in many of the components of synapses, such as presynaptic exocytotic proteins, endocytic proteins, calcium channels, postsynaptic receptors, and associated elements, must be properly organized to mediate transmission of signals, or can lead to visual problems [307]. It is interesting to note that disruption of some synaptic components of the secondary neurons (e.g., GRM6, GPR179, TRPM1, NYX, GNAO1, GNB5, and GNB3), while affecting function as assessed by ERG response, does not normally lead to PR degeneration [309]. This is also true of some presynaptic proteins, such as dystrophin [310] or dystroglycan [311]. However, disruption of some synaptic genes such as Ache, Cabp4, Cacnalf, Cacna2d4, and Unc119 does lead to PR degeneration. For example, a null allele of Ache [312], causes a $50 \%$ loss of PR nuclei between 1.5 and 2 months and $>80 \%$ by $6-8$ months. Although it was initially determined that the ACHE protein played an important role in hydrolyzing acetylcholine at synapses, its isoforms are now recognized to have far reaching structural functions [313]. Additionally, it has been shown that the loss of secondary neurons in the null allele model is likely to cause secondary PR cell loss [312]. Null or spontaneous alleles of synaptic genes that encode subunits of calcium channels that regulate the release of neurotransmitters, and the development and maturation of exocytic function of PR ribbon synapses, Cacna1f [314] and Cacna2d4 [315,316], respectively, show a slower rate of degeneration. By two months, there are approximately $10-25 \%$ of PR nuclei that have degenerated. CABP4, a protein that regulates calcium levels and neurotransmitter release at PR synapses, and modulates CACNA1F and other calcium channel activity shows a similar rate of PR degeneration of 10-25\% loss at 2 months [317]. UNC119, which localizes to PR synapses (and IS) and is hypothesized to play a role in neurotransmitter release, also leads to a relatively late onset, slower rate of PR degeneration [318]. Interestingly, Haeseleer has described an interaction between synaptic genes Cabp4 and Unc119 [319]. It is likely that other synaptic proteins will also lead to PR degeneration through either a primary or secondary effect and that the interactions among the synaptic proteins will play a significant role in determining the relative rate of the degenerative process. 


\subsubsection{Category 06: Channels and Transporters}

Ions such as sodium, potassium, and chloride, play important roles in the visual circuitry [320]. Their intracellular concentrations and movements within the cell, and between cells and the environment are exquisitely regulated by channels and transporters. Due to the importance of maintaining appropriate levels of these ions for proper function and maintenance of PRs, it is not surprising that disruption of these genes can lead to PR degeneration. Members of the ClC family of chloride channels, such as $\mathrm{Clcn} 2, \mathrm{Clcn} 3$, and $\mathrm{Clcn}$ 7, show particularly early and significant PR degeneration. Compared to other channels in this section, they appear to have an enriched expression in the RPE. A 50\% PR cell loss can be seen as early as 14-16 days in certain models with disruptions in these genes [231,321-323]. Indeed, rapid progression of PR cell loss is observed in mice carrying any of the following alleles: Clcn2 ${ }^{\text {nmf240 }}, C l c n 2^{t m 1 T j j}, C l c n 3^{t m 1 L a m b}, C l c n 3^{t m 1 T j j}, C l c n 7^{t m 1 T j j}, C l c n 7^{t m 2 T j j}$, and $C l c n 7^{t m 4.1 T j j}[231,321,322,324-327]$. A similar rapid and complete loss of PRs is seen when Atp1b2, a Na ${ }^{+} / \mathrm{K}^{+}$-ATPase thought to play a role in cell adhesion, is inactivated [328]. A targeted mutation in Slc6a6, which encodes a taurine/beta-alanine transporter, also leads to rapid, complete degeneration [329]. In contrast, inactivation of the bicarbonate, amino acid transporters, and $\mathrm{Na}^{+} / \mathrm{H}^{+}$ exchangers, Slc4a7, Slc7a14, and Slc9a8 results in a later onset, but still severe PR degeneration [330-332]. Mouse models bearing mutations affecting BSG, a protein that has a role in targeting monocarboxylate transporters such as SLC16A1 to the plasma membrane, or REEP6, which mediates trafficking of clathrin-coated vesicles from the ER to the plasma membrane at outer plexiform layer sites enriched for synaptic ribbon protein STX3, also fall in this latter late onset/severe category [333,334]. Asic3, an acid sensing $\mathrm{Na}^{+}$channel, Clcc1, an intracellular chloride channel, and Slc7a14, an intracellular arginine transporter, all cause moderately slow degeneration when mutated [331,335,336]. Slowly progressive PR cell loss is also caused by mutation of $M f s d 2 a$, which encodes a sodium-dependent lipid transporter responsible for maintaining a high DHA concentration in the retina is important for OS homeostasis, as discussed in Category 03 [337]. More data are needed to see if sensing and intracellular channels/transporters generally have milder phenotypes.

\subsubsection{Category 07: Adhesion and Cytoskeletal}

Proper structure of the retina is developed through protein interactions between cells and within cells. The spatial and laminar organization of the retina is maintained through junctional interactions between cells that impart mechanical support to maintain retinal architecture, a means for bidirectional communication (e.g., extracellular changes to the cell and from the cell to its environment), and together can form diffusion barriers. Within cells, cytoskeletal architecture is maintained through interactions of proteins with actin, intermediate filaments, and microtubules that serve to maintain cell morphology and polarity, and as discussed elsewhere, intracellular trafficking, contractility, motility, and cell division. Examples of disrupted proteins that lead to gaps between cell layers, presumably through aberrant adhesion, are mutations in Adam9 and Rs1. The null allele of Adam9, a single pass transmembrane protein with disintegrin and metalloprotease domains that has been shown to interact with a number of integrins [338], leads to aberrant adhesion between the apical processes of the RPE and OS and to late onset PR degeneration [339]. Likewise, mutations in RS1, a protein with a discoid domain, which has been implicated in cell adhesion and cell-cell interactions [340] lead to a splitting of the inner retinal layer and progressive PR loss [341-343]. RS1 binding to phospholipids on the membrane surface, together with other proteins [340] may provide a stabilizing scaffold that is important in cell-matrix, cell-cell, and cytoskeletal organization.

CRB1 and its interacting partners, such MPP3, MPP5, and PARD6A, have been shown to be important in establishing proper retinal lamination presumably through their essential roles in establishing cellular apical basal polarity [344]. A primary defect of a disruption in CRB1 is the fragmentation of the outer limiting membrane [345,346]. As reviewed previously [344,347], the outer limiting membrane consists of adherens/tight junctions formed in part by the CRB1 complexes between Müller glia and the rod or cone IS that form a diffusion barrier. Loss of components of the CRB1 
complexes (CRB1-MPP5-PATJ, CRB1-MPP5-MPDZ, and CRB1-PARD6A-MPP5-MPP3/MPP4) leads to lamination defects with formation of rosettes and a progressive loss of PRs. Although yet to be reported, it is likely that mutations in PATJ, PARD3, MPDZ, and MPP4 will lead to similar disease phenotypes, as a reduction in ERG response in MPDZ mutants [348] and a reduced ERG with abnormal retinal morphology have been indicated for PARD3 mutants [41,43].

Equally important to the function and maintenance of the retina are the intracellular components that make up the cytoskeletal cell structure. Disruption of proteins that interact with actin intracellularly have been shown to lead to PR degeneration. For example, CDC42, a small GTPase that is a key regulator of actin dynamics [349] leads to an early onset, progressive PR degeneration when disrupted. Models caused by mutations in FSCN2, an actin crosslinking protein [350,351], and by a hypomorphic variant of CTNNA1, a protein that coordinates cell surface cadherins with the intracellular actin filament network [352], show slow-paced PR cell loss. The proper localization of organelles within the cell is also mediated by the cytoskeletal architecture and can have an untoward effect when disrupted. For example, SYNE2, a nuclear outer membrane protein that binds to F-actin, tethers the nucleus to the cytoskeleton and is necessary for the structural integrity of the nucleus $[353,354]$. Without it, early onset, moderately paced PR cell loss occurs.

\subsubsection{Category 08: Signaling}

Molecules such as growth factors/cytokines, hormones, neurotransmitters, and extracellular matrix proteins, or alternatively, mechanical stimuli, are examples of signals used to communicate environmental changes to the cell. Surface or intracellular (e.g., nuclear) receptors recognize the signals and effect changes within the cell, often setting in motion amplifying transduction cascades that mediate responses such as activation or inhibition of protein activity or migration to different cellular localizations. Further, signals can also be transmitted from the cell to other cells, for example, through neurotransmitters. Since intra- and intercellular communication is crucial for the proper development or function of cells, it is not surprising that a large number of mutations in cellular signaling lead to defects in retinal development, which in turn affects PR survival. For example, vascular development is affected in mutants bearing disruptions in Fzd5, Lrp5, Ndp, and Tspan12-all components of the Wnt signaling pathway. Integral membrane frizzled receptors, of which they are 10, together with coreceptors, LRP5 and LRP6, mediate canonical Wnt signaling [355]. Thus, conditional Fzd5 null mutants develop microphthalmia, coloboma and persistent fetal vasculature, and late-onset progressive RD [356] and $L r p 5$ mutants exhibit similar vascular and retinal phenotypes [357]. Mice that are null for NDP, a ligand for FZD4, exhibit delayed retinal vasculature development, retrolental masses, disorganization of the ganglion cell layer, and occasionally focal areas of ONL absence at later stages of the disease [358]. TSPAN12, mediates NDP-FZD4-LRP5 signaling in the retinal vasculature, where it localizes, and a mutation leads to vascular defects that phenocopies disruptions in Ndp, Fzd4, and Lrp5, and at 3 months exhibits a 50\% loss of PRs [359]. In all of these models, it is likely that the loss of PRs is caused by the aberrant retinal vasculature having secondary effects on PRs. A review by Hackam suggests that Wnt signaling may affect the apoptotic pathway and neurotrophin release, dysregulation of which may affect PR survival [360]. MFRP, which bears a CRD domain shared by all frizzled proteins, also leads to PR degeneration when disrupted [361,362], as does the human knock-in allele, p.S163R, of its bicistronic partner, CTRP5 [363]. The exact role or function of either protein has yet to be fully elucidated.

Like the frizzled-associated proteins whose pathological effects on PRs are likely to be mediated through an aberrant retinal vasculature, other signaling molecules, Ptpn11 and Fyn, appear to mediate their effects on PRs through another cell type as well, in this case, Müller glia cells, and PRKQ through the RPE. A Six3-cre mediated conditional knockout of Ptnp11 [364] leads to altered ERK and MAPK signaling in Müller glia and alteration in their adhesive capabilities. FYN, a Src-kinase membrane associated tyrosine kinase, localizes to Müller glia cells, and FYN deficiency leads to altered adhesion properties of Müller cells and retinal dysmorphology [365]. PRKCQ, a serine threonine protein kinase, 
which localizes to the lateral surface of the RPE cells, causes a reduction in adhesion between the apical processes of the RPE and OSs when it is disrupted. The reduction in adhesion may be responsible for the retinal detachment and subsequent PR loss observed in this model [366].

The family of PI3Ks or phosphoinositide 3-kinases, made up of catalytic and regulatory subunits, function to phosphorylate the inositol ring of phosphatidylinositol and thereby regulate growth, proliferation, differentiation, motility, survival, and intracellular trafficking. For example, it mediates insulin-stimulated increase in glucose uptake and glycogen synthesis and responds to signals such as FGFRs and PDGFRs. Conditional knockouts of $P i k 3 c b$, encoding a catalytic subunit [367] and Pik3r1, encoding a regulatory subunit [368], using the cone-specific CRE, $\mathrm{Tg}(\mathrm{OPN} 1 \mathrm{LW}-\mathrm{cre}) 4 \mathrm{Yzl}$, lead to progressive cone PR loss. IRS2, necessary for the integration of signals from insulin and IGF1 receptors, causes an early-onset, moderately paced PR loss [369]. Additionally, a targeted conditional allele of PDGFRB developed diabetic retinopathy like features with angiogenesis, proliferative DR-like lesions, pericyte drop out, and eventual PR loss [370]. Disruption of MAP3K1, a serine/threonine kinase, which participates in the ERK, JNK, and NF-KB signaling pathways, leads to retinal laminar and vascular defects, aberrant RPE, and PR cell death [371]. SEMA4A, a transmembrane protein, also causes PR loss, most probably through its effects on endosomal sorting [372].

\subsection{Category 09: Transcription Factors}

In mice, cone and rod PRs are born and develop between approximately E12 and P0, and approximately E13.5 and P7, respectively, from the same multipotent retinal progenitor cell (RPC) pool [373]. PR development, orchestrated by a network of transcription factors, is divided into five phases: proliferation of multipotent RPCs, restriction of RPC competence, cell fate specification, expression of genes important for PR function, and finally, PR structural maturation [374,375]. RB1 and E2F1 function by controlling the G1 to S phase transition in the cell cycle; RB1 plays an inhibitory role until activated by phosphorylation, balancing cell proliferation and cell fate specification [376]. OTX2 is critical for fate determination, while CRX is necessary for terminal PR differentiation and acts at different steps in PR development. Transcriptional factors important for rod PR subtype specification include ROR $\beta, N R L$, and NR2E3, and for generation of the cone subtypes, TR $\beta 2$ and RXR $\gamma[374,375]$. Further, transcription factors regulate the expression of other transcription factors in the network (e.g., CRX interacts with $\mathrm{Nrl}$, Rorb, and Mef2d, to name a few, to mediate rod differentiation, cone differentiation, and proteins necessary for the maturation of the PR, respectively).

The importance of transcription factors in retinal development has been explored in many studies resulting in a number of mouse models with different disease phenotypes (MGI JAX). In many cases, disruption of transcription factors, especially those affecting earlier phases of PR development lead to a reduction in the total number of retinal cells generated. We have only included within this category those disrupted transcription factors that eventually lead to PR degeneration. Interestingly, the onset of degeneration of the PR transcription factor models is highly variable-14 days to 2 months of age-and appears to be dependent upon the method used to generate the model and possibly background strain, as variation of severity and onset differs among different models of the same gene. Interestingly, the $\mathrm{Cr} x$ models provide a series that recapitulate the clinical diagnoses of autosomal dominant cone-rod dystrophy, Leber congenital amaurosis, and late-onset dominant retinitis pigmentosa. Crx $x^{\text {Rip }}$ heterozygotes showed $34 \%$ degeneration at five weeks, compared to mice homozygous for the mutation, which reached $55 \%$ degeneration at the same age [377]. Cr $x^{t m 1.15 m g c}$ was also noted to have a heterozygous disease presentation more similar to a cone-rod dystrophy, while the homozygous mutant presented with a disease phenotype similar to Leber congenital amaurosis with $70 \%$ loss of PRs at one month of age [378].

Other transcriptional factors necessary for the proper maturation of the PRs, such as, MEF2D, shown to be important in regulating transcription of OS and synaptic proteins [379], or NRF1 [380], important in mitochondrial biogenesis also develop PR degeneration when disrupted. Finally, there are transcriptional factors that are important in the development or function of supporting cells such as 
ONECUT1 for horizontal cells [381] and MITF for RPE and/or choroidal melanocytes [382], which affect PR survival when disrupted.

\subsection{Category 10: DNA Repair, RNA Biogenesis, and Protein Modification}

Among the many disrupted genes that lead to PR degeneration, several instances have been documented in genes necessary for producing fully functional proteins, from transcription through post-translational modification. Defects in these genes are likely to impact the function of many other genes that they act upon, and hence, have a greater effect. Since they play a central and basic role, when disrupted they often lead to prenatal lethality in mice, and the adult phenotype is unknown unless a conditional knockout or hypomorphic allele is generated. For example, disruption of DNA repair genes such as Ercc1, RNA splicing genes such as Prpf3, Prpf6, Prpf8, Prpf31, and Bnc2, and miRNA processing genes, Dicer1 and Dgcr8 are prenatal lethal in a homozygous state [34,383]. In contrast, homozygous null alleles of Bmi1 [384] and Msi1 [385], both involved in repression of regulatory genes in embryonic development, are viable, suggesting potential compensatory mechanisms for the functional loss of these genes. Thus, germline, conditional or hypomorphic models were considered in this category.

Review of genes in this category suggested that a DNA damage response network to ensure transcription in the face of DNA lesions might be required for PR cell maintenance. DNA lesions, such as pyrimidine dimers, interstrand crosslinks, or double-strand breaks (DSBs), are induced by many mechanisms that include UV radiation or free radicals. Repair of such damage is essential for DNA replication and, of particular importance for long-lived post-mitotic neuronal cells, transcription [386-388]. Proteins encoded by Bmi1, Dgcr8, Dicer1, Elp1, Ercc1, Ercc6, Msi1, Sirt6, Top $2 b, U b b$, and Uchl3 are known to participate in the DNA damage response [387,389-398], some in transcription-coupled DNA repair. For example, BMI1 represses transcription at sites of UV-induced DNA damage to allow repair [389]; ELP1 is a required component of the Elongator complex [399], which couples RNA polymerase II to an alkyladenine glycosylase that initiates base excision repair [392]; ERCC6 promotes DSB repair in actively transcribed regions by displacing RNA polymerase from the lesion site [387], and DGCR8 interacts with both RNA polymerase II and ERCC6 to mediate transcription-coupled nucleotide excision repair of UV-induced DNA lesions [390]. Intriguingly, topoisomerase TOP2B, which creates DSBs during transcriptional activation [396], has been identified as a key regulator of transcription during the last stages of postnatal PR development [400]. Thus, DSBs in PR cells may arise in part from transcriptional activation of genes that encode components destined for the OS. Additionally supporting the importance of DNA repair to PR maintenance, Category 01 gene Atr encodes a master regulator of the DNA damage response that has surprisingly been linked to retinal degenerative disease and localized to the cilium [401]. Further, Category 03 gene Nmnat1 encodes an enzyme that synthesizes nicotinamide adenine dinucleotide in the nucleus, which may regulate the large-scale polyADP-ribosylation of protein targets at sites of DNA damage [402]. Mutations in the genes encoding these proteins all result in PR cell loss [230,384,385,400,401,403-411]. Mutations in five of these genes as included in Figure 6 (Cwc27, Ercc1, Ercc6, Sirt6, and Ubb) caused moderate to slow progression of PR cell loss ( $D_{50} \geq 2$ months), consistent with a steady accumulation of unresolved DNA damage with age. The rapid PR cell loss observed in $A t r^{t m 1 O f c}$ mice $\left(D_{50}=13\right.$ days) may reflect its direct involvement in OS development [401] in addition to the DNA damage response.

Due to the high percentage of alternatively spliced genes in the human retina [412,413], it is not surprising that mutations in mRNA splicing genes: PRPF3, PRPF4, PRPF6, PRPF8, PRPF31, PDAP1, and $B N C 2$ have been shown to lead to PR degeneration in humans [3]. In fact, in human retinal disease, $14 \%$ of disease genes are categorized as playing a role in RNA metabolism [383]. Interestingly, heterozygous humanized alleles of PRPF3 and PRPF8 and the null allele of Prpf 31 in mice do not recapitulate PR degeneration observed in humans but rather exhibit late-onset RPE degeneration [414]. In contrast, a hypomorphic allele of the mRNA splicing gene, Cwc27, with reduced viability, does lead 
to moderate onset PR degeneration [415]. The differences observed among species require additional studies to unravel the complexities that govern genetic interactions.

Post-translational modification, which occurs by adding modifying molecules to amino acids or removing or altering these modified amino acids, is important for proper folding, transport/trafficking, localization, function, regulation, and/or degradation of proteins. Examples of post-translational modifications include phosphorylation, glycosylation, acetylation, ubiquitination, sumoylation, methylation, and lipidation [416]. Kinases that affect activity by mediating phosphorylation states are described elsewhere, however, post-translational modification genes affecting glycosylation and lipidation/prenylation are prominent among those that lead to PR degeneration. For example, the encoded proteins of Fkrp, Large1, Pomt1, and PomgnT1, necessary for the glycosylation of alpha-dystroglycan, essential for formation of the dystroglycan complex and for proper retinal lamination, lead to moderate rates of PR degeneration when disrupted. Prenylation is critical for proper trafficking and localization of retinal proteins. Of the three genes important in the prenylation and postprenylation processes, conditional loss of Rce 1 leads to an absence of phosphodiesterase subunits PDE6A, PDE6B, and PDE6C from the rod OS, probably due to a failure to prenylate one or more of these proteins [417]. By contrast, ablation of Icmt does not appear to affect phosphodiesterase transport but rather results in lowered levels of prenylated proteins GNAT1, PDE6G, and GRK1 [418], which are essential PR proteins. The null mutation of farnesyl-diphosphate farnesyltransferase 1 , which adds a farnesyl group to the cysteine of the CAAX amino acid motif is prenatal lethal, but as a conditional tissue specific knockout may result in the same PR effects.

Two additional types of post-translational modification involve glycylation and glutamylation of proteins essential for normal connecting cilia function. Disruption of Ttll3, encoding a protein-glycine ligase necessary for glycylation of tubulin, results in an absence of glycylation in PR cells, shortening of the connecting cilia, and slow PR cell loss [419]. Interestingly, PR tubulin glutamylation increased in Ttll3 mutant mice. TTLL5, tubulin tyrosine ligase like 5, adds glutamate residues on proteins. Sun et al. [420] reported that Ttll5 disruption leads to late onset, slowly progressive PR cell loss that phenocopied retinal disease observed in Rpgr mutants. Perhaps this is not surprising as these investigators determined that TTLL5 glutamylates RPGR, a modification that is necessary for normal RPGR function in the PR cilium. Agtpbp1 encodes a metallocarboxypeptidase that deglutamylates target proteins. Its disruption in $p c d$ mutants leads to abnormal tubulin glutamylation [419] and an accumulation of vesicles in the interphotoreceptor space [421], indicating the importance of proper post-translational modification for PR survival.

\subsection{Category 11: Immune Response}

As resident immune cells, microglia survey the retina constantly, presumably with the goal of removing unwanted debris and responding to damage arising from environmental and/or genetic stressors. They respond to damage by eliciting various responses that can range from regenerative to inflammatory depending on the type of injury. Thus, although microglia are unlikely to be instigators in $\mathrm{RD}$, it may well be the case that microglia influence the severity of responses to ocular damage depending on mutations. Mutations in several genes central to the immune system lead to PR degeneration in mouse models. Aire ${ }^{t m 1.1 D o i}$ show early onset PR degeneration with $20 \%$ of ONL thickness loss at 10 weeks with rapid progression to $60 \%$ ONL thickness loss by 18 weeks [422]. C3ar $1^{\text {tm } 1 \text { Cge }}$ mutants show very slow PR degeneration with about $20 \%$ loss at 14 months [423]. Cd46 $6^{\text {tm1Atk }}$ show different rates of PR nuclei loss in male and female mice with $23 \%$ and $31 \%$ at 12 months of age, respectively [424]. Mutations in $C x 3 \mathrm{cr} 1$, normally expressed in immune cells including microglia, were associated with PR cell loss. Homozygous $C x 3 c r 1^{\text {tm1Litt }}$ [425] and Cx3cr ${ }^{\text {tm1Zm }}$ [426] mice on the same C57BL/6J background showed similar rates of PR degeneration with $30 \%$ and $40 \%$ loss, respectively, at $16-18$ months of age. However, $\mathrm{C} x 3 \mathrm{cr} 1^{\mathrm{tm} 1 \mathrm{Zm}}$ mice on the BALB/cJ background show complete nuclei loss at 4 months of age [426]. Cxcr5 ${ }^{\text {tm1Lipp }}$ causes late onset PR degeneration with $20 \%$ loss of ONL thickness at 17 months of age and RPE disorganization [427], whereas ablation of Irf3 and Igfbp3 showed mild PR degeneration 
at 2-4 months of age, about $10-14 \%$ [428,429]. Ccl2 and Ccr2 mutations also led to PR degeneration and fundus lesions, ONL loss in some areas and development of neovascular lesions, resembling phenotypes of AMD [430]. Cfh ${ }^{t m 1 M b o}$ was shown to have an impairment of rod and cone function by ERG and 29\% decreased thickness of Bruch's membrane; however, rod opsin was distributed normally

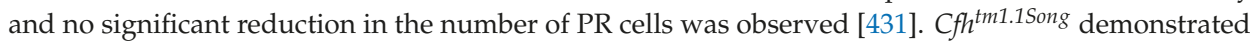
retinal whitening and cotton wool spots by fundus imaging [432]. Other genes involved in immune function that also showed PR degeneration as conditional knockouts encode transforming growth factor beta receptor II (Tgfbr2) [433] and aryl hydrocarbon receptor (Ahr) [434].

\subsection{Omitted Models with PR Abnormalities that May be of Interest}

Based on the exclusion criteria described in the Methods section, a number of models with PR abnormalities caused by single gene mutations were not included in our final Table S1. Since we narrowly defined PR degeneration models as post-developmental loss of PR nuclei, some models, which were described with only OS alterations or ERG differences, were not included. For example, mice bearing a spontaneous point mutation in the Ttc2 $6^{\text {hop }}$ [435] that leads to the generation of a stop codon, Tyr430Ter, were reported to show OS shortening at one year of age with no PR loss. Likewise, ectopic expression of cone opsins in rod OSs led to scotopic ERG abnormalities but not PR degeneration in Samd $7^{\text {tmlTFur }}$ mice at 12 months of age [436]. The many allelic variants that cause ERG abnormalities without PR cell loss are listed in the MGI database and can be accessed through a phenotype query.

\subsection{Factors Leading to Phenotypic Variability}

\subsubsection{Effects of Allelic Heterogeneity}

Allelic heterogeneity is frequently a cause of phenotypic variability. For mouse models, this is often encountered when comparing a knockout model with spontaneous or induced mutations that still allow a protein to be produced. The latter would primarily be hypomorphic alleles due to amino acid substitutions, some splicing mutations that leave alternate splice forms intact and some C-terminal truncating mutations, which may retain some protein function. Often the knockout allele will be the more severe, presumably because in addition to the loss of protein function, the loss of the protein itself may cause secondary defects such as the failure to form a molecular complex that normally needs the native protein to form.

Mutations in the voltage gated calcium channel, Cacna1f, cause congenital stationary night blindness in humans due to abnormal neurotransmitter release in PR synapses. A null mutation in the Cacnalf gene ( $\triangle$ Ex14-17) leads to an absent b-wave, abnormal PR synapses, lack of $\mathrm{Ca}^{2+}$ response in PR terminals and PR degeneration to 8 rows in the ONL at 8 months [314]. In contrast, an Ile756Thr amino acid substitution found in human patients and introduced into mouse, led to a different phenotype with reduced b-wave, some intact ribbon synapses, a strong abnormal $\mathrm{Ca}^{2+}$ response, and a more severe degeneration (3-4 rows at 8 months of age [314]). Here the human allele represents a gain-of-function mutation that in addition to the loss of the original enzyme activity results in a new activity, or causes cell stress, which then induces additional phenotypes and makes the disease presentation more severe.

Within an allelic series of amino acid substitutions there are also frequently gradations of phenotypic severity. If a protein has several functional domains, mutations in different domains may lead to distinct phenotypes. In addition, some mutations can lead to an abnormal tertiary structure of the protein. Such structural changes can lead to a failure to interact with binding partners or substrates/ligands or change the nature of such interactions [437]. Structural changes can also affect export of the protein from the endoplasmic reticulum (ER) and result in ER stress and eventually apoptosis of the cell [438].

One of the larger allelic series available is for human PRPH2 with more than 150 disease causing mutations reported [439]. Although only the secondary structure of the protein is available, some clustering of disease phenotypes is apparent. For example, the area around amino acids 190-220 
on the intradiscal loop 2 is enriched for mutations causing autosomal dominant retinitis pigmentosa. This area is thought to interact with ROM1. Mutations leading to macular degeneration are more frequently present between amino acids 142 and 172. However, some macular degeneration and autosomal dominant retinitis pigmentosa mutations are also found elsewhere in the protein $[439,440]$. Once a 3D structure is available, we may find that the disease specific mutations may well be in spatial proximity and a clearer picture of the genotype-phenotype relation may be revealed.

Allelic heterogeneity can also arise from the intron/exon structure of the gene itself. Many genes produce several distinct transcripts through alternative splicing of their exons [441]. These differing transcripts can each produce proteins, which possess unique functions. For example, the Rpgrip1 gene produces two splice variants that code for proteins that differ at their C-terminus, a full-length transcript and a shorter transcript encompassing exons 1-13 plus three additional C-terminal amino acids. An insertion between exons 14 and 15 of the full-length transcript leads to PRs with vertically stacked OS discs [442], whereas, a chemically induced mutation in the splice acceptor site in intron 6 that leads to a loss of both splice variant forms results in a failure to develop OSs altogether [443].

Despite the promise of genotype-phenotype correlation analyses to aid in the functional annotation of retinal proteins as well as in the diagnosis and prognosis of retinal degenerative diseases, few allelic series are yet available. In humans the analysis is complicated by the fact that environment and genetic background effects can confound the allelic effect. In animal models, large allelic series are not yet available.

Until recently allelic heterogeneity posed a problem for the generation of mouse models for human retinal diseases because only transgenesis and the generation of knockout models by homologous recombination were available. The removal of the gene products using knockouts can only model recessive or haploinsufficiency diseases, and often the complete lack of the protein will lead to embryonic lethality.

Transgenic models are associated with their own set of problems. Depending on the transgene integration site, the expression of the transgene can be reduced or cellularly restricted. Integration into an unrelated gene can disrupt expression of that gene and cause a phenotype that is not related to the transgene. The use of directed transgene insertion into safe sites, such as the Rosa26 locus (Gt(ROSA)26Sor) provides a workaround for some of these problems, although the choice of a promoter that faithfully mimics the native expression is still a difficult process. For these reasons, transgenic mouse models were not included in this review.

With the advent of CRISPR/Cas9 technology to produce precise cuts in genomic DNA, and the ability to perform gene editing through homology directed repair, it is now feasible to recreate human mutations in the mouse and directly probe for the phenotypic effects of allelic heterogeneity [444]. Comitato et al. present an interesting phenotype comparison of transgenic and knock-in rhodopsin P23H models [445].

\subsubsection{Effects of Genetic Interactions}

Gene interaction, or epistasis, is frequently observed during genetic analysis when two or more alleles at different loci combine to alter the onset, type, or severity of disease phenotypes. Such phenotype altering interactions arise from the organization of proteins and RNAs into macromolecular complexes and/or biochemical and regulatory pathways and networks. For example, consider hypomorphic mutations in two proteins that are components of a linear enzymatic pathway. Individually the reduced activity may not greatly impact the flux through the pathway, but combined in the same cell, the pathway flux may be reduced and become severe enough to induce a disease phenotype due to a lack of sufficient pathway product. Alternatively, a mutation may impair Pathway A, so that a disease phenotype arises. A second mutation may arise in a Pathway B that allows it to compensate for the malfunction in Pathway A and thus reduce the severity of the original disease phenotype. Mutations of this latter type of interacting mutations are called suppressor mutations and are extremely useful because they directly identify potential drug targets whose manipulation may be used to treat disease. 
In general, identification of genetic interactors can be useful for placing the primary mutated gene in a biological context and help to define its cellular and organismal function. Often, the known function of a gene and its biology can suggest candidate interacting genes. Similar to the first hypothetical interaction case above, mutations in two proteins involved in iron homeostasis, ceruloplasmin (CP), a ferroxidase associated with transferrin transport across the plasma membrane, and hephaestin (HEPH), implicated in iron transport across cells, individually do not show obvious PR degeneration. Combined in a double mutant mouse model, however, they lead to iron overload in the retina and subsequent RPE abnormalities and PR degeneration [446]. Another example involves two proteins necessary for retinoid recycling, ABCA4 and RDH8. Mutations in each alone do not show any phenotype; combined they cause all-trans-retinoid accumulation and PR degeneration [296]. Since previous studies had suggested that activation of TLR3 may lead to inflammation and mediating apoptosis [447], the authors explored the role of Tlr3 in their Abca4/Rdh8 double mutant model. Importantly, adding a targeted mutation of Tlr3 to make a triple mutant mouse resulted in rescue of PR cells [448]. Here then the Tlr3 mutation acts as a suppressor of the degenerative phenotype of the Abca4/Rdh8 double mutant.

Additional interacting gene pairs have been found that affect PR degeneration, among them

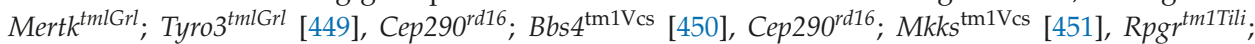
Cep290rd16 [452], Cngb1 ${ }^{\text {tm1.1Biel }}$; Cnga3 ${ }^{\text {tm1Biel }} ; \mathrm{Hcn1}^{\text {tm2Kndl }}$ [453], Crb1 ${ }^{\text {tm1.1Wij }}$ Crb2 ${ }^{\text {tm1.1Wij }}$ [454], Dio3 ${ }^{\text {tm1Stg; }}$ Dio2 ${ }^{\text {tm1Vag }}$ [455], and Ercc6 ${ }^{\text {tm1Goh }}$; and Xpatm1Hvs [456].

In addition to testing candidate interacting genes, methods have been developed to identify such interactors in an unbiased fashion that is illustrated below.

Effects of genetic background. For the calcium channel gene Cacna1f mentioned above, there is a third allele available. Chang et al. [457] reported the phenotype of the nob2 mutation, an out-of-frame insertion of a transposable element into the Cacnalf gene, which is predicted to cause a truncation after 32 amino acids. The authors demonstrated by western blot that this is a null mutation and no protein is detected. Compared to the $\Delta$ Ex14-17 null mutation, however, the phenotype of nob 2 is much milder with no apparent PR degradation [457]. The most likely explanation for this discrepancy can be deduced from the fact that the nob2 mutation arose on the AxB6 recombinant inbred strain, a strain whose DNA is composed of alternate segments derived from C57BL/6J and A/J. It is likely that the $\mathrm{A} / \mathrm{J}$ strain carries one or more modifier loci that suppress the PR degeneration induced by a Cacnalf null mutation.

Upon outcrossing an inbred strain carrying a mutation that leads to a particular phenotype with a different inbred strain, it is frequently observed that the phenotype of the offspring differs from that of the parents. This was often encountered in the past when knockout alleles were created in embryonic stem cells derived from strain $129 / \mathrm{Sv}$ and the founder animals were then made congenic on the C57BL/6 background. An early example is a study of a homozygous Rho knockout that was shown to lose PR nuclei significantly faster on the 129Sv background than on the C57BL/6 background [458]. Corresponding differences were also found in the number of apoptotic nuclei and in ERG responses. It was concluded that the B6 strain carries protective alleles of modifier genes that lead to a slower rate of PR degeneration [458]. Alternatively, it is also possible that 129Sv carries modifier alleles that accelerate degeneration.

Other inbred strains have also been reported to modify retinal phenotypes. For example, a targeted mutation of $R p 1$ (Rp1 $\left.1^{\text {tm } 1 E a p}\right)$ only showed moderate PR degeneration as an incipient congenic (N6) on the A/J strain background, but not on C57BL/6J or DBA/1J backgrounds [459]. ONL dysplasia and excess blue cone formation caused by loss of $\mathrm{Nr} 2 e 3$ in C57BL/6J are suppressed by the genetic backgrounds of CAST/EiJ, AKR/J, and NOD.NON-H2 ${ }^{\text {nb1 }}$ strains [460].

In principle, all inbred strains will carry modifier alleles. However, which strain modifies a particular mutation will depend on the primary mutation. It should be emphasized that an inbred strain represents a single genotype. In order to model the phenotypic spectrum of a human disease-causing mutation, many inbred strain backgrounds would have to be examined. Recently, advanced genetically 
diverse mouse populations have become available, such as the collaborative cross (CC) or the diversity outcross (DO) populations, that allow for more efficient modeling of human populations compared to the classical inbred strains [461,462].

Modifier screens. Modifier screens are a tool to identify genes that modify phenotypic traits caused by a particular mutation. The disease modifying properties of inbred strains have been used for many decades to identify the underlying modifier genes by using genetic crosses, marker assisted genetic mapping of modifying loci, and positional cloning or more recently high throughput whole exome or whole genome sequencing approaches. For example, when B6.Cg-Nr2e3 ${ }^{\text {rd7 }}$ homozygotes are outcrossed to CAST/EiJ, AKR/J, or NOD.NON-H2 ${ }^{n b 1}$ and then the F1 mice intercrossed, homozygous $\mathrm{Nr} 2 \mathrm{e}^{r d 7}$ mice of the F2 generation are found that unlike the parental B6.Cg-Nr2e $3^{r d 7}$ homozygotes have fewer spots on fundus examination and no PR layer dysplasia in histological sections [460]. This phenotypic variability is caused by the genetic interaction between the $\mathrm{Nr} 2 \mathrm{e}^{\text {rd7 }}$ disease allele and variants of so-called modifier genes that are specific to the outcross partner strain. Several quantitative trait loci (QTL) on chromosomes 7, 8, 11, and 19 were mapped [460]. Generation of a congenic line carrying the Chr11 modifier, along with further fine mapping, reduced the critical genomic interval to $3.3 \mathrm{cM}$. Several candidate genes were sequenced and a single nucleotide polymorphism was found in a nuclear receptor gene, Nr1d1, that is predicted to lead to an Arg409Gln amino acid change. Causality was confirmed by phenotypic rescue of the $r d 7$-associated phenotypes by in vivo electroporation of a wild-type Nr1d1 expression construct [463].

Several other modifiers have been mapped and identified based on inbred strain differences. For example, mapping crosses have been carried out for $r d 3$ (BALB/cJ and C57BL/6J, [464]), $r d 1$ (C3H/HeOu and FVB/N, [465]), Crb1 (C57BL/6N and C57BL/6JOlaHsd, Chr15, [466]), Mfrp (B6.C3Ga and CAST/EiJ, Chr 1, 6, and 11 [467]), and Tub and Tulp1 (C57BL/6J and AKR/J, Mtap1a, [468]).

Although not yet widely used as a means to explore retinal biology, a very efficient way to identify modifier genes is the use of a sensitized mutagenesis screen in which a male mouse carrying a mutation of interest is given a chemical mutagen and its offspring are examined for any change in the original phenotype. Offspring carrying a potential mutation is backcrossed to the unmutagenized parental inbred strain to test for heritability and to reduce the mutational load. Mutations are identified using whole exome sequencing of the pheno-deviant mouse. This approach avoids the limited genetic diversity of inbred strains since in principle all genes can be mutated. An example of the utility of mutagenesis to search for modifier genes is the identification of a suppressor mutation in Frmd $4 b$ that prevents the PR dysplasia and external limiting membrane fragmentation observed in $N r 2 e{ }^{r d 7}$ mutant mice [469].

\subsubsection{Effects of Environment on PR Degeneration}

PR cell loss has been shown to be induced by a number of environmental factors such as light, diet, and smoking in combination with particular genotypes. Perhaps not surprisingly, light exposure in some models bearing mutations in genes that function directly or in an ancillary fashion in the visual transduction pathway trend toward hastening PR degeneration [470,471]. For example, transgenic mice bearing the rhodopsin VPP mutation, widely used in visual transduction studies, is susceptible to light-exacerbated PR degeneration [472]. Likewise, mice carrying a homozygous Prom1 null mutation are particularly susceptible to light-induced degeneration. At eye opening, with exposure to light, degeneration initiates at P14, and all PRs are gone by P20, whereas dark rearing from P8 to P30 leads to significant preservation of PRs [471]. Dark-rearing has also been demonstrated to delay PR degeneration in Slc6a6 $6^{\text {tm } 1 \text { Dhau }}$ (10\% loss vs. 90\% loss in normal vivarium lighting at three weeks of age) [473] or have no effect in C57BL/6-Mitf ${ }^{m i-v i t} / J$ homozygotes [474]. In some situations, light may actually trigger the disease phenotype, as is the case in Sag knockout mice [198,199], with three Class B1 Rhodopsin missense mutations, Torm1 and Torm4 [157] or Torm144 [18], and in null mutation models of Rdh12 [475], Asic2 [476], Myo7a [477], Whrn [478], or Akt2 [479]. Sag mutants must be reared in the dark to observe any PR cells. Under normal vivarium lighting conditions, the other light-sensitive 
mouse models do not show PR degeneration or only a slight shortening of OS at one year of age, as in the those carrying Rho alleles Torm1, Torm4, or Torm144, and in retinol dehydrogenase (Rdh12) mutant mice. However, exposure to bright light or rearing under cyclic moderate-lighting, even subjecting mice to fundus examination, leads to PR degeneration. A comprehensive list of animal models and the effects of dark-rearing or light exposure can be found in reference [470].

Like light exposure, smoking and high fat intake have been proposed to have a negative impact on retinal function by increasing oxidative stress and inflammation in PR and RPE cells [480]. Smoking has been implicated as a major risk factor in the development of age-related macular degeneration in humans [481,482], and the results have been replicated in mouse models as well. Smoking leads to increased oxidative stress and inflammation in B6 mice [483] and in the presence of Nfe2l2 deficiency [484]. Likewise, combinations of smoking and high fat intake in the presence of an $A p o B$ mutation that promotes production of the APOB100 isoform [485] leads to significant loss of PRs [484]. Further, high-fat diet intake for certain genotypes, such as mutations of Ldlr [486] or certain alleles of Apoe [487], has been shown to compromise PR integrity in mice.

The majority of pharmacological or dietary interventions that have been reported in the relationship to PR degeneration in mouse models are associated with the goal of increasing vitamin A derivative availability [488-490] or reducing oxidative stress [491,492] in the retina. Heritable mutations in enzymes, such as LRAT or RPE65, required for processing of vitamin A within the retina are known to cause early onset RD due a deficiency of the 11-cis-retinal chromophore. Efficacy of treatment with 9-cis-retinal derivatives of mice with null mutations in Lrat and Rpe65 mice is thoroughly discussed in a review by Perusek and Maeda [488,489]. Administration of antioxidants has in some cases improved PR survival. Rs $1^{\text {tm1Web }}$ homozygous females or hemizygous males fed a diet high in DHA [493] or Pde $6 b^{r d 10}$ mice fed lutein and zeaxanthin [494] showed a significant PR preservation. Further, injections of a mixture of antioxidants_alpha tocopheral, ascorbic acid, alpha-lipoic acid, and/or Mn(III)tetrakis porphyrin-were able to slow the loss of cone/rod PRs in Pde6b $b^{r d 1}$ [495], and Pde6 $b^{r d 10}$ mice and in mice with a rhodopsin Q344ter mutation [492]. Environmental enhancement of $P d e 6 b^{r d 10}$ mice was able to significantly reduce PR loss presumably by reducing retinal oxidative stress [496].

\subsection{Relationship to Human Disease Genes}

Of the 273 retinal degenerative disease genes in RetNet [3] for which mouse homologs exist, mouse models are available for 110 or $40 \%$ of them, including both germline and conditional mutants (Figure 7). Through our survey, we found 120 additional genes, in which mutations lead to PR degeneration. These genes could serve as candidates for yet to be identified human retinal diseases. The available mouse models, for the most part, recapitulate the human disease phenotype well and permit mechanistic and therapeutic studies. However, apparent failures of mouse models do occur. When mutations in MFRP were first identified in humans [497], mice were thought to be a poor model because unlike humans [498], mice were previously reported to develop PR degeneration [499], and the microphthalmia and hyperopia found in human patients had not been reported in homozygous $M f r p^{r d 6}$ mice. In subsequent years, numerous human patients have been identified that do show a degenerative phenotype [500] and hyperopia was detected both in a mouse model carrying a human MFRP c.498_499insC allele [501] and the original Mfrp ${ }^{r d 6}$ mouse (our unpublished observations). An important family of deaf-blindness diseases, Usher syndrome, was also thought to be poorly recapitulated in mice, because early models like the shaker-1 mouse had only the characteristic hearing loss, but no retinal degeneration [502]. Later, however, it was found that moderate light exposure does result in photoreceptor degeneration in shaker-1 mice [477]. In addition, a knock-in of the Acadian USH1C c.216G >A mutation into the mouse Ush1c gene recapitulates both deafness and retinal degeneration phenotypes [503]. In many cases, discordance between the human and mouse phenotypes can be attributed to insufficient information about variation in the human disease, or to allelic effects (knockout vs. hypomorph or gain of function, expression of alternatively splice isoforms), or strain background (modifier genes) in the mouse models. Such shortcomings in mouse models can 
often be addressed by testing multiple models, including human disease alleles, and by using multiple genetic backgrounds.

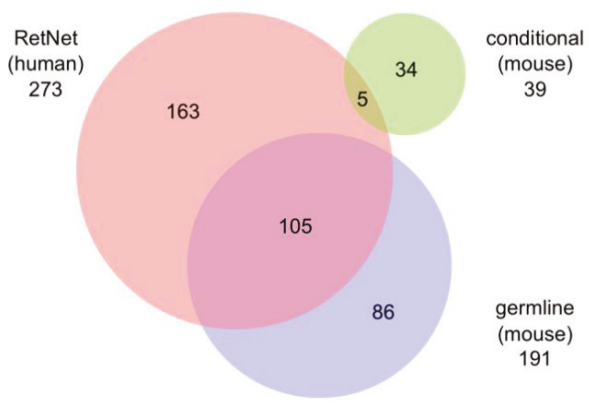

Figure 7. Comparison of the number of RD genes identified in human (RetNet) and mouse as listed in Table S1 and summarized in Figure 3. The total number of genes in the RetNet database that cause monogenic disease and have mouse homologs is indicated, as is the total for which conditional or germline mutations have been associated with PR cell loss in mice, as described in this review. Numbers within the overlapping areas of the diagram represent genes present in both RetNet and Table S1; the remaining numbers represent genes that are unique to the indicated category.

Although humans and mice share about $98 \%$ of their genes, species differences do exist and need to be considered when selecting a model. Examples of vision-related genes that mice lack are EYS, ARMS2, and CETP. Species differences are the result of different evolutionary histories; humans and mice have encountered different pathogens, resulting in adaptations of our respective immune systems. Mice have different nutritional requirements, resulting in differences in lipid metabolism. Additionally, mouse eyes are adapted to a nocturnal life, resulting in a rod dominated retina with no macula. Nevertheless, mice possess all of the same retinal cell types necessary for vision and the vast majority of the same genes, and even when missing genes are introduced into mice they result in relevant phenotypes. For example, in a transgenic mouse model for Stargardt-like macular degeneration 3 due to a mutation in Elovl4, PR cell loss occurs in the central retina in a pattern that resembles the human disease [504]. For the many retinal diseases still in need of models, including complex diseases such as AMD or diabetic retinopathy, it remains the case that valuable new insights into disease mechanism and basic eye biology can still be obtained from mouse studies.

\section{Discussion}

\subsection{Variability in Measuring PR Cell Loss}

We noted an extremely large variability in PR cell loss data presented in the various reports, which were based on several different types of measures, such as ONL thickness obtained from toluidine-stained plastic sections, hematoxylin and eosin-stained paraffin sections, or DAPI-stained cryosections; counts of rows of ONL nuclei in the same preparations; counts of the total number of ONL nuclei in a fixed retinal area; spider plots assessing ONL thickness over the full perimeter; ONL thickness from OCT B-scans. Cone numbers were assessed in sections or whole mounts stained with cone opsins, peanut agglutinin lectin, or cone arrestin. Methods to count cone cell nuclei efficiently might benefit from studies that examine the effect of mutations in genes that specifically affect cones. Perhaps more challenging is that many studies provided insufficient sample sizes or number of time points to assess the progression of PR cell loss. Some of this variability reflects the evolution of methods and quantitative approaches over several decades, and some may be attributed to the different choices each laboratory makes depending on what works best given resources and interests. However, to aid future efforts to compare PR cell loss between studies, we recommend that at least three ages be 
assessed, one prior to the onset of PR cell loss, and at least two within the age range where PR cells are declining exponentially (that is, between $95 \%$ and $5 \%$ of wild-type values), and that the data be quantified relative to gender-matched controls at these same ages.

Although a decrease in PR cell numbers as estimated above is widely accepted as evidence for RD, an alternative explanation may apply in some instances. Mcoln $1^{\text {tm1Sasl }}$ homozygotes exhibit an apparent decrease in PR cells to 54\% of wild-type at one month of age, a value that remained unchanged at two and six months [505]. TUNEL analysis revealed no increase in apoptosis at any age compared to controls, and both histology and OCT indicated a decrease in total retinal thickness. It is possible that the rapid initial decrease to a stable value may result from retinal thinning as the eye enlarges due to myopia during postnatal development, an occasionally observed feature of MCOLN1-associated disease in humans [506]. Methods are available to measure axial length in mice [507], which might be used to determine whether the observed change in nuclear layer thickness is due to ocular enlargement. Models in Table S1 with a similar phenotype include Guca1a ${ }^{\text {tm1.1Hunt }}$ and Ctnna1 ${ }^{\text {Tvrm5 }}$.

\subsection{Correlation of PR Cell Loss with Gene Function}

As a fortuitous consequence of our inquiry, in some cases, the progress of PR cell loss could be correlated with gene function within categories. For example, in the ciliary function and trafficking section we see trends in the onset and progression of RD depending on the role and location of gene products within the PR. Mice with mutations in genes involved in ciliogenesis or in transition zone protein complexes, typically result in a PR degeneration that begins at 2 weeks during OS biogenesis and progresses rapidly through OS maturation. Null mutations in genes that encode components of the IFT machinery tend to result in premature death or embryonic lethality, and conditional ablation of these genes in the retina typically leads to early and rapid PR loss. Models with disruptions in protein complexes with roles in BBSome assembly or regulation, protein/lipid trafficking, axoneme extension, or disc morphogenesis tend to have a moderate to slow degeneration. Lastly, mice with mutations that disturb basal body and pericentriolar anchoring and integrity such as the Usher periciliary membrane complex undergo a very slow form of RD, which results in partial PR function throughout the life of the mouse. It remains possible that the slower progression of PR cell loss, especially when associated with members of protein complexes, may be the result of genetic redundancy where multiple genes encode proteins that have similar biochemical functions.

It was also interesting to note that disruption of chloride channels appeared to be particularly deleterious to PR survival. Many of the models we identified with early and rapid progression of PR cell loss, including those affecting a subset of the ciliary genes discussed above or the OS components Prom1, Prph2, and Rho, appear to be required for OS assembly. Chloride channel defects resulted in similar progression, raising the possibility that chloride homeostasis is important for OS development. This idea is supported by evidence that chloride transport by the chloride channel ANO1 is required for ciliogenesis [508] and that control of intracellular chloride ion levels by this channel regulates the membrane organization of phosphatidylinositol 4,5-bisphosphate [509], a prominent lipid that regulates ciliary development $[131,510]$. Characterization of early OS development in mouse models defective in chloride channels CLCN2, CLCN3, or CLCN7 may provide mechanistic clues on the role of intracellular chloride in ciliogenesis.

Finally, our analysis revealed links between PR cell loss and a network of 13 genes known to participate in the cellular response to DNA damage, four of which have been directly associated with transcription-coupled DNA repair. Based on canonical indicators of DNA repair, such as the colocalization of phosphorylated histone H2AX and TRP53BP1 (also known as 53BP1) at DSBs, it has been reported that rod PR cells in mice lack a robust canonical DNA damage response, [241,511]. An attenuated response may reflect an adaptation to improve rod cell survival [241,511]. Nevertheless, mechanisms are present in rod cells to repair DNA damage, as evidenced by the robust levels of DNA repair factors [241] and by our results indicating that a network of DNA damage response genes is required for maintaining PR cell viability. Together, these results support the idea that a non-canonical 
DNA damage response pathway exists in rod PR cells [512]. Further study of the DNA damage response genes linked to PR cell loss in mice may be useful for elucidating this pathway.

\section{Conclusions}

This review highlights mouse models of monogenic retinal degenerative diseases that cause rod or cone PR cell loss. The models include germline mutations and conditional alleles, in which characterization of retinal phenotypes in germline mutations was not possible due to embryonic or perinatal lethality. By providing an extensive list of these models as well as a means of comparing their progression, we hope to benefit researchers who seek to optimize their experimental approaches.

Supplementary Materials: The following are available online at http:/www.mdpi.com/2073-4409/9/4/931/s1, Figure S1: OCT and fundus images of C57BL/6J control mice at various ages, Table S1: Monogenic mouse RD models with PR cell loss, Table S2: Description of gene/protein symbols used in the text and figures.

Author Contributions: Conceptualization, P.M.N., M.P.K.; methodology, M.P.K., B.C., P.M.N.; software, M.P.K.; formal analysis, M.P.K.; investigation, G.B.C., N.G., N.D., J.P., L.F.H., L.S., J.K.N., M.P.K., P.M.N.; resources, B.C., J.K.N., P.M.N.; data curation, G.B.C., N.G., N.D., J.P., L.F.H., L.S., J.K.N., M.P.K., P.M.N.; writing-original draft preparation, G.B.C., N.G., N.D., J.P., L.H., J.K.N., M.P.K., P.M.N.; writing-review and editing, G.B.C., N.G., J.K.N., M.P.K., P.M.N.; visualization, B.C., M.P.K.; supervision, J.K.N., P.M.N.; project administration, P.M.N.; funding acquisition, M.P.K., B.C., J.K.N., P.M.N. All authors have read and agree to the published version of the manuscript.

Funding: This work was funded in part by National Institutes of Health grants 5R01EY011996, 5R01EY027860, R01EY027305 (to P.M.N), 5R01EY028561 (to J.K.N.), 5R01EY019943 (to B.C.), and 5R21EY027894 (to M.P.K). N.D. was supported by the grant from the Royal Golden Jubilee (RGJ) Scholarship (PHD/0102/2559), Thailand Research Fund.

Acknowledgments: The authors thank James Kadin and Grace Stafford for help with scripts to filter PubMed data, Cynthia Smith for her contributions to Table S1 and for coordinating our efforts with those at MGI, Melissa Berry for assistance with nomenclature, Bernard Fitzmaurice and Wanda Hicks for fundus and/or OCT imaging, and Jane Cha for drawing Figure 1.

Conflicts of Interest: The authors declare no conflict of interest. The funders had no role in the design of the study; in the collection, analyses, or interpretation of data; in the writing of the manuscript, or in the decision to publish the results.

\section{References}

1. Cremers, F.P.M.; Boon, C.J.F.; Bujakowska, K.; Zeitz, C. Special Issue Introduction: Inherited Retinal Disease: Novel Candidate Genes, Genotype-Phenotype Correlations, and Inheritance Models. Genes (Basel) 2018, 9 , 215. [CrossRef] [PubMed]

2. Hanany, M.; Rivolta, C.; Sharon, D. Worldwide carrier frequency and genetic prevalence of autosomal recessive inherited retinal diseases. Proc. Natl. Acad. Sci. USA 2020, 117, 2710-2716. [CrossRef] [PubMed]

3. RetNet-Retinal Information Network. Available online: https://sph.uth.edu/retnet/home.htm (accessed on 8 December 2019).

4. Picaud, S.; Dalkara, D.; Marazova, K.; Goureau, O.; Roska, B.; Sahel, J.A. The primate model for understanding and restoring vision. Proc. Natl. Acad. Sci. USA 2019, 116, 26280-26287. [CrossRef] [PubMed]

5. Bunel, M.; Chaudieu, G.; Hamel, C.; Lagoutte, L.; Manes, G.; Botherel, N.; Brabet, P.; Pilorge, P.; Andre, C.; Quignon, P. Natural models for retinitis pigmentosa: Progressive retinal atrophy in dog breeds. Hum. Genet. 2019, 138, 441-453. [CrossRef] [PubMed]

6. Fletcher, E.L.; Jobling, A.I.; Vessey, K.A.; Luu, C.; Guymer, R.H.; Baird, P.N. Animal models of retinal disease. Prog. Mol. Biol. Transl. Sci. 2011, 100, 211-286. [CrossRef]

7. Veleri, S.; Lazar, C.H.; Chang, B.; Sieving, P.A.; Banin, E.; Swaroop, A. Biology and therapy of inherited retinal degenerative disease: Insights from mouse models. Dis. Model. Mech. 2015, 8, 109-129. [CrossRef]

8. Angueyra, J.M.; Kindt, K.S. Leveraging Zebrafish to Study Retinal Degenerations. Front. Cell Dev. Biol. 2018, 6, 110. [CrossRef]

9. Lehmann, M.; Knust, E.; Hebbar, S. Drosophila melanogaster: A Valuable Genetic Model Organism to Elucidate the Biology of Retinitis Pigmentosa. Methods Mol. Biol. 2019, 1834, 221-249. [CrossRef] 
10. Travis, G.H.; Brennan, M.B.; Danielson, P.E.; Kozak, C.A.; Sutcliffe, J.G. Identification of a photoreceptorspecific mRNA encoded by the gene responsible for retinal degeneration slow (rds). Nature 1989, 338, 70-73. [CrossRef]

11. Dryja, T.P.; McGee, T.L.; Reichel, E.; Hahn, L.B.; Cowley, G.S.; Yandell, D.W.; Sandberg, M.A.; Berson, E.L. A point mutation of the rhodopsin gene in one form of retinitis pigmentosa. Nature 1990, 343, 364-366. [CrossRef]

12. Bowes, C.; Li, T.; Danciger, M.; Baxter, L.C.; Applebury, M.L.; Farber, D.B. Retinal degeneration in the rd mouse is caused by a defect in the beta subunit of rod cGMP-phosphodiesterase. Nature 1990, 347, 677-680. [CrossRef] [PubMed]

13. Wheway, G.; Parry, D.A.; Johnson, C.A. The role of primary cilia in the development and disease of the retina. Organogenesis 2014, 10, 69-85. [CrossRef] [PubMed]

14. Baehr, W.; Hanke-Gogokhia, C.; Sharif, A.; Reed, M.; Dahl, T.; Frederick, J.M.; Ying, G. Insights into photoreceptor ciliogenesis revealed by animal models. Prog. Retin. Eye Res. 2019, 71, 26-56. [CrossRef] [PubMed]

15. Chang, B.; Hawes, N.L.; Hurd, R.E.; Davisson, M.T.; Nusinowitz, S.; Heckenlively, J.R. Retinal degeneration mutants in the mouse. Vis. Res. 2002, 42,517-525. [CrossRef]

16. Chang, B.; Hawes, N.L.; Hurd, R.E.; Wang, J.; Howell, D.; Davisson, M.T.; Roderick, T.H.; Nusinowitz, S.; Heckenlively, J.R. Mouse models of ocular diseases. Vis. NeuroSci. 2005, 22, 587-593. [CrossRef] [PubMed]

17. Won, J.; Shi, L.Y.; Hicks, W.; Wang, J.; Hurd, R.; Naggert, J.K.; Chang, B.; Nishina, P.M. Mouse model resources for vision research. J. Ophthalmol. 2011, 2011, 391384. [CrossRef]

18. Won, J.; Shi, L.Y.; Hicks, W.; Wang, J.; Naggert, J.K.; Nishina, P.M. Translational vision research models program. Adv. Exp. Med. Biol. 2012, 723, 391-397. [CrossRef]

19. Chang, B. Mouse models for studies of retinal degeneration and diseases. Methods Mol. Biol. 2013, 935, 27-39. [CrossRef]

20. Chang, B. Mouse Models as Tools to Identify Genetic Pathways for Retinal Degeneration, as Exemplified by Leber's Congenital Amaurosis. Methods Mol. Biol. 2016, 1438, 417-430. [CrossRef]

21. Krebs, M.P.; Collin, G.B.; Hicks, W.L.; Yu, M.; Charette, J.R.; Shi, L.Y.; Wang, J.; Naggert, J.K.; Peachey, N.S.; Nishina, P.M. Mouse models of human ocular disease for translational research. PLoS ONE 2017, 12, e0183837. [CrossRef]

22. Do, M.T.; Yau, K.W. Intrinsically photosensitive retinal ganglion cells. Physiol. Rev. 2010, 90, 1547-1581. [CrossRef] [PubMed]

23. Goldberg, A.F.; Moritz, O.L.; Williams, D.S. Molecular basis for photoreceptor outer segment architecture. Prog. Retin. Eye Res. 2016, 55, 52-81. [CrossRef] [PubMed]

24. Rachel, R.A.; Li, T.; Swaroop, A. Photoreceptor sensory cilia and ciliopathies: Focus on CEP290, RPGR and their interacting proteins. Cilia 2012, 1, 22. [CrossRef] [PubMed]

25. Wang, J.; O'Sullivan, M.L.; Mukherjee, D.; Punal, V.M.; Farsiu, S.; Kay, J.N. Anatomy and spatial organization of Muller glia in mouse retina. J. Comp. Neurol. 2017, 525, 1759-1777. [CrossRef]

26. Reichenbach, A.; Bringmann, A. Glia of the human retina. Glia 2019, 68, 768-796. [CrossRef]

27. Strauss, O. The retinal pigment epithelium in visual function. Physiol. Rev. 2005, 85, 845-881. [CrossRef]

28. Verbakel, S.K.; van Huet, R.A.C.; Boon, C.J.F.; den Hollander, A.I.; Collin, R.W.J.; Klaver, C.C.W.; Hoyng, C.B.; Roepman, R.; Klevering, B.J. Non-syndromic retinitis pigmentosa. Prog. Retin. Eye Res. 2018, 66, 157-186. [CrossRef]

29. Kumaran, N.; Moore, A.T.; Weleber, R.G.; Michaelides, M. Leber congenital amaurosis/early-onset severe retinal dystrophy: Clinical features, molecular genetics and therapeutic interventions. Br. J. Ophthalmol. 2017, 101, 1147-1154. [CrossRef]

30. Parisi, M.A. The molecular genetics of Joubert syndrome and related ciliopathies: The challenges of genetic and phenotypic heterogeneity. Transl. Sci. Rare Dis. 2019, 4, 25-49. [CrossRef]

31. Suspitsin, E.N.; Imyanitov, E.N. Bardet-Biedl Syndrome. Mol. Syndromol. 2016, 7, 62-71. [CrossRef]

32. Mathur, P.; Yang, J. Usher syndrome: Hearing loss, retinal degeneration and associated abnormalities. Biochim. Biophys. Acta 2015, 1852, 406-420. [CrossRef] [PubMed]

33. Kumaran, N.; Michaelides, M.; Smith, A.J.; Ali, R.R.; Bainbridge, J.W.B. Retinal gene therapy. Br. Med. Bull. 2018, 126, 13-25. [CrossRef] [PubMed] 
34. Mouse Genome Database (MGD) at the Mouse Genome Informatics website, The Jackson Laboratory, Bar Harbor, Maine. Available online: http://www.informatics.jax.org (accessed on 18 October 2019).

35. PubMed [Internet]. Bethesda (MD): National Library of Medicine (US), National Center for Biotechnology Information. Available online: https://www.ncbi.nlm.nih.gov/pubmed/ (accessed on 15 October 2019).

36. Clarke, G.; Collins, R.A.; Leavitt, B.R.; Andrews, D.F.; Hayden, M.R.; Lumsden, C.J.; McInnes, R.R. A one-hit model of cell death in inherited neuronal degenerations. Nature 2000, 406, 195-199. [CrossRef] [PubMed]

37. Krebs, M.P.; Xiao, M.; Sheppard, K.; Hicks, W.; Nishina, P.M. Bright-Field Imaging and Optical Coherence Tomography of the Mouse Posterior Eye. Methods Mol. Biol. 2016, 1438, 395-415. [CrossRef] [PubMed]

38. Krebs, M.P. Using Vascular Landmarks to Orient 3D Optical Coherence Tomography Images of the Mouse Eye. Curr. Protoc. Mouse Biol. 2017, 7, 176-190. [CrossRef]

39. Dyer, M.A.; Donovan, S.L.; Zhang, J.; Gray, J.; Ortiz, A.; Tenney, R.; Kong, J.; Allikmets, R.; Sohocki, M.M. Retinal degeneration in Aipl1-deficient mice: A new genetic model of Leber congenital amaurosis. Brain Res. Mol. Brain Res. 2004, 132, 208-220. [CrossRef]

40. Liu, X.; Bulgakov, O.V.; Wen, X.H.; Woodruff, M.L.; Pawlyk, B.; Yang, J.; Fain, G.L.; Sandberg, M.A.; Makino, C.L.; Li, T. AIPL1, the protein that is defective in Leber congenital amaurosis, is essential for the biosynthesis of retinal rod cGMP phosphodiesterase. Proc. Natl. Acad. Sci. USA 2004, 101, 13903-13908. [CrossRef]

41. Dickinson, M.E.; Flenniken, A.M.; Ji, X.; Teboul, L.; Wong, M.D.; White, J.K.; Meehan, T.F.; Weninger, W.J.; Westerberg, H.; Adissu, H.; et al. High-throughput discovery of novel developmental phenotypes. Nature 2016, 537, 508-514. [CrossRef]

42. Hanke-Gogokhia, C.; Wu, Z.; Gerstner, C.D.; Frederick, J.M.; Zhang, H.; Baehr, W. Arf-like Protein 3 (ARL3) Regulates Protein Trafficking and Ciliogenesis in Mouse Photoreceptors. J. Biol. Chem. 2016, 291, 7142-7155. [CrossRef]

43. International Mouse Phenotyping Consortium: Home-IMPC. Available online: https://www. mousephenotype.org/ (accessed on 22 December 2019).

44. Bujakowska, K.M.; Liu, Q.; Pierce, E.A. Photoreceptor Cilia and Retinal Ciliopathies. Cold Spring Harb. Perspect. Biol. 2017, 9, a028274. [CrossRef]

45. Gilliam, J.C.; Chang, J.T.; Sandoval, I.M.; Zhang, Y.; Li, T.; Pittler, S.J.; Chiu, W.; Wensel, T.G. Three-dimensional architecture of the rod sensory cilium and its disruption in retinal neurodegeneration. Cell 2012,151,1029-1041. [CrossRef] [PubMed]

46. Rohlich, P. The sensory cilium of retinal rods is analogous to the transitional zone of motile cilia. Cell Tiss. Res. 1975, 161, 421-430. [CrossRef] [PubMed]

47. Goncalves, J.; Pelletier, L. The Ciliary Transition Zone: Finding the Pieces and Assembling the Gate. Mol. Cells 2017, 40, 243-253. [CrossRef] [PubMed]

48. Seo, S.; Datta, P. Photoreceptor outer segment as a sink for membrane proteins: Hypothesis and implications in retinal ciliopathies. Hum. Mol. Genet. 2017, 26, R75-R82. [CrossRef]

49. Khanna, H. Photoreceptor Sensory Cilium: Traversing the Ciliary Gate. Cells 2015, 4, 674-686. [CrossRef]

50. Garcia-Gonzalo, F.R.; Corbit, K.C.; Sirerol-Piquer, M.S.; Ramaswami, G.; Otto, E.A.; Noriega, T.R.; Seol, A.D.; Robinson, J.F.; Bennett, C.L.; Josifova, D.J.; et al. A transition zone complex regulates mammalian ciliogenesis and ciliary membrane composition. Nat. Genet. 2011, 43, 776-784. [CrossRef]

51. Sedmak, T.; Wolfrum, U. Intraflagellar transport proteins in ciliogenesis of photoreceptor cells. Biol. Cell 2011, 103, 449-466. [CrossRef]

52. Salinas, R.Y.; Pearring, J.N.; Ding, J.D.; Spencer, W.J.; Hao, Y.; Arshavsky, V.Y. Photoreceptor discs form through peripherin-dependent suppression of ciliary ectosome release. J. Cell Biol. 2017, 216, 1489-1499. [CrossRef]

53. LaVail, M.M. Kinetics of rod outer segment renewal in the developing mouse retina. J. Cell Biol. 1973, 58, 650-661. [CrossRef]

54. De Robertis, E. Morphogenesis of the retinal rods; an electron microscope study. J. Biophys. Biochem. Cytol. 1956, 2, 209-218. [CrossRef]

55. Insinna, C.; Besharse, J.C. Intraflagellar transport and the sensory outer segment of vertebrate photoreceptors. Dev. Dyn. 2008, 237, 1982-1992. [CrossRef] [PubMed]

56. Rosenbaum, J.L.; Cole, D.G.; Diener, D.R. Intraflagellar transport: The eyes have it. J. Cell Biol. 1999, 144, 385-388. [CrossRef] 
57. Chuang, J.Z.; Zhao, Y.; Sung, C.H. SARA-regulated vesicular targeting underlies formation of the light-sensing organelle in mammalian rods. Cell 2007, 130, 535-547. [CrossRef] [PubMed]

58. Chuang, J.Z.; Hsu, Y.C.; Sung, C.H. Ultrastructural visualization of trans-ciliary rhodopsin cargoes in mammalian rods. Cilia 2015, 4, 4. [CrossRef] [PubMed]

59. Steinberg, R.H.; Fisher, S.K.; Anderson, D.H. Disc morphogenesis in vertebrate photoreceptors. J. Comp. Neurol. 1980, 190, 501-508. [CrossRef]

60. Ding, J.D.; Salinas, R.Y.; Arshavsky, V.Y. Discs of mammalian rod photoreceptors form through the membrane evagination mechanism. J. Cell Biol. 2015, 211, 495-502. [CrossRef]

61. Burgoyne, T.; Meschede, I.P.; Burden, J.J.; Bailly, M.; Seabra, M.C.; Futter, C.E. Rod disc renewal occurs by evagination of the ciliary plasma membrane that makes cadherin-based contacts with the inner segment. Proc. Natl. Acad. Sci. USA 2015, 112, 15922-15927. [CrossRef]

62. Young, R.W. The renewal of photoreceptor cell outer segments. J. Cell Biol. 1967, 33, 61-72. [CrossRef]

63. Jin, H.; White, S.R.; Shida, T.; Schulz, S.; Aguiar, M.; Gygi, S.P.; Bazan, J.F.; Nachury, M.V. The conserved Bardet-Biedl syndrome proteins assemble a coat that traffics membrane proteins to cilia. Cell 2010, 141, 1208-1219. [CrossRef]

64. Liew, G.M.; Ye, F.; Nager, A.R.; Murphy, J.P.; Lee, J.S.; Aguiar, M.; Breslow, D.K.; Gygi, S.P.; Nachury, M.V. The intraflagellar transport protein IFT27 promotes BBSome exit from cilia through the GTPase ARL6/BBS3. Dev. Cell 2014, 31, 265-278. [CrossRef]

65. Taub, D.G.; Liu, Q. The Role of Intraflagellar Transport in the Photoreceptor Sensory Cilium. Adv. Exp. Med. Biol. 2016, 854, 627-633. [CrossRef] [PubMed]

66. Pazour, G.J.; Baker, S.A.; Deane, J.A.; Cole, D.G.; Dickert, B.L.; Rosenbaum, J.L.; Witman, G.B.; Besharse, J.C. The intraflagellar transport protein, IFT88, is essential for vertebrate photoreceptor assembly and maintenance. J. Cell Biol. 2002, 157, 103-113. [CrossRef] [PubMed]

67. Jensen, V.L.; Leroux, M.R. Gates for soluble and membrane proteins, and two trafficking systems (IFT and LIFT), establish a dynamic ciliary signaling compartment. Curr. Opin. Cell Biol. 2017, 47, 83-91. [CrossRef] [PubMed]

68. Baehr, W. Membrane protein transport in photoreceptors: The function of PDEdelta: The Proctor lecture. Investig. Ophthalmol. Vis. Sci. 2014, 55, 8653-8666. [CrossRef]

69. Hsu, Y.; Garrison, J.E.; Kim, G.; Schmitz, A.R.; Searby, C.C.; Zhang, Q.; Datta, P.; Nishimura, D.Y.; Seo, S.; Sheffield, V.C. BBSome function is required for both the morphogenesis and maintenance of the photoreceptor outer segment. PLoS Genet. 2017, 13, e1007057. [CrossRef]

70. Jiang, L.; Wei, Y.; Ronquillo, C.C.; Marc, R.E.; Yoder, B.K.; Frederick, J.M.; Baehr, W. Heterotrimeric kinesin-2 (KIF3) mediates transition zone and axoneme formation of mouse photoreceptors. J. Biol. Chem. 2015, 290, 12765-12778. [CrossRef]

71. Ronquillo, C.C.; Hanke-Gogokhia, C.; Revelo, M.P.; Frederick, J.M.; Jiang, L.; Baehr, W. Ciliopathy-associated IQCB1/NPHP5 protein is required for mouse photoreceptor outer segment formation. FASEB J. 2016, 30, 3400-3412. [CrossRef]

72. Hanke-Gogokhia, C.; Wu, Z.; Sharif, A.; Yazigi, H.; Frederick, J.M.; Baehr, W. The guanine nucleotide exchange factor Arf-like protein 13b is essential for assembly of the mouse photoreceptor transition zone and outer segment. J. Biol. Chem. 2017, 292, 21442-21456. [CrossRef]

73. Cantagrel, V.; Silhavy, J.L.; Bielas, S.L.; Swistun, D.; Marsh, S.E.; Bertrand, J.Y.; Audollent, S.; Attie-Bitach, T.; Holden, K.R.; Dobyns, W.B.; et al. Mutations in the cilia gene ARL13B lead to the classical form of Joubert syndrome. Am. J. Hum. Genet. 2008, 83, 170-179. [CrossRef]

74. Caspary, T.; Larkins, C.E.; Anderson, K.V. The graded response to Sonic Hedgehog depends on cilia architecture. Dev. Cell 2007, 12, 767-778. [CrossRef]

75. Eblimit, A.; Agrawal, S.A.; Thomas, K.; Anastassov, I.A.; Abulikemu, T.; Moayedi, Y.; Mardon, G.; Chen, R. Conditional loss of Spata7 in photoreceptors causes progressive retinal degeneration in mice. Exp. Eye Res. 2018, 166, 120-130. [CrossRef] [PubMed]

76. Singla, V.; Reiter, J.F. The primary cilium as the cell's antenna: Signaling at a sensory organelle. Science 2006, 313, 629-633. [CrossRef]

77. Garcia-Gonzalo, F.R.; Reiter, J.F. Open Sesame: How Transition Fibers and the Transition Zone Control Ciliary Composition. Cold Spring Harb. Perspect. Biol. 2017, 9, a028134. [CrossRef] 
78. Shi, X.; Garcia, G., 3rd; Van De Weghe, J.C.; McGorty, R.; Pazour, G.J.; Doherty, D.; Huang, B.; Reiter, J.F. Super-resolution microscopy reveals that disruption of ciliary transition-zone architecture causes Joubert syndrome. Nat. Cell Biol. 2017, 19, 1178-1188. [CrossRef] [PubMed]

79. Williams, C.L.; Li, C.; Kida, K.; Inglis, P.N.; Mohan, S.; Semenec, L.; Bialas, N.J.; Stupay, R.M.; Chen, N.; Blacque, O.E.; et al. MKS and NPHP modules cooperate to establish basal body/transition zone membrane associations and ciliary gate function during ciliogenesis. J. Cell Biol. 2011, 192, 1023-1041. [CrossRef] [PubMed]

80. Knorz, V.J.; Spalluto, C.; Lessard, M.; Purvis, T.L.; Adigun, F.F.; Collin, G.B.; Hanley, N.A.; Wilson, D.I.; Hearn, T. Centriolar association of ALMS1 and likely centrosomal functions of the ALMS motif-containing proteins C10orf90 and KIAA1731. Mol. Biol. Cell 2010, 21, 3617-3629. [CrossRef] [PubMed]

81. Hearn, T.; Renforth, G.L.; Spalluto, C.; Hanley, N.A.; Piper, K.; Brickwood, S.; White, C.; Connolly, V.; Taylor, J.F.; Russell-Eggitt, I.; et al. Mutation of ALMS1, a large gene with a tandem repeat encoding 47 amino acids, causes Alstrom syndrome. Nat. Genet. 2002, 31, 79-83. [CrossRef]

82. Collin, G.B.; Marshall, J.D.; Ikeda, A.; So, W.V.; Russell-Eggitt, I.; Maffei, P.; Beck, S.; Boerkoel, C.F.; Sicolo, N.; Martin, M.; et al. Mutations in ALMS1 cause obesity, type 2 diabetes and neurosensory degeneration in Alstrom syndrome. Nat. Genet. 2002, 31, 74-78. [CrossRef]

83. Collin, G.B.; Cyr, E.; Bronson, R.; Marshall, J.D.; Gifford, E.J.; Hicks, W.; Murray, S.A.; Zheng, Q.Y.; Smith, R.S.; Nishina, P.M.; et al. Alms1-disrupted mice recapitulate human Alstrom syndrome. Hum. Mol. Genet. 2005, 14, 2323-2333. [CrossRef]

84. Brun, A.; Yu, X.; Obringer, C.; Ajoy, D.; Haser, E.; Stoetzel, C.; Roux, M.J.; Messaddeq, N.; Dollfus, H.; Marion, $\mathrm{V}$. In vivo phenotypic and molecular characterization of retinal degeneration in mouse models of three ciliopathies. Exp. Eye Res. 2019, 186, 107721. [CrossRef]

85. May-Simera, H.L.; Gumerson, J.D.; Gao, C.; Campos, M.; Cologna, S.M.; Beyer, T.; Boldt, K.; Kaya, K.D.; Patel, N.; Kretschmer, F.; et al. Loss of MACF1 Abolishes Ciliogenesis and Disrupts Apicobasal Polarity Establishment in the Retina. Cell Rep. 2016, 17, 1399-1413. [CrossRef] [PubMed]

86. Gerding, W.M.; Schreiber, S.; Schulte-Middelmann, T.; de Castro Marques, A.; Atorf, J.; Akkad, D.A.; Dekomien, G.; Kremers, J.; Dermietzel, R.; Gal, A.; et al. Ccdc66 null mutation causes retinal degeneration and dysfunction. Hum. Mol. Genet. 2011, 20, 3620-3631. [CrossRef] [PubMed]

87. Insolera, R.; Shao, W.; Airik, R.; Hildebrandt, F.; Shi, S.H. SDCCAG8 regulates pericentriolar material recruitment and neuronal migration in the developing cortex. Neuron 2014, 83, 805-822. [CrossRef] [PubMed]

88. Veleri, S.; Manjunath, S.H.; Fariss, R.N.; May-Simera, H.; Brooks, M.; Foskett, T.A.; Gao, C.; Longo, T.A.; Liu, P.; Nagashima, K.; et al. Ciliopathy-associated gene Cc2d2a promotes assembly of subdistal appendages on the mother centriole during cilia biogenesis. Nat. Commun. 2014, 5, 4207. [CrossRef]

89. Tallila, J.; Jakkula, E.; Peltonen, L.; Salonen, R.; Kestila, M. Identification of CC2D2A as a Meckel syndrome gene adds an important piece to the ciliopathy puzzle. Am. J. Hum. Genet. 2008, 82, 1361-1367. [CrossRef]

90. Gorden, N.T.; Arts, H.H.; Parisi, M.A.; Coene, K.L.; Letteboer, S.J.; van Beersum, S.E.; Mans, D.A.; Hikida, A.; Eckert, M.; Knutzen, D.; et al. CC2D2A is mutated in Joubert syndrome and interacts with the ciliopathy-associated basal body protein CEP290. Am. J. Hum. Genet. 2008, 83, 559-571. [CrossRef]

91. Mejecase, C.; Hummel, A.; Mohand-Said, S.; Andrieu, C.; El Shamieh, S.; Antonio, A.; Condroyer, C.; Boyard, F.; Foussard, M.; Blanchard, S.; et al. Whole exome sequencing resolves complex phenotype and identifies CC2D2A mutations underlying non-syndromic rod-cone dystrophy. Clin. Genet. 2019, 95, 329-333. [CrossRef]

92. Lewis, W.R.; Bales, K.L.; Revell, D.Z.; Croyle, M.J.; Engle, S.E.; Song, C.J.; Malarkey, E.B.; Uytingco, C.R.; Shan, D.; Antonellis, P.J.; et al. Mks6 mutations reveal tissue- and cell type-specific roles for the cilia transition zone. FASEB J. 2019, 33, 1440-1455. [CrossRef]

93. Sorusch, N.; Bauss, K.; Plutniok, J.; Samanta, A.; Knapp, B.; Nagel-Wolfrum, K.; Wolfrum, U. Characterization of the ternary Usher syndrome SANS/ush2a/whirlin protein complex. Hum. Mol. Genet. 2017, 26, 1157-1172. [CrossRef]

94. Liu, X.; Bulgakov, O.V.; Darrow, K.N.; Pawlyk, B.; Adamian, M.; Liberman, M.C.; Li, T. Usherin is required for maintenance of retinal photoreceptors and normal development of cochlear hair cells. Proc. Natl. Acad. Sci. USA 2007, 104, 4413-4418. [CrossRef] 
95. Yang, J.; Liu, X.; Zhao, Y.; Adamian, M.; Pawlyk, B.; Sun, X.; McMillan, D.R.; Liberman, M.C.; Li, T. Ablation of whirlin long isoform disrupts the USH2 protein complex and causes vision and hearing loss. PLoS Genet. 2010, 6, e1000955. [CrossRef] [PubMed]

96. Khattree, N.; Ritter, L.M.; Goldberg, A.F. Membrane curvature generation by a C-terminal amphipathic helix in peripherin-2/rds, a tetraspanin required for photoreceptor sensory cilium morphogenesis. J. Cell Sci. 2013, 126, 4659-4670. [CrossRef] [PubMed]

97. Molday, R.S.; Hicks, D.; Molday, L. Peripherin. A rim-specific membrane protein of rod outer segment discs. Investig. Ophthalmol. Vis. Sci. 1987, 28, 50-61. [PubMed]

98. Wood, C.R.; Huang, K.; Diener, D.R.; Rosenbaum, J.L. The cilium secretes bioactive ectosomes. Curr. Biol. 2013, 23, 906-911. [CrossRef]

99. Wood, C.R.; Rosenbaum, J.L. Ciliary ectosomes: Transmissions from the cell's antenna. Trends Cell Biol. 2015, 25, 276-285. [CrossRef] [PubMed]

100. Stuck, M.W.; Conley, S.M.; Naash, M.I. The Y141C knockin mutation in RDS leads to complex phenotypes in the mouse. Hum. Mol. Genet. 2014, 23, 6260-6274. [CrossRef]

101. Chakraborty, D.; Conley, S.M.; Zulliger, R.; Naash, M.I. The K153Del PRPH2 mutation differentially impacts photoreceptor structure and function. Hum. Mol. Genet. 2016, 25, 3500-3514. [CrossRef]

102. Sanyal, S.; De Ruiter, A.; Hawkins, R.K. Development and degeneration of retina in rds mutant mice: Light microscopy. J. Comp. Neurol. 1980, 194, 193-207. [CrossRef]

103. Sanyal, S.; Hawkins, R.K. Development and degeneration of retina in rds mutant mice: Effects of light on the rate of degeneration in albino and pigmented homozygous and heterozygous mutant and normal mice. Vis. Res. 1986, 26, 1177-1185. [CrossRef]

104. McNally, N.; Kenna, P.F.; Rancourt, D.; Ahmed, T.; Stitt, A.; Colledge, W.H.; Lloyd, D.G.; Palfi, A.; O’Neill, B.; Humphries, M.M.; et al. Murine model of autosomal dominant retinitis pigmentosa generated by targeted deletion at codon 307 of the rds-peripherin gene. Hum. Mol. Genet. 2002, 11, 1005-1016. [CrossRef]

105. Chakraborty, D.; Conley, S.M.; Al-Ubaidi, M.R.; Naash, M.I. Initiation of rod outer segment disc formation requires RDS. PLoS ONE 2014, 9, e98939. [CrossRef] [PubMed]

106. Kajiwara, K.; Berson, E.L.; Dryja, T.P. Digenic retinitis pigmentosa due to mutations at the unlinked peripherin/RDS and ROM1 loci. Science 1994, 264, 1604-1608. [CrossRef] [PubMed]

107. Clarke, G.; Goldberg, A.F.; Vidgen, D.; Collins, L.; Ploder, L.; Schwarz, L.; Molday, L.L.; Rossant, J.; Szel, A.; Molday, R.S.; et al. Rom-1 is required for rod photoreceptor viability and the regulation of disk morphogenesis. Nat. Genet. 2000, 25, 67-73. [CrossRef] [PubMed]

108. Sato, H.; Suzuki, T.; Ikeda, K.; Masuya, H.; Sezutsu, H.; Kaneda, H.; Kobayashi, K.; Miura, I.; Kurihara, Y.; Yokokura, S.; et al. A monogenic dominant mutation in Rom1 generated by N-ethyl-N-nitrosourea mutagenesis causes retinal degeneration in mice. Mol. Vis. 2010, 16, 378-391.

109. Spencer, W.J.; Pearring, J.N.; Salinas, R.Y.; Loiselle, D.R.; Skiba, N.P.; Arshavsky, V.Y. Progressive Rod-Cone Degeneration (PRCD) Protein Requires N-Terminal S-Acylation and Rhodopsin Binding for Photoreceptor Outer Segment Localization and Maintaining Intracellular Stability. Biochemistry 2016, 55, 5028-5037. [CrossRef]

110. Allon, G.; Mann, I.; Remez, L.; Sehn, E.; Rizel, L.; Nevet, M.J.; Perlman, I.; Wolfrum, U.; Ben-Yosef, T. PRCD is Concentrated at the Base of Photoreceptor Outer Segments and is Involved in Outer Segment Disc Formation. Hum. Mol. Genet. 2019, 28, 4078-4088. [CrossRef]

111. Zangerl, B.; Goldstein, O.; Philp, A.R.; Lindauer, S.J.; Pearce-Kelling, S.E.; Mullins, R.F.; Graphodatsky, A.S.; Ripoll, D.; Felix, J.S.; Stone, E.M.; et al. Identical mutation in a novel retinal gene causes progressive rod-cone degeneration in dogs and retinitis pigmentosa in humans. Genomics 2006, 88, 551-563. [CrossRef]

112. Spencer, W.J.; Ding, J.D.; Lewis, T.R.; Yu, C.; Phan, S.; Pearring, J.N.; Kim, K.Y.; Thor, A.; Mathew, R.; Kalnitsky, J.; et al. PRCD is essential for high-fidelity photoreceptor disc formation. Proc. Natl. Acad. Sci. USA 2019, 116, 13087-13096. [CrossRef]

113. Pedersen, L.B.; Rosenbaum, J.L. Intraflagellar transport (IFT) role in ciliary assembly, resorption and signalling. Curr. Top. Dev. Biol. 2008, 85, 23-61. [CrossRef]

114. Cortellino, S.; Wang, C.; Wang, B.; Bassi, M.R.; Caretti, E.; Champeval, D.; Calmont, A.; Jarnik, M.; Burch, J.; Zaret, K.S.; et al. Defective ciliogenesis, embryonic lethality and severe impairment of the Sonic Hedgehog pathway caused by inactivation of the mouse complex A intraflagellar transport gene Ift122/Wdr10, partially overlapping with the DNA repair gene Med1/Mbd4. Dev. Biol. 2009, 325, 225-237. [CrossRef] 
115. Murcia, N.S.; Richards, W.G.; Yoder, B.K.; Mucenski, M.L.; Dunlap, J.R.; Woychik, R.P. The Oak Ridge Polycystic Kidney (orpk) disease gene is required for left-right axis determination. Development 2000, 127, 2347-2355. [PubMed]

116. Stottmann, R.W.; Tran, P.V.; Turbe-Doan, A.; Beier, D.R. Ttc21b is required to restrict sonic hedgehog activity in the developing mouse forebrain. Dev. Biol. 2009, 335, 166-178. [CrossRef] [PubMed]

117. Gorivodsky, M.; Mukhopadhyay, M.; Wilsch-Braeuninger, M.; Phillips, M.; Teufel, A.; Kim, C.; Malik, N.; Huttner, W.; Westphal, H. Intraflagellar transport protein 172 is essential for primary cilia formation and plays a vital role in patterning the mammalian brain. Dev. Biol. 2009, 325, 24-32. [CrossRef] [PubMed]

118. Rix, S.; Calmont, A.; Scambler, P.J.; Beales, P.L. An Ift80 mouse model of short rib polydactyly syndromes shows defects in hedgehog signalling without loss or malformation of cilia. Hum. Mol. Genet. 2011, 20, 1306-1314. [CrossRef]

119. Berbari, N.F.; Kin, N.W.; Sharma, N.; Michaud, E.J.; Kesterson, R.A.; Yoder, B.K. Mutations in Traf3ip1 reveal defects in ciliogenesis, embryonic development, and altered cell size regulation. Dev. Biol. 2011, 360, 66-76. [CrossRef]

120. Bangs, F.; Anderson, K.V. Primary Cilia and Mammalian Hedgehog Signaling. Cold Spring Harb. Perspect. Biol. 2017, 9, a028175. [CrossRef]

121. Ko, H.W.; Liu, A.; Eggenschwiler, J.T. Analysis of hedgehog signaling in mouse intraflagellar transport mutants. Methods Cell Biol. 2009, 93, 347-369. [CrossRef]

122. Gupta, P.R.; Pendse, N.; Greenwald, S.H.; Leon, M.; Liu, Q.; Pierce, E.A.; Bujakowska, K.M. Ift172 conditional knock-out mice exhibit rapid retinal degeneration and protein trafficking defects. Hum. Mol. Genet. 2018, 27, 2012-2024. [CrossRef]

123. Keady, B.T.; Le, Y.Z.; Pazour, G.J. IFT20 is required for opsin trafficking and photoreceptor outer segment development. Mol. Biol. Cell 2011, 22, 921-930. [CrossRef]

124. Resh, M.D. Trafficking and signaling by fatty-acylated and prenylated proteins. Nat. Chem. Biol. 2006, 2, 584-590. [CrossRef]

125. Schwarz, N.; Hardcastle, A.J.; Cheetham, M.E. Arl3 and RP2 mediated assembly and traffic of membrane associated cilia proteins. Vis. Res. 2012, 75, 2-4. [CrossRef] [PubMed]

126. Li, L.; Khan, N.; Hurd, T.; Ghosh, A.K.; Cheng, C.; Molday, R.; Heckenlively, J.R.; Swaroop, A.; Khanna, H. Ablation of the X-linked retinitis pigmentosa $2(\mathrm{Rp} 2)$ gene in mice results in opsin mislocalization and photoreceptor degeneration. Investig. Ophthalmol. Vis. Sci. 2013, 54, 4503-4511. [CrossRef] [PubMed]

127. Zhang, H.; Hanke-Gogokhia, C.; Jiang, L.; Li, X.; Wang, P.; Gerstner, C.D.; Frederick, J.M.; Yang, Z.; Baehr, W. Mistrafficking of prenylated proteins causes retinitis pigmentosa 2. FASEB J. 2015, 29, 932-942. [CrossRef] [PubMed]

128. Wright, Z.C.; Singh, R.K.; Alpino, R.; Goldberg, A.F.; Sokolov, M.; Ramamurthy, V. ARL3 regulates trafficking of prenylated phototransduction proteins to the rod outer segment. Hum. Mol. Genet. 2016, 25, 2031-2044. [CrossRef]

129. Schrick, J.J.; Vogel, P.; Abuin, A.; Hampton, B.; Rice, D.S. ADP-ribosylation factor-like 3 is involved in kidney and photoreceptor development. Am. J. Pathol. 2006, 168, 1288-1298. [CrossRef]

130. Rao, K.N.; Zhang, W.; Li, L.; Anand, M.; Khanna, H. Prenylated retinal ciliopathy protein RPGR interacts with PDE6delta and regulates ciliary localization of Joubert syndrome-associated protein INPP5E. Hum. Mol. Genet. 2016, 25, 4533-4545. [CrossRef]

131. Xu, W.; Jin, M.; Hu, R.; Wang, H.; Zhang, F.; Yuan, S.; Cao, Y. The Joubert Syndrome Protein Inpp5e Controls Ciliogenesis by Regulating Phosphoinositides at the Apical Membrane. J. Am. Soc. Nephrol. 2017, 28, 118-129. [CrossRef]

132. Gillespie, P.G.; Prusti, R.K.; Apel, E.D.; Beavo, J.A. A soluble form of bovine rod photoreceptor phosphodiesterase has a novel 15-kDa subunit. J. Biol. Chem. 1989, 264, 12187-12193.

133. Thompson, D.A.; Khan, N.W.; Othman, M.I.; Chang, B.; Jia, L.; Grahek, G.; Wu, Z.; Hiriyanna, S.; Nellissery, J.; $\mathrm{Li}$, T.; et al. Rd9 is a naturally occurring mouse model of a common form of retinitis pigmentosa caused by mutations in RPGR-ORF15. PLOS ONE 2012, 7, e35865. [CrossRef]

134. Hong, D.H.; Pawlyk, B.S.; Shang, J.; Sandberg, M.A.; Berson, E.L.; Li, T. A retinitis pigmentosa GTPase regulator (RPGR)-deficient mouse model for X-linked retinitis pigmentosa (RP3). Proc. Natl. Acad. Sci. USA 2000, 97, 3649-3654. [CrossRef] 
135. Zhang, H.; Li, S.; Doan, T.; Rieke, F.; Detwiler, P.B.; Frederick, J.M.; Baehr, W. Deletion of PrBP/delta impedes transport of GRK1 and PDE6 catalytic subunits to photoreceptor outer segments. Proc. Natl. Acad. Sci. USA 2007, 104, 8857-8862. [CrossRef] [PubMed]

136. Ramamurthy, V.; Niemi, G.A.; Reh, T.A.; Hurley, J.B. Leber congenital amaurosis linked to AIPL1: A mouse model reveals destabilization of cGMP phosphodiesterase. Proc. Natl. Acad. Sci. USA 2004, 101, 13897-13902. [CrossRef] [PubMed]

137. Yadav, R.P.; Artemyev, N.O. AIPL1: A specialized chaperone for the phototransduction effector. Cell Signal. 2017, 40, 183-189. [CrossRef] [PubMed]

138. Scheidecker, S.; Etard, C.; Pierce, N.W.; Geoffroy, V.; Schaefer, E.; Muller, J.; Chennen, K.; Flori, E.; Pelletier, V.; Poch, O.; et al. Exome sequencing of Bardet-Biedl syndrome patient identifies a null mutation in the BBSome subunit BBIP1 (BBS18). J. Med. Genet. 2014, 51, 132-136. [CrossRef] [PubMed]

139. Eichers, E.R.; Abd-El-Barr, M.M.; Paylor, R.; Lewis, R.A.; Bi, W.; Lin, X.; Meehan, T.P.; Stockton, D.W.; Wu, S.M.; Lindsay, E.; et al. Phenotypic characterization of Bbs4 null mice reveals age-dependent penetrance and variable expressivity. Hum. Genet. 2006, 120, 211-226. [CrossRef] [PubMed]

140. Mykytyn, K.; Mullins, R.F.; Andrews, M.; Chiang, A.P.; Swiderski, R.E.; Yang, B.; Braun, T.; Casavant, T.; Stone, E.M.; Sheffield, V.C. Bardet-Biedl syndrome type 4 (BBS4)-null mice implicate Bbs4 in flagella formation but not global cilia assembly. Proc. Natl. Acad. Sci. USA 2004, 101, 8664-8669. [CrossRef] [PubMed]

141. Koch, K.W.; Dell'Orco, D. Protein and Signaling Networks in Vertebrate Photoreceptor Cells. Front. Mol. Neurosci. 2015, 8, 67. [CrossRef]

142. Pinto, L.H.; Vitaterna, M.H.; Shimomura, K.; Siepka, S.M.; McDearmon, E.L.; Fenner, D.; Lumayag, S.L.; Omura, C.; Andrews, A.W.; Baker, M.; et al. Generation, characterization, and molecular cloning of the Noerg-1 mutation of rhodopsin in the mouse. Vis. Neurosci. 2005, 22, 619-629. [CrossRef]

143. Liu, H.; Wang, M.; Xia, C.H.; Du, X.; Flannery, J.G.; Ridge, K.D.; Beutler, B.; Gong, X. Severe retinal degeneration caused by a novel rhodopsin mutation. Investig. Ophthalmol. Vis. Sci. 2010, 51, 1059-1065. [CrossRef]

144. Sakami, S.; Maeda, T.; Bereta, G.; Okano, K.; Golczak, M.; Sumaroka, A.; Roman, A.J.; Cideciyan, A.V.; Jacobson, S.G.; Palczewski, K. Probing mechanisms of photoreceptor degeneration in a new mouse model of the common form of autosomal dominant retinitis pigmentosa due to $\mathrm{P} 23 \mathrm{H}$ opsin mutations. J. Biol. Chem. 2011, 286, 10551-10567. [CrossRef]

145. Chiang, W.C.; Kroeger, H.; Sakami, S.; Messah, C.; Yasumura, D.; Matthes, M.T.; Coppinger, J.A.; Palczewski, K.; LaVail, M.M.; Lin, J.H. Robust Endoplasmic Reticulum-Associated Degradation of Rhodopsin Precedes Retinal Degeneration. Mol. Neurobiol. 2015, 52, 679-695. [CrossRef] [PubMed]

146. Comitato, A.; Schiroli, D.; Montanari, M.; Marigo, V. Calpain Activation Is the Major Cause of Cell Death in Photoreceptors Expressing a Rhodopsin Misfolding Mutation. Mol. Neurobiol. 2020, 57, 589-599. [CrossRef] [PubMed]

147. Athanasiou, D.; Aguila, M.; Bevilacqua, D.; Novoselov, S.S.; Parfitt, D.A.; Cheetham, M.E. The cell stress machinery and retinal degeneration. FEBS Lett. 2013, 587, 2008-2017. [CrossRef] [PubMed]

148. Athanasiou, D.; Aguila, M.; Bellingham, J.; Kanuga, N.; Adamson, P.; Cheetham, M.E. The role of the ER stress-response protein PERK in rhodopsin retinitis pigmentosa. Hum. Mol. Genet. 2017, 26, 4896-4905. [CrossRef]

149. Zhang, N.; Kolesnikov, A.V.; Jastrzebska, B.; Mustafi, D.; Sawada, O.; Maeda, T.; Genoud, C.; Engel, A.; Kefalov, V.J.; Palczewski, K. Autosomal recessive retinitis pigmentosa E150K opsin mice exhibit photoreceptor disorganization. J. Clin. Investig. 2013, 123, 121-137. [CrossRef]

150. Sakami, S.; Kolesnikov, A.V.; Kefalov, V.J.; Palczewski, K. P23H opsin knock-in mice reveal a novel step in retinal rod disc morphogenesis. Hum. Mol. Genet. 2014, 23, 1723-1741. [CrossRef]

151. Hollingsworth, T.J.; Gross, A.K. The severe autosomal dominant retinitis pigmentosa rhodopsin mutant Ter349Glu mislocalizes and induces rapid rod cell death. J. Biol. Chem. 2013, 288, 29047-29055. [CrossRef]

152. Humphries, M.M.; Rancourt, D.; Farrar, G.J.; Kenna, P.; Hazel, M.; Bush, R.A.; Sieving, P.A.; Sheils, D.M.; McNally, N.; Creighton, P.; et al. Retinopathy induced in mice by targeted disruption of the rhodopsin gene. Nat. Genet. 1997, 15, 216-219. [CrossRef]

153. Lem, J.; Krasnoperova, N.V.; Calvert, P.D.; Kosaras, B.; Cameron, D.A.; Nicolo, M.; Makino, C.L.; Sidman, R.L. Morphological, physiological, and biochemical changes in rhodopsin knockout mice. Proc. Natl. Acad. Sci. USA 1999, 96, 736-741. [CrossRef] 
154. Faber, S.; Roepman, R. Balancing the Photoreceptor Proteome: Proteostasis Network Therapeutics for Inherited Retinal Disease. Genes 2019, 10,557. [CrossRef]

155. Sancho-Pelluz, J.; Cui, X.; Lee, W.; Tsai, Y.T.; Wu, W.H.; Justus, S.; Washington, I.; Hsu, C.W.; Park, K.S.; Koch, S.; et al. Mechanisms of neurodegeneration in a preclinical autosomal dominant retinitis pigmentosa knock-in model with a Rho(D190N) mutation. Cell Mol. Life Sci. 2019, 76, 3657-3665. [CrossRef] [PubMed]

156. Park, P.S. Constitutively active rhodopsin and retinal disease. Adv. Pharmacol. 2014, 70, 1-36. [CrossRef] [PubMed]

157. Budzynski, E.; Gross, A.K.; McAlear, S.D.; Peachey, N.S.; Shukla, M.; He, F.; Edwards, M.; Won, J.; Hicks, W.L.; Wensel, T.G.; et al. Mutations of the opsin gene (Y102H and I307N) lead to light-induced degeneration of photoreceptors and constitutive activation of phototransduction in mice. J. Biol. Chem. 2010, 285, 14521-14533. [CrossRef] [PubMed]

158. Daniele, L.L.; Insinna, C.; Chance, R.; Wang, J.; Nikonov, S.S.; Pugh, E.N., Jr. A mouse M-opsin monochromat: Retinal cone photoreceptors have increased M-opsin expression when S-opsin is knocked out. Vis. Res. 2011, 51, 447-458. [CrossRef] [PubMed]

159. Zhang, Y.; Deng, W.T.; Du, W.; Zhu, P.; Li, J.; Xu, F.; Sun, J.; Gerstner, C.D.; Baehr, W.; Boye, S.L.; et al. Gene-based Therapy in a Mouse Model of Blue Cone Monochromacy. Sci. Rep. 2017, 7, 6690. [CrossRef] [PubMed]

160. Carter-Dawson, L.D.; LaVail, M.M.; Sidman, R.L. Differential effect of the rd mutation on rods and cones in the mouse retina. Investig. Ophthalmol. Vis. Sci. 1978, 17, 489-498. [PubMed]

161. Calvert, P.D.; Krasnoperova, N.V.; Lyubarsky, A.L.; Isayama, T.; Nicolo, M.; Kosaras, B.; Wong, G.; Gannon, K.S.; Margolskee, R.F.; Sidman, R.L.; et al. Phototransduction in transgenic mice after targeted deletion of the rod transducin alpha -subunit. Proc. Natl. Acad. Sci. USA 2000, 97, 13913-13918. [CrossRef]

162. Barber, A.C.; Hippert, C.; Duran, Y.; West, E.L.; Bainbridge, J.W.; Warre-Cornish, K.; Luhmann, U.F.; Lakowski, J.; Sowden, J.C.; Ali, R.R.; et al. Repair of the degenerate retina by photoreceptor transplantation. Proc. Natl. Acad. Sci. USA 2013, 110, 354-359. [CrossRef]

163. Miyamoto, M.; Aoki, M.; Sugimoto, S.; Kawasaki, K.; Imai, R. IRD1 and IRD2 mice, naturally occurring models of hereditary retinal dysfunction, show late-onset and progressive retinal degeneration. Curr. Eye Res. 2010, 35, 137-145. [CrossRef]

164. Mejecase, C.; Laurent-Coriat, C.; Mayer, C.; Poch, O.; Mohand-Said, S.; Prevot, C.; Antonio, A.; Boyard, F.; Condroyer, C.; Michiels, C.; et al. Identification of a Novel Homozygous Nonsense Mutation Confirms the Implication of GNAT1 in Rod-Cone Dystrophy. PLoS ONE 2016, 11, e0168271. [CrossRef]

165. Carrigan, M.; Duignan, E.; Humphries, P.; Palfi, A.; Kenna, P.F.; Farrar, G.J. A novel homozygous truncating GNAT1 mutation implicated in retinal degeneration. Br. J. Ophthalmol. 2016, 100, 495-500. [CrossRef] [PubMed]

166. Zenteno, J.C.; Garcia-Montano, L.A.; Cruz-Aguilar, M.; Ronquillo, J.; Rodas-Serrano, A.; Aguilar-Castul, L.; Matsui, R.; Vencedor-Meraz, C.I.; Arce-Gonzalez, R.; Graue-Wiechers, F.; et al. Extensive genic and allelic heterogeneity underlying inherited retinal dystrophies in Mexican patients molecularly analyzed by next-generation sequencing. Mol. Genet. Genomic Med. 2020, 8. [CrossRef] [PubMed]

167. Deng, W.T.; Sakurai, K.; Liu, J.; Dinculescu, A.; Li, J.; Pang, J.; Min, S.H.; Chiodo, V.A.; Boye, S.L.; Chang, B.; et al. Functional interchangeability of rod and cone transducin alpha-subunits. Proc. Natl. Acad. Sci. USA 2009, 106, 17681-17686. [CrossRef] [PubMed]

168. Chang, B.; Hawes, N.L.; Hurd, R.E.; Wang, J.; Davisson, M.T.; Nusinowitz, S.; Heckenlively, J.R. A New Mouse Model of Retinal Degeneration (rd17). Proceedings of ARVO Annual Meeting Abstract, Fort Lauderdale, FL, USA, 6-10 May 2007.

169. Lobanova, E.S.; Finkelstein, S.; Herrmann, R.; Chen, Y.M.; Kessler, C.; Michaud, N.A.; Trieu, L.H.; Strissel, K.J.; Burns, M.E.; Arshavsky, V.Y. Transducin gamma-subunit sets expression levels of alpha- and beta-subunits and is crucial for rod viability. J. Neurosci. 2008, 28, 3510-3520. [CrossRef] [PubMed]

170. Kolesnikov, A.V.; Rikimaru, L.; Hennig, A.K.; Lukasiewicz, P.D.; Fliesler, S.J.; Govardovskii, V.I.; Kefalov, V.J.; Kisselev, O.G. G-protein betagamma-complex is crucial for efficient signal amplification in vision. J. Neurosci. 2011, 31, 8067-8077. [CrossRef]

171. Lobanova, E.S.; Finkelstein, S.; Skiba, N.P.; Arshavsky, V.Y. Proteasome overload is a common stress factor in multiple forms of inherited retinal degeneration. Proc. Natl. Acad. Sci. USA 2013, 110, 9986-9991. [CrossRef] 
172. Jobling, A.I.; Vessey, K.A.; Waugh, M.; Mills, S.A.; Fletcher, E.L. A naturally occurring mouse model of achromatopsia: Characterization of the mutation in cone transducin and subsequent retinal phenotype. Investig. Ophthalmol. Vis. Sci. 2013, 54, 3350-3359. [CrossRef]

173. Chang, B.; Dacey, M.S.; Hawes, N.L.; Hitchcock, P.F.; Milam, A.H.; Atmaca-Sonmez, P.; Nusinowitz, S.; Heckenlively, J.R. Cone photoreceptor function loss-3, a novel mouse model of achromatopsia due to a mutation in Gnat2. Investig. Ophthalmol. Vis. Sci. 2006, 47, 5017-5021. [CrossRef]

174. Ronning, K.E.; Allina, G.P.; Miller, E.B.; Zawadzki, R.J.; Pugh, E.N., Jr.; Herrmann, R.; Burns, M.E. Loss of cone function without degeneration in a novel Gnat2 knock-out mouse. Exp. Eye Res. 2018, 171, 111-118. [CrossRef]

175. Hirji, N.; Aboshiha, J.; Georgiou, M.; Bainbridge, J.; Michaelides, M. Achromatopsia: Clinical features, molecular genetics, animal models and therapeutic options. Ophthalmic Genet. 2018, 39, 149-157. [CrossRef]

176. Michaelides, M.; Aligianis, I.A.; Holder, G.E.; Simunovic, M.; Mollon, J.D.; Maher, E.R.; Hunt, D.M.; Moore, A.T. Cone dystrophy phenotype associated with a frameshift mutation (M280fsX291) in the alpha-subunit of cone specific transducin (GNAT2). Br. J. Ophthalmol. 2003, 87, 1317-1320. [CrossRef]

177. Du, J.; An, J.; Linton, J.D.; Wang, Y.; Hurley, J.B. How Excessive cGMP Impacts Metabolic Proteins in Retinas at the Onset of Degeneration. Adv. Exp. Med. Biol. 2018, 1074, 289-295. [CrossRef]

178. Tolone, A.; Belhadj, S.; Rentsch, A.; Schwede, F.; Paquet-Durand, F. The cGMP Pathway and Inherited Photoreceptor Degeneration: Targets, Compounds, and Biomarkers. Genes 2019, 10, 453. [CrossRef] [PubMed]

179. Sothilingam, V.; Garcia Garrido, M.; Jiao, K.; Buena-Atienza, E.; Sahaboglu, A.; Trifunovic, D.; Balendran, S.; Koepfli, T.; Muhlfriedel, R.; Schon, C.; et al. Retinitis pigmentosa: Impact of different Pde6a point mutations on the disease phenotype. Hum. Mol. Genet. 2015, 24, 5486-5499. [CrossRef]

180. Sakamoto, K.; McCluskey, M.; Wensel, T.G.; Naggert, J.K.; Nishina, P.M. New mouse models for recessive retinitis pigmentosa caused by mutations in the Pde6a gene. Hum. Mol. Genet. 2009, 18, 178-192. [CrossRef]

181. Power, M.; Das, S.; Schutze, K.; Marigo, V.; Ekstrom, P.; Paquet-Durand, F. Cellular mechanisms of hereditary photoreceptor degeneration-Focus on cGMP. Prog. Retin. Eye Res. 2020, 74, 100772. [CrossRef] [PubMed]

182. Tsang, S.H.; Gouras, P.; Yamashita, C.K.; Kjeldbye, H.; Fisher, J.; Farber, D.B.; Goff, S.P. Retinal degeneration in mice lacking the gamma subunit of the rod cGMP phosphodiesterase. Science 1996, 272, 1026-1029. [CrossRef] [PubMed]

183. Thiadens, A.A.; Somervuo, V.; van den Born, L.I.; Roosing, S.; van Schooneveld, M.J.; Kuijpers, R.W.; van Moll-Ramirez, N.; Cremers, F.P.; Hoyng, C.B.; Klaver, C.C. Progressive loss of cones in achromatopsia: An imaging study using spectral-domain optical coherence tomography. Investig. Ophthalmol. Vis. Sci. 2010, 51, 5952-5957. [CrossRef] [PubMed]

184. Brennenstuhl, C.; Tanimoto, N.; Burkard, M.; Wagner, R.; Bolz, S.; Trifunovic, D.; Kabagema-Bilan, C.; Paquet-Durand, F.; Beck, S.C.; Huber, G.; et al. Targeted ablation of the Pde6h gene in mice reveals cross-species differences in cone and rod phototransduction protein isoform inventory. J. Biol. Chem. 2015, 290, 10242-10255. [CrossRef]

185. Kohl, S.; Coppieters, F.; Meire, F.; Schaich, S.; Roosing, S.; Brennenstuhl, C.; Bolz, S.; van Genderen, M.M.; Riemslag, F.C.; European Retinal Disease, C.; et al. A nonsense mutation in PDE6H causes autosomal-recessive incomplete achromatopsia. Am. J. Hum. Genet. 2012, 91, 527-532. [CrossRef]

186. Pedurupillay, C.R.; Landsend, E.C.; Vigeland, M.D.; Ansar, M.; Frengen, E.; Misceo, D.; Stromme, P. Segregation of Incomplete Achromatopsia and Alopecia Due to PDE6H and LPAR6 Variants in a Consanguineous Family from Pakistan. Genes 2016, 7, 41. [CrossRef]

187. Weitz, D.; Ficek, N.; Kremmer, E.; Bauer, P.J.; Kaupp, U.B. Subunit stoichiometry of the CNG channel of rod photoreceptors. Neuron 2002, 36, 881-889. [CrossRef]

188. Zheng, J.; Trudeau, M.C.; Zagotta, W.N. Rod cyclic nucleotide-gated channels have a stoichiometry of three CNGA1 subunits and one CNGB1 subunit. Neuron 2002, 36, 891-896. [CrossRef]

189. Huttl, S.; Michalakis, S.; Seeliger, M.; Luo, D.G.; Acar, N.; Geiger, H.; Hudl, K.; Mader, R.; Haverkamp, S.; Moser, M.; et al. Impaired channel targeting and retinal degeneration in mice lacking the cyclic nucleotide-gated channel subunit CNGB1. J. Neurosci. 2005, 25, 130-138. [CrossRef] [PubMed]

190. Vinberg, F.; Wang, T.; Molday, R.S.; Chen, J.; Kefalov, V.J. A new mouse model for stationary night blindness with mutant Slc24a1 explains the pathophysiology of the associated human disease. Hum. Mol. Genet. 2015, 24, 5915-5929. [CrossRef] [PubMed] 
191. Yang, R.B.; Robinson, S.W.; Xiong, W.H.; Yau, K.W.; Birch, D.G.; Garbers, D.L. Disruption of a retinal guanylyl cyclase gene leads to cone-specific dystrophy and paradoxical rod behavior. J. Neurosci. 1999, 19, 5889-5897. [CrossRef] [PubMed]

192. Bouzia, Z.; Georgiou, M.; Hull, S.; Robson, A.G.; Fujinami, K.; Rotsos, T.; Pontikos, N.; Arno, G.; Webster, A.R.; Hardcastle, A.J.; et al. GUCY2D-Associated Leber Congenital Amaurosis: A Retrospective Natural History Study in Preparation for Trials of Novel Therapies. Am. J. Ophthalmol. 2020, 210, 59-70. [CrossRef]

193. Baehr, W.; Karan, S.; Maeda, T.; Luo, D.G.; Li, S.; Bronson, J.D.; Watt, C.B.; Yau, K.W.; Frederick, J.M.; Palczewski, K. The function of guanylate cyclase 1 and guanylate cyclase 2 in rod and cone photoreceptors. J. Biol. Chem. 2007, 282, 8837-8847. [CrossRef]

194. Mendez, A.; Burns, M.E.; Sokal, I.; Dizhoor, A.M.; Baehr, W.; Palczewski, K.; Baylor, D.A.; Chen, J. Role of guanylate cyclase-activating proteins (GCAPs) in setting the flash sensitivity of rod photoreceptors. Proc. Natl. Acad. Sci. USA 2001, 98, 9948-9953. [CrossRef]

195. Buch, P.K.; Mihelec, M.; Cottrill, P.; Wilkie, S.E.; Pearson, R.A.; Duran, Y.; West, E.L.; Michaelides, M.; Ali, R.R.; Hunt, D.M. Dominant cone-rod dystrophy: A mouse model generated by gene targeting of the GCAP1/Guca1a gene. PLoS ONE 2011, 6, e18089. [CrossRef]

196. Marino, V.; Dal Cortivo, G.; Oppici, E.; Maltese, P.E.; D’Esposito, F.; Manara, E.; Ziccardi, L.; Falsini, B.; Magli, A.; Bertelli, M.; et al. A novel p.(Glu111Val) missense mutation in GUCA1A associated with cone-rod dystrophy leads to impaired calcium sensing and perturbed second messenger homeostasis in photoreceptors. Hum. Mol. Genet. 2018, 27, 4204-4217. [CrossRef] [PubMed]

197. Chen, C.K.; Burns, M.E.; Spencer, M.; Niemi, G.A.; Chen, J.; Hurley, J.B.; Baylor, D.A.; Simon, M.I. Abnormal photoresponses and light-induced apoptosis in rods lacking rhodopsin kinase. Proc. Natl. Acad. Sci. USA 1999, 96, 3718-3722. [CrossRef] [PubMed]

198. Xu, J.; Dodd, R.L.; Makino, C.L.; Simon, M.I.; Baylor, D.A.; Chen, J. Prolonged photoresponses in transgenic mouse rods lacking arrestin. Nature 1997, 389, 505-509. [CrossRef] [PubMed]

199. Chen, J.; Simon, M.I.; Matthes, M.T.; Yasumura, D.; LaVail, M.M. Increased susceptibility to light damage in an arrestin knockout mouse model of Oguchi disease (stationary night blindness). Investig. Ophthalmol. Vis. Sci. 1999, 40, 2978-2982. [PubMed]

200. Charette, J.R.; Samuels, I.S.; Yu, M.; Stone, L.; Hicks, W.; Shi, L.Y.; Krebs, M.P.; Naggert, J.K.; Nishina, P.M.; Peachey, N.S. A Chemical Mutagenesis Screen Identifies Mouse Models with ERG Defects. Adv. Exp. Med. Biol. 2016, 854, 177-183. [CrossRef] [PubMed]

201. Rajappa, M.; Goyal, A.; Kaur, J. Inherited metabolic disorders involving the eye: A clinico-biochemical perspective. Eye 2010, 24, 507-518. [CrossRef] [PubMed]

202. Poll-The, B.T.; Maillette de Buy Wenniger-Prick, C.J. The eye in metabolic diseases: Clues to diagnosis. Eur. J. Paediatr. Neurol. 2011, 15, 197-204. [CrossRef]

203. Wright, A.F.; Chakarova, C.F.; Abd El-Aziz, M.M.; Bhattacharya, S.S. Photoreceptor degeneration: Genetic and mechanistic dissection of a complex trait. Nat. Rev. Genet. 2010, 11, 273-284. [CrossRef]

204. Fliesler, S.J.; Anderson, R.E. Chemistry and metabolism of lipids in the vertebrate retina. Prog. Lipid Res. 1983, 22, 79-131. [CrossRef]

205. Giusto, N.M.; Pasquare, S.J.; Salvador, G.A.; Ilincheta de Boschero, M.G. Lipid second messengers and related enzymes in vertebrate rod outer segments. J. Lipid Res. 2010, 51, 685-700. [CrossRef]

206. Niu, S.L.; Mitchell, D.C.; Litman, B.J. Manipulation of cholesterol levels in rod disk membranes by methyl-beta-cyclodextrin: Effects on receptor activation. J. Biol. Chem. 2002, 277, 20139-20145. [CrossRef] [PubMed]

207. Bretillon, L.; Thuret, G.; Gregoire, S.; Acar, N.; Joffre, C.; Bron, A.M.; Gain, P.; Creuzot-Garcher, C.P. Lipid and fatty acid profile of the retina, retinal pigment epithelium/choroid, and the lacrimal gland, and associations with adipose tissue fatty acids in human subjects. Exp. Eye Res. 2008, 87, 521-528. [CrossRef]

208. Fliesler, S.J.; Bretillon, L. The ins and outs of cholesterol in the vertebrate retina. J. Lipid Res. 2010, 51, 3399-3413. [CrossRef]

209. German, O.L.; Agnolazza, D.L.; Politi, L.E.; Rotstein, N.P. Light, lipids and photoreceptor survival: Live or let die? Photochem. Photobiol. Sci. 2015, 14, 1737-1753. [CrossRef] [PubMed] 
210. Shindou, H.; Koso, H.; Sasaki, J.; Nakanishi, H.; Sagara, H.; Nakagawa, K.M.; Takahashi, Y.; Hishikawa, D.; Iizuka-Hishikawa, Y.; Tokumasu, F.; et al. Docosahexaenoic acid preserves visual function by maintaining correct disc morphology in retinal photoreceptor cells. J. Biol. Chem. 2017, 292, 12054-12064. [CrossRef] [PubMed]

211. Lobanova, E.S.; Schuhmann, K.; Finkelstein, S.; Lewis, T.R.; Cady, M.A.; Hao, Y.; Keuthan, C.; Ash, J.D.; Burns, M.E.; Shevchenko, A.; et al. Disrupted Blood-Retina Lysophosphatidylcholine Transport Impairs Photoreceptor Health But Not Visual Signal Transduction. J. Neurosci. 2019, 39, 9689-9701. [CrossRef] [PubMed]

212. Pham, T.L.; He, J.; Kakazu, A.H.; Jun, B.; Bazan, N.G.; Bazan, H.E.P. Defining a mechanistic link between pigment epithelium-derived factor, docosahexaenoic acid, and corneal nerve regeneration. J. Biol. Chem. 2017, 292, 18486-18499. [CrossRef]

213. Comitato, A.; Subramanian, P.; Turchiano, G.; Montanari, M.; Becerra, S.P.; Marigo, V. Pigment epithelium-derived factor hinders photoreceptor cell death by reducing intracellular calcium in the degenerating retina. Cell Death Dis. 2018, 9, 560. [CrossRef]

214. Bernstein, P.S.; Tammur, J.; Singh, N.; Hutchinson, A.; Dixon, M.; Pappas, C.M.; Zabriskie, N.A.; Zhang, K.; Petrukhin, K.; Leppert, M.; et al. Diverse macular dystrophy phenotype caused by a novel complex mutation in the ELOVL4 gene. Investig. Ophthalmol. Vis. Sci. 2001, 42, 3331-3336.

215. Vasireddy, V.; Jablonski, M.M.; Mandal, M.N.; Raz-Prag, D.; Wang, X.F.; Nizol, L.; Iannaccone, A.; Musch, D.C.; Bush, R.A.; Salem, N., Jr.; et al. Elovl4 5-bp-deletion knock-in mice develop progressive photoreceptor degeneration. Investig. Ophthalmol. Vis. Sci. 2006, 47, 4558-4568. [CrossRef]

216. Friedman, J.S.; Chang, B.; Krauth, D.S.; Lopez, I.; Waseem, N.H.; Hurd, R.E.; Feathers, K.L.; Branham, K.E.; Shaw, M.; Thomas, G.E.; et al. Loss of lysophosphatidylcholine acyltransferase 1 leads to photoreceptor degeneration in rd11 mice. Proc. Natl. Acad. Sci. USA 2010, 107, 15523-15528. [CrossRef] [PubMed]

217. Perkovic, T.; Duh, D.; Peterlin, B.; Gregoric, J. The Str mouse as a model for incontinentia pigmenti. Pflugers Arch. 2000, 440, R53-R54. [CrossRef] [PubMed]

218. Coleman, J.A.; Zhu, X.; Djajadi, H.R.; Molday, L.L.; Smith, R.S.; Libby, R.T.; John, S.W.; Molday, R.S Phospholipid flippase ATP8A2 is required for normal visual and auditory function and photoreceptor and spiral ganglion cell survival. J. Cell Sci. 2014, 127, 1138-1149. [CrossRef] [PubMed]

219. Bryde, S.; Hennrich, H.; Verhulst, P.M.; Devaux, P.F.; Lenoir, G.; Holthuis, J.C. CDC50 proteins are critical components of the human class-1 P4-ATPase transport machinery. J. Biol. Chem. 2010, 285, 40562-40572. [CrossRef]

220. van der Velden, L.M.; Wichers, C.G.; van Breevoort, A.E.; Coleman, J.A.; Molday, R.S.; Berger, R.; Klomp, L.W.; van de Graaf, S.F. Heteromeric interactions required for abundance and subcellular localization of human CDC50 proteins and class 1 P4-ATPases. J. Biol. Chem. 2010, 285, 40088-40096. [CrossRef]

221. Zhang, L.; Yang, Y.; Li, S.; Zhang, S.; Zhu, X.; Tai, Z.; Yang, M.; Liu, Y.; Guo, X.; Chen, B.; et al. Loss of Tmem30a leads to photoreceptor degeneration. Sci. Rep. 2017, 7, 9296. [CrossRef]

222. Wong-Riley, M.T. Energy metabolism of the visual system. Eye Brain 2010, 2, 99-116. [CrossRef] [PubMed]

223. Du, J.; Rountree, A.; Cleghorn, W.M.; Contreras, L.; Lindsay, K.J.; Sadilek, M.; Gu, H.; Djukovic, D.; Raftery, D.; Satrustegui, J.; et al. Phototransduction Influences Metabolic Flux and Nucleotide Metabolism in Mouse Retina. J. Biol. Chem. 2016, 291, 4698-4710. [CrossRef]

224. Joyal, J.S.; Sun, Y.; Gantner, M.L.; Shao, Z.; Evans, L.P.; Saba, N.; Fredrick, T.; Burnim, S.; Kim, J.S.; Patel, G.; et al. Retinal lipid and glucose metabolism dictates angiogenesis through the lipid sensor Ffar1. Nat. Med. 2016, 22, 439-445. [CrossRef]

225. Zhang, L.; Sun, Z.; Zhao, P.; Huang, L.; Xu, M.; Yang, Y.; Chen, X.; Lu, F.; Zhang, X.; Wang, H.; et al. Whole-exome sequencing revealed HKDC1 as a candidate gene associated with autosomal-recessive retinitis pigmentosa. Hum. Mol. Genet. 2018, 27, 4157-4168. [CrossRef]

226. Xia, C.H.; Lu, E.; Liu, H.; Du, X.; Beutler, B.; Gong, X. The role of Vldlr in intraretinal angiogenesis in mice. Investig. Ophthalmol. Vis. Sci. 2011, 52, 6572-6579. [CrossRef] [PubMed]

227. Hu, W.; Jiang, A.; Liang, J.; Meng, H.; Chang, B.; Gao, H.; Qiao, X. Expression of VLDLR in the retina and evolution of subretinal neovascularization in the knockout mouse model's retinal angiomatous proliferation. Investig. Ophthalmol. Vis. Sci. 2008, 49, 407-415. [CrossRef] [PubMed] 
228. Chen, Y.; Hu, Y.; Moiseyev, G.; Zhou, K.K.; Chen, D.; Ma, J.X. Photoreceptor degeneration and retinal inflammation induced by very low-density lipoprotein receptor deficiency. Microvasc. Res. 2009, 78, 119-127. [CrossRef] [PubMed]

229. Lin, J.B.; Kubota, S.; Ban, N.; Yoshida, M.; Santeford, A.; Sene, A.; Nakamura, R.; Zapata, N.; Kubota, M.; Tsubota, K.; et al. NAMPT-Mediated NAD(+) Biosynthesis Is Essential for Vision In Mice. Cell Rep. 2016, 17, 69-85. [CrossRef]

230. Greenwald, S.H.; Charette, J.R.; Staniszewska, M.; Shi, L.Y.; Brown, S.D.M.; Stone, L.; Liu, Q.; Hicks, W.L.; Collin, G.B.; Bowl, M.R.; et al. Mouse Models of NMNAT1-Leber Congenital Amaurosis (LCA9) Recapitulate Key Features of the Human Disease. Am. J. Pathol. 2016, 186, 1925-1938. [CrossRef]

231. Bosl, M.R.; Stein, V.; Hubner, C.; Zdebik, A.A.; Jordt, S.E.; Mukhopadhyay, A.K.; Davidoff, M.S.; Holstein, A.F.; Jentsch, T.J. Male germ cells and photoreceptors, both dependent on close cell-cell interactions, degenerate upon ClC-2 Cl(-) channel disruption. EMBO J. 2001, 20, 1289-1299. [CrossRef]

232. Ng, L.; Lyubarsky, A.; Nikonov, S.S.; Ma, M.; Srinivas, M.; Kefas, B.; St Germain, D.L.; Hernandez, A.; Pugh, E.N., Jr.; Forrest, D. Type 3 deiodinase, a thyroid-hormone-inactivating enzyme, controls survival and maturation of cone photoreceptors. J. Neurosci. 2010, 30, 3347-3357. [CrossRef]

233. Ng, L.; Hurley, J.B.; Dierks, B.; Srinivas, M.; Salto, C.; Vennstrom, B.; Reh, T.A.; Forrest, D. A thyroid hormone receptor that is required for the development of green cone photoreceptors. Nat. Genet. 2001, 27, 94-98. [CrossRef]

234. Gianesini, C.; Hiragaki, S.; Laurent, V.; Hicks, D.; Tosini, G. Cone Viability Is Affected by Disruption of Melatonin Receptors Signaling. Investig. Ophthalmol. Vis. Sci. 2016, 57, 94-104. [CrossRef]

235. Baba, K.; Pozdeyev, N.; Mazzoni, F.; Contreras-Alcantara, S.; Liu, C.; Kasamatsu, M.; Martinez-Merlos, T.; Strettoi, E.; Iuvone, P.M.; Tosini, G. Melatonin modulates visual function and cell viability in the mouse retina via the MT1 melatonin receptor. Proc. Natl. Acad. Sci. USA 2009, 106, 15043-15048. [CrossRef]

236. Chen, Y.; Mehta, G.; Vasiliou, V. Antioxidant defenses in the ocular surface. Ocul. Surf. 2009, 7, 176-185. [CrossRef]

237. Nita, M.; Grzybowski, A. The Role of the Reactive Oxygen Species and Oxidative Stress in the Pathomechanism of the Age-Related Ocular Diseases and Other Pathologies of the Anterior and Posterior Eye Segments in Adults. Oxid. Med. Cell Longev. 2016, 2016, 3164734. [CrossRef] [PubMed]

238. Chen, H.; Lukas, T.J.; Du, N.; Suyeoka, G.; Neufeld, A.H. Dysfunction of the retinal pigment epithelium with age: Increased iron decreases phagocytosis and lysosomal activity. Investig. Ophthalmol. Vis. Sci. 2009, 50, 1895-1902. [CrossRef] [PubMed]

239. Blasiak, J.; Glowacki, S.; Kauppinen, A.; Kaarniranta, K. Mitochondrial and nuclear DNA damage and repair in age-related macular degeneration. Int. J. Mol. Sci. 2013, 14, 2996-3010. [CrossRef]

240. Tan, B.L.; Norhaizan, M.E.; Liew, W.P.; Sulaiman Rahman, H. Antioxidant and Oxidative Stress: A Mutual Interplay in Age-Related Diseases. Front. Pharmacol. 2018, 9, 1162. [CrossRef]

241. Frohns, A.; Frohns, F.; Naumann, S.C.; Layer, P.G.; Lobrich, M. Inefficient double-strand break repair in murine rod photoreceptors with inverted heterochromatin organization. Curr. Biol. 2014, 24, 1080-1090. [CrossRef]

242. Blasiak, J.; Petrovski, G.; Vereb, Z.; Facsko, A.; Kaarniranta, K. Oxidative stress, hypoxia, and autophagy in the neovascular processes of age-related macular degeneration. Biomed. Res. Int. 2014, 2014, 768026. [CrossRef]

243. Tokarz, P.; Kaarniranta, K.; Blasiak, J. Role of antioxidant enzymes and small molecular weight antioxidants in the pathogenesis of age-related macular degeneration (AMD). Biogerontology 2013, 14, 461-482. [CrossRef]

244. Hashizume, K.; Hirasawa, M.; Imamura, Y.; Noda, S.; Shimizu, T.; Shinoda, K.; Kurihara, T.; Noda, K.; Ozawa, Y.; Ishida, S.; et al. Retinal dysfunction and progressive retinal cell death in SOD1-deficient mice. Am. J. Pathol. 2008, 172, 1325-1331. [CrossRef]

245. Biswal, M.R.; Ildefonso, C.J.; Mao, H.; Seo, S.J.; Wang, Z.; Li, H.; Le, Y.Z.; Lewin, A.S. Conditional Induction of Oxidative Stress in RPE: A Mouse Model of Progressive Retinal Degeneration. Adv. Exp. Med. Biol. 2016, 854, 31-37. [CrossRef]

246. Cronin, T.; Raffelsberger, W.; Lee-Rivera, I.; Jaillard, C.; Niepon, M.L.; Kinzel, B.; Clerin, E.; Petrosian, A.; Picaud, S.; Poch, O.; et al. The disruption of the rod-derived cone viability gene leads to photoreceptor dysfunction and susceptibility to oxidative stress. Cell Death Differ. 2010, 17, 1199-1210. [CrossRef] [PubMed] 
247. Jaillard, C.; Mouret, A.; Niepon, M.L.; Clerin, E.; Yang, Y.; Lee-Rivera, I.; Ait-Ali, N.; Millet-Puel, G.; Cronin, T.; Sedmak, T.; et al. Nxnl2 splicing results in dual functions in neuronal cell survival and maintenance of cell integrity. Hum. Mol. Genet. 2012, 21, 2298-2311. [CrossRef] [PubMed]

248. Yokota, T.; Igarashi, K.; Uchihara, T.; Jishage, K.; Tomita, H.; Inaba, A.; Li, Y.; Arita, M.; Suzuki, H.; Mizusawa, H.; et al. Delayed-onset ataxia in mice lacking alpha -tocopherol transfer protein: Model for neuronal degeneration caused by chronic oxidative stress. Proc. Natl. Acad. Sci. USA 2001, 98, 15185-15190. [CrossRef] [PubMed]

249. Mukherjee, A.B.; Appu, A.P.; Sadhukhan, T.; Casey, S.; Mondal, A.; Zhang, Z.; Bagh, M.B. Emerging new roles of the lysosome and neuronal ceroid lipofuscinoses. Mol. Neurodegener. 2019, 14, 4. [CrossRef] [PubMed]

250. Schulze, H.; Kolter, T.; Sandhoff, K. Principles of lysosomal membrane degradation: Cellular topology and biochemistry of lysosomal lipid degradation. Biochim. Biophys. Acta 2009, 1793, 674-683. [CrossRef] [PubMed]

251. Birch, D.G. Retinal degeneration in retinitis pigmentosa and neuronal ceroid lipofuscinosis: An overview. Mol. Genet. Metab. 1999, 66, 356-366. [CrossRef]

252. Ostergaard, J.R. Juvenile neuronal ceroid lipofuscinosis (Batten disease): Current insights. Degener. Neurol. Neuromuscul. Dis. 2016, 6, 73-83. [CrossRef]

253. Leinonen, H.; Keksa-Goldsteine, V.; Ragauskas, S.; Kohlmann, P.; Singh, Y.; Savchenko, E.; Puranen, J.; Malm, T.; Kalesnykas, G.; Koistinaho, J.; et al. Retinal Degeneration In A Mouse Model Of CLN5 Disease Is Associated With Compromised Autophagy. Sci. Rep. 2017, 7, 1597. [CrossRef]

254. Bartsch, U.; Galliciotti, G.; Jofre, G.F.; Jankowiak, W.; Hagel, C.; Braulke, T. Apoptotic photoreceptor loss and altered expression of lysosomal proteins in the nclf mouse model of neuronal ceroid lipofuscinosis. Investig. Ophthalmol. Vis. Sci. 2013, 54, 6952-6959. [CrossRef]

255. Jankowiak, W.; Brandenstein, L.; Dulz, S.; Hagel, C.; Storch, S.; Bartsch, U. Retinal Degeneration in Mice Deficient in the Lysosomal Membrane Protein CLN7. Investig. Ophthalmol. Vis. Sci. 2016, 57, 4989-4998. [CrossRef]

256. Chang, B.; Bronson, R.T.; Hawes, N.L.; Roderick, T.H.; Peng, C.; Hageman, G.S.; Heckenlively, J.R. Retinal degeneration in motor neuron degeneration: A mouse model of ceroid lipofuscinosis. Investig. Ophthalmol. Vis. Sci. 1994, 35, 1071-1076. [PubMed]

257. Hafler, B.P.; Klein, Z.A.; Jimmy Zhou, Z.; Strittmatter, S.M. Progressive retinal degeneration and accumulation of autofluorescent lipopigments in Progranulin deficient mice. Brain Res. 2014, 1588, 168-174. [CrossRef] [PubMed]

258. Heldermon, C.D.; Hennig, A.K.; Ohlemiller, K.K.; Ogilvie, J.M.; Herzog, E.D.; Breidenbach, A.; Vogler, C.; Wozniak, D.F.; Sands, M.S. Development of sensory, motor and behavioral deficits in the murine model of Sanfilippo syndrome type B. PLoS ONE 2007, 2, e772. [CrossRef] [PubMed]

259. Gelfman, C.M.; Vogel, P.; Issa, T.M.; Turner, C.A.; Lee, W.S.; Kornfeld, S.; Rice, D.S. Mice lacking alpha/beta subunits of GlcNAc-1-phosphotransferase exhibit growth retardation, retinal degeneration, and secretory cell lesions. Investig. Ophthalmol. Vis. Sci. 2007, 48, 5221-5228. [CrossRef] [PubMed]

260. Kevany, B.M.; Palczewski, K. Phagocytosis of retinal rod and cone photoreceptors. Physiology 2010, 25, 8-15. [CrossRef] [PubMed]

261. Prasad, D.; Rothlin, C.V.; Burrola, P.; Burstyn-Cohen, T.; Lu, Q.; Garcia de Frutos, P.; Lemke, G. TAM receptor function in the retinal pigment epithelium. Mol. Cell Neurosci. 2006, 33, 96-108. [CrossRef] [PubMed]

262. Duncan, J.L.; LaVail, M.M.; Yasumura, D.; Matthes, M.T.; Yang, H.; Trautmann, N.; Chappelow, A.V.; Feng, W.; Earp, H.S.; Matsushima, G.K.; et al. An RCS-like retinal dystrophy phenotype in mer knockout mice. Investig. Ophthalmol. Vis. Sci. 2003, 44, 826-838. [CrossRef]

263. Houssier, M.; Raoul, W.; Lavalette, S.; Keller, N.; Guillonneau, X.; Baragatti, B.; Jonet, L.; Jeanny, J.C.; Behar-Cohen, F.; Coceani, F.; et al. CD36 deficiency leads to choroidal involution via COX2 down-regulation in rodents. PLoS Med. 2008, 5, e39. [CrossRef]

264. Ying, G.; Boldt, K.; Ueffing, M.; Gerstner, C.D.; Frederick, J.M.; Baehr, W. The small GTPase RAB28 is required for phagocytosis of cone outer segments by the murine retinal pigmented epithelium. J. Biol. Chem. 2018, 293, 17546-17558. [CrossRef]

265. Lin, W.; Xu, G. Autophagy: A Role in the Apoptosis, Survival, Inflammation, and Development of the Retina. Ophthalmic Res. 2019, 61, 65-72. [CrossRef] 
266. Seranova, E.; Connolly, K.J.; Zatyka, M.; Rosenstock, T.R.; Barrett, T.; Tuxworth, R.I.; Sarkar, S. Dysregulation of autophagy as a common mechanism in lysosomal storage diseases. Essays Biochem. 2017, 61, 733-749. [CrossRef] [PubMed]

267. Byrne, S.; Jansen, L.; JM, U.K.-I.; Siddiqui, A.; Lidov, H.G.; Bodi, I.; Smith, L.; Mein, R.; Cullup, T.; Dionisi-Vici, C.; et al. EPG5-related Vici syndrome: A paradigm of neurodevelopmental disorders with defective autophagy. Brain 2016, 139, 765-781. [CrossRef] [PubMed]

268. Smucker, W.D.; Kontak, J.R. Adverse drug reactions causing hospital admission in an elderly population: Experience with a decision algorithm. J. Am. Board Fam. Pract. 1990, 3, 105-109. [PubMed]

269. Kim, J.Y.; Zhao, H.; Martinez, J.; Doggett, T.A.; Kolesnikov, A.V.; Tang, P.H.; Ablonczy, Z.; Chan, C.C.; Zhou, Z.; Green, D.R.; et al. Noncanonical autophagy promotes the visual cycle. Cell 2013, 154, 365-376. [CrossRef] [PubMed]

270. Mohlin, C.; Taylor, L.; Ghosh, F.; Johansson, K. Autophagy and ER-stress contribute to photoreceptor degeneration in cultured adult porcine retina. Brain Res. 2014, 1585, 167-183. [CrossRef]

271. Chen, Y.; Sawada, O.; Kohno, H.; Le, Y.Z.; Subauste, C.; Maeda, T.; Maeda, A. Autophagy protects the retina from light-induced degeneration. J. Biol. Chem. 2013, 288, 7506-7518. [CrossRef]

272. Falk, M.J. Neurodevelopmental manifestations of mitochondrial disease. J. Dev. Behav. Pediatr. 2010, 31, 610-621. [CrossRef]

273. Davies, V.J.; Powell, K.A.; White, K.E.; Yip, W.; Hogan, V.; Hollins, A.J.; Davies, J.R.; Piechota, M.; Brownstein, D.G.; Moat, S.J.; et al. A missense mutation in the murine Opa3 gene models human Costeff syndrome. Brain 2008, 131, 368-380. [CrossRef]

274. Findlay, A.S.; Carter, R.N.; Starbuck, B.; McKie, L.; Novakova, K.; Budd, P.S.; Keighren, M.A.; Marsh, J.A.; Cross, S.H.; Simon, M.M.; et al. Mouse Idh3a mutations cause retinal degeneration and reduced mitochondrial function. Dis. Model. Mech. 2018, 11, 036426. [CrossRef]

275. Bruschi, M.; Petretto, A.; Caicci, F.; Bartolucci, M.; Calzia, D.; Santucci, L.; Manni, L.; Ramenghi, L.A.; Ghiggeri, G.; Traverso, C.E.; et al. Proteome of Bovine Mitochondria and Rod Outer Segment Disks: Commonalities and Differences. J. Proteome Res. 2018, 17, 918-925. [CrossRef]

276. Calzia, D.; Barabino, S.; Bianchini, P.; Garbarino, G.; Oneto, M.; Caicci, F.; Diaspro, A.; Tacchetti, C.; Manni, L.; Candiani, S.; et al. New findings in ATP supply in rod outer segments: Insights for retinopathies. Biol. Cell 2013, 105, 345-358. [CrossRef] [PubMed]

277. Funk, R.H.; Schumann, U.; Engelmann, K.; Becker, K.A.; Roehlecke, C. Blue light induced retinal oxidative stress: Implications for macular degeneration. World J. Ophthalmol. 2014, 4, 29-34. [CrossRef]

278. Calzia, D.; Garbarino, G.; Caicci, F.; Manni, L.; Candiani, S.; Ravera, S.; Morelli, A.; Traverso, C.E.; Panfoli, I. Functional expression of electron transport chain complexes in mouse rod outer segments. Biochimie 2014, 102, 78-82. [CrossRef] [PubMed]

279. Roehlecke, C.; Schumann, U.; Ader, M.; Brunssen, C.; Bramke, S.; Morawietz, H.; Funk, R.H. Stress reaction in outer segments of photoreceptors after blue light irradiation. PLoS ONE 2013, 8, e71570. [CrossRef] [PubMed]

280. Wanders, R.J.; Waterham, H.R.; Ferdinandusse, S. Metabolic Interplay between Peroxisomes and Other Subcellular Organelles Including Mitochondria and the Endoplasmic Reticulum. Front. Cell Dev. Biol. 2015, 3, 83. [CrossRef]

281. Folz, S.J.; Trobe, J.D. The peroxisome and the eye. Surv. Ophthalmol. 1991, 35, 353-368. [CrossRef]

282. Das, Y.; Roose, N.; De Groef, L.; Fransen, M.; Moons, L.; Van Veldhoven, P.P.; Baes, M. Differential distribution of peroxisomal proteins points to specific roles of peroxisomes in the murine retina. Mol. Cell Biochem. 2019, 456, 53-62. [CrossRef]

283. Hiebler, S.; Masuda, T.; Hacia, J.G.; Moser, A.B.; Faust, P.L.; Liu, A.; Chowdhury, N.; Huang, N.; Lauer, A.; Bennett, J.; et al. The Pex1-G844D mouse: A model for mild human Zellweger spectrum disorder. Mol. Genet. Metab. 2014, 111, 522-532. [CrossRef]

284. Pang, J.J.; Chang, B.; Hawes, N.L.; Hurd, R.E.; Davisson, M.T.; Li, J.; Noorwez, S.M.; Malhotra, R.; McDowell, J.H.; Kaushal, S.; et al. Retinal degeneration 12 (rd12): A new, spontaneously arising mouse model for human Leber congenital amaurosis (LCA). Mol. Vis. 2005, 11, 152-162.

285. Wright, C.B.; Chrenek, M.A.; Feng, W.; Getz, S.E.; Duncan, T.; Pardue, M.T.; Feng, Y.; Redmond, T.M.; Boatright, J.H.; Nickerson, J.M. The Rpe65 rd12 allele exerts a semidominant negative effect on vision in mice. Investig. Ophthalmol. Vis. Sci. 2014, 55, 2500-2515. [CrossRef] 
286. Redmond, T.M.; Yu, S.; Lee, E.; Bok, D.; Hamasaki, D.; Chen, N.; Goletz, P.; Ma, J.X.; Crouch, R.K.; Pfeifer, K. Rpe65 is necessary for production of 11-cis-vitamin A in the retinal visual cycle. Nat. Genet. 1998, 20, 344-351. [CrossRef] [PubMed]

287. Tanabu, R.; Sato, K.; Monai, N.; Yamauchi, K.; Gonome, T.; Xie, Y.; Takahashi, S.; Ishiguro, S.I.; Nakazawa, M. The findings of optical coherence tomography of retinal degeneration in relation to the morphological and electroretinographic features in RPE65-/- mice. PLoS ONE 2019, 14, e0210439. [CrossRef] [PubMed]

288. Woodruff, M.L.; Wang, Z.; Chung, H.Y.; Redmond, T.M.; Fain, G.L.; Lem, J. Spontaneous activity of opsin apoprotein is a cause of Leber congenital amaurosis. Nat. Genet. 2003, 35, 158-164. [CrossRef] [PubMed]

289. Samardzija, M.; von Lintig, J.; Tanimoto, N.; Oberhauser, V.; Thiersch, M.; Reme, C.E.; Seeliger, M.; Grimm, C.; Wenzel, A. R91W mutation in Rpe65 leads to milder early-onset retinal dystrophy due to the generation of low levels of 11-cis-retinal. Hum. Mol. Genet. 2008, 17, 281-292. [CrossRef] [PubMed]

290. Choi, E.H.; Suh, S.; Sander, C.L.; Hernandez, C.J.O.; Bulman, E.R.; Khadka, N.; Dong, Z.; Shi, W.; Palczewski, K.; Kiser, P.D. Insights into the pathogenesis of dominant retinitis pigmentosa associated with a D477G mutation in RPE65. Hum. Mol. Genet. 2018, 27, 2225-2243. [CrossRef]

291. Radu, R.A.; Yuan, Q.; Hu, J.; Peng, J.H.; Lloyd, M.; Nusinowitz, S.; Bok, D.; Travis, G.H. Accelerated accumulation of lipofuscin pigments in the RPE of a mouse model for ABCA4-mediated retinal dystrophies following Vitamin A supplementation. Investig. Ophthalmol. Vis. Sci. 2008, 49, 3821-3829. [CrossRef]

292. Weng, J.; Mata, N.L.; Azarian, S.M.; Tzekov, R.T.; Birch, D.G.; Travis, G.H. Insights into the function of Rim protein in photoreceptors and etiology of Stargardt's disease from the phenotype in abcr knockout mice. Cell 1999, 98, 13-23. [CrossRef]

293. Molday, L.L.; Wahl, D.; Sarunic, M.V.; Molday, R.S. Localization and functional characterization of the p.Asn965Ser (N965S) ABCA4 variant in mice reveal pathogenic mechanisms underlying Stargardt macular degeneration. Hum. Mol. Genet. 2018, 27, 295-306. [CrossRef]

294. Zhang, N.; Tsybovsky, Y.; Kolesnikov, A.V.; Rozanowska, M.; Swider, M.; Schwartz, S.B.; Stone, E.M.; Palczewska, G.; Maeda, A.; Kefalov, V.J.; et al. Protein misfolding and the pathogenesis of ABCA4-associated retinal degenerations. Hum. Mol. Genet. 2015, 24, 3220-3237. [CrossRef]

295. Wu, L.; Ueda, K.; Nagasaki, T.; Sparrow, J.R. Light damage in Abca4 and Rpe65rd12 mice. Investig. Ophthalmol. Vis. Sci. 2014, 55, 1910-1918. [CrossRef]

296. Maeda, A.; Maeda, T.; Golczak, M.; Palczewski, K. Retinopathy in mice induced by disrupted all-trans-retinal clearance. J. Biol. Chem. 2008, 283, 26684-26693. [CrossRef]

297. Chen, Y.; Okano, K.; Maeda, T.; Chauhan, V.; Golczak, M.; Maeda, A.; Palczewski, K. Mechanism of all-trans-retinal toxicity with implications for stargardt disease and age-related macular degeneration. J. Biol. Chem. 2012, 287, 5059-5069. [CrossRef] [PubMed]

298. Okano, K.; Maeda, A.; Chen, Y.; Chauhan, V.; Tang, J.; Palczewska, G.; Sakai, T.; Tsuneoka, H.; Palczewski, K.; Maeda, T. Retinal cone and rod photoreceptor cells exhibit differential susceptibility to light-induced damage. J. Neurochem. 2012, 121, 146-156. [CrossRef] [PubMed]

299. Batten, M.L.; Imanishi, Y.; Maeda, T.; Tu, D.C.; Moise, A.R.; Bronson, D.; Possin, D.; Van Gelder, R.N.; Baehr, W.; Palczewski, K. Lecithin-retinol acyltransferase is essential for accumulation of all-trans-retinyl esters in the eye and in the liver. J. Biol. Chem. 2004, 279, 10422-10432. [CrossRef] [PubMed]

300. Ruiz, A.; Ghyselinck, N.B.; Mata, N.; Nusinowitz, S.; Lloyd, M.; Dennefeld, C.; Chambon, P.; Bok, D. Somatic ablation of the Lrat gene in the mouse retinal pigment epithelium drastically reduces its retinoid storage. Investig. Ophthalmol. Vis. Sci. 2007, 48, 5377-5387. [CrossRef]

301. Liou, G.I.; Fei, Y.; Peachey, N.S.; Matragoon, S.; Wei, S.; Blaner, W.S.; Wang, Y.; Liu, C.; Gottesman, M.E.; Ripps, H. Early onset photoreceptor abnormalities induced by targeted disruption of the interphotoreceptor retinoid-binding protein gene. J. Neurosci. 1998, 18, 4511-4520. [CrossRef]

302. Shen, J.; Shi, D.; Suzuki, T.; Xia, Z.; Zhang, H.; Araki, K.; Wakana, S.; Takeda, N.; Yamamura, K.; Jin, S.; et al. Severe ocular phenotypes in Rbp4-deficient mice in the C57BL/6 genetic background. Lab. Investig. 2016, 96, 680-691. [CrossRef]

303. Ruiz, A.; Mark, M.; Jacobs, H.; Klopfenstein, M.; Hu, J.; Lloyd, M.; Habib, S.; Tosha, C.; Radu, R.A.; Ghyselinck, N.B.; et al. Retinoid content, visual responses, and ocular morphology are compromised in the retinas of mice lacking the retinol-binding protein receptor, STRA6. Investig. Ophthalmol. Vis. Sci. 2012, 53, 3027-3039. [CrossRef] 
304. Amengual, J.; Zhang, N.; Kemerer, M.; Maeda, T.; Palczewski, K.; Von Lintig, J. STRA6 is critical for cellular vitamin A uptake and homeostasis. Hum. Mol. Genet. 2014, 23, 5402-5417. [CrossRef]

305. Wu, S.M. Synaptic transmission in the outer retina. Annu. Rev. Physiol. 1994, 56, 141-168. [CrossRef]

306. Wu, S.M. Synaptic organization of the vertebrate retina: General principles and species-specific variations: The Friedenwald lecture. Investig. Ophthalmol. Vis. Sci. 2010, 51, 1263-1274. [CrossRef] [PubMed]

307. Mercer, A.J.; Thoreson, W.B. The dynamic architecture of photoreceptor ribbon synapses: Cytoskeletal, extracellular matrix, and intramembrane proteins. Vis. Neurosci. 2011, 28, 453-471. [CrossRef] [PubMed]

308. Furukawa, T.; Ueno, A.; Omori, Y. Molecular mechanisms underlying selective synapse formation of vertebrate retinal photoreceptor cells. Cell Mol. Life Sci. 2019, 77, 1251-1266. [CrossRef] [PubMed]

309. Pardue, M.T.; Peachey, N.S. Mouse b-wave mutants. Doc. Ophthalmol. 2014, 128, 77-89. [CrossRef] [PubMed]

310. Pillers, D.A.; Weleber, R.G.; Woodward, W.R.; Green, D.G.; Chapman, V.M.; Ray, P.N. mdxCv3 mouse is a model for electroretinography of Duchenne/Becker muscular dystrophy. Investig. Ophthalmol. Vis. Sci. 1995, $36,462-466$.

311. Satz, J.S.; Philp, A.R.; Nguyen, H.; Kusano, H.; Lee, J.; Turk, R.; Riker, M.J.; Hernandez, J.; Weiss, R.M.; Anderson, M.G.; et al. Visual impairment in the absence of dystroglycan. J. Neurosci. 2009, 29, 13136-13146. [CrossRef]

312. Bytyqi, A.H.; Lockridge, O.; Duysen, E.; Wang, Y.; Wolfrum, U.; Layer, P.G. Impaired formation of the inner retina in an AChE knockout mouse results in degeneration of all photoreceptors. Eur. J. Neurosci. 2004, 20, 2953-2962. [CrossRef]

313. Grisaru, D.; Sternfeld, M.; Eldor, A.; Glick, D.; Soreq, H. Structural roles of acetylcholinesterase variants in biology and pathology. Eur. J. Biochem. 1999, 264, 672-686. [CrossRef]

314. Regus-Leidig, H.; Atorf, J.; Feigenspan, A.; Kremers, J.; Maw, M.A.; Brandstatter, J.H. Photoreceptor degeneration in two mouse models for congenital stationary night blindness type 2. PLoS ONE 2014, 9, e86769. [CrossRef]

315. Kerov, V.; Laird, J.G.; Joiner, M.L.; Knecht, S.; Soh, D.; Hagen, J.; Gardner, S.H.; Gutierrez, W.; Yoshimatsu, T.; Bhattarai, S.; et al. alpha2delta-4 Is Required for the Molecular and Structural Organization of Rod and Cone Photoreceptor Synapses. J. Neurosci. 2018, 38, 6145-6160. [CrossRef]

316. Ruether, K.; Grosse, J.; Matthiessen, E.; Hoffmann, K.; Hartmann, C. Abnormalities of the photoreceptorbipolar cell synapse in a substrain of C57BL/10 mice. Investig. Ophthalmol. Vis. Sci. 2000, 41, 4039-4047. [PubMed]

317. Haeseleer, F.; Imanishi, Y.; Maeda, T.; Possin, D.E.; Maeda, A.; Lee, A.; Rieke, F.; Palczewski, K. Essential role of Ca2+-binding protein 4, a Cav1.4 channel regulator, in photoreceptor synaptic function. Nat. Neurosci. 2004, 7, 1079-1087. [CrossRef] [PubMed]

318. Ishiba, Y.; Higashide, T.; Mori, N.; Kobayashi, A.; Kubota, S.; McLaren, M.J.; Satoh, H.; Wong, F.; Inana, G. Targeted inactivation of synaptic HRG4 (UNC119) causes dysfunction in the distal photoreceptor and slow retinal degeneration, revealing a new function. Exp. Eye Res. 2007, 84, 473-485. [CrossRef] [PubMed]

319. Haeseleer, F. Interaction and colocalization of CaBP4 and Unc119 (MRG4) in photoreceptors. Investig. Ophthalmol. Vis. Sci. 2008, 49, 2366-2375. [CrossRef]

320. Giblin, J.P.; Comes, N.; Strauss, O.; Gasull, X. Ion Channels in the Eye: Involvement in Ocular Pathologies. Adv. Protein Chem. Struct. Biol. 2016, 104, 157-231. [CrossRef]

321. Edwards, M.M.; Marin de Evsikova, C.; Collin, G.B.; Gifford, E.; Wu, J.; Hicks, W.L.; Whiting, C.; Varvel, N.H.; Maphis, N.; Lamb, B.T.; et al. Photoreceptor degeneration, azoospermia, leukoencephalopathy, and abnormal RPE cell function in mice expressing an early stop mutation in CLCN2. Investig. Ophthalmol. Vis. Sci. 2010, 51, 3264-3272. [CrossRef]

322. Stobrawa, S.M.; Breiderhoff, T.; Takamori, S.; Engel, D.; Schweizer, M.; Zdebik, A.A.; Bosl, M.R.; Ruether, K.; Jahn, H.; Draguhn, A.; et al. Disruption of ClC-3, a chloride channel expressed on synaptic vesicles, leads to a loss of the hippocampus. Neuron 2001, 29, 185-196. [CrossRef]

323. Rajan, I.; Read, R.; Small, D.L.; Perrard, J.; Vogel, P. An alternative splicing variant in Clcn7-/- mice prevents osteopetrosis but not neural and retinal degeneration. Vet. Pathol. 2011, 48, 663-675. [CrossRef]

324. Dickerson, L.W.; Bonthius, D.J.; Schutte, B.C.; Yang, B.; Barna, T.J.; Bailey, M.C.; Nehrke, K.; Williamson, R.A.; Lamb, F.S. Altered GABAergic function accompanies hippocampal degeneration in mice lacking $\mathrm{ClC}-3$ voltage-gated chloride channels. Brain Res. 2002, 958, 227-250. [CrossRef] 
325. Kornak, U.; Kasper, D.; Bosl, M.R.; Kaiser, E.; Schweizer, M.; Schulz, A.; Friedrich, W.; Delling, G.; Jentsch, T.J. Loss of the ClC-7 chloride channel leads to osteopetrosis in mice and man. Cell 2001, 104, 205-215. [CrossRef]

326. Kasper, D.; Planells-Cases, R.; Fuhrmann, J.C.; Scheel, O.; Zeitz, O.; Ruether, K.; Schmitt, A.; Poet, M.; Steinfeld, R.; Schweizer, M.; et al. Loss of the chloride channel ClC-7 leads to lysosomal storage disease and neurodegeneration. EMBO J. 2005, 24, 1079-1091. [CrossRef] [PubMed]

327. Weinert, S.; Jabs, S.; Hohensee, S.; Chan, W.L.; Kornak, U.; Jentsch, T.J. Transport activity and presence of ClC-7/Ostm1 complex account for different cellular functions. EMBO Rep. 2014, 15, 784-791. [CrossRef] [PubMed]

328. Weber, P.; Bartsch, U.; Schachner, M.; Montag, D. Na,K-ATPase subunit beta1 knock-in prevents lethality of beta2 deficiency in mice. J. Neurosci. 1998, 18, 9192-9203. [CrossRef] [PubMed]

329. Heller-Stilb, B.; van Roeyen, C.; Rascher, K.; Hartwig, H.G.; Huth, A.; Seeliger, M.W.; Warskulat, U.; Haussinger, D. Disruption of the taurine transporter gene (taut) leads to retinal degeneration in mice. FASEB J. 2002, 16, 231-233. [CrossRef] [PubMed]

330. Bok, D.; Galbraith, G.; Lopez, I.; Woodruff, M.; Nusinowitz, S.; BeltrandelRio, H.; Huang, W.; Zhao, S.; Geske, R.; Montgomery, C.; et al. Blindness and auditory impairment caused by loss of the sodium bicarbonate cotransporter NBC3. Nat. Genet. 2003, 34, 313-319. [CrossRef]

331. Jin, Z.B.; Huang, X.F.; Lv, J.N.; Xiang, L.; Li, D.Q.; Chen, J.; Huang, C.; Wu, J.; Lu, F.; Qu, J. SLC7A14 linked to autosomal recessive retinitis pigmentosa. Nat. Commun. 2014, 5, 3517. [CrossRef]

332. Jadeja, S.; Barnard, A.R.; McKie, L.; Cross, S.H.; White, J.K.; Sanger Mouse Genetics, P.; Robertson, M.; Budd, P.S.; MacLaren, R.E.; Jackson, I.J. Mouse slc9a8 mutants exhibit retinal defects due to retinal pigmented epithelium dysfunction. Investig. Ophthalmol. Vis. Sci. 2015, 56, 3015-3026. [CrossRef]

333. Hori, K.; Katayama, N.; Kachi, S.; Kondo, M.; Kadomatsu, K.; Usukura, J.; Muramatsu, T.; Mori, S.; Miyake, Y. Retinal dysfunction in basigin deficiency. Investig. Ophthalmol. Vis. Sci. 2000, 41, 3128-3133.

334. Veleri, S.; Nellissery, J.; Mishra, B.; Manjunath, S.H.; Brooks, M.J.; Dong, L.; Nagashima, K.; Qian, H.; Gao, C.; Sergeev, Y.V.; et al. REEP6 mediates trafficking of a subset of Clathrin-coated vesicles and is critical for rod photoreceptor function and survival. Hum. Mol. Genet. 2017, 26, 2218-2230. [CrossRef]

335. Ettaiche, M.; Deval, E.; Pagnotta, S.; Lazdunski, M.; Lingueglia, E. Acid-sensing ion channel 3 in retinal function and survival. Investig. Ophthalmol. Vis. Sci. 2009, 50, 2417-2426. [CrossRef]

336. Li, L.; Jiao, X.; D’Atri, I.; Ono, F.; Nelson, R.; Chan, C.C.; Nakaya, N.; Ma, Z.; Ma, Y.; Cai, X.; et al. Mutation in the intracellular chloride channel CLCC1 associated with autosomal recessive retinitis pigmentosa. PLoS Genet. 2018, 14, e1007504. [CrossRef] [PubMed]

337. Wong, B.H.; Chan, J.P.; Cazenave-Gassiot, A.; Poh, R.W.; Foo, J.C.; Galam, D.L.; Ghosh, S.; Nguyen, L.N.; Barathi, V.A.; Yeo, S.W.; et al. Mfsd2a Is a Transporter for the Essential omega-3 Fatty Acid Docosahexaenoic Acid (DHA) in Eye and Is Important for Photoreceptor Cell Development. J. Biol. Chem. 2016, 291, 10501-10514. [CrossRef] [PubMed]

338. Mahimkar, R.M.; Visaya, O.; Pollock, A.S.; Lovett, D.H. The disintegrin domain of ADAM9: A ligand for multiple beta1 renal integrins. Biochem. J. 2005, 385, 461-468. [CrossRef] [PubMed]

339. Parry, D.A.; Toomes, C.; Bida, L.; Danciger, M.; Towns, K.V.; McKibbin, M.; Jacobson, S.G.; Logan, C.V.; Ali, M.; Bond, J.; et al. Loss of the metalloprotease ADAM9 leads to cone-rod dystrophy in humans and retinal degeneration in mice. Am. J. Hum. Genet. 2009, 84, 683-691. [CrossRef] [PubMed]

340. Vijayasarathy, C.; Ziccardi, L.; Sieving, P.A. Biology of retinoschisin. Adv. Exp. Med. Biol. 2012, 723, 513-518. [CrossRef]

341. Jablonski, M.M.; Dalke, C.; Wang, X.; Lu, L.; Manly, K.F.; Pretsch, W.; Favor, J.; Pardue, M.T.; Rinchik, E.M.; Williams, R.W.; et al. An ENU-induced mutation in Rs1h causes disruption of retinal structure and function. Mol. Vis. 2005, 11, 569-581.

342. Han, J.; Farmer, S.R.; Kirkland, J.L.; Corkey, B.E.; Yoon, R.; Pirtskhalava, T.; Ido, Y.; Guo, W. Octanoate attenuates adipogenesis in 3T3-L1 preadipocytes. J. Nutr. 2002, 132, 904-910. [CrossRef]

343. Zeng, Y.; Takada, Y.; Kjellstrom, S.; Hiriyanna, K.; Tanikawa, A.; Wawrousek, E.; Smaoui, N.; Caruso, R.; Bush, R.A.; Sieving, P.A. RS-1 Gene Delivery to an Adult Rs1h Knockout Mouse Model Restores ERG b-Wave with Reversal of the Electronegative Waveform of X-Linked Retinoschisis. Investig. Ophthalmol. Vis. Sci. 2004, 45, 3279-3285. [CrossRef]

344. Quinn, P.M.; Pellissier, L.P.; Wijnholds, J. The CRB1 Complex: Following the Trail of Crumbs to a Feasible Gene Therapy Strategy. Front. Neurosci. 2017, 11, 175. [CrossRef] 
345. Mehalow, A.K.; Kameya, S.; Smith, R.S.; Hawes, N.L.; Denegre, J.M.; Young, J.A.; Bechtold, L.; Haider, N.B.; Tepass, U.; Heckenlively, J.R.; et al. CRB1 is essential for external limiting membrane integrity and photoreceptor morphogenesis in the mammalian retina. Hum. Mol. Genet. 2003, 12, 2179-2189. [CrossRef]

346. van de Pavert, S.A.; Kantardzhieva, A.; Malysheva, A.; Meuleman, J.; Versteeg, I.; Levelt, C.; Klooster, J.; Geiger, S.; Seeliger, M.W.; Rashbass, P.; et al. Crumbs homologue 1 is required for maintenance of photoreceptor cell polarization and adhesion during light exposure. J. Cell Sci. 2004, 117, 4169-4177. [CrossRef] [PubMed]

347. Quinn, P.M.J.; Wijnholds, J. Retinogenesis of the Human Fetal Retina: An Apical Polarity Perspective. Genes 2019, 10, 987. [CrossRef]

348. Moore, B.A.; Leonard, B.C.; Sebbag, L.; Edwards, S.G.; Cooper, A.; Imai, D.M.; Straiton, E.; Santos, L.; Reilly, C.; Griffey, S.M.; et al. Identification of genes required for eye development by high-throughput screening of mouse knockouts. Commun. Biol. 2018, 1, 236. [CrossRef] [PubMed]

349. Watson, J.R.; Owen, D.; Mott, H.R. Cdc42 in actin dynamics: An ordered pathway governed by complex equilibria and directional effector handover. Small GTPases 2017, 8, 237-244. [CrossRef]

350. Yokokura, S.; Wada, Y.; Nakai, S.; Sato, H.; Yao, R.; Yamanaka, H.; Ito, S.; Sagara, Y.; Takahashi, M.; Nakamura, Y.; et al. Targeted disruption of FSCN2 gene induces retinopathy in mice. Investig. Ophthalmol. Vis. Sci. 2005, 46, 2905-2915. [CrossRef] [PubMed]

351. Liu, X.; Zhao, M.; Xie, Y.; Li, P.; Wang, O.; Zhou, B.; Yang, L.; Nie, Y.; Cheng, L.; Song, X.; et al. Null Mutation of the Fascin2 Gene by TALEN Leading to Progressive Hearing Loss and Retinal Degeneration in C57BL/6J Mice. G3 2018, 8, 3221-3230. [CrossRef]

352. Saksens, N.T.; Krebs, M.P.; Schoenmaker-Koller, F.E.; Hicks, W.; Yu, M.; Shi, L.; Rowe, L.; Collin, G.B.; Charette, J.R.; Letteboer, S.J.; et al. Mutations in CTNNA1 cause butterfly-shaped pigment dystrophy and perturbed retinal pigment epithelium integrity. Nat. Genet. 2016, 48, 144-151. [CrossRef]

353. Maddox, D.M.; Collin, G.B.; Ikeda, A.; Pratt, C.H.; Ikeda, S.; Johnson, B.A.; Hurd, R.E.; Shopland, L.S.; Naggert, J.K.; Chang, B.; et al. A Mutation in Syne2 Causes Early Retinal Defects in Photoreceptors, Secondary Neurons, and Muller Glia. Investig. Ophthalmol. Vis. Sci. 2015, 56, 3776-3787. [CrossRef]

354. Yu, J.; Lei, K.; Zhou, M.; Craft, C.M.; Xu, G.; Xu, T.; Zhuang, Y.; Xu, R.; Han, M. KASH protein Syne-2/Nesprin-2 and SUN proteins SUN1/2 mediate nuclear migration during mammalian retinal development. Hum. Mol. Genet. 2011, 20, 1061-1073. [CrossRef]

355. Gordon, M.D.; Nusse, R. Wnt signaling: Multiple pathways, multiple receptors, and multiple transcription factors. J. Biol. Chem. 2006, 281, 22429-22433. [CrossRef]

356. Liu, C.; Nathans, J. An essential role for frizzled 5 in mammalian ocular development. Development 2008, 135, 3567-3576. [CrossRef] [PubMed]

357. Wang, Z.; Liu, C.H.; Sun, Y.; Gong, Y.; Favazza, T.L.; Morss, P.C.; Saba, N.J.; Fredrick, T.W.; He, X.; Akula, J.D.; et al. Pharmacologic Activation of Wnt Signaling by Lithium Normalizes Retinal Vasculature in a Murine Model of Familial Exudative Vitreoretinopathy. Am. J. Pathol. 2016, 186, 2588-2600. [CrossRef] [PubMed]

358. Berger, W.; van de Pol, D.; Bachner, D.; Oerlemans, F.; Winkens, H.; Hameister, H.; Wieringa, B.; Hendriks, W.; Ropers, H.H. An animal model for Norrie disease (ND): Gene targeting of the mouse ND gene. Hum. Mol. Genet. 1996, 5, 51-59. [CrossRef] [PubMed]

359. Junge, H.J.; Yang, S.; Burton, J.B.; Paes, K.; Shu, X.; French, D.M.; Costa, M.; Rice, D.S.; Ye, W. TSPAN12 regulates retinal vascular development by promoting Norrin- but not Wnt-induced FZD4/beta-catenin signaling. Cell 2009, 139, 299-311. [CrossRef] [PubMed]

360. Hackam, A.S. The Wnt signaling pathway in retinal degenerations. IUBMB Life 2005, 57, 381-388. [CrossRef]

361. Hawes, N.L.; Chang, B.; Hageman, G.S.; Nusinowitz, S.; Nishina, P.M.; Schneider, B.S.; Smith, R.S.; Roderick, T.H.; Davisson, M.T.; Heckenlively, J.R. Retinal degeneration 6 (rd6): A new mouse model for human retinitis punctata albescens. Investig. Ophthalmol. Vis. Sci. 2000, 41, 3149-3157.

362. Hawkes, W.G. Bibliography: Nurses and smoking. J. N. Y. State Nurses Assoc. 1990, $21,14$.

363. Chavali, V.R.; Khan, N.W.; Cukras, C.A.; Bartsch, D.U.; Jablonski, M.M.; Ayyagari, R. A CTRP5 gene S163R mutation knock-in mouse model for late-onset retinal degeneration. Hum. Mol. Genet. 2011, 20, 2000-2014. [CrossRef]

364. Cai, Z.; Simons, D.L.; Fu, X.Y.; Feng, G.S.; Wu, S.M.; Zhang, X. Loss of Shp2-mediated mitogen-activated protein kinase signaling in Muller glial cells results in retinal degeneration. Mol. Cell Biol. 2011, 31, 2973-2983. [CrossRef] 
365. Chavez-Solano, M.; Ibarra-Sanchez, A.; Trevino, M.; Gonzalez-Espinosa, C.; Lamas, M. Fyn kinase genetic ablation causes structural abnormalities in mature retina and defective Muller cell function. Mol. Cell Neurosci. 2016, 72, 91-100. [CrossRef]

366. Ji, X.; Liu, Y.; Hurd, R.; Wang, J.; Fitzmaurice, B.; Nishina, P.M.; Chang, B. Retinal Pigment Epithelium Atrophy 1 (rpea1): A New Mouse Model With Retinal Detachment Caused by a Disruption of Protein Kinase C, theta. Investig. Ophthalmol. Vis. Sci. 2016, 57, 877-888. [CrossRef]

367. Ivanovic, I.; Anderson, R.E.; Le, Y.Z.; Fliesler, S.J.; Sherry, D.M.; Rajala, R.V. Deletion of the p85alpha regulatory subunit of phosphoinositide 3-kinase in cone photoreceptor cells results in cone photoreceptor degeneration. Investig. Ophthalmol. Vis. Sci. 2011, 52, 3775-3783. [CrossRef] [PubMed]

368. Rajala, R.V.; Ranjo-Bishop, M.; Wang, Y.; Rajala, A.; Anderson, R.E. The p110alpha isoform of phosphoinositide 3-kinase is essential for cone photoreceptor survival. Biochimie 2015, 112, 35-40. [CrossRef] [PubMed]

369. Yi, X.; Schubert, M.; Peachey, N.S.; Suzuma, K.; Burks, D.J.; Kushner, J.A.; Suzuma, I.; Cahill, C.; Flint, C.L.; Dow, M.A.; et al. Insulin receptor substrate 2 is essential for maturation and survival of photoreceptor cells. J. Neurosci. 2005, 25, 1240-1248. [CrossRef] [PubMed]

370. Kitahara, H.; Kajikawa, S.; Ishii, Y.; Yamamoto, S.; Hamashima, T.; Azuma, E.; Sato, H.; Matsushima, T.; Shibuya, M.; Shimada, Y.; et al. The Novel Pathogenesis of Retinopathy Mediated by Multiple RTK Signals is Uncovered in Newly Developed Mouse Model. EBioMedicine 2018, 31, 190-201. [CrossRef]

371. Mongan, M.; Wang, J.; Liu, H.; Fan, Y.; Jin, C.; Kao, W.Y.; Xia, Y. Loss of MAP3K1 enhances proliferation and apoptosis during retinal development. Development 2011, 138, 4001-4012. [CrossRef]

372. Toyofuku, T.; Nojima, S.; Ishikawa, T.; Takamatsu, H.; Tsujimura, T.; Uemura, A.; Matsuda, J.; Seki, T.; Kumanogoh, A. Endosomal sorting by Semaphorin 4A in retinal pigment epithelium supports photoreceptor survival. Genes Dev. 2012, 26, 816-829. [CrossRef]

373. Carter-Dawson, L.D.; LaVail, M.M. Rods and cones in the mouse retina. II. Autoradiographic analysis of cell generation using tritiated thymidine. J. Comp. Neurol. 1979, 188, 263-272. [CrossRef]

374. Swaroop, A.; Kim, D.; Forrest, D. Transcriptional regulation of photoreceptor development and homeostasis in the mammalian retina. Nat. Rev. Neurosci. 2010, 11, 563-576. [CrossRef]

375. Brzezinski, J.A.; Reh, T.A. Photoreceptor cell fate specification in vertebrates. Development 2015, 142, 3263-3273. [CrossRef]

376. Khidr, L.; Chen, P.L. RB, the conductor that orchestrates life, death and differentiation. Oncogene 2006, 25, 5210-5219. [CrossRef] [PubMed]

377. Roger, J.E.; Hiriyanna, A.; Gotoh, N.; Hao, H.; Cheng, D.F.; Ratnapriya, R.; Kautzmann, M.A.; Chang, B.; Swaroop, A. OTX2 loss causes rod differentiation defect in CRX-associated congenital blindness. J. Clin. Investig. 2014, 124, 631-643. [CrossRef] [PubMed]

378. Tran, N.M.; Zhang, A.; Zhang, X.; Huecker, J.B.; Hennig, A.K.; Chen, S. Mechanistically distinct mouse models for CRX-associated retinopathy. PLoS Genet. 2014, 10, e1004111. [CrossRef] [PubMed]

379. Omori, Y.; Kitamura, T.; Yoshida, S.; Kuwahara, R.; Chaya, T.; Irie, S.; Furukawa, T. Mef2d is essential for the maturation and integrity of retinal photoreceptor and bipolar cells. Genes Cells 2015, 20, 408-426. [CrossRef]

380. Kiyama, T.; Chen, C.K.; Wang, S.W.; Pan, P.; Ju, Z.; Wang, J.; Takada, S.; Klein, W.H.; Mao, C.A. Essential roles of mitochondrial biogenesis regulator Nrf1 in retinal development and homeostasis. Mol. Neurodegener. 2018, 13, 56. [CrossRef] [PubMed]

381. Wu, F.; Li, R.; Umino, Y.; Kaczynski, T.J.; Sapkota, D.; Li, S.; Xiang, M.; Fliesler, S.J.; Sherry, D.M.; Gannon, M.; et al. Onecut1 is essential for horizontal cell genesis and retinal integrity. J. Neurosci. 2013, 33, 13053-13065. [CrossRef]

382. Goding, C.R.; Arnheiter, H. MITF-the first 25 years. Genes Dev. 2019, 33, 983-1007. [CrossRef]

383. Zelinger, L.; Swaroop, A. RNA Biology in Retinal Development and Disease. Trends Genet. 2018, 34, 341-351. [CrossRef]

384. Barabino, A.; Plamondon, V.; Abdouh, M.; Chatoo, W.; Flamier, A.; Hanna, R.; Zhou, S.; Motoyama, N.; Hebert, M.; Lavoie, J.; et al. Loss of Bmil causes anomalies in retinal development and degeneration of cone photoreceptors. Development 2016, 143, 1571-1584. [CrossRef]

385. Susaki, K.; Kaneko, J.; Yamano, Y.; Nakamura, K.; Inami, W.; Yoshikawa, T.; Ozawa, Y.; Shibata, S.; Matsuzaki, O.; Okano, H.; et al. Musashi-1, an RNA-binding protein, is indispensable for survival of photoreceptors. Exp. Eye Res. 2009, 88, 347-355. [CrossRef] 
386. McKinnon, P.J. Maintaining genome stability in the nervous system. Nat. Neurosci. 2013, 16, 1523-1529. [CrossRef] [PubMed]

387. Gregersen, L.H.; Svejstrup, J.Q. The Cellular Response to Transcription-Blocking DNA Damage. Trends Biochem. Sci. 2018, 43, 327-341. [CrossRef] [PubMed]

388. Lans, H.; Hoeijmakers, J.H.J.; Vermeulen, W.; Marteijn, J.A. The DNA damage response to transcription stress. Nat. Rev. Mol. Cell Biol. 2019, 20, 766-784. [CrossRef] [PubMed]

389. Sanchez, A.; De Vivo, A.; Uprety, N.; Kim, J.; Stevens, S.M., Jr.; Kee, Y. BMI1-UBR5 axis regulates transcriptional repression at damaged chromatin. Proc. Natl. Acad. Sci. USA 2016, 113, 11243-11248. [CrossRef] [PubMed]

390. Calses, P.C.; Dhillon, K.K.; Tucker, N.; Chi, Y.; Huang, J.W.; Kawasumi, M.; Nghiem, P.; Wang, Y.; Clurman, B.E.; Jacquemont, C.; et al. DGCR8 Mediates Repair of UV-Induced DNA Damage Independently of RNA Processing. Cell Rep. 2017, 19, 162-174. [CrossRef] [PubMed]

391. Burger, K.; Schlackow, M.; Potts, M.; Hester, S.; Mohammed, S.; Gullerova, M. Nuclear phosphorylated Dicer processes double-stranded RNA in response to DNA damage. J. Cell Biol. 2017, 216, 2373-2389. [CrossRef]

392. Montaldo, N.P.; Bordin, D.L.; Brambilla, A.; Rosinger, M.; Fordyce Martin, S.L.; Bjoras, K.O.; Bradamante, S.; Aas, P.A.; Furrer, A.; Olsen, L.C.; et al. Alkyladenine DNA glycosylase associates with transcription elongation to coordinate DNA repair with gene expression. Nat. Commun. 2019, 10, 5460. [CrossRef]

393. Faridounnia, M.; Folkers, G.E.; Boelens, R. Function and Interactions of ERCC1-XPF in DNA Damage Response. Molecules 2018, 23, 3205. [CrossRef]

394. de Araujo, P.R.; Gorthi, A.; da Silva, A.E.; Tonapi, S.S.; Vo, D.T.; Burns, S.C.; Qiao, M.; Uren, P.J.; Yuan, Z.M.; Bishop, A.J.; et al. Musashi1 Impacts Radio-Resistance in Glioblastoma by Controlling DNA-Protein Kinase Catalytic Subunit. Am. J. Pathol. 2016, 186, 2271-2278. [CrossRef]

395. Onn, L.; Portillo, M.; Ilic, S.; Cleitman, G.; Stein, D.; Kaluski, S.; Shirat, I.; Slobodnik, Z.; Einav, M.; Erdel, F.; et al. SIRT6 is a DNA double-strand break sensor. Elife 2020, 9. [CrossRef]

396. Calderwood, S.K. A critical role for topoisomerase Ilb and DNA double strand breaks in transcription. Transcription 2016, 7, 75-83. [CrossRef] [PubMed]

397. Aleksandrov, R.; Dotchev, A.; Poser, I.; Krastev, D.; Georgiev, G.; Panova, G.; Babukov, Y.; Danovski, G.; Dyankova, T.; Hubatsch, L.; et al. Protein Dynamics in Complex DNA Lesions. Mol. Cell 2018, 69, 1046.e1045-1061.e1045. [CrossRef]

398. Nishi, R.; Wijnhoven, P.W.G.; Kimura, Y.; Matsui, M.; Konietzny, R.; Wu, Q.; Nakamura, K.; Blundell, T.L.; Kessler, B.M. The deubiquitylating enzyme UCHL3 regulates Ku80 retention at sites of DNA damage. Sci. Rep. 2018, 8, 17891. [CrossRef] [PubMed]

399. Xu, H.; Lin, Z.; Li, F.; Diao, W.; Dong, C.; Zhou, H.; Xie, X.; Wang, Z.; Shen, Y.; Long, J. Dimerization of elongator protein 1 is essential for Elongator complex assembly. Proc. Natl. Acad. Sci. USA 2015, 112, 10697-10702. [CrossRef] [PubMed]

400. Li, Y.; Hao, H.; Swerdel, M.R.; Cho, H.Y.; Lee, K.B.; Hart, R.P.; Lyu, Y.L.; Cai, L. Top2b is involved in the formation of outer segment and synapse during late-stage photoreceptor differentiation by controlling key genes of photoreceptor transcriptional regulatory network. J. Neurosci. Res. 2017, 95, 1951-1964. [CrossRef] [PubMed]

401. Valdes-Sanchez, L.; De la Cerda, B.; Diaz-Corrales, F.J.; Massalini, S.; Chakarova, C.F.; Wright, A.F.; Bhattacharya, S.S. ATR localizes to the photoreceptor connecting cilium and deficiency leads to severe photoreceptor degeneration in mice. Hum. Mol. Genet. 2013, 22, 1507-1515. [CrossRef]

402. Bian, C.; Zhang, C.; Luo, T.; Vyas, A.; Chen, S.H.; Liu, C.; Kassab, M.A.; Yang, Y.; Kong, M.; Yu, X. NADP(+) is an endogenous PARP inhibitor in DNA damage response and tumor suppression. Nat. Commun. 2019, 10, 693. [CrossRef]

403. Sundermeier, T.R.; Sakami, S.; Sahu, B.; Howell, S.J.; Gao, S.; Dong, Z.; Golczak, M.; Maeda, A.; Palczewski, K. MicroRNA-processing Enzymes Are Essential for Survival and Function of Mature Retinal Pigmented Epithelial Cells in Mice. J. Biol. Chem. 2017, 292, 3366-3378. [CrossRef]

404. Damiani, D.; Alexander, J.J.; O’Rourke, J.R.; McManus, M.; Jadhav, A.P.; Cepko, C.L.; Hauswirth, W.W.; Harfe, B.D.; Strettoi, E. Dicer inactivation leads to progressive functional and structural degeneration of the mouse retina. J. Neurosci. 2008, 28, 4878-4887. [CrossRef] [PubMed]

405. Ueki, Y.; Ramirez, G.; Salcedo, E.; Stabio, M.E.; Lefcort, F. Loss of Ikbkap Causes Slow, Progressive Retinal Degeneration in a Mouse Model of Familial Dysautonomia. eNeuro 2016, 3. [CrossRef] [PubMed] 
406. Spoor, M.; Nagtegaal, A.P.; Ridwan, Y.; Borgesius, N.Z.; van Alphen, B.; van der Pluijm, I.; Hoeijmakers, J.H.; Frens, M.A.; Borst, J.G. Accelerated loss of hearing and vision in the DNA-repair deficient Ercc1(delta/-) mouse. Mech. Ageing Dev. 2012, 133, 59-67. [CrossRef] [PubMed]

407. Gorgels, T.G.; van der Pluijm, I.; Brandt, R.M.; Garinis, G.A.; van Steeg, H.; van den Aardweg, G.; Jansen, G.H.; Ruijter, J.M.; Bergen, A.A.; van Norren, D.; et al. Retinal degeneration and ionizing radiation hypersensitivity in a mouse model for Cockayne syndrome. Mol. Cell Biol. 2007, 27, 1433-1441. [CrossRef] [PubMed]

408. Eblimit, A.; Zaneveld, S.A.; Liu, W.; Thomas, K.; Wang, K.; Li, Y.; Mardon, G.; Chen, R. NMNAT1 E257K variant, associated with Leber Congenital Amaurosis (LCA9), causes a mild retinal degeneration phenotype. Exp. Eye Res. 2018, 173, 32-43. [CrossRef]

409. Peshti, V.; Obolensky, A.; Nahum, L.; Kanfi, Y.; Rathaus, M.; Avraham, M.; Tinman, S.; Alt, F.W.; Banin, E.; Cohen, H.Y. Characterization of physiological defects in adult SIRT6-/- mice. PLoS ONE 2017, 12, e0176371. [CrossRef] [PubMed]

410. Lim, D.; Park, C.W.; Ryu, K.Y.; Chung, H. Disruption of the polyubiquitin gene Ubb causes retinal degeneration in mice. Biochem. Biophys. Res. Commun. 2019, 513, 35-40. [CrossRef]

411. Semenova, E.; Wang, X.; Jablonski, M.M.; Levorse, J.; Tilghman, S.M. An engineered 800 kilobase deletion of Uchl3 and Lmo7 on mouse chromosome 14 causes defects in viability, postnatal growth and degeneration of muscle and retina. Hum. Mol. Genet. 2003, 12, 1301-1312. [CrossRef]

412. Pinelli, M.; Carissimo, A.; Cutillo, L.; Lai, C.H.; Mutarelli, M.; Moretti, M.N.; Singh, M.V.; Karali, M.; Carrella, D.; Pizzo, M.; et al. An atlas of gene expression and gene co-regulation in the human retina. Nucleic Acids Res. 2016, 44, 5773-5784. [CrossRef]

413. Hoshino, A.; Ratnapriya, R.; Brooks, M.J.; Chaitankar, V.; Wilken, M.S.; Zhang, C.; Starostik, M.R.; Gieser, L.; La Torre, A.; Nishio, M.; et al. Molecular Anatomy of the Developing Human Retina. Dev. Cell 2017, 43, 763.e764-779.e764. [CrossRef]

414. Graziotto, J.J.; Farkas, M.H.; Bujakowska, K.; Deramaudt, B.M.; Zhang, Q.; Nandrot, E.F.; Inglehearn, C.F.; Bhattacharya, S.S.; Pierce, E.A. Three gene-targeted mouse models of RNA splicing factor RP show late-onset RPE and retinal degeneration. Investig. Ophthalmol. Vis. Sci. 2011, 52, 190-198. [CrossRef]

415. Xu, M.; Xie, Y.A.; Abouzeid, H.; Gordon, C.T.; Fiorentino, A.; Sun, Z.; Lehman, A.; Osman, I.S.; Dharmat, R.; Riveiro-Alvarez, R.; et al. Mutations in the Spliceosome Component CWC27 Cause Retinal Degeneration with or without Additional Developmental Anomalies. Am. J. Hum. Genet. 2017, 100, 592-604. [CrossRef]

416. Chen, B.J.; Lam, T.C.; Liu, L.Q.; To, C.H. Post-translational modifications and their applications in eye research (Review). Mol. Med. Rep. 2017, 15, 3923-3935. [CrossRef] [PubMed]

417. Christiansen, J.R.; Kolandaivelu, S.; Bergo, M.O.; Ramamurthy, V. RAS-converting enzyme 1-mediated endoproteolysis is required for trafficking of rod phosphodiesterase 6 to photoreceptor outer segments. Proc. Natl. Acad. Sci. USA 2011, 108, 8862-8866. [CrossRef] [PubMed]

418. Christiansen, J.R.; Pendse, N.D.; Kolandaivelu, S.; Bergo, M.O.; Young, S.G.; Ramamurthy, V. Deficiency of Isoprenylcysteine Carboxyl Methyltransferase (ICMT) Leads to Progressive Loss of Photoreceptor Function. J. Neurosci. 2016, 36, 5107-5114. [CrossRef]

419. Bosch Grau, M.; Masson, C.; Gadadhar, S.; Rocha, C.; Tort, O.; Marques Sousa, P.; Vacher, S.; Bieche, I.; Janke, C. Alterations in the balance of tubulin glycylation and glutamylation in photoreceptors leads to retinal degeneration. J. Cell Sci. 2017, 130, 938-949. [CrossRef] [PubMed]

420. Sun, X.; Park, J.H.; Gumerson, J.; Wu, Z.; Swaroop, A.; Qian, H.; Roll-Mecak, A.; Li, T. Loss of RPGR glutamylation underlies the pathogenic mechanism of retinal dystrophy caused by TTLL5 mutations. Proc. Natl. Acad. Sci. USA 2016, 113, E2925-E2934. [CrossRef] [PubMed]

421. Blanks, J.C.; Spee, C. Retinal degeneration in the pcd/pcd mutant mouse: Accumulation of spherules in the interphotoreceptor space. Exp. Eye Res. 1992, 54, 637-644. [CrossRef]

422. Chen, J.; Qian, H.; Horai, R.; Chan, C.C.; Falick, Y.; Caspi, R.R. Comparative analysis of induced vs. spontaneous models of autoimmune uveitis targeting the interphotoreceptor retinoid binding protein. PLoS ONE 2013, 8, e72161. [CrossRef]

423. Yu, M.; Zou, W.; Peachey, N.S.; McIntyre, T.M.; Liu, J. A novel role of complement in retinal degeneration. Investig. Ophthalmol. Vis. Sci. 2012, 53, 7684-7692. [CrossRef]

424. Lyzogubov, V.V.; Bora, P.S.; Wu, X.; Horn, L.E.; de Roque, R.; Rudolf, X.V.; Atkinson, J.P.; Bora, N.S. The Complement Regulatory Protein CD46 Deficient Mouse Spontaneously Develops Dry-Type Age-Related Macular Degeneration-Like Phenotype. Am. J. Pathol. 2016, 186, 2088-2104. [CrossRef] 
425. Jobling, A.I.; Waugh, M.; Vessey, K.A.; Phipps, J.A.; Trogrlic, L.; Greferath, U.; Mills, S.A.; Tan, Z.L.; Ward, M.M.; Fletcher, E.L. The Role of the Microglial Cx3cr1 Pathway in the Postnatal Maturation of Retinal Photoreceptors. J. Neurosci. 2018, 38, 4708-4723. [CrossRef]

426. Combadiere, C.; Feumi, C.; Raoul, W.; Keller, N.; Rodero, M.; Pezard, A.; Lavalette, S.; Houssier, M.; Jonet, L.; Picard, E.; et al. CX3CR1-dependent subretinal microglia cell accumulation is associated with cardinal features of age-related macular degeneration. J. Clin. Investig. 2007, 117, 2920-2928. [CrossRef]

427. Huang, H.; Liu, Y.; Wang, L.; Li, W. Age-related macular degeneration phenotypes are associated with increased tumor necrosis-alpha and subretinal immune cells in aged Cxcr5 knockout mice. PLoS ONE 2017, 12, e0173716. [CrossRef] [PubMed]

428. Zhang, X.; Zhu, J.; Chen, X.; Jie-Qiong, Z.; Li, X.; Luo, L.; Huang, H.; Liu, W.; Zhou, X.; Yan, J.; et al. Interferon Regulatory Factor 3 Deficiency Induces Age-Related Alterations of the Retina in Young and Old Mice. Front. Cell Neurosci. 2019, 13, 272. [CrossRef] [PubMed]

429. Zhang, Q.; Jiang, Y.; Miller, M.J.; Peng, B.; Liu, L.; Soderland, C.; Tang, J.; Kern, T.S.; Pintar, J.; Steinle, J.J. IGFBP-3 and TNF-alpha regulate retinal endothelial cell apoptosis. Investig. Ophthalmol. Vis. Sci. 2013, 54, 5376-5384. [CrossRef] [PubMed]

430. Ambati, J.; Anand, A.; Fernandez, S.; Sakurai, E.; Lynn, B.C.; Kuziel, W.A.; Rollins, B.J.; Ambati, B.K. An animal model of age-related macular degeneration in senescent Ccl-2- or Ccr-2-deficient mice. Nat. Med. 2003, 9, 1390-1397. [CrossRef] [PubMed]

431. Coffey, P.J.; Gias, C.; McDermott, C.J.; Lundh, P.; Pickering, M.C.; Sethi, C.; Bird, A.; Fitzke, F.W.; Maass, A.; Chen, L.L.; et al. Complement factor $\mathrm{H}$ deficiency in aged mice causes retinal abnormalities and visual dysfunction. Proc. Natl. Acad. Sci. USA 2007, 104, 16651-16656. [CrossRef]

432. Ueda, Y.; Mohammed, I.; Song, D.; Gullipalli, D.; Zhou, L.; Sato, S.; Wang, Y.; Gupta, S.; Cheng, Z.; Wang, H.; et al. Murine systemic thrombophilia and hemolytic uremic syndrome from a factor $\mathrm{H}$ point mutation. Blood 2017, 129, 1184-1196. [CrossRef]

433. Ma, W.; Silverman, S.M.; Zhao, L.; Villasmil, R.; Campos, M.M.; Amaral, J.; Wong, W.T. Absence of TGFbeta signaling in retinal microglia induces retinal degeneration and exacerbates choroidal neovascularization. Elife 2019, 8. [CrossRef]

434. Zhou, Y.; Li, S.; Huang, L.; Yang, Y.; Zhang, L.; Yang, M.; Liu, W.; Ramasamy, K.; Jiang, Z.; Sundaresan, P.; et al. A splicing mutation in aryl hydrocarbon receptor associated with retinitis pigmentosa. Hum. Mol. Genet. 2018, 27, 2563-2572. [CrossRef]

435. Swiderski, R.E.; Nakano, Y.; Mullins, R.F.; Seo, S.; Banfi, B. A mutation in the mouse ttc26 gene leads to impaired hedgehog signaling. PLoS Genet. 2014, 10, e1004689. [CrossRef]

436. Omori, Y.; Kubo, S.; Kon, T.; Furuhashi, M.; Narita, H.; Kominami, T.; Ueno, A.; Tsutsumi, R.; Chaya, T.; Yamamoto, H.; et al. Samd7 is a cell type-specific PRC1 component essential for establishing retinal rod photoreceptor identity. Proc. Natl. Acad. Sci. USA 2017, 114, E8264-E8273. [CrossRef] [PubMed]

437. Behnen, P.; Felline, A.; Comitato, A.; Di Salvo, M.T.; Raimondi, F.; Gulati, S.; Kahremany, S.; Palczewski, K.; Marigo, V.; Fanelli, F. A Small Chaperone Improves Folding and Routing of Rhodopsin Mutants Linked to Inherited Blindness. iScience 2018, 4, 1-19. [CrossRef] [PubMed]

438. Kroeger, H.; Chiang, W.C.; Felden, J.; Nguyen, A.; Lin, J.H. ER stress and unfolded protein response in ocular health and disease. FEBS J. 2019, 286, 399-412. [CrossRef] [PubMed]

439. Stuck, M.W.; Conley, S.M.; Naash, M.I. PRPH2/RDS and ROM-1: Historical context, current views and future considerations. Prog. Retin. Eye Res. 2016, 52, 47-63. [CrossRef]

440. Boon, C.J.; den Hollander, A.I.; Hoyng, C.B.; Cremers, F.P.; Klevering, B.J.; Keunen, J.E. The spectrum of retinal dystrophies caused by mutations in the peripherin/RDS gene. Prog. Retin. Eye Res. 2008, 27, 213-235. [CrossRef]

441. Liu, M.M.; Zack, D.J. Alternative splicing and retinal degeneration. Clin. Genet. 2013, 84, 142-149. [CrossRef]

442. Zhao, Y.; Hong, D.H.; Pawlyk, B.; Yue, G.; Adamian, M.; Grynberg, M.; Godzik, A.; Li, T. The retinitis pigmentosa GTPase regulator (RPGR)- interacting protein: Subserving RPGR function and participating in disk morphogenesis. Proc. Natl. Acad. Sci. USA 2003, 100, 3965-3970. [CrossRef]

443. Won, J.; Gifford, E.; Smith, R.S.; Yi, H.; Ferreira, P.A.; Hicks, W.L.; Li, T.; Naggert, J.K.; Nishina, P.M. RPGRIP1 is essential for normal rod photoreceptor outer segment elaboration and morphogenesis. Hum. Mol. Genet. 2009, 18, 4329-4339. [CrossRef] 
444. Zhang, C.; Quan, R.; Wang, J. Development and application of CRISPR/Cas9 technologies in genomic editing. Hum. Mol. Genet. 2018, 27, R79-R88. [CrossRef]

445. Comitato, A.; Schiroli, D.; La Marca, C.; Marigo, V. Differential Contribution of Calcium-Activated Proteases and ER-Stress in Three Mouse Models of Retinitis Pigmentosa Expressing P23H Mutant RHO. Adv. Exp. Med. Biol. 2019, 1185, 311-316. [CrossRef]

446. Hahn, P.; Qian, Y.; Dentchev, T.; Chen, L.; Beard, J.; Harris, Z.L.; Dunaief, J.L. Disruption of ceruloplasmin and hephaestin in mice causes retinal iron overload and retinal degeneration with features of age-related macular degeneration. Proc. Natl. Acad. Sci. USA 2004, 101, 13850-13855. [CrossRef]

447. Kumar, M.V.; Nagineni, C.N.; Chin, M.S.; Hooks, J.J.; Detrick, B. Innate immunity in the retina: Toll-like receptor (TLR) signaling in human retinal pigment epithelial cells. J. Neuroimmunol. 2004, 153, 7-15. [CrossRef] [PubMed]

448. Shiose, S.; Chen, Y.; Okano, K.; Roy, S.; Kohno, H.; Tang, J.; Pearlman, E.; Maeda, T.; Palczewski, K.; Maeda, A. Toll-like receptor 3 is required for development of retinopathy caused by impaired all-trans-retinal clearance in mice. J. Biol. Chem. 2011, 286, 15543-15555. [CrossRef] [PubMed]

449. Vollrath, D.; Yasumura, D.; Benchorin, G.; Matthes, M.T.; Feng, W.; Nguyen, N.M.; Sedano, C.D.; Calton, M.A.; LaVail, M.M. Tyro3 Modulates Mertk-Associated Retinal Degeneration. PLoS Genet. 2015, 11, e1005723. [CrossRef] [PubMed]

450. Zhang, Y.; Seo, S.; Bhattarai, S.; Bugge, K.; Searby, C.C.; Zhang, Q.; Drack, A.V.; Stone, E.M.; Sheffield, V.C. BBS mutations modify phenotypic expression of CEP290-related ciliopathies. Hum. Mol. Genet. 2014, 23, 40-51. [CrossRef] [PubMed]

451. Rachel, R.A.; May-Simera, H.L.; Veleri, S.; Gotoh, N.; Choi, B.Y.; Murga-Zamalloa, C.; McIntyre, J.C.; Marek, J.; Lopez, I.; Hackett, A.N.; et al. Combining Cep290 and Mkks ciliopathy alleles in mice rescues sensory defects and restores ciliogenesis. J. Clin. Investig. 2012, 122, 1233-1245. [CrossRef] [PubMed]

452. Rao, K.N.; Zhang, W.; Li, L.; Ronquillo, C.; Baehr, W.; Khanna, H. Ciliopathy-associated protein CEP290 modifies the severity of retinal degeneration due to loss of RPGR. Hum. Mol. Genet. 2016, 25, 2005-2012. [CrossRef]

453. Schon, C.; Asteriti, S.; Koch, S.; Sothilingam, V.; Garcia Garrido, M.; Tanimoto, N.; Herms, J.; Seeliger, M.W.; Cangiano, L.; Biel, M.; et al. Loss of HCN1 enhances disease progression in mouse models of CNG channel-linked retinitis pigmentosa and achromatopsia. Hum. Mol. Genet. 2016, 25, 1165-1175. [CrossRef]

454. Quinn, P.M.; Mulder, A.A.; Henrique Alves, C.; Desrosiers, M.; de Vries, S.I.; Klooster, J.; Dalkara, D.; Koster, A.J.; Jost, C.R.; Wijnholds, J. Loss of CRB2 in Muller glial cells modifies a CRB1-associated retinitis pigmentosa phenotype into a Leber congenital amaurosis phenotype. Hum. Mol. Genet. 2019, 28, 105-123. [CrossRef]

455. Ng, L.; Liu, H.; St Germain, D.L.; Hernandez, A.; Forrest, D. Deletion of the Thyroid Hormone-Activating Type 2 Deiodinase Rescues Cone Photoreceptor Degeneration but Not Deafness in Mice Lacking Type 3 Deiodinase. Endocrinology 2017, 158, 1999-2010. [CrossRef]

456. van der Pluijm, I.; Garinis, G.A.; Brandt, R.M.; Gorgels, T.G.; Wijnhoven, S.W.; Diderich, K.E.; de Wit, J.; Mitchell, J.R.; van Oostrom, C.; Beems, R.; et al. Impaired genome maintenance suppresses the growth hormone-insulin-like growth factor 1 axis in mice with Cockayne syndrome. PLoS Biol. 2007, 5, e2. [CrossRef]

457. Chang, B.; Heckenlively, J.R.; Bayley, P.R.; Brecha, N.C.; Davisson, M.T.; Hawes, N.L.; Hirano, A.A.; Hurd, R.E.; Ikeda, A.; Johnson, B.A.; et al. The nob2 mouse, a null mutation in Cacna1f: Anatomical and functional abnormalities in the outer retina and their consequences on ganglion cell visual responses. Vis. Neurosci. 2006, 23, 11-24. [CrossRef] [PubMed]

458. Humphries, M.M.; Kiang, S.; McNally, N.; Donovan, M.A.; Sieving, P.A.; Bush, R.A.; Machida, S.; Cotter, T.; Hobson, A.; Farrar, J.; et al. Comparative structural and functional analysis of photoreceptor neurons of Rho-/- mice reveal increased survival on C57BL/6J in comparison to $129 \mathrm{~Sv}$ genetic background. Vis. Neurosci. 2001, 18, 437-443. [CrossRef]

459. Liu, Q.; Saveliev, A.; Pierce, E.A. The severity of retinal degeneration in Rp1h gene-targeted mice is dependent on genetic background. Investig. Ophthalmol. Vis. Sci. 2009, 50, 1566-1574. [CrossRef] [PubMed]

460. Haider, N.B.; Zhang, W.; Hurd, R.; Ikeda, A.; Nystuen, A.M.; Naggert, J.K.; Nishina, P.M. Mapping of genetic modifiers of Nr2e3 rd7/rd7 that suppress retinal degeneration and restore blue cone cells to normal quantity. Mamm. Genome 2008, 19, 145-154. [CrossRef] 
461. Threadgill, D.W.; Miller, D.R.; Churchill, G.A.; de Villena, F.P. The collaborative cross: A recombinant inbred mouse population for the systems genetic era. ILAR J. 2011, 52, 24-31. [CrossRef]

462. Churchill, G.A.; Gatti, D.M.; Munger, S.C.; Svenson, K.L. The Diversity Outbred mouse population. Mamm. Genome 2012, 23, 713-718. [CrossRef]

463. Cruz, N.M.; Yuan, Y.; Leehy, B.D.; Baid, R.; Kompella, U.; DeAngelis, M.M.; Escher, P.; Haider, N.B. Modifier genes as therapeutics: The nuclear hormone receptor Rev Erb alpha (Nr1d1) rescues Nr2e3 associated retinal disease. PLoS ONE 2014, 9, e87942. [CrossRef]

464. Danciger, M.; Ogando, D.; Yang, H.; Matthes, M.T.; Yu, N.; Ahern, K.; Yasumura, D.; Williams, R.W.; Lavail, M.M. Genetic modifiers of retinal degeneration in the rd3 mouse. Investig. Ophthalmol. Vis. Sci. 2008, 49, 2863-2869. [CrossRef]

465. van Wyk, M.; Schneider, S.; Kleinlogel, S. Variable phenotypic expressivity in inbred retinal degeneration mouse lines: A comparative study of $\mathrm{C} 3 \mathrm{H} / \mathrm{HeOu}$ and FVB/N rd1 mice. Mol. Vis. 2015, 21, 811-827.

466. Luhmann, U.F.; Carvalho, L.S.; Holthaus, S.M.; Cowing, J.A.; Greenaway, S.; Chu, C.J.; Herrmann, P.; Smith, A.J.; Munro, P.M.; Potter, P.; et al. The severity of retinal pathology in homozygous Crb1rd8/rd8 mice is dependent on additional genetic factors. Hum. Mol. Genet. 2015, 24, 128-141. [CrossRef] [PubMed]

467. Won, J.; Charette, J.R.; Philip, V.M.; Stearns, T.M.; Zhang, W.; Naggert, J.K.; Krebs, M.P.; Nishina, P.M. Genetic modifier loci of mouse Mfrp(rd6) identified by quantitative trait locus analysis. Exp. Eye Res. 2014, 118, 30-35. [CrossRef] [PubMed]

468. Maddox, D.M.; Ikeda, S.; Ikeda, A.; Zhang, W.; Krebs, M.P.; Nishina, P.M.; Naggert, J.K. An allele of microtubule-associated protein 1A (Mtap1a) reduces photoreceptor degeneration in Tulp1 and Tub Mutant Mice. Investig. Ophthalmol. Vis. Sci. 2012, 53, 1663-1669. [CrossRef]

469. Kong, Y.; Zhao, L.; Charette, J.R.; Hicks, W.L.; Stone, L.; Nishina, P.M.; Naggert, J.K. An FRMD4B variant suppresses dysplastic photoreceptor lesions in models of enhanced S-cone syndrome and of Nrl deficiency. Hum. Mol. Genet. 2018, 27, 3340-3352. [CrossRef] [PubMed]

470. Paskowitz, D.M.; LaVail, M.M.; Duncan, J.L. Light and inherited retinal degeneration. Br. J. Ophthalmol. 2006, 90, 1060-1066. [CrossRef] [PubMed]

471. Dellett, M.; Sasai, N.; Nishide, K.; Becker, S.; Papadaki, V.; Limb, G.A.; Moore, A.T.; Kondo, T.; Ohnuma, S. Genetic background and light-dependent progression of photoreceptor cell degeneration in Prominin-1 knockout mice. Investig. Ophthalmol. Vis. Sci. 2014, 56, 164-176. [CrossRef]

472. Naash, M.L.; Peachey, N.S.; Li, Z.Y.; Gryczan, C.C.; Goto, Y.; Blanks, J.; Milam, A.H.; Ripps, H. Light-induced acceleration of photoreceptor degeneration in transgenic mice expressing mutant rhodopsin. Investig. Ophthalmol. Vis. Sci. 1996, 37, 775-782.

473. Rascher, K.; Servos, G.; Berthold, G.; Hartwig, H.G.; Warskulat, U.; Heller-Stilb, B.; Haussinger, D. Light deprivation slows but does not prevent the loss of photoreceptors in taurine transporter knockout mice. Vis. Res. 2004, 44, 2091-2100. [CrossRef]

474. Smith, S.B.; Cope, B.K.; McCoy, J.R. Effects of dark-rearing on the retinal degeneration of the C57BL/6-mivit/mivit mouse. Exp. Eye Res. 1994, 58, 77-84. [CrossRef]

475. Maeda, A.; Maeda, T.; Imanishi, Y.; Sun, W.; Jastrzebska, B.; Hatala, D.A.; Winkens, H.J.; Hofmann, K.P.; Janssen, J.J.; Baehr, W.; et al. Retinol dehydrogenase (RDH12) protects photoreceptors from light-induced degeneration in mice. J. Biol. Chem. 2006, 281, 37697-37704. [CrossRef]

476. Ettaiche, M.; Guy, N.; Hofman, P.; Lazdunski, M.; Waldmann, R. Acid-sensing ion channel 2 is important for retinal function and protects against light-induced retinal degeneration. J. Neurosci. 2004, 24, 1005-1012. [CrossRef] [PubMed]

477. Peng, Y.W.; Zallocchi, M.; Wang, W.M.; Delimont, D.; Cosgrove, D. Moderate light-induced degeneration of rod photoreceptors with delayed transducin translocation in shaker1 mice. Investig. Ophthalmol. Vis. Sci. 2011, 52, 6421-6427. [CrossRef] [PubMed]

478. Tian, M.; Wang, W.; Delimont, D.; Cheung, L.; Zallocchi, M.; Cosgrove, D.; Peng, Y.W. Photoreceptors in whirler mice show defective transducin translocation and are susceptible to short-term light/dark changes-induced degeneration. Exp. Eye Res. 2014, 118, 145-153. [CrossRef] [PubMed]

479. Li, G.; Anderson, R.E.; Tomita, H.; Adler, R.; Liu, X.; Zack, D.J.; Rajala, R.V. Nonredundant role of Akt2 for neuroprotection of rod photoreceptor cells from light-induced cell death. J. Neurosci. 2007, 27, $203-211$. [CrossRef] 
480. Datta, S.; Cano, M.; Ebrahimi, K.; Wang, L.; Handa, J.T. The impact of oxidative stress and inflammation on RPE degeneration in non-neovascular AMD. Prog. Retin. Eye Res. 2017, 60, 201-218. [CrossRef]

481. Khan, J.C.; Thurlby, D.A.; Shahid, H.; Clayton, D.G.; Yates, J.R.; Bradley, M.; Moore, A.T.; Bird, A.C.; Genetic Factors in AMD Study. Smoking and age related macular degeneration: The number of pack years of cigarette smoking is a major determinant of risk for both geographic atrophy and choroidal neovascularisation. $\mathrm{Br}$. J. Ophthalmol. 2006, 90, 75-80. [CrossRef]

482. Cano, M.; Thimmalappula, R.; Fujihara, M.; Nagai, N.; Sporn, M.; Wang, A.L.; Neufeld, A.H.; Biswal, S.; Handa, J.T. Cigarette smoking, oxidative stress, the anti-oxidant response through Nrf2 signaling, and Age-related Macular Degeneration. Vis. Res. 2010, 50, 652-664. [CrossRef]

483. Fujihara, M.; Nagai, N.; Sussan, T.E.; Biswal, S.; Handa, J.T. Chronic cigarette smoke causes oxidative damage and apoptosis to retinal pigmented epithelial cells in mice. PLoS ONE 2008, 3, e3119. [CrossRef]

484. Ebrahimi, K.B.; Cano, M.; Rhee, J.; Datta, S.; Wang, L.; Handa, J.T. Oxidative Stress Induces an Interactive Decline in Wnt and Nrf2 Signaling in Degenerating Retinal Pigment Epithelium. Antioxid. Redox Signal. 2018, 29, 389-407. [CrossRef]

485. Farese, R.V., Jr.; Veniant, M.M.; Cham, C.M.; Flynn, L.M.; Pierotti, V.; Loring, J.F.; Traber, M.; Ruland, S.; Stokowski, R.S.; Huszar, D.; et al. Phenotypic analysis of mice expressing exclusively apolipoprotein B48 or apolipoprotein B100. Proc. Natl. Acad. Sci. USA 1996, 93, 6393-6398. [CrossRef]

486. Schmidt-Erfurth, U.; Rudolf, M.; Funk, M.; Hofmann-Rummelt, C.; Franz-Haas, N.S.; Aherrahrou, Z.; Schlotzer-Schrehardt, U. Ultrastructural changes in a murine model of graded Bruch membrane lipoidal degeneration and corresponding VEGF164 detection. Investig. Ophthalmol. Vis. Sci. 2008, 49, 390-398. [CrossRef] [PubMed]

487. Malek, G.; Johnson, L.V.; Mace, B.E.; Saloupis, P.; Schmechel, D.E.; Rickman, D.W.; Toth, C.A.; Sullivan, P.M.; Bowes Rickman, C. Apolipoprotein E allele-dependent pathogenesis: A model for age-related retinal degeneration. Proc. Natl. Acad. Sci. USA 2005, 102, 11900-11905. [CrossRef] [PubMed]

488. Perusek, L.; Maeda, T. Vitamin A derivatives as treatment options for retinal degenerative diseases. Nutrients 2013, 5, 2646-2666. [CrossRef] [PubMed]

489. Perusek, L.; Maeda, A.; Maeda, T. Supplementation with vitamin a derivatives to rescue vision in animal models of degenerative retinal diseases. Methods Mol. Biol. 2015, 1271, 345-362. [CrossRef]

490. Guadagni, V.; Novelli, E.; Piano, I.; Gargini, C.; Strettoi, E. Pharmacological approaches to retinitis pigmentosa: A laboratory perspective. Prog. Retin. Eye Res. 2015, 48, 62-81. [CrossRef] [PubMed]

491. Cai, X.; McGinnis, J.F. Oxidative stress: The achilles' heel of neurodegenerative diseases of the retina. Front. Biosci. Landmark. Ed. 2012, 17, 1976-1995. [CrossRef]

492. Komeima, K.; Rogers, B.S.; Campochiaro, P.A. Antioxidants slow photoreceptor cell death in mouse models of retinitis pigmentosa. J. Cell Physiol. 2007, 213, 809-815. [CrossRef]

493. Ebert, S.; Weigelt, K.; Walczak, Y.; Drobnik, W.; Mauerer, R.; Hume, D.A.; Weber, B.H.; Langmann, T. Docosahexaenoic acid attenuates microglial activation and delays early retinal degeneration. J. Neurochem. 2009, 110, 1863-1875. [CrossRef]

494. Yu, M.; Yan, W.; Beight, C. Lutein and Zeaxanthin Isomers Reduce Photoreceptor Degeneration in the Pde6b (rd10) Mouse Model of Retinitis Pigmentosa. Biomed. Res. Int. 2018, 2018, 4374087. [CrossRef]

495. Komeima, K.; Rogers, B.S.; Lu, L.; Campochiaro, P.A. Antioxidants reduce cone cell death in a model of retinitis pigmentosa. Proc. Natl. Acad. Sci. USA 2006, 103, 11300-11305. [CrossRef]

496. Barone, I.; Novelli, E.; Piano, I.; Gargini, C.; Strettoi, E. Environmental enrichment extends photoreceptor survival and visual function in a mouse model of retinitis pigmentosa. PLoS ONE 2012, 7, e50726. [CrossRef]

497. Sundin, O.H.; Leppert, G.S.; Silva, E.D.; Yang, J.M.; Dharmaraj, S.; Maumenee, I.H.; Santos, L.C.; Parsa, C.F.; Traboulsi, E.I.; Broman, K.W.; et al. Extreme hyperopia is the result of null mutations in MFRP, which encodes a Frizzled-related protein. Proc. Natl. Acad. Sci. USA 2005, 102, 9553-9558. [CrossRef] [PubMed]

498. Sundin, O.H. The mouse's eye and Mfrp: Not quite human. Ophthalmic Genet. 2005, 26, 153-155. [CrossRef] [PubMed]

499. Kameya, S.; Hawes, N.L.; Chang, B.; Heckenlively, J.R.; Naggert, J.K.; Nishina, P.M. Mfrp, a gene encoding a frizzled related protein, is mutated in the mouse retinal degeneration 6. Hum. Mol. Genet. 2002, 11, 1879-1886. [CrossRef] [PubMed] 
500. Almoallem, B.; Arno, G.; De Zaeytijd, J.; Verdin, H.; Balikova, I.; Casteels, I.; de Ravel, T.; Hull, S.; Suzani, M.; Destree, A.; et al. The majority of autosomal recessive nanophthalmos and posterior microphthalmia can be attributed to biallelic sequence and structural variants in MFRP and PRSS56. Sci. Rep. 2020, 10, 1289. [CrossRef] [PubMed]

501. Chekuri, A.; Sahu, B.; Chavali, V.R.M.; Voronchikhina, M.; Soto-Hermida, A.; Suk, J.J.; Alapati, A.N.; Bartsch, D.U.; Ayala-Ramirez, R.; Zenteno, J.C.; et al. Long-Term Effects of Gene Therapy in a Novel Mouse Model of Human MFRP-Associated Retinopathy. Hum. Gene Ther. 2019, 30, 632-650. [CrossRef] [PubMed]

502. Gibson, F.; Walsh, J.; Mburu, P.; Varela, A.; Brown, K.A.; Antonio, M.; Beisel, K.W.; Steel, K.P.; Brown, S.D. A type VII myosin encoded by the mouse deafness gene shaker-1. Nature 1995, 374, 62-64. [CrossRef]

503. Lentz, J.J.; Gordon, W.C.; Farris, H.E.; MacDonald, G.H.; Cunningham, D.E.; Robbins, C.A.; Tempel, B.L.; Bazan, N.G.; Rubel, E.W.; Oesterle, E.C.; et al. Deafness and retinal degeneration in a novel USH1C knock-in mouse model. Dev. Neurobiol. 2010, 70, 253-267. [CrossRef]

504. Karan, G.; Lillo, C.; Yang, Z.; Cameron, D.J.; Locke, K.G.; Zhao, Y.; Thirumalaichary, S.; Li, C.; Birch, D.G.; Vollmer-Snarr, H.R.; et al. Lipofuscin accumulation, abnormal electrophysiology, and photoreceptor degeneration in mutant ELOVL4 transgenic mice: A model for macular degeneration. Proc. Natl. Acad. Sci. USA 2005, 102, 4164-4169. [CrossRef]

505. Grishchuk, Y.; Stember, K.G.; Matsunaga, A.; Olivares, A.M.; Cruz, N.M.; King, V.E.; Humphrey, D.M.; Wang, S.L.; Muzikansky, A.; Betensky, R.A.; et al. Retinal Dystrophy and Optic Nerve Pathology in the Mouse Model of Mucolipidosis IV. Am. J. Pathol. 2016, 186, 199-209. [CrossRef]

506. Amir, N.; Zlotogora, J.; Bach, G. Mucolipidosis type IV: Clinical spectrum and natural history. Pediatrics 1987, 79, 953-959. [PubMed]

507. Park, H.; Qazi, Y.; Tan, C.; Jabbar, S.B.; Cao, Y.; Schmid, G.; Pardue, M.T. Assessment of axial length measurements in mouse eyes. Optom. Vis. Sci. 2012, 89, 296-303. [CrossRef]

508. Ruppersburg, C.C.; Hartzell, H.C. The Ca2+-activated Cl- channel ANO1/TMEM16A regulates primary ciliogenesis. Mol. Biol. Cell 2014, 25, 1793-1807. [CrossRef] [PubMed]

509. He, M.; Ye, W.; Wang, W.J.; Sison, E.S.; Jan, Y.N.; Jan, L.Y. Cytoplasmic Cl(-) couples membrane remodeling to epithelial morphogenesis. Proc. Natl. Acad. Sci. USA 2017, 114, E11161-E11169. [CrossRef] [PubMed]

510. Gupta, A.; Fabian, L.; Brill, J.A. Phosphatidylinositol 4,5-bisphosphate regulates cilium transition zone maturation in Drosophila melanogaster. J. Cell Sci. 2018, 131. [CrossRef]

511. Bhatia, V.; Valdes-Sanchez, L.; Rodriguez-Martinez, D.; Bhattacharya, S.S. Formation of 53BP1 foci and ATM activation under oxidative stress is facilitated by RNA:DNA hybrids and loss of ATM-53BP1 expression promotes photoreceptor cell survival in mice. F1000Res 2018, 7, 1233. [CrossRef]

512. Muller, B.; Ellinwood, N.M.; Lorenz, B.; Stieger, K. Detection of DNA Double Strand Breaks by gammaH2AX Does Not Result in 53bp1 Recruitment in Mouse Retinal Tissues. Front. Neurosci. 2018, 12, 286. [CrossRef]

(C) 2020 by the authors. Licensee MDPI, Basel, Switzerland. This article is an open access article distributed under the terms and conditions of the Creative Commons Attribution (CC BY) license (http://creativecommons.org/licenses/by/4.0/). 



\title{
Review \\ Large Animal Models of Inherited Retinal Degenerations: A Review
}

\author{
Paige A. Winkler, Laurence M. Occelli and Simon M. Petersen-Jones * \\ Department of Small Animal Clinical Sciences, Veterinary Medical Center, Michigan State University, East \\ Lansing, MI 48824, USA; winkle38@msu.edu (P.A.W.); occelli@msu.edu (L.M.O.) \\ * Correspondence: peter315@msu.edu; Tel.: +1-517-353-3278
}

Received: 1 March 2020; Accepted: 31 March 2020; Published: 3 April 2020

\begin{abstract}
Studies utilizing large animal models of inherited retinal degeneration (IRD) have proven important in not only the development of translational therapeutic approaches, but also in improving our understanding of disease mechanisms. The dog is the predominant species utilized because spontaneous IRD is common in the canine pet population. Cats are also a source of spontaneous IRDs. Other large animal models with spontaneous IRDs include sheep, horses and non-human primates (NHP). The pig has also proven valuable due to the ease in which transgenic animals can be generated and work is ongoing to produce engineered models of other large animal species including NHP. These large animal models offer important advantages over the widely used laboratory rodent models. The globe size and dimensions more closely parallel those of humans and, most importantly, they have a retinal region of high cone density and denser photoreceptor packing for high acuity vision. Laboratory rodents lack such a retinal region and, as macular disease is a critical cause for vision loss in humans, having a comparable retinal region in model species is particularly important. This review will discuss several large animal models which have been used to study disease mechanisms relevant for the equivalent human IRD.
\end{abstract}

Keywords: large animal model; inherited retinal disease; progressive retinal atrophy; retinitis pigmentosa; Leber congenital amaurosis; achromatopsia; congenital stationary night blindness

\section{Introduction}

Large animal models for inherited retinal degenerations (IRDs) have been identified within populations of dogs, cats, sheep, horses and, more recently, non-human primates (NHP). Many different IRDs have been identified in pedigree dogs, most of which mimic retinitis pigmentosa (RP) or Leber congenital amaurosis (LCA). The term "progressive retinal atrophy" (PRA) is used to describe this group of photoreceptor degenerations. In some instances, more detailed descriptive terms such as rod-cone dysplasia or progressive rod cone degeneration have been used. Common dog breeding practices have tended to bring out recessive conditions and have made the pedigree dog a rich source of models for inherited disease, including IRDs. Engineered large animal IRD models such as transgenic pigs have also been produced [1]. With advances in gene editing technologies, further models are likely to be produced in species such as pigs and NHPs and possibly even cats and dogs. The advantages of large animal models over laboratory rodent models of IRDs include the similarity in the size of the eye to that of man [2]. This is of particular importance for the development of translational therapies because it allows identical surgical delivery approaches to be used in the animal model to those that will be eventually used in human patients. NHPs are obviously close relatives to humans, making them attractive models. However, only a few spontaneous IRDs in primates have been identified [3,4], although steps are being taken to identify more animals with disease-causing mutations and to use genome editing to generate additional models with mutations in genes of importance, either for 
germline transmission (see [5] for a review) or somatic gene knockout [6]. Another major advantage of large animal models is the presence of a retinal region equivalent to the macula. Laboratory mice and rats are nocturnal rodents that do not have a macula equivalent. The macula is of major importance for high acuity vision and some conditions specifically or differentially affect that retinal region compared to the peripheral retina. Macular dystrophies have been associated with mutations in a number of different genes (see $[7,8]$ for reviews), some of which have relevant large animal models which are discussed below, including Stargardt Disease ( $A B C A 4$ mutations), Best Disease (BEST1 mutations) and in some patients with RDH5 mutations. Age-related macular degeneration (AMD) is a major cause of vision loss and has genetic and environmental contributors [9,10]. Screening of primate colonies for animals with lesions comparable to AMD have been performed [11] (for a review, including primate models, see Pennesi et al. [12]). The large animal model species considered here have an area centralis with high photoreceptor density, including, importantly, cones that are equivalent to the human macula [13]. While NHP also have a fovea, most of the other model species do not, although the dog has been reported to have a small concentration of cones in the center of the area centralis, referred to as a "bouquet" of cones [14].

There are several important instances where laboratory rodent engineered models fail to recapitulate the human disease; important examples including $A B C A 4$-Stargardt disease, RDH5-retinopathy, or where the gene involved is not present in the mouse or rat genome e.g., EYS [15].

The disadvantages of the large animal model species tend to be cost, generation of sufficient animals due to the slower reproduction, and because of the longer lifespan (compared to laboratory rodents), the disease course may be longer.

Large animal models have also been important in therapy development. The first proof-of-concept gene augmentation therapy that eventually led to the FDA approval of the first gene therapy product, Luxturna, for the treatment of LCA due to RPE65 mutations, was performed in a dog model [16].

Table 1 shows a list of IRDs in large animal models and their identified mutations. Retinal layers and the genes discussed in detail within the text are shown in Figure 1. This review will cover the models in which the studies have contributed to the understanding of the mechanism of disease and/or protein structure and function in greater detail. 


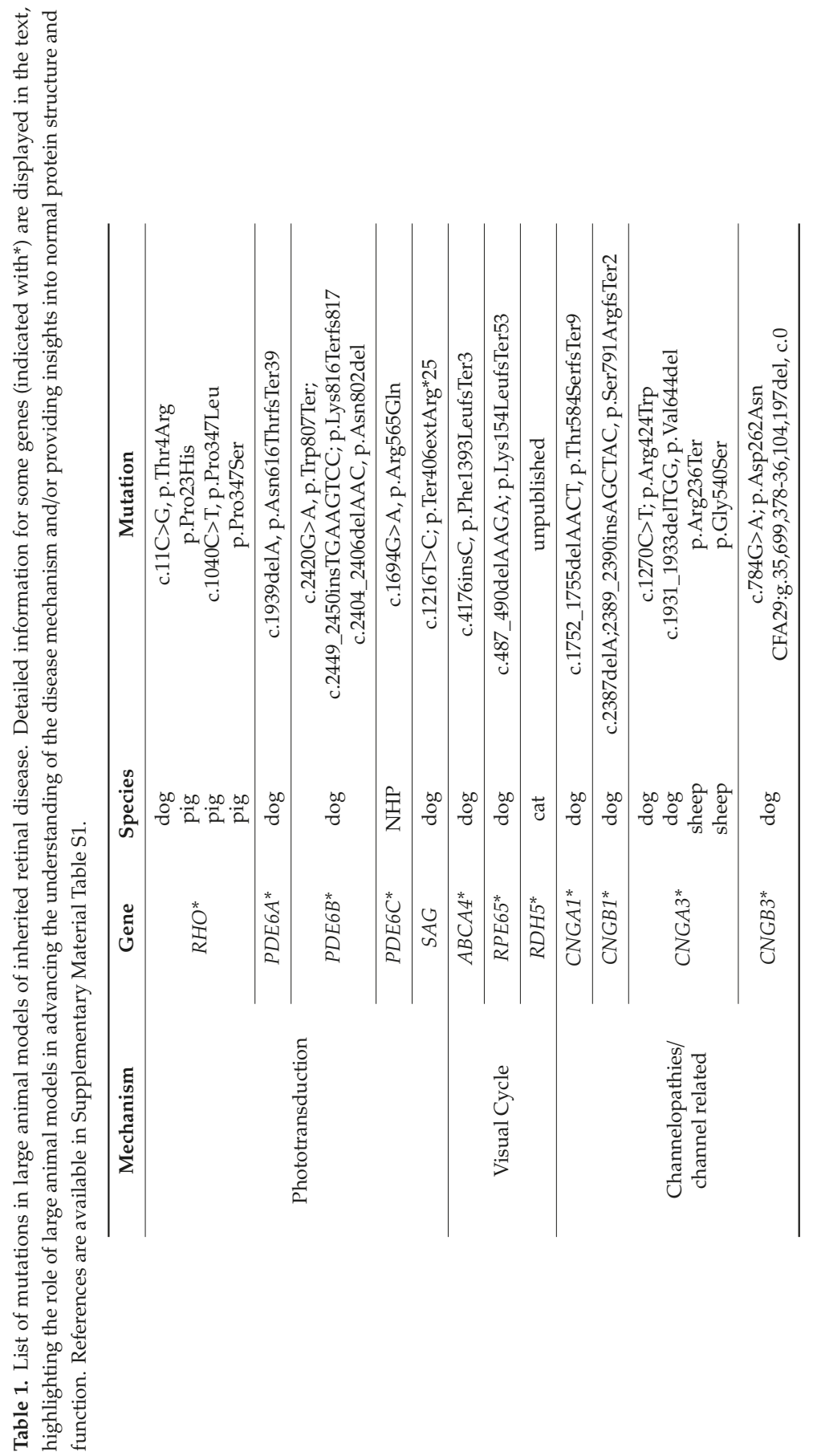




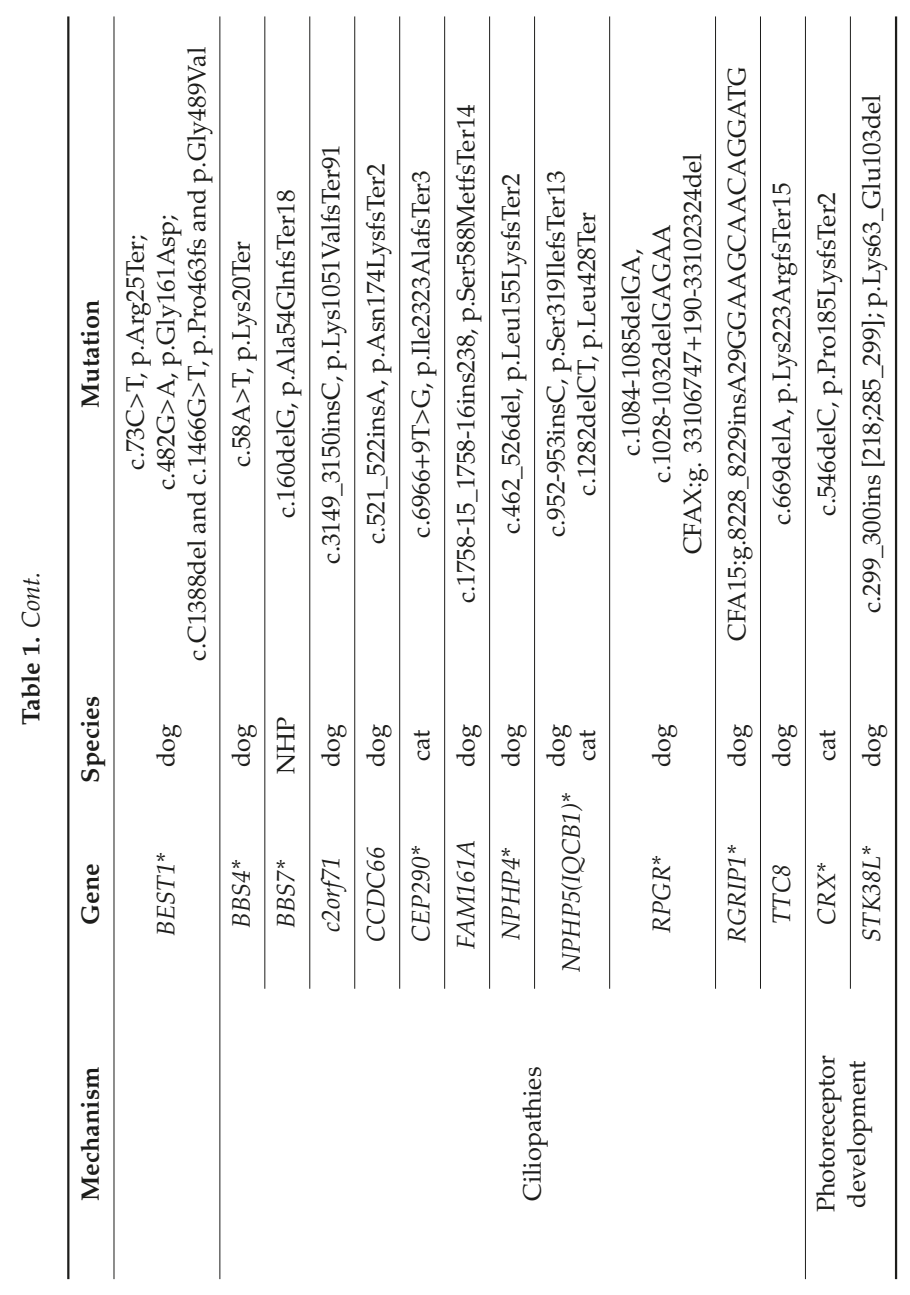


Cells 2020, 9, 882

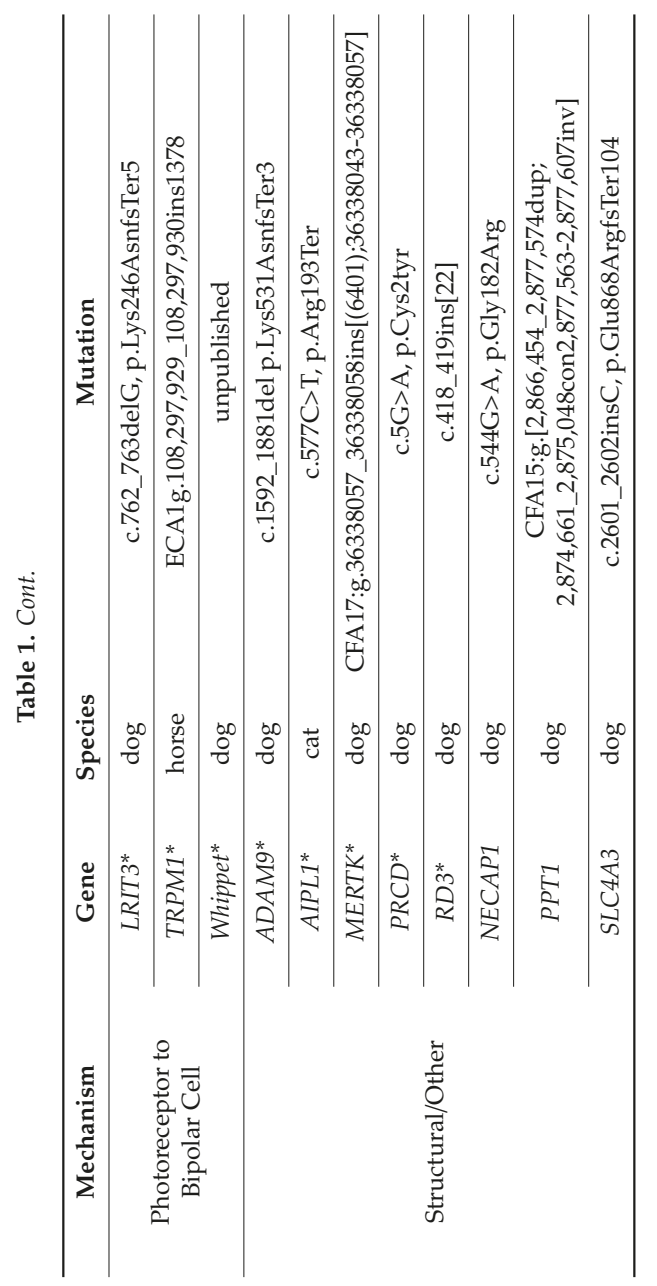




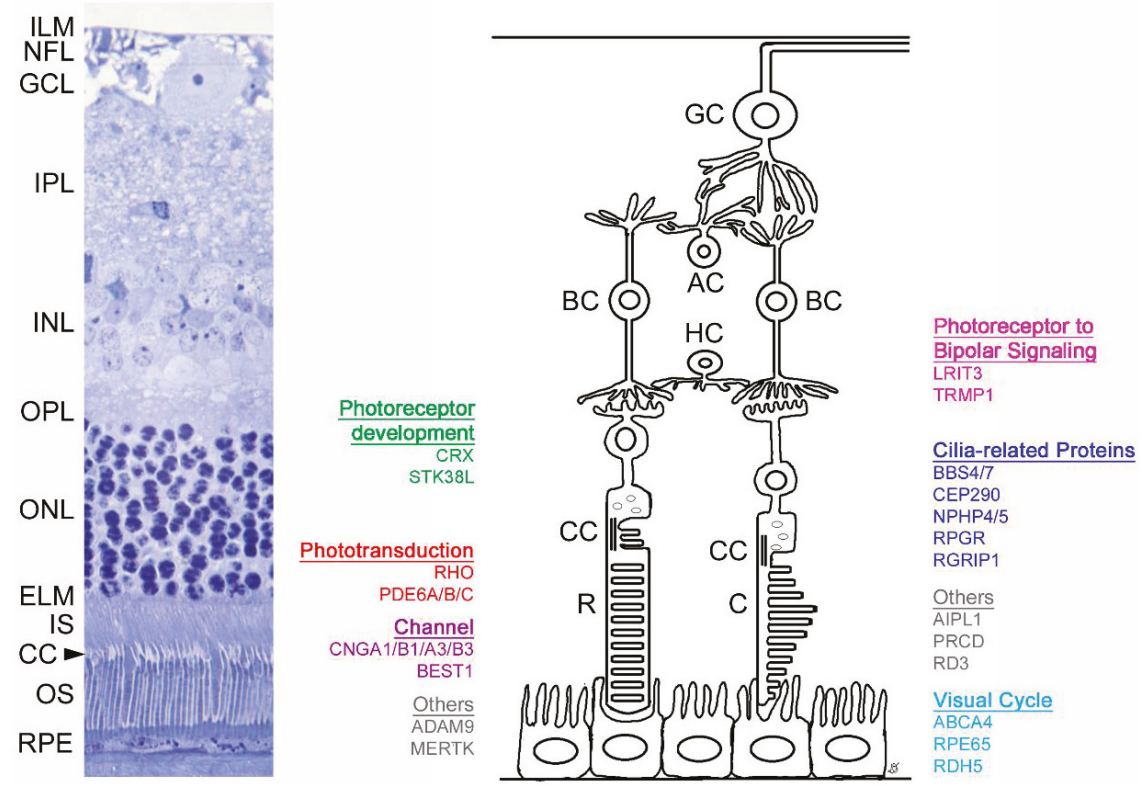

Figure 1. Schematic of retinal layers and associated genes discussed within this review. The left image shows a histologic section of a feline retina (with comparable anatomy to the human retina). The right panel depicts a schematic showing the genes detailed in this review and their site of expression, grouped per biological process. Inner limiting membrane (ILM), nerve fiber layer (NFL), ganglion cell layer (GCL), inner plexiform layer (IPL), inner nuclear layer (INL), outer plexifom layer (OPL), outer nuclear layer (ONL), external limiting membrane (ELM), photoreceptor inner segment (IS), connecting cilium (CC), photoreceptor outer segment (OS), retinal pigmentary epithelium (RPE). Ganglion cell (GC), amacrine cell (AC), bipolar cell (BC), horizontal cell (HC), rod (R), cone (C).

\section{Mutations in Phototransduction Genes}

There are spontaneously occurring large animal models with mutations in the genes of the phototransduction cascade, and rhodopsin $(R H O)$ transgenic pig models have been generated. Studies of these models have contributed to the understanding of how the mutation of these genes leads to photoreceptor death.

\section{1. $\mathrm{RHO}$}

Mutations in $R H O$ can result in a range of phenotypes, most commonly autosomal dominant $\mathrm{RP}$ (adRP), but also autosomal recessive RP (arRP) and congenital stationary night blindness (CSNB). Animal models and in vitro studies have allowed $R H O$ mutations to be divided into seven classes (see [17] for a recent review).

\subsubsection{Dog Model}

A spontaneous dog model of $R H O$ adRP has been identified [18]. The c.11C $>\mathrm{G}$, p.Thr4Arg mutant $\operatorname{dog}\left(R h o^{T 4 R}\right)$ develops a retinal degeneration that is greatly exacerbated by light exposure [19]. The phenotype of this dog model closely resembles that of the human p.Thr4Lys RHO mutation which results in dominant RP [20]. Studies of the $R h o^{T 4 R}$ dog have advanced the understanding of the disease mechanism underlying class four $\mathrm{RHO}$ mutations (those with altered post-translational modification and stability [17]). 
RHO has seven transmembrane loops with intradiscal and cytoplasmic loops and in the dark-adapted state is combined with the chromophore 11-cis-retinal. The $\mathrm{N}$-terminal of the protein, which is affected by the p.Thr4Lys mutation, is positioned within the lumen of the outer segment discs and creates a "cap" over one of the extracellular loops. This cap contributes towards thermal stability and receptor activation of the protein; it also protects the chromophore to opsin protein covalent bond from hydrolysis. Important for the cap role of the N-terminal is glycosylation at N2 and N15. The p.Thr4Arg mutation interferes with glycosylation at the N2 site, altering the cap role. The monoglycosylated rhodopsin is expressed and is trafficked to the outer segment; however, it loses the chromophore faster than the normal meta-rhodopsin II and interacts poorly with the G-protein [21]. The $R h o^{T 4 R}$ dog has light dependent degeneration similar to the sector RP seen with some rhodopsin mutations. In sector $\mathrm{RP}$, the inferior retina is more severely affected as this region gets more light exposure [22]. There is increasing awareness of the need to reduce light exposure to patients with certain RHO mutations [23]. Studies suggested that the unliganded form of the mutant opsin has a detrimental effect because of the loss of its structural integrity. Further evidence to support this was provided by cross breeding $R h o^{T 4 R}$ dogs with the Rpe65 ${ }^{-/-}$dog to produce $R h o^{T 4 R /+} R p e 65^{-/-}$ dogs which lack 11-cis-retinal chromophore (due to the lack of Rpe65 function) and thus have only unliganded mutant rod opsin (i.e., have a lack of rod opsin combined with 11-cis-retinal) and show a greatly accelerated rate of retinal degeneration compared to $R h 0^{T 4 R /+} R p e 65^{+/+}$dogs (see details on the Rpe65 ${ }^{-/-}$dog below) [21].

\subsubsection{Pig Models}

A number of Rho transgenic pigs have been generated, representing different human mutations: p.Pro23His [24], p.Pro347Leu [25] and p.Pro347Ser [26]. Studies using the p.Pro347Leu pigs showed the development of ectopic cone to rod bipolar cell synapses [27] and also interference with the cone to OFF-bipolar cell connection maturation [28]. The potential contribution of oxidative stress to cone death was demonstrated in the model [29]. The light responses of single rod photoreceptors of p.Pro347Leu and p.Pro347Ser transgenic pigs have been studied by suction pipette recording. The recording revealed protracted recovery of the photoresponse and a progressive reduction in the time to peak of the response with reduced sensitivity. This work suggests that the mutant rhodopsin reaches the outer segment and that the substitution at Pro347 interferes with inactivation of the activated form of Rho. The resulting hypothesis was that the carboxyl end of Rho may be involved in the binding of rhodopsin kinase. Mutations at Pro347 may reduce the stability of the carboxyl end attachment to rhodopsin kinase, potentially slowing phosphorylation and the subsequent binding of arrestin [30].

The p.Pro23His pig model has been used to study factors associated with cones developing dormancy and whether they can be reactivated. There are stages of retinal degeneration where the degenerating cones lose inner and outer segments. The remaining cell bodies are described as dormant cones. One hypothesis is that a lack of glucose supply to the cones as a result of loss of surrounding rods leads to cone dormancy. Experiments to either introduce rod precursors or to supply glucose to the subretinal space resulted in reactivation of the dormant cones suggesting mechanisms for the treatment of later-stage IRDs [31].

\subsection{Phosphodiesterase 6 Genes}

Mutations in genes encoding for the subunits forming the rod specific cyclic guanosine monophosphate (cGMP) phosphodiesterase (PDE) holoenzyme cause about 36,000 cases of autosomal recessive RP worldwide in humans, leading to the early onset of night blindness and retinal degeneration [32].

The rod PDE heteromeric holoenzyme has a catalytic core made of PDE6A and PDE6B subunits combined with two inhibitory gamma subunits [33]. Mutation in the gene encoding the alpha subunit of cGMP-PDE (Pde6a) causes PRA in the Cardigan Welsh corgi dog [34,35] and is a model of RP43 in humans, which accounts for 3\% to $4 \%$ of recessive RP in North America [36,37]. Mutations in the gene 
encoding the beta subunit of cGMP-PDE (Pde6b) have been identified in a few dog breeds (see below), including the Irish setter dog [38], which is a model for RP40 in humans and represents about $3 \%$ to $5 \%$ of the recessive forms of RP $[39,40]$. Disease mechanisms for these canine models are detailed below.

\subsubsection{PDE6A}

A dog model with a null mutation in the gene encoding for Pde6a has been identified. It has a relatively severe phenotype and is a model for RP43 in humans [34,35]. This form of PRA was given the name rod-cone dysplasia type 3 ( $r c d 3)$. The frameshift mutation, c.1939delA. p.Asn616ThrfsTer39, results in the absence of Pde6a in the affected dog retina $\left(P_{d e 6 a^{--}}\right)$[35]. Western blot analysis shows the absence of all Pde6 subunits, showing the requirement of Pde6a for stability and normal trafficking of Pde6b [35]. In the absence of the Pde6 holoenzyme, the cGMP hydrolyzing activity is absent and cGMP accumulates in the rod photoreceptors [41]. Increased cGMP is a well-established cause of photoreceptor cell death, likely due to the increased influx of calcium ions into the outer segment [42], triggering apoptosis [43]. The rod outer segments fail to mature in Pde6a $a^{-/-}$dogs and the genetically unaffected cones have stunted outer segments, which is reflected in a reduction in cone electroretinogram (ERG) a-waves early in the disease process [35]. Following the death of rod photoreceptors, there is a progressive loss of cones, which is reflected in the declining cone ERG amplitudes which eventually become undetectable at about one year of age (SMPJ unpublished data). Adeno-associated gene therapy was able to rescue rod function and promote cone function, as well as preserve retinal morphology [41,44].

\subsubsection{PDE6B}

The catalytic beta subunit (PDE6B) of the cGMP-PDE heteromeric holoenzyme is another essential component of the rod photoreceptor phototransduction cascade located in the outer segments [33]. Mutations in the gene encoding for Pde6b causes PRA (rod-cone dysplasia type 1, rcd1) in the Irish Setter dog $[38,45,46]$ which is a model for RP40 in humans. RP40 is one of the most common autosomal recessive RPs leading to blindness in midlife in humans. As with the Pde6a ${ }^{-/-} \operatorname{dog}$, rod photoreceptors are affected first, then cones later in the disease $[47,48]$. The Pde6b $b^{-/}$dog phenotype has an autosomal recessive mode of inheritance and is caused by a nonsense mutation (c.2420G >A, p.Trp807Ter) [38,45]. This truncated protein would lack the C-terminal domain that is required for posttranslational processing and membrane association. The failure of phosphodiesterase activity due to a lack of function in Pde6b leads to elevated cGMP levels from an early age $[47,49]$. The elevation of cGMP in rods as they develop outer segments results in the halting of outer segment elongation followed by rod degeneration, starting in the central retina first, then spreading to the entire retina following the same pattern of rod maturation. The cone outer segment development is also halted and, with the loss of the rods, the genetically unaffected cone photoreceptors also progressively degenerate $[46,47]$. Interestingly, in contrast to the Pde6a ${ }^{-/}$dog retina where the lack of Pde6a leads to the absence of both alpha and beta Pde6 subunits, the Pde6 $b^{-/-}$dog retina has a detectable Pde6a subunit in Western blot, prior to rod degeneration [38]. Therefore, it appears that the alpha subunit is necessary for the beta subunit to be maintained, while the beta subunit is not essential for the maintenance of the alpha subunit. Adeno-associated gene therapy has been shown to halt retinal degeneration in the $r c d 1$ dog [50].

Mutations in Pde6b have been identified in at least two other breeds of dogs (Sloughi and American Staffordshire terriers; see Table 1 for mutation information) [51,52].

\subsubsection{PDE6C}

Recently, a NHP spontaneous achromatopsia model was identified with a missense mutation in a cone phosphodiesterase subunit gene (Pde6c; c.1694G >A, p.Arg565Gln) [4]. Affected animals had behavioral changes, reflecting the photophobia seen in human subjects. They also had macula changes including foveal thinning and a subtle bullseye maculopathy. In vitro studies suggested that 
the mutant protein was expressed and colocalized with its chaperones, Aipl1 and P. However, the mutation alters the catalytic domain, meaning that the mutant protein fails to hydrolyze cGMP.

\section{Visual Cycle}

\section{1. $A B C A 4$}

Stargardt disease is an inherited macular dystrophy which affects one in 8,000-10,000 people. It is the most common inherited macular dystrophy and there is currently no cure. It results from mutations in the gene $A B C A 4$. Mutations in $A B C A 4$ also result in cone-rod dystrophies and RP. ABCA4 is an ATP-dependent flippase expressed in the photoreceptor disc membrane and is necessary in the visual cycle for its transport of N-retinylidene-phosphatidylethanolamine and phosphatidylethanolamine out of the lumen into the cytoplasm [53].

A 1 bp insertion in Abca4 was identified in a family group of Labrador retriever dogs resulting in a frameshift and premature stop codon [54]. This mutation causes a decrease in the mRNA transcript and the loss of the full-length protein. As seen in humans, there is an accumulation of lipofuscin in the RPE cells. Cone and rod photoreceptors both had abnormal function and were decreased in number in older affected dogs (10+ years). The development of a colony of these dogs as a model for therapy will have a substantial impact on the treatment of humans with Stargardt disease because mouse $\mathrm{Abca4}^{-/}$ models lack a phenotype $[54,55]$. The phenotype in the dog appears to be milder than that seen in human subjects, with $A B C A 4$ mutations reflecting species differences [56]. Early biomarkers of retinal changes in the affected dogs will facilitate the use of the Abca4 mutant dog as a model for human Stargardt disease.

\subsection{RPE65}

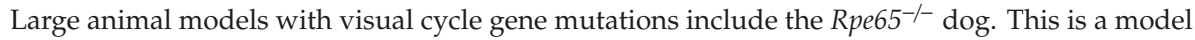
for LCA2. This model was crucial in the development of translational gene augmentation therapy which led to the first FDA-approved gene therapy product. The first animal injected with a therapeutic vector for LCA2 was an $R p e 65^{-/-}$dog. Therapies were developed by three independent groups and consisted in each instance of recombinant adeno-associated virus vectors packaged with RPE65 cDNA. The precise details of promoters and other features such as polyadenylation signals and enhancers differed between the groups. Four groups with colonies of Rpe $65^{-/}$dogs reported successful restoration of rod and cone function [16,57-59]. Loss of rod photoreceptors in the Rpe65 $5^{--}$dog was slow and gene therapy showed ERG rescue even in middle-age [60]. Studies showed that S-cones were sensitive to the lack of normal 11-cis-retinal supply and s-cone opsin immunoreactivity was lost at an early age [61]. There were some phenotypic differences between the Rpe $65^{-/}$dog colonies, with one showing early photoreceptor degeneration in the area centralis (canine equivalent of the human macula) [62]. Trials in human subjects have not resulted in the same restoration of function shown by the dramatic improvement in ERG responses seen in dog and mouse models. A possible explanation for this species difference in therapy efficacy was provided by a comparison of the Rpe65 function of primates and dogs. This suggested that primates require a higher level of Rpe65 than dogs for the function of the visual cycle and that the current therapy might not result in adequate levels of enzymatic function in humans [63].

\section{3. $\mathrm{RDH5}$}

Recently, a cat model with a mutation in another visual cycle gene, $R d h 5$, has been identified by our group [64]. Rdh5 functions to convert 11-cis-retinol to 11-cis-retinal for transport to photoreceptors for reforming the visual pigments. In humans, RDH5 mutations cause a variety of phenotypes. Fundus albipuncatatus is the predominant phenotype [65] but a subset of patients have macular atrophy [66-69] or cone dystrophy [70]. The Rdh5-mutant cat promises to be a valuable model because the $R d h 5^{-1-}$ mouse lacks a phenotype and does not recapitulate RDH5-retinopathy in human patients [71]. In 
contrast, the cat model, similar to affected humans, shows a very delayed recovery of photoreceptor function following light exposure and recapitulates the RDH5-macular atrophy phenotype.

\section{Channelopathies/Channel-Related Mutations}

Mutations that affect channel protein structure and function resulting in disease are termed channelopathies. Spontaneously occurring retinal channelopathies have been identified in dogs resulting from mutations in the cyclic nucleotide-gated ion channels (CNG) of the rod and cone photoreceptors and the anion channel bestrophin 1 (BEST1).

CNG channels in the rod and cone photoreceptors are directly involved in phototransduction. The CNG channels expressed in the rod photoreceptor consist of four subunits: three CNGA1 and one CNGB1 [72-74]. The CNG channel in cone photoreceptors consists of CNGA3 and CNGB3 proteins, in a 3:1 or 2:2 ratio (the stoichiometry of the CNGA3/CNGB3 channel is under debate) [72]. Damaging mutations in the rod CNG channels result in RP, while mutations in cone CNG channels result in achromatopsia.

BEST1 is a homopentameric channel expressed in the retinal pigment epithelium (RPE) and involved in anion transport and intracellular calcium homeostasis [75]. Mutations in BEST1 result in a collection of retinal diseases. Mutations in BEST1 often result in Best Vitelliform Macular Dystrophy, but the age of onset, mode of inheritance, disease characteristics and prognosis can vary [75].

\subsection{CNGA1}

In humans, damaging mutations in CNGA1 result in RP49 representing 1\% or less of arRP cases [39]. A 4 bp deletion in Cngal was identified in Shetland Sheepdogs with PRA. The mutation causes a frameshift and a premature stop codon in a highly conserved region of the protein [76].

\section{2. $C N G B 1$}

There are three splice variants of CNGB1 expressed in the retina, glutamic acid rich proteins (GARPs) GARP1, GARP2 and CNGB1a. The full-length protein, CNGB1a, is part of the heterotetrameric CNG channel of the rod photoreceptor [72]. A complex mutation in Cngb1 was identified in Papillon dogs with PRA. The mutation identified in these dogs is a 6 bp insertion accompanied by a 1 bp deletion, predicted to result in a frameshift and a premature stop codon, six amino acids downstream [77]. Upon further analysis of the Cngbla transcript in the $\mathrm{Cngb1}^{-/}$dogs, it was found that the mutation led to the skipping of exon 26, resulting in a premature stop codon early in exon 27. A truncated Cngbla protein is produced, but does not form channels with Cnga1 and remains in the inner segments of the rod photoreceptors [78]. Mutations in CNGB1 cause RP45 in humans, which represents less than $4 \%$ of arRP cases [39].

Human RP45 patients with mutations downstream of the GARP splice variants, the mouse knockout model (Cngb1-X26) and the Cngb1 ${ }^{-/}$dog have similar phenotypes [78,79]. The canine model shows a slow loss of rod photoreceptors and the relative preservation of cones, particularly in the area centralis and visual streak. Cone function decreases as the disease progresses, as assessed by ERG, but cone-led vision remains normal, at least up to four years [77]. Preliminary gene therapy trials have shown that treatment at 3.5-6.5 months of age in affected dogs rescues vision and slows the progression of the disease in the treated areas [78].

Both the slow disease progression and the large treatment window (in dogs and anticipated in humans [80]) make the $\mathrm{Cngb1^{-/ }}$ dog an ideal model for studying therapies and outcome measures.

\section{3. $C N G A 3$}

About $25 \%$ of human achromatopsia cases are caused by damaging mutations in the alpha subunit of the cone CNG channel (CNGA3) [81]. Two canine models of achromatopsia were identified with mutations in CNGA3. The mutations, p.Arg424Trp and p.Val644del (R424W and V644del, respectively), provided intriguing mutation sites for in vitro testing. Modeling of these mutations lead to further 
insights into CNG channel gating and subunit interactions [82]. The residue R424 is located in the S6 transmembrane helix and forms a salt bridge with the residue E306 in the S4-S5 linker. Protein modeling of the canine R424W mutation found the disruption of this salt bridge plays an important role in protein folding, subunit assembly and channel gating. In vitro expression studies of the R424W mutation showed increased mislocalization of the mutant CNGA3 protein and the mutant channels did not produce cyclic nucleotide-activated currents [82].

The canine V644del mutation is in the C-terminal leucine zipper (CLZ) domain, which is involved in channel assembly and stability. The one amino acid deletion shifts all subsequent residue interactions within the domain. In vitro studies in a heterologous expression system showed evidence of mislocalization and a decrease in the total number of active channels, but some mutant channels $(\sim 60 \%)$ reached the cell membrane and had normal cyclic nucleotide-activated currents [82].

Two spontaneous sheep models of Cnga3 achromatopsia have been identified [83]. The first identified has a nonsense mutation (p.Arg236Ter) and the second a missense mutation (p.Gly540Ser) [84]. Adeno-associated virus gene therapy was able to restore cone function in both models $[84,85]$.

\section{4. $C N G B 3$}

Two unique mutations were identified in the canine $\mathrm{Cngb3}$ gene- - a genomic deletion that removes the entire $\mathrm{Cngb3}$ gene ( $\mathrm{Cngb3}^{--}$) and a missense mutation (p. Asp262Asn; D262N). The missense mutation was identified in German Shorthaired Pointers, while the genomic deletion was initially found in Alaskan Malamutes and later found in other breeds [86,87]. Mutations in CNGB3 are responsible for at least $50 \%$ of achromatopsia cases in humans, making it an ideal target for therapies $[87,88]$.

The D262N mutation in Cngb3 inspired investigation into the Tri-Asp motif that is conserved in CNG channels [89]. When this mutation was introduced to heterologously expressed CNGA3, it abolished homomeric channel function and was responsible for mislocalization of the protein. When the mutation was introduced in CNGB3 in coexpression studies with CNGA3, the response to cyclic nucleotides was reduced but still present and consistent with CNGA3 homomeric channel function. These experiments show the Tri-Asp motif is necessary for proper cone CNG channel formation, but raises the question why mutations in CNGB3 result in the loss of cone function and achromatopsia when CNGA3 can form a functioning homomeric channel in the absence of CNGB3. Further work in retinal tissue and/or cone photoreceptors will be needed to elucidate this [89].

Delivery of a human CNGB3 gene via rAAV5 vector to both $\mathrm{Cngb3}^{-/}$and the D262N mutant dogs showed that the vector could be targeted to the medium and long wavelength $(\mathrm{M} / \mathrm{L})$ cones via a human red cone opsin promoter and the rescue was dependent on the age of the dog and the promotor, not on the mutation type [90].

\subsection{BEST1}

Best vitelliform macular dystrophy (Best disease, BVMD) has autosomal dominant inheritance and is the most common disease associated with mutations in the gene BEST1. Four other disease phenotypes have been described in association with BEST1 mutations: adult onset vitelliform macular dystrophy, autosomal recessive bestrophinopathy, autosomal dominant vitreoretinochoroidopathy and retinitis pigmentosa. BVMD is characterized by at least one vitelliform lesion in the macula but can present with multiple lesions. The disease slowly progresses to degenerate the RPE and retina in the affected regions, resulting in vision impairment [75]. In dogs, a similar disease has been described, termed canine multifocal retinopathy (CMR) and is caused by mutations in the Best1 gene. In contrast to the BVMD in humans, CMR due to Best1 mutations is an autosomal recessive disease and has a consistent and predictable disease phenotype. This consistency and the detailed natural history of the disease lends well to measuring the outcomes of translatable therapies.

Initially, two Best1 mutations were identified in dogs: p.Arg25Ter (Great Pyrenees and mastiff-related breeds, cmr1) and p.Gly161Asp (Coton de Tulear, cmr2), with the former resulting in a premature stop codon and, presumably, a lack of Best1 protein [91]. Analysis of additional breeds 
with CMR identified another breed (Lapponian herder, cmr3) with two deleterious mutations in exon 10, a $1 \mathrm{bp}$ deletion leading to a frameshift and premature stop codon (p.Pro463fs) and a missense mutation (p.Gly489Val) [92]. Interestingly, the phenotype resulting from the three mutations in dogs is indistinguishable. The dogs present with multifocal regions of retinal separation with a pink or tan-colored subretinal fluid, which eventually leads to retinal degeneration. Using optical coherence tomography to image eyes from $c m r 1 / c m r 1, c m r 3 / c m r 3$ and $c m r 1 / c m r 3$ dogs in vivo, the earliest detectable sign of the disease is at $\sim 11$ weeks of age in which there is a retinal elevation in the fovea-like region of the area centralis. As the disease progresses, this retinal elevation becomes a macrodetachment, surrounded by microdetachments. Rates of progression, detachment location and number varied, but the disease was typically localized to the more cone-rich regions of the retina [93].

Immunohistochemistry of CMR canine tissues showed a lack of RPE apical microvilli at the cone photoreceptor/RPE interface along with accumulated lipofuscin within the RPE. The loss of the RPE apical processes results in a loss of all direct contact of the cones to the RPE, severely impacting the physiological role the RPE has on retinal maintenance. It is hypothesized that the lack of RPE apical processes and subsequent weakened interphotoreceptor matrix is instrumental in the characteristic detachments and lesions observed in diseases caused by BEST1 mutations [93,94]. Interestingly, microdetachments were identified in response to light exposure in pre-clinical CMR dogs. The detachments occurred between the photoreceptor inner/outer segments and the RPE/tapetum interface. These light-induced detachments occurred within minutes and increased in response to time of light exposure and would resolve within $24 \mathrm{~h}$.

Using a rAAV2 vector delivered subretinally, CMR dogs were treated by gene augmentation with wildtype canine or human BEST1. Macro and microdetachments were resolved and RPE microvilli ensheathment of cone photoreceptors returned within the treatment area. This positive outcome was retained in the dogs for as long as 207 weeks post injection. The same outcome was present regardless of age of treatment (within 27-69 weeks of age), the stage of detachments or mutation ( $\mathrm{cmr} 1 / \mathrm{cmr} 1$, $\mathrm{cmr} 3 / \mathrm{cmr} 3$ or $\mathrm{cmr} 1 / \mathrm{cmr} 3$ ). These results show promise for the treatment of human patients with BEST1 mutations [93].

\section{Cilia-Related Proteins (Ciliopathies)}

Photoreceptors are non-motile sensory ciliated cells. The connecting cilium between inner and outer segment is critical for the transport of proteins to and from the outer segment. Many proteins are expressed in this region of photoreceptors and act to control the trafficking of proteins. Ciliopathies is a general term used to group conditions resulting from mutations in cilia proteins. As cilia are present in many cell types, mutations in cilia proteins can cause syndromic disease where retinal degeneration is present, accompanied by disorders in other organ systems. Mutations that have a milder effect on function may result in a photoreceptor-only phenotype such as LCA or RP. A number of large animal models with mutations in cilia related genes have been identified (Table 1).

\subsection{BBS4}

A mutation in the Bbs4, (Bardet-Biedl syndrome 4) gene, was identified in Hungarian Puli dogs with PRA. This mutation (c.58A $>$ T, p.Lys20Ter) is predicted to result in nonsense mediated decay of the Bbs4 transcript. BBS4 is one of the BBS genes involved in cilia function. These dogs showed a typical PRA phenotype along with noted obesity and abnormal sperm, although more affected dogs may need to be examined before confirming that this is a syndromic BBS model [95].

\subsection{BBS7}

An NHP model of Bardet-Biedl syndrome has been recently described. A mutation in exon 3 of the Bbs7 gene c.160delG (p.Ala54GlnfsTer18) that is predicted to produce a truncated non-functional protein was identified. As with BBS4, BBS7 is involved in cilia function. Typically in humans, mutations in BBS genes cause a syndromic condition. The affected NHPs had several features of BBS, including 
retinal atrophy, which was most severe centrally; the affected animals had smaller brains, renal disease, and the males had small testes. This is the first described model of BBS in NHP and shares many characteristics with BBS patients with truncating mutations [3].

\subsection{CEP290}

This cat model, $r d A c$ (retinal degeneration, Abyssinian cat) has been studied in detail for many years. Compared to most CEP290 (centrosomal protein 290) mutations in other species, the cat model has a mild phenotype. The onset and rate of photoreceptor degeneration in the cat with the sparing of the area centralis makes it a model for RP $[96,97]$. In humans, CEP290 mutations can result in a spectrum of phenotypes including lethality, severe syndromic disease (e.g., Joubert syndrome) and most commonly LCA; see [98] for a review. Milder phenotypes such as RP are less common. In the cat, an intronic mutation (c.6966+9T>G) leads to the introduction of a stronger splice acceptor site (the wildtype splice acceptor is a GC rather than the much more commonly used GT). The mutation strengthens an adjacent GT, which, when used, adds 4 bps to the exon 50 sequence, the resulting frameshift introduces a premature stop codon [99]. The truncated protein escapes nonsense mediated decay and is expressed. In addition, the wildtype acceptor site is still used for a percentage of the transcripts (unpublished data). The combination of a truncated protein with some remaining function combined with a low-level production of full-length transcripts explains the mild phenotype. CEP290 is too large to be packaged in an adeno-associated virus vector and one therapeutic approach being investigated is the use of a truncated transcript so that a miniprotein may have partial function and convert a severe phenotype into a milder phenotype similar to that of the cat.

\subsection{NPHP4}

Wire-haired dachshunds have a cone-rod dystrophy resulting from a truncating mutation in Nphp4. The identified Nphp4 mutation was a deletion involving exon 5/intron 5 that led to skipping of exon 5 and a premature truncation in exon 6 of 30 (c.462_526del, p.Leu155LysfsTer2) [100]. A colony was established from a single founder male [101]. Puppies had miotic pupils and cone-mediated ERGs were reduced prior to retinal maturation. Furthermore, they did not show the normal increase in amplitudes with retinal maturation and further declined in amplitude rapidly. The amplitudes of the rod-driven responses were less severely affected, but were lost with age [102,103]. Interestingly, the condition is non-syndromic in dogs, just presenting as a cone-rod dystrophy. Human patients with NPHP4 mutations do not always develop a retinal phenotype, but typically have nephronophthisis, with some patients having Senior-Løken syndrome, which combines the renal phenotype with a retinal dystrophy (see for [104] a review). This phenotype difference may represent a species difference, with

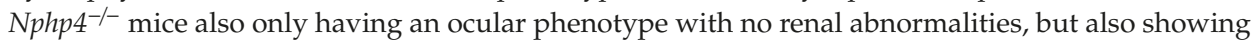
male infertility [105].

\subsection{NPHP5 (IQCB1)}

A mutation was identified in the NPHP5 gene (aka IQCB1) resulting in a cone-rod dystrophy in American pit bull terrier dogs ( $c r d 2)$ modeling non-syndromic LCA. This mutation (c.952-953insC, p.Ser319llefsTer13) results in a frameshift and a premature stop codon [52]. At 6 weeks of age, the $c r d 2$ dogs had no cone function and abnormal rod function. Morphologically, the cones completely lacked outer segments, while the rods developed disorganized outer segments. Despite the lack of cone function, the cones are retained within the central retina up to 33 weeks of age [106]. Adeno-associated gene therapy restores and improves photoreceptor function and preserves the outer retina layer [107].

\section{6. $R P G R$}

Retinitis pigmentosa GTPase regulator (RPGR) localizes to the connecting cilium and mutations within the RPGR gene account for $>80 \%$ of $\mathrm{X}$-linked RP (XLRP). There are two major retinal isoforms, one encoded by exons 1-19 and a second isoform that consists of exons 1-15 and a retained portion of intron 
15 (ORF15; see [108] for a summary). ORF15 is a mutation hotspot [109]. Three different Rpgr mutations cause $\mathrm{X}$-linked retinal degeneration in dogs. One is caused by a genomic deletion [110] and two are due to microdeletions in ORF15 and provide two distinct mechanistic models for RPGR-XLRP [111]. The first, known in the dog as XLPRA1, has a 5 bp deletion and a premature stop codon [111]. This is a relatively late onset and slowly progressive degeneration [112]. The second form, XLPRA2, has a 2 bp deletion with a frameshift resulting in the addition of 34 basic residues. In vitro studies showed that the mutant protein aggregated in the endoplasmic reticulum and is hypothesized to have a toxic effect [111]. The phenotype presents as an early onset degeneration with early changes being outer segment disruption and opsin (rod and cone) mislocalization. Photoreceptor cell death was shown to occur in a biphasic manner with two distinct phases of cell death with evidence of remodeling occurring [113]. Studies of the heterozygous females revealed that there are patches of diseased retina, presumably resulting from regions where random X-inactivation has resulted in expression of the mutant allele and patches of unaffected retina (where the wildtype allele is expressed). In the earlier onset XLPRA2 migration of adjacent photoreceptors into regions where rod photoreceptors died occurs, showing retinal plasticity in the younger animals. This remodeling was not described in the XLPRA1, where patches of degeneration occur at a later age [114].

\subsection{RPGRIP1}

Retinitis pigmentosa GTPase regulator-interacting protein 1 (RPGRIP1) localizes to the connecting cilium, where it interacts with RPGR. Mutations in RPGRIP1 are associated with autosomal recessive LCA. A cone-rod dystrophy form of PRA in a colony of miniature longhaired Dachshunds was reported to be due to an insertion in Rpgrip1 [115] and the rescue of the phenotype by gene therapy was achieved [116]. When miniature longhaired Dachshunds in pet homes were investigated, the Rpgrip1 insertion did not appear to segregate with disease status [117]. Further studies have shown that two other loci influence the development of the phenotype [118-120]. This is an example of the potential effect of modifying loci on phenotype.

\section{Photoreceptor Development}

\section{1. $C R X$}

The CRX gene encodes an OTX-like homeodomain transcription factor which is essential for photoreceptor development, maturation and survival [121-124]. Transcription factors are essential for control of the maturation of progenitor cells [123-126]. Only one spontaneously occurring large animal model of a retinal transcription factor homeobox mutation has been described, the $C r x^{R d y}$ cat $[127,128]$. The heterozygous animal has a severe phenotype of cone-led retinal dystrophy and is a model for one form of severe early childhood onset blindness (LCA7). While most mutations in CRX result in LCA7, other phenotypes including cone-rod dystrophy, RP and macular degeneration have been described [129-131]. CRX mutations have been reported to account for between $0.6 \%$ and $2.35 \%$ of LCA [131-134].

As with other transcription factors, CRX has a characteristic structure with a DNA binding domain (homeodomain) near its $\mathrm{N}$-terminal and a transactivation domain at the C-terminal. The $\mathrm{Cr} x^{R d y /+}$ mutant cat models Class III CRX mutations, which are antimorphic frameshift/nonsense mutations with intact DNA binding, but a lack of target gene transactivation [135]. The $\mathrm{Cr} x^{R d y /+}$ cat mutation is caused by a 1 bp deletion (c.546delC; p.Pro185LysfsTer2) in the final exon of the Crx gene [127]. This mutant mRNA avoids nonsense mediated decay and produces a truncated Crx protein with an intact DNA-binding domain but disrupted transactivation domain [128]. Both the mutant transcript and protein accumulate at higher levels than the wildtype versions, possibly due to increased stability of the mutant mRNA compared to the wildtype. In vitro studies showed that the mutant mRNA fails to activate its own promoter [128]. This suggests that the mutant protein has a dominant negative effect by binding promoter recognition sites, but fails in its transactivation function. The result is 
misregulated gene expression; for example, Rho and cone arrestin (Arr3) mRNA levels are severely decreased. Truncated photoreceptor outer segments are produced, but the photoreceptors fail to fully mature [128]. A decreased rod ERG is detectable, which shows evidence of partial maturation before this is halted and the ERG amplitudes decline, paralleling rod degeneration. Cone function is more severely affected, with no cone ERG responses being detectable [128].

\subsection{STK38L}

An early retinal degeneration was described in Norwegian Elkhounds in 1987 [136] in which the dogs present with vision impairment in low light as early as 6 weeks of age. The causal mutation was found to be a short interspersed element (SINE) insertion in exon 4 of $S t k 38 l$, a gene that had not been associated with retinal degenerations before its discovery in the Norwegian Elkhounds [137]. The mRNA transcript is produced in the affected dog but does not contain exon 4 . Interestingly, at $\sim 6$ weeks of age, the expression of the mutant $S t k 38 l$ transcript is similar to control dogs, but expression increases at $\sim 8$ and $\sim 12$ weeks [138].

Detailed histological experiments were performed on affected canine retina to understand the role of Stk381 in retinal development and how the lack of the functional protein results in retinal degeneration. The retina in $\sim 4$ week old Stk38l-affected dogs is normal, but by $\sim 8$ weeks of age the rod photoreceptors begin to show mislocalization of Rho, irregularities in the outer segments and increased TUNEL labeling. However, this increase in apoptotic cells is not accompanied by a thinning of the outer nuclear layer (ONL), as would be expected from loss of rod photoreceptors. The maintenance of ONL thickness is a result of proliferation of rod-like photoreceptors which express both Rho and cone S-opsin. After $\sim 14$ weeks of age, this proliferation ceases and the ONL begins to thin as the photoreceptors die, resulting in the loss of at least $50 \%$ of the ONL rows by 48 weeks [138].

The Stk38l-affected dogs provide an interesting and unique model of retinal degeneration in which differentiated photoreceptors either apoptose or proliferate for a short amount of time before retinal degeneration progresses.

\section{Photoreceptor to Bipolar Cell Signaling}

\subsection{LRIT3}

Recently, a dog model of recessively inherited CSNB has been identified due to a mutation in leucine-rich-repeat, immunoglobulin-like transmembrane-domain 3 gene (LRIT3). Mutations in LRIT3 in humans causes a form of CSNB [139]. The ERG of the affected dogs shows a lack of ON-bipolar cell function with preservation of cone OFF-bipolar contributions [140-142]. There is currently debate about the positioning of the LRIT3 protein which had been described as being in the synaptic tips of the bipolar cells; however, a recent publication showed that it was presynaptic, being present in photoreceptors, but bridged the synapse to influence the positioning of post-synaptic glutamate signaling proteins [143]. The new availability of a large animal model may facilitate further investigations.

\subsection{TRPM1}

Studies of Appaloosa horses with CSNB contributed to the identification of the role of transient receptor potential cation channel subfamily M, member 1 (Trpm1) in bipolar cell signaling [144,145]. The night-blind Appaloosa horse was first recognized as an animal model for the Schubert-Bornschein type of CSNB in the 1970s [146]. The study published at that time reported a lack of an ERG b-wave and night blindness but no retinal degeneration or obvious morphological abnormality of the photoreceptor synapses with second order neurons [146]. The correlation of CSNB in the Appaloosa with the Leopard complex spotting coat color was recognized [147]. This coat color is governed at a single gene locus with animals homozygous for the Leopard complex spotting associated allele (LP) also having CSNB [147]. When the LP locus was mapped, Trpm1, which is also expressed in melanocytes, was identified as a positional candidate gene and showed markedly reduced expression in retina and 
skin from affected animals [148]. Investigation of TRPM1 in humans with complete CSNB identified mutations in TRPM1 [149-151] and its role in ON-bipolar cell signaling was identified.

The LP mutation was identified as a retroviral insertion in intron 1 of equine $\operatorname{Trpm} 1$ that disrupts gene transcription by causing premature polyadenylation [152].

\subsection{Whippet Dog Model of Incomplete CSNB with Retinal Degeneration}

A dog model of cone-rod synaptic dysfunction, which has been described by some authors as a form of incomplete CSNB, has been identified [153]. The affected dogs lack an ERG b-wave and also lack cone OFF-bipolar cell attributable ERG components [154]. Interestingly, the dog model develops a progressive retinal degeneration, which is not reported as a feature of the condition in humans [155].

\section{Structural/Other}

Retinal degenerations can occur due to mutations in genes involved in the structure or maintenance of the retina.

\subsection{ADAM9}

ADAM9 (a disintegrin and metalloprotease domain, family member 9) mutations are associated with cone-rod dystrophies in humans and dogs. A genomic deletion in Adam 9 was identified in Irish Glen of Imaal Terriers, resulting in a premature stop codon and the loss of the full-length protein. Similar to the mouse model, histological sections show that the RPE cells do not invest in the outer segments of the photoreceptors. This mutation effects both the rod and cone photoreceptors, but the cones are more severely affected throughout the course of the disease $[156,157]$. Further work is needed to understand the role of ADAM9 in the retinal structure and RPE maintenance of photoreceptors.

\subsection{AIPL1}

Mutations in the gene encoding aryl hydrocarbon receptor-interacting protein-like 1 (AIPL1) causes LCA4 accounting for about $3 \%$ to $7 \%$ of autosomal recessive LCA [132,134,158-160]. The AIPL1 gene encodes for a protein expressed in the photoreceptor and pineal gland [161]. In photoreceptors, AIPL1 is concentrated in the tip of the inner segment in proximity to the connecting cilia, acting as a co-chaperone involved in the folding, assembly and transport of the retinal cGMP phosphodiesterase (PDE6) heteromeric holoenzyme within photoreceptor outer segments [162-166].

One spontaneous occurring feline large animal model of LCA4 currently exists, the Persian cat [167]. This feline model, similar to human patients with LCA4, presents as an autosomal recessive severe retinal dystrophy. The disease is characterized by a very early photoreceptor loss, progressing to severe retinal degeneration before adulthood. This leads to very early blindness [167]. The feline phenotype is caused by a nonsense mutation at position c.577C $>$ T producing a stop codon (p.Arg193Ter), leading to the production of a truncated non-functional protein [168]. The precise mechanisms of the severe phenotype in the feline model are yet to be investigated. The feline model represents a valuable large animal model for mechanistic studies underlying AIPL1-LCA in humans.

\subsection{MERTK}

Mutations in MERTK (MER proto-concogene, tyrosine kinase) account for about 1\% of arRP. Mertk was found to be overexpressed (six-fold) in Swedish Vallhund dogs with a retinal dystrophy [169]. After whole genome sequencing of an affected dog, an intact LINE-1 insertion was identified in intron one of Mertk. A LINE-1 retrotransposon is a long interspersed repetitive element that is found in all mammalian genomes [170]. It is unclear how this insertion results in overexpression of the Mertk transcript and subsequent development of retinal disease. More in-depth investigation of this mechanism will aid a better understanding of how retrotransposon insertions can modify gene 
regulation/expression, ultimately leading to disease, in addition to understanding the specific role of overexpression of the MERTK transcript in the context of retinal disease [171].

\section{4. $P R C D$}

Progressive rod-cone degeneration ( $P r C d$ ) was a formerly unknown gene that was mapped and sequenced first in dogs with progressive rod-cone degeneration (PRCD) PRA [172]. Dogs with this form of PRA had been studied in detail over many years and many different breeds of dog are affected. The mutation is p.Cys2Tyr, which is in a highly conserved region of the gene. An identical mutation was found in a human RP patient. Additional mutations also located in exon 1 of $P R C D$ have been identified in RP patients [172,173]. Since the discovery of $P r c d$, a mouse knockout model has been generated and experiments have shown Prcd's involvement in photoreceptor disc formation and maintenance [174-176]. Studies in the PRCD dog showed an early ultrastructural change that may have resulted from this purported function. This was the development of vesicular profiles adjacent to outer segments being consistent with abnormality in disc formation, furthermore studies also showed affected dogs had slower photoreceptor disc renewal than normal controls [177]

\section{5. $R D 3$}

Mutations in RD3 result in LCA12 in humans, a severe retinal degeneration in mice and rod-cone dysplasia type $2(r c d 2)$ in Collie dogs [178-180]. RD3 is thought to act as competitor for guanylyl cyclase-activating proteins, preventing premature cyclase activity in inner segments [181].

Collie dogs have a $22 \mathrm{bp}$ insertion in the $R d 3$ gene resulting in altered amino acids and an extended open reading frame [182]. Three splice variants of the gene were identified in the canine retina. In the $R d 3$-mutant Collie, the rod and cone photoreceptors outer segments do not fully develop and the retina degenerates rapidly. By 6 months, there are no photoreceptor inner or outer segments and the outer nuclear layer contains only one layer of nuclei [183].

\section{Conclusions}

IRDs that lead to visual impairment and blindness show considerable heterogeneity, where even similar disease phenotypes can result from mutations in a wide range of different genes. Closer examination of the broad phenotypes such as RP and LCA show the genetic heterogeneity with altered function of a range of genes leading to photoreceptor loss. Large numbers of different mutations within a single gene exist and can result in a range of phenotypic differences.

The mechanisms by which the gene mutation leads to cell death differs between genes and between mutations within individual genes. Laboratory rodents have been the workhorses for studying disease mechanisms because of the ease with which their genome can be engineered, the short reproductive time and relatively low costs of maintenance. They have allowed identification of disease pathways and allowed the study of how and why degeneration occurs. However, laboratory rodents have very significant shortcomings. Rodents are nocturnal animals with a different pattern of distribution of photoreceptors to humans. The large animal models discussed in this review have retinal photoreceptor distribution that is much closer to that of humans. This is particularly important when considering conditions that have a major effect on the macula. Large animal models have also been of value for the development and testing of translational therapies. They are particularly valuable for this because the larger eye size and proportions of lens and vitreous is much closer to that in humans, allowing for identical surgical approaches for trials of translational therapies. The several, mostly spontaneously occurring, large animal models mentioned here have provided valuable information about disease phenotypes and disease mechanisms. They offer great potential for even more detailed studies to understand how photoreceptor function and survivability is affected by the gene mutations and to study the extensive inner retinal remodeling and glial cell activation that occurs in IRDs. Modeling of IRDs using retinal organoids developed from induced pluripotent stem cells from IRD patients is an exciting field of research. These potentially allow the study of the effect of the exact mutation that is 
present in the patient and in a human cultured tissue. However, they do not yet fully recapitulate the specific environment within the retina of a living animal, so, while showing great promise, they cannot yet replace whole animal studies. Improving our understanding of how and why photoreceptors die may suggest novel therapies to preserve function and slow down vision loss. There remain untapped populations of companion animals (dogs and cats) with spontaneous IRDs, with new potential models being identified with increasing frequency. The advances in gene editing also expand the opportunity to develop additional large animal models with specific mutations that even more accurately model human IRDs.

Supplementary Materials: The following are available online at http://www.mdpi.com/2073-4409/9/4/882/s1, Table S1: List of mutations in large animal models of inherited retinal disease and references.

Author Contributions: All authors contributed to the conception, design and writing of the manuscript. All authors have read and agreed to the published version of the manuscript.

Funding: The NIH (EY027285) and the Donald R. Myers and William E. Dunlap Endowment for Canine Health, to Simon Petersen-Jones, provided support for the work done in this review.

Conflicts of Interest: The authors declare no conflict of interest.

\section{References}

1. Fan, N.; Lai, L. Genetically Modified Pig Models for Human Diseases. J. Genet. Genom. 2013, 40, 67-73. [CrossRef] [PubMed]

2. Petersen-Jones, S.M. Drug and gene therapy of hereditary retinal disease in dog and cat models. Drug Discov. Today Dis. Model. 2013, 10, e215-e223. [CrossRef]

3. Peterson, S.M.; McGill, T.J.; Puthussery, T.; Stoddard, J.; Renner, L.; Lewis, A.D.; Colgin, L.M.; Gayet, J.; Wang, X.; Prongay, K.; et al. Bardet-Biedl Syndrome in rhesus macaques: A nonhuman primate model of retinitis pigmentosa. Exp. Eye Res. 2019, 189, 107825. [CrossRef]

4. Moshiri, A.; Chen, R.; Kim, S.; Harris, R.A.; Li, Y.; Raveendran, M.; Davis, S.; Liang, Q.; Pomerantz, O.; Wang, J.; et al. A nonhuman primate model of inherited retinal disease. J. Clin. Investig. 2019, 129, 863-874. [CrossRef]

5. Luo, X.; Li, M.; Su, B. Application of the genome editing tool CRISPR/Cas9 in non-human primates. Zool. Res. 2016, 37, 214-219.

6. Kang, Y.; Chu, C.; Wang, F.; Niu, Y. CRISPR/Cas9-mediated genome editing in nonhuman primates. Dis. Model. Mech. 2019, 12, dmm039982. [CrossRef] [PubMed]

7. Rahman, N.; Georgiou, M.; Khan, K.N.; Michaelides, M. Macular dystrophies: Clinical and imaging features, molecular genetics and therapeutic options. Br. J. Ophthalmol. 2019, 104, 451-460. [CrossRef]

8. Michaelides, M.; Hunt, D.M.; Moore, A.T. The genetics of inherited macular dystrophies. J. Med. Genet. 2003, 40, 641-650. [CrossRef]

9. Fritsche, L.G.; Igl, W.; Bailey, J.N.C.; Grassmann, F.; Sengupta, S.; Bragg-Gresham, J.L.; Burdon, K.P.; Hebbring, S.J.; Wen, C.; Gorski, M.; et al. A large genome-wide association study of age-related macular degeneration highlights contributions of rare and common variants. Nat. Genet. 2015, 48, 134-143. [CrossRef]

10. Heesterbeek, T.J.; Lorés-Motta, L.; Hoyng, C.B.; Lechanteur, Y.T.E.; Hollander, A.I.D. Risk factors for progression of age-related macular degeneration. Ophthalmic Physiol. Opt. 2020, 40, 140-170. [CrossRef]

11. Nishiguchi, K.; Yokoyama, Y.; Fujii, Y.; Furukawa, T.; Ono, F.; Shimozawa, N.; Togo, M.; Suzuki, M.; Nakazawa, T. Association between drusen and blood test results in a colony of 1174 monkeys. Acta Ophthalmol. 2015, 93, 93. [CrossRef]

12. Pennesi, M.E.; Neuringer, M.; Courtney, R.J. Animal models of age related macular degeneration. Mol. Asp. Med. 2012, 33, 487-509. [CrossRef] [PubMed]

13. Mowat, F.M.; Petersen-Jones, S.M.; Williamson, H.; Williams, D.L.; Luthert, P.J.; Ali, R.R.; Bainbridge, J.W. Topographical characterization of cone photoreceptors and the area centralis of the canine retina. Mol. Vis. 2008, 14, 2518-2527. [PubMed]

14. Beltran, W.A.; Cideciyan, A.V.; Guziewicz, K.E.; Iwabe, S.; Swider, M.; Scott, E.M.; Savina, S.V.; Ruthel, G.; Stefano, F.; Zhang, L.; et al. Canine Retina Has a Primate Fovea-Like Bouquet of Cone Photoreceptors Which Is Affected by Inherited Macular Degenerations. PLoS ONE 2014, 9, e90390. [CrossRef] 
15. El-Aziz, M.M.A.; Barragan, I.; O’Driscoll, C.A.; Goodstadt, L.; Prigmore, E.; Borrego, S.; Mena, M.; Pieras, J.I.; El-Ashry, M.F.; Abu Safieh, L.; et al. EYS, encoding an ortholog of Drosophila spacemaker, is mutated in autosomal recessive retinitis pigmentosa. Nat. Genet. 2008, 40, 1285-1287. [CrossRef]

16. Acland, G.M.; Aguirre, G.D.; Ray, J.; Zhang, Q.; Aleman, T.S.; Cideciyan, A.V.; Pearce-Kelling, S.E.; Anand, V.; Zeng, Y.; Maguire, A.M.; et al. Gene therapy restores vision in a canine model of childhood blindness. Nat. Genet. 2001, 28, 92-95. [CrossRef]

17. Athanasiou, D.; Aguila, M.; Bellingham, J.; Li, W.; McCulley, C.; Reeves, P.J.; Cheetham, M.E. The molecular and cellular basis of rhodopsin retinitis pigmentosa reveals potential strategies for therapy. Prog. Retin. Eye Res. 2017, 62, 1-23. [CrossRef]

18. Kijas, J.W.; Cideciyan, A.V.; Aleman, T.S.; Pianta, M.; Pearce-Kelling, S.E.; Miller, B.J.; Jacobson, S.G.; Aguirre, G.D.; Acland, G.M. Naturally occurring rhodopsin mutation in the dog causes retinal dysfunction and degeneration mimicking human dominant retinitis pigmentosa. Proc. Natl. Acad. Sci. USA 2002, 99, 6328-6333. [CrossRef]

19. Iwabe, S.; Ying, G.-S.; Aguirre, G.D.; Beltran, W.A. Assessment of visual function and retinal structure following acute light exposure in the light sensitive T4R rhodopsin mutant dog. Exp. Eye Res. 2016, 146, 341-353. [CrossRef]

20. Born, L.I.V.D.; Van Schooneveld, M.J.; De Jong, L.A.M.S.; Riemslag, F.C.C.; DeJong, P.T.V.M.; Gal, A.; Bleeker-Wagemakers, E.M. Thr4Lys rhodopsin mutation is associated with autosomal dominant retinitis pigmentosa of the cone-rod type in a small Dutch family. Ophthalmic Genet. 1994, 15, 51-60. [CrossRef]

21. Zhu, L.; Jang, G.-F.; Jastrzebska, B.; Filipek, S.; Pearce-Kelling, S.E.; Aguirre, G.D.; Stenkamp, R.E.; Acland, G.M.; Palczewski, K. A naturally occurring mutation of the opsin gene (T4R) in dogs affects glycosylation and stability of the G protein-coupled receptor. J. Boil. Chem. 2004, 279, 53828-53839. [CrossRef] [PubMed]

22. Heckenlively, J.R.; Rodriguez, J.A.; Daiger, S.P. Autosomal Dominant Sectoral Retinitis Pigmentosa. Arch. Ophthalmol. 1991, 109, 84. [CrossRef] [PubMed]

23. Orlans, H.O.; MacLaren, R.E. Comment on: 'Sector retinitis pigmentosa caused by mutations of the RHO gene'. Eye 2019, 1-2. [CrossRef] [PubMed]

24. Ross, J.W.; De Castro, J.P.F.; Zhao, J.; Samuel, M.; Walters, E.; Rios, C.; Bray-Ward, P.; Jones, B.W.; Marc, R.E.; Wang, W.; et al. Generation of an Inbred Miniature Pig Model of Retinitis Pigmentosa. Investig. Opthalmol. Vis. Sci. 2012, 53, 501-507. [CrossRef] [PubMed]

25. Petters, R.M.; Alexander, C.A.; Wells, K.D.; Collins, E.B.; Sommer, J.; Blanton, M.R.; Rojas, G.; Hao, Y.; Flowers, W.L.; Banin, E.; et al. Genetically engineered large animal model for studying cone photoreceptor survival and degeneration in retinitis pigmentosa. Nat. Biotechnol. 1997, 15, 965-970. [CrossRef]

26. Kraft, T.; Allen, D.; Petters, R.M.; Hao, Y.; Peng, Y.-W.; Wong, F. Altered light responses of single rod photoreceptors in transgenic pigs expressing P347L or P347S rhodopsin. Mol. Vis. 2005, 11, 1246-1256.

27. Peng, Y.-W.; Hao, Y.; Petters, R.M.; Wong, F. Ectopic synaptogenesis in the mammalian retina caused by rod photoreceptor-specific mutations. Nat. Neurosci. 2000, 3, 1121-1127. [CrossRef]

28. Banin, E.; Cideciyan, A.V.; Aleman, T.S.; Petters, R.M.; Wong, F.; Milam, A.H.; Jacobson, S.G. Retinal Rod Photoreceptor-Specific Gene Mutation Perturbs Cone Pathway Development. Neuron 1999, 23, 549-557. [CrossRef]

29. Shen, J.; Yang, X.; Dong, A.; Petters, R.M.; Peng, Y.-W.; Wong, F.; Campochiaro, P.A. Oxidative damage is a potential cause of cone cell death in retinitis pigmentosa. J. Cell. Physiol. 2005, 203, 457-464. [CrossRef]

30. Sommer, J.R.; Wong, F.; Petters, R.M. Phenotypic stability of Pro347Leu rhodopsin transgenic pigs as indicated by photoreceptor cell degeneration. Transgenic Res. 2011, 20, 1391-1395. [CrossRef]

31. Wang, W.; Lee, S.J.; Scott, P.A.; Lu, X.; Emery, D.; Liu, Y.; Ezashi, T.; Roberts, M.R.; Ross, J.W.; Kaplan, H.J.; et al. Two-Step Reactivation of Dormant Cones in Retinitis Pigmentosa. Cell Rep. 2016, 15, 372-385. [CrossRef] [PubMed]

32. Daiger, S.P.; Bowne, S.J.; Sullivan, L.S. Perspective on Genes and Mutations Causing Retinitis Pigmentosa. Arch. Ophthalmol. 2007, 125, 151-158. [CrossRef] [PubMed]

33. Baehr, W.; Devlin, M.J.; Applebury, M.L. Isolation and characterization of cGMP phosphodiesterase from bovine rod outer segments. J. Boil. Chem. 1979, 254, 11669-11677.

34. Petersen-Jones, S.M.; Entz, D.D.; Sargan, D.R. cGMP phosphodiesterase- $\alpha$ mutation causes progressive retinal atrophy in the Cardigan Welsh corgi dog. Investig. Ophthalmol. Vis. Sci. 1999, 40, 1637-1644. 
35. Tuntivanich, N.; Pittler, S.J.; Fischer, A.J.; Omar, G.; Kiupel, M.; Weber, A.; Yao, S.; Steibel, J.P.; Khan, N.W.; Petersen-Jones, S.M. Characterization of a canine model of autosomal recessive retinitis pigmentosa due to a PDE6A mutation. Investig. Opthalmol. Vis. Sci. 2008, 50, 801-813. [CrossRef] [PubMed]

36. Dryja, T.P.; Finn, J.T.; Peng, Y.W.; McGee, T.L.; Berson, E.L.; Yau, K.W. Mutations in the gene encoding the alpha subunit of the rod cGMP-gated channel in autosomal recessive retinitis pigmentosa. Proc. Natl. Acad. Sci. USA 1995, 92, 10177-10181. [CrossRef]

37. Huang, S.H.; Pittler, S.J.; Huang, X.; Oliveira, L.; Berson, E.L.; Dryja, T.P. Autosomal recessive retinitis pigmentosa caused by mutations in the $\alpha$ subunit of rod cGMP phosphodiesterase. Nat. Genet. 1995, 11, 468-471. [CrossRef]

38. Suber, M.L.; Pittler, S.J.; Qin, N.; Wright, G.C.; Holcombe, V.; Lee, R.H.; Craft, C.M.; Lolley, R.N.; Baehr, W.; Hurwitz, R.L. Irish setter dogs affected with rod/cone dysplasia contain a nonsense mutation in the rod cGMP phosphodiesterase beta-subunit gene. Proc. Natl. Acad. Sci. USA 1993, 90, 3968-3972. [CrossRef]

39. Hartong, D.T.; Berson, E.L.; Dryja, T.P. Retinitis pigmentosa. Lancet 2006, 368, 1795-1809. [CrossRef]

40. McLaughlin, M.E.; Ehrhart, T.L.; Berson, E.L.; Dryja, T.P. Mutation spectrum of the gene encoding the beta subunit of rod phosphodiesterase among patients with autosomal recessive retinitis pigmentosa. Proc. Natl. Acad. Sci. USA 1995, 92, 3249-3253. [CrossRef]

41. Occelli, L.M.; Schön, C.; Seeliger, M.W.; Biel, M.; Michalakis, S.; Petersen-Jones, S.M.; The RD-Cure Consortium. Gene Supplementation Rescues Rod Function and Preserves Photoreceptor and Retinal Morphology in Dogs, Leading the Way Toward Treating Human PDE6A-Retinitis Pigmentosa. Hum. Gene Ther. 2017, 28, 1189-1201. [CrossRef] [PubMed]

42. Arango-Gonzalez, B.; Trifunović, D.; Sahaboglu, A.; Kranz, K.; Michalakis, S.; Farinelli, P.; Koch, S.; Koch, F.; Cottet, S.; Janssen-Bienhold, U.; et al. Identification of a Common Non-Apoptotic Cell Death Mechanism in Hereditary Retinal Degeneration. PLoS ONE 2014, 9, e112142. [CrossRef] [PubMed]

43. Wensel, T.G.; Zhang, Z.; Anastassov, I.; Gilliam, J.C.; He, F.; Schmid, M.F.; Robichaux, M.A. Structural and molecular bases of rod photoreceptor morphogenesis and disease. Prog. Retin. Eye Res. 2016, 55, 32-51. [CrossRef] [PubMed]

44. Mowat, F.M.; Occelli, L.M.; Bartoe, J.T.; Gervais, K.J.; Bruewer, A.R.; Querubin, J.; Dinculescu, A.; Boye, S.L.; Hauswirth, W.; Petersen-Jones, S.M. Gene Therapy in a Large Animal Model of PDE6A-Retinitis Pigmentosa. Front. Mol. Neurosci. 2017, 11, 342. [CrossRef]

45. Clements, P.J.M.; Gregory, C.Y.; Petersen-Jones, S.M.; Sargan, D.R.; Bhattacharya, S.S. Confirmation of the rod cGMP phophodiesterase á-subunit (PDEá) nonsense mutation in affected rcd-1 Irish setters in the UK and development of a diagnostic test. Curr. Eye Res. 1993, 12, 861-866. [CrossRef]

46. Farber, D.B.; Danciger, J.S.; Aguirre, G. The beta subunit of cyclic GMP phosphodiesterase mRNA is deficient in canine rod-cone dysplasia 1. Neuron 1992, 9, 349-356. [CrossRef]

47. Aguirre, G.; Farber, D.; Lolley, R.; O’Brien, P.; Alligood, J.; Fletcher, R.T.; Chader, G. Retinal degenerations in the dog III abnormal cyclic nucleotide metabolism in rod-cone dysplasia. Exp. Eye Res. 1982, 35, 625-642. [CrossRef]

48. Aguirre, G.D.; Rubin, L.F. Rod-cone dysplasia (progressive retinal atrophy) in Irish setters. J. Am. Veter Med. Assoc. 1975, 166, 157-164.

49. Aquirre, G.; Farber, D.; Lolley, R.; Fletcher, R.; Chader, G. Rod-cone dysplasia in Irish setters: A defect in cyclic GMP metabolism in visual cells. Science 1978, 201, 1133-1134. [CrossRef]

50. Pichard, V.; Provost, N.; Mendes-Madeira, A.; Libeau, L.; Hulin, P.; Tshilenge, K.-T.; Biget, M.; Ameline, B.; Deschamps, J.-Y.; Weber, M.; et al. AAV-mediated Gene Therapy Halts Retinal Degeneration in PDE6ß-deficient Dogs. Mol. Ther. 2016, 24, 867-876. [CrossRef]

51. Dekomien, G.; Runte, M.; Gödde, R.; Epplen, J.T. Generalized progressive retinal atrophy of Sloughi dogs is due to an 8-bp insertion in exon 21 of the PDE6B gene. Cytogenet. Cell Genet. 2000, 90, 261-267. [CrossRef] [PubMed]

52. Goldstein, O.; Mezey, J.G.; Schweitzer, P.A.; Boyko, A.R.; Gao, C.; Bustamante, C.D.; Jordan, J.A.; Aguirre, G.D.; Acland, G.M. IQCB1 and PDE6B Mutations Cause Similar Early Onset Retinal Degenerations in Two Closely Related Terrier Dog Breeds. Investig. Opthalmol. Vis. Sci. 2013, 54, 7005-7019. [CrossRef] [PubMed]

53. Quazi, F.; Lenevich, S.; Molday, R.S. ABCA4 is an N-retinylidene-phosphatidylethanolamine and phosphatidylethanolamine importer. Nat. Commun. 2012, 3, 925. [CrossRef] [PubMed] 
54. Mäkeläinen, S.; Gòdia, M.; Hellsand, M.; Viluma, A.; Hahn, D.; Makdoumi, K.; Zeiss, C.J.; Mellersh, C.; Ricketts, S.L.; Narfström, K.; et al. An ABCA4 loss-of-function mutation causes a canine form of Stargardt disease. PLoS Genet. 2019, 15, e1007873. [CrossRef] [PubMed]

55. Maeda, A.; Maeda, T.; Golczak, M.; Palczewski, K. Retinopathy in mice induced by disrupted all-trans-retinal clearance. J. Boil. Chem. 2008, 283, 26684-26693. [CrossRef]

56. Tsang, S.H.; Sharma, T. Stargardt Disease. In Atlas of Inherited Retinal Diseases; Tsang, S.H., Sharma, T., Eds.; Springer International Publishing: Cham, Switzerland, 2018; pp. 139-151. [CrossRef]

57. Le Meur, G.; Stieger, K.; Smith, A.J.; Weber, M.; Deschamps, J.Y.; Nivard, D.; Mendes-Madeira, A.; Provost, N.; Péréon, Y.; Cherel, Y.; et al. Restoration of vision in RPE65-deficient Briard dogs using an AAV serotype 4 vector that specifically targets the retinal pigmented epithelium. Gene Ther. 2006, 14, 292-303. [CrossRef]

58. Narfström, K.; Katz, M.L.; Bragadottir, R.; Seeliger, M.; Boulanger, A.; Redmond, T.M.; Caro, L.; Lai, C.-M.; Rakoczy, P.E. Functional and structural recovery of the retina after gene therapy in the RPE65 null mutation dog. Investig. Opthalmol. Vis. Sci. 2003, 44, 1663-1672. [CrossRef]

59. Annear, M.J.; Bartoe, J.T.; Barker, S.E.; Smith, A.J.; Curran, P.G.; Bainbridge, J.W.; Ali, R.R.; Petersen-Jones, S.M. Gene therapy in the second eye of RPE65-deficient dogs improves retinal function. Gene Ther. 2010, 18, 53-61. [CrossRef]

60. Annear, M.J.; Mowat, F.M.; Bartoe, J.T.; Querubin, J.; Azam, S.A.; Basche, M.; Curran, P.G.; Smith, A.; Bainbridge, J.W.; Ali, R.R.; et al. Successful Gene Therapy in Older Rpe65-Deficient Dogs Following Subretinal Injection of an Adeno-Associated Vector Expressing RPE65. Hum. Gene Ther. 2013, 24, 883-893. [CrossRef]

61. Mowat, F.M.; Breuwer, A.R.; Bartoe, J.T.; Annear, M.J.; Zhang, Z.; Smith, A.J.; Bainbridge, J.W.; Petersen-Jones, S.M.; Ali, R.R. RPE65 gene therapy slows cone loss in Rpe65-deficient dogs. Gene Ther. 2012, 20, 545-555. [CrossRef]

62. Mowat, F.M.; Gervais, K.J.; Occelli, L.M.; Annear, M.J.; Querubin, J.; Bainbridge, J.W.; Smith, A.J.; Ali, R.R.; Petersen-Jones, S.M. Early-Onset Progressive Degeneration of the Area Centralis in RPE65-Deficient Dogs. Investig. Opthalmol. Vis. Sci. 2017, 58, 3268. [CrossRef] [PubMed]

63. Bainbridge, J.W.; Mehat, M.S.; Sundaram, V.; Robbie, S.J.; Barker, S.E.; Ripamonti, C.; Georgiadis, A.; Mowat, F.M.; Beattie, S.G.; Gardner, P.; et al. Long-Term Effect of Gene Therapy on Leber's Congenital Amaurosis. N. Engl. J. Med. 2015, 372, 1887-1897. [CrossRef] [PubMed]

64. Petersen-Jones, S.M.; Occelli, L.; Winkler, P.; Minella, A.; Sun, K.; Lyons, L.; Daruwalla, A.; Kiser, P.; Palczewski, K. New large animal model for RDH5-associated retinopathies. Investig. Ophthalmol. Vis. Sci. 2019, 60, 458 .

65. Gonzalez-Fernandez, F.; Kurz, D.; Bao, Y.; Newman, S.; Conway, B.P.; Young, J.E.; Han, D.P.; Khani, S.C. 11-cis retinol dehydrogenase mutations as a major cause of the congenital night-blindness disorder known as fundus albipunctatus. Mol. Vis. 1999, 5, 41. [PubMed]

66. Hotta, K.; Nakamura, M.; Kondo, M.; Ito, S.; Terasaki, H.; Miyake, Y.; Hida, T. Macular dystrophy in a Japanese family with fundus albipunctatus. Am. J. Ophthalmol. 2003, 135, 917-919. [CrossRef]

67. Nakamura, M.; Miyake, Y. Macular dystrophy in a 9-year-old boy with fundus albipunctatus. Am. J. Ophthalmol. 2002, 133, 278-280. [CrossRef]

68. Nakamura, M.; Skalet, J.; Miyake, Y. RDH5 gene mutations and electroretinogram in fundus albipunctatus with or without macular dystrophy: RDH5 mutations and ERG in fundus albipunctatus. Doc. Ophthalmol. 2003, 107, 3-11. [CrossRef]

69. Yamamoto, H.; Yakushijin, K.; Kusuhara, S.; Escaño, M.F.T.; Nagai, A.; Negi, A. A novel RDH5 gene mutation in a patient with fundus albipunctatus presenting with macular atrophy and fading white dots. Am. J. Ophthalmol. 2003, 136, 572-574. [CrossRef]

70. Kuehlewein, L.; Nasser, F.; Gloeckle, N.; Kohl, S.; Zrenner, E. Fundus albipunctatus associated with cone dysfunction. Retin. Cases Brief Rep. 2017, 11, S73-S76. [CrossRef]

71. Kim, T.S.; Maeda, A.; Maeda, T.; Heinlein, C.; Kedishvili, N.; Palczewski, K.; Nelson, P.S. Delayed dark adaptation in 11-cis-retinol dehydrogenase-deficient mice: A role of RDH11 in visual processes in vivo. J. Boil. Chem. 2005, 280, 8694-8704. [CrossRef]

72. Kaupp, U.B.; Seifert, R. Cyclic Nucleotide-Gated Ion Channels. Physiol. Rev. 2002, 82, 769-824. [CrossRef] [PubMed] 
73. Zheng, J.; Trudeau, M.C.; Zagotta, W.N. Rod cyclic nucleotide-gated channels have a stoichiometry of three CNGA1 subunits and one CNGB1 subunit. Neuron 2002, 36, 891-896. [CrossRef]

74. Zhong, H.; Molday, L.L.; Molday, R.S.; Yau, K.-W. The heteromeric cyclic nucleotide-gated channel adopts a 3A:1B stoichiometry. Nature 2002, 420, 193-198. [CrossRef] [PubMed]

75. Johnson, A.A.; Guziewicz, K.E.; Lee, C.J.; Kalathur, R.C.; Pulido, J.S.; Marmorstein, L.Y.; Marmorstein, A.D. Bestrophin 1 and retinal disease. Prog. Retin. Eye Res. 2017, 58, 45-69. [CrossRef] [PubMed]

76. Wiik, A.C.; Ropstad, E.O.; Ekesten, B.; Karlstam, L.; Wade, C.; Lingaas, F. Progressive retinal atrophy in Shetland sheepdog is associated with a mutation in theCNGA1gene. Anim. Genet. 2015, 46, 515-521. [CrossRef] [PubMed]

77. Winkler, P.A.; Ekenstedt, K.J.; Occelli, L.M.; Frattaroli, A.V.; Bartoe, J.T.; Venta, P.J.; Petersen-Jones, S.M. A Large Animal Model for CNGB1 Autosomal Recessive Retinitis Pigmentosa. PLoS ONE 2013, 8, 7222. [CrossRef]

78. Petersen-Jones, S.M.; Occelli, L.M.; Winkler, P.A.; Lee, W.; Sparrow, J.R.; Tsukikawa, M.; Boye, S.L.; Chiodo, V.; Capasso, J.E.; Becirovic, E.; et al. Patients and animal models of CNG $\beta 1$-deficient retinitis pigmentosa support gene augmentation approach. J. Clin. Investig. 2017, 128, 190-206. [CrossRef]

79. Hüttl, S.; Michalakis, S.; Seeliger, M.; Luo, N.-G.; Acar, N.; Geiger, H.; Hudl, K.; Mader, R.; Haverkamp, S.; Moser, M.; et al. Impaired channel targeting and retinal degeneration in mice lacking the cyclic nucleotide-gated channel subunit CNGB1. J. Neurosci. 2005, 25, 130-138. [CrossRef]

80. Hull, S.; Attanasio, M.; Arno, G.; Carss, K.; Robson, A.; Thompson, D.; Plagnol, V.; Michaelides, M.; Holder, G.E.; Henderson, R.H.; et al. Clinical Characterization of CNGB1-Related Autosomal Recessive Retinitis Pigmentosa. JAMA Ophthalmol. 2017, 135, 137-144. [CrossRef]

81. Wissinger, B.; Gamer, D.; Jägle, H.; Giorda, R.; Marx, T.; Mayer, S.; Tippmann, S.; Broghammer, M.; Jurklies, B.; Rosenberg, T.; et al. CNGA3 Mutations in Hereditary Cone Photoreceptor Disorders. Am. J. Hum. Genet. 2001, 69, 722-737. [CrossRef]

82. Tanaka, N.; Dutrow, E.; Miyadera, K.; Delemotte, L.; MacDermaid, C.; Reinstein, S.L.; Crumley, W.R.; Dixon, C.J.; Casal, M.L.; Klein, M.L.; et al. Canine CNGA3 Gene Mutations Provide Novel Insights into Human Achromatopsia-Associated Channelopathies and Treatment. PLoS ONE 2015, 10, e0138943. [CrossRef] [PubMed]

83. Reicher, S.; Seroussi, E.; Gootwine, E. A mutation in gene CNGA3 is associated with day blindness in sheep. Genomics 2010, 95, 101-104. [CrossRef] [PubMed]

84. Gootwine, E.; Abu-Siam, M.; Obolensky, A.; Rosov, A.; Honig, H.; Nitzan, T.; Shirak, A.; Ezra-Elia, R.; Yamin, E.; Banin, E.; et al. Gene Augmentation Therapy for a Missense Substitution in the cGMP-Binding Domain of Ovine CNGA3 Gene Restores Vision in Day-Blind Sheep. Investig. Opthalmol. Vis. Sci. 2017, 58, 1577-1584. [CrossRef] [PubMed]

85. Gootwine, E.; Ofri, R.; Banin, E.; Obolensky, A.; Averbukh, E.; Ezra-Elia, R.; Ross, M.; Honig, H.; Rosov, A.; Yamin, E.; et al. Safety and Efficacy Evaluation of rAAV2tYF-PR1.7-hCNGA3 Vector Delivered by Subretinal Injection in CNGA3 Mutant Achromatopsia Sheep. Hum. Gene Ther. Clin. Dev. 2017, 28, 96-107. [CrossRef] [PubMed]

86. Sidjanin, D.J.; Lowe, J.K.; McElwee, J.; Milne, B.S.; Phippen, T.M.; Sargan, D.R.; Aguirre, G.D.; Acland, G.M.; Ostrander, E.A. Canine CNGB3 mutations establish cone degeneration as orthologous to the human achromatopsia locus ACHM3. Hum. Mol. Genet. 2002, 11, 1823-1833. [CrossRef]

87. Yeh, C.Y.; Goldstein, O.; Kukekova, A.; Holley, D.; Knollinger, A.M.; Huson, H.J.; Pearce-Kelling, S.E.; Acland, G.M.; Komáromy, A.M. Genomic deletion of CNGB3 is identical by descent in multiple canine breeds and causes achromatopsia. BMC Genet. 2013, 14, 27. [CrossRef]

88. Kohl, S.; Varsányi, B.; Antunes, G.A.; Baumann, B.; Hoyng, C.B.; Jägle, H.; Rosenberg, T.; Kellner, U.; Lorenz, B.; Salati, R.; et al. CNGB3 mutations account for $50 \%$ of all cases with autosomal recessive achromatopsia. Eur. J. Hum. Genet. 2004, 13, 302-308. [CrossRef]

89. Tanaka, N.; Delemotte, L.; Klein, M.L.; Komáromy, A.M.; Tanaka, J.C. A Cyclic Nucleotide-Gated Channel Mutation Associated with Canine Daylight Blindness Provides Insight into a Role for the S2 Segment Tri-Asp motif in Channel Biogenesis. PLoS ONE 2014, 9, e88768. [CrossRef]

90. Ye, G.-J.; Komáromy, A.M.; Zeiss, C.; Calcedo, R.; Harman, C.D.; Koehl, K.L.; Stewart, G.A.; Iwabe, S.; Chiodo, V.A.; Hauswirth, W.; et al. Safety and Efficacy of AAV5 Vectors Expressing Human or Canine CNGB3 in CNGB3-Mutant Dogs. Hum. Gene Ther. Clin. Dev. 2017, 28, 197-207. [CrossRef] 
91. Guziewicz, K.E.; Zangerl, B.; Lindauer, S.J.; Mullins, R.F.; Sandmeyer, L.S.; Grahn, B.H.; Stone, E.M.; Acland, G.M.; Aguirre, G.D. Bestrophin gene mutations cause canine multifocal retinopathy: A novel animal model for best disease. Investig. Opthalmol. Vis. Sci. 2007, 48, 1959-1967. [CrossRef]

92. Zangerl, B.; Wickström, K.; Slavik, J.; Lindauer, S.J.; Ahonen, S.; Schelling, C.; Lohi, H.; Guziewicz, K.E.; Aguirre, G.D. Assessment of canine BEST1 variations identifies new mutations and establishes an independent bestrophinopathy model (cmr3). Mol. Vis. 2010, 16, 2791-2804. [PubMed]

93. Guziewicz, K.E.; Cideciyan, A.V.; Beltran, W.A.; Komaromy, A.M.; Dufour, V.L.; Swider, M.; Iwabe, S.; Sumaroka, A.; Kendrick, B.T.; Ruthel, G.; et al. BEST1 gene therapy corrects a diffuse retina-wide microdetachment modulated by light exposure. Proc. Natl. Acad. Sci. USA 2018, 115, E2839-E2848. [CrossRef] [PubMed]

94. Guziewicz, K.E.; McTish, E.; Dufour, V.L.; Zorych, K.; Dhingra, A.; Boesze-Battaglia, K.; Aguirre, G.D. Underdeveloped RPE Apical Domain Underlies Lesion Formation in Canine Bestrophinopathies. Single Mol. Single Cell Seq. 2018, 1074, 309-315.

95. Chew, T.; Haase, B.; Bathgate, R.; Willet, C.; Kaukonen, M.K.; Mascord, L.J.; Lohi, H.; Wade, C. A Coding Variant in the Gene Bardet-Biedl Syndrome 4 (BBS4) Is Associated with a Novel Form of Canine Progressive Retinal Atrophy. G3 Genes Genomes Genet. 2017, 7, 2327-2335. [CrossRef] [PubMed]

96. Narfström, K. Progressive retinal atrophy in the Abyssinian cat. Clinical characteristics. Investig. Ophthalmol. Vis. Sci. 1985, 26, 193-200.

97. Minella, A.L.; Occelli, L.M.; Narfström, K.; Petersen-Jones, S.M. Central retinal preservation in rdAc cats. Veter Ophthalmol. 2017, 21, 224-232. [CrossRef]

98. Coppieters, F.; Lefever, S.; Leroy, B.P.; De Baere, E. CEP290, a gene with many faces: Mutation overview and presentation of CEP290base. Hum. Mutat. 2010,31, 1097-1108. [CrossRef]

99. Menotti-Raymond, M.; David, V.A.; Schäffer, A.A.; Stephens, R.; Wells, D.; Kumar-Singh, R.; O’Brien, S.; Narfström, K. Mutation in CEP290 Discovered for Cat Model of Human Retinal Degeneration. J. Hered. 2007, 98, 211-220. [CrossRef]

100. Wiik, A.C.; Wade, C.; Biagi, T.; Ropstad, E.-O.; Bjerkås, E.; Lindblad-Toh, K.; Lingaas, F. A deletion in nephronophthisis 4 (NPHP4) is associated with recessive cone-rod dystrophy in standard wire-haired dachshund. Genome Res. 2008, 18, 1415-1421. [CrossRef]

101. Ropstad, E.O.; Narfström, K.; Lingaas, F.; Wiik, C.; Bruun, A.; Bjerkas, E. Functional and Structural Changes in the Retina of Wire-Haired Dachshunds with Early-Onset Cone-Rod Dystrophy. Investig. Opthalmol. Vis. Sci. 2008, 49, 1106-1115. [CrossRef]

102. Ropstad, E.O.; Bjerkås, E.; Narfström, K. Electroretinographic findings in the Standard Wire Haired Dachshund with inherited early onset cone-rod dystrophy. Doc. Ophthalmol. 2006, 114, 27-36. [CrossRef] [PubMed]

103. Ropstad, E.O.; Bjerkås, E.; Narfström, K. Clinical findings in early onset cone-rod dystrophy in the Standard Wire-haired Dachshund. Veter Ophthalmol. 2007, 10, 69-75. [CrossRef] [PubMed]

104. Ronquillo, C.; Bernstein, P.S.; Baehr, W. Senior-Løken syndrome: A syndromic form of retinal dystrophy associated with nephronophthisis. Vis. Res. 2012, 75, 88-97. [CrossRef] [PubMed]

105. Won, J.; De Evsikova, C.M.; Smith, R.S.; Hicks, W.L.; Edwards, M.M.; Longo-Guess, C.; Li, T.; Naggert, J.K.; Nishina, P.M. NPHP4 is necessary for normal photoreceptor ribbon synapse maintenance and outer segment formation, and for sperm development. Hum. Mol. Genet. 2010, 20, 482-496. [CrossRef]

106. Downs, L.M.; Scott, E.M.; Cideciyan, A.V.; Iwabe, S.; Dufour, V.L.; Gardiner, K.L.; Genini, S.; Marinho, L.F.; Sumaroka, A.; Kosyk, M.S.; et al. Overlap of abnormal photoreceptor development and progressive degeneration in Leber congenital amaurosis caused by NPHP5 mutation. Hum. Mol. Genet. 2016, 25, 4211-4226. [CrossRef]

107. Aguirre, G.D.; Cideciyan, A.V.; Boye, S.L.; Iwabe, S.; Dufour, V.; Swider, M.; Roszak, K.; Hauswirth, W.W.; Jacobson, S.G.; Beltran, W.A. Long-term preservation of photoreceptor function and structure following early-stage treatment by AAV-mediated gene augmentation in canine model of NPHP5 Leber congenital amaurosis. Investig. Ophthalmol. Vis. Sci. 2018, 59, 6006.

108. Khanna, H. More Than Meets the Eye: Current Understanding of RPGR Function. In Advances in Experimental Medicine and Biology; Springer: Cham, Switzerland, 2018; pp. 521-538. 
109. Vervoort, R.; Lennon, A.; Bird, A.C.; Tulloch, B.; Axton, R.; Miano, M.G.; Meindl, A.; Meitinger, T.; Ciccodicola, A.; Wright, A.F. Mutational hot spot within a new RPGR exon in X-linked retinitis pigmentosa. Nat. Genet. 2000, 25, 462-466. [CrossRef]

110. Kropatsch, R.; Akkad, D.A.; Frank, M.; Rosenhagen, C.; Altmüller, J.; Nürnberg, P.; Epplen, J.T.; Dekomien, G. A large deletion in RPGR causes XLPRA in Weimaraner dogs. Canine Genet. Epidemiol. 2016, 3, 7. [CrossRef]

111. Zhang, Q.; Acland, G.M.; Wu, W.X.; Johnson, J.; Pearce-Kelling, S.; Tulloch, B.; Vervoort, R.; Wright, A.F.; Aguirre, G.D. Different RPGR exon ORF15 mutations in Canids provide insights into photoreceptor cell degeneration. Hum. Mol. Genet. 2002, 11, 993-1003. [CrossRef]

112. Zeiss, C.J.; Acland, G.M.; Aguirre, G.D. Retinal pathology of canine X-linked progressive retinal atrophy, the locus homologue of RP3. Investig. Ophthalmol. Vis. Sci. 1999, 40, 3292-3304.

113. Beltran, W.A.; Hammond, P.; Acland, G.M.; Aguirre, G.D. A Frameshift Mutation in RPGR Exon ORF15 Causes Photoreceptor Degeneration and Inner Retina Remodeling in a Model of X-Linked Retinitis Pigmentosa. Investig. Opthalmol. Vis. Sci. 2006, 47, 1669-1681. [CrossRef] [PubMed]

114. Beltran, W.A.; Acland, G.M.; Aguirre, G.D. Age-dependent disease expression determines remodeling of the retinal mosaic in carriers of RPGR exon ORF15 mutations. Investig. Opthalmol. Vis. Sci. 2009, 50, 3985-3995. [CrossRef] [PubMed]

115. Mellersh, C.; Boursnell, M.; Pettitt, L.; Ryder, E.; Holmes, N.; Grafham, D.; Forman, O.; Sampson, J.; Barnett, K.; Blanton, S.; et al. Canine RPGRIP1 mutation establishes cone-rod dystrophy in miniature longhaired dachshunds as a homologue of human Leber congenital amaurosis. Genomics 2006, 88, $293-301$. [CrossRef] [PubMed]

116. Lhériteau, E.; Petit, L.; Weber, M.; Le Meur, G.; Deschamps, J.-Y.; Libeau, L.; Mendes-Madeira, A.; Guihal, C.; François, A.; Guyon, R.; et al. Successful Gene Therapy in the RPGRIP1-deficient Dog: A Large Model of Cone-Rod Dystrophy. Mol. Ther. 2013, 22, 265-277. [CrossRef] [PubMed]

117. Kuznetsova, T.; Iwabe, S.; Boesze-Battaglia, K.; Pearce-Kelling, S.; Chang-Min, Y.; McDaid, K.; Miyadera, K.; Komaromy, A.; Aguirre, G.D. Exclusion of RPGRIP1 ins44 from Primary Causal Association with Early-Onset Cone-Rod Dystrophy in Dogs. Investig. Opthalmol. Vis. Sci. 2012, 53, 5486-5501. [CrossRef]

118. Forman, O.P.; Hitti, R.J.; Boursnell, M.; Miyadera, K.; Sargan, D.R.; Mellersh, C. Canine genome assembly correction facilitates identification of a MAP9 deletion as a potential age of onset modifier for RPGRIP1-associated canine retinal degeneration. Mamm. Genome 2016, 27, 237-245. [CrossRef]

119. Miyadera, K.; Kato, K.; Boursnell, M.; Mellersh, C.; Sargan, D.R. Genome-wide association study in RPGRIP1 $^{-/-}$dogs identifies a modifier locus that determines the onset of retinal degeneration. Mamm. Genome 2011, 23, 212-223. [CrossRef]

120. Miyadera, K.; Murgiano, L.; Spector, C.; Marinho, F.P.; Dufour, V.; Das, R.G.; Brooks, M.; Swaroop, A.; Aguirre, G.D. Isolated population helps tease out a third locus underlying a multigenic form of canine RPGRIP1 cone-rod dystrophy. Investig. Ophthalmol. Vis. Sci. 2018, 59, 1438.

121. Chau, K.-Y.; Chen, S.; Zack, D.J.; Ono, S.J. Functional Domains of the Cone-Rod Homeobox (CRX) Transcription Factor. J. Boil. Chem. 2000, 275, 37264-37270. [CrossRef]

122. Morrow, E.M.; Furukawa, T.; Raviola, E.; Cepko, C.L. Synaptogenesis and outer segment formation are perturbed in the neural retina of Crx mutant mice. BMC Neurosci. 2005, 6, 5. [CrossRef]

123. Chen, S.; Wang, Q.-L.; Nie, Z.; Sun, H.; Lennon, G.; Copeland, N.; Gilbert, D.J.; Jenkins, N.A.; Zack, D.J. Crx, a novel Otx-like paired-homeodomain protein, binds to and transactivates photoreceptor cell-specific genes. Neuron 1997, 19, 1017-1030. [CrossRef]

124. Furukawa, T.; Morrow, E.M.; Cepko, C.L. Crx, a novel otx-like homeobox gene, shows photoreceptor-specific expression and regulates photoreceptor differentiation. Cell 1997, 91, 531-541. [CrossRef]

125. Hennig, A.K.; Peng, G.-H.; Chen, S. Regulation of photoreceptor gene expression by Crx-associated transcription factor network. Brain Res. 2007, 1192, 114-133. [CrossRef] [PubMed]

126. Peng, G.-H.; Chen, S. Crx activates opsin transcription by recruiting HAT-containing co-activators and promoting histone acetylation. Hum. Mol. Genet. 2007, 16, 2433-2452. [CrossRef] [PubMed]

127. Menotti-Raymond, M.; Deckman, K.H.; David, V.; Myrkalo, J.; O’Brien, S.J.; Narfström, K. Mutation discovered in a feline model of human congenital retinal blinding disease. Investig. Opthalmol. Vis. Sci. 2010, 51, 2852-2859. [CrossRef] [PubMed] 
128. Occelli, L.M.; Tran, N.M.; Narfström, K.; Chen, S.; Petersen-Jones, S.M. CrxRdy Cat: A Large Animal Model for CRX-Associated Leber Congenital Amaurosis. Investig. Opthalmol. Vis. Sci. 2016, 57, 3780-3792. [CrossRef]

129. Sohocki, M.M.; Sullivan, L.S.; Mintz-Hittner, H.A.; Birch, D.G.; Heckenlively, J.R.; Freund, C.L.; McInnes, R.R.; Daiger, S.P. A range of clinical phenotypes associated with mutations in CRX, a photoreceptor transcription-factor gene. Am. J. Hum. Genet. 1998, 63, 1307-1315. [CrossRef]

130. Hull, S.; Arno, G.; Plagnol, V.; Chamney, S.; Russell-Eggitt, I.; Thompson, D.; Ramsden, S.C.; Black, G.C.; Robson, A.; Holder, G.E.; et al. The Phenotypic Variability of Retinal Dystrophies Associated With Mutations in CRX, With Report of a Novel Macular Dystrophy Phenotype. Investig. Opthalmol. Vis. Sci. 2014, 55, 6934-6944. [CrossRef]

131. Huang, L.; Xiao, X.; Li, S.; Jia, X.; Wang, P.; Guo, X.; Zhang, Q. CRX variants in cone-rod dystrophy and mutation overview. Biochem. Biophys. Res. Commun. 2012, 426, 498-503. [CrossRef]

132. Hanein, S.; Perrault, I.; Gerber, S.; Tanguy, G.; Barbet, F.; Ducroq, D.; Calvas, P.; Dollfus, H.; Hamel, C.; Löppönen, T.; et al. Leber congenital amaurosis: Comprehensive survey of the genetic heterogeneity, refinement of the clinical definition, and genotype-phenotype correlations as a strategy for molecular diagnosis. Hum. Mutat. 2004, 23, 306-317. [CrossRef]

133. Kumaran, N.; Pennesi, M.E.; Yang, P.; Trzupek, K.M.; Schlechter, C.; Moore, A.T.; Weleber, R.G.; Michaelides, M. Leber Congenital Amaurosis/Early-Onset Severe Retinal Dystrophy Overview. In GeneReviews $((R))$; Adam, M.P., Ardinger, H.H., Pagon, R.A., Wallace, S.E., Bean, L.J.H., Stephens, K., Amemiya, A., Eds.; University of Washington: Seattle, WA, USA, 1993.

134. Hollander, A.I.D.; Roepman, R.; Koenekoop, R.K.; Cremers, F.P. Leber congenital amaurosis: Genes, proteins and disease mechanisms. Prog. Retin. Eye Res. 2008, 27, 391-419. [CrossRef] [PubMed]

135. Tran, N.M.; Chen, S. Mechanisms of blindness: Animal models provide insight into distinct CRX-associated retinopathies. Dev. Dyn. 2014, 243, 1153-1166. [CrossRef]

136. Acland, G.M.; Aguirre, G.D. Retinal degenerations in the dog: IV. Early retinal degeneration (erd) in the Norwegian elkhound. Exp. Eye Res. 1987, 44, 491-521. [CrossRef]

137. Goldstein, O.; Kukekova, A.V.; Aguirre, G.D.; Acland, G.M. Exonic SINE insertion in STK38L causes canine early retinal degeneration (erd). Genomics 2010, 96, 362-368. [CrossRef] [PubMed]

138. Berta, Á.I.; Boesze-Battaglia, K.; Genini, S.; Goldstein, O.; O’Brien, P.J.; Szél, Á.; Acland, G.M.; Beltran, W.A.; Aguirre, G.D. Photoreceptor Cell Death, Proliferation and Formation of Hybrid Rod/S-Cone Photoreceptors in the Degenerating STK38L Mutant Retina. PLoS ONE 2011, 6, e24074. [CrossRef] [PubMed]

139. Zeitz, C.; Jacobson, S.G.; Hamel, C.P.; Bujakowska, K.M.; Neuillé, M.; Orhan, E.; Zanlonghi, X.; Lancelot, M.-E.; Michiels, C.; Schwartz, S.B.; et al. Whole-Exome Sequencing Identifies LRIT3 Mutations as a Cause of Autosomal-Recessive Complete Congenital Stationary Night Blindness. Am. J. Hum. Genet. 2013, 92, 67-75. [CrossRef]

140. Kondo, M.; Das, R.; Imai, R.; Santana, E.; Nakashita, T.; Imawaka, M.; Ueda, K.; Ohtsuka, H.; Sakai, K.; Aihara, T.; et al. A Naturally Occurring Canine Model of Autosomal Recessive Congenital Stationary Night Blindness. PLoS ONE 2015, 10, e0137072. [CrossRef]

141. Das, R.; Becker, D.; Jagannathan, V.; Goldstein, O.; Santana, E.; Carlin, K.; Sudharsan, R.; Leeb, T.; Nishizawa, Y.; Kondo, M.; et al. Genome-wide association study and whole-genome sequencing identify a deletion in LRIT3 associated with canine congenital stationary night blindness. Sci. Rep. 2019, 9, 14166. [CrossRef]

142. Oh, A.; Loew, E.R.; Foster, M.L.; Davidson, M.G.; English, R.V.; Gervais, K.J.; Herring, I.; Mowat, F.M. Phenotypic characterization of complete CSNB in the inbred research beagle: How common is CSNB in research and companion dogs? Doc. Ophthalmol. 2018, 137, 87-101. [CrossRef]

143. Hasan, N.; Pangeni, G.; Cobb, C.A.; Ray, T.A.; Nettesheim, E.R.; Ertel, K.J.; Lipinski, D.M.; McCall, M.A.; Gregg, R.G. Presynaptic Expression of LRIT3 Transsynaptically Organizes the Postsynaptic Glutamate Signaling Complex Containing TRPM1. Cell Rep. 2019, 27, 3107-3116. [CrossRef]

144. Morgans, C.; Zhang, J.; Jeffrey, B.G.; Nelson, S.M.; Burke, N.S.; Duvoisin, R.M.; Brown, R.L. TRPM1 is required for the depolarizing light response in retinal ON-bipolar cells. Proc. Natl. Acad. Sci. USA 2009, 106, 19174-19178. [CrossRef] [PubMed]

145. Morgans, C.; Brown, R.L.; Duvoisin, R.M. TRPM1: The endpoint of the mGluR6 signal transduction cascade in retinal ON-bipolar cells. BioEssays 2010, 32, 609-614. [CrossRef] [PubMed] 
146. Witzel, D.A.; Smith, E.L.; Wilson, R.D.; Aguirre, G.D. Congenital stationary night blindness: An animal model. Investig. Ophthalmol. Vis. Sci. 1978, 17, 788-795.

147. Sandmeyer, L.S.; Breaux, C.B.; Archer, S.; Grahn, B.H. Clinical and electroretinographic characteristics of congenital stationary night blindness in the Appaloosa and the association with the leopard complex. Veter Ophthalmol. 2007, 10, 368-375. [CrossRef] [PubMed]

148. Bellone, R.; Brooks, S.A.; Sandmeyer, L.; Murphy, B.A.; Forsyth, G.; Archer, S.; Bailey, E.; Grahn, B. Differential Gene Expression of TRPM1, the Potential Cause of Congenital Stationary Night Blindness and Coat Spotting Patterns (LP) in the Appaloosa Horse (Equus caballus). Genetics 2008, 179, 1861-1870. [CrossRef] [PubMed]

149. Audo, I.; Kohl, S.; Leroy, B.P.; Munier, F.L.; Guillonneau, X.; Mohand-Saïd, S.; Bujakowska, K.M.; Nandrot, E.F.; Lorenz, B.; Preising, M.; et al. TRPM1 Is Mutated in Patients with Autosomal-Recessive Complete Congenital Stationary Night Blindness. Am. J. Hum. Genet. 2009, 85, 720-729. [CrossRef]

150. Li, Z.; Sergouniotis, P.I.; Michaelides, M.; Mackay, D.; Wright, G.A.; Devery, S.; Moore, A.T.; Holder, G.E.; Robson, A.; Webster, A.R. Recessive Mutations of the Gene TRPM1 Abrogate ON Bipolar Cell Function and Cause Complete Congenital Stationary Night Blindness in Humans. Am. J. Hum. Genet. 2009, 85, 711-719. [CrossRef]

151. Van Genderen, M.M.; Bijveld, M.M.; Claassen, Y.B.; Florijn, R.J.; Pearring, J.N.; Meire, F.M.; McCall, M.A.; Riemslag, F.C.; Gregg, R.G.; Bergen, A.A.; et al. Mutations in TRPM1 Are a Common Cause of Complete Congenital Stationary Night Blindness. Amer. J. Human Genet. 2009, 85, 730-736. [CrossRef]

152. Bellone, R.; Holl, H.; Setaluri, V.; Devi, S.; Maddodi, N.; Archer, S.; Sandmeyer, L.; Ludwig, A.; Förster, D.; Pruvost, M.; et al. Evidence for a Retroviral Insertion in TRPM1 as the Cause of Congenital Stationary Night Blindness and Leopard Complex Spotting in the Horse. PLoS ONE 2013, 8, e78280. [CrossRef]

153. Littink, K.W.; Van Genderen, M.M.; Collin, R.W.; Roosing, S.; De Brouwer, A.P.M.; Riemslag, F.C.C.; Venselaar, H.; Thiadens, A.A.H.J.; Hoyng, C.; Rohrschneider, K.; et al. A Novel Homozygous Nonsense Mutation inCABP4Causes Congenital Cone-Rod Synaptic Disorder. Investig. Opthalmol. Vis. Sci. 2009, 50, 2344-2350. [CrossRef]

154. Marinho, L.L.P.; Occelli, L.M.; Pasmanter, N.; Somma, A.T.; Montiani-Ferreira, F.; Petersen-Jones, S.M. Autosomal recessive night blindness with progressive photoreceptor degeneration in a dog model. Investig. Ophthalmol. Vis. Sci. 2019, 60, 465.

155. Somma, A.T.; Moreno, J.C.D.; Sato, M.T.; Rodrigues, B.D.; Occelli, L.M.; Bacellar-Galdino, M.; Petersen-Jones, S.M.; Montiani-Ferreira, F. Characterization of a novel form of progressive retinal atrophy in Whippet dogs: A clinical, electroretinographic, and breeding study. Veter Ophthalmol. 2016, 20, 450-459. [CrossRef] [PubMed]

156. Kropatsch, R.; Petrasch-Parwez, E.; Seelow, D.; Schlichting, A.; Gerding, W.M.; Akkad, D.A.; Epplen, J.T.; Dekomien, G. Generalized progressive retinal atrophy in the Irish Glen of Imaal Terrier is associated with a deletion in the ADAM9 gene. Mol. Cell. Probes 2010, 24, 357-363. [CrossRef] [PubMed]

157. Goldstein, O.; Mezey, J.G.; Boyko, A.R.; Gao, C.; Wang, W.; Bustamante, C.D.; Anguish, L.J.; Jordan, J.A.; Pearce-Kelling, S.E.; Aguirre, G.D.; et al. An ADAM9 mutation in canine cone-rod dystrophy 3 establishes homology with human cone-rod dystrophy 9. Mol. Vis. 2010, 16, 1549-1569. [PubMed]

158. Sohocki, M.M.; Perrault, I.; Leroy, B.P.; Payne, A.; Dharmaraj, S.; Bhattacharya, S.S.; Kaplan, J.; Maumenee, I.H.; Koenekoop, R.; Meire, F.M.; et al. Prevalence of AIPL1 Mutations in Inherited Retinal Degenerative Disease. Mol. Genet. Metab. 2000, 70, 142-150. [CrossRef]

159. Van Der Spuy, J.; Kim, J.H.; Yu, Y.S.; Szel, A.; Luthert, P.J.; Clark, B.J.; Cheetham, M.E. The expression of the Leber congenital amaurosis protein AIPL1 coincides with rod and cone photoreceptor development. Investig. Opthalmol. Vis. Sci. 2003, 44, 5396-5403. [CrossRef]

160. Kumaran, N.; Moore, A.T.; Weleber, R.G.; Michaelides, M. Leber congenital amaurosis/early-onset severe retinal dystrophy: Clinical features, molecular genetics and therapeutic interventions. Br. J. Ophthalmol. 2017, 101, 1147-1154. [CrossRef]

161. Sohocki, M.M.; Bowne, S.J.; Sullivan, L.S.; Blackshaw, S.; Cepko, C.L.; Payne, A.M.; Bhattacharya, S.S.; Khaliq, S.; Mehdi, S.Q.; Birch, D.G.; et al. Mutations in a new photoreceptor-pineal gene on 17p cause Leber congenital amaurosis. Nat. Genet. 2000, 24, 79-83. [CrossRef]

162. Gopalakrishna, K.N.; Boyd, K.; Yadav, R.P.; Artemyev, N.O. Aryl Hydrocarbon Receptor-interacting Protein-like 1 Is an Obligate Chaperone of Phosphodiesterase 6 and Is Assisted by the $\gamma$-Subunit of Its Client. J. Boil. Chem. 2016, 291, 16282-16291. [CrossRef] 
163. Hidalgo-De-Quintana, J.; Evans, R.J.; Cheetham, M.E.; Van Der Spuy, J. The Leber congenital amaurosis protein AIPL1 functions as part of a chaperone heterocomplex. Investig. Opthalmol. Vis. Sci. 2008, 49, 2878-2887. [CrossRef]

164. Ramamurthy, V.; Niemi, G.A.; Reh, T.A.; Hurley, J.B. Leber congenital amaurosis linked to AIPL1: A mouse model reveals destabilization of cGMP phosphodiesterase. Proc. Natl. Acad. Sci. USA 2004, 101, 13897-13902. [CrossRef]

165. Yadav, R.P.; Artemyev, N.O. AIPL1: A specialized chaperone for the phototransduction effector. Cell. Signal. 2017, 40, 183-189. [CrossRef]

166. Kolandaivelu, S.; Singh, R.K.; Ramamurthy, V. AIPL1, A protein linked to blindness, is essential for the stability of enzymes mediating cGMP metabolism in cone photoreceptor cells. Hum. Mol. Genet. 2013, 23, 1002-1012. [CrossRef]

167. Rah, H.; Maggs, D.J.; Blankenship, T.N.; Narfström, K.; Lyons, L.A. Early-Onset, Autosomal Recessive, Progressive Retinal Atrophy in Persian Cats. Investig. Opthalmol. Vis. Sci. 2005, 46, 1742-1747. [CrossRef] [PubMed]

168. Lyons, L.A.; Creighton, E.K.; Alhaddad, H.; Beale, H.; Grahn, R.; Rah, H.; Maggs, D.J.; Helps, C.; Gandolfi, B. Whole genome sequencing in cats, identifies new models for blindness in AIPL1 and somite segmentation in HES7. BMC Genom. 2016, 17, 265. [CrossRef] [PubMed]

169. Ahonen, S.; Arumilli, M.; Seppälä, E.; Hakosalo, O.; Kaukonen, M.K.; Komaromy, A.M.; Lohi, H. Increased Expression of MERTK is Associated with a Unique Form of Canine Retinopathy. PLoS ONE 2014, 9, e114552. [CrossRef] [PubMed]

170. Fanning, T.; Singer, M. LINE-1: A mammalian transposable element. Biochim. Biophys. Acta (BBA) Gene Struct. Expr. 1987, 910, 203-212. [CrossRef]

171. Everson, R.; Pettitt, L.; Forman, O.P.; Dower-Tylee, O.; McLaughlin, B.; Ahonen, S.; Kaukonen, M.; Komaromy, A.M.; Lohi, H.; Mellersh, C.S.; et al. An intronic LINE-1 insertion in MERTK is strongly associated with retinopathy in Swedish Vallhund dogs. PLoS ONE 2017, 12, e0183021. [CrossRef] [PubMed]

172. Zangerl, B.; Goldstein, O.; Philp, A.R.; Lindauer, S.J.; Pearce-Kelling, S.E.; Mullins, R.F.; Graphodatsky, A.; Ripoll, D.; Felix, J.S.; Stone, E.M.; et al. Identical mutation in a novel retinal gene causes progressive rod-cone degeneration in dogs and retinitis pigmentosa in humans. Genomics 2006, 88, 551-563. [CrossRef]

173. Nevet, M.J.; Shalev, S.A.; Zlotogora, J.; Mazzawi, N.; Ben-Yosef, T. Identification of a prevalent founder mutation in an Israeli Muslim Arab village confirms the role of PRCD in the aetiology of retinitis pigmentosa in humans. J. Med. Genet. 2010, 47, 533-537. [CrossRef]

174. Allon, G.; Mann, I.; Remez, L.; Sehn, E.; Rizel, L.; Nevet, M.J.; Perlman, I.; Wolfrum, U.; Ben-Yosef, T. PRCD is Concentrated at the Base of Photoreceptor Outer Segments and is Involved in Outer Segment Disc Formation. Hum. Mol. Genet. 2019, 28, 4078-4088. [CrossRef] [PubMed]

175. Spencer, W.J.; Pearring, J.N.; Salinas, R.Y.; Loiselle, D.R.; Skiba, N.P.; Arshavsky, V.Y. Progressive Rod-Cone Degeneration (PRCD) Protein Requires N-Terminal S-Acylation and Rhodopsin Binding for Photoreceptor Outer Segment Localization and Maintaining Intracellular Stability. Biochemistry 2016, 55, 5028-5037. [CrossRef]

176. Spencer, W.J.; Ding, J.-D.; Lewis, T.R.; Yu, C.; Phan, S.; Pearring, J.N.; Kim, K.-Y.; Thor, A.; Mathew, R.; Kalnitsky, J.; et al. PRCD is essential for high-fidelity photoreceptor disc formation. Proc. Natl. Acad. Sci. USA 2019, 116, 13087-13096. [CrossRef]

177. Aguirre, G.D.; Alligood, J.; O’Brien, P.; Buyukmihci, N. Pathogenesis of progressive rod-cone degneration in miniature poodles. Investig. Ophthalmol. Vis. Sci. 1982, 23, 610-630.

178. Friedman, J.S.; Chang, B.; Kannabiran, C.; Chakarova, C.; Singh, H.; Jalali, S.; Hawes, N.L.; Branham, K.; Othman, M.; Filippova, E.; et al. Premature Truncation of a Novel Protein, RD3, Exhibiting Subnuclear Localization Is Associated with Retinal Degeneration. Am. J. Hum. Genet. 2006, 79, 1059-1070. [CrossRef] [PubMed]

179. Wolf, E.D.; Vainisi, S.J.; Santos-Anderson, R. Rod-cone dysplasia in the collie. J. Am. Veter Med Assoc. 1978, 173, 1331-1333.

180. Woodford, B.; Liu, Y.; Fletcher, R.; Chader, G.; Farber, D.; Santos-Anderson, R.; Tso, M.O. Cyclic nucleotide metabolism in inherited retinopathy in collies: A biochemical and histochemical study. Exp. Eye Res. 1982, 34, 703-714. [CrossRef] 
181. Dizhoor, A.M.; Olshevskaya, E.V.; Peshenko, I.V. Retinal guanylyl cyclase activation by calcium sensor proteins mediates photoreceptor degeneration in an rd3 mouse model of congenital human blindness. J. Boil. Chem. 2019, 294, 13729-13739. [CrossRef]

182. Kukekova, A.V.; Goldstein, O.; Johnson, J.; Richardson, M.A.; Pearce-Kelling, S.E.; Swaroop, A.; Friedman, J.S.; Aguirre, G.D.; Acland, G.M. Canine RD3 mutation establishes rod-cone dysplasia type 2 (rcd2) as ortholog of human and murine rd3. Mamm. Genome 2009, 20, 109-123. [CrossRef]

183. Santos-Anderson, R.M.; Tso, M.O.; Wolf, E.D. An inherited retinopathy in collies. A light and electron microscopic study. Investig. Ophthalmol. Vis. Sci. 1980, 19, 1282-1294.

(C) 2020 by the authors. Licensee MDPI, Basel, Switzerland. This article is an open access article distributed under the terms and conditions of the Creative Commons Attribution (CC BY) license (http://creativecommons.org/licenses/by/4.0/). 


\title{
The Interplay between Peripherin 2 Complex Formation and Degenerative Retinal Diseases
}

\author{
Lars Tebbe, Mashal Kakakhel, Mustafa S. Makia, Muayyad R. Al-Ubaidi * and Muna I. Naash * \\ Department of Biomedical Engineering, University of Houston, Houston, TX 77204, USA; \\ ltebbe@Central.UH.EDU (L.T.); mkakakhe@central.uh.edu (M.K.); msmakia@Central.UH.EDU (M.S.M.) \\ * Correspondence: malubaid@central.uh.edu (M.R.A.-U.); mnaash@central.uh.edu (M.I.N.); \\ Tel.: +1-713-743-1651 (M.I.N.)
}

Received: 4 February 2020; Accepted: 20 March 2020; Published: 24 March 2020

\begin{abstract}
Peripherin 2 (Prph2) is a photoreceptor-specific tetraspanin protein present in the outer segment (OS) rims of rod and cone photoreceptors. It shares many common features with other tetraspanins, including a large intradiscal loop which contains several cysteines. This loop enables Prph2 to associate with itself to form homo-oligomers or with its homologue, rod outer segment membrane protein 1 (Rom1) to form hetero-tetramers and hetero-octamers. Mutations in PRPH2 cause a multitude of retinal diseases including autosomal dominant retinitis pigmentosa (RP) or cone dominant macular dystrophies. The importance of Prph2 for photoreceptor development, maintenance and function is underscored by the fact that its absence results in a failure to initialize OS formation in rods and formation of severely disorganized OS membranous structures in cones. Although the exact role of Rom 1 has not been well studied, it has been concluded that it is not necessary for disc morphogenesis but is required for fine tuning OS disc size and structure. Pathogenic mutations in PRPH2 often result in complex and multifactorial phenotypes, involving not just photoreceptors, as has historically been reasoned, but also secondary effects on the retinal pigment epithelium (RPE) and retinal/choroidal vasculature. The ability of Prph2 to form complexes was identified as a key requirement for the development and maintenance of OS structure and function. Studies using mouse models of pathogenic Prph 2 mutations established a connection between changes in complex formation and disease phenotypes. Although progress has been made in the development of therapeutic approaches for retinal diseases in general, the highly complex interplay of functions mediated by Prph 2 and the precise regulation of these complexes made it difficult, thus far, to develop a suitable Prph2-specific therapy. Here we describe the latest results obtained in Prph2-associated research and how mouse models provided new insights into the pathogenesis of its related diseases. Furthermore, we give an overview on the current status of the development of therapeutic solutions.
\end{abstract}

Keywords: peripherin 2; retinal degeneration; retina; tetraspanin; photoreceptor

\section{Introduction}

Tetraspanins represent a family of highly conserved membrane proteins involved in a variety of functions. These functions include membrane organization and compartmentalization, cell signaling, adhesion and migration [1-4]. Structurally, tetraspanins consist of a short cytoplasmic $\mathrm{N}$-terminus, four transmembrane domains (TM), one small and one large extracellular loop (EC1 and EC2, respectively), as well as a short cytoplasmic C-terminus [3,5,6]. The EC2 loop can be further divided in a conserved and a variable region $[7,8]$. The highly conserved part of the EC2 loop was shown to mediate the dimerization of tetraspanin proteins, while the variable region is required for specific interactions of the tetraspanins with their variable interaction partners [7-10]. Tetraspanin interactions can be divided into three levels of interactions [1]. The primary level consists of direct associations between two tetraspanin proteins as well as between tetraspanin and a non-tetraspanin interacting 
partners, interactions that resist the treatment with strong detergents [1,4]. Binding of these primary complexes to each other represents the secondary level which is indirect and in some cases supported by palmitoylation $[1,4,11]$. In the third level, tetraspanins can indirectly associate with their interacting partners to form large insoluble complexes, which only resist mild detergents like CHAPS $[1,4,12,13]$. The ability of tetraspanins to interact with each other and with other partners allows them to form tetraspanin-enriched microdomains known as the tetraspanin web [4,14].

Peripherin 2 (Prph2, formally known as retinal degeneration slow, RDS) represents a photoreceptor-specific tetraspanin. It is a structural protein that is critical for the proper development of rod and cone outer segments (ROS and COS, respectively) and thus for vision $[15,16]$. The main function of Prph2, in the morphogenesis of ROS and COS, is to promote membrane curvature, flattening and fusion; processes that are required for the rim formation of the OS discs and lamella [15,17-20]. Like the other members of the tetraspanin family, Prph2 contains two loops, referred to as intradiscal loops (D1 and D2, respectively). The D2 loop is equivalent to the EC2 loop of other tetraspanins and mediates the bulk of interactions of Prph2 [21-23]. Prph2 interacts with another photoreceptor-specific tetraspanin, called rod outer segment membrane protein 1 (Rom1) [24]. Prph2 forms non-covalently associated homo- and hetero-tetramers with Rom1. These tetramers associate and form covalently linked intermediate complexes. In addition to that, Prph2 is able to form higher order complexes consisting of several Prph2 homo-tetramers. Interestingly, hetero-tetramers including Rom-1 are excluded from these higher order complexes $[21,25,26]$. Formation of these complexes is required for Prph2 to promote the development of rim domains essential for OS formation $[18,27,28]$. In line with its key role in OS formation, mutations in PRPH2 are connected with a multitude of retinal diseases ranging from autosomal dominant retinitis pigmentosa (ADRP) to macular degeneration (MD) [29]. The associated phenotypes show a high degree of variability of onset and phenotype even between patients carrying the same mutation. In addition to the degeneration of the retina, some Prph2 related diseases were found to cause secondary effects on the retinal pigment epithelium (RPE) or the choroid [29-31].

This review describes the importance of Prph2 in the development of both ROS and COS. Recent data provided new insight into the steps necessary for OS morphogenesis and the role of Prph2 in orchestrating these events. Furthermore, we will summarize how the precise formation and regulation of Prph2/Rom1 complex affects the function of Prph2 in rods versus cones. Finally, we will discuss the animal models expressing pathogenic Prph 2 mutations and provide a short summary about therapeutic approaches so far aimed to treat Prph2 related diseases.

\section{The Role of Prph2 in Photoreceptor Outer Segment Morphogenesis}

Since mice lacking Prph2 (Prph2 ${ }^{-/-}$) fail to form ROS, it became clear that the major function of Prph2 is in the initialization and elaboration of ROS [32]. Recent data confirmed this function and identified the C-terminus of Prph2 as the motif responsible for the initialization of ROS formation [33,34]. The photoreceptor outer segment (OS) represents a highly specialized form of a modified primary cilium [35]. The initial steps in the development of the OS are similar to the development of other primary cilia, starting with the attachment of the ciliary vesicle to the basal body [36-38]. After this initial step, the elongation of the ciliary axoneme and the expansion of the plasma membrane happen in a synchronous fashion. A recent study showed that these initial steps in ciliogenesis take place in both wild type (WT) and $\mathrm{Prph}^{-{ }^{-}}$photoreceptors, an observation which was in line with previous data showing the presence of an intact connecting cilium (CC) in the Prph $2^{-/}$photoreceptors $[32,33,39]$. Additionally, the same study revealed that the Prph2 C-terminus mediates suppression of the formation of ectosomes at the distal part of the CC in rod photoreceptors [33]. Ectosomes are thought to play an important role in the processes of ciliary resorption, regulation of the ciliary protein content and regulation of signal transduction [40-44]. The ability of Prph2 C-terminus to suppress the release of ectosomes offers an explanation to why photoreceptor cilia are able to form the highly complexed intra-ciliary membranous structures like that of the ROS [33]. Further evidence for a key role of 
the Prph2 C-terminus in the initialization of ROS formation was provided by a recent study that generated a chimeric protein composed of the C-terminus of Prph2 fused to the body of Rom1, the non-glycosylated homologue of Prph2 [34]. Expression of the chimeric protein was sufficient to initialize the formation of ROS structures but failed to elaborate them proving that the Prph2 C-terminus is responsible for the formation of the initial membrane outgrowth while the other parts of Prph2 are required for the development of the highly organized structure of the OS.

$\mathrm{Prph}^{+/-}$mice show defects in both function and structure of photoreceptor OS, which proves that the role of Prph2 is not restricted to the initialization of OS formation but also important for its structural development and maintenance [16]. In mature ROS discs, Prph2 is restricted to the rim [15]. In addition, previous studies demonstrated that Prph2 displayed membrane fusion activity, which led to the theory that Prph2 is involved in the shaping of the closed rim of fully developed discs in ROS $[45,46]$. Indeed, a function of Prph2 in inducing the curvature of the plasma membrane was observed in several studies $[25,33,47,48]$. While all these studies agree that Prph2 is involved in the generation of membrane curvature, they are contradictory in defining the exact region of Prph2 responsible for this activity. Two studies pinpointed the membrane curvature inducing activity to an $\alpha$-helix motif located in the C-terminus of Prph2 [47,48], while a third study argues that this motif is rather preventing membrane curvature than inducing it [25]. A recent study found evidence for a function of the tetraspanin core of Prph2 in generating membrane curvature while the C-terminus was unable to perform this function [33]. The localization of Prph2 in newly formed ROS discs provided further evidence for the importance of Prph2 in membrane curvature and disc closure [17]. Newly formed discs in the ROS have a close rim at the site adjacent to the axoneme of the photoreceptor cilium while the opposing side of the disc remains open [49]. In these newly formed discs, Prph2 is restricted to the axonemal closed side of the disc [17]. A recent study confirmed the localization of Prph2 on the axonemal side of newly formed discs in the ROS [50]. An additional finding in this study was that Prph2 is more abundant at the closed rim of newly formed discs than at the rim of mature discs, indicating that Prph2 is not only involved in the closure of this rim but also required for the initialization of the formation of new discs.

The membrane curvature of the open end of newly formed discs in ROS is opposite to the curvature on the closed rim, and thus unlikely to allow the binding of Prph2 [26]. This led to the question of whether a different transmembrane protein may promote the curvature of the open ends. Prominin-1 was identified as a potential candidate involved in the shaping of newly synthetized discs in ROS. Prominin-1 is a membrane protein with five transmembrane domains that localizes at the open end of newly synthesized discs in ROS, thus opposite to the localization of Prph2 [51-55]. The vital role of Prominin-1 in the structural integrity of the photoreceptor OS is supported by the finding that mutations in Prominin- 1 are connected to retinal diseases causing degeneration of the photoreceptors [54,56-59]. Earlier studies on Prominin-1 demonstrated that it shows a binding preference for curved membranes, but does not induce the curvature itself $[60,61]$. Thus, Prominin- 1 is a promising candidate for the maintenance of the curvature on the open end, while the protein responsible for inducing the curvature at these ends remains elusive. A second question, which remains open thus far, is what mechanism restricts Prominin-1 to the newly synthesized discs? When ROS discs mature and reach their final diameter, the open ends close; presumably through the activity of Prph2 and become separated from the plasma membrane of ROS. While Prph2 is located on the entire rim of these closed discs, Prominin-1 is no longer found after the closure of the now matured disc [55].

While rod OS discs are closed and separated from the plasma membrane, cone OSs on the other hand contain open discs also referred to as lamellae and are contiguous with the plasma membrane. Although amphibian COS consists exclusively of open discs, the number of open discs in mammalian COS varies depending on the species [38,62-65]. The localization of Prph2 in the open discs of COS is comparable with its localization in the newly synthesized ROS discs, with Prph2 being restricted to the side adjacent to the axoneme of the photoreceptor cilium [55]. The discs in COS display a closed rim formation on the axonemal sides. Prominin-1 is localized on the open side, in line with its localization 
in the newly formed ROS. Studying the role of Prph2 in the development of cones in mice has proven to be difficult due to the low percentage of cone photoreceptors in the murine retina, whereby only $\sim 3-5 \%$ of photoreceptors are cones. We have analyzed the effect of loss of Prph 2 in the background of an $\mathrm{Nrl}$ knockout $\left(\mathrm{Nrl}^{--}\right)$, in which all rods are converted to S-cone-like cells [66-68]. Here the cones in the double knockout mice $\left(\mathrm{Prph}^{-/-} \mathrm{Nrl}^{-/-}\right)$displayed disorganized COSs lacking the flattened lamellae characteristic of WT COS [69]. Furthermore, this study provided evidence for the trafficking of many OS proteins to this altered OS structure, indicating that the transport of those proteins towards the OS does not rely on Prph2 [68]. The ability of $\mathrm{Prph}^{-/-}$retinas to form disorganized COSs argues for a different role of Prph2 in cones than in rods. As mentioned above, ROSs fail to develop in the Prph $2^{-/}$mice, thus demonstrating a vital role for Prph2 in the initialization of the formation of this complex intra-ciliary membrane structure in ROS. The discovery of a role for the C-terminal region of Prph2 in inhibiting the release of ectosomes from the rod photoreceptor cilia [33] added new interesting aspect to the process of the rod OS formation. Future studies are needed to determine if this function of $\operatorname{Prph} 2$ is preserved in cones.

The identification of Rom1 as a Prph2 interactor initiated a multitude of studies investigating the complex formation between Prph2 and Rom1 [24,26,70]. Like Prph2, Rom1 is a photoreceptor-specific membrane protein and a member of the tetraspanin superfamily [71]. While only sharing a sequence identity of 35\% with Prph2, both proteins form a highly conserved and similar secondary and tertiary structures with four transmembrane domains and two intradiscal loops [24]. Prph2 and Rom1 form heteromeric and homomeric tetrameric complexes, held together by non-covalent bonding mediated by the second intradiscal (D2) loop [21-23]. These tetramers can assemble into intermediate complexes consisting of both hetero- and homotetramers to establish a mix of covalent and non-covalent linked complexes [72]. These intermediate complexes were found to include at least two tetramers. In addition to the intermediate complexes, higher order complexes containing only Prph2 were also identified [72]. The core of both intermediate and higher order complexes consists of tetramers [21,25]. Interestingly Rom1 is excluded from these higher order complexes [21,25]. How this exclusion is mediated is not fully understood. A possible explanation is that Prph2 can utilize both the conventional secretory pathway through the Golgi apparatus as well as the unconventional pathway which bypasses the Golgi apparatus [26,73]. The presence of Rom 1 results in more Prph2 being transported through the conventional pathway [34]. It is likely that this level of sorting during the initial steps of protein trafficking provides a possible mechanism mediating the exclusion of Rom1 from higher order complexes. Studies verifying this mechanism are a possible direction for future work. The higher order complexes are localized at the closed rim of discs indicating an important role for them in the membrane folding necessary to close the disc rim [25,74]. Biochemical evidence supported the idea that during the formation of Prph2/Rom1 complexes, intermolecular disulfide bonding between Prph2 and Rom1 are formed $[72,75,76]$.

The ability of Prph2 and Rom1 to form complexes was proven to be vital for their function in OS formation $[18,27,74]$. The cysteine at position 150 (C150) in the D2 loop of Prph2 was found to be critical for the formation of higher order Prph2/Rom1 complexes [21,28,77]. C150 of Prph2 forms an intermolecular disulfide bond with C150 of another Prph2 to make homodimers or with C153 of Rom1, thus allowing the formation of Prph2/Rom1 heterodimers and heteromeric tetramers. A knock-in mouse expressing a point mutation at C150 of Prph2 (C150S) provided further evidence for the importance of this cysteine in the formation of higher order complexes and thus for the role of Prph2 and Rom1 in the development of the OS [78]. The formation of intermediate as well as higher order complexes was fully abolished by the C150S mutation and both ROS and COS were severely disorganized. Despite being disorganized, the OS structure of the C150S mice were somewhat better when compared to $\mathrm{Prph}^{+/-}$while no functional improvement was observed, indicating that the ability of Prph 2 to form intermediate complexes with Rom- 1 as well as its ability to form higher order complexes consisting of Prph2 homo-tetramers is not only relevant for maintaining the OS structure but also for its function [78]. 
The development of higher order complexes seems to be expendable for the initiation of OS formation, since generation of both ROS and COS is initiated in the C150S mutant retinas that are devoid of these complexes. Comparable results were obtained for a mutation deleting lysine at

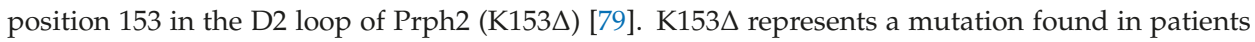
resulting in variable phenotypes ranging from ADRP to more cone specific MD [80]. This mutation also obliterates the ability of Prph2 to form higher order complexes [79]. The initiation of ROS and COS structures was unaffected while both displayed structural and functional decline. While the C150S and K153 $\Delta$ mutations prevent the formation of higher order complexes, some pathogenic mutations of Prph2 lead to the formation of an abnormal high molecular weight aggregates that include both Prph2 and Rom1 [81,82]. A patient mutation causing the exchange of the tyrosine at position 141 for a cysteine (Y141C), effectively adding another cysteine in the D2 loop, causes mostly defects in the macula but some cases of RP were also reported $[30,82,83]$. The Prph2 and Rom1 complexes in Y141C knockin retinas were significantly altered, showing the formation of abnormal high molecular weight aggregates containing both Prph2 and Rom1. This is a remarkable finding considering that Rom1 is normally excluded from higher order complexes [82]. These mice display structural and functional defects in ROS and COS. The formation of abnormal high molecular weight aggregates could also be found in another patient mutation in which tryptophan in Prph2 is substituted by arginine at position 172 (R172W) [81]. Transgenic mice carrying this mutation displayed the formation of high molecular weight aggregates of Rom1 while Prph2 complexes were not affected. Additionally, these high molecular weight Rom1 aggregates were more abundant in cones, a result that is in line with the decline of cone function in this mouse model [81].

Taken together, these results demonstrate that the exact regulation of the Prph2/Rom1 complex formation is critical for proper OS development, maintenance and disc size in both rods and cones as well as for their function. For OS initiation, proper complex formation seems to be irrelevant. It is worth noting that these studies point to a striking difference between rods and cones with regard to how they are affected by the different Prph 2 mutations. Mutations, which primarily affect the rods, seem to be resulting from haploinsufficiency or loss-of-function effects $[78,84,85]$, while those effecting cones seem to be more susceptible to changes in the complex formation, likely due to gain-of-function defects $[78,79,81,82,86-88]$. Another differentiation between rods and cones is how they process Rom1. While ablation of Prph2 results in the complete loss of ROS and severely disorganized COS, elimination of Rom1 $\left(\mathrm{Rom}^{-/-}\right)$is less severe, indicating that Rom1's role is more in fine tuning of disc sizing [27]. It is worth noting that most studies considering these functions were performed in WT murine rods. A recent study performed on a $\mathrm{Nrl}^{-/-}$background using the K153 $\Delta$ mutation of Prph2 demonstrated a loss of Prph2/Rom1 interaction specifically in cones [79]. In order to unravel the precise differences in the roles of Rom 1 in rods versus cones, further studies are needed.

The D2 loop of Prph2 contains a motif for N-linked glycosylation, a motif which is absent in the D2 loop of Rom1 [24]. In rods, the N-glycosylation on Prph2 was found to be expendable for ROS [89]. In transgenic mice expressing the unglycosylated form of Prph2 specifically in rods, the interaction with Rom1 or the formation of complexes was not affected. Furthermore, expressing unglycosylated Prph2 on a Prph $2^{-/-}$background achieved a full rescue of the phenotype, further proving that the $\mathrm{N}$-glycosylation of Prph2 is unnecessary for its function in rods [89]. A knockin mouse model expressing unglycosylated Prph2 [75] recapitulated the rod results observed by Kedzierski et al. [89]. However, while rods were unaffected, cone function was found to decrease significantly in these mice. Furthermore, a significant decrease in Prph2 and Rom1 levels in the cones of the knockin mice was also observed. These results further highlight the differential functional roles of Prph2 in rods versus cones.

Apart from its interaction with Rom1, Prph2 was found to interact with additional OS specific proteins. One of those interactors is with cyclic nucleotide gated channel B1a (CNGB1a) [90,91]. CNGB1a was shown to be relevant for the correct sizing and alignment of the rod discs, supporting the hypothesis that the interaction between Prph2 and CNGB1a plays a role in the proper shaping of the discs [92,93]. Additionally, Prph2 was also found to directly interact with rhodopsin (Rho), thus forming 
a complex of Prph2, CNGB1a and Rho to anchor the rim of the discs to the OS plasma membrane and the rims of adjacent discs. It has been hypothesized that these interactions are responsible for correct alignment and stacking of the discs [94]. This was supported by findings that co-depletion of these three proteins exacerbated both functional and structural defects in rod photoreceptors [95].

\section{Insight from Mouse Models into the Pathophysiology of Prph2 Mutations}

\subsection{Transgenic Mouse Models of Prph2 Mutations}

Genetically engineered mouse models expressing pathogenic mutations of Prph2 have proven to be valuable tools in studying the pathophysiology of Prph2 related blinding diseases. These models have been generated as transgenic expressing Prph2 under the control of heterologous promoters that in some cases are specific to rods, cones or both.

\subsection{1. $\operatorname{Prph} 2^{\mathrm{R} 172 \mathrm{~W}}$}

One of the most common disease-causing mutations of $P R P H 2$ is the substitution of tryptophan to arginine at position 172 (R172W) [96-99]. Patients carrying this mutation display a decrease in cone function, while rods' function remains unaffected [96]. The R172W mutation is located in the D2 loop, which is the region that promotes complex formation and where the vast majority of pathogenic $P R P H 2$ mutations are located [22,87]. Interestingly, the R172W mutation is located outside the part of the D2 loop involved in the formation of complexes [22]. Expressing the R172W mutation in the presence of the full complement of WT Prph2 led initially to normal rod function and structure [87]. In contrast, the retina of these mice exhibited a significant loss in the number of blue and green cones and associated with a decrease in their photopic responses. When expressed on Prph $2^{+-}$background, the R172W mutation led to a late-onset reduction in the number of rod cells and in scotopic responses, besides the cone phenotype. This varies from the cone exclusive phenotype observed in patients carrying this mutation and is most likely due to haploinsufficiency of Prph2. Expression of the R172W mutation in the $\mathrm{Nrl}^{-/-}$retina reproduced the functional decline in the photopic responses and provided evidence as to how the R172W mutation disrupted COS structures by causing the formation of abnormal high molecular weight Prph2/Rom1 aggregates [81]. This result demonstrates how the functional decline of the cones occurs in patients carrying this mutation.

\subsection{2. $\operatorname{Prph} 2^{\mathrm{C} 214 \mathrm{~S}}$}

The substitution of a cysteine at position 214 in Prph2 with a serine (C214S) was shown to cause ADRP, a degeneration of rods followed by a late-onset degeneration of cones [100]. Expression of the C214S transgene in the presence of the full complement of WT Prph2 mice failed to produce any phenotype, proving that the phenotype observed in patients is through a mechanism other

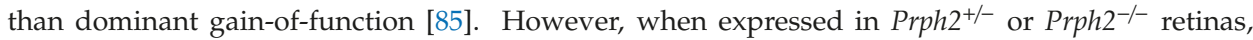
the C214S protein failed to rescue the functional and structural phenotypes in both rods and cones, indicating that the $\mathrm{C} 214 \mathrm{~S}$ mutation is resulting in a loss of function. An interesting finding was that the C214S mutant Prph2 was unable to interact with Rom1, indicating an alteration in the formation of complexes caused by this mutation. Although an ample amount of Prph2 C214S transgene message was detected, only a trace amount of the mutant protein was identified, which led to the conclusion of a loss-of-function phenotype associated with the C214S mutation. It is unclear whether the low levels of mutant protein are due to a low rate of synthesis or instability of the mutant protein.

\subsection{3. $\operatorname{Prph} 2^{\mathrm{P} 216 \mathrm{~L}}$}

A substitution of a proline at position 216 in Prph2 with a leucine (P216L) was found to cause $\mathrm{RP}$ in patients [101]. Expression of P216L in mice in presence of WT Prph2 $\left(\operatorname{Prph}^{+/+}\right)$levels led to an age-dependent significant decrease in scotopic responses and shortened rod OSs with normal disc alignments, indicative of a dominant-gain-of-function effect [84]. Moreover, in the presence of one 
WT allele of $\operatorname{Prph} 2\left(\operatorname{Prph} 2^{+-}\right)$, expression of the P216L caused a significant decrease in rod function compared to $\mathrm{Prph}^{+/-}$mice. In these mice, a more severe OS shortening and disorganization of the discs could be observed in presence of the P216L mutant protein. Interestingly, expression of the P216L protein in a $\mathrm{Prph}^{-/-}$retina caused the formation of small, highly ROS, which represents a limited rescue when compared to the complete absence of ROS in $\operatorname{Prph}^{-/-}$. Taken together, these results prove that the P216L mutation in Prph2 results in a dominant-gain-of effect, in agreement with the rod dominant phenotype observed in patients.

\subsection{4. $\operatorname{Prph} 2^{\mathrm{L} 185 \mathrm{P}}$}

The substitution of leucine at position 185 to proline (L185P) in Prph2 causes a rare digenic form of RP. Patients heterozygous for both the L185P mutation and a null mutation in Rom1 exhibit symptoms of RP, while carriers of any of the two mutations do not show a phenotype [102,103]. Transgenic mice expressing the L185P mutation in a $P r p h 2^{+/-}$or a digenic $\operatorname{Prph}^{+/-} / \operatorname{Rom~}^{+/-}$background displayed a late onset thinning of the outer nuclear layer (ONL) concomitant with reduced scotopic electroretinograms (ERG) [104].

The transgenic mouse models described above provided valuable insights into the pathophysiology of Prph2 related diseases. Since the promoters used to express the transgene are heterologous, often levels of expression of the transgene differ considerably from that of the endogenous leading to a varied phenotype.

\subsection{Prph2 Knockin Mouse Models}

The variation in expression levels of the transgene observed in transgenic mouse models is particularly problematic in cases of reduced levels of the expressed protein since reductions in the expression level of WT Prph2 were shown to result in haploinsufficiency leading to severe retinal defects $[16,105]$. Haploinsufficiency makes it hard to distinguish between the effects seen in transgenic mice that are due to the mutation or those resulting from the reduced levels of expressed protein. In order to overcome this, recent studies relied on Prph2 knockin mouse models for a set of mutations found in patients of Prph2 related diseases. Below are the models currently presented in the literature and comparison of their retinal phenotypes to patient's phenotype carrying the same mutation.

\subsubsection{Prph $2^{307 /+}$ and Prph $2^{307 / 307}$}

A deletion of a single base pair at codon 307 in human PRPH2 results in a slow progressing form of ADRP [106]. A mouse model in which a targeted single base deletion at codon 307 in Prph 2 was introduced and showed a severe decline in photoreceptor survival and function [107]. ERG revealed a decrease in photoreceptor function in heterozygous animals starting at two months of age, while no ERG responses were detected in the homozygous animals even at one month of age. Retinal phenotypes in the heterozygous or homozygous mice were more severe than in age-matched $\mathrm{Prph} 2^{+/-}$and $\mathrm{Prph} 2^{-{ }_{-}}$ mice, respectively. Thus, the deletion mutation resulted in a strong dominant gain-of-function effect. This knockin model represents a drastic case of a retinal phenotype that differs greatly from that observed in the patients whereby the structural and functional decline is slow, starting in the fifth or sixth decades of life. The mouse model, on the other hand, displays a rapid degeneration with an early onset. The deletion at codon 307 in the human $P R P H 2$ gene causes a frameshift and creates a stop codon after the addition of 16 amino acids. If the resulting mutant protein is stable, it is expected to be 26 amino acid shorter than the wildtype. It is likely the case since the phenotype seen in patients is mild due to gain-of-function defect, but if the mutant protein is unstable, then the phenotype would be loss-of-function defect similar to that of haploinsufficiency. However, in the mouse genome, such one base deletion at codon 307 is predicted to result in the alteration of the last 40 amino acids of the $C$ terminus of the protein and the addition of 11 extra amino acids. This results in the translation of 51 amino acids after codon 307 in the mouse [107]. The different effects on the translated protein caused by the deletion in codon 307 observed between human and mouse explains 
the differences in the severity of the retinal phenotypes. It is important to note that retinal phenotype in the homozygous mouse is reported to be worse than the complete-loss-of-function phenotype seen in the Prph 2 null mice. This observation suggests that the mouse phenotype is likely a combination of lossand gain-of-function defects and that the toxicity beyond that seen in the Prph2 knockout is probably the outcome of the latter. Since the mouse model was not assessed for the presence of the mutant protein, at this point, it is unclear whether the toxicity arises from the absence of the protein, the 51 altered amino acids at the C-terminus or the lack of endogenous 40 amino acids at the C-terminus. Obviously, the addition of 16 altered amino acids in the human PRPH2 and shortening the protein by 26 amino acids at the C-terminus have less drastic effect on the retina than those modifications occurred in the mouse protein.

\subsubsection{Prph $2^{\mathrm{C} 213 \mathrm{Y} /+}$ and $\operatorname{Prph} 2^{\mathrm{C} 213 \mathrm{Y} / \mathrm{C} 213 \mathrm{Y}}$}

The C213Y mutation is located in the D2 loop of Prph2, in the motif that mediates intramolecular and intermolecular disulfide bonds, thus responsible for the formation of Prph2/Prph2 and Prph2/Rom1 tetramers as well as for the formation of intermediate and higher order complexes [77]. The heterozygous mice (Prph $2^{\mathrm{C} 213 \mathrm{Y} /+}$ ) displayed a shortened and disorganized ROS. When compared to Prph ${ }^{+/-}$mice, the ROS of Prph $2^{\mathrm{C} 213 Y /+}$ mice showed a slight improvement in the stacking and alignment of the discs (Figure 1A,B) [76].

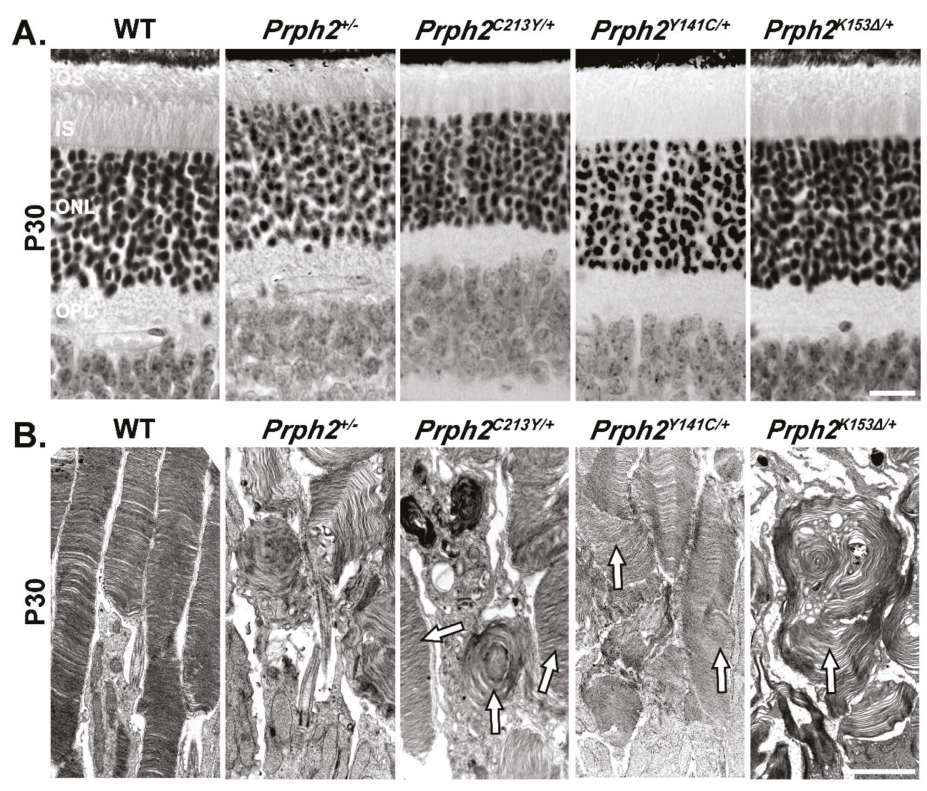

Figure 1. Mutations in the mouse Prph2 gene lead to varying degrees of photoreceptor degeneration. (A) Representative light microscopic images from hematoxylin and eosin stained retinal sections at P30 aligned at the upper edge of the retinal pigment epithelium (RPE). (B) Transmission electron microscopic (TEM) images of the interface between the IS and OS of photoreceptors of the indicated genotypes. OS, outer segments; IS, inner segment; ONL, outer nuclear layer; OPL, outer plexiform layer. Scale bars: $20 \mu \mathrm{m}$ for A and $2 \mu \mathrm{m}$ for B. Eyes used in this study were dissected, fixed and embedded as previously described [79]. Images were captured at 40× and converted to black and white using ZEN Image Analysis software. The plastic-embedding and TEM methods were as described previously [85]. Images were adjusted and cropped using Adobe Photoshop CS5. 
This is evident from the well stacked OS discs seen in some photoreceptors while others looked like whorls similar to those seen in the Prph $2^{+/}$(arrows in Figure 1). Scotopic ERG responses of the Prph $2^{\mathrm{C} 213 Y /+}$ mice were significantly reduced starting at P30 (Figure 2) and persist all the way to P365. The scotopic response was nearly completely abolished in $\operatorname{Prph} 2^{\mathrm{C} 213 Y / C 213 Y}$ animals and the OS was almost non-existent. The photopic responses were significantly decreased in both heterozygous and homozygous animals at P30. At later time points, the photopic response decreased further in the Prph $2^{\mathrm{C} 213 Y /+}$ mice and was completely absent in the Prph $2^{\mathrm{C} 213 Y / C 213 Y}$ animals. A key finding in this study was that the Prph $2^{\mathrm{C} 213 Y /+}$ mice, that represent the genotype present in patients, showed better retinal structure despite the fact that both of rod and cone ERG responses were reduced when compared to Prph $2^{+/-}$animals.

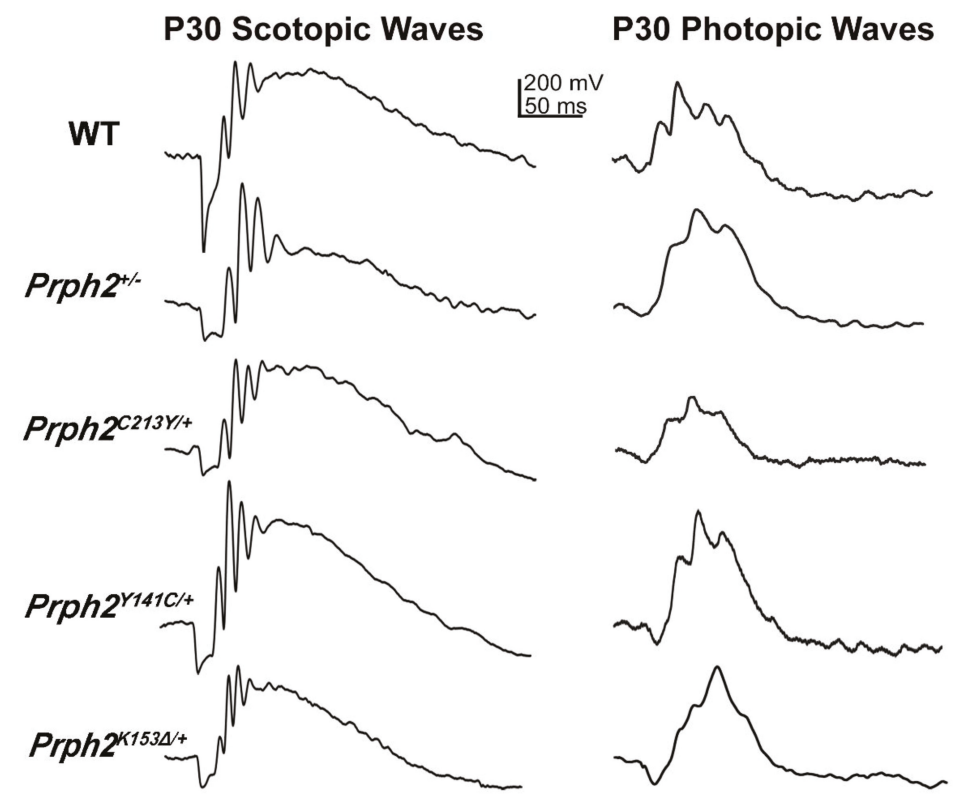

Figure 2. Mutations in Prph2 hinder OS function assessed via scotopic and photopic electroretinograms (ERGs) at P30. Full-field ERGs were recorded under scotopic and photopic conditions. Shown are representative ERG waveforms from the indicated genotypes at P30. Full-field ERG tests were performed as previously described [85]. After overnight dark adaptation, mice were anesthetized and their pupils dilated. ERGs were recorded with a UTAS system (LKC, Gaithersburg, MD, USA). Waveforms were exported into GraphPad software to obtain wave traces and then exported into Photoshop using a uniform scale.

A key requirement for the function of Prph2 is its ability to interact with Rom1 and to form oligomeric complexes [18,27,74]. Co-immunoprecipitation (co-IP) experiments using retinal lysate from Prph $2^{\mathrm{C} 213 Y / \mathrm{C} 213 Y}$ mice revealed the inability of Prph2 ${ }^{\mathrm{C} 213 \mathrm{Y}}$ to interact with Rom1. The homomeric interaction of Prph2 was also reduced in these mice as evident from reduced ability of Prph2 ${ }^{\mathrm{C} 213 \mathrm{Y}}$ to form intermediate and higher order complexes as determined by sucrose gradient velocity sedimentation [76]. The reduction in oligomeric complexes offers an explanation for the functional decline observed in both $\operatorname{Prph} 2^{\mathrm{C} 213 Y /+}$ and $\operatorname{Prph} 2^{\mathrm{C} 213 Y / \mathrm{C} 213 Y}$ mice and no abnormal high molecular weight aggregates were observed under non-reducing conditions. Prph $2^{\mathrm{C} 213 Y /+}$ retina retains some Prph2 in the IS, shown by immunofluorescence (IF) staining of Prph2 (green and arrows in Figure 3) and IS maker syntaxin 3B (STX3B) (red, Figure 3). However, Prph $2^{\mathrm{C} 213 Y / C 213 Y}$ lacks the ability to form complexes which leads to 
complete retention of $\operatorname{Prph} 2$ in the inner segments (IS), and perinuclear region while a smaller amount of Rom1 was retained in the IS [76].

Patients carrying the C213Y mutation in PRPH2 display a butterfly-shaped pattern/macular dystrophy, while a rod dominant RP phenotype is absent [29,108-110]. The knockin mouse model for the C213Y displays functional defects in both rods and cones. While the defects in the cones are in line with the defects observed in the cone rich macula of the patients, the defects in the rods displayed by the model are not. The difference between phenotypes in patients and the mouse model may be due to the fact that the mouse retina consists almost exclusively of rod photoreceptors. Since the murine retina lacks a macula, it was not possible to reproduce the butterfly shaped macular dystrophy in the mouse model. One interesting finding in the Prph2 ${ }^{\mathrm{C} 213 \mathrm{Y}}$ mouse model was the observation of a yellow flecking in the fundus of both $\operatorname{Prph} 2^{\mathrm{C} 213 Y /+}$ and $\operatorname{Prph} 2^{\mathrm{C} 213 Y / C 213 Y}$ mice at P180. This phenotype mimics funduscopic anomalies found in patients carrying the C213Y mutation [110].

Gene supplementation was performed by crossing a WT Prph2 overexpressing mouse line (NMP) onto hetero- $\left(\operatorname{Prph} 2^{\mathrm{C} 213 \mathrm{Y} /+}\right)$ or homozygous (Prph $2^{\mathrm{C} 213 \mathrm{Y} / \mathrm{C} 213 \mathrm{Y}}$ ) mice. The defects observed in the protein trafficking as well as in OS structure were rescued in $\operatorname{Prph} 2^{\mathrm{C} 213 Y}$ mice. A rescue on the functional level however could not be observed [76]. These results show that the presence of the mutant protein has a detrimental effect on photoreceptor function.

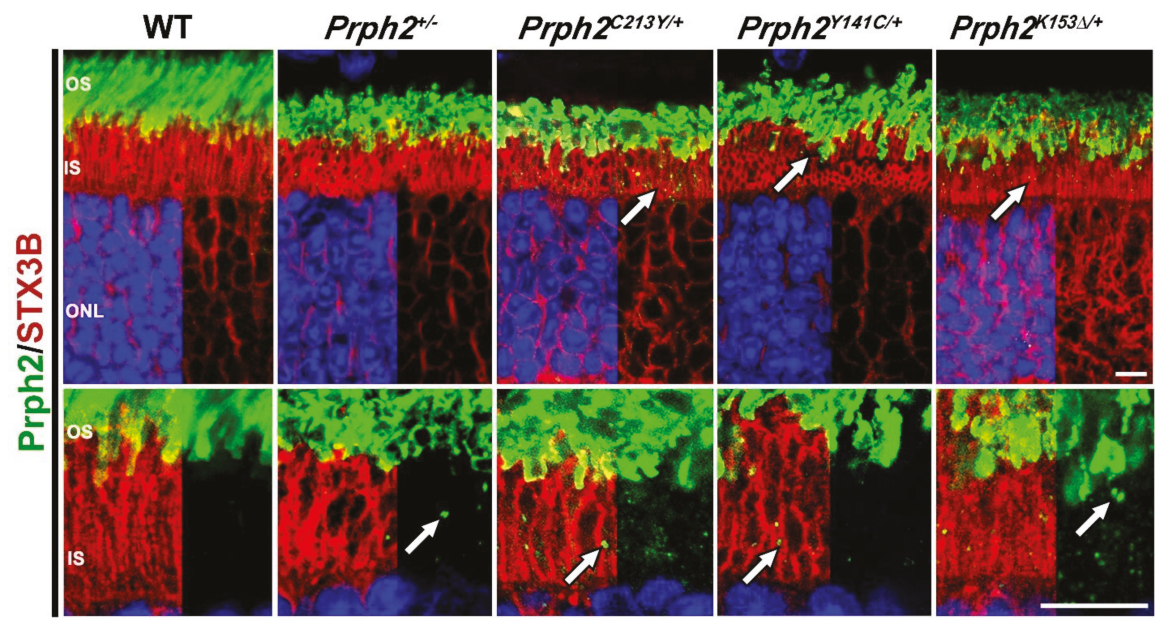

Figure 3. Mutated Prph2 protein traffics to the OS while a small pool is retained in the inner segment. Retinal sections at P30 from the indicated genotypes were probed with antibodies against Prph2 (green) and syntaxin 3B (STX3B) (red). Arrows indicate regions of mislocalization of Prph2. OS, outer segments; IS, inner segment; ONL, outer nuclear layer. Scale bar: $20 \mu \mathrm{m}$. Primary antibodies used for immunostaining were polyclonal antibody against Prph2 C-terminus (Prph2-CT) [22] and monoclonal antibody against STX3B [111] (inner segment marker) diluted at (1:1000). AlexaFluor conjugated secondary antibodies (Alexa 488 Rabbit and Alexa 555 Mouse, Life Technologies/ThermoFisher) were used at a dilution of 1:1000 for 2 hours at room temperature. Images were captured on a ZEISS Confocal LSM 900 microscope equipped with a Zeiss Axiocam (Zeiss, Jena, Germany) using a 63× (oil, 1.4 NA) objective. Images were then processed using ZEN Image Analysis software (Zeiss, Jena, Germany). All images shown are orthogonally projected from an eight slice confocal z-stack.

\subsubsection{Prph $2^{\mathrm{Y} 141 \mathrm{C} /+}$ and $\operatorname{Prph} 2^{\mathrm{Y} 141 \mathrm{C} / \mathrm{Y} 141 \mathrm{C}}$}

Like the C213Y mutation, the Y141C mutation of Prph2 is also located in the D2 loop. The OSs in the Y141C knockin mouse model (Prph2 $2^{Y 141 C /+}$ and Prph2 ${ }^{Y 141 C / 1141 C}$ for heterozygous and homozygous animals, respectively) displayed structural anomalies. In $\operatorname{Prph}^{\mathrm{Y} 141 \mathrm{C} /+}$ mice, the OS was shortened 
with some structural alterations of the discs, including lengthening and vesicular structures [82]. When compared to the $\mathrm{Prph}^{+/-}$mice, the structure was better conserved in the Prph $2^{\mathrm{Y} 141 \mathrm{C} /+}$ retina (Figure 1B). In the Prph2 $2^{Y 141 C / 1141 C}$ mice, OS formation is initialized but neither mature discs nor lamellae could be observed. Instead, the OS contained flattened whorl shaped membranous structures with vesicular arrangements lining up adjacent to them. Scotopic ERG revealed a significant decrease in rod function displayed by the Prph $2^{\mathrm{Y} 141 \mathrm{C} /+}$ mice at P30 (Figure 2). Interestingly, the scotopic ERG did not deteriorate further at P180 [82].

Non-reducing SDS-PAGE/immunoblots of $\mathrm{Prph}^{\mathrm{Y141C/+}}$ retinal lysates showed abnormal high molecular weight aggregates at $250 \mathrm{kDA}$. This was further evaluated by sucrose gradient velocity sedimentation experiments which revealed the formation of the expected intermediate and higher order complexes with an aberrant high molecular weight band indicating that the Y141C mutation results in the formation of abnormal large aggregates [82]. In the Prph2 ${ }^{Y 141 C / Y 141 C}$ retinas, the observed phenotype was even more pronounced with an increase in the aberrant high molecular weight aggregates at the expense of the formation of the normal intermediate and higher order complexes. While in WT mice Rom1 is normally excluded from higher order complexes, in the mutant retina, a portion of Rom1 was incorporated in the aberrant high molecular weight aggregates. Surprisingly though, mutant Prph2 was transported correctly to the OS which is shown by IF staining of Prph2 (green) and IS marker STX3B (red) (Figure 3). However, the formation of these high molecular weight aggregates likely interfered with the normal function of Prph2 and likely Rom1 in the OS. This offers an explanation for the structural and functional phenotypes observed in the knockin mice.

Patients carrying the Y141C mutation in PRPH2 display primarily defects in the macula with some reported cases of more rod-associated phenotypes such as night blindness and RP [30,82,83]. The Prph $2^{Y 141 C /+}$ mice, which represent patients' genotype, exhibit a decline in rods' function, mimicking the observed rod phenotype in some patients. Again, the difference between the knockin model and the patient's phenotypes can be seen in the cone function. The most prominent phenotype found in patients with the Y141C mutation is the functional and structural decline in cones. The Prph $2^{\mathrm{Y} 141 \mathrm{C} / \mathrm{+}}$ mice on the other hand displayed only a slight functional decline in the photopic ERG which was not found to be significant [82], indicating that the cone function in these mice is not severely affected. While the heterozygous mice do not show significant decline in cone function, they do display a flecking in funduscopic analyses [82], which mimics findings in the fundus of patients. Recently, a study was undertaken to identify a potential explanation for the huge variation in phenotypes observed in patients by determining the role Rom1 plays in the observed phenotype [88]. Mice heterozygous or homozygous for the Y141C mutation were crossed with Rom1 knockout mice to produce mice

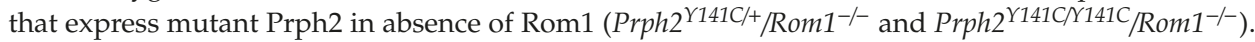
Absence of Rom 1 abolished the formation of the abnormal high molecular weight aggregates and accumulation of mutant Prph2 in the IS and ONL in Prph2 $2^{\gamma 141 C / 1141 C} /$ Rom1 $^{-/-}$retinas [88]. The depletion of Rom1 also changed the symptoms seen in the Y141C model. While the photopic ERG amplitudes in

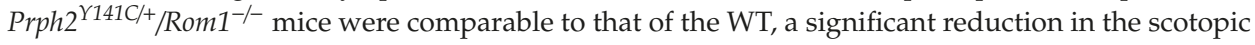
ERG responses were observed [88].

When compared to the cone-rod functional defects noted for the Prph $2^{Y 141 C /+}$ mice, $\operatorname{Prph} 2^{\mathrm{Y} 141 \mathrm{C} /+} / \mathrm{Rom}^{-/-}$mice mainly displayed a rod-dominant functional defect. In addition, the funduscopic anomalies were almost completely abolished in the Prph $2^{\mathrm{Y141C} /+} / \mathrm{Rom}^{-/-}$mice. The results obtained in this study prove that alteration in the level of Rom 1 can change the phenotype caused by a pathogenic Prph 2 mutation, and thus potentially provide an explanation to the variable phenotypes seen in patients carrying the same PRPH2 mutation. Further studies combining Prph2 mutations and mutations in Rom 1 should provide further insights into the complexity of phenotypes seen in the various $P R P H 2$ related diseases. 


\subsection{4. $\operatorname{Prph} 2^{\mathrm{K} 153 \Delta /+}$ and $\operatorname{Prph} 2^{\mathrm{K} 153 \Delta / \mathrm{K} 153 \Delta}$}

Another mutation in PRPH2 that leads to variable phenotypes among patients is the deletion of codon 153 (K153 $\Delta$ ) that results in the elimination of the lysine at position 153 in the D2 loop of Prph2. This mutation is found to associate with RP, pattern dystrophy and fundus flavimaculatus [80]. K153 $\Delta$-Prph2 knockin mouse model was generated and provided evidence that the mutant protein cannot form the complexes required for OS formation [79]. The heterozygous knockin mice $\left(\operatorname{Prph} 2^{\mathrm{K} 153 \Delta /+}\right)$ displayed a shortened OS with minor structural defects at P30 [79] (Figure 1). At P180, these animals also exhibited a reduction in ONL thickness without further structural deterioration of the OS [79]. The overall Prph2 protein level in these mice was around $80 \%$ compared to the level in WT mice, thus demonstrating that the observed structural defects are most likely caused by the dominant effect of the mutant protein rather than due to haploinsufficiency.

The heterozygous animals displayed a significant progressive reduction in scotopic responses that started as early as P30 (Figure 2) and worsened with age. The photopic response in the Prph2 ${ }^{\mathrm{K} 153 \Delta /+}$ was also significantly reduced at P30, albeit this reduction did not worsen as the animals aged (Figure 2). In the homozygous animals, the scotopic and photopic responses were minimal at P30. Biochemical

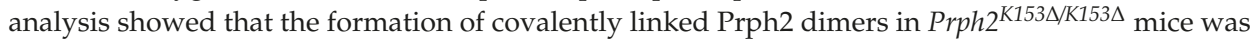
abolished while Rom1 homodimers were present [79].

Due to the observed effects on cone function, $\operatorname{Prph} 2^{\mathrm{K} 153 \Delta /+}$ animals were crossed into the $\mathrm{Nrl}{ }^{-/-}$ background in order to assess the effects of the K153 $\Delta$ mutation on cones. Photoreceptor cells in the resulting $\operatorname{Prph} 2^{\mathrm{K} 153 \mathrm{\Delta} /{ }^{+}} / \mathrm{Nr} l^{-/-}$retina displayed a highly disrupted structure with many photoreceptors having no lamellae what so ever. Interestingly, the mutant Prph2 was able to interact with Rom1 in the rod-dominant $\operatorname{Prph} 2^{\mathrm{K} 153 \Delta / K 153 \Delta}$ retinas, but this interaction was abolished in $\operatorname{Prph} 2^{\mathrm{K} 153 \Delta / K 153 \Delta} / \mathrm{Nrl}{ }^{-/-}$ retina, indicating a defect in the Prph2/Rom1 interaction specific to cones [79]. Sucrose gradient velocity sedimentation showed no significant alteration in complex formation in Prph $2^{\mathrm{K} 153 \Delta /+}$ retina when compared to WT while in the Prph $2^{K 153 \Delta / K 153 \Delta}$ retina, the formation of intermediate and higher order complexes is abolished [79]. Here, both Prph2 and Rom1 are restricted to the tetramer fractions. The same was observed in the $\operatorname{Prph} 2^{\mathrm{K} 153 \Delta / K 153 \Delta} / \mathrm{Nr} l^{-/-}$retinas. Sedimentation profile showed that the amount of higher order complexes in the $\operatorname{Prph} 2^{\mathrm{K} 153 \Delta /+} / \mathrm{Nrl}^{-/-}$retina was reduced, while unaffected in $\operatorname{Prph} 2^{\mathrm{K} 153 \Delta /+}$ retina [79]. This provides further evidence for a differential role of the lysine at position 153 in rods and cones, and hence emphasizes the notion for potential varied roles for Prph2 in rods versus cones. Localization studies performed in Prph $2^{\mathrm{K} 153 \Delta /+}$ mice demonstrated that most of Prph2 and Rom1 were successfully transported to the OS with some amount of Prph2 mislocalized to the IS (arrows in Figure 3). Small amount of rhodopsin (Rho) and M-opsin were also found to be mislocalized to the ONL and outer plexiform layer (OPL) in this model [79].

In the Prph $2^{K 153 \Delta / K 153 \Delta}$ retinas, the majority of Rho and Prph2, but not Rom1, were found to be mislocalized to the ONL. As stated above, patients with the K153 $\Delta$ mutation exhibit a highly variable phenotype, ranging from rod dominant RP to more cone related defects in the macula [80]. The K153 $\Delta$ knockin mouse model displayed functional and structural defects in both rods and cones, and thus mimics the phenotype seen in patients carrying this mutation. While the lack of a macula in the murine retina made it impossible to observe the macular pattern dystrophy often found in patients [80], the knockin mouse still showed funduscopic anomalies [79] which are characteristic of the pattern dystrophy in patients. Prph $2^{\mathrm{K} 153 \Delta /+}$ mice show a flecking in the fundus at P180 which is more severe in the Prph $2^{K 153 \Delta / K 153 \Delta}$ mice. This phenotype does not deteriorate further in P365 heterozygous animals while the flecking was replaced by large splotches in the homozygous animals [79].

Gene supplementation using the NMP mouse that over-express Prph2 (NMP/Prph $2^{K 153 \Delta /+}$ and $N M P / P r p h 2^{K 153 \Delta / K 153 \Delta}$ ) rescued the structural defects but failed to rescue the functional decline seen in scotopic and photopic ERGs [79]. This indicates that the presence of the mutant protein alone is sufficient to deteriorate the photoreceptor function and suggest that gene silencing along with gene augmentation is the best strategy for this model. 
The knockin models for $P R P H 2$ related patient mutations proved to be very useful in highlighting dominant-effects of the Prph 2 mutations. In general, the models were more successful in mimicking patient phenotypes related to a decline in rod function. Patient phenotypes related to functional defects in the cones or pattern dystrophies in the macula were more difficult to reproduce in mice due to the lack of a macula and a lower overall percentage of cones in the murine retina. Crossing the knockin mouse models with $\mathrm{Nrl}^{-/}$mice, as done in the studies with the K153 $\Delta$ model [79], has proven to be a successful approach in studying the effects of the mutation on cones in detail. While the knockin models could not reproduce the macular pattern dystrophy, they successfully reproduced the funduscopic aberrations, which connect with the pattern dystrophy.

\subsubsection{Prph $2^{\mathrm{N} 229 \mathrm{~S} /+}$ and $\operatorname{Prph} 2^{\mathrm{N} 229 \mathrm{~S} / \mathrm{N} 229}$}

The knockin mouse that alters the N-linked glycosylation at asparagine 229 (N229S) in the D2 loop was used to study if Prph2 glycosylation plays a role in its interaction with Rom1. Heterozygous mice $\left(\operatorname{Prph} 2^{\mathrm{N} 229 S /+}\right)$ did not have any significant changes in structure or function of the OS [75]. However, homozygous mice (Prph $2^{\text {N229S/N229S }}$ ) displayed a late onset thinning of the outer nuclear layer (ONL) and occasional abnormal disc staking in cones and a slightly reduced photopic ERG at P180. Since Prph2 could not be glycosylated, higher order complexes were decreased and there was an increase of Prph2 and Rom-1 in the intermediate complexes [75]. Therefore, it was concluded that the glycosylation plays a major part in regulating the interaction between Prph2 and Rom1, which is critical for cone health.

Tables 1 and 2 summarize the phenotypes associated with the Prph2 knockin models, highlighting rod and cone structure, Scotopic and Photopic ERG, complex formation and protein localization. It also summarizes fundus observations, and patient's phenotypes whether mainly rod- or cone-specific defects or the combination of the two. Figures 1 and 2 show structural and functional differences between Prph2 heterozygous knockin mutations, while Figure 3 is a representative IF showing retinal localization of Prph2 among the models.

Table 1. Prph 2 mutations and correlating phenotypes.

\begin{tabular}{|c|c|c|c|c|c|c|}
\hline Genotype & $\operatorname{Prph} 2^{+/-}$ & Prph2-/- & $\operatorname{Prph} 2^{K 153 \Delta /+}$ & $\operatorname{Prph} 2^{K 153 \Delta / K 153 \Delta}$ & Prph2 $2^{Y 141 C /+}$ & Prph2 $2^{Y 141 C / Y 141 C}$ \\
\hline $\begin{array}{c}\text { Mouse Rod } \\
\text { Structure }\end{array}$ & $\begin{array}{l}\text { Short OSs } \\
\text { with whorl } \\
\text { structures } \\
\text { and ONL } \\
\text { thinning at } \\
\text { P180. }\end{array}$ & $\begin{array}{c}\text { No OS } \\
\text { structures. }\end{array}$ & $\begin{array}{c}\text { Short OSs } \\
\text { with whorl } \\
\text { structures } \\
\text { and ONL } \\
\text { thinning at } \\
\text { P180. }\end{array}$ & $\begin{array}{l}\text { Almost no Oss } \\
\text { with rare whorl } \\
\text { structures and } \\
\text { ONL thinning at } \\
\text { P30, more severe } \\
\text { at P180. }\end{array}$ & $\begin{array}{c}\text { Short OS with } \\
\text { longer discs and } \\
\text { accumulation of } \\
\text { vesicular } \\
\text { structures, no } \\
\text { ONL thinning at } \\
\text { P30, ONL } \\
\text { thinning at P180. }\end{array}$ & $\begin{array}{l}\text { Small OS with } \\
\text { flattened whorls } \\
\text { and vesicular } \\
\text { structures at P30, } \\
\text { no OS present at } \\
\text { P180, ONL } \\
\text { thinning at P30, } \\
\text { more severe at } \\
\text { P180. }\end{array}$ \\
\hline $\begin{array}{l}\text { Mouse } \\
\text { Cone } \\
\text { Structure }\end{array}$ & $\begin{array}{l}\text { Oss with } \\
\text { whorl } \\
\text { structures }\end{array}$ & $\begin{array}{l}\text { Open OS } \\
\text { with no } \\
\text { lamella }\end{array}$ & $\begin{array}{l}\text { Occasional } \\
\text { whorl } \\
\text { shaped OS, } \\
\text { mostly open } \\
\text { OS with no } \\
\text { lamella }\end{array}$ & $\begin{array}{c}\text { Occasional COS } \\
\text { seen but mostly } \\
\text { absent. }\end{array}$ & $\begin{array}{c}\text { Abnormal and } \\
\text { short COS } \\
\text { structures. }\end{array}$ & $\begin{array}{c}\text { Occasional COS } \\
\text { seen but mostly } \\
\text { absent. }\end{array}$ \\
\hline $\begin{array}{l}\text { Scotopic } \\
\text { ERG }\end{array}$ & $\begin{array}{l}57 \% \text { and } 33 \% \\
\text { reduction in } \\
\text { a- and } \\
\text { b-wave at } \\
\text { P30. } \\
74 \% \text { and } 48 \% \\
\text { reduction in } \\
\text { a- and } \\
\text { b-waves at } \\
\text { P180, } \\
\text { respectively. }\end{array}$ & $\begin{array}{c}96 \% \text { and } \\
93 \% \\
\text { reduction } \\
\text { in a- and } \\
\text { b-wave at } \\
\text { P30, } \\
\text { respectively. }\end{array}$ & $\begin{array}{c}63 \% \text { and } 49 \% \\
\text { reduction in } \\
\text { a- and } \\
\text { b-wave at } \\
\text { P30. } \\
83 \% \text { and at } \\
60 \% \\
\text { reduction in } \\
\text { a- and } \\
\text { b-wave at } \\
\text { P180, } \\
\text { respectively. }\end{array}$ & $\begin{array}{c}90 \% \text { and } 89 \% \\
\text { reduction in a- } \\
\text { and b-wave at } \\
\text { P30, } \\
\text { respectively. }\end{array}$ & $\begin{array}{c}54 \% \text { and } 27 \% \\
\text { reduction in a- } \\
\text { and b-wave at } \\
\text { P30 } \\
50 \% \text { and } 25 \% \\
\text { reduction in a- } \\
\text { and b-wave at } \\
\text { P180, } \\
\text { respectively. }\end{array}$ & $\begin{array}{c}90 \% \text { and } 78 \% \\
\text { reduction in a- } \\
\text { and b-wave at } \mathrm{P} 30 . \\
95 \% \text { and } 94 \% \\
\text { reduction in a- } \\
\text { and b-wave at } \\
\text { P180, respectively. }\end{array}$ \\
\hline
\end{tabular}


Table 1. Cont.

\begin{tabular}{|c|c|c|c|c|c|c|}
\hline Genotype & Prph2+/- & Prph2-/- & $\operatorname{Prph} 2^{K 153 \Delta /+}$ & $\operatorname{Prph} 2^{K 153 \Delta / K 153 \Delta}$ & $\operatorname{Prph} 2^{1141 C /+}$ & $\operatorname{Prph} 2^{Y 141 C / Y 141 C}$ \\
\hline $\begin{array}{l}\text { Photopic } \\
\text { ERG }\end{array}$ & $\begin{array}{c}\text { Photopic } \\
\text { b-wave } \\
\text { comparable } \\
\text { to WT at P30. } \\
35 \% \\
\text { reduction in } \\
\text { photopic } \\
\text { b-wave at } \\
\text { P180. }\end{array}$ & $\begin{array}{c}91 \% \\
\text { reduction } \\
\text { in b-wave } \\
\text { at } \mathrm{P} 30 .\end{array}$ & $\begin{array}{c}24 \% \\
\text { reduction in } \\
\text { b-wave P30 } \\
\text { and } 50 \% \text { at } \\
\mathrm{P} 180 .\end{array}$ & $\begin{array}{l}64 \% \text { reduction in } \\
\text { b-wave at } \mathrm{P} 30 .\end{array}$ & $\begin{array}{l}10 \% \text { reduction in } \\
\mathrm{b} \text {-wave at } \mathrm{P} 30 \\
\text { and } \mathrm{P} 180 .\end{array}$ & $\begin{array}{c}64 \% \text { reduction in } \\
\text { b-wave at } \mathrm{P} 30 \text { and } \\
90 \% \text { at } \mathrm{P} 180 .\end{array}$ \\
\hline $\begin{array}{l}\text { Complex } \\
\text { formation }\end{array}$ & $\begin{array}{c}\text { Prph2 } \\
\text { complexes } \\
\text { and } \\
\text { distribution } \\
\text { unchanged, } \\
50 \% \text { less } \\
\text { Prph2 and } \\
\text { Rom1. } \\
\text { On NR }{ }^{\mathrm{L}--} \\
\text { background, } \\
\text { higher order } \\
\text { complexes } \\
\text { decreased. }\end{array}$ & $\begin{array}{l}\text { No Prph2 } \\
\text { present, } \\
\text { Rom1 still } \\
\text { present but } \\
\text { at lesser } \\
\text { amount. }\end{array}$ & $\begin{array}{c}\text { Prph2 } \\
\text { complexes } \\
\text { and } \\
\text { distribution } \\
\text { unchanged, } \\
\text { while Rom1 } \\
\text { shifted } \\
\text { towards } \\
\text { tetramers. } \\
\text { On NRL } \text { NR }^{-/} \\
\text {background, } \\
\text { higher order } \\
\text { complexes } \\
\text { decreased, } \\
\text { shift of } \\
\text { Prph2 and } \\
\text { Rom1 } \\
\text { towards } \\
\text { intermediate } \\
\text { complexes. }\end{array}$ & $\begin{array}{l}\text { No Prph2 } \\
\text { dimers were } \\
\text { formed, while } \\
\text { Rom1 dimers } \\
\text { were still } \\
\text { formed. } \\
\text { Prph2 interacted } \\
\text { with Rom1. } \\
\text { Prph2 and Rom1 } \\
\text { restricted to } \\
\text { tetramers. } \\
\text { On NRL-- } \\
\text { background, no } \\
\text { Prph2 dimers } \\
\text { were formed, } \\
\text { while Rom1 } \\
\text { dimers were still } \\
\text { formed. Prph2 } \\
\text { did not interact } \\
\text { with Rom1. } \\
\text { Prph2 and Rom1 } \\
\text { restricted to } \\
\text { tetramers. }\end{array}$ & $\begin{array}{c}\text { Prph2 } \\
\text { occasionally } \\
\text { found in } \\
\text { abnormal high } \\
\text { molecular } \\
\text { weight } \\
\text { aggregates. } \\
\text { Abnormal } \\
\text { aggregates were } \\
\text { held together by } \\
\text { intermolecular } \\
\text { disulfide bonds. } \\
\text { Rom1 also } \\
\text { present in } \\
\text { abnormal } \\
\text { aggregates. } \\
\text { Intermediate } \\
\text { and higher order } \\
\text { complexes } \\
\text { formed but } \\
\text { abnormal high } \\
\text { molecular } \\
\text { weight } \\
\text { aggregates also } \\
\text { present. }\end{array}$ & $\begin{array}{c}\text { Prph2 almost } \\
\text { exclusively found } \\
\text { in abnormal high } \\
\text { molecular weight } \\
\text { aggregates. } \\
\text { Abnormal } \\
\text { aggregates were } \\
\text { held together by } \\
\text { intermolecular } \\
\text { disulfide bonds. } \\
\text { Rom1 also present } \\
\text { in abnormal } \\
\text { aggregates. } \\
\text { Intermediate and } \\
\text { higher order } \\
\text { complexes } \\
\text { reduced in favor } \\
\text { of the abnormal } \\
\text { high molecular } \\
\text { weight aggregates. }\end{array}$ \\
\hline $\begin{array}{c}\text { Protein } \\
\text { localization }\end{array}$ & $\begin{array}{l}\text { Some } \\
\text { rhodopsin } \\
\text { detected in } \\
\text { the IS and } \\
\text { ONL. }\end{array}$ & $\begin{array}{l}\text { Rhodopsin } \\
\text { mislocalized } \\
\text { to IS and } \\
\text { ONL. }\end{array}$ & $\begin{array}{c}\text { Small } \\
\text { amount of } \\
\text { rhodopsin } \\
\text { and M-opsin } \\
\text { mislocalized } \\
\text { in the IS and } \\
\text { ONL. }\end{array}$ & $\begin{array}{l}\text { Huge amount of } \\
\text { rhodopsin and } \\
\text { Prph2 } \\
\text { mislocalized in } \\
\text { the IS and ONL. }\end{array}$ & NA & NA \\
\hline Fundus & $\begin{array}{c}\text { No } \\
\text { abnormality }\end{array}$ & $\begin{array}{l}\text { Flecking } \\
\text { and } \\
\text { splotches } \\
\text { at P360 } \\
\text { and older. }\end{array}$ & $\begin{array}{l}\text { Flecking at } \\
\text { P180 and no } \\
\text { change at } \\
\text { P365. }\end{array}$ & $\begin{array}{l}\text { Severe flecking } \\
\text { at P180 and big } \\
\text { splotches at } \\
\text { P365. }\end{array}$ & Flecking at P180. & Flecking at P180. \\
\hline $\begin{array}{l}\text { Rod defect } \\
\text { in patients }\end{array}$ & NA & NA & $\mathrm{RP}$ & NA & $\begin{array}{l}\text { Night blindness } \\
\text { and RP reported } \\
\text { in some patients. }\end{array}$ & NA \\
\hline $\begin{array}{l}\text { Cone defect } \\
\text { in patients }\end{array}$ & NA & NA & $\begin{array}{c}\text { Pattern } \\
\text { dystrophy } \\
\text { and fundus } \\
\text { flavimaculs. }\end{array}$ & NA & $\begin{array}{c}\text { Pattern } \\
\text { dystrophy } \\
\text { changed fundus } \\
\text { in macula. }\end{array}$ & NA \\
\hline Reference & {$[26,76,79]$} & {$[26,76,79]$} & [79] & [79] & [82] & [82] \\
\hline
\end{tabular}




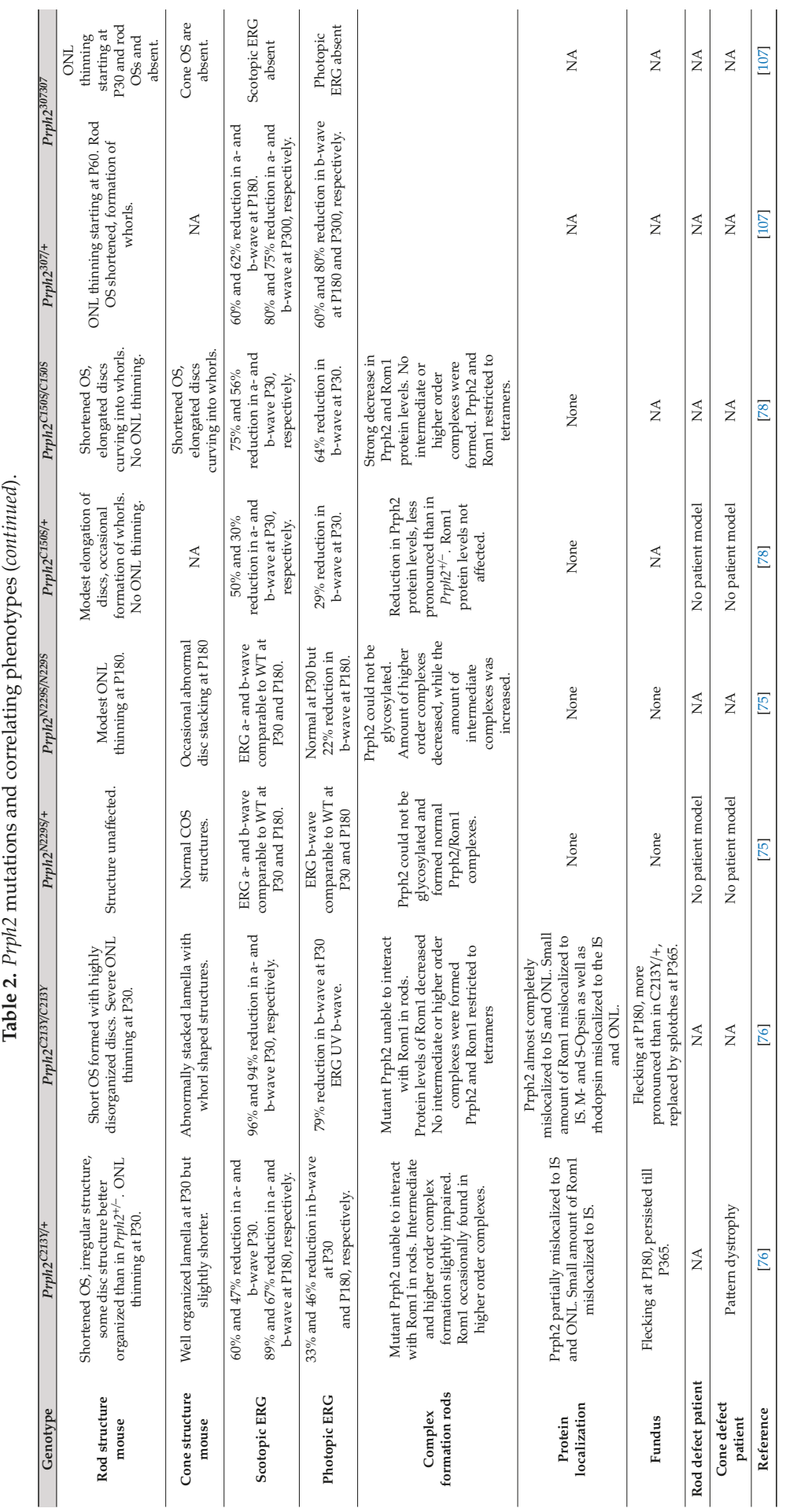




\section{Gene Therapy of PRPH2 Mutations}

There is a vast variety of $P R P H 2$ mutations associated with autosomal dominant retinal degenerative diseases, including RP, several forms of macular dystrophy and cone rod dystrophies [29]. Gene therapy seems to be a promising approach in the treatment of those PRPH2 associated diseases. The small size of the Prph 2 cDNA, which is approximately $1.1 \mathrm{~kb}$, is advantageous since many vectors used for gene therapy can only carry DNA of a limited size. Despite the variety of feasible approaches available for the gene therapy of Prph2, thus far no treatment ready for clinical trials has been developed.

The expression of the WT Prph2 in the background of pathogenic Prph 2 mutations represents one of the most promising approaches. Pioneer studies using transgenic mice expressing WT Prph2 under different promoters in a $\mathrm{Prph} 2^{+/-}$or $\mathrm{Prph}^{-/-}$background revealed a significant increase in both structural and functional properties of rods and cones [105,112]. Replacing Prph2 in Prph $2^{-/}$ mice utilizing adeno-associated virus (AAV) carrying Prph2 regulated under the rhodopsin promoter provided promising results [113]. Here the subretinal injection of the AAV resulted in a partial rescue of ROS structure as well as scotopic ERG response. A follow up study was able to show that repeated injections with the AAV resulted in an even more pronounced rescue of the phenotype with an increase in the scotopic b-wave response observed in the injected mice [114]. In this study, several time points after the injections were analyzed in order to validate whether the observed rescue was long lasting. This analyses revealed that the rescue achieved by the AAV injection was lost after 15 weeks post injection [114]. In addition to the loss of the rescue with time, the amplitude of the scotopic b-wave was significantly lower than in WT mice and the scotopic a-wave was not improved by the injection of the AAV. A reason for this is that the injection of the AAV in mice only resulted in a low transduction rate of roughly $30 \%$ [114]. An improvement in the transduction rate will thus be necessary in order to develop a viable AAV based gene therapy.

Nanoparticles (NP) represent a second promising approach in transferring WT Prph2. These particles were found to be well tolerated by the retina, even after multiple injections, and have a high DNA capacity up to $14 \mathrm{~kb}$, as tested in the eye [115-120]. NP carrying full-length murine Prph2 cDNA under the control of either rod or cone specific IRBP promoters or ubiquitous chicken beta actin promoter was injected in the retina of $\mathrm{Prph}^{+/-}$mice [121]. The injection resulted in a partial rescue of OS structure and also prevented the thinning of the ONL. These rescue effects lasted 15 months post injection and were most pronounced near the site of injection [121]. While the NP treatment could overcome the decline in rescue overtime, the effect on the photoreceptor function remained limited. $\mathrm{NP}$ injection resulted in a small yet insignificant improvement in the scotopic a-wave but a significant improvement in the photopic b-wave. The results obtained with the different promoters used were comparable. The small benefit of the NP injection observed in the functional tests might be due to an incomplete distribution of the NPs in the retina. In line with this, the structural improvements observed after NP injection were best close to the site of injection. Improving on the distribution and uptake of the used vector in the retina, regardless if AAVs or NPs are used, seems to be a necessary next step in order to achieve a more pronounced functional rescue.

The studies above described the replacement of Prph2 in either $\mathrm{Prph}^{+/-}$or Prph2 $2^{-/}$mice, thus in a scenario whereby Prph2 is either absent or haploinsufficient. However, most patients suffer from a dominant mutation in PRPH2. In order to analyze the efficiency of treatment in a scenario closer to actual PRPH2 related diseases, the knockin mouse models carrying a disease related Prph 2 mutation were analyzed. Both $\operatorname{Prph} 2^{\mathrm{K} 153 \Delta /+}$ and $\operatorname{Prph} 2^{\mathrm{C} 213 \mathrm{Y} /+}$ mice were crossed with a normal-Prph2-overexpressing mouse line (NMP) [76,79]. The resulting $\operatorname{Prph} 2^{\mathrm{K} 153 \Delta /+} / \mathrm{NMP}^{+/-}$and $\operatorname{Prph} 2^{\mathrm{C} 213 \mathrm{Y} /+} / \mathrm{NMP}^{+/-}$mice displayed a rescue in OS structure, protein expression levels and trafficking, but no rescue in both rod and cone functions [76]. In both cases, the presence of the mutant protein continues to affect the photoreceptor function. This is due to dominant-gain-of-function effects caused by these mutations. These results show that, in addition to gene augmentation therapy, silencing the mutant Prph2 allele is essential for ultimate rescue. A combined approach of short hairpin (sh)-RNA mediated knockdown and expression of a shRNA resistant protein has been performed 
in models for ADRP carrying pathogenic mutations of rhodopsin [122,123]. Here, both WT and mutant Rhodopsin were knocked down by sh-RNA carried in AAV together with a sh-RNA resistant rhodopsin. The expression of the sh-RNA resistant rhodopsin following the knockdown rescued the phenotype. Studies combining the knockdown of mutant Prph2 with the expression of WT Prph2 provided first promising results thus far showing partial functional and structural rescue in mice expressing pathogenic mutations of Prph2 [124,125].

The examples above show that while progress was made in the gene therapy of $P R P H 2$ related diseases, there are many factors, which need to be considered for the development of a successful therapy. Further complications might arise from the fact that the functional role of Prph2 seems to vary in rods and cones. In addition to that, secondary effects of PRPH2 mutations on the RPE and the choroid could be observed [29-31]. A high variability in the clinical phenotypes displayed by patients, even when carrying the same mutation, represents another challenge, which has to be overcome in order to treat $P R P H 2$ related diseases.

\section{Conclusions and Perspectives}

Prph2 plays a key role in the maintenance as well as the development of photoreceptor OS. While Prph2 is found to be vital for both rods and cones, there seem to be distinct differences in its function in these two types of photoreceptor cells. A complete knockout of Prph2 results in the complete absence of ROS while COS formation is still initialized even though the resulting COS are severely disorganized and lack lamellae and discs. This begs the question, which protein is mediating the formation of the COS and if it works in an interplay with Prph2. The OS of both rods and cones represent a highly modified primary cilium. The Prph2 mediated suppression of ectosomes shedding from the photoreceptor cilium was found to be a prerequisite for the formation of the ROS. Thus far there is no study demonstrating a similar mechanism in the formation of COS. The investigation of the formation of ectosomes and membrane dynamics in the development of COS in WT and Prph $2^{-/-}$ mice might be a potential approach for further studies aiming to unravel the different function of Prph 2 in ROS versus COS. While the function of Prph2 in the initialization of ROS and COS varies, it seems to be indispensable for the correct shaping, sizing and stacking of discs and lamellae in both. Furthermore, not only the presence of Prph 2 is needed for developing and maintaining these structures but also the precise regulation in the formation of the different Prph2/Rom1 and Prph2/Prph2 complexes. Changes in the ratio of the tetramers, intermediate complexes and higher order complexes were proven detrimental to the function as well as the structure of the photoreceptors. The transgenic and knockin mouse models discussed here proved the importance of the precise regulation of the complex formation. Each of the knockin mouse models carrying a pathogenic Prph 2 mutation displayed an altered ratio of the different Prph2/Rom1 and Prph2 complexes resulting in disorganized OS structure and decreased response found in ERG measurements. Interestingly rods and cones were differently affected by the alterations in complex formation, providing further evidence for the differential use of Prph2 by the two photoreceptor cell types. In addition to that, the mouse models used helped identify whether a mutation results in a loss-of-function or a gain-of-function effect.

While there is agreement in the numerous studies concerning the ratio between tetramers, intermediate and higher order complexes and its vital importance for OS structure and function, the precise roles of the different complexes are still not fully understood. In the C150S knockin mouse model, both Prph2 and Rom1 are found exclusively as tetramers, while intermediate and higher order complexes are absent. Still the formation of both ROS and COS is initiated, proving that the tetramers alone are sufficient to initiate OS formation. Homozygous animals fail to form disc and lamellae, indicating that the intermediate and higher order complexes are more likely to be involved in the formation of the rim, membrane closure and disc stacking. The knockout of Rom1 showed minor effects on disc alignment, sizing and stacking while the disc rim formation is unaffected. Rom1 is found in non-covalent hetero-tetramers and in intermediate complexes but it is excluded from higher order Prph 2 complexes. It seems plausible that the Prph2 higher order complexes, which are 
unaffected in the Rom 1 knockout retinas, mediate the membrane curvature and rim closure, while the intermediate hetero complexes support disc spacing, sizing and alignment. Additional studies targeting the formation of the different complexes specifically and analyzing their function in rods and cones are needed to further pinpoint the roles of the different Prph2 and Rom1complexes.

Progress in the development of a feasible gene therapy of Prph2 related diseases has been made in recent years. Transducing the photoreceptors of Prph $2^{-/-}$and $P r p h 2^{+/}$mice either via AAVs or NPs showed a partial rescue of the knockout phenotype, even though the magnitude of the rescue and (in case of the AAVs) the persistence of the rescue effect is still low. The small magnitude of the rescue is most likely due to a low transduction rate of photoreceptors by the vectors used. Improving the transduction rate achieved by the vector as well as its distribution in the eye is one obstacle which needs to be overcome in future attempts. A second obstacle is that pathogenic PRPH2 mutations do not result in a knockout scenario, but instead in a scenario where the mutant protein is still expressed. The knockin mouse models summarized in this review have proven to be a valid approach in understanding how the presence of the mutant protein affects both rod and cone photoreceptors, and also how stable the mutant protein is. In addition, these models helped to identify mutations, which result in dominant effects. Using a sh-RNA-mediated knockdown of both WT and mutant proteins followed by the expression of a sh-RNA resistant form of the WT protein provided a promising approach in the therapy of dominant gain-of-function mutations of Rho and Prph2. The fact that rods and cones utilize Prph2 differently could lead to further complications; this issue needs to be taken into consideration when developing effective therapeutic strategies for $P R P H 2$ related diseases. Testing these strategies in the murine models adds another layer of complication due to the low percentage of cones, which makes it difficult to address cone-dominant phenotypes in mouse models. Analyzing Prph 2 mutations in the $\mathrm{Nrl}^{-/-}$background provides an approach for studying functional and structural effects of mutation specifically on cones. The lack of a macula in the murine retina continues to be the rate limiting factor which prevents conclusive studies aimed at developing therapies for mutations associated with macular defects and pattern dystrophy using mouse models. In addition to this, several PRPH2 mutations were found to cause secondary effects in the choroid and RPE. The reasons for these secondary defects as well as their impact on the pathogenesis of PRPH2 associated diseases are not well understood. Characterizing these secondary defects has to be achieved in future studies in order to devise a successful therapy.

Author Contributions: L.T. wrote original draft of the manuscript. M.S.M. performed electroretinography and histology. M.K. performed immunohistochemistry, developed the tables and participate in the writing. M.I.N., M.R.A.-U. designed the experiments, reviewed and edited the manuscript. All authors have read and agreed on the final version of the manuscript.

Funding: This research is supported by a grant from the National Institutes of Health (R01 EY10609-MIN, MRA).

Conflicts of Interest: The authors declare no conflicts of interest.

\section{References}

1. Hemler, M.E. Tetraspanin functions and associated microdomains. Nat. Rev. Mol. Cell Biol. 2005, 6, 801-811. [CrossRef]

2. Levy, S.; Shoham, T. The tetraspanin web modulates immune-signalling complexes. Nat. Rev. Immunol. 2005, 5, 136-148. [CrossRef] [PubMed]

3. Charrin, S.; Jouannet, S.; Boucheix, C.; Rubinstein, E. Tetraspanins at a glance. J. Cell Sci. 2014, 127, 3641-3648. [CrossRef]

4. Van Deventer, S.J.; Dunlock, V.E.; van Spriel, A.B. Molecular interactions shaping the tetraspanin web. Biochem. Soc. Trans. 2017, 45, 741-750. [CrossRef] [PubMed]

5. Boucheix, C.; Rubinstein, E. Tetraspanins. Cell. Mol. Life Sci. Cmls 2001, 58, 1189-1205. [CrossRef] [PubMed]

6. Hemler, M.E. Tetraspanin proteins mediate cellular penetration, invasion, and fusion events and define a novel type of membrane microdomain. Annu. Rev. Cell Dev. Biol. 2003, 19, 397-422. [CrossRef] 
7. Kitadokoro, K.; Bordo, D.; Galli, G.; Petracca, R.; Falugi, F.; Abrignani, S.; Grandi, G.; Bolognesi, M. CD81 extracellular domain 3D structure: Insight into the tetraspanin superfamily structural motifs. Embo. J. 2001, 20, 12-18. [CrossRef]

8. Murru, L.; Moretto, E.; Martano, G.; Passafaro, M. Tetraspanins shape the synapse. Mol. Cell. Neurosci. 2018, 91, 76-81. [CrossRef]

9. Stipp, C.S.; Kolesnikova, T.V.; Hemler, M.E. Functional domains in tetraspanin proteins. Trends Biochem. Sci. 2003, 28, 106-112. [CrossRef]

10. DeSalle, R.; Mares, R.; Garcia-España, A. Evolution of cysteine patterns in the large extracellular loop of tetraspanins from animals, fungi, plants and single-celled eukaryotes. Mol. Phylogenetics Evol. 2010, 56, 486-491. [CrossRef]

11. Yang, X.; Kovalenko, O.V.; Tang, W.; Claas, C.; Stipp, C.S.; Hemler, M.E. Palmitoylation supports assembly and function of integrin-tetraspanin complexes. J. Cell Biol. 2004, 167, 1231-1240. [CrossRef]

12. Skubitz, K.M.; Campbell, K.D.; Skubitz, A.P. CD63 associates with CD11/CD18 in large detergent-resistant complexes after translocation to the cell surface in human neutrophils. Febs Lett. 2000, 469, 52-56. [CrossRef]

13. Claas, C.; Stipp, C.S.; Hemler, M.E. Evaluation of prototype transmembrane 4 superfamily protein complexes and their relation to lipid rafts. J. Biol. Chem. 2001, 276, 7974-7984. [CrossRef] [PubMed]

14. Hemler, M.E. Specific tetraspanin functions. J. Cell Biol. 2001, 155, 1103-1107. [CrossRef] [PubMed]

15. Molday, R.S.; Hicks, D.; Molday, L. Peripherin. A rim-specific membrane protein of rod outer segment discs. Invest. Ophthalmol. Vis. Sci. 1987, 28, 50-61. [PubMed]

16. Cheng, T.; Peachey, N.S.; Li, S.; Goto, Y.; Cao, Y.; Naash, M.I. The effect of peripherin/rds haploinsufficiency on rod and cone photoreceptors. J. Neurosci. Off. J. Soc. Neurosci. 1997, 17, 8118-8128. [CrossRef]

17. Arikawa, K.; Molday, L.L.; Molday, R.S.; Williams, D.S. Localization of peripherin/rds in the disk membranes of cone and rod photoreceptors: Relationship to disk membrane morphogenesis and retinal degeneration. J. Cell Biol. 1992, 116, 659-667. [CrossRef] [PubMed]

18. Wrigley, J.D.; Ahmed, T.; Nevett, C.L.; Findlay, J.B. Peripherin/rds influences membrane vesicle morphology. Implications for retinopathies. J. Biol. Chem. 2000, 275, 13191-13194. [CrossRef]

19. Boesze-Battaglia, K. Fusion between retinal rod outer segment membranes and model membranes: Functional assays and role for peripherin/rds. Methods Enzymol. 2000, 316, 65-86.

20. Conley, S.M.; Cai, X.; Naash, M.I. Nonviral ocular gene therapy: Assessment and future directions. Curr. Opin. Mol. Ther. 2008, 10, 456-463.

21. Goldberg, A.F.; Molday, R.S. Subunit composition of the peripherin/rds-rom-1 disk rim complex from rod photoreceptors: Hydrodynamic evidence for a tetrameric quaternary structure. Biochemistry 1996, 35, 6144-6149. [CrossRef] [PubMed]

22. Ding, X.Q.; Stricker, H.M.; Naash, M.I. Role of the second intradiscal loop of peripherin/rds in homo and hetero associations. Biochemistry 2005, 44, 4897-4904. [CrossRef] [PubMed]

23. Chakraborty, D.; Conley, S.M.; Stuck, M.W.; Naash, M.I. Differences in RDS trafficking, assembly and function in cones versus rods: Insights from studies of C150S-RDS. Hum. Mol. Genet. 2010, 19, 4799-4812. [CrossRef] [PubMed]

24. Bascom, R.A.; Manara, S.; Collins, L.; Molday, R.S.; Kalnins, V.I.; McInnes, R.R. Cloning of the cDNA for a novel photoreceptor membrane protein (rom-1) identifies a disk rim protein family implicated in human retinopathies. Neuron 1992, 8, 1171-1184. [CrossRef]

25. Kevany, B.M.; Tsybovsky, Y.; Campuzano, I.D.; Schnier, P.D.; Engel, A.; Palczewski, K. Structural and functional analysis of the native peripherin-ROM1 complex isolated from photoreceptor cells. J. Biol. Chem. 2013, 288, 36272-36284. [CrossRef]

26. Stuck, M.W.; Conley, S.M.; Naash, M.I. PRPH2/RDS and ROM-1: Historical context, current views and future considerations. Prog Retin Eye Res. 2016, 52, 47-63. [CrossRef]

27. Clarke, G.; Goldberg, A.F.; Vidgen, D.; Collins, L.; Ploder, L.; Schwarz, L.; Molday, L.L.; Rossant, J.; Szel, A.; Molday, R.S.; et al. Rom-1 is required for rod photoreceptor viability and the regulation of disk morphogenesis. Nat. Genet. 2000, 25, 67-73. [CrossRef]

28. Chakraborty, D.; Ding, X.Q.; Conley, S.M.; Fliesler, S.J.; Naash, M.I. Differential requirements for retinal degeneration slow intermolecular disulfide-linked oligomerization in rods versus cones. Hum. Mol. Genet. 2009, 18, 797-808. [CrossRef] 
29. Boon, C.J.; den Hollander, A.I.; Hoyng, C.B.; Cremers, F.P.; Klevering, B.J.; Keunen, J.E. The spectrum of retinal dystrophies caused by mutations in the peripherin/RDS gene. Prog Retin Eye Res. 2008, 27, 213-235. [CrossRef]

30. Francis, P.J.; Schultz, D.W.; Gregory, A.M.; Schain, M.B.; Barra, R.; Majewski, J.; Ott, J.; Acott, T.; Weleber, R.G.; Klein, M.L. Genetic and phenotypic heterogeneity in pattern dystrophy. Br. J. Ophthalmol. 2005, 89, 1115-1119. [CrossRef]

31. Gocho, K.; Akeo, K.; Itoh, N.; Kameya, S.; Hayashi, T.; Katagiri, S.; Gekka, T.; Ohkuma, Y.; Tsuneoka, H.; Takahashi, H. High-Resolution Adaptive Optics Retinal Image Analysis at Early Stage Central Areolar Choroidal Dystrophy With PRPH2 Mutation. Ophthalmic Surg. Lasers Imaging Retin. 2016, 47, 1115-1126. [CrossRef] [PubMed]

32. Jansen, H.G.; Sanyal, S. Development and degeneration of retina in rds mutant mice: Electron microscopy. J. Comp. Neurol. 1984, 224, 71-84. [CrossRef] [PubMed]

33. Salinas, R.Y.; Pearring, J.N.; Ding, J.D.; Spencer, W.J.; Hao, Y.; Arshavsky, V.Y. Photoreceptor discs form through peripherin-dependent suppression of ciliary ectosome release. J. Cell Biol. 2017, 216, 1489-1499. [CrossRef] [PubMed]

34. Conley, S.M.; Stuck, M.W.; Watson, J.N.; Zulliger, R.; Burnett, J.L.; Naash, M.I. Prph2 initiates outer segment morphogenesis but maturation requires Prph2/Rom1 oligomerization. Hum. Mol. Genet. 2019, 28, 459-475. [CrossRef]

35. May-Simera, H.; Nagel-Wolfrum, K.; Wolfrum, U. Cilia - The sensory antennae in the eye. Prog. Retin. Eye Res. 2017, 60, 144-180. [CrossRef]

36. Sorokin, S. Centrioles and the formation of rudimentary cilia by fibroblasts and smooth muscle cells. J. Cell Biol. 1962, 15, 363-377. [CrossRef]

37. Sedmak, T.; Wolfrum, U. Intraflagellar transport proteins in ciliogenesis of photoreceptor cells. Biol. Cell 2011, 103, 449-466. [CrossRef]

38. Pearring, J.N.; Salinas, R.Y.; Baker, S.A.; Arshavsky, V.Y. Protein sorting, targeting and trafficking in photoreceptor cells. Prog. Retin. Eye Res. 2013, 36, 24-51. [CrossRef]

39. Chakraborty, D.; Conley, S.M.; Al-Ubaidi, M.R.; Naash, M.I. Initiation of rod outer segment disc formation requires RDS. PLoS ONE 2014, 9, e98939. [CrossRef]

40. Wang, J.; Silva, M.; Haas, L.A.; Morsci, N.S.; Nguyen, K.C.; Hall, D.H.; Barr, M.M. C. elegans ciliated sensory neurons release extracellular vesicles that function in animal communication. Curr. Biol. 2014, 24, 519-525. [CrossRef]

41. Cao, M.; Ning, J.; Hernandez-Lara, C.I.; Belzile, O.; Wang, Q.; Dutcher, S.K.; Liu, Y.; Snell, W.J. Uni-directional ciliary membrane protein trafficking by a cytoplasmic retrograde IFT motor and ciliary ectosome shedding. eLife 2015, 4, e05242. [CrossRef] [PubMed]

42. Wang, J.; Barr, M.M. Ciliary Extracellular Vesicles: Txt Msg Organelles. Cell. Mol. Neurobiol. 2016, 36, 449-457. [CrossRef] [PubMed]

43. Nager, A.R.; Goldstein, J.S.; Herranz-Perez, V.; Portran, D.; Ye, F.; Garcia-Verdugo, J.M.; Nachury, M.V. An Actin Network Dispatches Ciliary GPCRs into Extracellular Vesicles to Modulate Signaling. Cell 2017, 168, 252-263.e214. [CrossRef] [PubMed]

44. Phua, S.C.; Chiba, S.; Suzuki, M.; Su, E.; Roberson, E.C.; Pusapati, G.V.; Setou, M.; Rohatgi, R.; Reiter, J.F.; Ikegami, K.; et al. Dynamic Remodeling of Membrane Composition Drives Cell Cycle through Primary Cilia Excision. Cell 2017, 168, 264-279.e215. [CrossRef] [PubMed]

45. Boesze-Battaglia, K.; Lamba, O.P.; Napoli, A.A., Jr.; Sinha, S.; Guo, Y.; Boesze-Battagliaa, K.; Stefano, F.P.; Milstein, M.L.; Kimler, V.A.; Ghatak, C.; et al. Fusion between retinal rod outer segment membranes and model membranes: A role for photoreceptor peripherin/rds Peripherin/rds fusogenic function correlates with subunit assembly An inducible amphipathic helix within the intrinsically disordered C terminus can participate in membrane curvature generation by peripherin-2/rds. Biochemistry 1998, 37, 9477-9487. [PubMed]

46. Boesze-Battagliaa, K.; Stefano, F.P. Peripherin/rds fusogenic function correlates with subunit assembly. Exp. Eye Res. 2002, 75, 227-231. [CrossRef]

47. Khattree, N.; Ritter, L.M.; Goldberg, A.F. Membrane curvature generation by a C-terminal amphipathic helix in peripherin-2/rds, a tetraspanin required for photoreceptor sensory cilium morphogenesis. J. Cell Sci. 2013, 126, 4659-4670. [CrossRef] 
48. Milstein, M.L.; Kimler, V.A.; Ghatak, C.; Ladokhin, A.S.; Goldberg, A.F.X. An inducible amphipathic helix within the intrinsically disordered $C$ terminus can participate in membrane curvature generation by peripherin-2/rds. J. Biol. Chem. 2017, 292, 7850-7865. [CrossRef]

49. Steinberg, R.H.; Fisher, S.K.; Anderson, D.H. Disc morphogenesis in vertebrate photoreceptors. J. Comp. Neurol. 1980, 190, 501-508. [CrossRef]

50. Ding, J.D.; Salinas, R.Y.; Arshavsky, V.Y. Discs of mammalian rod photoreceptors form through the membrane evagination mechanism. J. Cell Biol. 2015, 211, 495-502. [CrossRef]

51. Miraglia, S.; Godfrey, W.; Yin, A.H.; Atkins, K.; Warnke, R.; Holden, J.T.; Bray, R.A.; Waller, E.K.; Buck, D.W. A novel five-transmembrane hematopoietic stem cell antigen: Isolation, characterization, and molecular cloning. Blood 1997, 90, 5013-5021. [CrossRef] [PubMed]

52. Weigmann, A.; Corbeil, D.; Hellwig, A.; Huttner, W.B. Prominin, a novel microvilli-specific polytopic membrane protein of the apical surface of epithelial cells, is targeted to plasmalemmal protrusions of non-epithelial cells. Proc. Natl. Acad. Sci. USA 1997, 94, 12425-12430. [CrossRef]

53. Yin, A.H.; Miraglia, S.; Zanjani, E.D.; Almeida-Porada, G.; Ogawa, M.; Leary, A.G.; Olweus, J.; Kearney, J.; Buck, D.W. AC133, a novel marker for human hematopoietic stem and progenitor cells. Blood 1997, 90, 5002-5012. [CrossRef]

54. Maw, M.A.; Corbeil, D.; Koch, J.; Hellwig, A.; Wilson-Wheeler, J.C.; Bridges, R.J.; Kumaramanickavel, G.; John, S.; Nancarrow, D.; Roper, K.; et al. A frameshift mutation in prominin (mouse)-like 1 causes human retinal degeneration. Hum. Mol. Genet. 2000, 9, 27-34. [CrossRef] [PubMed]

55. Han, Z.; Anderson, D.W.; Papermaster, D.S. Prominin-1 localizes to the open rims of outer segment lamellae in Xenopus laevis rod and cone photoreceptors. Investig. Ophthalmol. Vis. Sci. 2012, 53, 361-373. [CrossRef] [PubMed]

56. Yang, Z.; Chen, Y.; Lillo, C.; Chien, J.; Yu, Z.; Michaelides, M.; Klein, M.; Howes, K.A.; Li, Y.; Kaminoh, Y.; et al. Mutant prominin 1 found in patients with macular degeneration disrupts photoreceptor disk morphogenesis in mice. J. Clin. Investig. 2008, 118, 2908-2916. [CrossRef]

57. Zacchigna, S.; Oh, H.; Wilsch-Brauninger, M.; Missol-Kolka, E.; Jaszai, J.; Jansen, S.; Tanimoto, N.; Tonagel, F.; Seeliger, M.; Huttner, W.B.; et al. Loss of the cholesterol-binding protein prominin-1/CD133 causes disk dysmorphogenesis and photoreceptor degeneration. J. Neurosci. Off. J. Soc. Neurosci. 2009, 29, 2297-2308. [CrossRef]

58. Collison, F.T.; Fishman, G.A.; Nagasaki, T.; Zernant, J.; McAnany, J.J.; Park, J.C.; Allikmets, R. Characteristic Ocular Features in Cases of Autosomal Recessive PROM1 Cone-Rod Dystrophy. Investig. Ophthalmol. Vis. Sci. 2019, 60, 2347-2356. [CrossRef]

59. Liang, J.; She, X.; Chen, J.; Zhai, Y.; Liu, Y.; Zheng, K.; Gong, Y.; Zhu, H.; Luo, X.; Sun, X. Identification of novel PROM1 mutations responsible for autosomal recessive maculopathy with rod-cone dystrophy. Graefe's Arch. Clin. Exp. Ophthalmol. 2019, 257, 619-628. [CrossRef]

60. Huttner, W.B.; Zimmerberg, J. Implications of lipid microdomains for membrane curvature, budding and fission. Curr. Opin. Cell Biol. 2001, 13, 478-484. [CrossRef]

61. Iglic, A.; Hagerstrand, H.; Veranic, P.; Plemenitas, A.; Kralj-Iglic, V. Curvature-induced accumulation of anisotropic membrane components and raft formation in cylindrical membrane protrusions. J. Theor. Biol. 2006, 240, 368-373. [CrossRef] [PubMed]

62. Cohen, A.I. Further studies on the question of the patency of saccules in outer segments of vertebrate photoreceptors. Vis. Res. 1970, 10, 445-453. [CrossRef]

63. Anderson, D.H.; Fisher, S.K.; Steinberg, R.H. Mammalian cones: Disc shedding, phagocytosis, and renewal. Investig. Ophthalmol. Vis. Sci. 1978, 17, 117-133. [PubMed]

64. Bunt, A.H. Fine structure and radioautography of rabbit photoreceptor cells. Investig. Ophthalmol. Vis. Sci. 1978, 17, 90-104

65. Carter-Dawson, L.D.; LaVail, M.M. Rods and cones in the mouse retina. I. Structural analysis using light and electron microscopy. J. Comp. Neurol. 1979, 188, 245-262. [CrossRef]

66. Mears, A.J.; Kondo, M.; Swain, P.K.; Takada, Y.; Bush, R.A.; Saunders, T.L.; Sieving, P.A.; Swaroop, A. Nrl is required for rod photoreceptor development. Nat. Genet. 2001, 29, 447-452. [CrossRef]

67. Daniele, L.L.; Lillo, C.; Lyubarsky, A.L.; Nikonov, S.S.; Philp, N.; Mears, A.J.; Swaroop, A.; Williams, D.S.; Pugh, E.N., Jr. Cone-like morphological, molecular, and electrophysiological features of the photoreceptors of the Nrl knockout mouse. Investig. Ophthalmol. Vis. Sci. 2005, 46, 2156-2167. [CrossRef] 
68. Conley, S.M.; Al-Ubaidi, M.R.; Han, Z.; Naash, M.I. Rim formation is not a prerequisite for distribution of cone photoreceptor outer segment proteins. FASEB J. 2014, 28, 3468-3479. [CrossRef]

69. Farjo, R.; Skaggs, J.S.; Nagel, B.A.; Quiambao, A.B.; Nash, Z.A.; Fliesler, S.J.; Naash, M.I. Retention of function without normal disc morphogenesis occurs in cone but not rod photoreceptors. J. Cell Biol. 2006, 173, 59-68. [CrossRef]

70. Moritz, O.L.; Molday, R.S. Molecular cloning, membrane topology, and localization of bovine rom-1 in rod and cone photoreceptor cells. Investig. Ophthalmol. Vis. Sci 1996, 37, 352-362.

71. Conley, S.M.; Stuck, M.W.; Naash, M.I. Structural and functional relationships between photoreceptor tetraspanins and other superfamily members. Cell. Mol. Life Sci. 2012, 69, 1035-1047. [CrossRef] [PubMed]

72. Loewen, C.J.; Molday, R.S. Disulfide-mediated oligomerization of Peripherin/Rds and Rom-1 in photoreceptor disk membranes. Implications for photoreceptor outer segment morphogenesis and degeneration. J. Biol. Chem. 2000, 275, 5370-5378. [CrossRef] [PubMed]

73. Tian, G.; Ropelewski, P.; Nemet, I.; Lee, R.; Lodowski, K.H.; Imanishi, Y. An unconventional secretory pathway mediates the cilia targeting of peripherin/rds. J. Neurosci. Off. J. Soc. Neurosci. 2014, 34, 992-1006. [CrossRef] [PubMed]

74. Chakraborty, D.; Ding, X.Q.; Fliesler, S.J.; Naash, M.I. Outer segment oligomerization of Rds: Evidence from mouse models and subcellular fractionation. Biochemistry 2008, 47, 1144-1156. [CrossRef] [PubMed]

75. Stuck, M.W.; Conley, S.M.; Naash, M.I. Retinal Degeneration Slow (RDS) Glycosylation Plays a Role in Cone Function and in the Regulation of RDS.ROM-1 Protein Complex Formation. J. Biol. Chem. 2015, 290, 27901-27913. [CrossRef] [PubMed]

76. Chakraborty, D.; Strayve, D.G.; Makia, M.S.; Conley, S.M.; Kakahel, M.; Al-Ubaidi, M.R.; Naash, M.I. Novel molecular mechanisms for Prph2-associated pattern dystrophy. FASEB J. 2020, 34, 1211-1230. [CrossRef]

77. Goldberg, A.F.; Loewen, C.J.; Molday, R.S. Cysteine residues of photoreceptor peripherin/rds: Role in subunit assembly and autosomal dominant retinitis pigmentosa. Biochemistry 1998, 37, 680-685. [CrossRef]

78. Zulliger, R.; Conley, S.M.; Mwoyosvi, M.L.; Al-Ubaidi, M.R.; Naash, M.I. Oligomerization of Prph2 and Rom1 is essential for photoreceptor outer segment formation. Hum. Mol. Genet. 2018, 27, 3507-3518. [CrossRef]

79. Chakraborty, D.; Conley, S.M.; Zulliger, R.; Naash, M.I. The K153Del PRPH2 mutation differentially impacts photoreceptor structure and function. Hum. Mol. Genet. 2016, 25, 3500-3514. [CrossRef]

80. Weleber, R.G.; Carr, R.E.; Murphey, W.H.; Sheffield, V.C.; Stone, E.M. Phenotypic variation including retinitis pigmentosa, pattern dystrophy, and fundus flavimaculatus in a single family with a deletion of codon 153 or 154 of the peripherin/RDS gene. Arch. Ophthalmol. 1993, 111, 1531-1542. [CrossRef]

81. Conley, S.M.; Stuck, M.W.; Burnett, J.L.; Chakraborty, D.; Azadi, S.; Fliesler, S.J.; Naash, M.I. Insights into the mechanisms of macular degeneration associated with the R172W mutation in RDS. Hum. Mol. Genet. 2014, 23, 3102-3114. [CrossRef] [PubMed]

82. Stuck, M.W.; Conley, S.M.; Naash, M.I. The Y141C knockin mutation in RDS leads to complex phenotypes in the mouse. Hum. Mol. Genet. 2014, 23, 6260-6274. [CrossRef] [PubMed]

83. Khani, S.C.; Karoukis, A.J.; Young, J.E.; Ambasudhan, R.; Burch, T.; Stockton, R.; Lewis, R.A.; Sullivan, L.S.; Daiger, S.P.; Reichel, E.; et al. Late-onset autosomal dominant macular dystrophy with choroidal neovascularization and nonexudative maculopathy associated with mutation in the RDS gene. Investig. Ophthalmol. Vis. Sci. 2003, 44, 3570-3577. [CrossRef] [PubMed]

84. Kedzierski, W.; Lloyd, M.; Birch, D.G.; Bok, D.; Travis, G.H. Generation and analysis of transgenic mice expressing P216L-substituted rds/peripherin in rod photoreceptors. Investig. Ophthalmol. Vis. Sci. 1997, 38, 498-509.

85. Stricker, H.M.; Ding, X.Q.; Quiambao, A.; Fliesler, S.J.; Naash, M.I. The Cys ${ }^{214} \rightarrow$ Ser mutation in peripherin/rds causes a loss-of-function phenotype in transgenic mice. Biochem. J. 2005, 388, 605-613. [CrossRef]

86. Ding, X.Q.; Nour, M.; Ritter, L.M.; Goldberg, A.F.; Fliesler, S.J.; Naash, M.I. The R172W mutation in peripherin/rds causes a cone-rod dystrophy in transgenic mice. Hum. Mol. Genet. 2004, 13, 2075-2087. [CrossRef]

87. Conley, S.; Nour, M.; Fliesler, S.J.; Naash, M.I. Late-onset cone photoreceptor degeneration induced by R172W mutation in Rds and partial rescue by gene supplementation. Investig. Ophthalmol. Vis. Sci. 2007, 48, 5397-5407. [CrossRef] 
88. Conley, S.M.; Stuck, M.W.; Watson, J.N.; Naash, M.I. Rom1 converts Y141C-Prph2-associated pattern dystrophy to retinitis pigmentosa. Hum. Mol. Genet. 2017, 26, 509-518. [CrossRef]

89. Kedzierski, W.; Bok, D.; Travis, G.H. Transgenic analysis of rds/peripherin N-glycosylation: Effect on dimerization, interaction with rom1, and rescue of the rds null phenotype. J. Neurochem. 1999, 72, 430-438. [CrossRef]

90. Poetsch, A.; Molday, L.L.; Molday, R.S. The cGMP-gated channel and related glutamic acid-rich proteins interact with peripherin-2 at the rim region of rod photoreceptor disc membranes. J. Biol. Chem. 2001, 276, 48009-48016. [CrossRef]

91. Ritter, L.M.; Khattree, N.; Tam, B.; Moritz, O.L.; Schmitz, F.; Goldberg, A.F. In situ visualization of protein interactions in sensory neurons: Glutamic acid-rich proteins (GARPs) play differential roles for photoreceptor outer segment scaffolding. J. Neurosci. Off. J. Soc. Neurosci. 2011, 31, 11231-11243. [CrossRef] [PubMed]

92. Zhang, Y.; Molday, L.L.; Molday, R.S.; Sarfare, S.S.; Woodruff, M.L.; Fain, G.L.; Kraft, T.W.; Pittler, S.J. Knockout of GARPs and the beta-subunit of the rod cGMP-gated channel disrupts disk morphogenesis and rod outer segment structural integrity. J. Cell Sci. 2009, 122, 1192-1200. [CrossRef] [PubMed]

93. Gilliam, J.C.; Chang, J.T.; Sandoval, I.M.; Zhang, Y.; Li, T.; Pittler, S.J.; Chiu, W.; Wensel, T.G. Three-dimensional architecture of the rod sensory cilium and its disruption in retinal neurodegeneration. Cell 2012,151,1029-1041. [CrossRef] [PubMed]

94. Becirovic, E.; Nguyen, O.N.; Paparizos, C.; Butz, E.S.; Stern-Schneider, G.; Wolfrum, U.; Hauck, S.M.; Ueffing, M.; Wahl-Schott, C.; Michalakis, S.; et al. Peripherin-2 couples rhodopsin to the CNG channel in outer segments of rod photoreceptors. Hum. Mol. Genet. 2014, 23, 5989-5997. [CrossRef] [PubMed]

95. Chakraborty, D.; Conley, S.M.; Pittler, S.J.; Naash, M.I. Role of RDS and Rhodopsin in Cngb1-Related Retinal Degeneration. Investig. Ophthalmol. Vis. Sci. 2016, 57, 787-797. [CrossRef] [PubMed]

96. Wroblewski, J.J.; Wells, J.A., 3rd; Eckstein, A.; Fitzke, F.; Jubb, C.; Keen, T.J.; Inglehearn, C.; Bhattacharya, S.; Arden, G.B.; Jay, M.; et al. Macular dystrophy associated with mutations at codon 172 in the human retinal degeneration slow gene. Ophthalmology 1994, 101, 12-22. [CrossRef]

97. Piguet, B.; Heon, E.; Munier, F.L.; Grounauer, P.A.; Niemeyer, G.; Butler, N.; Schorderet, D.F.; Sheffield, V.C.; Stone, E.M. Full characterization of the maculopathy associated with an Arg-172-Trp mutation in the RDS/peripherin gene. Ophthalmic Genet. 1996, 17, 175-186. [CrossRef]

98. Payne, A.M.; Downes, S.M.; Bessant, D.A.; Bird, A.C.; Bhattacharya, S.S. Founder effect, seen in the British population, of the 172 peripherin/RDS mutation-and further refinement of genetic positioning of the peripherin/RDS gene. Am. J. Hum. Genet. 1998, 62, 192-195. [CrossRef]

99. Downes, S.M.; Fitzke, F.W.; Holder, G.E.; Payne, A.M.; Bessant, D.A.; Bhattacharya, S.S.; Bird, A.C. Clinical features of codon 172 RDS macular dystrophy: Similar phenotype in 12 families. Arch. Ophthalmol. 1999, 117, 1373-1383. [CrossRef]

100. Saga, M.; Mashima, Y.; Akeo, K.; Oguchi, Y.; Kudoh, J.; Shimizu, N. A novel Cys-214-Ser mutation in the peripherin/RDS gene in a Japanese family with autosomal dominant retinitis pigmentosa. Hum. Genet. 1993, 92, 519-521. [CrossRef]

101. Kajiwara, K.; Hahn, L.B.; Mukai, S.; Travis, G.H.; Berson, E.L.; Dryja, T.P. Mutations in the human retinal degeneration slow gene in autosomal dominant retinitis pigmentosa. Nature 1991, 354, 480-483. [CrossRef]

102. Kajiwara, K.; Berson, E.L.; Dryja, T.P. Digenic retinitis pigmentosa due to mutations at the unlinked peripherin/RDS and ROM1 loci. Science 1994, 264, 1604-1608. [CrossRef] [PubMed]

103. Dryja, T.P.; Hahn, L.B.; Kajiwara, K.; Berson, E.L. Dominant and digenic mutations in the peripherin/RDS and ROM1 genes in retinitis pigmentosa. Investig. Ophthalmol. Vis. Sci. 1997, 38, 1972-1982. [PubMed]

104. Kedzierski, W.; Nusinowitz, S.; Birch, D.; Clarke, G.; McInnes, R.R.; Bok, D.; Travis, G.H. Deficiency of rds/peripherin causes photoreceptor death in mouse models of digenic and dominant retinitis pigmentosa. Proc. Natl. Acad. Sci. USA 2001, 98, 7718-7723. [CrossRef] [PubMed]

105. Nour, M.; Ding, X.Q.; Stricker, H.; Fliesler, S.J.; Naash, M.I. Modulating expression of peripherin/rds in transgenic mice: Critical levels and the effect of overexpression. Investig. Ophthalmol. Vis. Sci. 2004, 45, 2514-2521. [CrossRef] [PubMed]

106. Apfelstedt-Sylla, E.; Theischen, M.; Ruther, K.; Wedemann, H.; Gal, A.; Zrenner, E. Extensive intrafamilial and interfamilial phenotypic variation among patients with autosomal dominant retinal dystrophy and mutations in the human RDS/peripherin gene. Br. J. Ophthalmol. 1995, 79, 28-34. [CrossRef] 
107. McNally, N.; Kenna, P.F.; Rancourt, D.; Ahmed, T.; Stitt, A.; Colledge, W.H.; Lloyd, D.G.; Palfi, A.; O’Neill, B.; Humphries, M.M.; et al. Murine model of autosomal dominant retinitis pigmentosa generated by targeted deletion at codon 307 of the rds-peripherin gene. Hum. Mol. Genet. 2002, 11, 1005-1016. [CrossRef]

108. Nichols, B.E.; Sheffield, V.C.; Vandenburgh, K.; Drack, A.V.; Kimura, A.E.; Stone, E.M. Butterfly-shaped pigment dystrophy of the fovea caused by a point mutation in codon 167 of the RDS gene. Nat. Genet. 1993, 3, 202-207. [CrossRef]

109. Fossarello, M.; Bertini, C.; Galantuomo, M.S.; Cao, A.; Serra, A.; Pirastu, M. Deletion in the peripherin/RDS gene in two unrelated Sardinian families with autosomal dominant butterfly-shaped macular dystrophy. Arch. Ophthalmol. 1996, 114, 448-456. [CrossRef]

110. Zhang, K.; Garibaldi, D.C.; Li, Y.; Green, W.R.; Zack, D.J. Butterfly-shaped pattern dystrophy: A genetic, clinical, and histopathological report. Arch. Ophthalmol. 2002, 120, 485-490. [CrossRef]

111. Zulliger, R.; Conley, S.M.; Mwoyosvi, M.L.; Stuck, M.W.; Azadi, S.; Naash, M.I. SNAREs Interact with Retinal Degeneration Slow and Rod Outer Segment Membrane Protein-1 during Conventional and Unconventional Outer Segment Targeting. PLoS ONE 2015, 10, e0138508. [CrossRef] [PubMed]

112. Travis, G.H.; Groshan, K.R.; Lloyd, M.; Bok, D. Complete rescue of photoreceptor dysplasia and degeneration in transgenic retinal degeneration slow (rds) mice. Neuron 1992, 9, 113-119. [CrossRef]

113. Ali, R.R.; Sarra, G.M.; Stephens, C.; Alwis, M.D.; Bainbridge, J.W.; Munro, P.M.; Fauser, S.; Reichel, M.B.; Kinnon, C.; Hunt, D.M.; et al. Restoration of photoreceptor ultrastructure and function in retinal degeneration slow mice by gene therapy. Nat. Genet. 2000, 25, 306-310. [CrossRef] [PubMed]

114. Schlichtenbrede, F.C.; da Cruz, L.; Stephens, C.; Smith, A.J.; Georgiadis, A.; Thrasher, A.J.; Bainbridge, J.W.; Seeliger, M.W.; Ali, R.R. Long-term evaluation of retinal function in Prph2Rd2/Rd2 mice following AAV-mediated gene replacement therapy. J. Gene Med. 2003, 5, 757-764. [CrossRef] [PubMed]

115. Ding, X.Q.; Quiambao, A.B.; Fitzgerald, J.B.; Cooper, M.J.; Conley, S.M.; Naash, M.I. Ocular delivery of compacted DNA-nanoparticles does not elicit toxicity in the mouse retina. PLoS ONE 2009, 4, e7410. [CrossRef]

116. Cai, X.; Conley, S.M.; Nash, Z.; Fliesler, S.J.; Cooper, M.J.; Naash, M.I. Gene delivery to mitotic and postmitotic photoreceptors via compacted DNA nanoparticles results in improved phenotype in a mouse model of retinitis pigmentosa. FASEB J. 2010, 24, 1178-1191. [CrossRef]

117. Han, Z.; Conley, S.M.; Makkia, R.S.; Cooper, M.J.; Naash, M.I. DNA nanoparticle-mediated ABCA4 delivery rescues Stargardt dystrophy in mice. J. Clin. Investig. 2012, 122, 3221-3226. [CrossRef]

118. Han, Z.; Conley, S.M.; Makkia, R.; Guo, J.; Cooper, M.J.; Naash, M.I. Comparative analysis of DNA nanoparticles and AAVs for ocular gene delivery. PLoS ONE 2012, 7, e52189. [CrossRef]

119. Han, Z.; Koirala, A.; Makkia, R.; Cooper, M.J.; Naash, M.I. Direct gene transfer with compacted DNA nanoparticles in retinal pigment epithelial cells: Expression, repeat delivery and lack of toxicity. Nanomedicine 2012, 7, 521-539. [CrossRef]

120. Conley, S.M.; Naash, M.I. Gene therapy for PRPH2-associated ocular disease: Challenges and prospects. Cold Spring Harb. Perspect. Med. 2014, 4, a017376. [CrossRef]

121. Cai, X.; Nash, Z.; Conley, S.M.; Fliesler, S.J.; Cooper, M.J.; Naash, M.I. A partial structural and functional rescue of a retinitis pigmentosa model with compacted DNA nanoparticles. PLoS ONE 2009, 4, e5290. [CrossRef] [PubMed]

122. Mao, H.; Gorbatyuk, M.S.; Rossmiller, B.; Hauswirth, W.W.; Lewin, A.S. Long-term rescue of retinal structure and function by rhodopsin RNA replacement with a single adeno-associated viral vector in $\mathrm{P} 23 \mathrm{H} \mathrm{RHO}$ transgenic mice. Hum. Gene Ther. 2012, 23, 356-366. [CrossRef] [PubMed]

123. Cideciyan, A.V.; Sudharsan, R.; Dufour, V.L.; Massengill, M.T.; Iwabe, S.; Swider, M.; Lisi, B.; Sumaroka, A.; Marinho, L.F.; Appelbaum, T.; et al. Mutation-independent rhodopsin gene therapy by knockdown and replacement with a single AAV vector. Proc. Natl. Acad. Sci. USA 2018, 115, E8547-E8556. [CrossRef] [PubMed] 
124. Palfi, A.; Ader, M.; Kiang, A.S.; Millington-Ward, S.; Clark, G.; O’Reilly, M.; McMahon, H.P.; Kenna, P.F.; Humphries, P.; Farrar, G.J. RNAi-based suppression and replacement of rds-peripherin in retinal organotypic culture. Hum. Mutat. 2006, 27, 260-268. [CrossRef]

125. Petrs-Silva, H.; Yasumura, D.; Matthes, M.T.; LaVail, M.M.; Lewin, A.S.; Hauswirth, W.W. Suppression of rds expression by siRNA and gene replacement strategies for gene therapy using rAAV vector. Adv. Exp. Med. Biol. 2012, 723, 215-223.

(C) 2020 by the authors. Licensee MDPI, Basel, Switzerland. This article is an open access article distributed under the terms and conditions of the Creative Commons Attribution (CC BY) license (http://creativecommons.org/licenses/by/4.0/). 



\title{
Phosphoinositides in Retinal Function and Disease
}

\author{
Theodore G. Wensel
}

Verna and Marrs McLean Department of Biochemistry and Molecular Biology, Baylor College of Medicine, Houston, TX 77030, USA; twensel@bcm.edu

Received: 1 March 2020; Accepted: 30 March 2020; Published: 2 April 2020

\begin{abstract}
Phosphatidylinositol and its phosphorylated derivatives, the phosphoinositides, play many important roles in all eukaryotic cells. These include modulation of physical properties of membranes, activation or inhibition of membrane-associated proteins, recruitment of peripheral membrane proteins that act as effectors, and control of membrane trafficking. They also serve as precursors for important second messengers, inositol $(1,4,5)$ trisphosphate and diacylglycerol. Animal models and human diseases involving defects in phosphoinositide regulatory pathways have revealed their importance for function in the mammalian retina and retinal pigmented epithelium. New technologies for localizing, measuring and genetically manipulating them are revealing new information about their importance for the function and health of the vertebrate retina.
\end{abstract}

Keywords: phosphoinositides; retinal lipids; membrane trafficking

\section{Introduction}

Phosphoinositides are membrane phospholipids with the six-member cyclic polyol myo-inositol, $(\mathrm{CHOH})_{6}$, or O-phosphorylated forms of inositol, as their headgroup (Figure 1). The phosphorylated forms are of low abundance in eukaryotic cells of all types, generally comprising $1 \%$ or less of total phospholipid. Nevertheless, they play critical roles in cellular regulation, and defects in their synthesis and regulation lead to devastating diseases [1-6]. The retina is clearly no exception to this generality, but surprisingly few details have been worked out in what is arguably one of the most extensively studied tissues in our bodies, about the regulation and regulatory roles of retinal phosphoinositides. Recent technological advances make it possible to make substantial advances in this field in the next few years. 


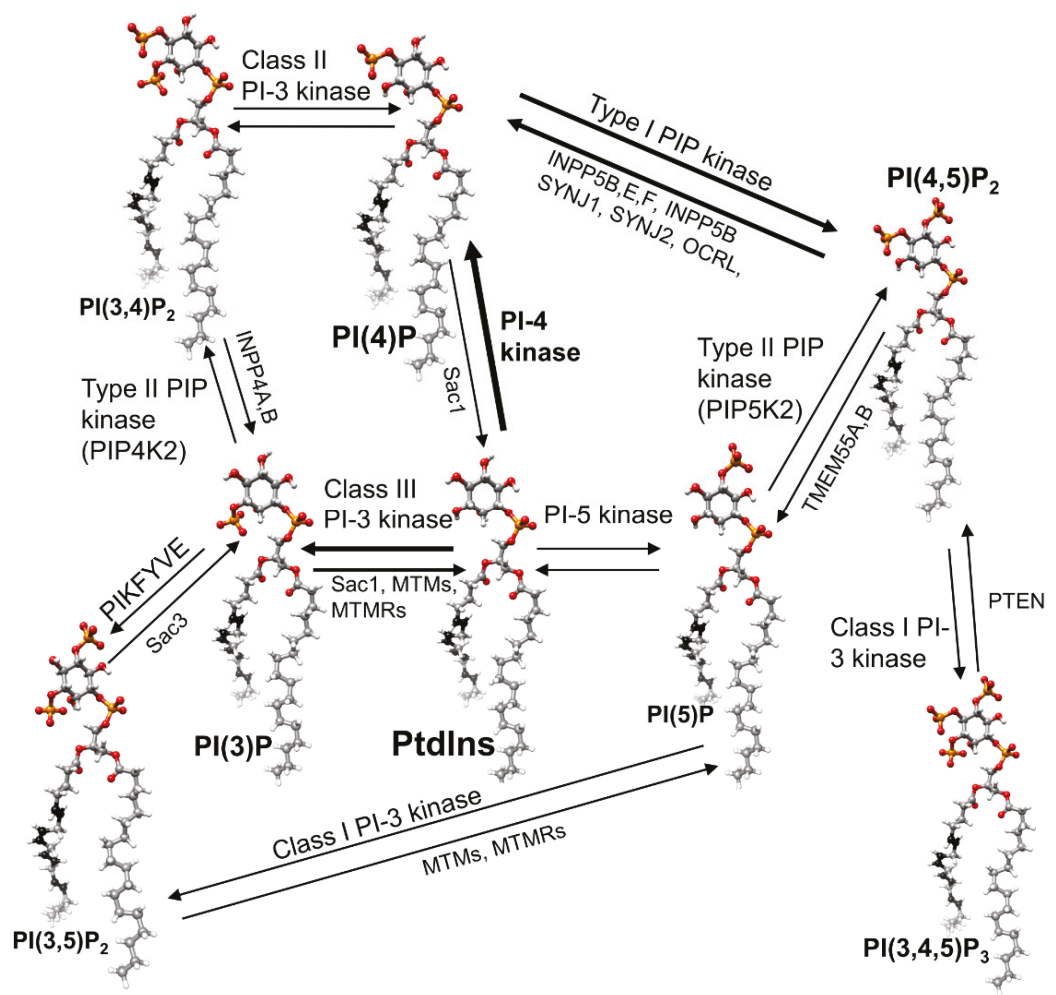

Figure 1. Structures of the cellular phosphoinositides and enzymes responsible for their synthesis and interconversion. Phosphoinositides (PI) species are shown with the acyl chains most commonly found on phosphatidylinositol, which is the starting point for all the others, arachidonic acid (20:4) and stearic acid (C18). Relative font sizes correlate with relative abundance.

\section{Chemical Structures of Phosphoinositides}

Phosphoinositides (abbreviated here as "PI") include phosphatidylinositol (PtdIns), in which the $1^{\prime}$-position of the inositol ring is attached via a phosphodiester bond to the $s n-3$ position of $(1,2)$ diacyl glycerol, and derivatives of PtdIns with one, two, or three phosphates attached in various combinations to the $4^{\prime}, 5^{\prime}$ or $3^{\prime}$ hydroxyls of PtdIns. Including PtdIns, a total of eight different PI head groups are commonly found in eukaryotic cells (Figure 1) [4,5].

\section{Phosphatidylinositol Content in the Retina and RPE}

PtdIns is a relatively substantial component of the membranes of most cells in metazoans, with mole fractions ranging from $\sim 4 \%-20 \%$ of total phospholipid [4]. Early reports stated that retina phospholipids contained 4.4\%-6.4\% PtdIns across six different mammalian species [7-9]. Interestingly, rod outer segment (ROS) membranes were found to have much lower levels, $1.5 \%$ to $2.5 \%$ in bovine and $2.1 \%$ in frog [10-13]. Retinal pigment epithelial cells (RPE), in contrast, have higher levels of PtdIns, at $6.5 \%$ of total phospholipid [14], while ER membranes isolated from bovine retinas contain $9.6 \%$ PtdIns [10]. The general picture that emerges is that total PtdIns content in total retinal membranes outside of outer segments and that of RPE are more or less "normal" as compared to other tissues and cell types, consistent with the notion that widespread roles of PI are likely to be conserved in many retinal cell types. In contrast, ROS have an unusually low PtdIns content, suggesting different roles 
for this lipid class in that organelle. This idea is consistent with findings discussed below, indicating different lipid compositions in plasma membranes as compared to ciliary membranes.

\section{Content of Minor Phosphoinositides in the Retina and RPE}

As a negatively charged lipid, which typically contains one polyunsaturated side chain fatty acid, such as arachidonic acid [8], PtdIns has a major impact on the physical properties of the membrane domains containing it. In contrast, the phosphorylated forms are much less abundant, and exert their influence on cell physiology largely through interactions with proteins with high-affinity and high-specificity PI-binding domains [15-17]. There have been few measurements of phosphorylated PI in retina or cells isolated from retina, although there have been several papers addressing the turnover of PtdIns and other PI, or activity of enzymes involved in PI metabolism [18-21]. The likely reason for the absence of information on PI levels is their low abundance and the lack of sensitivity provided by conventional methods for lipid analysis. More sensitive techniques have been developed recently, including one based on recombinant phosphoinositide-binding domains fused to an epitope tag, allowing sensitive detection by enzyme-linked immunosorbent assays (ELISAs) and measurement of chemiluminescence [22]. This technique was used to quantify $\mathrm{PI}(3) \mathrm{P}$ and $\mathrm{PI}(3,4,5) \mathrm{P}_{3}$ in preparations of rod cells that contain both outer segments and fragments of the inner segments and demonstrated levels of PI(3)P at $0.0035 \mathrm{~mol} \%$ of total phospholipid under illumination conditions that yielded the highest levels of that lipid, and at least 10 -fold lower (i.e., undetectable) levels of $\mathrm{PI}(3,4,5) \mathrm{P}_{3}$. PI(4)P and $\mathrm{PI}(4,5) \mathrm{P}_{2}$ are, in general, found at much higher levels than the 3-phosphorylated PI, but even those appear to be present in rods at very low levels, which are only about 10-fold higher than the levels of $\mathrm{PI}(3) \mathrm{P}$, i.e., on the order of $0.04 \mathrm{~mol} \%$ (He and Wensel, unpublished observations).

\section{Comparison to Other Tissues and Cell Types}

These numbers are comparable to those found in other eukaryotic cells, reported as PtdIns3P, 0.002\% of phospholipid mass; PtdIns4P, 0.05\%; PtdIns5P, 0.002\%; PtdIns(4,5)P, 0.05\%; PtdIns(3,4)P, $0.0001 \%$; PtdIns $(3,5) \mathrm{P}, 0.0001 \%$ [17], see also references in [23]. A more recent mass spectrometry study reported PI $(3,4,5) 3$ levels as 50 -fold or more lower than those of the more common PIs in mammalian U87MG cells [24]. An interesting observation derived from measurements of PI levels in cultured cells is that, generally, they do not change greatly upon activation with extracellular stimuli; for the more common forms, PtdIns, $\mathrm{PI}(4) \mathrm{P}, \mathrm{PI}(4,5) \mathrm{P}_{2}$ and $\mathrm{PI}(3) \mathrm{P}$, the change is generally less than $30 \%$ [17]. The implication, for $\mathrm{PI}(4,5) \mathrm{P}_{2}$, which is rapidly degraded to form $\mathrm{InsP}_{3}$ and diacylglycerol upon activation of phospholipase $C$ isozymes by G-protein-coupled receptors or growth factor receptors $[25,26]$, is that homeostatic mechanisms are in place to regenerate rapidly the pools of both $\mathrm{PI}(4,5) \mathrm{P}_{2}$ and its precursor PI(4)P upon PLC activation. In contrast, $\mathrm{PI}(3,4,5) \mathrm{P}_{3}$ level changes due to receptor stimulation are generally too low to have a major impact on levels of its precursor, $\mathrm{PI}(4,5) \mathrm{P}_{2}$.

\section{Importance of PI Generally}

Despite their low abundance, phosphoinositides play major roles in regulation of cell signaling and membrane dynamics, and are essential for control of a wide range of processes including development, proliferation and differentiation, membrane excitability, exocytosis, phagocytosis, cell motility, and detection of extracellular signals [4]. These functional roles are primarily mediated by a plethora of enzymes, scaffold proteins and complex-nucleating proteins containing phosphoinositide-binding domains of high affinity and high specificity. Genetic defects in the enzymes responsible for their regulation are, in most cases, lethal at the embryo stage, except for some cases of redundancy (i.e., more than one gene encoding enzymes or PI-binding proteins with similar activities). Cell-type specific deletion of these enzymes leads to more specific pathologies, e.g., neurodegeneration in the case of the type III PI-3 kinase, Vps34 (also known as PIK3C3) [27,28]. Some of these enzymes, such as type I PI-3 kinases, can act as oncogenes when mutations disrupt their regulation, and are considered prime targets for cancer chemotherapy, whereas others, such as the phosphoinositide phosphatase, PTEN, 
are considered tumor suppressor genes [29]; these therapeutic targets are present in the retina, where their roles in retinal function, and any effects of drugs targeting them are unknown.

\section{Importance of Phosphoinositides for Dynamics and Functions of Membranes in Retina and RPE}

Among the most important roles of phosphoinositides and their protein effectors are those mediating intracellular membrane traffic, by directing membrane proteins and lipids from one compartment to another in response to cellular needs and changing environments. For example, PI(3)P is found in early and recycling endosomes, and is important for recruiting key proteins that regulate trafficking to these compartments [30]. It is also important for autophagy, a survival-promoting pathway leading to the lysosomal degradation of organelles [31]. PI(4)P is enriched in the Golgi apparatus, and is thought to be important for membrane trafficking through the Golgi compartments and from the Golgi to the plasma membrane and other subcellular compartments [32], likely including disk membranes. $\mathrm{PI}(4,5) \mathrm{P}_{2}$ plays a key role in clathrin-mediated endocytosis, and serves to direct many effector proteins to the plasma membrane where it is primarily found [33].

$\mathrm{PI}(4,5) \mathrm{P}_{2}$ and other phosphoinositides directly regulate the activity of ion channels, transporters and enzymes in membranes [34,35]. In addition, $\mathrm{PI}(4,5) \mathrm{P}_{2}$ serves as the substrate for phosphoinositide-specific phospholipase $\mathrm{C}$, leading to production of the important second messengers, inositol $(1,4,5)$ trisphosphate $\left(\mathrm{InsP}_{3}\right)$ and diacylglycerol $[25,26,36,37]$. Phosphoinositides are also critically important for regulating interactions between membranes and cytoskeletal elements [38,39]. Changes in these interactions are critical for cell growth and mobility, and for remodeling of intracellular structures. They may well play a role in cytoskeleton-dependent disk morphogenesis in rods.

\section{Membrane Trafficking in Retina and RPE}

Every aspect of retinal biology depends heavily on the correct organization and composition of highly specialized membranes, from the unique disc membranes of the photoreceptor sensory cilia, to the ribbon synapses of rods, cones, and bipolar cells, to the apical processes of RPE (retinal pigmented epithelium) cells, uniquely tuned to the detection and engulfment of shed outer segment fragments. The formation, maintenance, and functions of these membranes rely heavily on the phosphorylated phosphoinositides [2-4,40]. Despite the intense interest in phosphoinositide research in recent decades, surprisingly little is known about their regulation and functional roles in the retina, although it is known that disruption of phosphoinositide regulation can lead to blindness in human patients and animal models [41-48]. The membranes and pathways they regulate are known to be essential for the function and health of the retina as well as for disease processes and cellular responses to disease states.

One of the reasons for the dearth of knowledge has been a lack of tools for studying these very low-abundance lipids within the multiple cell types of the retina and the adjacent retinal pigment epithelium. Recently, tools developed by the broader PI field have begun to be applied to the unique challenges and opportunities posed by the retina [22,49]. Doing so will have enormous impact on our understanding of the cell biology of the retina and its disruption in disease. This can help to inform the design and optimization of therapies aimed at treating and preventing retinal dysfunction and degeneration.

\section{Features of the Retina that Make It Ideal for Studies of Phosphoinositide Regulation In Vivo}

The field of phosphoinositide regulation has long been dominated by studies in cultured immortalized cell lines, giving rise to a critical need for elucidation of their physiological regulation in terminally differentiated neurons. For this reason, in vivo studies have broad significance for this field. There are several features of the retina that make it particularly amenable to studies of phosphoinositide regulation in vivo. These include the ability to assay both structure and function non-invasively, the ease of making cell-type-specific knockouts, the ability to isolate rod cells for either biochemical analysis or ultrastructure determination, an extensive understanding of biochemistry and cell biology, 
especially of rods, which exceeds that of any of other vertebrate neurons, and a wealth of knowledge of RPE cell biology.

\section{Importance of Phosphoinositides for Membrane Trafficking in Retina}

Despite years of study, no convincing evidence has accumulated for an important role for phosphoinositides in the phototransduction cascade. In contrast, a steady stream of evidence supports a central role for these lipids in membrane trafficking and sorting in all mammalian cell types, just as a critical role in photoreceptors for membrane sorting and trafficking has long been established $[3,50,51]$. A review article in 2011 covered advances in understanding the roles of phosphoinositides in photoreceptors [6]. It is highly likely that events such as endocytosis and exocytosis, endosomal sorting, membrane budding, post-Golgi vesicle trafficking, and disk morphogenesis all depend on phosphoinositide dynamics, and published reports support a role for $\mathrm{PI}(3) \mathrm{P}$ and $\mathrm{PI}(4,5) \mathrm{P}_{2}$ in rhodopsin trafficking [52,53]. For example, $\mathrm{PI}(4,5) \mathrm{P}_{2}$-binding proteins, ezrin and moesin, were reported to colocalize with Rac1 and Rab8 on rhodopsin transport carrier vesicles at the site of their fusion with the plasma membrane. A recent report suggesting the involvement of actin-nucleating proteins Arp2/Arp3 in basal disc extension [54] potentially implicates local pools or $\mathrm{PI}(4,5) \mathrm{P}_{2}$, which are known to be critical for their function $[55,56]$.

In cone photoreceptors, ablation of a type I PI-3 kinase leads to enhanced sensitivity to light damage [57]. Mutations in the phosphoinositide phosphatase, synaptojanin 1 lead to defects in synaptic vesicle trafficking in cone cells [44].

\section{Retinal Cilia and Phosphoinositides}

Phosphoinositides, and especially $\mathrm{PI}(4,5) \mathrm{P}_{2}$ and $\mathrm{PI}(4) \mathrm{P}$, have been proposed to play important roles in assembly, disassembly, and regulation of primary cilia [52,58-62]. The lipid content of cilia is different from that of the plasma membrane, and a membrane diffusion barrier surrounding the cilium has been demonstrated (see [63] for a review). Two PI-5-phosphatases, INPP5E and OCRL, essential for cilium function have been reported to be localized to the cilium $[41,64-66]$

\section{Phosphoinositides and the BBSome}

The BBSome, a heterooctameric protein complex involved in ciliary trafficking, whose defects lead to the blinding ciliopathy, Bardet-Biedl syndrome, binds to membranes, and shows a preference for acidic lipids, including phosphoinositides [67,68], and isolated BBS5 which contains pleckstrin homology domains, binds to phosphoinositides, especially PI(3)P [68]. More recently, it was shown that a core BBSome complex containing BBS 1, 4, 5, 8, 9 and 18 and a smaller sub-complex lacking BBS1 and BBS5 bind phosphoinositides with similar specificities. A caveat for these studies is that the commercial "PIP strips" used have local surface densities of phosphoinositides that far exceed anything found under physiological conditions, so further investigation of phosphoinositide-binding of the BBSome and its sub-complexes is warranted.

\section{Autophagy and other Stress Responses Involving Redirection of Membrane Traffic and Phosphoinositides}

It has been reported that light exposure induces elements of the autophagy pathway in rods and that autophagy plays an important role in photoreceptor homeostasis [69-73]. This pathway has been suggested to be a neuroprotective one that forestalls apoptosis under conditions of stress [69,74]. This process may be part of a more general neuroprotective response involving re-direction of membrane traffic and phosphoinositides. As discussed below, Synaptojanin-1 has been implicated in autophagy in zebrafish cones [75]. 


\section{Evidence for Effects of Light on Phosphoinositide Metabolism}

A number of early reports in the 1980s suggested that light had measurable impacts on PI metabolism in photoreceptors or the retina generally. Based on measurement of ${ }^{32} \mathrm{Pi}$ incorporated into PtdIns by metabolic labeling, it was reported that exposure of isolated frog retina to light decreased levels of PIP 2 by $14 \%$ after $5 \mathrm{~s}$ and $37 \%$ after $15 \mathrm{~s}$, while levels of PI(4)P, PtdIns and other acidic lipids remained essentially constant [76]. Subsequent publications from the same group reported increased levels of ${ }^{3} \mathrm{H}$ inositol and ${ }^{32} \mathrm{P}$ into phosphoinositides upon illumination [77]. They also reported $\mathrm{PI}(4,5) \mathrm{P}_{2}$-specific phospholipase C (PLC) activity in frog photoreceptors, and PLC immunoreactivity in bovine rod outer segments [78,79], as well as PI-kinase and PIP-kinase activities in frog ROS [19]. Another group found that exposure of rat retinas to light led to decreased staining of rod outer segments with anti-PI $(4,5) \mathrm{P}_{2}$ antibodies $[80,81]$. The caveats of those experiments are that it is known that physical properties of outer segment membranes and their protein composition are altered by bright light exposure, and that it is difficult to establish the specificity of such antibody staining. In addition to light, reports on regulation of photoreceptor PLC by $\mathrm{Ca}^{2+}$ [82] or by subunits of the phototransduction $\mathrm{G}$ protein, transducin [83], were published, also suggesting a possible influence of light exposure, which is known to control levels of $\mathrm{Ca}^{2+}$ and active and inactive forms of transducin subunits.

In contrast, another group [84] reported that bovine rod outer segments have very little PIP kinase activity as compared to the rest of the retina (consistent with the previously reported low level of $\mathrm{PI}(4,5) \mathrm{P}_{2}$ in outer segment membranes), and that light adaptation had no measurable effect on phosphoinositide metabolism as compared to in vivo dark adaptation. Yet another group, using metabolic labeling with $\left[{ }^{3} \mathrm{H}\right]$ inositol, reported that light led to decreases in $\mathrm{PIP}_{2}$ levels without generation of Ins $\mathrm{P}_{3}$, suggesting light-dependent activity of a phosphatase rather than of phospholipase $C$ [85]. In vitro studies demonstrated that the presence of $\mathrm{PI}(4,5) \mathrm{P}_{2}$ in membranes could affect the activity of components of the phototransduction cascade [86,87], including the cGMP-gated cation channel and the cGMP phosphodiesterase-transducin complex. The physiological relevance of effects of $\mathrm{PI}(4,5) \mathrm{P}_{2}$ on phototransduction or of effects of light on $\mathrm{PI}(4,5) \mathrm{P}_{2}$ metabolism remains untested, and it seems to be possible to explain the entire time-course of rod light responses without invoking any participation by phosphoinositide metabolism [88]. Antibodies specific for isoforms of phospholipase $C$ or $G_{\alpha q / 11}$ PLC-coupled subunits revealed the presence of PLC $\beta 4$ and $G_{\alpha q / 11}$ in rod outer segments, and $G_{\alpha q}$ and other PKC isoforms elsewhere in the retina [89]. The functional roles of these proteins in outer segments are not known. There have been suggestions that slower effects of prolonged light exposure, such as arrestin translocation from the inner to outer segments of rod cells, may be mediated by a phospholipase $\mathrm{C}$ cascade [90].

\section{Light Regulation of PI-3 Kinase}

Interest has turned toward Type I PI-3 kinase and PI(3,4,5) $\mathrm{P}_{3}$, with reports of effects of light on the activity of this enzyme in bovine rod outer segments [91,92], which were reported to be mediated by light-stimulated activation and tyrosine phosphorylation of the insulin receptor [93-95]. Deletion of the p $85 \alpha$ regulatory subunit of Type I PI-3 kinase in cone cells resulted in progressive degeneration of cones, without observable effects on rod survival [57]. Likewise, cone-specific inactivation of the gene encoding the $\mathrm{p} 110 \alpha$ catalytic subunit also resulted in defects in cone survival [96].

In contrast, Type I PI-3 kinase and its product, $\mathrm{PI}(3,4,5) \mathrm{P}_{3}$, seem to be much less important for rod function. Rod-specific ablation of the p $85 \alpha$ gene using two different rod-specific Cre transgenes yielded no obvious defects in retinal morphology or rod cell survival [22,97], although modest effects on kinetics of light response recovery and arrestin translocation were reported in one case [97]. Quantitative analysis of $\mathrm{PI}(3,4,5) \mathrm{P}_{3}$ levels in rods isolated from dark-adapted or light-adapted retinas revealed levels of this phosphoinositide of more than one order magnitude lower than those found for PI(3)P in the light, or at least two orders of magnitude lower than light-stimulated levels of $\mathrm{PI}(4,5) \mathrm{P}_{2}$ [22]. 


\section{2. $\mathrm{PI}(4,5) \mathrm{P}_{2}$ and Phospholipase $\mathrm{C}$ in Intrinsically Photosensitive Ganglion Cells}

In addition to image-forming light detection mediated by rods and cones, the vertebrate retina also contains intrinsically photosensitive retinal ganglion cells. These contain phototransduction cascades reminiscent of that found in invertebrate rhabdomeric photoreceptors [98-104]. Light activates melanopsin, encoded by the Opn4 gene, a visual pigment which is more closely related to invertebrate opsins than to vertebrate opsin [105]. In M1-type ganglion cells, melanopsin photoisomerization leads to activation of a $\mathrm{G}_{\alpha \mathrm{q} / 11 / 14}$ class $\mathrm{G}$-protein, which activates the phosphoinositide-specific phospholipase C isoform, PLC $\beta$; the phospholipase presumably acts on $\mathrm{PI}(4,5) \mathrm{P}_{2}$ as in other cell types, including Drosophila photoreceptors, which contain a homologous phospholipase, to produce diacylglycerol and $\operatorname{InsP}_{3} . \mathrm{PI}(4,5) \mathrm{P}_{2}$ hydrolysis, in turn, leads to the activation of the cation channels TRPC6 and TRPC7 [104]. In M4 ganglion cells, a different phototransduction cascade involving cyclic nucleotides and cyclic nucleotide-regulated $\mathrm{HCN}$ channels, whereas in M2 ganglion cells, both of these cascades operate [102].

\section{Studies of PI Metabolism in the RPE}

A variety of extracellular stimuli acting on tyrosine kinase-associated receptors or G protein-coupled-receptors have been reported to stimulate release of inositol phosphates in cultured RPE cells, presumably derived from PLC action of $\mathrm{PI}(4,5) \mathrm{P}_{2}$, on a timescale of tens of minutes; effective stimuli included fetal bovine serum, agonists for muscarinic, histamine, and serotonin, peptides, including bradykinin, arginine vasopressin, bombesin and oxytocin, [106-109]. The physiological relevance of these observations was not explored, but an in vivo study using frogs demonstrated dramatic acceleration of inositol phosphate release, especially of $\mathrm{InsP}_{3}$, following stimulation by light [21]. Acutely isolated rat RPE cells were reported to release $\mathrm{InsP}_{3}$ in response to induction of phagocytosis by addition of isolated rod outer segments [110]; this $\mathrm{InsP}_{3}$ release was not observed in cells from Royal College of Surgeon (RCS) rats, which have a defect in OS phagocytosis due to a deficiency in the receptor tyrosine kinase, MERTK [111]. As in many other cell types, insulin has been reported to stimulate activity of Type I PI-3 kinase to produce PI $(3,4,5) \mathrm{P}_{3}[95,112,113]$. Responses to hypoxia $[112,114,115]$ and elevated glucose $[116,117]$ are also reported to involve this pathway in RPE.

A number of important processes in RPE are known to rely on phosphoinositides, but how they are regulated in these cells is not well understood. Phagocytosis, autophagy, endocytosis and endosome processing, establishment of epithelial cell polarity and extension of microvilli membranes are all known to critically depend on phosphoinositides. For example, both autophagy and phagocytosis involve the recruitment of the ubiquitin-like protein, LC3 [118-120], whose recruitment to membranes depends on PI(3)P. Phagocytosis is also thought to require $\mathrm{PI}(4,5) \mathrm{P}_{2}$ and lysosomal fusion may involve other phosphoinositides such as $\mathrm{PI}(3,5) \mathrm{P}_{2}$ and $\mathrm{PI}(5) \mathrm{P}[22,121-125]$.

\section{Phosphoinositide Kinases and Phosphatase}

In mammals, there are 47 genes encoding 19 PI-kinase and PIP-kinases and 28 PIP phosphatases [126].

\subsection{Kinases}

The kinase isoforms are divided into three major families: PI 4-kinases (PI4Ks), the PI 3-kinases (PI3Ks), and PIP (PIP) kinases (PIPKs) [127]. The nomenclature for these is a bit confusing, as some enzymes termed "PI-kinases" actually act primarily as PIP kinases. For example, Type I PI-3 kinases primarily use $\mathrm{PI}(4,5) \mathrm{P}_{2}$ as their substrate to produce $\mathrm{PI}(3,4,5) \mathrm{P}_{3}$, and there is little evidence for a substantial portion of cellular PI(3)P being formed by these enzymes. When the Type III PI-3 kinase, Vps34, was knocked out in mouse rod cells and phosphoinositide levels measured, the results suggested complete ablation of PI(3)P despite the presence of the Type I enzyme [22]. Several of these have been reported to be expressed in retina or RPE at the level of protein, mRNA or enzyme activity and most, 
if not all, are likely present at some level; however, as far as their functions, only the Type I and Type III PI-3 kinase have been studied using gene knockouts in the retina and RPE $[22,49,57,128]$. Global knockouts have been produced for the $\alpha, \beta$, and $\gamma$ isoforms of Type I PIP-kinases, which are the major source of $\mathrm{PI}(4,5) \mathrm{P}_{2}$ in most cells [16]. Of these, the $\gamma$ isoform seems to have the highest expression in the retina [129] and in other neurons $[130,131]$ and leads to severe neuronal phenotypes and early postnatal mortality when knocked out [132]. Type II PIP-kinases have been observed in retina and are reported to be regulated by tyrosine phosphorylation [133]. The proteins encoded by the mouse genes, Pip4k2a, Pip4k2b, Pip4k2c, Pip5k1a, and Pip5k1c were all observed in a proteomic study of mouse retina [134].

\subsection{Phosphatases}

The phosphoinositide phosphatases encoded by Inpp1, Inpp4a, Inpp4b, Inpp5e, Mtmr2 and Synj1 (synaptojanin-1) have also been detected in retina by proteomics [134]. As noted below, defects in synaptojanin-1 and INPP5E are associated with retinal defects, as is the phosphoinositide 5-phosphatase, OCRL (oculocerebrorenal syndrome of Lowe) $[135,136]$. Among the other enzymes detected, almost none were enriched in the rod outer segment fraction as compared to the rest of the retina, consistent with the relatively low PI content of that organelle. One exception was Pip4k2c, the PI(5)P-4-kinase Type II $\gamma$ isoform. Inactivation of the mouse Pip $4 k c 2$ gene was found to lead to hyperactivation of the immune system, but the retinal phenotype was not examined [137].

\section{Retinal Phenotypes of Genetic Defects in Genes Related to Phosphoinositide Metabolism and Signaling}

Rods and cones have a very high rate of metabolism and biosynthesis of membrane components, due to the energetic demands of the phototransduction cascade and the daily shedding of $\sim 10 \%$ of the disk membranes (based on observation of a rate of $9 \%-13 \%$ in the rhesus monkey [138], which have to be engulfed and recycled by the RPE. As highly polarized cells, their function and health depend critically on efficient and accurate transport of the correct proteins and lipids to the correct compartments. A host of human blinding diseases have been linked to defects in membrane transport and sorting $[139,140]$. In RPE cells, massive amounts of membrane traffic are associated with their role as professional phagophores. Defects in RPE phagocytosis, as in MERTK deficiency [111,141] and Bestrophin deficiency [142-145], cause retinal degeneration in humans and animal models and have been proposed to play a role in age-related macular degeneration [146].

\subsection{Phosphatases and Inherited Retinopathies}

Inherited defects in the phosphoinositide phosphatase, INPP5E, are associated with the multi-syndromic ciliopathies, Joubert syndrome and Bardet-Biedl syndrome [147-150], and with retinal degeneration. The substrates for this phosphatase are $\mathrm{PI}(4,5) \mathrm{P}_{2}$ and $\mathrm{PI}(3,4,5) \mathrm{P}_{3}$, and its critical role in the cilia suggests that regulating levels of $\mathrm{PI}(4,5) \mathrm{P}_{2}$ may be important in ciliogenesis and cilium stability.

Synaptojanins are phosphoinositide phosphatases associated with synaptic function [151-153], including vesicle uncoating and endocytosis. Synaptojanin-1 deficiency causes severe cone defects in zebrafish and has been implicated in the regulation of autophagy in cones [45,46,75].

Defects in another phosphoinositide phosphatase, OCRL, are associated with a severe ciliopathy, oculocerebrorenal syndrome of Lowe [136]. Symptoms of this disease include glaucoma, a disease of the retina.

\subsection{PI3NM3}

The phosphatidyl inositol transfer protein PIT3NM3 (aka CCL118) is the mammalian homologue of the Drosophila retinal degeneration gene, $r d g B$, and defects in it are associated with autosomal dominant cone-rod dystrophy CORD5 [154-159]. The protein has also been implicated in cancer 
metastasis [160]. In zebrafish, the Class I phosphatidylinositol transfer protein $\beta$ isoform, Pitpnb, is essential for biogenesis and maintenance of double cones [161].

\subsection{PI Binding Proteins}

Proteins with phosphoinositide-binding domains have also been associated with retinal disease. These include the product of the $t u b / T U B$ gene $[162,163]$ and a related protein, TULP1 $[47,164-166]$. Tub was discovered as the protein encoded by a gene whose mutation in tubby mice causes obesity, deafness and blindness. The widely expressed members of the tubby family, including TULP1-TULP4, have a characteristic carboxyl-terminal tubby domain consisting of an alpha helix surrounded by a beta barrel which has been shown to bind to specific phosphoinositides [167]. Both Tub and Tulp1 are expressed in the retina, and TULP1 mutations cause retinitis pigmentosa $[47,168]$.

\section{Conclusions}

While the overall field of phosphoinositide regulation and signaling has made dramatic advances in recent years, our understanding of specific regulatory pathways in specific cell types of the retina remains limited. The emergence of new technologies for cell-type-specific gene manipulation, along with imaging techniques for subcellular localization of phosphoinositides, their regulatory enzymes and their effector proteins, and the development of more sensitive methods for measuring PI levels, bodes well for rapid progress in understanding the roles of retinal phosphoinositides in the coming years.

Funding: The author's research in this area has been supported by NIH grants R01-EY007981, R01-EY025218 and the Robert A. Welch Foundation, Q0035.

Conflicts of Interest: The author declares no conflicts of interest relevant to this paper.

\section{References}

1. Phan, T.K.; Williams, S.A.; Bindra, G.K.; Lay, F.T.; Poon, I.K.H.; Hulett, M.D. Phosphoinositides: Multipurpose cellular lipids with emerging roles in cell death. Cell Death Differ. 2019, 26, 781-793. [CrossRef] [PubMed]

2. Dickson, E.J.; Hille, B. Understanding phosphoinositides: Rare, dynamic, and essential membrane phospholipids. Biochem. J. 2019, 476, 1-23. [CrossRef] [PubMed]

3. Schink, K.O.; Tan, K.W.; Stenmark, H. Phosphoinositides in Control of Membrane Dynamics. Annu. Rev. Cell Dev. Biol. 2016, 32, 143-171. [CrossRef] [PubMed]

4. Balla, T. Phosphoinositides: Tiny lipids with giant impact on cell regulation. Physiol. Rev. 2013, 93, 1019-1137. [CrossRef]

5. Maffucci, T. An introduction to phosphoinositides. Curr. Top. Microbiol. Immunol. 2012, 362, 1-42. [CrossRef]

6. Brockerhoff, S.E. Phosphoinositides and photoreceptors. Mol. Neurobiol. 2011, 44, 420-425. [CrossRef]

7. Broekhuyse, R.M. Phospholipids in tissues of the eye. I. Isolation, characterization and quantitative analysis by two-dimensional thin-layer chromatography of diacyl and vinyl-ether phospholipids. Biochim. Biophys. Acta 1968, 152, 307-315. [CrossRef]

8. Anderson, R.E.; Feldman, L.S.; Feldman, G.L. Lipids of ocular tissues. II. The phospholipids of mature bovine and rabbit whole retina. Biochim. Biophys. Acta 1970, 202, 367-373. [CrossRef]

9. Anderson, R.E. Lipids of ocular tissues. IV. A comparison of the phospholipids from the retina of six mammalian species. Exp. Eye Res. 1970, 10, 339-344. [CrossRef]

10. Anderson, R.E.; Maude, M.B.; Zimmerman, W. Lipids of ocular tissues-X. Lipid composition of subcellular fractions of bovine retina. Vision Res. 1975, 15, 1087-1090. [CrossRef]

11. Anderson, R.E.; Maude, M.B. Phospholipids of bovine outer segments. Biochemistry 1970, 9, 3624-3628. [CrossRef] [PubMed]

12. Nielsen, N.C.; Fleischer, S.; McConnell, D.G. Lipid composition of bovine retinal outer segment fragments. Biochim. Biophys. Acta 1970, 211, 10-19. [CrossRef]

13. Anderson, R.E.; Risk, M. Lipids of ocular tissues. IX. The phospholipids of frog photoreceptor membranes. Vision Res. 1974, 14, 129-131. [CrossRef] 
14. Anderson, R.E.; Lissandrello, P.M.; Maude, M.B.; Matthes, M.T. Lipids of bovine retinal pigment epithelium. Exp. Eye Res. 1976, 23, 149-157. [CrossRef]

15. Balla, T. Inositol-lipid binding motifs: Signal integrators through protein-lipid and protein-protein interactions. J. Cell Sci. 2005, 118, 2093-2104. [CrossRef] [PubMed]

16. Choi, S.; Thapa, N.; Tan, X.; Hedman, A.C.; Anderson, R.A. PIP kinases define PI4,5P(2)signaling specificity by association with effectors. Biochim. Biophys. Acta 2015, 1851, 711-723. [CrossRef]

17. Lemmon, M.A. Membrane recognition by phospholipid-binding domains. Nat. Rev. Mol. Cell Biol. 2008, 9, 99-111. [CrossRef]

18. Anderson, R.E.; Maude, M.B.; Kelleher, P.A. Metabolism of phosphatidylinositol in the frog retina. Biochim. Biophys. Acta 1980, 620, 236-246. [CrossRef]

19. Choe, H.G.; Ghalayini, A.J.; Anderson, R.E. Phosphoinositide metabolism in frog rod outer segments. Exp. Eye Res. 1990, 51, 167-176. [CrossRef]

20. Gehm, B.D.; Mc Connell, D.G. Phosphoinositide synthesis in bovine rod outer segments. Biochemistry 1990, 29, 5442-5446. [CrossRef]

21. Rodriguez de Turco, E.B.; Gordon, W.C.; Bazan, N.G. Light stimulates in vivo inositol lipid turnover in frog retinal pigment epithelial cells at the onset of shedding and phagocytosis of photoreceptor membranes. Exp. Eye Res. 1992, 55, 719-725. [CrossRef]

22. He, F.; Agosto, M.A.; Anastassov, I.A.; Tse, D.Y.; Wu, S.M.; Wensel, T.G. Phosphatidylinositol-3-phosphate is light-regulated and essential for survival in retinal rods. Sci. Rep. 2016, 6, 26978. [CrossRef]

23. Stephens, L.R.; Jackson, T.R.; Hawkins, P.T. Agonist-stimulated synthesis of phosphatidylinositol(3,4,5)-trisphosphate: A new intracellular signalling system? Biochim. Biophys. Acta 1993, 1179, 27-75. [CrossRef]

24. Bui, H.H.; Sanders, P.E.; Bodenmiller, D.; Kuo, M.S.; Donoho, G.P.; Fischl, A.S. Direct analysis of PI(3,4,5)P3 using liquid chromatography electrospray ionization tandem mass spectrometry. Anal. Biochem. 2018, 547, 66-76. [CrossRef]

25. Kadamur, G.; Ross, E.M. Mammalian phospholipase C. Annu. Rev. Physiol. 2013, 75, 127-154. [CrossRef]

26. Trebak, M.; Lemonnier, L.; Smyth, J.T.; Vazquez, G.; Putney, J.W., Jr. Phospholipase C-coupled receptors and activation of TRPC channels. Handb Exp. Pharmacol. 2007, 593-614.

27. Wang, L.; Budolfson, K.; Wang, F. Pik3c3 deletion in pyramidal neurons results in loss of synapses, extensive gliosis and progressive neurodegeneration. Neuroscience 2011, 172, 427-442. [CrossRef]

28. Zhou, X.; Wang, L.; Hasegawa, H.; Amin, P.; Han, B.X.; Kaneko, S.; He, Y.; Wang, F. Deletion of PIK3C3/Vps34 in sensory neurons causes rapid neurodegeneration by disrupting the endosomal but not the autophagic pathway. Proc. Natl. Acad. Sci. USA 2010, 107, 9424-9429. [CrossRef]

29. Carnero, A.; Blanco-Aparicio, C.; Renner, O.; Link, W.; Leal, J.F. The PTEN/PI3K/AKT signalling pathway in cancer, therapeutic implications. Curr. Cancer Drug Targets 2008, 8, 187-198. [CrossRef]

30. Carpentier, S.; N'Kuli, F.; Grieco, G.; Van Der Smissen, P.; Janssens, V.; Emonard, H.; Bilanges, B.; Vanhaesebroeck, B.; Gaide Chevronnay, H.P.; Pierreux, C.E.; et al. Class III phosphoinositide 3-kinase/VPS34 and dynamin are critical for apical endocytic recycling. Traffic 2013, 14, 933-948. [CrossRef]

31. Dall'Armi, C.; Devereaux, K.A.; Di Paolo, G. The role of lipids in the control of autophagy. Curr. Biol. 2013, 23, R33-R45. [CrossRef]

32. Lenoir, M.; Overduin, M. PtdIns(4)P signalling and recognition systems. Adv. Exp. Med. Biol. 2013, 991, 59-83. [CrossRef]

33. McLaughlin, S.; Wang, J.; Gambhir, A.; Murray, D. PIP(2) and proteins: Interactions, organization, and information flow. Annu. Rev. Biophys. Biomol. Struct. 2002, 31, 151-175. [CrossRef]

34. Falkenburger, B.H.; Jensen, J.B.; Dickson, E.J.; Suh, B.C.; Hille, B. Phosphoinositides: Lipid regulators of membrane proteins. J. Physiol. 2010, 588, 3179-3185. [CrossRef]

35. Hilgemann, D.W.; Feng, S.; Nasuhoglu, C. The complex and intriguing lives of PIP2 with ion channels and transporters. Sci. STKE 2001, 2001, re19. [CrossRef]

36. Fukami, K.; Inanobe, S.; Kanemaru, K.; Nakamura, Y. Phospholipase C is a key enzyme regulating intracellular calcium and modulating the phosphoinositide balance. Prog. Lipid Res. 2010, 49, 429-437. [CrossRef]

37. Vines, C.M. Phospholipase C. Adv. Exp. Med. Biol. 2012, 740, 235-254. [CrossRef]

38. Shewan, A.; Eastburn, D.J.; Mostov, K. Phosphoinositides in cell architecture. Cold Spring Harb. Perspect. Biol. 2011, 3, a004796. [CrossRef] 
39. Yin, H.L.; Janmey, P.A. Phosphoinositide regulation of the actin cytoskeleton. Annu. Rev. Physiol. 2003, 65, 761-789. [CrossRef]

40. Hille, B.; Dickson, E.J.; Kruse, M.; Vivas, O.; Suh, B.C. Phosphoinositides regulate ion channels. Biochim. Biophys. Acta 2015, 1851, 844-856. [CrossRef]

41. Bielas, S.L.; Silhavy, J.L.; Brancati, F.; Kisseleva, M.V.; Al-Gazali, L.; Sztriha, L.; Bayoumi, R.A.; Zaki, M.S.; Abdel-Aleem, A.; Rosti, R.O.; et al. Mutations in INPP5E, encoding inositol polyphosphate-5-phosphatase E, link phosphatidyl inositol signaling to the ciliopathies. Nat. Genet. 2009, 41, 1032-1036. [CrossRef]

42. Jacoby, M.; Cox, J.J.; Gayral, S.; Hampshire, D.J.; Ayub, M.; Blockmans, M.; Pernot, E.; Kisseleva, M.V.; Compere, P.; Schiffmann, S.N.; et al. INPP5E mutations cause primary cilium signaling defects, ciliary instability and ciliopathies in human and mouse. Nat. Genet. 2009, 41, 1027-1031. [CrossRef]

43. Saar, K.; Al-Gazali, L.; Sztriha, L.; Rueschendorf, F.; Nur, E.K.M.; Reis, A.; Bayoumi, R. Homozygosity mapping in families with Joubert syndrome identifies a locus on chromosome $9 q 34.3$ and evidence for genetic heterogeneity. Am. J. Hum. Genet. 1999, 65, 1666-1671. [CrossRef] [PubMed]

44. George, A.A.; Hayden, S.; Holzhausen, L.C.; Ma, E.Y.; Suzuki, S.C.; Brockerhoff, S.E. Synaptojanin 1 is required for endolysosomal trafficking of synaptic proteins in cone photoreceptor inner segments. PLoS ONE 2014, 9, e84394. [CrossRef] [PubMed]

45. Holzhausen, L.C.; Lewis, A.A.; Cheong, K.K.; Brockerhoff, S.E. Differential role for synaptojanin 1 in rod and cone photoreceptors. J. Comp. Neurol. 2009, 517, 633-644. [CrossRef]

46. Van Epps, H.A.; Hayashi, M.; Lucast, L.; Stearns, G.W.; Hurley, J.B.; De Camilli, P.; Brockerhoff, S.E. The zebrafish nrc mutant reveals a role for the polyphosphoinositide phosphatase synaptojanin 1 in cone photoreceptor ribbon anchoring. J. Neurosci. 2004, 24, 8641-8650. [CrossRef]

47. Hagstrom, S.A.; North, M.A.; Nishina, P.L.; Berson, E.L.; Dryja, T.P. Recessive mutations in the gene encoding the tubby-like protein TULP1 in patients with retinitis pigmentosa. Nat. Genet. 1998, 18, 174-176. [CrossRef]

48. Jacobson, S.G.; Cideciyan, A.V.; Huang, W.C.; Sumaroka, A.; Roman, A.J.; Schwartz, S.B.; Luo, X.; Sheplock, R.; Dauber, J.M.; Swider, M.; et al. TULP1 mutations causing early-onset retinal degeneration: Preserved but insensitive macular cones. Invest. Ophthalmol. Vis. Sci. 2014, 55, 5354-5364. [CrossRef]

49. He, F.; Nichols, R.M.; Kailasam, L.; Wensel, T.G.; Agosto, M.A. Critical Role for Phosphatidylinositol-3 Kinase Vps34/PIK3C3 in ON-Bipolar Cells. Invest. Ophthalmol. Vis. Sci. 2019, 60, 2861-2874. [CrossRef]

50. Hammond, G.R.; Hong, Y. Phosphoinositides and Membrane Targeting in Cell Polarity. Cold Spring Harb. Perspect. Biol. 2018, 10. [CrossRef]

51. Mayinger, P. Phosphoinositides and vesicular membrane traffic. Biochim. Biophys. Acta 2012, 1821, 1104-1113. [CrossRef] [PubMed]

52. Chuang, J.Z.; Zhao, Y.; Sung, C.H. SARA-regulated vesicular targeting underlies formation of the light-sensing organelle in mammalian rods. Cell 2007, 130, 535-547. [CrossRef] [PubMed]

53. Deretic, D.; Traverso, V.; Parkins, N.; Jackson, F.; Rodriguez de Turco, E.B.; Ransom, N. Phosphoinositides, ezrin/moesin, and rac1 regulate fusion of rhodopsin transport carriers in retinal photoreceptors. Mol. Biol. Cell 2004, 15, 359-370. [CrossRef] [PubMed]

54. Spencer, W.J.; Lewis, T.R.; Phan, S.; Cady, M.A.; Serebrovskaya, E.O.; Schneider, N.F.; Kim, K.Y.; Cameron, L.A.; Skiba, N.P.; Ellisman, M.H.; et al. Photoreceptor disc membranes are formed through an Arp2/3-dependent lamellipodium-like mechanism. Proc. Natl. Acad. Sci. USA 2019. [CrossRef]

55. Bucki, R.; Wang, Y.H.; Yang, C.; Kandy, S.K.; Fatunmbi, O.; Bradley, R.; Pogoda, K.; Svitkina, T.; Radhakrishnan, R.; Janmey, P.A. Lateral distribution of phosphatidylinositol 4,5-bisphosphate in membranes regulates formin- and ARP2/3-mediated actin nucleation. J. Biol. Chem. 2019, 294, 4704-4722. [CrossRef]

56. Daste, F.; Walrant, A.; Holst, M.R.; Gadsby, J.R.; Mason, J.; Lee, J.E.; Brook, D.; Mettlen, M.; Larsson, E.; Lee, S.F.; et al. Control of actin polymerization via the coincidence of phosphoinositides and high membrane curvature. J. Cell Biol. 2017, 216, 3745-3765. [CrossRef]

57. Ivanovic, I.; Anderson, R.E.; Le, Y.Z.; Fliesler, S.J.; Sherry, D.M.; Rajala, R.V. Deletion of the p85alpha regulatory subunit of phosphoinositide 3-kinase in cone photoreceptor cells results in cone photoreceptor degeneration. Invest. Ophthalmol. Vis. Sci. 2011, 52, 3775-3783. [CrossRef]

58. Xu, W.; Jin, M.; Huang, W.; Wang, H.; Hu, R.; Li, J.; Cao, Y. Apical PtdIns(4,5)P2 is required for ciliogenesis and suppression of polycystic kidney disease. FASEB J. 2019, 33, 2848-2857. [CrossRef]

59. Phua, S.C.; Nihongaki, Y.; Inoue, T. Autonomy declared by primary cilia through compartmentalization of membrane phosphoinositides. Curr. Opin. Cell Biol. 2018, 50, 72-78. [CrossRef] 
60. Park, J.; Lee, N.; Kavoussi, A.; Seo, J.T.; Kim, C.H.; Moon, S.J. Ciliary Phosphoinositide Regulates Ciliary Protein Trafficking in Drosophila. Cell Rep. 2015, 13, 2808-2816. [CrossRef]

61. Garcia-Gonzalo, F.R.; Phua, S.C.; Roberson, E.C.; Garcia, G., 3rd; Abedin, M.; Schurmans, S.; Inoue, T.; Reiter, J.F. Phosphoinositides Regulate Ciliary Protein Trafficking to Modulate Hedgehog Signaling. Dev. Cell 2015, 34, 400-409. [CrossRef] [PubMed]

62. Vieira, O.V.; Gaus, K.; Verkade, P.; Fullekrug, J.; Vaz, W.L.; Simons, K. FAPP2, cilium formation, and compartmentalization of the apical membrane in polarized Madin-Darby canine kidney (MDCK) cells. Proc. Natl. Acad. Sci. USA 2006, 103, 18556-18561. [CrossRef] [PubMed]

63. Nachury, M.V.; Seeley, E.S.; Jin, H. Trafficking to the ciliary membrane: How to get across the periciliary diffusion barrier? Annu. Rev. Cell Dev. Biol. 2010, 26, 59-87. [CrossRef] [PubMed]

64. Luo, N.; West, C.C.; Murga-Zamalloa, C.A.; Sun, L.; Anderson, R.M.; Wells, C.D.; Weinreb, R.N.; Travers, J.B.; Khanna, H.; Sun, Y. OCRL localizes to the primary cilium: A new role for cilia in Lowe syndrome. Hum. Mol. Genet. 2012, 21, 3333-3344. [CrossRef] [PubMed]

65. Coon, B.G.; Hernandez, V.; Madhivanan, K.; Mukherjee, D.; Hanna, C.B.; Barinaga-Rementeria Ramirez, I.; Lowe, M.; Beales, P.L.; Aguilar, R.C. The Lowe syndrome protein OCRL1 is involved in primary cilia assembly. Hum. Mol. Genet. 2012, 21, 1835-1847. [CrossRef] [PubMed]

66. Humbert, M.C.; Weihbrecht, K.; Searby, C.C.; Li, Y.; Pope, R.M.; Sheffield, V.C.; Seo, S. ARL13B, PDE6D, and CEP164 form a functional network for INPP5E ciliary targeting. Proc. Natl. Acad. Sci. USA 2012, 109, 19691-19696. [CrossRef] [PubMed]

67. Jin, H.; White, S.R.; Shida, T.; Schulz, S.; Aguiar, M.; Gygi, S.P.; Bazan, J.F.; Nachury, M.V. The conserved Bardet-Biedl syndrome proteins assemble a coat that traffics membrane proteins to cilia. Cell 2010, 141, 1208-1219. [CrossRef]

68. Nachury, M.V.; Loktev, A.V.; Zhang, Q.; Westlake, C.J.; Peranen, J.; Merdes, A.; Slusarski, D.C.; Scheller, R.H.; Bazan, J.F.; Sheffield, V.C.; et al. A core complex of BBS proteins cooperates with the GTPase Rab8 to promote ciliary membrane biogenesis. Cell 2007, 129, 1201-1213. [CrossRef]

69. Chinskey, N.D.; Zheng, Q.D.; Zacks, D.N. Control of Photoreceptor Autophagy after Retinal Detachment: The Switch from Survival to Death. Invest. Ophthalmol. Vis. Sci. 2014, 55, 688-695. [CrossRef]

70. Reme, C.; Drinker, C.K.; Aeberhard, B. Modification of autophagic degradation by medium- and illumination conditions in frog visual cells in vitro. Doc. Ophthalmol. 1984, 56, 377-383. [CrossRef]

71. Reme, C. Autophagy in rods and cones of the vertebrate retina. Dev. Ophthalmol. 1981, 4, 101-148.

72. Reme, C.E.; Sulser, M. Diurnal variation of autophagy in rod visual cells in the rat. Albrecht Von Graefes Arch. Klin. Exp. Ophthalmol. 1977, 203, 261-270. [CrossRef]

73. Chen, Y.; Sawada, O.; Kohno, H.; Le, Y.Z.; Subauste, C.; Maeda, T.; Maeda, A. Autophagy protects the retina from light-induced degeneration. J. Biol. Chem. 2013, 288, 7506-7518. [CrossRef] [PubMed]

74. Besirli, C.G.; Chinskey, N.D.; Zheng, Q.D.; Zacks, D.N. Autophagy activation in the injured photoreceptor inhibits fas-mediated apoptosis. Invest. Ophthalmol. Vis. Sci. 2011, 52, 4193-4199. [CrossRef] [PubMed]

75. George, A.A.; Hayden, S.; Stanton, G.R.; Brockerhoff, S.E. Arf6 and the 5'phosphatase of synaptojanin 1 regulate autophagy in cone photoreceptors. Bioessays 2016, 38 (Suppl. 1), S119-S135. [CrossRef]

76. Ghalayini, A.; Anderson, R.E. Phosphatidylinositol 4,5-bisphosphate: Light-mediated breakdown in the vertebrate retina. Biochem. Biophys. Res. Commun. 1984, 124, 503-506. [CrossRef]

77. Anderson, R.E.; Maude, M.B.; Pu, G.A.; Hollyfield, J.G. Effect of light on the metabolism of lipids in the rat retina. J. Neurochem. 1985, 44, 773-778. [CrossRef] [PubMed]

78. Tarver, A.P.; Anderson, R.E. Phospholipase C activity and substrate specificity in frog photoreceptors. Exp. Eye Res. 1988, 46, 29-35. [CrossRef]

79. Ghalayini, A.J.; Tarver, A.P.; Mackin, W.M.; Koutz, C.A.; Anderson, R.E. Identification and immunolocalization of phospholipase C in bovine rod outer segments. J. Neurochem. 1991, 57, 1405-1412. [CrossRef]

80. Das, N.D.; Yoshioka, T.; Samuelson, D.; Shichi, H. Immunocytochemical localization of phosphatidylinositol-4,5-bisphosphate in dark- and light-adapted rat retinas. Cell Struct. Funct. 1986, 11, 53-63. [CrossRef]

81. Das, N.D.; Yoshioka, T.; Samuelson, D.; Cohen, R.J.; Shichi, H. Immunochemical evidence for the light-regulated modulation of phosphatidylinositol-4,5-bisphosphate in rat photoreceptor cells. Cell Struct. Funct. 1987, 12, 471-481. [CrossRef] [PubMed] 
82. Panfoli, I.; Morelli, A.; Pepe, I. Calcium ion-regulated phospholipase C activity in bovine rod outer segments. Biochem. Biophys. Res. Commun. 1990, 173, 283-288. [CrossRef]

83. Grigorjev, I.V.; Grits, A.I.; Artamonov, I.D.; Baranova, L.A.; Volotovski, I.D. betagamma-Transducin stimulates hydrolysis and synthesis of phosphatidylinositol 4,5-bisphosphate in bovine rod outer segment membranes. Biochim. Biophys. Acta 1996, 1310, 131-136. [CrossRef]

84. Van Rooijen, L.A.; Bazan, N.G. The inositide cycle in bovine photoreceptor membranes. Life Sci. 1986, 38, 1685-1693. [CrossRef]

85. Millar, F.A.; Fisher, S.C.; Muir, C.A.; Edwards, E.; Hawthorne, J.N. Polyphosphoinositide hydrolysis in response to light stimulation of rat and chick retina and retinal rod outer segments. Biochim. Biophys. Acta 1988, 970, 205-211. [CrossRef]

86. He, F.; Mao, M.; Wensel, T.G. Enhancement of phototransduction g protein-effector interactions by phosphoinositides. J. Biol. Chem. 2004, 279, 8986-8990. [CrossRef]

87. Womack, K.B.; Gordon, S.E.; He, F.; Wensel, T.G.; Lu, C.C.; Hilgemann, D.W. Do phosphatidylinositides modulate vertebrate phototransduction? J. Neurosci. 2000, 20, 2792-2799. [CrossRef]

88. Gross, O.P.; Pugh, E.N., Jr.; Burns, M.E. Spatiotemporal cGMP dynamics in living mouse rods. Biophys J. 2012, 102, 1775-1784. [CrossRef]

89. Peng, Y.W.; Rhee, S.G.; Yu, W.P.; Ho, Y.K.; Schoen, T.; Chader, G.J.; Yau, K.W. Identification of components of a phosphoinositide signaling pathway in retinal rod outer segments. Proc. Natl. Acad. Sci. USA 1997, 94, 1995-2000. [CrossRef]

90. Orisme, W.; Li, J.; Goldmann, T.; Bolch, S.; Wolfrum, U.; Smith, W.C. Light-dependent translocation of arrestin in rod photoreceptors is signaled through a phospholipase C cascade and requires ATP. Cell Signal. 2010, 22, 447-456. [CrossRef]

91. Guo, X.; Ghalayini, A.J.; Chen, H.; Anderson, R.E. Phosphatidylinositol 3-kinase in bovine photoreceptor rod outer segments. Invest. Ophthalmol. Vis. Sci. 1997, 38, 1873-1882. [PubMed]

92. Guo, X.X.; Huang, Z.; Bell, M.W.; Chen, H.; Anderson, R.E. Tyrosine phosphorylation is involved in phosphatidylinositol 3-kinase activation in bovine rod outer segments. Mol. Vis. 2000, 6, 216-221. [PubMed]

93. Rajala, R.V.; McClellan, M.E.; Chan, M.D.; Tsiokas, L.; Anderson, R.E. Interaction of the retinal insulin receptor beta-subunit with the p85 subunit of phosphoinositide 3-kinase. Biochemistry 2004, 43, 5637-5650. [CrossRef] [PubMed]

94. Rajala, R.V.; Anderson, R.E. Light regulation of the insulin receptor in the retina. Mol. Neurobiol. 2003, 28, 123-138. [CrossRef]

95. Rajala, R.V.; McClellan, M.E.; Ash, J.D.; Anderson, R.E. In vivo regulation of phosphoinositide 3-kinase in retina through light-induced tyrosine phosphorylation of the insulin receptor beta-subunit. J. Biol. Chem. 2002, 277, 43319-43326. [CrossRef] [PubMed]

96. Rajala, R.V.; Ranjo-Bishop, M.; Wang, Y.; Rajala, A.; Anderson, R.E. The p110alpha isoform of phosphoinositide 3-kinase is essential for cone photoreceptor survival. Biochimie 2015, 112, 35-40. [CrossRef] [PubMed]

97. Ivanovic, I.; Allen, D.T.; Dighe, R.; Le, Y.Z.; Anderson, R.E.; Rajala, R.V. Phosphoinositide 3-kinase signaling in retinal rod photoreceptors. Invest. Ophthalmol. Vis. Sci. 2011, 52, 6355-6362. [CrossRef]

98. Detwiler, P.B. Phototransduction in Retinal Ganglion Cells. Yale J. Biol. Med. 2018, 91, 49-52.

99. Graham, D.M.; Wong, K.Y.; Shapiro, P.; Frederick, C.; Pattabiraman, K.; Berson, D.M. Melanopsin ganglion cells use a membrane-associated rhabdomeric phototransduction cascade. J. Neurophysiol. 2008, 99, 2522-2532. [CrossRef]

100. Hardie, R.C. Photosensitive TRPs. Handb. Exp. Pharmacol. 2014, 223, 795-826. [CrossRef]

101. Isoldi, M.C.; Rollag, M.D.; Castrucci, A.M.; Provencio, I. Rhabdomeric phototransduction initiated by the vertebrate photopigment melanopsin. Proc. Natl. Acad. Sci. USA 2005, 102, 1217-1221. [CrossRef] [PubMed]

102. Jiang, Z.; Yue, W.W.S.; Chen, L.; Sheng, Y.; Yau, K.W. Cyclic-Nucleotide- and HCN-Channel-Mediated Phototransduction in Intrinsically Photosensitive Retinal Ganglion Cells. Cell 2018, 175, 652-664 e612. [CrossRef]

103. Montell, C. Drosophila visual transduction. Trends Neurosci. 2012, 35, 356-363. [CrossRef] [PubMed]

104. Xue, T.; Do, M.T.; Riccio, A.; Jiang, Z.; Hsieh, J.; Wang, H.C.; Merbs, S.L.; Welsbie, D.S.; Yoshioka, T.; Weissgerber, P.; et al. Melanopsin signalling in mammalian iris and retina. Nature 2011, 479, 67-73. [CrossRef] [PubMed]

105. Arendt, D. Evolution of eyes and photoreceptor cell types. Int. J. Dev. Biol. 2003, 47, 563-571. [PubMed] 
106. Kuriyama, S.; Ohuchi, T.; Yoshimura, N.; Honda, Y. Growth factor-induced cytosolic calcium ion transients in cultured human retinal pigment epithelial cells. Invest. Ophthalmol. Vis. Sci. 1991, 32, 2882-2890.

107. Osborne, N.N.; FitzGibbon, F.; Schwartz, G. Muscarinic acetylcholine receptor-mediated phosphoinositide turnover in cultured human retinal pigment epithelium cells. Vision Res. 1991, 31, 1119-1127. [CrossRef]

108. Feldman, E.L.; Randolph, A.E.; Johnston, G.C.; DelMonte, M.A.; Greene, D.A. Receptor-coupled phosphoinositide hydrolysis in human retinal pigment epithelium. J. Neurochem. 1991, 56, 2094-2100. [CrossRef]

109. York, N.; Halbach, P.; Chiu, M.A.; Bird, I.M.; Pillers, D.M.; Pattnaik, B.R. Oxytocin (OXT)-stimulated inhibition of Kir7.1 activity is through PIP2-dependent $\mathrm{Ca}(2+)$ response of the oxytocin receptor in the retinal pigment epithelium in vitro. Cell Signal. 2017, 37, 93-102. [CrossRef]

110. Heth, C.A.; Marescalchi, P.A. Inositol triphosphate generation in cultured rat retinal pigment epithelium. Invest. Ophthalmol. Vis. Sci. 1994, 35, 409-416.

111. D'Cruz, P.M.; Yasumura, D.; Weir, J.; Matthes, M.T.; Abderrahim, H.; LaVail, M.M.; Vollrath, D. Mutation of the receptor tyrosine kinase gene Mertk in the retinal dystrophic RCS rat. Hum. Mol. Genet. 2000, 9, 645-651. [CrossRef] [PubMed]

112. Treins, C.; Giorgetti-Peraldi, S.; Murdaca, J.; Semenza, G.L.; Van Obberghen, E. Insulin stimulates hypoxia-inducible factor 1 through a phosphatidylinositol 3-kinase/target of rapamycin-dependent signaling pathway. J. Biol. Chem. 2002, 277, 27975-27981. [CrossRef] [PubMed]

113. Geraldes, P.; Yagi, K.; Ohshiro, Y.; He, Z.; Maeno, Y.; Yamamoto-Hiraoka, J.; Rask-Madsen, C.; Chung, S.W.; Perrella, M.A.; King, G.L. Selective regulation of heme oxygenase-1 expression and function by insulin through IRS1/phosphoinositide 3-kinase/Akt-2 pathway. J. Biol. Chem. 2008, 283, 34327-34336. [CrossRef]

114. Mwaikambo, B.R.; Yang, C.; Chemtob, S.; Hardy, P. Hypoxia up-regulates CD36 expression and function via hypoxia-inducible factor-1- and phosphatidylinositol 3-kinase-dependent mechanisms. J. Biol. Chem. 2009, 284, 26695-26707. [CrossRef]

115. Yang, X.M.; Wang, Y.S.; Zhang, J.; Li, Y.; Xu, J.F.; Zhu, J.; Zhao, W.; Chu, D.K.; Wiedemann, P. Role of PI3K/Akt and MEK/ERK in mediating hypoxia-induced expression of HIF-1alpha and VEGF in laser-induced rat choroidal neovascularization. Invest. Ophthalmol. Vis. Sci. 2009, 50, 1873-1879. [CrossRef]

116. Kim, D.I.; Lim, S.K.; Park, M.J.; Han, H.J.; Kim, G.Y.; Park, S.H. The involvement of phosphatidylinositol 3-kinase/Akt signaling in high glucose-induced downregulation of GLUT-1 expression in ARPE cells. Life Sci. 2007, 80, 626-632. [CrossRef]

117. Qin, D.; Zhang, G.M.; Xu, X.; Wang, L.Y. The PI3K/Akt signaling pathway mediates the high glucose-induced expression of extracellular matrix molecules in human retinal pigment epithelial cells. J. Diabetes Res. 2015, 2015, 920280. [CrossRef]

118. Ferguson, T.A.; Green, D.R. Autophagy and phagocytosis converge for better vision. Autophagy 2014, 10, 165-167. [CrossRef]

119. Kim, J.Y.; Zhao, H.; Martinez, J.; Doggett, T.A.; Kolesnikov, A.V.; Tang, P.H.; Ablonczy, Z.; Chan, C.C.; Zhou, Z.; Green, D.R.; et al. Noncanonical autophagy promotes the visual cycle. Cell 2013, 154, 365-376. [CrossRef]

120. Muniz-Feliciano, L.; Doggett, T.A.; Zhou, Z.; Ferguson, T.A. RUBCN/rubicon and EGFR regulate lysosomal degradative processes in the retinal pigment epithelium (RPE) of the eye. Autophagy 2017, 13, 2072-2085. [CrossRef]

121. Shaw, J.D.; Hama, H.; Sohrabi, F.; DeWald, D.B.; Wendland, B. PtdIns(3,5)P2 is required for delivery of endocytic cargo into the multivesicular body. Traffic 2003, 4, 479-490. [CrossRef]

122. Ketel, K.; Krauss, M.; Nicot, A.S.; Puchkov, D.; Wieffer, M.; Muller, R.; Subramanian, D.; Schultz, C.; Laporte, J.; Haucke, V. A phosphoinositide conversion mechanism for exit from endosomes. Nature 2016, 529, 408-412. [CrossRef]

123. Isobe, Y.; Nigorikawa, K.; Tsurumi, G.; Takemasu, S.; Takasuga, S.; Kofuji, S.; Hazeki, K. PIKfyve accelerates phagosome acidification through activation of TRPML1 while arrests aberrant vacuolation independent of the Ca2+ channel. J. Biochem. 2019, 165, 75-84. [CrossRef]

124. Ikonomov, O.C.; Sbrissa, D.; Shisheva, A. Localized PtdIns 3,5-P2 synthesis to regulate early endosome dynamics and fusion. Am. J. Physiol. Cell Physiol. 2006, 291, C393-C404. [CrossRef] 
125. Dong, X.P.; Shen, D.; Wang, X.; Dawson, T.; Li, X.; Zhang, Q.; Cheng, X.; Zhang, Y.; Weisman, L.S.; Delling, M.; et al. $\mathrm{PI}(3,5) \mathrm{P}(2)$ controls membrane trafficking by direct activation of mucolipin $\mathrm{Ca}(2+)$ release channels in the endolysosome. Nat. Commun. 2010, 1, 38. [CrossRef]

126. Sasaki, T.; Takasuga, S.; Sasaki, J.; Kofuji, S.; Eguchi, S.; Yamazaki, M.; Suzuki, A. Mammalian phosphoinositide kinases and phosphatases. Prog. Lipid Res. 2009, 48, 307-343. [CrossRef]

127. Brown, J.R.; Auger, K.R. Phylogenomics of phosphoinositide lipid kinases: Perspectives on the evolution of second messenger signaling and drug discovery. BMC Evol. Biol. 2011, 11, 4. [CrossRef]

128. Azadi, S.; Brush, R.S.; Anderson, R.E.; Rajala, R.V. Class I Phosphoinositide 3-Kinase Exerts a Differential Role on Cell Survival and Cell Trafficking in Retina. Adv. Exp. Med. Biol. 2016, 854, 363-369. [CrossRef]

129. Sakagami, H.; Katsumata, O.; Hara, Y.; Tamaki, H.; Fukaya, M. Preferential localization of type I phosphatidylinositol 4-phosphate 5-kinase gamma at the periactive zone of mouse photoreceptor ribbon synapses. Brain Res. 2014, 1586, 23-33. [CrossRef]

130. Wenk, M.R.; Pellegrini, L.; Klenchin, V.A.; Di Paolo, G.; Chang, S.; Daniell, L.; Arioka, M.; Martin, T.F.; De Camilli, P. PIP kinase Igamma is the major PI(4,5)P(2) synthesizing enzyme at the synapse. Neuron 2001, 32, 79-88. [CrossRef]

131. Wright, B.D.; Loo, L.; Street, S.E.; Ma, A.; Taylor-Blake, B.; Stashko, M.A.; Jin, J.; Janzen, W.P.; Frye, S.V.; Zylka, M.J. The lipid kinase PIP5K1C regulates pain signaling and sensitization. Neuron 2014, 82, 836-847. [CrossRef]

132. Di Paolo, G.; Moskowitz, H.S.; Gipson, K.; Wenk, M.R.; Voronov, S.; Obayashi, M.; Flavell, R.; Fitzsimonds, R.M.; Ryan, T.A.; De Camilli, P. Impaired PtdIns(4,5)P2 synthesis in nerve terminals produces defects in synaptic vesicle trafficking. Nature 2004, 431, 415-422. [CrossRef]

133. Huang, Z.; Guo, X.X.; Chen, S.X.; Alvarez, K.M.; Bell, M.W.; Anderson, R.E. Regulation of type II phosphatidylinositol phosphate kinase by tyrosine phosphorylation in bovine rod outer segments. Biochemistry 2001, 40, 4550-4559. [CrossRef]

134. Zhao, L.; Chen, Y.; Bajaj, A.O.; Eblimit, A.; Xu, M.; Soens, Z.T.; Wang, F.; Ge, Z.; Jung, S.Y.; He, F.; et al. Integrative subcellular proteomic analysis allows accurate prediction of human disease-causing genes. Genome Res. 2016, 26, 660-669. [CrossRef]

135. Prosseda, P.P.; Luo, N.; Wang, B.; Alvarado, J.A.; Hu, Y.; Sun, Y. Loss of OCRL increases ciliary PI(4,5)P2 in Lowe oculocerebrorenal syndrome. J. Cell Sci. 2017, 130, 3447-3454. [CrossRef]

136. Attree, O.; Olivos, I.M.; Okabe, I.; Bailey, L.C.; Nelson, D.L.; Lewis, R.A.; McInnes, R.R.; Nussbaum, R.L. The Lowe's oculocerebrorenal syndrome gene encodes a protein highly homologous to inositol polyphosphate-5-phosphatase. Nature 1992, 358, 239-242. [CrossRef]

137. Shim, H.; Wu, C.; Ramsamooj, S.; Bosch, K.N.; Chen, Z.; Emerling, B.M.; Yun, J.; Liu, H.; Choo-Wing, R.; Yang, Z.; et al. Deletion of the gene Pip4k2c, a novel phosphatidylinositol kinase, results in hyperactivation of the immune system. Proc. Natl. Acad. Sci. USA 2016, 113, 7596-7601. [CrossRef]

138. Young, R.W. The renewal of rod and cone outer segments in the rhesus monkey. J. Cell Biol. 1971, 49, 303-318. [CrossRef]

139. Adams, N.A.; Awadein, A.; Toma, H.S. The retinal ciliopathies. Ophthalmic Genet. 2007, 28, 113-125. [CrossRef]

140. Wheway, G.; Parry, D.A.; Johnson, C.A. The role of primary cilia in the development and disease of the retina. Organogenesis 2013, 10, 69-85. [CrossRef]

141. Gal, A.; Li, Y.; Thompson, D.A.; Weir, J.; Orth, U.; Jacobson, S.G.; Apfelstedt-Sylla, E.; Vollrath, D. Mutations in MERTK, the human orthologue of the RCS rat retinal dystrophy gene, cause retinitis pigmentosa. Nat. Genet. 2000, 26, 270-271. [CrossRef]

142. Marmorstein, A.D.; Johnson, A.A.; Bachman, L.A.; Andrews-Pfannkoch, C.; Knudsen, T.; Gilles, B.J.; Hill, M.; Gandhi, J.K.; Marmorstein, L.Y.; Pulido, J.S. Mutant Best1 Expression and Impaired Phagocytosis in an iPSC Model of Autosomal Recessive Bestrophinopathy. Sci Rep. 2018, 8, 4487. [CrossRef]

143. Strauss, O.; Reichhart, N.; Gomez, N.M.; Muller, C. Contribution of Ion Channels in Calcium Signaling Regulating Phagocytosis: MaxiK, Cav1.3 and Bestrophin-1. Adv. Exp. Med. Biol. 2016, 854, 739-744. [CrossRef]

144. Muller, C.; Mas Gomez, N.; Ruth, P.; Strauss, O. CaV1.3 L-type channels, maxiK Ca(2+)-dependent K(+) channels and bestrophin-1 regulate rhythmic photoreceptor outer segment phagocytosis by retinal pigment epithelial cells. Cell Signal. 2014, 26, 968-978. [CrossRef] 
145. Xiao, Q.; Hartzell, H.C.; Yu, K. Bestrophins and retinopathies. Pflugers Arch. 2010, 460, 559-569. [CrossRef]

146. Strauss, O. The retinal pigment epithelium in visual function. Physiol. Rev. 2005, 85, 845-881. [CrossRef]

147. Luo, N.; Lu, J.; Sun, Y. Evidence of a role of inositol polyphosphate 5-phosphatase INPP5E in cilia formation in zebrafish. Vision Res. 2012, 75, 98-107. [CrossRef]

148. Travaglini, L.; Brancati, F.; Silhavy, J.; Iannicelli, M.; Nickerson, E.; Elkhartoufi, N.; Scott, E.; Spencer, E.; Gabriel, S.; Thomas, S.; et al. Phenotypic spectrum and prevalence of INPP5E mutations in Joubert syndrome and related disorders. Eur. J. Hum. Genet. 2013, 21, 1074-1078. [CrossRef]

149. Plotnikova, O.V.; Seo, S.; Cottle, D.L.; Conduit, S.; Hakim, S.; Dyson, J.M.; Mitchell, C.A.; Smyth, I.M. INPP5E interacts with AURKA, linking phosphoinositide signaling to primary cilium stability. J. Cell Sci. 2015, 128, 364-372. [CrossRef]

150. Xu, W.; Jin, M.; Hu, R.; Wang, H.; Zhang, F.; Yuan, S.; Cao, Y. The Joubert Syndrome Protein Inpp5e Controls Ciliogenesis by Regulating Phosphoinositides at the Apical Membrane. J. Am. Soc. Nephrol. 2017, 28, 118-129. [CrossRef]

151. Verstreken, P.; Koh, T.W.; Schulze, K.L.; Zhai, R.G.; Hiesinger, P.R.; Zhou, Y.; Mehta, S.Q.; Cao, Y.; Roos, J.; Bellen, H.J. Synaptojanin is recruited by endophilin to promote synaptic vesicle uncoating. Neuron 2003, 40, 733-748. [CrossRef]

152. Chi, Y.; Zhou, B.; Wang, W.Q.; Chung, S.K.; Kwon, Y.U.; Ahn, Y.H.; Chang, Y.T.; Tsujishita, Y.; Hurley, J.H.; Zhang, Z.Y. Comparative mechanistic and substrate specificity study of inositol polyphosphate 5-phosphatase Schizosaccharomyces pombe Synaptojanin and SHIP2. J. Biol. Chem. 2004, 279, 44987-44995. [CrossRef]

153. Chang-Ileto, B.; Frere, S.G.; Chan, R.B.; Voronov, S.V.; Roux, A.; Di Paolo, G. Synaptojanin 1-mediated $\mathrm{PI}(4,5) \mathrm{P} 2$ hydrolysis is modulated by membrane curvature and facilitates membrane fission. Dev. Cell 2011, 20, 206-218. [CrossRef] [PubMed]

154. Lev, S.; Hernandez, J.; Martinez, R.; Chen, A.; Plowman, G.; Schlessinger, J. Identification of a novel family of targets of PYK2 related to Drosophila retinal degeneration B (rdgB) protein. Mol. Cell Biol. 1999, 19, 2278-2288. [CrossRef]

155. Tian, D.; Lev, S. Cellular and developmental distribution of human homologues of the Drosophilia rdgB protein in the rat retina. Invest. Ophthalmol. Vis. Sci. 2002, 43, 1946-1953.

156. Kohn, L.; Kadzhaev, K.; Burstedt, M.S.I.; Haraldsson, S.; Hallberg, B.; Sandgren, O.; Golovleva, I. Mutation in the PYK2-binding domain of PITPNM3 causes autosomal dominant cone dystrophy (CORD5) in two Swedish families. Eur. J. Hum. Genet. 2007, 15, 664-671. [CrossRef]

157. Kohn, L.; Kadzhaev, K.; Burstedt, M.S.I.; Haraldsson, S.; Sandgren, O.; Golovleva, I. Mutation in the PYK2-binding domain of PITPNM3 causes autosomal dominant cone dystrophy (CORD5) in two Swedish families. Rencent Adv. Exp. Med. Biol. 2008, 613, 229-234. [CrossRef]

158. Kohn, L.; Kohl, S.; Bowne, S.J.; Sullivan, L.S.; Kellner, U.; Daiger, S.P.; Sandgren, O.; Golovleva, I. PITPNM3 is an uncommon cause of cone and cone-rod dystrophies. Ophthalmic Genet. 2010, 31, 139-140. [CrossRef]

159. Bakhoum, M.F.; Sengillo, J.D.; Cui, X.; Tsang, S.H. Autoimmune retinopathy in a patient with a missense mutation in PITPNM3. Retin Cases Brief. Rep. 2018, 12 (Suppl. 1), S72-S75. [CrossRef]

160. Lin, Z.; Li, W.; Zhang, H.; Wu, W.; Peng, Y.; Zeng, Y.; Wan, Y.; Wang, J.; Ouyang, N. CCL18/PITPNM3 enhances migration, invasion, and EMT through the NF-kappaB signaling pathway in hepatocellular carcinoma. Tumour Biol. 2016, 37, 3461-3468. [CrossRef]

161. Ile, K.E.; Kassen, S.; Cao, C.; Vihtehlic, T.; Shah, S.D.; Mousley, C.J.; Alb, J.G., Jr.; Huijbregts, R.P.; Stearns, G.W.; Brockerhoff, S.E.; et al. Zebrafish class 1 phosphatidylinositol transfer proteins: PITPbeta and double cone cell outer segment integrity in retina. Traffic 2010, 11, 1151-1167. [CrossRef] [PubMed]

162. Chang, B.; Hawes, N.L.; Hurd, R.E.; Davisson, M.T.; Nusinowitz, S.; Heckenlively, J.R. Retinal degeneration mutants in the mouse. Vision Res. 2002, 42, 517-525. [CrossRef]

163. Borman, A.D.; Pearce, L.R.; Mackay, D.S.; Nagel-Wolfrum, K.; Davidson, A.E.; Henderson, R.; Garg, S.; Waseem, N.H.; Webster, A.R.; Plagnol, V.; et al. A homozygous mutation in the TUB gene associated with retinal dystrophy and obesity. Hum. Mutat. 2014, 35, 289-293. [CrossRef] [PubMed]

164. Hagstrom, S.A.; Watson, R.F.; Pauer, G.J.; Grossman, G.H. Tulp1 is involved in specific photoreceptor protein transport pathways. Adv. Exp. Med. Biol. 2012, 723, 783-789. [CrossRef] [PubMed]

165. Xi, Q.; Pauer, G.J.; Traboulsi, E.I.; Hagstrom, S.A. Mutation screen of the TUB gene in patients with retinitis pigmentosa and Leber congenital amaurosis. Exp. Eye Res. 2006, 83, 569-573. [CrossRef] [PubMed] 
166. Xi, Q.; Pauer, G.J.; West, K.A.; Crabb, J.W.; Hagstrom, S.A. Retinal degeneration caused by mutations in TULP1. Adv. Exp. Med. Biol. 2003, 533, 303-308. [CrossRef]

167. Mukhopadhyay, S.; Jackson, P.K. The tubby family proteins. Genome Biol. 2011, 12, 225. [CrossRef]

168. Hagstrom, S.A.; Duyao, M.; North, M.A.; Li, T. Retinal degeneration in tulp1-/- mice: Vesicular accumulation in the interphotoreceptor matrix. Invest. Ophthalmol. Vis. Sci. 1999, 40, 2795-2802.

(C) 2020 by the author. Licensee MDPI, Basel, Switzerland. This article is an open access article distributed under the terms and conditions of the Creative Commons Attribution (CC BY) license (http://creativecommons.org/licenses/by/4.0/). 


\title{
From Rust to Quantum Biology: The Role of Iron in Retina Physiopathology
}

\author{
Emilie Picard ${ }^{1, *}$, Alejandra Daruich ${ }^{1,2}$, Jenny Youale ${ }^{1}$, Yves Courtois ${ }^{1}$ and \\ Francine Behar-Cohen ${ }^{1,3}$ \\ 1 Centre de Recherche des Cordeliers, INSERM, Sorbonne Université, USPC, Université Paris Descartes, \\ Team 17, F-75006 Paris, France; adaruich.matet@gmail.com (A.D.); youale.j@hotmail.fr (J.Y.); \\ courtois.yves@numericable.com (Y.C.); francine.behar@gmail.com (F.B.-C.) \\ 2 Ophthalmology Department, Necker-Enfants Malades University Hospital, APHP, 75015 Paris, France \\ 3 Ophtalmopole, Cochin Hospital, AP-HP, Assistance Publique Hôpitaux de Paris, 24 rue du Faubourg \\ Saint-Jacques, 75014 Paris, France \\ * Correspondence: picardemilie@gmail.com; Tel.: +331-44-27-81-82
}

Received: 17 February 2020; Accepted: 9 March 2020; Published: 13 March 2020

\begin{abstract}
Iron is essential for cell survival and function. It is a transition metal, that could change its oxidation state from $\mathrm{Fe}^{2+}$ to $\mathrm{Fe}^{3+}$ involving an electron transfer, the key of vital functions but also organ dysfunctions. The goal of this review is to illustrate the primordial role of iron and local iron homeostasis in retinal physiology and vision, as well as the pathological consequences of iron excess in animal models of retinal degeneration and in human retinal diseases. We summarize evidence of the potential therapeutic effect of iron chelation in retinal diseases and especially the interest of transferrin, a ubiquitous endogenous iron-binding protein, having the ability to treat or delay degenerative retinal diseases.
\end{abstract}

Keywords: iron; retina; transferrin

\section{Introduction}

Iron is a major element in biology. Besides its well-known role in prebiotic conditions after the rise of oxygen in the atmosphere, its insolubility led to the development of many mechanisms to allow the primitive cells and organisms to use it. They are driven by the transition of ferrous iron $\left(\mathrm{Fe}^{2+}\right)$ to ferric iron $\left(\mathrm{Fe}^{3+}\right)$ involving an electron which is particularly available and is the basis of vital functions and dysfunctions in the organs.

In this review, we analyze several of the well-known or recently discovered functions of iron in the eye, mainly in the retina, and the most promising approaches to regulate it and improve a large number of its negative side effects which can lead to vision impairment. We will focus on the main functions of transferrin (TF) as a partner in the systemic and cellular mechanisms that underlie the regulation of iron homeostasis and its disorders. 


\section{The Retina Structure and Retinal Oxygen Supply}

The eye is a complex and confined organ formed by different compartments and structures essential for the transmission and focus of photons from the cornea to the photoreceptors (PRs), which convert them into an electrical signal transmitted to the brain. The neural retina comprises the PRs, cones, rods, the interneurons, the ganglion cells, the glial cells such as retinal Müller's glial cells (MGC), astrocytes, and microglia (Figure 1). The retina is vascularized by two separate vascular systems, the retinal vessels, branches of the central retinal artery that vascularize the inner retinal layers, and the choroidal vessels, branches of the ciliary arteries that supply the avascular PR layer through the retinal pigment epithelium (RPE) cells. In primates and human, visual acuity, photopic vision, and color vision are ensured by the macula, a highly specialized retinal area that comprises less than $5 \%$ of the total retinal surface, located at the center of the visual axis. The center of the macula, the fovea, is devoid of retinal vessels and composed exclusively of cones and MGC cells.

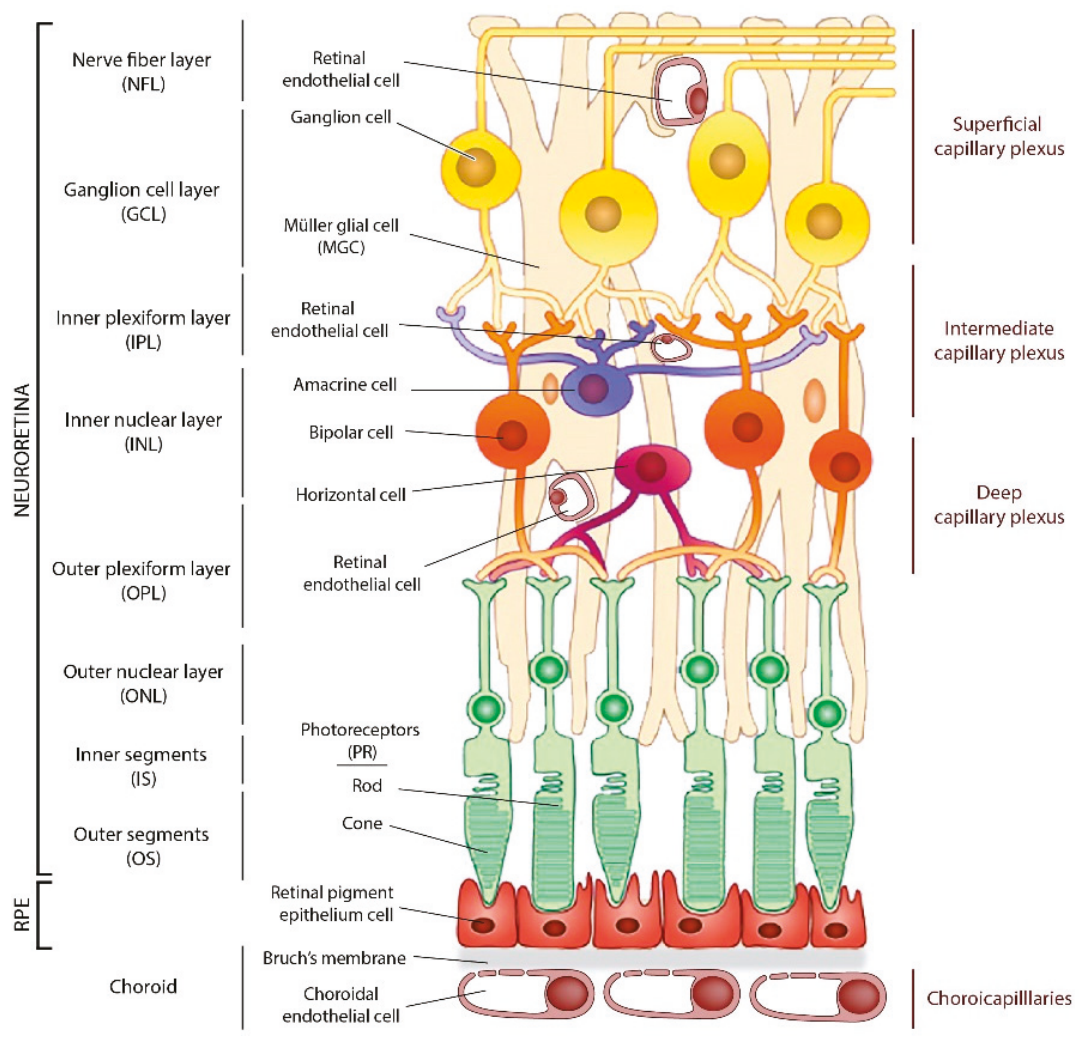

Figure 1. Schematic drawing of the cellular components of the retina. Legend Figure 1: There are three retinal vascular plexuses tightly coordinated with retinal neurons and a choroid plexus underlying RPE. GCL: Ganglion cell layer; NFL: Nerve fiber layer; INL: Inner nuclear layer; IPL: Inner plexiform layer; MGC: Müller glial cell; ONL: Outer nuclear layer; OPL: Outer plexiform layer; OS: Outer segments; IS: Inner segments; PR: Photoreceptors; RPE, retinal pigment epithelium. 
The retina is separated from the circulation by two blood-retinal barriers (BRB). The inner BRB consists of a neuro-glio-vascular complex formed by tight junctions between endothelial cells of the retinal capillaries, pericytes, astrocytes, MGC, and microglia [1]. The outer BRB is formed by the tight-junction monolayer of RPE cells that are in close contact with the choriocapillaries, which control exchanges through diaphragmed fenestrations [1]. Oxygen is the most supply-limited metabolite in the retina [2]. Its supply to the retina is ensured by the choroid, which provides oxygen to the outer retina, whilst the retinal circulation provides the oxygen requirements of the inner retina. In normal condition, the level of oxygen tension $\left(\mathrm{Po}_{2}\right)$ in the outer retina is ten times lower than in the inner retina [3]. Oxygen and glucose consumption are metabolized to lactate, while aerobic glycolysis dominates energy production in the outer retina. Several factors modify $\mathrm{Po}_{2}$ level and utilization at the cellular level: the retinal depth, the light, and hyperoxia [4,5]. PRs have almost all mitochondria in their inner segments far from blood vessels. Light decreases oxygen utilization on the outer retina as much as by a factor of two and increase $\mathrm{Po}_{2}$. Hyperoxia dramatically increases $\mathrm{Po}_{2}$ in the retina with the increase higher in outer retina compared to inner retina. The development and maintenance of retinal vasculature is regulated by $\alpha$ subunits of hypoxia-inducible factor (HIF), which induce genes required for retinal homeostasis, such as vascular endothelial growth factor (VEGF) under hypoxic conditions [6]. HIF proteins, which act as regulators of oxygen homeostasis, also depend on iron for their activity, and they regulate genes involved in iron metabolism [7]. Hyperoxia is deleterious to the outer retina as the oxygen leads to the formation of reactive oxygen species (ROS) according to the Fenton and Haber-Weiss reaction catalyzed by iron to generate $\mathrm{RO}^{\circ}$ radical (review in [3]). Iron and oxygen are thus closely linked in retinal metabolism in health and disease conditions.

\section{Iron Homeostasis in the Retina}

\subsection{Distribution of Iron in the Retina}

Iron is widely and unevenly distributed throughout the adult rat retina. The highest concentrations of iron were observed by proton-induced X-ray emission in the choroid, the RPE, and the inner segments of the PR. PR outer segments also contain iron, as inclusions inside the discs [8]. Iron and iron-related parameter (total iron binding capacity, TF and TF saturation percentage) distribution in the eye are different between diurnal and nocturnal animals. In cow and pig retina, iron concentration is higher than in rat retina, suggesting that the nocturnal habit of living could influence iron-related parameters in the retina [9]. The iron level also varies during retinal development and aging. Moos et al. have shown that in rats, iron entry is very high during retinal development and maturation, then decreases in adulthood, and increases again with aging [10]. In rodents, there are gender and strain-specific influences on iron regulation in the neural retina [11]. Human sex-associated differences in iron levels have also been reported, women having more retinal iron than men at all ages [12]. With aging, iron deposits are found in the RPE/choroid complex in rats and in the stroma of the choroid in non-human primate regardless of serum iron concentration [13]. Increased iron levels in the retina have also been reported in human eyes with age [12]. In rodent eyes, both neural retina and RPE/choroid present an increase of iron concentration which is associated with modifications of iron-related proteins mRNA and protein levels $[14,15]$. 


\subsection{Proteins Involved in Retinal Iron Homeostasis}

\subsubsection{General Iron Homeostasis}

Under physiological conditions, almost all non-heme iron $\left(\mathrm{Fe}^{3+}\right)$ in the circulation is transported bound to TF (transferrin-bound iron: TBI) with high affinity (for review on cellular iron metabolism [16]). At cellular level, the TF with two $\mathrm{Fe}^{3+}$ (holo-TF) is bound by its receptor (TFR1), and the complex is internalized. The $\mathrm{Fe}^{3+}$ is released from TF in the endosome under the effect of acidification through the action of an ATP-dependent proton pump. Iron is then reduced into the ferrous form, $\mathrm{Fe}^{2+}$, by endosomal ferrireductase six transmembrane epithelial antigen of the prostate 3 (STEAP3) and exported by divalent metal transporter 1 (DMT1) in the cytosol where it contributes to the labile iron pool (LIP). In case of iron overload, TF is saturated with iron, and the non-transferrin-bound ferrous iron (NTBI) could be up-taken by iron importers such as the ZRT/IRT-like proteins (ZIPs) or DMT1 and joined intracellular LIP. The LIP consists of a transitory pool of iron species associated with a variety of ligands with low affinity (citrate, phosphate, inorganics irons) and easily oxidized, in transit to be distributed to the organelles (in particularly in mitochondria or nucleus) for cell metabolism in iron requiring proteins, stored or released.

In non-erythroid cells, the majority of iron is stored in ferritin (FT). Composed of 24 subunits of both heavy (HFT) and light (LFT) chains, FT forms a tissue-specific heterocomplex which can store up to $4500 \mathrm{Fe}^{3+}$. $\mathrm{Fe}^{2+}$ from LIP is transported to FT by Poly(rC)-binding proteins (PCBP1 and PCBP2) and is oxidized by ferroxidase activity of HFT in an oxygen-dependent manner. Then $\mathrm{Fe}^{3+}$ is stored in the cavity formed by LFT. Iron is released from FT in a controlled manner by autophagy involving a cargo receptor, the nuclear receptor coactivator 4 (NCOA4).

The only known mammalian iron exporter is ferroportin (FPN), a transmembrane protein which requires a multicopper ferroxidase to convert $\mathrm{Fe}^{2+}$ to $\mathrm{Fe}^{3+}$, allowing binding to TF. Hephaestin (HEPC), ceruloplasmin (CP), amyloid-beta precursor protein (APP), and the newly identified zyloklopen (ZP) are co-localized with FPN at the surface membrane or secreted.

\subsubsection{Iron Flux in Retina}

A local retinal homeostasis of iron, independent from the systemic regulation, is suspected by the fact that main proteins involved in iron homeostasis, previously confined to systemic expression, are locally synthesized in the retina (Table 1). In addition, the outer and inner BRB prevents the entry of large quantities of iron into the eye in case of systemic iron overload. The study of mouse models invalidated for iron homeostasis proteins (Table 1) led to a hypothetical model in which iron entry and homeostasis in the retina is divided into two compartments, carried by the RPE and MGC, delimited by the external limiting membrane, and having limited exchanges in physiological condition. 


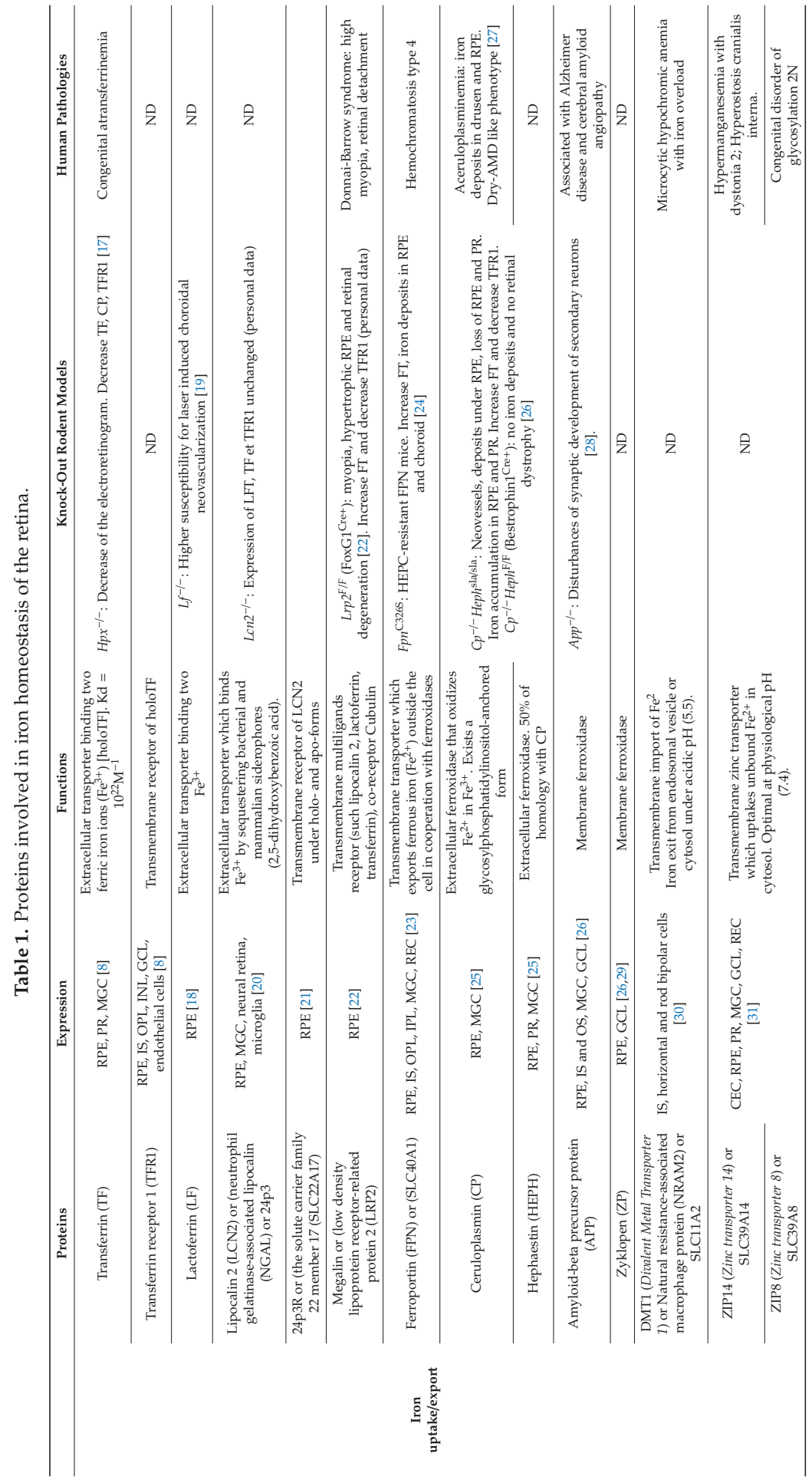




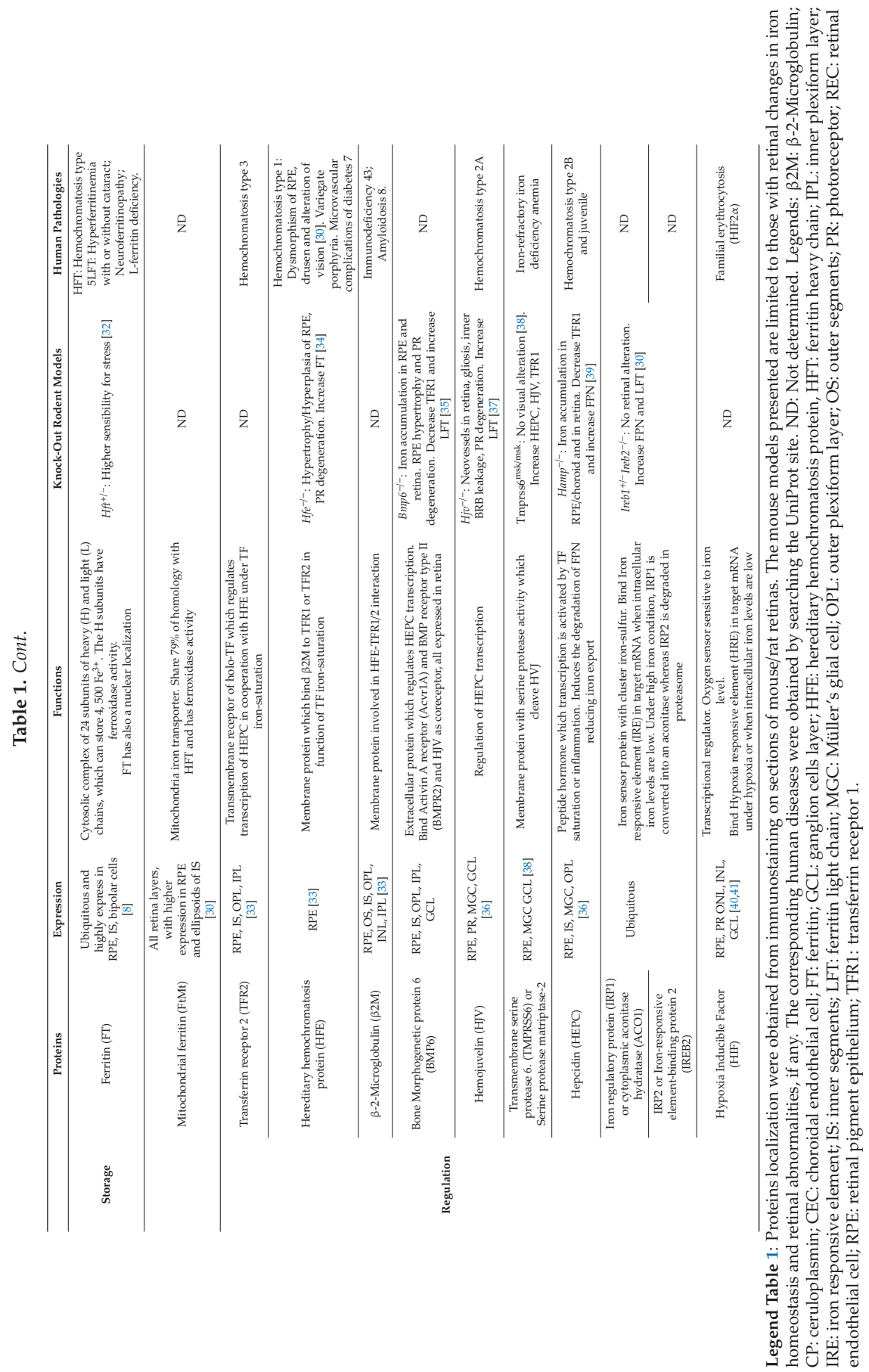


Transferrin-Bound Iron Transport in the Retina

The RPE imports iron bound to TF from the choriocapillaries through the transcytosis of TFR1 present at the basal membrane of the RPE (Figure 2). The transcytosis of the TF/TFR1 complex along microtubules via galectin 4 and Rab11a [42] has been described in vitro. The presence of TFR1 on the apical side of RPE is ambiguous and suggests that TF/TFR1 transcytosis or a potential iron-TF uptake by RPE could egress iron from the outer retina to choriocapillaries. Six hours after an intravitreal injection of holoTF tagged with a fluorochrome, TF is localized in RPE and choroid, which favors the later hypothesis [43]. Another iron entry in RPE is the phagocytosis of PR outer segments which contains high quantities of iron [8]. Once in RPE cytosol, iron is stored in FT and in melanosomes [44]. The release of iron from cell is possible through FPN present at the basal membrane of RPE and a multicopper ferroxidase. HEPH, CP, and APP but not ZP are expressed in RPE [26].

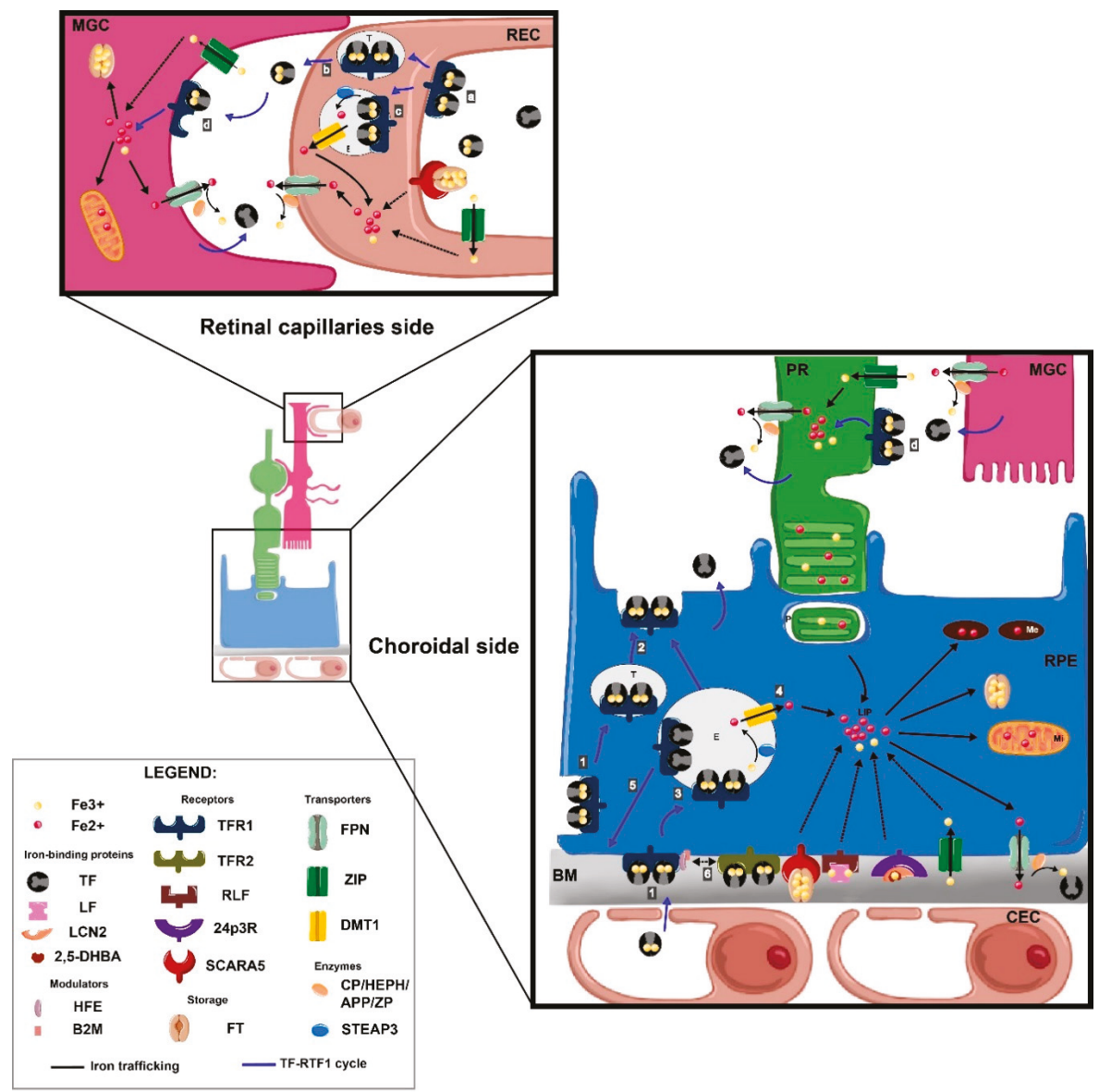

Figure 2. Iron uptake from capillaries and transport in the retina. Legend Figure 2: Under physiological condition, non-heme iron $\left(\mathrm{Fe}^{3+}\right)$ in the circulation is transported bound to transferrin (TF). A. At the 
choroidal side, $\mathrm{Fe}^{3+}$ linked to TF is captured by its receptor 1 (TFR1) (1) at the basolateral level of the retinal pigment epithelium (RPE) (blue arrows). The internalized TF/TFR complex is transported to the apical pole by transcytosis (T) (2) or to the endosome (E) (3). In this case, $\mathrm{Fe}^{3+}$ is released from TF and reduced by the metaloreductase six transmembrane epithelial antigen of the prostate 3 (STEAP3) to ferrous iron $\left(\mathrm{Fe}^{2+}\right)$ and then exported to the cytosol by the transporter divalent metal transporter 1 (DMT1) where it constitutes the free iron pool (LIP) (4). TF and TRF1 are recycled to membrane (5). Iron is then transported from LIP to the organelles as needed, either stored in ferritin (FT) and melanosomes (Me), or exported by ferroportin (FPN) coupled to ferroxidases such as ceruloplasmin $(\mathrm{CP})$ or hephaestin (HEPH), amyloid-beta precursor protein (APP) or zyloklopen (ZP) (black arrows). Hemochromatosis protein (HFE) and beta-2 microglobulin (B2M) associated to TFR1, shift to TFR2 in case of iron overload (saturation of TF) and activate hepcidin (HEPC) transcription (6). B. At retinal capillaries side, $\mathrm{Fe}^{3+}$ bound to TF is up-taken by TFR1 at the luminal side of retinal endothelial cells (REC) (a), and TF/TFR1 pass directly through transcytosis into the retina (b) or endocytosed then exported by FPN (c). TF synthetized by RPE, Müller glial cells (MGC) or photoreceptors (PR) up-taken retinal iron (d) and distributed it throughout the retina, especially to PR. Phototransduction performed on the outer segments of PR is a highly iron-dependent process. PR uptake $\mathrm{Fe}^{3+}$ bound to TF by TFR1 presents in inner segments and export it by FPN or by phagocytosis $(\mathrm{P})$ of the outer segments of PR by RPE. TF-independent iron delivery to the retina can occur, especially in case of systemic iron dysregulation (black dotted lines). Serum FT has a specific receptor, the scavenger receptor class A, member 5 (SCARA5) localizes at the basal membrane of RPE, luminal side of REC, PR and MGC. Lactoferrin (LF), a member of TF superfamily and its receptors (LFR) are present in RPE. Fe $\mathrm{F}^{3+}$ captured by a siderophore (2,5-dihydroxybenzoic acid (2,5-DHBA)) is bound by lipocalin 2 (LCN2) and its receptors (24p3R) in RPE. The non-TF-bound iron (NTBI) is up-taken by MGC, REC, PR and RPE by DMT1 or ZRT/IRT-like proteins (ZIP) importers. BM: Bruch's membrane; CEC: choroidal endothelial cell; E: endosome; Me: melanosome; MGC: Müller's glial cell; MI: mitochondria; P: phagosome; PR: photoreceptor; REC: retinal endothelial cell; RPE: retinal pigment epithelium; T: transcytosis.

In the inner retinal layers, iron is imported through retinal endothelial cells (REC) which express TFR1 at their luminal side [45]. Two mechanisms of iron transfer across the abluminal membrane of REC into the retina are evoked: TF/TFR1 transcytosis or/and TF/TFR1 endocytosis following by iron released from endosome and iron export by FPN. The abluminal membrane of REC expresses FPN colocalized with HEPC, CP, and APP [24,31]. Iron is bound by TF and distributed to the retina or the vitreous. With its unique position extending from the vitreous to PRs and its capacity to synthetize TF [46] and to express FPN [24], MGC plays a crucial role in the distribution of iron from the inner retina to the inner segment of the PR.

Iron presents in the non-vascularized subretinal space, between the apical side of RPE and the PR, is mainly bound to TF, secreted by PRs and RPE, and up-taken by PRs for their highly metabolism activities, by TFR1 express at inner segments. The PR inner segment is the iron storage compartment for PR segments, where both FT chains and mitochondrial FT are highly concentrated [30]. Iron export from the PR is ensured mostly by FPN also present in inner segments, CP and HEPH being poorly involved in favor of APP [26].

\section{Non-Transferrin-Bound Iron Transport in the Retina}

Whereas iron-bound TF is the main transport system to cross the BRB, transferrin-independent iron delivery to the retina can occur (Figure 1). Serum FT, exclusively composed of LFT has specific receptor, the scavenger receptor class A, member 5 (SCARA5) expressed in cytoplasm and nucleus in retinal endothelial cells, ganglion cells, astrocytes, the inner nuclear layer, MGC, microglia, outer nuclear layer, cones segments and RPE. After intravenous injection, serum FT remained confined in retinal endothelial cells in the inner retina [47]. Another protein possibly involved in iron transport is lactoferrin (LF), a multifunctional protein which shares $65 \%$ of homology with TF. It is synthesized in various human ocular tissues mainly in the RPE but not in the neural retina [18]. LF receptors have 
not been studied in the eye but are present in the brain [48]. Lipocalin 2 (LCN2) does not bind to iron directly, but through interaction with siderophores (catecholate and carboxylate) as cofactors [49] could also be implicated in iron transport in the retina [20]. $\beta$-hydroxybutyrate dehydrogenase-2 (Bdh2), an enzyme that is critical for the synthesis of 2,5-dihydroxybenzoic acid (2,5-DHBA), the mammalian siderophore, is found throughout the retina in all cell layers, including ganglion cells, MGC, and RPE cells [50]. Two major membrane-bound receptors for LCN2, megalin and 24p3R, have been identified in RPE [21,22]. Although LCN2 is being recognized as an important factor in retinal diseases [21], its exact contribution in iron retinal transport in health and diseases remain to be determined.

In case of iron overload, the NTBI could be up taken by iron importer ZIP or DMT1. This could explain why in retinal iron overload models, iron continues to accumulate despite the reduced expression of TFR1 in retina and RPE [31]. The specific localization of DMT1 in PRs and bipolar and horizontal cells suggests that it could be involved in providing iron to these cells, for phototransduction or neurotransmitter synthesis [30] ZIP8 and ZIP14 are expressed in RPE, choroid, REC, choroidal endothelial cells (CEC), ganglion cells, PRs, and MGC. At a high degree of TF saturation in the retina, there is a decreased ZIP14 expression whereas ZIP8 expression remains stable [31].

\subsubsection{Iron Regulation in Retina}

Cellular iron uptake and release and the intracellular LIP size are tightly controlled. Transcriptional, post-transcriptional, and post-translational processes regulate iron homeostatic proteins (for a review, see [51]). The main mechanisms of intra- and extra-cellular regulation of iron levels are limited to two extremely controlled systems.

The first system includes iron regulatory proteins (IRP) 1 and 2-intracellular iron regulatory proteins which, depending on the amount of iron, bind iron responsive element (IRE) sequences present on the mRNAs of iron homeostasis proteins such as FPN, TFR1, FT, and DMT1. Depending on the position of the IRE site, IRP controls their translation or degradation. Under conditions of increased cellular iron, IRP1 loses its IRE-binding activity by acquiring an iron in the $4 \mathrm{Fe}-4 \mathrm{~S}$ cluster, whereas IRP2, is degraded by proteasome. In this condition, $t f r 1$ and $d m t 1$ mRNA are degraded, whereas $f t, f p n$, and hif-2 $\alpha$ mRNA are translated. The localization of IRP1 and IRP2 has not yet been identified in the retina but their expressions are ubiquitous in mammalian cells. Mice with $\operatorname{Irp} 1^{+/-} \operatorname{Irp} 2^{-/-}$genotype show more severe neurodegenerative disease than Irp $2^{-/-}$animals [30]. These IRP deficient retinas have increased FPN and FT in the inner segments, MGC endfeet, and inner retina compared to age and strain matched wild type retinas, suggesting that FPN and FT levels are regulated by IRPs in the retina [23]. In a model of light induced retinal degeneration, $2 \mathrm{~h}$ after light exposure, Irp 2 but not Irp1 mRNA increased in the retina [32].

The second system focuses on hepcidin (HEPC), a peptide hormone principally synthetized by the liver. However, HEPC is also synthesized by PR, RPE, and MGC [39]. It is activated by two cellular signaling pathways induced by excess of iron, the transferrin receptor 2 (TFR2)/Human homeostatic iron regulator protein (HFE) pathway and the Bone Morphogenetic protein (BMP6)/Mothers against decapentaplegic homolog 1 (SMAD) pathway. When the TF saturation is high at the basolateral level of the RPE, the HFE is released from TFR1 and binds to TFR2, which activates the transcription of HEPC. BMP6 secreted by the retina and the RPE, binds to its receptors coupled to hemojuvelin (HJV) protein at the apical level of RPE in order to activate the synthesis of HEPC [35]. HEPC binds to the extracellular domain of FPN on the cell surface, leading to its internalization and degradation, effectively preventing cellular iron export and limiting the amount of iron that gets into the extracellular fluid. The specific deletion of HEPC in the retina does not lead to age-associated retinal iron accumulation, whereas liver-specific HEPC silencing leads to early serum, RPE, and retina iron accumulation followed by retinal degeneration [52].

Finally, the hypoxia inducible factor (HIF) acts as a transcription factor for certain iron homeostasis genes such as the $T f, t f r 1, D m t 1, F p n$, and $C p$ genes by binding to a specific hypoxia-responsive element (HRE) site present on their mRNAs. Expression and degradation of HIF are also dependent on iron. In 
fact, $\mathrm{Fe}^{2+}$ is the cofactor of prolyl hydroxylase involved in the degradation of $\mathrm{HIF}-1 \alpha$, and at the same time HIF- $2 \alpha$ has an IRE sequence in the $5^{\prime}$ UTR of its mRNA, which in the condition of iron deficiency, inhibits its translation. Nuclear staining of HIF- $1 \alpha$ was observed in the GCL, the inner nuclear layer and the outer nuclear layer in human and rat [41]. Under retinal hypoxia, both HIF- $1 \alpha$ and HIF- $2 \alpha$ are activated but have cell specific expression within the inner retina. Specifically HIF- $2 \alpha$ activation seems to play a key role in regulating the response of MGC to hypoxia [53].

\section{Physiopathological Role of Iron in the Retina}

\subsection{Iron in Cellular Metabolism/Functions}

\subsubsection{Iron as a Fe-S Structural Motif Involved in Various Cellular Machinery Proteins}

Iron sulfur (Fe-S) proteins are characterized by the presence of Fe-S clusters localized in different cell compartments (for review [54]). IRP1 is a Fe-S cluster that participates in sensing and regulating iron homeostasis in the retina. Frataxin is a nuclear-encoded mitochondrial protein involved in Fe-S cluster assembly, heme synthesis, and intracellular iron homeostasis. Frataxin is an allosteric activator which binds to this assembly complex [55]. It is present in the retina [56] and in the RPE [57] and could be responsible for retinal neurodegeneration induced by defective mitochondrial function [58]. In addition, Fe-S clusters may act as biological sensors by their binding properties to molecular oxygen and nitric oxide [59] both critical for the retinal physiology and pathology.

\subsubsection{Iron in Nucleic Acids Machinery, Cell Proliferation, and DNA Repair}

A recent review has reported the multiple implications of iron in DNA synthesis and repair, as well as in RNA metabolism [60]. Cytosolic and nuclear Fe-S proteins intervene in the genome stability [61]. Iron has been implicated in DNA synthesis and repair as a cofactor of sirtuin 2, an histone deacetylase, involved in iron homeostasis [62]. Sirtuin 2 maintains cellular iron levels by binding the nuclear factor erythroid-2-related factor 2 (NRF2) leading to a reduction in total and nuclear NRF2 levels. NRF2 is a transcription factor that plays key roles in retinal antioxidant and detoxification responses and has been linked with the development of age-related macular degeneration (AMD) [63].

Mitochondria are a major source of ROS and mitochondrial DNA is very susceptible to oxidative damage [64]. In RPE cells, mitochondrial DNA is damaged by hydrogen peroxide [65]. Deletions in mitochondrial DNA occurred in function of age in human neural retina [66], and the accumulation of age-related mitochondrial mutations in the eye has been correlated with a decrease in ATP production and increase ROS output, leading to oxidative stress, inflammation, and degradation [67].

\subsubsection{Iron in Oxygen Transport and Regulation}

Hemoglobin is synthetized in the retina [68]. It is one of the main protein synthesized in primary cultures of human RPE and secreted in vivo through the basolateral membrane [69].

Under physiological condition, free hemoglobin is bound by haptoglobin, but in case of massive hemolysis, hemoglobin releases free heme which binds hemopexin. Both hemopexin and haptoglobin have been described in the human retina [70,71]. The mRNAs for both haptoglobin and hemopexin were detected in the neural retina and PR as well as ganglion cells but not in RPE cells.

Neuroglobin is a highly conserved oxygen-binding protein reviewed in [72] and highly expressed in the retina. Its role is to facilitate oxygen metabolism, being localized in mitochondria. Hemin, the ferric chloride salt of heme enhances neuroglobin expression and protects animal model of $\mathrm{N}$-methyl-N-nitrosourea-induced retinal degeneration [73]. In this model, hemin protects also cones from apoptosis. Neuroglobin has also been associated with retinal damage induced by light [74] which may reflect the changes in iron metabolism first described with light on retina [32]. It has also been associated with VEGF expression and thus could participate in retinal angiogenesis [75]. 
Heme, $\mathrm{Fe}^{2+}$ protoporphyrin IX, the prosthetic group of hemoproteins including hemoglobin, neuroglobin, oxidases/peroxidases, or cytochromes can be released after auto-oxidation. Heme transporter proteins also intervene in iron metabolism in the retina, and their dysregulation could potentially cause oxidative cell damage. All three heme transporters feline leukemia virus subgroup $C$ receptor (FLVCR), breast cancer resistance protein (BCRP), and proton-coupled folate transporter (PCFT/HCP-1) are expressed in the retina and RPE. In the RPE, the expression of FLVCR is restricted to the apical membrane and the expression of BCRP and PCFT to the basolateral membrane. In cases of iron overload, the expression of FLVCR and PCFT is upregulated and BCRP is downregulated, suggesting an important role of heme transporter proteins in retinal iron regulation [76].

\subsubsection{Iron and Visual Function}

The involvement of iron in the vision cycle was discovered with the characterization of the enzyme RPE65, as an iron-dependent isomerohydrolase [77]. RPE65, abundant in the RPE [78], ensures the isomerization and hydrolysis of all-trans retinyl ester to 11-cis retinol. RPE65 is essential for vision, and mutations in rpe65 genes induce Leber congenital amaurosis, a form of retinitis pigmentosa that leads to blindness [79]. Recently, RPE65 was also shown to intervene in the production of meso-zeaxantin, an ocular specific carotenoid which protects the fovea from oxidative stress [80].

An alternate pathway for 11-cis retinol recycling has been described in MGC by isomerases 1 or 2 that also appear to be iron dependent [81]. Few studies have analyzed the iron flux in the retina with the diurnal cycle conversely to what has been performed in brain in mice $[82,83]$. Among the sensory guanylate cyclase proteins and signaling network, guanylyl cyclase activating protein 5 is the only protein that binds strongly $\mathrm{Fe}^{2+}$ in zebrafish [84]. It is proposed as redox sensor in visual transduction.

Phototransduction depends on the phagocytosis of outer segments from PR by the RPE. The constant release of the outer segments from PR and their digestion during phagocytosis by RPE implies membrane biogenesis, a process which needs iron as a cofactor of fatty acid desaturase [85]. Royal College Surgeon (RCS) rats invalided for the phagocytosis protein Myeloid-epithelial-reproductive tyrosine kinase (MERTK) have increased iron in retina and particularly in RPE phagosomes and also increased retinal FT and TF expression [86].

Iron is also involved in neurotransmitters secretion as it regulates glutamate secretion by RPE cells via the cytosolic aconitase pathway [87]. Dopamine biosynthesis in specialized amacrine cells results from the conversion of the amino acid L-tyrosine in L-3,4-dihydroxyphenylalanine (L-DOPA) using oxygen and $\mathrm{Fe}^{2+}$ [88]. Synaptosomal nerve-associated protein 25 (SNAP-25) is a Fe-S protein involved in synapse vesicle fusion with plasma membranes highly present in retina [89].

A significant number of ATP binding cassette $(\mathrm{ABC})$ transporters, involved in lipid trafficking in retinal cells, have been linked to severe genetic ocular diseases [90]. ABCA4 is present in the PR and transports 11-cis and all-trans isomers of $N$-retinylidene-phosphatidylethanolamine across disc membranes, preventing the accumulation of toxic bisretinoid lipofuscin compounds in PR and RPE cells. In Abca4 null mutant mouse which presents accumulation of N-retinylidineN-ethanolamine (A2E) bisretinoids and lipofuscin in the RPE, intracellular iron accumulation is also observed which contributes to enhancing oxidative cell death [91]. The intracellular accumulation of iron in cells of the RPE in culture decreases the expression of the transporters of cholesterol ABCA1/ABCG1, increasing the level of pro-inflammatory cholesterol in retina [50].

\subsection{The Dark Side of Iron}

\subsubsection{The Crucial Role of Iron in Oxidative Stress-Mediated Damages in the Retina}

The ability of iron to change easily its valence and switch between the $\mathrm{Fe}^{2+}$ and $\mathrm{Fe}^{3+}$ forms, providing or accepting electrons, respectively, ensures a privileged position in living matter as mediator of key biochemical reactions. However, the presence of free labile iron in cell or NTBI in circulation is prone to generate highly ROS in the Fenton/Haber-Weiss reaction. 


$$
\begin{aligned}
& \mathrm{Fe}^{2+}+\mathrm{H}_{2} \mathrm{O}_{2} \rightarrow \mathrm{Fe}^{3+}+\mathrm{OH}^{-}+\mathrm{HO}^{\prime}: \text { Fenton reaction } \\
& \mathrm{O}_{2}^{--}+\mathrm{Fe}^{3+} \rightarrow \mathrm{O}_{2}+\mathrm{Fe}^{2+}: \text { Haber-Weiss reaction } \\
& \mathrm{O}_{2}{ }^{--}+\mathrm{H}_{2} \mathrm{O}_{2} \stackrel{\mathrm{Fe}^{2+} ; \mathrm{Fe}^{3+}}{\rightarrow} \mathrm{O}_{2}+\mathrm{OH}^{-}+\mathrm{HO}: \text { Fenton/Haber-Weiss reaction }
\end{aligned}
$$

The toxicity of free iron has been extensively studied on neuronal and retinal cells, and they are not sensitive to the same doses of iron $[46,92,93]$, the cones being the most sensitive to iron [94]. In RPE cells, the interaction of iron with bisretinoids and lipofuscin induces cell damage and retinal degeneration [91]. Conversely, melanin can bind large amounts of iron to preserve the RPE and the choroid from a pro-oxidant environment, intensified by light exposure. However, with age, the accumulation of iron in melanosomes associated with a reduction in the amount of melanin in RPE promotes the formation of free radicals [95]. Exposure of RPE cells to high non-lethal doses of iron leads to a decrease in phagocytic and lysosomal activity [15], favoring the accumulation of breakdown products of Vitamin A (lipofuscin) leading to the formation of glycation end products (AGE) present in drusen, RPE, and Bruch's membrane of AMD patients [91]. In addition, phagocytosis of PR discs, peroxidized by ferrous ions, damage the membranes of phagosomes and lysosomes in RPE cells in culture $[15,96]$.

In hypoxic conditions, an efflux of iron from RPE to the basolateral direction [97] could explain, at least in part, that PRs tolerate better hypoxia than hyperoxia [98]. $\mathrm{Fe}^{2+}$ contributes also to light-induced PR cell death through the production of hydroxyl radicals [99]. The ascorbate-Fe ${ }^{2+}$ complex induce lipid peroxidation in rod outer segment membranes and subsequently damage proteins such as rhodopsin by carbonylation or loss of thiol groups [100]. Finally, free heme can be also a source of redox-active iron and therefore highly toxic for the retina and for RPE cells [101].

In optic neuropathy, such as glaucoma, several mechanisms involved in ganglion cell death seem to be enhanced by iron-dependent oxidative stress [102,103].

Iron is thus a key component of oxidative-induced damages in the retina and in the RPE and involved in major cell death mechanisms.

\subsubsection{Retinal Cells Death Mechanisms in Iron Overload}

Iron overload, induced experimentally by the implantation of iron particles in rat vitreous cavity caused apoptosis (TUNEL-positive nuclei) in the outer nuclear layer after only 2 days [104]. Rat retinal explant exposed to iron showed an early increase of necrotic markers, such as lactate deshydrogenase, receptor-interacting serine/threonine-protein (RIP) kinase, and incorporation of propidium iodide, even before intraretinal iron accumulation was detected. Using retinal organo-culture, it was observed that iron deposits in retinal explants induced a shift from necrosis to apoptosis with activation of caspase 3 and TUNEL-positive nuclei [105]. Increased intraocular iron levels following intravitreal $\mathrm{FeSO}_{4}$ injection caused oxidative damage of $\mathrm{PR}$, as shown by the increase of superoxide radicals; hydroxynonenal, a marker of lipid peroxidation; and increased expression of heme oxygenase 1 [94]. Retinal iron overload also activates the NOD-like receptor family, pyrin domain containing 3 (NLRP3) inflammasome signaling pathway. In fact, the expression levels of NLRP3, activated caspase-1, a downstream target of NLRP3, and interleukin (IL) 1ß were higher in the retinas of HFE KO mice, a model of genetic iron overload [106]. Ferroptosis, a newly characterized form of necrosis, is induced by the accumulation of iron in degenerative diseases and has been described in RPE cells in culture subjected to oxidative stress [107]. Glutathione depletion also induced ferroptosis, autophagy, and premature senescence in RPE cells [108].

\subsubsection{Inflammation}

The general implication of iron in inflammation has been recently reviewed, and it will not be detailed here [109]. In RPE cells, the intracellular accumulation of iron activated the NRLP3 inflammasome pathway via the repression of the degradation of aluRNA by double-stranded RNA-specific endoribonuclease (DICER1). This mechanism involved the sequestration of the cofactor 
PCBP2 [110] and has been advocated in AMD. It has been also reported that iron induces the synthesis of complement C3 by activation of the Extracellular signal-regulated kinases (ERK)/SMAD3/CCAAT Enhancer Binding Protein Delta (CEBPD) 48 pathway [111]. The complement factor C5 carries between 13 and 15 iron atoms necessary for its conversion into an active form C5b by C 5 convertase, a complex formed from the cleavage products of C3 [112], which shows the importance of iron in complement pathways activation, a recognized risk factor for AMD [113].

The prion protein $\left(\mathrm{PrP}^{\mathrm{C}}\right)$, the principal protein implicated in the pathogenesis of human and animal prion disorders, is also implied in retinal degeneration due to iron metabolism dysfunction. This neuronal protein is expressed in many tissues of the eye, such as the retina and the cornea trabeculum. $\mathrm{PrPC}^{\mathrm{C}}$ is also expressed on the basolateral membrane of RPE, where it facilitates uptake of iron from choriocapillaries to neuroretina by functioning as a ferrireductase partner for divalent metal transporters. PrP-scrapie $(\operatorname{PrP}(\mathrm{Sc}))$, a misfolded isoform of this $\mathrm{PrP}^{\mathrm{C}}$ accumulates in the neuroretina resulting in iron accumulation [114].

In the brain, IL6 produced by microglia in response to lipopolysaccharide, induced the production of HEPC by astrocytes [115]. HEPC prevented the iron overload-activated neuronal apoptosis [115]. LPS induced also an HFE-independent expression of HEPC in MGC and in the RPE, both in vitro and in vivo. The increase in HEPC levels in retinal cells, occurring with a decrease in FPN levels, led to oxidative stress and apoptosis within the retina in vivo [116]. On the other hand, in both in vitro and in vivo models of amyloid $\beta$-induced pathology, HEPC downregulates the inflammatory and pro-oxidant processes in astrocytes and microglia and protected neurons from cell death [117]. Microglia and MGC activation associated with reactive gliosis has been observed in HJV knockout mice ( $\mathrm{Hjv}^{-1-}$ mice) with aging and subsequent retinal iron accumulation [37].

The role of LCN2 has been suspected in AMD where its expression is increased in aqueous humor and in the infiltrating cells present in the retina and choroid [118]. An age-related increase in LCN2 was described in RPE cells of Beta-crystallin A3 (Cryba1) conditional knockout mouse, a model of AMD associated with chronic inflammation response [49], but the exact implication of LCN2 in iron metabolism in these models remains to be studied.

\subsubsection{Angiogenesis}

Increased iron levels in the retina could also have a role in the development of new vessels by inhibiting the anti-angiogenic effect of cleaved high molecular weight kininogen (Hka) [119], promoting the expression of succinate receptor 1 (SUCNR1 or GPR91) [120] which stimulates production of pro-angiogenic factors VEGF and angiopoietin [121]. In $\mathrm{Hjv}^{-1-}$ mice, that leads to abnormal retinal iron overload. Proliferation of new leaky blood vessels in the vitreous was associated with reactive gliosis involving MGC and microglia [37]. In addition to proliferation by migratory cells, intravitreal hemoglobin also stimulates a transient proliferation in cells of the RPE and possibly in some supportive cells of the neural retina, such as MGC and astrocytes [122]. Iron also plays a role in HIF transcriptional regulation of pro-angiogenic genes [51]

\section{Role of Iron in Retinal Diseases}

\subsection{Siderosis and Retinal Hemorrhages}

Eye siderosis is probably the first known manifestation of iron toxicity for the eye. The presence of a foreign body containing iron inside the eyeball leads to various clinical complications including heterochromia of the iris, mydriasis, cataract, and retinal and RPE atrophy. Electroretinography analysis shows a decrease in a and $\mathrm{b}$ wave amplitudes, due to the progressive degeneration of the cones and rods (for a review, see [123]). The increase in iron can be observed histologically as a granular structure with FT or hemosiderin into cells. The level of vitreous iron also increases [124].

Retinal hemorrhages are present in several retinal pathologies, such as exudative AMD, diabetic retinopathy, or myopic degeneration, and they are particularly deleterious for vision when located 
in the subretinal space. Vision loss is dependent on the size of the hemorrhage and the ability of the tissue to shed blood $[125,126]$. During sub-macular hemorrhage early PR damage has been reported within $24 \mathrm{~h}$ [127]. In rabbits, the injection of their own blood into the subretinal space leads to a progressive degeneration of the PRs from one day after injection until a total destruction at 7 days, with an accumulation of iron in the outer segments of PR and in RPE [128]. The increased release of iron from hemoglobin induces peroxidation of unsaturated phospholipids, which are extensively present in the retina and affects particularly retinal neurons compared to the retinal glial cells [129].

\subsection{Retinal Manifestations of Inherited Iron Disorders}

Iron can accumulate in the retina of patients with inherited diseases involving mutations in genes encoding proteins of iron homeostasis, which can cause an imbalance in the metabolism of retinal iron. The most common hereditary hemochromatosis is related to a mutation in the Hfe gene resulting in excessive absorption of iron by the intestine and its accumulation in the organs. Mutations in the Tfr2, Fpn, Hjv, and Hepc genes are also involved in the development of hemochromatosis. The clinical findings associated to the retinal iron accumulation in these patients, as well as the impact on visual function, are quite rarely reported due to the variability of penetrance and the existence of a treatment reducing systemic iron overload. However, iron deposits and other changes in the RPE as well as visual acuity loss have been already reported [130].

Aceruloplasminemia is an autosomal recessive disorder caused by mutations in the Cp gene, resulting in a defect in the export of iron from cells. The retina, brain, and pancreas are overloaded with iron, leading to the clinical consequences as retinal degeneration, dementia, and diabetes. Several cases associating yellow discoloration of the fundus, atrophy of the RPE, and drusen-like deposits in the macula have been described. In post-mortem sections, an accumulation of iron associated with an enlarged RPE and loss of pigment of the RPE was observed (for a review, see [27]).

In animal models invalidated for the genes coding for iron-related proteins, an accumulation of iron in the RPE and PR is systematically observed as well as abnormalities in the RPE and PR degeneration $[25,35,39,52,131]$.

Studies carried out in aging rodents have shown that the increase in iron intakes in food or by intravenous injection leads to local iron deposits in the choroid, the RPE, and the segments of PR, as well as deposits of complement C3 in the Bruch's membrane, hypertrophy, and vacuolation of RPE and changes in the choriocapillaries [132,133].

\subsection{Age-Related Macular Degeneration}

AMD is a leading cause of worldwide blindness in the elderly population, affecting 200 million individuals by 2020 and nearly 300 million by 2040 [134]. The pathological aging of the macula can cause dry or non-neovascular and wet or neovascular AMD. At the early stage, accumulation of extracellular material forms drusen between the basal lamina of the RPE and the inner layer of Bruch's membrane in the eye. At the late stages, degeneration of the PR overlying the drusen can cause severe central vision loss in the dry form, whilst formation of new abnormal blood vessels from the choroid growing into the retina can cause subretinal fluid accumulation and bleeding. The wet form progresses rapidly and is responsible for $90 \%$ of severe vision loss associated with AMD. The pathogenesis of AMD is multifactorial, with genetic and environmental factors such as smoking. It is associated with dysregulations in the angiogenic, oxidative stress, lipid, inflammatory, and complement pathways [135]. Patients with early AMD have more iron in the macula than healthy patients. Iron deposits are found in the melanosomes of the choroid and the RPE, in the central layer of the calcified Bruch's membrane, in the drusen, and at the level of the PR [136]. Part of this iron, found in the pathological retina of AMD patients, is in the toxic free form [137]. Patients with dry AMD have more than twice the concentration of iron in their aqueous humor than in patients with cataract surgery [138]. The macular region of AMD patients with geographic atrophy showed an increase in the expression of proteins involved in iron homeostasis such as TF, FT, and FPN in the PR layer and feet MGC [139]. TF and CP mRNAs 
are increased in the two advanced forms of AMD [140]. In the serum of patients with the different forms of AMD, a significant increase in TF and TFR1 and a significant decrease in the concentration of soluble FT were observed while iron levels were unchanged [141]. Several polymorphisms of the iron homeostasis genes have been associated with risk factors for AMD: Tfr1, Tfr2 (obesity, tobacco) [142], Dmt1 [143], Irp1 and Irp2 [143], and heme oxygenases 1 and 2 (HO1/2) [144]. A recent study has shown that the expression of several miRNA, small non-coding RNA molecules binding in 3'UTR genes, was modified in the serum of AMD patients, especially those controlling the translation of the TFR1 and DMT1 proteins [145].

\subsection{Diabetic Retinopathy}

Diabetic retinopathy is a vision-threatening complication of diabetes affecting approximately 93 million in the middle-aged and elderly populations [146]. Chronic hyperglycemia causes progressive damage to retinal cells and to the retinal capillaries, leading to ischemia, VEGF-mediated retinal vascular abnormalization, and neovascular vessels that leak and bleed into the retina. Macular edema is also a major cause of vision loss in diabetic retinopathy [1]. Clinical reports have shown the link between iron levels in the vitreous and proliferative diabetic retinopathy [124,147]. A strong iron label was observed in the RPE and outer plexiform layer of patients with diabetic retinopathy [148]. In a mouse model of diabetic retinopathy, higher iron concentrations in the retina led to an increased expression of renin by a mechanism dependent on the GPR91 receptor [106].

\subsection{Glaucoma Neuropathy}

Glaucoma is increasingly a cause of irreversible blindness in the world. Its global prevalence is expected to be 76 million by 2020 and 112 million by 2040. Progressive damage to the optic nerve, leading to severe vision loss results from increased ocular pressure and other multiple favoring factors $[149,150]$. Although the link between iron and glaucoma is not yet fully understood, there is a change in iron homeostasis in glaucomatous eyes. TF concentration is increased in the aqueous humor [151], and mRNA of TF are increased in retina [152]. Whilst no differences were found in iron levels in aqueous humor of patients with primary open-angle glaucoma [153], serum levels of iron and FT were significantly increased [154,155], and serum CP level was lower [156]. A glaucomatous mice model had lower retinal iron concentrations than pre-glaucomatous DBA/2J and age-matched C57Bl/6J mice [157]. The expression of FT, CP, and TF was increased in monkey and rat glaucoma models [152,158]. In addition, the role of glutamate excitotoxicity in the pathogenesis of glaucoma is well documented; yet, there seems to be a link between the toxicity of glutamate and the increase in the entry of iron into neurons [159], and iron chelation seems to protect neurons against excitotoxicity and intraocular pressure-induced toxicity $[160,161]$. A mutation in the autophagy receptor optineurin is associated with the pathogenesis of glaucoma. It induces the degradation of the TFR1 and the Rab12-dependent autophagy mechanism leading to retinal ganglion cell death. The addition of iron in this model reduces cell death [162]. It seems that iron metabolism is dysregulated in glaucoma, but the exact role of iron is optic nerve damage and remains to be studied in the pathogenesis of glaucoma.

\subsection{Inherited Retinal Dystrophies and Associated Diseases}

Retinitis pigmentosa affects approximately 1.8 to 2.4 million people around the world. The disease is characterized by degeneration of the PR and progressive complete blindness [163]. Although iron has been shown to accumulate in several models of retinal degeneration, as in rd10 mouse or RCS rat [86,164], the direct link between iron and retinitis pigmentosa has not been established in human disease.

Macular telangiectasia type 2 (MacTel 2) is a complex macular disease, characterized by abnormal perifoveal vessels (telangiectasia), loss of retinal organization, and ultimately loss of macula function. MacTel2 is the only human disease recognized as primarily associated with MGC cells loss. It has been shown that iron accumulates in the retina of patients with MacTel 2. In a murine model of MGC 
ablation that mimics part of MacTel 2 phenotype, there is also an accumulation of iron in retina and in the RPE [148]. Knowing the importance of MGC cells in the regulation of iron levels in the retina, it could be hypothesized that iron accumulates in MacTel 2 as a consequence of MGC loss in the fovea [165].

\section{Iron Neutralization as a Therapeutic Strategy for Retinal Diseases}

\subsection{Chemical Chelators}

Whether iron dysmetabolism in the retina is a cause or a consequence of various retinal diseases, iron accumulation is pathogenic, and its neutralization was shown to protect the retina from oxidative damage and retinal cell death in various models using different neutralizing strategies [133] (Table 2). As early as in the 1970s, an iron chelator, Deferroxamine, was used in humans to reduce the amount of "rust" deposited on the eye with satisfactory results. Used in many other models of retinal degeneration (retinitis pigmentosa [166] or light-induced retinal damage models [167]), this chelator reduces the iron load and preserves the retina. Other chelators, such as Deferriprone, have shown significant protection of the retina in mice with impaired mechanisms of iron homeostasis [168-171]. These chemical chelators are mainly used clinically to treat hemosiderosis induced by frequent transfusions. Administered orally, subcutaneously or intramuscularly, they could led to several eye side effects, including vision loss $[172,173]$. These side effects could be explained because chemical iron chelators also bind the iron necessary for RPE and PR function [133,174].

As highlighted in a recent review [175], the clinical use of chemical chelators is complex because they should (1) target only the organ or tissue which is affected by the iron excess; (2) have a sufficient half-life; (3) cross the different barriers that surround the tissue; and (4) have a rapid elimination route.

Table 2. Comparation between chemical iron chelators and transferrin in clinical use.

\begin{tabular}{|c|c|c|c|c|}
\hline & Deferoxamine & Deferiprone & Deferasirox & Transferrin \\
\hline Iron Binding & $1: 1$ & $3: 1$ & $2: 1$ & $2: 1$ \\
\hline $\begin{array}{c}\text { Route of } \\
\text { administration }\end{array}$ & $\begin{array}{l}\text { Sub-cutaneous (every 8-12h) } \\
\text { Intravenous (IV) (5 days/week) }\end{array}$ & Oral (t.i.d) & Oral (q.d) & Intravenous [176] \\
\hline $\begin{array}{l}\text { Half-Life(after IV } \\
\text { administration) }\end{array}$ & 20-30 min & $3-4 \mathrm{~h}$ & $8-16 \mathrm{~h}$ & $4-8 \mathrm{~d}$ [176] \\
\hline Excretion & Urinary/fecal & Urinary & Fecal & Unknown \\
\hline $\begin{array}{l}\text { Usual Doses } \\
\text { (mg/Kg/d) }\end{array}$ & $25-60$ [177] & 75-100 [177] & $20-40$ [177] & 100 [176] \\
\hline Clinical Use & $\begin{array}{l}\text { Acute iron intoxicationChronic } \\
\text { iron overload }\end{array}$ & $\begin{array}{c}\text { Chronic iron } \\
\text { overload }\end{array}$ & $\begin{array}{l}\text { Chronic iron } \\
\text { overload }\end{array}$ & $\begin{array}{c}\text { Atransferrinemia [178] } \\
\text { Haematological stem } \\
\text { cell transplant [176] }\end{array}$ \\
\hline Ocular Side effects & $\begin{array}{l}\text { Pigmentary retinopathy [173], } \\
\text { visual loss [179], impaired night } \\
\text { vision [180], optic neuritis [173] } \\
\text { and cataract [172]. }\end{array}$ & $\begin{array}{l}\text { Diplopia [181], } \\
\text { cataract [182] and } \\
\text { possible retinal } \\
\text { toxicity [183]. }\end{array}$ & $\begin{array}{l}\text { Lens opacities [184] } \\
\text { and retinal } \\
\text { disorders [185] }\end{array}$ & $\begin{array}{c}\text { No adverse effects } \\
\text { observed }\end{array}$ \\
\hline
\end{tabular}

Legend Table 2: Tid: 3 times a day; q.d: once a day.

\subsection{Natural Chelators}

Other natural molecules generally coming from plants, such as curcumin, polyphenols, and flavonoids, are iron chelators and have shown effectiveness in mouse models of retinal degeneration (for a review, see $[186,187]$ ).

\subsection{Transferrin}

TF is part of the TF superfamily, which also includes lactoferrin, melanotransferrin, and ovotransferrin, which are found in many species of both mammals and invertebrates. It consists in two lobes, each binding a $\mathrm{Fe}^{3+}$ atom with a very high affinity $\left(10^{22} \mathrm{M}^{-1}\right)$. Its primary role is to 
maintain an environment devoid of free iron. TF synthesized by RPE, PR, and neuronal cells is found in the aqueous and vitreous humors $[8,105]$. By single-cell RNA sequencing of human neural retina, mRNA for TF was enriched in peripheral retina compared to fovea [188]. Its expression is amplified during inflammation or immunity to increase the buffering capacity of iron. In light-induced retinal degeneration, TF and TFR1 mRNA increased in retina immediately after light exposure and then decreased at basal level. One day after light exposure, TF was increased, whereas TFR1 was reduced compared to not illuminated mice [32]. TF has long been of therapeutic interest due to its antimicrobial capacity and the ubiquitous presence of TFR1 allowing penetration of the blood-brain barrier [189]. TF has also been used successfully in humans in iron metabolism pathologies and for its cytoprotective capacity [190].

Our laboratory is interested in the potential of TF for the treatment of retinal pathologies (Table 3). Our work has shown that administration of the iron-free form (apoTF) by intraperitoneal injections in rd10 mice, a model of retinitis pigmentosa, preserves PRs better compared to the use of other chelators or antioxidants $[46,164]$. Injected into the vitreous, TF is present throughout the neural retina (MGC) and is eliminated via its receptors by RPE and the choroid without any immunogenic or toxic effect on the retina $[46,105]$. Thus, TF administered in a model of light-induced degeneration, allows the restoration of iron homeostasis, decreases iron accumulation, reduces inflammation and apoptosis, and preserves PRs and visual function [43]. In an ex vivo model of retinal detachment, TF inhibits the degenerative processes activated by the iron excess by reducing necrosis, apoptosis, gliosis, and oxidative stress. In vivo, human TF constitutively expressed in transgenic mice (TG) reduces loss of cones, cleavage of caspase 3, an apoptosis effector, DNA breaks, and necrosis (Figure 3). In rats, TF injected at the time of the detachment, reduces retinal edema, cell death and preserves PRs. In addition to its ability to reduce the accumulation of iron in the retina following detachment, TF also acts on other cellular pathways, no doubt through its interaction with molecular partners which remain to be discovered [105].

Table 3. Transferrin as a therapeutic drug in retinal diseases models.

\begin{tabular}{|c|c|c|c|c|}
\hline Model Experiment & Physiopathology & Administration Mode & Therapeutic Action of Transferrin & References \\
\hline $\begin{array}{l}\text { Primary culture of } \\
\text { Müller glial cells. }\end{array}$ & Iron exposure & $\begin{array}{l}\text { Cell isolation from transgenic } \\
\text { mice carrying the human } \\
\text { transferrin gene (TghTF) }\end{array}$ & $\begin{array}{l}\text { Cell number preservation. Lower necrosis revealed by } \\
\text { lactate dehydrogenase release. Inhibition of mRNA TF } \\
\text { diminution. }\end{array}$ & [46] \\
\hline $\begin{array}{l}\text { Primary culture of } \\
\text { Müller glial cells }\end{array}$ & Iron exposure & $\begin{array}{l}\text { Addition of apo- or } \\
\text { holo-human TF }\end{array}$ & $\begin{array}{c}\text { Dose-dependent cell number preservation by apo-but not } \\
\text { holo-human TF }\end{array}$ & [46] \\
\hline rd10 mice & $\begin{array}{c}\text { Model of retinitis } \\
\text { pigmentosa presenting iron } \\
\text { accumulation in } \\
\text { photoreceptors (PR) }\end{array}$ & $\begin{array}{l}\text { Crossing rd10 mice with } \\
\text { TghTF mice }\end{array}$ & $\begin{array}{l}\text { Preservation of retinal histology (outer and inner nuclear } \\
\text { layers thickness). } \\
\text { Less apoptotic-positive retinal cells.Conservation of rods } \\
\text { and cones morphology }\end{array}$ & [164] \\
\hline rd10 mice & $\begin{array}{l}\text { Model of retinitis } \\
\text { pigmentosa presenting iron } \\
\text { accumulation in PR }\end{array}$ & $\begin{array}{l}\text { Daily intraperitoneal } \\
\text { injections of apo-human TF }\end{array}$ & $\begin{array}{c}\text { Dose-dependent preservation of retinal histology (outer and } \\
\text { inner nuclear layers thickness). } \\
\text { Less apoptotic-positive retinal cells. } \\
\text { Conservation of rods and cones morphology }\end{array}$ & [164] \\
\hline $\begin{array}{l}\text { Light-induced } \\
\text { degeneration }\end{array}$ & $\begin{array}{l}\text { Model of acute degenerative } \\
\text { retina }\end{array}$ & $\begin{array}{l}\text { Intravitreal injection of } \\
\text { apo-human TF before and } \\
\text { after light-induced } \\
\text { degeneration }\end{array}$ & $\begin{array}{c}\text { Preservation of retinal histology and functions. } \\
\text { Preservation of ONL thickness and PR morphology. } \\
\text { Lower ONL apoptotic- positive cells.Regulation of iron } \\
\text { homeostasis balance. } \\
\text { Lower retinal iron accumulation and oxidative stress. } \\
\text { Regulation of retina inflammation and diminution of } \\
\text { microglial cells activation in outer retina. }\end{array}$ & [43] \\
\hline $\begin{array}{l}\text { Light-induced } \\
\text { degeneration }\end{array}$ & $\begin{array}{l}\text { Model of acute degenerative } \\
\text { retina }\end{array}$ & $\begin{array}{l}\text { Electrotransfer of cDNA of } \\
\text { human TF for in oculo } \\
\text { production }\end{array}$ & Preservation of retinal histology and ONL layer thickness. & [43] \\
\hline $\mathrm{P} 23 \mathrm{H}$ rats & $\begin{array}{l}\text { Model of retinitis } \\
\text { pigmentosa }\end{array}$ & $\begin{array}{l}\text { Electrotransfer of cDNA of } \\
\text { human TF for in oculo } \\
\text { production }\end{array}$ & Preservation of retinal histology and ONL layer thickness. & [43] \\
\hline $\begin{array}{l}\text { Bone } \\
\text { morphogenetic } \\
\text { protein } 6 \text { mice }\end{array}$ & $\begin{array}{l}\text { Model of hemochromatosis } \\
\text { with retinal iron } \\
\text { accumulation }\end{array}$ & $\begin{array}{l}\text { Intraperitoneal and } \\
\text { intravitreal injections of } \\
\text { apo-human TF }\end{array}$ & $\begin{array}{l}\text { Diminution of iron accumulation in retina pigment } \\
\text { epithelium }\end{array}$ & [43] \\
\hline
\end{tabular}


Table 3. Cont.

\begin{tabular}{|c|c|c|c|c|}
\hline Model Experiment & Physiopathology & Administration Mode & Therapeutic Action of Transferrin & References \\
\hline $\begin{array}{l}\text { Retinal explant of } \\
\text { mice }\end{array}$ & $\begin{array}{l}\text { Retinal detachment with } \\
\text { iron exposure }\end{array}$ & Retinas from TghTF & $\begin{array}{c}\text { Preservation of cones number and rod outer segments } \\
\text { length. } \\
\text { Lower necrosis } \\
\text { Prevention of iron retinal accumulation }\end{array}$ & [105] \\
\hline $\begin{array}{l}\text { Retinal explant of } \\
\text { rats }\end{array}$ & $\begin{array}{l}\text { Retinal detachment with } \\
\text { iron exposure }\end{array}$ & $\begin{array}{l}\text { Addition of apo-human TF } \\
\text { after iron exposure }\end{array}$ & $\begin{array}{c}\text { Preservation of rhodopsin expression level and cones } \\
\text { number } \\
\text { Lower necrosis and apoptosis } \\
\text { Prevention of retinal iron accumulation }\end{array}$ & [105] \\
\hline $\begin{array}{l}\text { Subretinal injection } \\
\text { of hyaluronic acid } \\
\text { in mice }\end{array}$ & $\begin{array}{l}\text { Retinal detachment } \\
\text { presenting iron } \\
\text { accumulation in subretinal } \\
\text { space }\end{array}$ & TghTF mice & $\begin{array}{l}\text { Preservation of retinal histology, rods outer segments length } \\
\text { and number of cones Diminution of retinal oedema and } \\
\text { Müller glial cells activation } \\
\text { Lower apoptosis and necrosis } \\
\text { Regulation of pathways involved in biological functions }\end{array}$ & [105] \\
\hline $\begin{array}{l}\text { Subretinal injection } \\
\text { of hyaluronic acid } \\
\text { in rats }\end{array}$ & $\begin{array}{l}\text { Retinal detachment } \\
\text { presenting iron } \\
\text { accumulation in subretinal } \\
\text { space }\end{array}$ & $\begin{array}{l}\text { Intravitreal injection of } \\
\text { apo-hTF }\end{array}$ & $\begin{array}{c}\text { Preservation of retinal histology, rods outer segments length } \\
\text { Diminution of retinal oedema }\end{array}$ & [105] \\
\hline
\end{tabular}

Legend Table 3: ApoTF: transferrin without iron; HoloTF: transferrin binding iron; INL: inner nuclear layer; ONL: outer nuclear layer; PR: photoreceptors; TF: transferrin; TghTf: transgenic mice carrying the complete human transferrin gene.
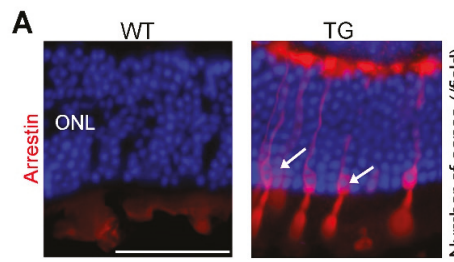

B

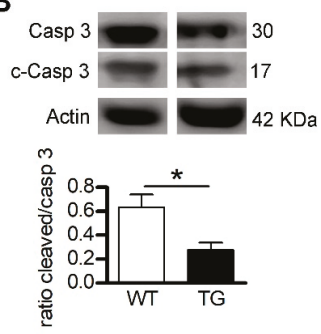

D

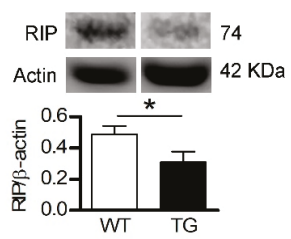

c

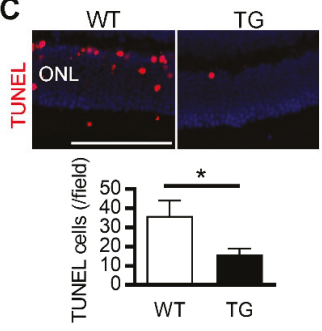

Figure 3. Transferrin expression preserves the detached retina. Legend Figure 3: After retinal detachment (RD), photoreceptors died by apoptosis and necrosis. Transgenic mice (TG) expressing human transferrin (TF) were used to demonstrate the protective effects of TF. (A) Arrestin staining revealed cones in retinal sections of TG mice (arrows) after RD. Cone number was higher in TG compared with WT mice. (B) The ratio of cleaved/pro-caspase 3 protein level was lower in TG mice compared to WT mice after RD. (C) The number of nuclei positive apoptotic-DNA breaks, stained by TUNEL, was reduced in TG mice compared to WT mice. (D) Necrotic RIP kinase protein level was reduced in TG mice compared with WT mice. All values are represented as the mean \pm SEM. Mann-Whitney test $(n=3-6),{ }^{*} p \leq 0.025$. ONL: Outer nuclear layer. Scale bar, $100 \mu \mathrm{m}$. From [105]. Reprinted with permission from AAAS. 


\section{Conclusions}

Iron is one of the most common elements on Earth. Two-hundred years ago, it was discovered that, after desiccation, the residual ashes of an aged human retina could be mobilized by a magnet (as quoted in [191]). Nowadays the chemical study of iron structure and its outer electrons has been revealed by the discovery of quantum effects of iron electron in biology. The best illustrations have been described by Cedric Weber who demonstrated that very specific quantum effects are involved to explain the energy in the binding of iron to oxygen and CO to hemoglobin [192]. This transition metal plays a main role in retinal physiology, but overload leads to retinal degeneration and loss of function. Iron chelation is a potential therapeutic target to prevent retinal degeneration. TF, as an endogenous iron binding protein, avoids toxic effects of iron depletion and activates additional neuroprotective pathways.

Author Contributions: Conceptualization: E.P., Y.C., F.B.-C.; literature search: E.P., A.D.; J.Y., Y.C., F.B.-C.; figure and tables: E.P., J.Y.; writing-original draft preparation E.P., A.D.; Y.C., F.B.-C.; writing-review and editing: E.P., A.D.; Y.C., F.B.-C. All authors have read and agreed to the published version of the manuscript.

Funding: This research received no external funding.

Acknowledgments: We sincerely thank Jean-Claude Jeanny and Marina Yefimova for their significant contribution in the initial description of iron and iron-related proteins in retina.

Conflicts of Interest: Y.C., E.P. and F.B.C. are cited as inventor on a patent for the use of transferrin for the treatment of eye diseases.

\begin{tabular}{|c|c|}
\hline $\mathrm{ABC}$ & ATP binding cassette \\
\hline AMD & Age-Related Macular Degeneration \\
\hline $\mathrm{APP}$ & amyloid-beta precursor protein \\
\hline BMP & Bone Morphogenetic Protein \\
\hline BRB & Blood Retinal Barrier \\
\hline CEC & Choroidal Endothelial Cell \\
\hline $\mathrm{CP}$ & Ceruloplasmin \\
\hline $\mathrm{DFO}$ & Deferroxamine \\
\hline DMT1 & divalent metal transporter 1 \\
\hline Fe-S & Cluster Iron-Sulfur \\
\hline FPN & Ferroportin \\
\hline FT & Ferritin \\
\hline HEPC & Hepcidin \\
\hline HEPH & Hephaestin \\
\hline HFE & Hemochromatosis protein \\
\hline HFT & Heavy Ferritin chin \\
\hline HIF & Hypoxia Inducible Factor \\
\hline HJV & Hemojuveline \\
\hline HRE & Hypoxia Responsive Element \\
\hline IL & Interleukin \\
\hline IRE & Iron Responsive Element \\
\hline IRP & Iron Regulatory Protein \\
\hline LCN2 & Lipocalin 2 \\
\hline LF & Lactoferrin \\
\hline LFT & Light Ferritin chain \\
\hline LIP & Labile Iron Pool \\
\hline MacTel 2 & Macular telangiectasia type 2 \\
\hline MGC & Muller Glial cell \\
\hline NTBI & Non-Transferrin Bound Iron \\
\hline
\end{tabular}




$\begin{array}{ll}\text { NLRP3 } & \text { NOD-like receptor family, pyrin domain containing } 3 \\ \text { PCBP } & \text { Poly(rC)-binding proteins } \\ \text { PrPC } & \text { Prion protein } \\ \text { PR } & \text { photoreceptor } \\ \text { REC } & \text { Retinal Endothelial Cell } \\ \text { ROS } & \text { Reactive Oxygen Species } \\ \text { RPE } & \text { Retinal Pigment Epithelium } \\ \text { SCARA5 } & \text { Scavenger receptor class A, member } 5 \\ \text { SMAD } & \text { Mothers against decapentaplegic homolog } 1 \\ \text { TBI } & \text { Transferrin Bound Iron } \\ \text { TF } & \text { Transferrin } \\ \text { TFR } & \text { Transferrin Receptor } \\ \text { VEGF } & \text { Vascular Endothelial Growth Factor } \\ \text { ZP } & \text { Zyloklopen } \\ \text { ZIP } & \text { ZRT/IRT-like proteins }\end{array}$

\section{References}

1. Daruich, A.; Matet, A.; Moulin, A.; Kowalczuk, L.; Nicolas, M.; Sellam, A.; Rothschild, P.-R.; Omri, S.; Gélizé, E.; Jonet, L.; et al. Mechanisms of macular edema: Beyond the surface. Prog. Retin. Eye Res. 2018, 63, 20-68. [CrossRef] [PubMed]

2. Anderson, B.; Saltzman, H.A. RETINAL OXYGEN UTILIZATION MEASURED BY HYPERBARIC BLACKOUT. Arch. Ophthalmol. 1964, 72, 792-795. [CrossRef] [PubMed]

3. Linsenmeier, R.A.; Zhang, H.F. Retinal oxygen: From animals to humans. Prog. Retin. Eye Res. 2017, 58, 115-151. [CrossRef] [PubMed]

4. Wang, L.; Törnquist, P.; Bill, A. Glucose metabolism in pig outer retina in light and darkness. Acta Physiol. Scand. 1997, 160, 75-81. [CrossRef] [PubMed]

5. Hurley, J.B.; Lindsay, K.J.; Du, J. Glucose, lactate, and shuttling of metabolites in vertebrate retinas. J. Neurosci. Res. 2015, 93, 1079-1092. [CrossRef] [PubMed]

6. Kurihara, T. Development and pathological changes of neurovascular unit regulated by hypoxia response in the retina. Prog. Brain Res. 2016, 225, 201-211.

7. Yang, L.; Wang, D.; Wang, X.-T.; Lu, Y.-P.; Zhu, L. The roles of hypoxia-inducible Factor-1 and iron regulatory protein 1 in iron uptake induced by acute hypoxia. Biochem. Biophys. Res. Commun. 2018, 507, 128-135. [CrossRef]

8. Yefimova, M.G.; Jeanny, J.C.; Guillonneau, X.; Keller, N.; Nguyen-Legros, J.; Sergeant, C.; Guillou, F.; Courtois, Y. Iron, ferritin, transferrin, and transferrin receptor in the adult rat retina. Investig. Ophthalmol. Vis. Sci. 2000, 41, 2343-2351.

9. Garcia-Castineiras, S. Iron, the retina and the lens: A focused review. Exp. Eye Res. 2010, 90, 664-678. [CrossRef]

10. Moos, T.; Bernth, N.; Courtois, Y.; Morgan, E.H. Developmental iron uptake and axonal transport in the retina of the rat. Mol. Cell. Neurosci. 2011, 46, 607-613. [CrossRef]

11. Hahn, P.; Song, Y.; Ying, G.S.; He, X.; Beard, J.; Dunaief, J.L. Age-dependent and gender-specific changes in mouse tissue iron by strain. Exp. Gerontol. 2009, 44, 594-600. [CrossRef] [PubMed]

12. Hahn, P.; Ying, G.S.; Beard, J.; Dunaief, J.L. Iron levels in human retina: Sex difference and increase with age. Neuroreport 2006, 17, 1803-1806. [CrossRef] [PubMed]

13. Ugarte, M.; Osborne, N.N.; Brown, L.A.; Bishop, P.N. Iron, zinc, and copper in retinal physiology and disease. Surv. Ophthalmol. 2013, 58, 585-609. [CrossRef] [PubMed]

14. Chen, H.; Liu, B.; Lukas, T.J.; Suyeoka, G.; Wu, G.; Neufeld, A.H. Changes in iron-regulatory proteins in the aged rodent neural retina. Neurobiol. Aging 2009, 30, 1865-1876. [CrossRef] [PubMed]

15. Chen, H.; Lukas, T.J.; Du, N.; Suyeoka, G.; Neufeld, A.H. Dysfunction of the retinal pigment epithelium with age: Increased iron decreases phagocytosis and lysosomal activity. Investig. Ophthalmol. Vis. Sci. 2009, 50, 1895-1902. [CrossRef] [PubMed] 
16. Lane, D.J.R.; Merlot, A.M.; Huang, M.L.-H.; Bae, D.-H.; Jansson, P.J.; Sahni, S.; Kalinowski, D.S.; Richardson, D.R. Cellular iron uptake, trafficking and metabolism: Key molecules and mechanisms and their roles in disease. Biochim. Biophys. Acta (BBA) - Mol. Cell Res. 2015, 1853, 1130-1144. [CrossRef]

17. Lederman, M.; Obolensky, A.; Grunin, M.; Banin, E.; Chowers, I. Retinal Function and Structure in the Hypotransferrinemic Mouse. Investig. Opthalmol. Vis. Sci. 2012, 53, 605. [CrossRef]

18. Rageh, A.A.; Ferrington, D.A.; Roehrich, H.; Yuan, C.; Terluk, M.R.; Nelson, E.F.; Montezuma, S.R. Lactoferrin Expression in Human and Murine Ocular Tissue. Curr. Eye Res. 2016, 41, 883-889. [CrossRef]

19. Montezuma, S.R.; Dolezal, L.D.; Rageh, A.A.; Mar, K.; Jordan, M.; Ferrington, D.A. Lactoferrin Reduces Chorioretinal Damage in the Murine Laser Model of Choroidal Neovascularization. Curr. Eye Res. 2015, 40, 946-953. [CrossRef]

20. Parmar, T.; Parmar, V.M.; Arai, E.; Sahu, B.; Perusek, L.; Maeda, A. Acute Stress Responses Are Early Molecular Events of Retinal Degeneration in Abca4-/-Rdh8-/- Mice After Light Exposure. Investig. Ophthalmol. Vis. Sci. 2016, 57, 3257-3267. [CrossRef]

21. Parmar, T.; Parmar, V.M.; Perusek, L.; Georges, A.; Takahashi, M.; Crabb, J.W.; Maeda, A. Lipocalin 2 Plays an Important Role in Regulating Inflammation in Retinal Degeneration. J. Immunol. 2018, 200, 3128-3141. [CrossRef] [PubMed]

22. Cases, O.; Obry, A.; Ben-Yacoub, S.; Augustin, S.; Joseph, A.; Toutirais, G.; Simonutti, M.; Christ, A.; Cosette, P.; Kozyraki, R. Impaired vitreous composition and retinal pigment epithelium function in the FoxG1::LRP2 myopic mice. Biochim. Biophys. Acta 2017, 1863, 1242-1254. [CrossRef] [PubMed]

23. Hahn, P.; Dentchev, T.; Qian, Y.; Rouault, T.; Harris, Z.L.; Dunaief, J.L. Immunolocalization and regulation of iron handling proteins ferritin and ferroportin in the retina. Mol. Vis. 2004, 10, 598-607. [PubMed]

24. Theurl, M.; Song, D.; Clark, E.; Sterling, J.; Grieco, S.; Altamura, S.; Galy, B.; Hentze, M.; Muckenthaler, M.U.; Dunaief, J.L. Mice with hepcidin-resistant ferroportin accumulate iron in the retina. FASEB J. 2016, 30, 813-823. [CrossRef] [PubMed]

25. Hahn, P.; Qian, Y.; Dentchev, T.; Chen, L.; Beard, J.; Harris, Z.L.; Dunaief, J.L. Disruption of ceruloplasmin and hephaestin in mice causes retinal iron overload and retinal degeneration with features of age-related macular degeneration. Proc. Natl. Acad. Sci. USA 2004, 101, 13850-13855. [CrossRef]

26. Wolkow, N.; Song, D.; Song, Y.; Chu, S.; Hadziahmetovic, M.; Lee, J.C.; Iacovelli, J.; Grieco, S.; Dunaief, J.L. Ferroxidase hephaestin's cell-autonomous role in the retinal pigment epithelium. Am. J. Pathol. 2012, 180, 1614-1624. [CrossRef]

27. Wolkow, N.; Song, Y.; Wu, T.-D.; Qian, J.; Guerquin-Kern, J.-L.; Dunaief, J.L. Aceruloplasminemia: Retinal histopathologic manifestations and iron-mediated melanosome degradation. Arch. Ophthalmol. 2011, 129, 1466-1474. [CrossRef]

28. Dinet, V.; An, N.; Ciccotosto, G.D.; Bruban, J.; Maoui, A.; Bellingham, S.A.; Hill, A.F.; Andersen, O.M.; Nykjaer, A.; Jonet, L.; et al. APP involvement in retinogenesis of mice. Acta Neuropathol. 2011, 121, 351-363. [CrossRef]

29. Chen, H.; Attieh, Z.K.; Syed, B.A.; Kuo, Y.; Stevens, V.; Fuqua, B.K.; Andersen, H.S.; Naylor, C.E.; Evans, R.W.; Gambling, L.; et al. Identification of Zyklopen, a New Member of the Vertebrate Multicopper Ferroxidase Family, and Characterization in Rodents and Human Cells123. J. Nutr. 2010, 140, 1728-1735. [CrossRef]

30. He, X.; Hahn, P.; Iacovelli, J.; Wong, R.; King, C.; Bhisitkul, R.; Massaro-Giordano, M.; Dunaief, J.L. Iron homeostasis and toxicity in retinal degeneration. Prog. Retin. Eye Res. 2007, 26, 649-673. [CrossRef]

31. Sterling, J.; Guttha, S.; Song, Y.; Song, D.; Hadziahmetovic, M.; Dunaief, J.L. Iron importers Zip8 and Zip14 are expressed in retina and regulated by retinal iron levels. Exp. Eye Res. 2017, 155, 15-23. [CrossRef] [PubMed]

32. Picard, E.; Ranchon-Cole, I.; Jonet, L.; Beaumont, C.; Behar-Cohen, F.; Courtois, Y.; Jeanny, J.-C. Light-induced retinal degeneration correlates with changes in iron metabolism gene expression, ferritin level, and aging. Investig. Ophthalmol. Vis. Sci. 2011, 52, 1261-1274. [CrossRef] [PubMed]

33. Martin, P.M.; Gnana-Prakasam, J.P.; Roon, P.; Smith, R.G.; Smith, S.B.; Ganapathy, V. Expression and polarized localization of the hemochromatosis gene product HFE in retinal pigment epithelium. Investig. Ophthalmol. Vis. Sci. 2006, 47, 4238-4244. [CrossRef] [PubMed]

34. Gnana-Prakasam, J.P.; Thangaraju, M.; Liu, K.; Ha, Y.; Martin, P.M.; Smith, S.B.; Ganapathy, V. Absence of iron-regulatory protein Hfe results in hyperproliferation of retinal pigment epithelium: Role of cystine/glutamate exchanger. Biochem. J. 2009, 424, 243-252. [CrossRef] [PubMed] 
35. Hadziahmetovic, M.; Song, Y.; Wolkow, N.; Iacovelli, J.; Kautz, L.; Roth, M.P.; Dunaief, J.L. Bmp6 regulates retinal iron homeostasis and has altered expression in age-related macular degeneration. Am. J. Pathol. 2011, 179, 335-348. [CrossRef] [PubMed]

36. Gnana-Prakasam, J.P.; Zhang, M.; Martin, P.M.; Atherton, S.S.; Smith, S.B.; Ganapathy, V. Expression of the iron-regulatory protein haemojuvelin in retina and its regulation during cytomegalovirus infection. Biochem. J. 2009, 419, 533-543. [CrossRef] [PubMed]

37. Tawfik, A.; Gnana-Prakasam, J.P.; Smith, S.B.; Ganapathy, V. Deletion of hemojuvelin, an iron-regulatory protein, in mice results in abnormal angiogenesis and vasculogenesis in retina along with reactive gliosis. Investig. Ophthalmol. Vis. Sci. 2014, 55, 3616-3625. [CrossRef] [PubMed]

38. Gnana-Prakasam, J.P.; Baldowski, R.B.; Ananth, S.; Martin, P.M.; Smith, S.B.; Ganapathy, V. Retinal expression of the serine protease matriptase-2 (Tmprss6) and its role in retinal iron homeostasis. Mol. Vis. 2014, 20, 561-574. [PubMed]

39. Hadziahmetovic, M.; Song, Y.; Ponnuru, P.; Iacovelli, J.; Hunter, A.; Haddad, N.; Beard, J.; Connor, J.R.; Vaulont, S.; Dunaief, J.L. Age-Dependent Retinal Iron Accumulation and Degeneration in Hepcidin Knockout Mice. Investig. Ophthalmol. Vis. Sci. 2011, 52, 109-118. [CrossRef] [PubMed]

40. Kast, B.; Schori, C.; Grimm, C. Hypoxic preconditioning protects photoreceptors against light damage independently of hypoxia inducible transcription factors in rods. Exp. Eye Res. 2016, 146, 60-71. [CrossRef]

41. Hughes, J.M.; Groot, A.J.; van der Groep, P.; Sersansie, R.; Vooijs, M.; van Diest, P.J.; Van Noorden, C.J.F.; Schlingemann, R.O.; Klaassen, I. Active HIF-1 in the normal human retina. J. Histochem. Cytochem. 2010, 58, 247-254. [CrossRef]

42. Perez Bay, A.E.; Schreiner, R.; Benedicto, I.; Rodriguez-Boulan, E.J. Galectin-4-mediated transcytosis of transferrin receptor. J. Cell. Sci. 2014, 127, 4457-4469. [CrossRef] [PubMed]

43. Picard, E.; Le Rouzic, Q.; Oudar, A.; Berdugo, M.; El Sanharawi, M.; Andrieu-Soler, C.; Naud, M.C.; Jonet, L.; Latour, C.; Klein, C.; et al. Targeting iron-mediated retinal degeneration by local delivery of transferrin. Free Radic. Biol. Med. 2015, 89, 1105-1121. [CrossRef] [PubMed]

44. Kaczara, P.; Zaręba, M.; Herrnreiter, A.; Skumatz, C.M.B.; Żądło, A.; Sarna, T.; Burke, J.M. Melanosome-iron interactions within retinal pigment epithelium-derived cells. Pigment Cell Melanoma Res. 2012, 25, 804-814. [CrossRef] [PubMed]

45. Baumann, B.H.; Shu, W.; Song, Y.; Simpson, E.M.; Lakhal-Littleton, S.; Dunaief, J.L. Ferroportin-mediated iron export from vascular endothelial cells in retina and brain. Exp. Eye Res. 2019, 187, 107728. [CrossRef] [PubMed]

46. Picard, E.; Fontaine, I.; Jonet, L.; Guillou, F.; Behar-Cohen, F.; Courtois, Y.; Jeanny, J.C. The protective role of transferrin in Muller glial cells after iron-induced toxicity. Mol. Vis. 2008, 14, 928-941. [PubMed]

47. Mendes-Jorge, L.; Ramos, D.; Valença, A.; López-Luppo, M.; Pires, V.M.R.; Catita, J.; Nacher, V.; Navarro, M.; Carretero, A.; Rodriguez-Baeza, A.; et al. Correction: L-Ferritin Binding to Scara5: A New Iron Traffic Pathway Potentially Implicated in Retinopathy. PLoS ONE 2017, 12, e0180288. [CrossRef]

48. Rousseau, E.; Michel, P.P.; Hirsch, E.C. The Iron-Binding Protein Lactoferrin Protects Vulnerable Dopamine Neurons from Degeneration by Preserving Mitochondrial Calcium Homeostasis. Mol. Pharm. 2013, 84, 888-898. [CrossRef]

49. Valapala, M.; Edwards, M.; Hose, S.; Grebe, R.; Bhutto, I.A.; Cano, M.; Berger, T.; Mak, T.W.; Wawrousek, E.; Handa, J.T;; et al. Increased Lipocalin-2 in the retinal pigment epithelium of Cryba1 cKO mice is associated with a chronic inflammatory response. Aging Cell 2014, 13, 1091-1094. [CrossRef]

50. Ananth, S.; Gnana-Prakasam, J.P.; Bhutia, Y.D.; Veeranan-Karmegam, R.; Martin, P.M.; Smith, S.B.; Ganapathy, V. Regulation of the cholesterol efflux transporters ABCA1 and ABCG1 in retina in hemochromatosis and by the endogenous siderophore 2,5-dihydroxybenzoic acid. Biochim. Biophys. Acta (BBA) - Mol. Basis Dis. 2014, 1842, 603-612. [CrossRef]

51. Anderson, C.P.; Shen, M.; Eisenstein, R.S.; Leibold, E.A. Mammalian iron metabolism and its control by iron regulatory proteins. Biochim. Biophys. Acta 2012, 1823, 1468-1483. [CrossRef] [PubMed]

52. Baumann, B.H.; Shu, W.; Song, Y.; Sterling, J.; Kozmik, Z.; Lakhal-Littleton, S.; Dunaief, J.L. Liver-Specific, but Not Retina-Specific, Hepcidin Knockout Causes Retinal Iron Accumulation and Degeneration. Am. J. Pathol. 2019, 189, 1814-1830. [CrossRef] [PubMed] 
53. Mowat, F.M.; Luhmann, U.F.O.; Smith, A.J.; Lange, C.; Duran, Y.; Harten, S.; Shukla, D.; Maxwell, P.H.; Ali, R.R.; Bainbridge, J.W.B. HIF-1alpha and HIF-2alpha Are Differentially Activated in Distinct Cell Populations in Retinal Ischaemia. PLoS ONE 2010, 5, e11103. [CrossRef] [PubMed]

54. Maio, N.; Rouault, T.A. Iron-sulfur cluster biogenesis in mammalian cells: New insights into the molecular mechanisms of cluster delivery. Biochim. Biophys. Acta 2015, 1853, 1493-1512. [CrossRef]

55. Das, D.; Patra, S.; Bridwell-Rabb, J.; Barondeau, D.P. Mechanism of frataxin "bypass" in human iron-sulfur cluster biosynthesis with implications for Friedreich's ataxia. J. Biol. Chem. 2019, 294, 9276-9284. [CrossRef] [PubMed]

56. Efimova, M.G.; Trottier, Y. Distribution of frataxin in eye retina of normal mice and of transgenic R7E mice with retinal degeneration. J. Evol. Biochem. Phys. 2010, 46, 414-417. [CrossRef]

57. Crombie, D.E.; Van Bergen, N.; Davidson, K.C.; Anjomani Virmouni, S.; Mckelvie, P.A.; Chrysostomou, V.; Conquest, A.; Corben, L.A.; Pook, M.A.; Kulkarni, T.; et al. Characterization of the retinal pigment epithelium in Friedreich ataxia. Biochem. Biophys. Rep. 2015, 4, 141-147. [CrossRef]

58. Rouault, T.A.; Maio, N. Biogenesis and functions of mammalian iron-sulfur proteins in the regulation of iron homeostasis and pivotal metabolic pathways. J. Biol. Chem. 2017, 292, 12744-12753. [CrossRef]

59. Crack, J.C.; Green, J.; Thomson, A.J.; Brun, N.E.L. Iron-Sulfur Clusters as Biological Sensors: The Chemistry of Reactions with Molecular Oxygen and Nitric Oxide. Acc. Chem. Res. 2014, 47, 3196-3205. [CrossRef]

60. Puig, S.; Ramos-Alonso, L.; Romero, A.M.; Martínez-Pastor, M.T. The elemental role of iron in DNA synthesis and repair. Metallomics 2017, 9, 1483-1500. [CrossRef]

61. Paul, V.D.; Lill, R. Biogenesis of cytosolic and nuclear iron-sulfur proteins and their role in genome stability. Biochim. Biophys. Acta 2015, 1853, 1528-1539. [CrossRef] [PubMed]

62. Luo, H.; Zhou, M.; Ji, K.; Zhuang, J.; Dang, W.; Fu, S.; Sun, T.; Zhang, X. Expression of Sirtuins in the Retinal Neurons of Mice, Rats, and Humans. Front. Aging Neurosci. 2017, 9, 366. [CrossRef] [PubMed]

63. Zhao, Z.; Chen, Y.; Wang, J.; Sternberg, P.; Freeman, M.L.; Grossniklaus, H.E.; Cai, J. Age-Related Retinopathy in NRF2-Deficient Mice. PLoS ONE 2011, 6, e19456. [CrossRef]

64. Alexeyev, M.; Shokolenko, I.; Wilson, G.; LeDoux,S. The Maintenance of Mitochondrial DNA Integrity-Critical Analysis and Update. Cold Spring Harb. Perspect. Biol. 2013, 5, a012641. [CrossRef] [PubMed]

65. Ballinger, S.W.; Van Houten, B.; Jin, G.F.; Conklin, C.A.; Godley, B.F. Hydrogen peroxide causes significant mitochondrial DNA damage in human RPE cells. Exp. Eye Res. 1999, 68, 765-772. [CrossRef] [PubMed]

66. Barreau, E.; Brossas, J.-Y.; Courtois, Y.; Treton, J.A. Accumulation of Mitochondrial DNA Deletions in Human Retina During Aging. Investig. Ophthalmol. Vis. Sci. 1996, 37, 384-391.

67. Gkotsi, D.; Begum, R.; Salt, T.; Lascaratos, G.; Hogg, C.; Chau, K.-Y.; Schapira, A.H.V.; Jeffery, G. Recharging mitochondrial batteries in old eyes. Near infra-red increases ATP. Exp. Eye Res. 2014, 122, 50-53. [CrossRef]

68. Tezel, T.H.; Geng, L.; Lato, E.B.; Schaal, S.; Liu, Y.; Dean, D.; Klein, J.B.; Kaplan, H.J. Synthesis and Secretion of Hemoglobin by Retinal Pigment Epithelium. Investig. Opthalmol. Vis. Sci. 2009, 50, 1911. [CrossRef]

69. Promsote, W.; Makala, L.; Li, B.; Smith, S.B.; Singh, N.; Ganapathy, V.; Pace, B.S.; Martin, P.M. Monomethylfumarate Induces $\gamma$-Globin Expression and Fetal Hemoglobin Production in Cultured Human Retinal Pigment Epithelial (RPE) and Erythroid Cells, and in Intact Retina. Investig. Opthalmol. Vis. Sci. 2014, 55, 5382. [CrossRef]

70. Hunt, R.C.; Hunt, D.M.; Gaur, N.; Smith, A. Hemopexin in the human retina: Protection of the retina against heme-mediated toxicity. J. Cell. Physiol. 1996, 168, 71-80. [CrossRef]

71. Chen, W.; Lu, H.; Dutt, K.; Smith, A.; Hunt, D.M.; Hunt, R.C. Expression of the protective proteins hemopexin and haptoglobin by cells of the neural retina. Exp. Eye Res. 1998, 67, 83-93. [CrossRef] [PubMed]

72. Ascenzi, P.; di Masi, A.; Leboffe, L.; Fiocchetti, M.; Nuzzo, M.T.; Brunori, M.; Marino, M. Neuroglobin: From structure to function in health and disease. Mol. Asp. Med. 2016, 52, 1-48. [CrossRef] [PubMed]

73. Tao, Y.; Ma, Z.; Liu, B.; Fang, W.; Qin, L.; Huang, Y.F.; Wang, L.; Gao, Y. Hemin supports the survival of photoreceptors injured by N-Methyl-N-nitrosourea: The contributory role of neuroglobin in photoreceptor degeneration. Brain Res. 2018, 1678, 47-55. [CrossRef] [PubMed]

74. Yu, Z.-L.; Qiu, S.; Chen, X.-C.; Dai, Z.-H.; Huang, Y.-C.; Li, Y.-N.; Cai, R.-H.; Lei, H.-T.; Gu, H.-Y. Neuroglobin - A potential biological marker of retinal damage induced by LED light. Neuroscience 2014, 270, $158-167$. [CrossRef]

75. Jin, K.; Mao, X.; Xie, L.; Greenberg, D.A. Interactions between Vascular Endothelial Growth Factor and Neuroglobin. Neurosci. Lett. 2012, 519, 47-50. [CrossRef] 
76. Gnana-Prakasam, J.P.; Reddy, S.K.; Veeranan-Karmegam, R.; Smith, S.B.; Martin, P.M.; Ganapathy, V. Polarized distribution of heme transporters in retinal pigment epithelium and their regulation in the iron-overload disease hemochromatosis. Investig. Ophthalmol. Vis. Sci. 2011, 52, 9279-9286. [CrossRef]

77. Moiseyev, G.; Takahashi, Y.; Chen, Y.; Gentleman, S.; Redmond, T.M.; Crouch, R.K.; Ma, J.-X. RPE65 is an iron(II)-dependent isomerohydrolase in the retinoid visual cycle. J. Biol. Chem. 2006, 281, 2835-2840. [CrossRef]

78. Hamel, C.P.; Tsilou, E.; Pfeffer, B.A.; Hooks, J.J.; Detrick, B.; Redmond, T.M. Molecular cloning and expression of RPE65, a novel retinal pigment epithelium-specific microsomal protein that is post-transcriptionally regulated in vitro. J. Biol. Chem. 1993, 268, 15751-15757.

79. Marlhens, F.; Bareil, C.; Griffoin, J.M.; Zrenner, E.; Amalric, P.; Eliaou, C.; Liu, S.Y.; Harris, E.; Redmond, T.M.; Arnaud, B.; et al. Mutations in RPE65 cause Leber's congenital amaurosis. Nat. Genet. 1997, 17, 139-141. [CrossRef]

80. Shyam, R.; Gorusupudi, A.; Nelson, K.; Horvath, M.P.; Bernstein, P.S. RPE65 has an additional function as the lutein to meso -zeaxanthin isomerase in the vertebrate eye. Proc. Natl. Acad. Sci. USA 2017, 114, 10882-10887. [CrossRef]

81. Betts-Obregon, B.S.; Gonzalez-Fernandez, F.; Tsin, A.T. Interphotoreceptor retinoid-binding protein (IRBP) promotes retinol uptake and release by rat Müller cells (rMC-1) in vitro: Implications for the cone visual cycle. Investig. Ophthalmol. Vis. Sci. 2014, 55, 6265-6271. [CrossRef]

82. Unger, E.L.; Earley, C.J.; Beard, J.L. Diurnal cycle influences peripheral and brain iron levels in mice. J. Appl. Physiol. 2009, 106, 187-193. [CrossRef] [PubMed]

83. Unger, E.L.; Jones, B.C.; Bianco, L.E.; Allen, R.P.; Earley, C.J. Diurnal variations in brain iron concentrations in BXD RI mice. Neuroscience 2014, 263, 54-59. [CrossRef] [PubMed]

84. Lim, S.; Scholten, A.; Manchala, G.; Cudia, D.; Zlomke-Sell, S.-K.; Koch, K.-W.; Ames, J.B. Structural Characterization of Ferrous Ion Binding to Retinal Guanylate Cyclase Activator Protein 5 from Zebrafish Photoreceptors. Biochemistry 2017, 56, 6652-6661. [CrossRef]

85. Shichi, H. Microsomal electron transfer system of bovine retinal pigment epithelium. Exp. Eye Res. 1969, 8 , 60-68. [CrossRef]

86. Yefimova, M.G.; Jeanny, J.-C.; Keller, N.; Sergeant, C.; Guillonneau, X.; Beaumont, C.; Courtois, Y. Impaired retinal iron homeostasis associated with defective phagocytosis in Royal College of Surgeons rats. Investig. Ophthalmol. Vis. Sci. 2002, 43, 537-545.

87. McGahan, M.C.; Harned, J.; Mukunnemkeril, M.; Goralska, M.; Fleisher, L.; Ferrell, J.B. Iron alters glutamate secretion by regulating cytosolic aconitase activity. Am. J. Physiol. Cell Physiol. 2005, 288, C1117-C1124. [CrossRef]

88. Kaushik, P.; Gorin, F.; Vali, S. Dynamics of tyrosine hydroxylase mediated regulation of dopamine synthesis. J. Comput. Neurosci. 2007, 22, 147-160. [CrossRef]

89. Huang, Q.; Hong, X.; Hao, Q. SNAP-25 is also an iron-sulfur protein. FEBS Lett. 2008, 582, 1431-1436. [CrossRef]

90. Molday, R.S. Insights into the Molecular Properties of ABCA4 and Its Role in the Visual Cycle and Stargardt Disease. In Progress in Molecular Biology and Translational Science; Elsevier: Amsterdam, The Netherlands, 2015; Volume 134, pp. 415-431. ISBN 978-0-12-801059-4.

91. Ueda, K.; Kim, H.J.; Zhao, J.; Song, Y.; Dunaief, J.L.; Sparrow, J.R. Iron promotes oxidative cell death caused by bisretinoids of retina. Proc. Natl. Acad. Sci. USA 2018, 115, 4963-4968. [CrossRef]

92. Lucius, R.; Sievers, J. Postnatal retinal ganglion cells in vitro: Protection against reactive oxygen species (ROS)-induced axonal degeneration by cocultured astrocytes. Brain Res. 1996, 743, 56-62. [CrossRef]

93. Kurz, T.; Karlsson, M.; Brunk, U.T.; Nilsson, S.E.; Frennesson, C. ARPE-19 retinal pigment epithelial cells are highly resistant to oxidative stress and exercise strict control over their lysosomal redox-active iron. Autophagy 2009, 5, 494-501. [CrossRef] [PubMed]

94. Rogers, B.S.; Symons, R.C.A.; Komeima, K.; Shen, J.; Xiao, W.; Swaim, M.E.; Gong, Y.Y.; Kachi, S.; Campochiaro, P.A. Differential sensitivity of cones to iron-mediated oxidative damage. Investig. Ophthalmol. Vis. Sci. 2007, 48, 438-445. [CrossRef] [PubMed]

95. Różanowski, B.; Burke, J.M.; Boulton, M.E.; Sarna, T.; Różanowska, M. Human RPE Melanosomes Protect from Photosensitized and Iron-Mediated Oxidation but Become Pro-oxidant in the Presence of Iron upon Photodegradation. Investig. Ophthalmol. Vis. Sci. 2008, 49, 2838-2847. [CrossRef] 
96. Akeo, K.; Hiramitsu, T.; Yorifuji, H.; Okisaka, S. Membranes of retinal pigment epithelial cells in vitro are damaged in the phagocytotic process of the photoreceptor outer segment discs peroxidized by ferrous ions. Pigment Cell Res. 2002, 15, 341-347. [CrossRef]

97. Harned, J.; Nagar, S.; McGahan, M.C. Hypoxia controls iron metabolism and glutamate secretion in retinal pigmented epithelial cells. Biochim. Biophys. Acta 2014, 1840, 3138-3144. [CrossRef]

98. Reiner, A.; Fitzgerald, M.E.C.; Del Mar, N.; Li, C. Neural control of choroidal blood flow. Prog. Retin. Eye Res. 2018, 64, 96-130. [CrossRef]

99. Imamura, T.; Hirayama, T.; Tsuruma, K.; Shimazawa, M.; Nagasawa, H.; Hara, H. Hydroxyl radicals cause fluctuation in intracellular ferrous ion levels upon light exposure during photoreceptor cell death. Exp. Eye Res. 2014, 129, 24-30. [CrossRef]

100. Guajardo, M.H.; Terrasa, A.M.; Catalá, A. Lipid-protein modifications during ascorbate-Fe2+ peroxidation of photoreceptor membranes: Protective effect of melatonin. J. Pineal Res. 2006, 41, 201-210. [CrossRef]

101. Hunt, R.C.; Handy, I.; Smith, A. Heme-mediated reactive oxygen species toxicity to retinal pigment epithelial cells is reduced by hemopexin. J. Cell. Physiol. 1996, 168, 81-86. [CrossRef]

102. Tian, Y.; He, Y.; Song, W.; Zhang, E.; Xia, X. Neuroprotective effect of deferoxamine on N-methyl-d-aspartate-induced excitotoxicity in RGC-5 cells. Acta Biochim. Biophys. Sin. (Shanghai) 2017, 49, 827-834. [CrossRef] [PubMed]

103. Thaler, S.; Fiedorowicz, M.; Rejdak, R.; Choragiewicz, T.J.; Sulejczak, D.; Stopa, P.; Zarnowski, T.; Zrenner, E.; Grieb, P.; Schuettauf, F. Neuroprotective effects of tempol on retinal ganglion cells in a partial optic nerve crush rat model with and without iron load. Exp. Eye Res. 2010, 90, 254-260. [CrossRef] [PubMed]

104. Wang, Z.J.; Lam, K.W.; Lam, T.T.; Tso, M.O. Iron-induced apoptosis in the photoreceptor cells of rats. Investig. Ophthalmol. Vis. Sci. 1998, 39, 631-633. [PubMed]

105. Daruich, A.; Le Rouzic, Q.; Jonet, L.; Naud, M.-C.; Kowalczuk, L.; Pournaras, J.-A.; Boatright, J.H.; Thomas, A.; Turck, N.; Moulin, A.; et al. Iron is neurotoxic in retinal detachment and transferrin confers neuroprotection. Sci. Adv. 2019, 5, eaau9940. [CrossRef]

106. Chaudhary, K.; Promsote, W.; Ananth, S.; Veeranan-Karmegam, R.; Tawfik, A.; Arjunan, P.; Martin, P.; Smith, S.B.; Thangaraju, M.; Kisselev, O.; et al. Iron Overload Accelerates the Progression of Diabetic Retinopathy in Association with Increased Retinal Renin Expression. Sci. Rep. 2018, 8, 3025. [CrossRef]

107. Totsuka, K.; Ueta, T.; Uchida, T.; Roggia, M.F.; Nakagawa, S.; Vavvas, D.G.; Honjo, M.; Aihara, M. Oxidative stress induces ferroptotic cell death in retinal pigment epithelial cells. Exp. Eye Res. 2019, 181, 316-324. [CrossRef]

108. Sun, Y.; Zheng, Y.; Wang, C.; Liu, Y. Glutathione depletion induces ferroptosis, autophagy, and premature cell senescence in retinal pigment epithelial cells. Cell Death Dis. 2018, 9, 753. [CrossRef]

109. Muckenthaler, M.U.; Rivella, S.; Hentze, M.W.; Galy, B. A Red Carpet for Iron Metabolism. Cell 2017, 168, 344-361. [CrossRef]

110. Gelfand, B.D.; Wright, C.B.; Kim, Y.; Yasuma, T.; Yasuma, R.; Li, S.; Fowler, B.J.; Bastos-Carvalho, A.; Kerur, N.; Uittenbogaard, A.; et al. Iron Toxicity in the Retina Requires Alu RNA and the NLRP3 Inflammasome. Cell Rep. 2015, 11, 1686-1693. [CrossRef]

111. Li, Y.; Song, D.; Song, Y.; Zhao, L.; Wolkow, N.; Tobias, J.W.; Song, W.; Dunaief, J.L. Iron-induced Local Complement Component 3 (C3) Up-regulation via Non-canonical Transforming Growth Factor (TGF)-beta Signaling in the Retinal Pigment Epithelium. J. Biol. Chem 2015, 290, 11918-11934. [CrossRef]

112. Vogi, W.; Nolte, R.; Brunahl, D. Binding of iron to the 5th component of human complement directs oxygen radical-mediated conversion to specific sites and causes nonenzymic activation. Complement Inflamm 1991, 8 , 313-319.

113. Toomey, C.B.; Johnson, L.V.; Bowes Rickman, C. Complement factor H in AMD: Bridging genetic associations and pathobiology. Prog. Retin. Eye Res. 2018, 62, 38-57. [CrossRef] [PubMed]

114. Asthana, A.; Baksi, S.; Ashok, A.; Karmakar, S.; Mammadova, N.; Kokemuller, R.; Greenlee, M.H.; Kong, Q.; Singh, N. Prion protein facilitates retinal iron uptake and is cleaved at the $\beta$-site: Implications for retinal iron homeostasis in prion disorders. Sci. Rep. 2017, 7, 9600. [CrossRef] [PubMed]

115. You, L.-H.; Yan, C.-Z.; Zheng, B.-J.; Ci, Y.-Z.; Chang, S.-Y.; Yu, P.; Gao, G.-F.; Li, H.-Y.; Dong, T.-Y.; Chang, Y.-Z. Astrocyte hepcidin is a key factor in LPS-induced neuronal apoptosis. Cell Death Dis. 2017, 8, e2676. [CrossRef] [PubMed] 
116. Gnana-Prakasam, J.P.; Martin, P.M.; Mysona, B.A.; Roon, P.; Smith, S.B.; Ganapathy, V. Hepcidin expression in mouse retina and its regulation via lipopolysaccharide/Toll-like receptor-4 pathway independent of Hfe. Biochem. J. 2008, 411, 79-88. [CrossRef] [PubMed]

117. Urrutia, P.J.; Hirsch, E.C.; González-Billault, C.; Núñez, M.T. Hepcidin attenuates amyloid beta-induced inflammatory and pro-oxidant responses in astrocytes and microglia. J. Neurochem. 2017, 142, 140-152. [CrossRef]

118. Ghosh, S.; Shang, P.; Yazdankhah, M.; Bhutto, I.; Hose, S.; Montezuma, S.R.; Luo, T.; Chattopadhyay, S.; Qian, J.; Lutty, G.A.; et al. Activating the AKT2-nuclear factor-kB-lipocalin-2 axis elicits an inflammatory response in age-related macular degeneration: Lipocalin-2 as an indicator of early AMD. J. Pathol. 2017, 241, 583-588. [CrossRef]

119. Coffman, L.G.; Brown, J.C.; Johnson, D.A.; Parthasarathy, N.; D’Agostino, R.B.; Lively, M.O.; Hua, X.; Tilley, S.L.; Muller-Esterl, W.; Willingham, M.C.; et al. Cleavage of high-molecular-weight kininogen by elastase and tryptase is inhibited by ferritin. Am. J. Physiol. Lung Cell Mol. Physiol. 2008, 294, L505-L515. [CrossRef]

120. Gnana-Prakasam, J.P.; Ananth, S.; Prasad, P.D.; Zhang, M.; Atherton, S.S.; Martin, P.M.; Smith, S.B.; Ganapathy, V. Expression and iron-dependent regulation of succinate receptor GPR91 in retinal pigment epithelium. Investig. Ophthalmol. Vis. Sci. 2011, 52, 3751-3758. [CrossRef]

121. Arjunan, P.; Gnanaprakasam, J.P.; Ananth, S.; Romej, M.A.; Rajalakshmi, V.-K.; Prasad, P.D.; Martin, P.M.; Gurusamy, M.; Thangaraju, M.; Bhutia, Y.D.; et al. Increased Retinal Expression of the Pro-Angiogenic Receptor GPR91 via BMP6 in a Mouse Model of Juvenile Hemochromatosis. Investig. Ophthalmol. Vis. Sci. 2016, 57, 1612-1619. [CrossRef]

122. Burke, J.M.; Smith, J.M. Retinal proliferation in response to vitreous hemoglobin or iron. Investig. Ophthalmol. Vis. Sci. 1981, 20, 582-592. [PubMed]

123. Loporchio, D.; Mukkamala, L.; Gorukanti, K.; Zarbin, M.; Langer, P.; Bhagat, N. Intraocular foreign bodies: A review. Surv. Ophthalmol. 2016, 61, 582-596. [CrossRef] [PubMed]

124. Konerirajapuram, N.S.; Coral, K.; Punitham, R.; Sharma, T.; Kasinathan, N.; Sivaramakrishnan, R. Trace elements iron, copper and zinc in vitreous of patients with various vitreoretinal diseases. Indian J. Ophthalmol. 2004, 52, 145-148. [PubMed]

125. Conart, J.-B.; Berrod, J.-P. [Non-traumatic vitreous hemorrhage]. J. Fr. Ophtalmol. 2016, 39, $219-225$. [CrossRef] [PubMed]

126. Levin, A.V. Retinal hemorrhage in abusive head trauma. Pediatrics 2010, 126, 961-970. [CrossRef]

127. Casini, G.; Loiudice, P.; Menchini, M.; Sartini, F.; De Cillà, S.; Figus, M.; Nardi, M. Traumatic submacular hemorrhage: Available treatment options and synthesis of the literature. Int. J. Retin. Vitr. 2019, 5, 48. [CrossRef]

128. Bhisitkul, R.B.; Winn, B.J.; Lee, O.-T.; Wong, J.; de Souza Pereira, D.; Porco, T.C.; He, X.; Hahn, P.; Dunaief, J.L. Neuroprotective effect of intravitreal triamcinolone acetonide against photoreceptor apoptosis in a rabbit model of subretinal hemorrhage. Investig. Ophthalmol. Vis. Sci. 2008, 49, 4071-4077. [CrossRef]

129. Chen-Roetling, J.; Regan, K.A.; Regan, R.F. Protective effect of vitreous against hemoglobin neurotoxicity. Biochem. Biophys. Res. Commun. 2018, 503, 152-156. [CrossRef]

130. Zerbib, J.; Pierre-Kahn, V.; Sikorav, A.; Oubraham, H.; Sayag, D.; Lobstein, F.; Massonnet-Castel, S.; Haymann-Gawrilow, P.; Souied, E.H. Unusual retinopathy associated with hemochromatosis. Retin Cases Brief Rep. 2015, 9, 190-194. [CrossRef]

131. Gnana-Prakasam, J.P.; Tawfik, A.; Romej, M.; Ananth, S.; Martin, P.M.; Smith, S.B.; Ganapathy, V. Iron-mediated retinal degeneration in haemojuvelin-knockout mice. Biochem. J. 2012, 441, 599-608. [CrossRef]

132. Kumar, P.; Nag, T.C.; Jha, K.A.; Dey, S.K.; Kathpalia, P.; Maurya, M.; Gupta, C.L.; Bhatia, J.; Roy, T.S.; Wadhwa, S. Experimental oral iron administration: Histological investigations and expressions of iron handling proteins in rat retina with aging. Toxicology 2017, 392, 22-31. [CrossRef]

133. Shu, W.; Dunaief, J.L. Potential Treatment of Retinal Diseases with Iron Chelators. Pharmaceuticals (Basel) 2018, 11, 112. [CrossRef] [PubMed]

134. Wong, W.L.; Su, X.; Li, X.; Cheung, C.M.G.; Klein, R.; Cheng, C.-Y.; Wong, T.Y. Global prevalence of age-related macular degeneration and disease burden projection for 2020 and 2040: A systematic review and meta-analysis. Lancet Glob. Health 2014, 2, e106-e116. [CrossRef] 
135. Handa, J.T.; Bowes Rickman, C.; Dick, A.D.; Gorin, M.B.; Miller, J.W.; Toth, C.A.; Ueffing, M.; Zarbin, M.; Farrer, L.A. A systems biology approach towards understanding and treating non-neovascular age-related macular degeneration. Nat. Commun. 2019, 10, 3347. [CrossRef] [PubMed]

136. Biesemeier, A.; Yoeruek, E.; Eibl, O.; Schraermeyer, U. Iron accumulation in Bruch's membrane and melanosomes of donor eyes with age-related macular degeneration. Exp. Eye Res. 2015, 137, 39-49. [CrossRef]

137. Hahn, P.; Milam, A.H.; Dunaief, J.L. Maculas Affected by Age-Related Macular Degeneration Contain Increased Chelatable Iron in the Retinal Pigment Epithelium and Bruch's Membrane. Arch. Ophthalmol. 2003, 121, 1099-1105. [CrossRef]

138. Junemann, A.G.; Stopa, P.; Michalke, B.; Chaudhri, A.; Reulbach, U.; Huchzermeyer, C.; Schlotzer-Schrehardt, U.; Kruse, F.E.; Zrenner, E.; Rejdak, R. Levels of aqueous humor trace elements in patients with non-exsudative age-related macular degeneration: A case-control study. PLoS ONE 2013, 8, e56734. [CrossRef]

139. Dentchev, T.; Hahn, P.; Dunaief, J.L. Strong labeling for iron and the iron-handling proteins ferritin and ferroportin in the photoreceptor layer in age-related macular degeneration. Arch Ophthalmol. 2005, 123, 1745-1746. [CrossRef]

140. Chowers, I.; Wong, R.; Dentchev, T.; Farkas, R.H.; Iacovelli, J.; Gunatilaka, T.L.; Medeiros, N.E.; Presley, J.B.; Campochiaro, P.A.; Curcio, C.A.; et al. The iron carrier transferrin is upregulated in retinas from patients with age-related macular degeneration. Investig. Ophthalmol. Vis. Sci. 2006, 47, 2135-2140. [CrossRef]

141. Čolak, E.; Žorić, L.; Radosavljević, A.; Ignjatović, S. The Association of Serum Iron-Binding Proteins and the Antioxidant Parameter Levels in Age-Related Macular Degeneration. Curr. Eye Res. 2018, 43, 659-665. [CrossRef]

142. Wysokinski, D.; Danisz, K.; Pawlowska, E.; Dorecka, M.; Romaniuk, D.; Robaszkiewicz, J.; Szaflik, M.; Szaflik, J.; Blasiak, J.; Szaflik, J.P. Transferrin receptor levels and polymorphism of its gene in age-related macular degeneration. Acta Biochim. Pol. 2015, 62, 177-184. [CrossRef] [PubMed]

143. Synowiec, E.; Pogorzelska, M.; Blasiak, J.; Szaflik, J.; Szaflik, J.P. Genetic polymorphism of the iron-regulatory protein-1 and -2 genes in age-related macular degeneration. Mol. Biol. Rep. 2012, 39, 7077-7087. [CrossRef] [PubMed]

144. Synowiec, E.; Szaflik, J.; Chmielewska, M.; Wozniak, K.; Sklodowska, A.; Waszczyk, M.; Dorecka, M.; Blasiak, J.; Szaflik, J.P. An association between polymorphism of the heme oxygenase-1 and -2 genes and age-related macular degeneration. Mol. Biol. Rep. 2012, 39, 2081-2087. [CrossRef] [PubMed]

145. Szemraj, M.; Oszajca, K.; Szemraj, J.; Jurowski, P. MicroRNA Expression Analysis in Serum of Patients with Congenital Hemochromatosis and Age-Related Macular Degeneration (AMD). Med. Sci. Monit. 2017, 23, 4050-4060. [CrossRef] [PubMed]

146. Ding, J.; Wong, T.Y. Current epidemiology of diabetic retinopathy and diabetic macular edema. Curr. Diab. Rep. 2012, 12, 346-354. [CrossRef] [PubMed]

147. Ciudin, A.; Hernández, C.; Simó, R. Iron overload in diabetic retinopathy: A cause or a consequence of impaired mechanisms? Exp. Diabetes Res. 2010, 2010, 714108. [CrossRef]

148. Baumann, B.; Sterling, J.; Song, Y.; Song, D.; Fruttiger, M.; Gillies, M.; Shen, W.; Dunaief, J.L. Conditional Müller Cell Ablation Leads to Retinal Iron Accumulation. Investig. Ophthalmol. Vis. Sci. 2017, 58, 4223-4234. [CrossRef]

149. Weinreb, R.N.; Aung, T.; Medeiros, F.A. The pathophysiology and treatment of glaucoma: A review. JAMA 2014, 311, 1901-1911. [CrossRef]

150. Wang, H.-W.; Sun, P.; Chen, Y.; Jiang, L.-P.; Wu, H.-P.; Zhang, W.; Gao, F. Research progress on human genes involved in the pathogenesis of glaucoma (Review). Mol. Med. Rep. 2018, 18, 656-674. [CrossRef]

151. Tripathi, R.C.; Borisuth, N.S.; Tripathi, B.J.; Gotsis, S.S. Quantitative and qualitative analyses of transferrin in aqueous humor from patients with primary and secondary glaucomas. Investig. Ophthalmol. Vis. Sci. 1992, 33, 2866-2873.

152. Farkas, R.H.; Chowers, I.; Hackam, A.S.; Kageyama, M.; Nickells, R.W.; Otteson, D.C.; Duh, E.J.; Wang, C.; Valenta, D.F.; Gunatilaka, T.L.; et al. Increased expression of iron-regulating genes in monkey and human glaucoma. Investig. Ophthalmol. Vis. Sci. 2004, 45, 1410-1417. [CrossRef] [PubMed] 
153. Hohberger, B.; Chaudhri, M.A.; Michalke, B.; Lucio, M.; Nowomiejska, K.; Schlötzer-Schrehardt, U.; Grieb, P.; Rejdak, R.; Jünemann, A.G.M. Levels of aqueous humor trace elements in patients with open-angle glaucoma. J. Trace Elem. Med. Biol. 2018, 45, 150-155. [CrossRef] [PubMed]

154. Fick, A.; Jünemann, A.; Michalke, B.; Lucio, M.; Hohberger, B. Levels of serum trace elements in patients with primary open-angle glaucoma. J. Trace Elem. Med. Biol. 2019, 53, 129-134. [CrossRef] [PubMed]

155. Lin, S.-C.; Wang, S.Y.; Yoo, C.; Singh, K.; Lin, S.C. Association between serum ferritin and glaucoma in the South Korean population. JAMA Ophthalmol. 2014, 132, 1414-1420. [CrossRef] [PubMed]

156. Sarnat-Kucharczyk, M.; Rokicki, W.; Zalejska-Fiolka, J.; Pojda-Wilczek, D.; Mrukwa-Kominek, E. Determination of Serum Ceruloplasmin Concentration in Patients with Primary Open Angle Glaucoma with Cataract and Patients with Cataract Only: A Pilot Study. Med. Sci. Monit. 2016, 22, 1384-1388. [CrossRef] [PubMed]

157. DeToma, A.S.; Dengler-Crish, C.M.; Deb, A.; Braymer, J.J.; Penner-Hahn, J.E.; van der Schyf, C.J.; Lim, M.H.; Crish, S.D. Abnormal metal levels in the primary visual pathway of the DBA/2J mouse model of glaucoma. Biometals 2014, 27, 1291-1301. [CrossRef]

158. Anders, F.; Teister, J.; Funke, S.; Pfeiffer, N.; Grus, F.; Solon, T.; Prokosch, V. Proteomic profiling reveals crucial retinal protein alterations in the early phase of an experimental glaucoma model. Graefes Arch. Clin. Exp. Ophthalmol. 2017, 255, 1395-1407. [CrossRef]

159. Cheah, J.H.; Kim, S.F.; Hester, L.D.; Clancy, K.W.; Patterson, S.E.; Papadopoulos, V.; Snyder, S.H. NMDA receptor-nitric oxide transmission mediates neuronal iron homeostasis via the GTPase Dexras1. Neuron 2006, 51, 431-440. [CrossRef]

160. Liu, P.; Zhang, M.; Shoeb, M.; Hogan, D.; Tang, L.; Syed, M.F.; Wang, C.Z.; Campbell, G.A.; Ansari, N.H. Metal chelator combined with permeability enhancer ameliorates oxidative stress-associated neurodegeneration in rat eyes with elevated intraocular pressure. Free Radic. Biol. Med. 2014, 69, 289-299. [CrossRef]

161. Sakamoto, K.; Suzuki, T.; Takahashi, K.; Koguchi, T.; Hirayama, T.; Mori, A.; Nakahara, T.; Nagasawa, H.; Ishii, K. Iron-chelating agents attenuate NMDA-Induced neuronal injury via reduction of oxidative stress in the rat retina. Exp. Eye Res. 2018, 171, 30-36. [CrossRef]

162. Sirohi, K.; Chalasani, M.L.S.; Sudhakar, C.; Kumari, A.; Radha, V.; Swarup, G. M98K-OPTN induces transferrin receptor degradation and RAB12-mediated autophagic death in retinal ganglion cells. Autophagy 2013, 9, 510-527. [CrossRef] [PubMed]

163. Hamel, C. Retinitis pigmentosa. Orphanet. J. Rare Dis. 2006, 1, 40. [CrossRef] [PubMed]

164. Picard, E.; Jonet, L.; Sergeant, C.; Vesvres, M.H.; Behar-Cohen, F.; Courtois, Y.; Jeanny, J.C. Overexpressed or intraperitoneally injected human transferrin prevents photoreceptor degeneration in rd10 mice. Mol. Vis. 2010, 16, 2612-2625. [PubMed]

165. Scerri, T.S.; Quaglieri, A.; Cai, C.; Zernant, J.; Matsunami, N.; Baird, L.; Scheppke, L.; Bonelli, R.; Yannuzzi, L.A.; Friedlander, M.; et al. Genome-wide analyses identify common variants associated with macular telangiectasia type 2. Nat. Genet. 2017, 49, 559-567. [CrossRef] [PubMed]

166. Obolensky, A.; Berenshtein, E.; Lederman, M.; Bulvik, B.; Alper-Pinus, R.; Yaul, R.; Deleon, E.; Chowers, I.; Chevion, M.; Banin, E. Zinc-desferrioxamine attenuates retinal degeneration in the rd10 mouse model of retinitis pigmentosa. Free Radic. Biol. Med. 2011, 51, 1482-1491. [CrossRef]

167. Li, Z.L.; Lam, S.; Tso, M.O. Desferrioxamine ameliorates retinal photic injury in albino rats. Curr. Eye Res. 1991, 10, 133-144. [CrossRef]

168. Hadziahmetovic, M.; Song, Y.; Wolkow, N.; Iacovelli, J.; Grieco, S.; Lee, J.; Lyubarsky, A.; Pratico, D.; Connelly, J.; Spino, M.; et al. The Oral Iron Chelator Deferiprone Protects against Iron Overload-Induced Retinal Degeneration. Investig. Ophthalmol. Vis. Sci. 2011, 52, 959-968. [CrossRef]

169. Song, D.; Zhao, L.; Li, Y.; Hadziahmetovic, M.; Song, Y.; Dunaief, J.L. The oral iron chelator deferiprone protects against iron overload-induced retinal degeneration in Hepcidin knockout mice. Investig. Ophthalmol. Vis. Sci. 2014, 55, 4525-4532. [CrossRef]

170. Song, D.; Song, Y.; Hadziahmetovic, M.; Zhong, Y.; Dunaief, J.L. Systemic administration of the iron chelator deferiprone protects against light-induced photoreceptor degeneration in the mouse retina. Free Radic. Biol. Med. 2012, 53, 64-71. [CrossRef]

171. Hadziahmetovic, M.; Pajic, M.; Grieco, S.; Song, Y.; Song, D.; Li, Y.; Cwanger, A.; Iacovelli, J.; Chu, S.; Ying, G.-S.; et al. The Oral Iron Chelator Deferiprone Protects Against Retinal Degeneration Induced through Diverse Mechanisms. Transl. Vis. Sci. Technol. 2012, 1, 7. [CrossRef] 
172. Arora, A.; Wren, S.; Gregory Evans, K. Desferrioxamine related maculopathy: A case report. Am. J. Hematol. 2004, 76, 386-388. [CrossRef] [PubMed]

173. Lakhanpal, V.; Schocket, S.S.; Jiji, R. Deferoxamine (Desferal)-induced toxic retinal pigmentary degeneration and presumed optic neuropathy. Ophthalmology 1984, 91, 443-451. [CrossRef]

174. Jauregui, R.; Park, K.S.; Bassuk, A.G.; Mahajan, V.B.; Tsang, S.H. Deferoxamine-induced electronegative ERG responses. Doc. Ophthalmol. 2018, 137, 15-23. [CrossRef] [PubMed]

175. Mobarra, N.; Shanaki, M.; Ehteram, H.; Nasiri, H.; Sahmani, M.; Saeidi, M.; Goudarzi, M.; Pourkarim, H.; Azad, M. A Review on Iron Chelators in Treatment of Iron Overload Syndromes. Int. J. Hematol. Oncol. Stem Cell Res. 2016, 10, 239-247. [PubMed]

176. Sahlstedt, L.; von Bonsdorff, L.; Ebeling, F.; Ruutu, T.; Parkkinen, J. Effective binding of free iron by a single intravenous dose of human apotransferrin in haematological stem cell transplant patients. Br. J. Haematol. 2002, 119, 547-553. [CrossRef]

177. Brittenham, G.M. Iron-chelating therapy for transfusional iron overload. N. Engl. J. Med. 2011, 364, 146-156. [CrossRef]

178. Goya, N.; Miyazaki, S.; Kodate, S.; Ushio, B. A family of congenital atransferrinemia. Blood 1972, 40, $239-245$. [CrossRef]

179. Simon, S.; Athanasiov, P.A.; Jain, R.; Raymond, G.; Gilhotra, J.S. Desferrioxamine-related ocular toxicity: A case report. Indian J. Ophthalmol. 2012, 60, 315-317. [CrossRef]

180. Di Nicola, M.; Barteselli, G.; Dell'Arti, L.; Ratiglia, R.; Viola, F. Functional and Structural Abnormalities in Deferoxamine Retinopathy: A Review of the Literature. Biomed. Res. Int. 2015, 2015, 249617. [CrossRef]

181. Beau-Salinas, F.; Guitteny, M.A.; Donadieu, J.; Jonville-Bera, A.P.; Autret-Leca, E. High doses of deferiprone may be associated with cerebellar syndrome. BMJ 2009, 338, a2319. [CrossRef]

182. Mehdizadeh, M.; Nowroozzadeh, M.H. Posterior subcapsular opacity in two patients with thalassaemia major following deferiprone consumption. Clin. Exp. Optom. 2009, 92, 392-394. [CrossRef]

183. Taneja, R.; Malik, P.; Sharma, M.; Agarwal, M.C. Multiple transfused thalassemia major: Ocular manifestations in a hospital-based population. Indian J. Ophthalmol. 2010, 58, 125-130. [PubMed]

184. Masera, N.; Rescaldani, C.; Azzolini, M.; Vimercati, C.; Tavecchia, L.; Masera, G.; De Molfetta, V.; Arpa, P. Development of lens opacities with peculiar characteristics in patients affected by thalassemia major on chelating treatment with deferasirox (ICL670) at the Pediatric Clinic in Monza, Italy. Haematologica 2008, 93, e9-e10. [CrossRef] [PubMed]

185. Pan, Y.; Keane, P.A.; Sadun, A.A.; Fawzi, A.A. Optical coherence tomography findings in deferasirox-related maculopathy. Retin Cases Brief Rep. 2010, 4, 229-232. [CrossRef]

186. Farajipour, H.; Rahimian, S.; Taghizadeh, M. Curcumin: A new candidate for retinal disease therapy? J. Cell. Biochem. 2018, 120, 6886-6893. [CrossRef]

187. Majumdar, S.; Srirangam, R. Potential of the Bioflavonoids in the Prevention/Treatment of Ocular Disorders. J. Pharm. Pharmacol. 2010, 62, 951-965. [CrossRef] [PubMed]

188. Voigt, A.P.; Whitmore, S.S.; Flamme-Wiese, M.J.; Riker, M.J.; Wiley, L.A.; Tucker, B.A.; Stone, E.M.; Mullins, R.F.; Scheetz, T.E. Molecular characterization of foveal versus peripheral human retina by single-cell RNA sequencing. Exp. Eye Res. 2019, 184, 234-242. [CrossRef]

189. Qian, Z.M.; Li, H.; Sun, H.; Ho, K. Targeted drug delivery via the transferrin receptor-mediated endocytosis pathway. Pharm. Rev. 2002, 54, 561-587. [CrossRef]

190. Gomme, P.T.; McCann, K.B.; Bertolini, J. Transferrin: Structure, function and potential therapeutic actions. Drug Discov. Today 2005, 10, 267-273. [CrossRef]

191. de Jong, P.T.V.M. A Historical Analysis of the Quest for the Origins of Aging Macula Disorder, the Tissues Involved, and Its Terminology. Ophthalmol. Eye. Dis. 2016, 8, 5-14. [CrossRef]

192. Weber, C.; Cole, D.J.; O'Regan, D.D.; Payne, M.C. Renormalization of myoglobin-ligand binding energetics by quantum many-body effects. PNAS 2014, 111, 5790-5795. [CrossRef] [PubMed]

(C) 2020 by the authors. Licensee MDPI, Basel, Switzerland. This article is an open access article distributed under the terms and conditions of the Creative Commons Attribution (CC BY) license (http://creativecommons.org/licenses/by/4.0/). 



\title{
Review \\ Noble Metals and Soft Bio-Inspired Nanoparticles in Retinal Diseases Treatment: A Perspective
}

\author{
Valeria De Matteis ${ }^{1, *}$ and Loris Rizzello ${ }^{2,3,4}$ \\ 1 Department of Mathematics and Physics "Ennio De Giorgi”, University of Salento, Via Arnesano, \\ 73100 Lecce, Italy \\ 2 Department of Chemistry, University College London, 20 Gordon Street, London WC1H 0AJ, UK; \\ lrizzello@ibecbarcelona.eu \\ 3 Institute for Bioengineering of Catalonia (IBEC), The Barcelona Institute of Science and Technology, Baldiri \\ Reixac 10-12, 08028 Barcelona, Spain \\ 4 Department of Pharmaceutical Sciences, University of Milan, via Mangiagalli 25, 20133 Milano, Italy \\ * Correspondence: valeria.dematteis@unisalento.it
}

Received: 31 January 2020; Accepted: 8 March 2020; Published: 10 March 2020

\begin{abstract}
We are witnessing an exponential increase in the use of different nanomaterials in a plethora of biomedical fields. We are all aware of how nanoparticles (NPs) have influenced and revolutionized the way we supply drugs or how to use them as therapeutic agents thanks to their tunable physico-chemical properties. However, there is still a niche of applications where NP have not yet been widely explored. This is the field of ocular delivery and NP-based therapy, which characterizes the topic of the current review. In particular, many efforts are being made to develop nanosystems capable of reaching deeper sections of the eye such as the retina. Particular attention will be given here to noble metal (gold and silver), and to polymeric nanoparticles, systems consisting of lipid bilayers such as liposomes or vesicles based on nonionic surfactant. We will report here the most relevant literature on the use of different types of NPs for an efficient delivery of drugs and bio-macromolecules to the eyes or as active therapeutic tools.
\end{abstract}

Keywords: retinal diseases; noble metals NPs; bio-inspired NPs; drug delivery

\section{Introduction}

The human eye is a unique and fragile structure prone to different types of pathologies. It is characterized by an anterior and the posterior segment [1]. Pupil, cornea, iris, ciliary body, aqueous humor and lens make up the anterior segment, whereas vitreous humor, macula, retina, choroid, and optic nerve are parts of the posterior segment [1]. The macula is a small area of the retina that contains photoreceptor, special light-sensitive cells, connected to a neuronal network [2]. Retina contains five types of neurons: photoreceptor, bipolar, ganglion, horizontal, and amacrine cells (Figure 1) [3,4]. The retina is the nerve layer that receives visual information, creating impulses that are transmitted through the optic nerve to the visual cortex in the brain [5]. Two types of photoreceptor cells exist: the rods and the cones. It is estimated that there are approximately 120 million rods and about six million cones in the human eye. The unique properties of photoreceptor cells expose them to a variety of genetic and environmental threats. Any dysfunction or deterioration of vital ocular tissue has a dramatic impact on a patient's quality of life. Therefore, the retina is susceptible to several diseases that result in visual loss and blindness. As countries become wealthier and the per capita income increases, prevalence of blindness decreases, and causes of blindness change. In a poor African country, the major of blinding conditions are likely to be cataract and corneal scar. In the middle-income countries such as Latin America, the leading causes of blindness are glaucoma and diabetic retinopathy. Because cataract surgery is more readily available, fewer people become blind from cataract [6]. In wealthy countries, 
glaucoma and cataract continue to be very common and important conditions, but most of the blindness is due to retinal degenerative diseases (RDDs) [7], an heterogeneous group of phatologies characterized by progressive death of photoreceptors. RDDs can be caused by defects in proteins involved in photo transduction, synaptic transmission, retinal pigment epithelial (RPE) integrity function, intracellular trafficking and cilia function. It can be also related to toxic accumulation of retinoids. Each of these RDDs can lead to visual loss or complete blindness [8].

The most common RDDs include age-related macular degeneration (AMD), diabetic retinopathy $(\mathrm{DR})$, and retinitis pigmentosa (RP). Cytomegalovirus (CMV) retinitis, proliferative vitreoretinopathy, Stargardt disease, and retinoblastoma can also occur and are usually associated with toxic retinoid biogenesis and accumulation [9]. Retinal diseases are already the most common cause of childhood blindness [10] with an incidence of 1.5 million people suffering worldwide. RP is characterized by a progressive loss of photoreceptors and its specific mechanisms are not fully understood. Following the loss of photoreceptor functionality, a retinal remodeling phenomenon is triggered as the final step, which then culminates with cell death and topological restructuring of the retina. The progression of retinal remodeling is similar to negative plasticity that occurs in some pathologies like trauma and epilepsy constituting substantial impediments to rescue strategies of all types [11]. Another process that involve the retina segment is neovascularization, a pathological phenomenon that provokes vision loss causing blindness due to its correlation with diabetic retinopathy and AMD [12]. In the neovascularization phenomenon, endothelial growth factor (VEGF) pathway has a key role [13,14].

Ocular diseases, and retinal diseases in particular, are still approached using rather invasive treatments, which are also not completely effective. The development of efficacious therapeutics is thus a primary research goal. So far, many delivery strategies have been explored, and the progress in the field of molecular genetics has led to the identification of genes involved in retinal disruption. For this reason, gene delivery systems represent one of the most promising approaches [15,16]. Delivery approaches can be broadly classified in two groups, whether they are based on a viral or non-viral method of delivery. Each system comes with advantages and disadvantages. For instance, although Adeno-Associated Virus (AAV) based vectors have high gene transduction efficiency, they face a major obstacle in terms of biosafety for clinical applications. The development of effective non-viral approaches for ocular disease is thus crucial. However, how to adequately deliver therapeutic agents to the back of eye (i.e., retina) remains a challenge. With the development of nanotechnology and bioengineering science, a wide range of innovations in this area have been achieved. This holds the promise for developing more effective ocular drug delivery systems.

In this review, we carefully report the last studies in which noble metal and soft (bioinspired) NPs were applied as new delivery agents for treatment of ocular disease in vitro and in vivo. We also provide perspective on opportunities and challenges for future nanotechnology in retinal drug delivery and therapies. 
A
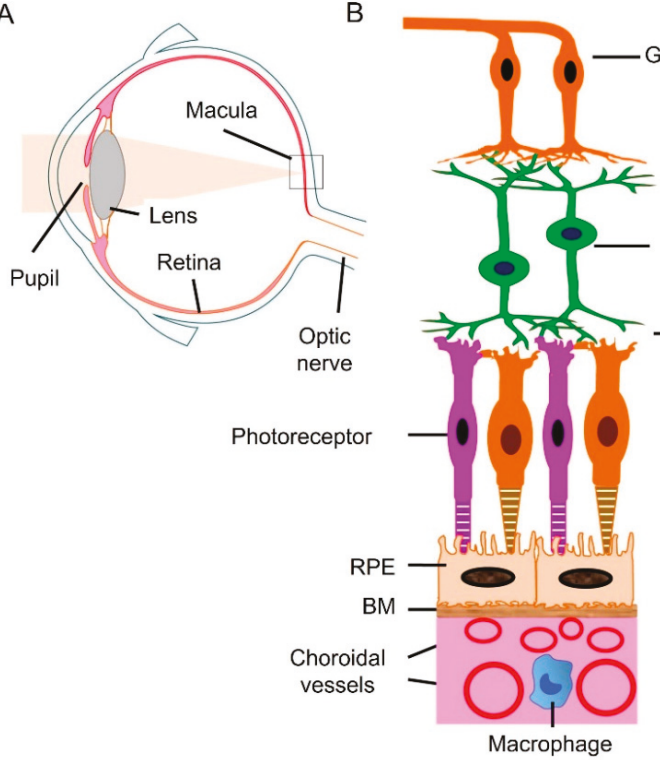

B
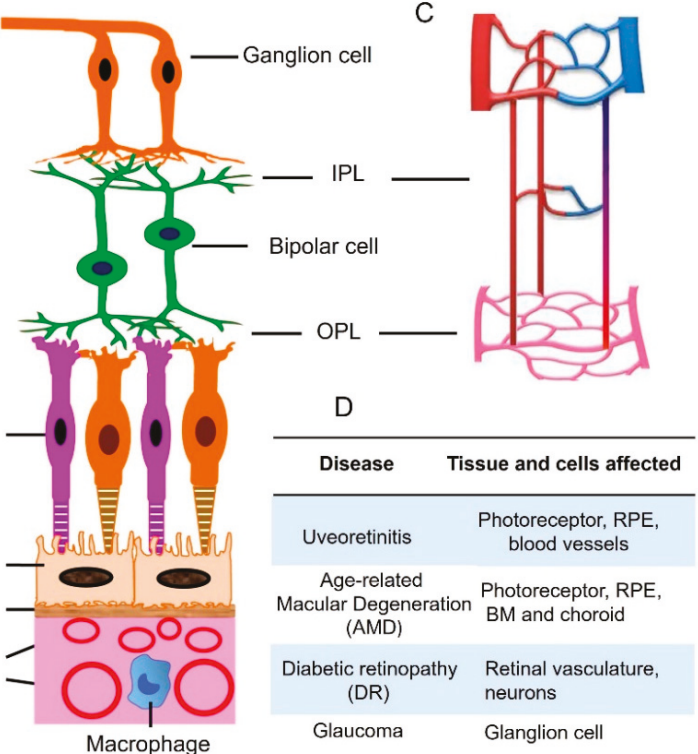

Figure 1. Retinal neuronal and vascular structure and retinal disease. (A) Diagram of a human eye. Light passes through the pupil and is focused by lens onto macula of the retinal layer at the back of the eye. (B) Retina consists of three layers of neurons, photoreceptor, bipolar, and ganglion cells. The retinal pigment epithelial (RPE) monolayer together with Bruch's membrane (BM) form outer blood retinal barrier that separates the neuroretina from the choroid. Choroidal circulation provides oxygen and nutrients to outer retina. (C) The retina has an interconnected network of three vascular layers located in the ganglion cell/nerve fiber layer, inner plexiform layer (IPL), and outer plexiform layer (OPL). (D) retinal tissue and cells that are affected under different disease conditions. Adapted from reference [17].

\section{Drug Ocular Administrations}

The delivery of drugs in the eyes presents many difficulties. As in the case of the brain, it presents multiple physical boundaries: corneal and conjunctival epithelium, blood-aqueous barriers (BAB), and blood-retinal barriers (BRB). These barriers finely control the transit of molecules and fluids across eye and, in most cases, the drug is unable to enrich the deeper layers. The BRB consists of two different barriers: (i) the outer BRB characterized by endothelial cells lining the choroidal vasculature (fenestrated blood vessels) and by tight-junction-coupled RPE cells, and (ii) inner BRB. The latter is made by endothelial cells in conjunction with pericytes, astrocytes, and Müller glial cells. The inner BRB shows a lot of similarities with blood-brain barrier (BBB) with the exception of Müller glia [18]. In general, the most common drug administration is through eye drops and suspensions' that target only the anterior segment of eye, mainly due to the patient compliance. With such a method, less than $5 \%$ of topically applied dose is delivered to deeper ocular tissues, such as in vitreous cavity [19]. The chemical nature of bioactive molecules also influences the uptake. For example, BRB is selectively permeable to lipophilic molecules in the presence of specific eye drugs transporters. In this way, the formulation of new therapies should take in consideration this limitation [20]. Ocular transporters are divided in two groups: the solute carrier (SLC) family and the ATP-binding cassette $(\mathrm{ABC})$ family. The electrochemical gradient was used by SLC transporters to induce the uptake of molecules through cell membrane, whereas ABC transporters employ ATP [21]. However, physiological eye phenomena, such as tear turnover, reflex blinking, ocular static, or lacrimal drainage reduce the action of the drug and consequently the eye drop administration fails to treat 
retina disease [22]. As a matter of fact, in unstimulated conditions, total aqueous tear volume is $7 \mu \mathrm{L}$ and the normal tear turn-over is about $1.2 \mu \mathrm{L} / \mathrm{min}$ [23]. Thus, the precorneal drug half-life is about 3 minutes [24]. Therefore, treatment of posterior segment injuries still remains a challenge. For all the reasons explained above, medical research seeks alternative routes such as intravitreal injections. This method consists of direct administration into vitreus via pars plana using a needle. In this way, a high concentration of drug is guaranteed at the retina level, but the molecule half-life depends on its molecular weight. In fact, proteins and peptides characterized by high molecular weight (ranging from $40 \mathrm{kDa}$ to $70 \mathrm{kDa}$ ) and steric hindrance showed longer retention [25,26]. However, a lot of complications (hemorrhages, retinal detachment, cataracts) can manifest after injection [27]. An alternative method is periocular route (Figure 2) that was demonstrated to be an effective route to direct drugs to the posterior eye segment consisting of subconjunctival, sub-tenon, retrobulbar, peribulbar, and posterior juxtascleral [28]. Administration of drug by sub-tenon injection was found to be more suitable as demonstrated by Ghate et al. [29], where sodium fluorescein was used in rabbits' eyes by subocular administration. The study concluded that injection of drug via sub-tenon resulted in highest vitreous concentration of sodium fluorescein (two sodium fluorescein (NaF) concentrations, $2.5 \mathrm{mg}$ in $0.1 \mathrm{~mL}$ (c1) and $2.5 \mathrm{mg}$ in $0.5 \mathrm{~mL}$ (c2)) compared to the other routes. However, also in this case, a lot of adverse effects have been observed, such as strabismus, hyphema, and intraocular pressure [30].

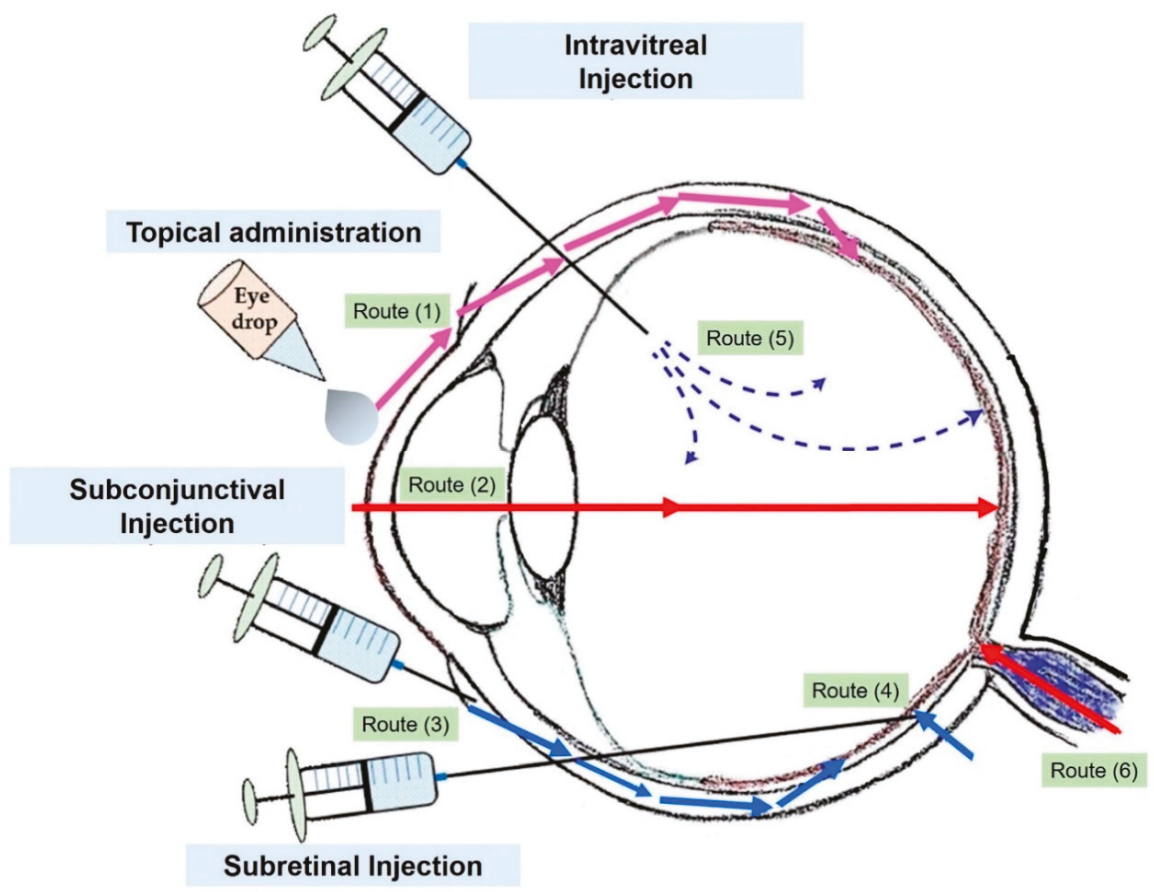

Figure 2. Common drug administration routes through the eye. Topical administration $(\mathbf{1}, \mathbf{2})$, subconjunctival injection (periocular route) (3), subretinal injection (4), and intravitreal injection (5). Adapted from reference [31].

\section{Physico-Chemical Properties of Nanomaterials}

A "Nanomaterial" is defined as a "material with any external dimension in the nanoscale or having internal structure or surface structure in the nanoscale" (ISO, 2010) [32]. Similarly, a definition of "nanoparticle" is a "nano-object with all three external dimensions in the nanoscale" where nanoscale was defined as size ranging from $1 \mathrm{~nm}$ to $100 \mathrm{~nm}$ (ISO, 2008) [33]. NPs can be obtained by many synthetic 
routes, starting from chemical elements such as carbon, metals, metal oxide, biological molecules, and polymers [34]. The biological effects are strongly affected by physico-chemical properties of NPs such as surface charge, size, shape, and solubility. In addition, they exhibit greater surface area per unit mass compared to bulk materials [35]. Because of the above mentioned properties and of the material they are made, NPs are ideal tools to treat retinal disease as active components of the therapy without the help of drugs [36,37]. In addition, NPs with a smaller size $(<20 \mathrm{~nm})$ are demonstrated to have the ability to cross eye barriers including cornea, conjunctiva, and BRB [38,39]. NPs for ocular therapy include inorganic NPs (metal oxide and noble metal NPs), as well as soft-biopolymer-based NPs. Metal NPs are more suitable as active therapy tools (for their intrinsic properties), and soft-NPs are more efficient in encapsulating drugs and macromolecules due to their ability to form aqueous-suspended vesicles having a hollow lumen. All these nanomaterials are different in charge, shape, and size, but they are able to be internalized by cells to treat retinal disease [40]. Finally, recent applications of soft nanorobot (with size range in the molecular scale) have been studied. These structures have the capability to deliver active biomolecules in ocular sections due to their ability to make changes in a controlled and predictable manner to the environment following external stimuli [41].

\subsection{Noble Metal NPs: The Case of Gold ( $A u)$ and Silver $(A g)$}

Noble metals, especially AuNPs and AgNPs, are characterized by unique optical properties. For this reason they are used for a variety of applications owing to collective oscillations of conduction electrons coupled with incident light [42-45]. This phenomenon, known as Localized Surface Plasmon Resonance (LSPR) is strongly influenced by NPs' shape and the metal they are formed. The surface plasmon resonance bands of metal NPs can be tuned from visible to Near Infrared Region (NIR), which is a typical wavelength to penetrate and analyze biological tissues [46]. Even if AuNPs are the most studied in nanomedicine field due to their good chemical stability and well-controlled size/surface functionalization and biocompatibility, AgNPs have the advantage of possessing an antibacterial and antiangiogenesis effect $[47,48]$.

\subsection{Au and AgNPs: Safety Studies in In Vitro and In Vivo Retinal Models}

\subsubsection{AuNPs}

The use of novel nanomaterials necessitates a better understanding of potential adverse effects on biological entities [49]. These effects are strictly dependent on physico-chemical properties of NPs and evaluation of cytotoxicity is critical, especially from a medical field application perspective [50]. A lot of studies were conducted to assess biocompatibility in order to ensure safe drug release with low toxicity. In general, high degree of biocompatibility is verified when NPs interact with the living organisms without triggering unacceptable toxic, thrombogenic, carcinogenic, and immunogenic responses [51].

The unique physico-chemical properties of AuNPs make them a powerful tool in the biomedical field. They are widely used in photothermal treatment of cancer and in drug and gene delivery. For their synthesis, auric salts such as $\mathrm{HAuCl}_{4}$ are used as starting materials to obtain stable AuNPs with a size ranging from 1-100 nm (Brust or Turkevish [52,53]). In recent years, green syntheses of AuNPs has also been developed to reduce solvents and reduce/cap agents' toxicity so that AuNPs can be synthetized from plant extracts or biomass $[54,55]$. AuNPs are easy to functionalize with a wide range of ligands ranging to polymer, DNA, peptides, RNA, and fluorescent molecules particularly suitable for bio-imaging applications [56]. Furthermore, various cytotoxicity studies demonstrated their low toxicity due to the inert nature of $\mathrm{Au}[57,58]$.

Regarding the biodistribution, a recent work demonstrated a rapid elimination of colloidal AuNPs from blood circulation in a rats model, and liver and spleen accumulation without inducing adverse effects or inflammation [59]. Au comprised in passion fruit-like nanoarchitectures (NAs) were studied in murine models demonstrating the excretion by renal and biliary pathways without nephrotoxicity up to $150 \mathrm{mg} \mathrm{kg}^{-1}$ [60]. Some studies reported pro-inflammatory cytokines expressions induced by 
AuNPs in different organs of rats [61] and mice [62]. However, the cytotoxic effects were temporary and normalized in a short time. The biocompatibility of $\mathrm{Au}$ is strictly correlated to their size, shape, concentration, as well as surface area as demonstrated by Biswas's group [63]. They investigated the effects of Au spheres $(5,10,20,30,50$, and $100 \mathrm{~nm})$, cubes $(50 \mathrm{~nm})$, and rods $(10 \times 90 \mathrm{~nm})$ in cells in adult retinal pigment epithelial cell line 19 (ARPE-19). The cubes with an average diameter of $50 \mathrm{~nm}$ and spheres (50-100 nm) resulted in being biocompatible up to $750 \mu \mathrm{g}$ of concentration. NPs with a smaller size $(<30 \mathrm{~nm})$ and nanorods $(10 \mathrm{~nm})$ showed higher toxicity, but they are more able to be uptaked by cells. Kim et al. [64] used C57BL/6 mice to demonstrate the ability of AuNPs (20 nm) to cross BRB and enriched retinal layers after intravenous administration. NPs greatly accumulated in neurons $(75 \pm 5 \%)$, followed by endothelial cells $(17 \pm 6 \%)$ and peri-endothelial glial cells $(8 \pm 3 \%)$ without affecting both viability of endothelial cells, astrocytes, retinoblastoma cells, and expression of functional biomolecules. After five weeks of post administration, the thickness of retinal layers was undisturbed without any damage. Bakri et al. [65] used Dutch-belted rabbits to test biocompatibility of AuNPs. They used two groups of rabbits receiving a $67 \mu \mathrm{mol} / 0.1 \mathrm{~mL}$ and $670 \mu \mathrm{mol} / 0.1 \mathrm{~mL}$ of AuNPs into one eye respectively by intravitreal injection. After one month, evident inflammation or adverse effects were not detected also at a high dosage. Soderstjerna et al. [66] used AuNPs and AgNPs with 19 and $89 \mathrm{~nm}$ citrate capped to test biocompatibility in in vitro cultured retinas using $800 \mathrm{NPs} / \mathrm{cell}$. The retina tissue model offered a sustained long-term retinal organization and controlled serum-free conditions. This study demonstrated that low concentrations of Au and AgNPs have adverse effects on retina and can induce apoptosis and oxidative stress. A strong connection between NPs hydrodynamic diameter, surface functionalization/concentration and the biological effects is evident. In particular, the authors showed that NPs size should be large enough to avoid their fast flow into circulation, but small enough to be administrated and easy cross the BRB [67].

Starting from this assumption, the application of AuNPs is influenced by these parameters that should be taken in consideration to customize nanotools for a specific biomedical application.

\subsubsection{AgNPs}

Numerous in vitro and in vivo studies demonstrated the toxicity of AgNPs that perturbs several cellular pathways [4]. The most accepted mechanism through which they induce side effects, despite still under investigation, seems to be related to Ag ions released from NPs surface [68-70]. In terms of production process, AgNPs can be synthetized by different chemical routes such as chemical-based reduction [71], sol-gel, or green synthesis [72,73].

Jun et al. [74] used zebrafish embryos as model organism to test their delivery at an ocular level. They investigated the toxicity of AgNPs at concentration of $0.4 \mathrm{mg} / \mathrm{L}$ and observed down- expressions of different lens crystalline genes by unknown mechanisms that either induced apoptosis or blocked nuclear DNA or RNA export.

Sriram et al. [75] demonstrated the cytotoxicity and Reactive Oxygen Species (ROS)-induced apoptosis after exposure of AgNPs with two different size (22.4 and $42.5 \mathrm{~nm}$ ) in bovine retinal endothelial cells (BRECs) finding size-dependent toxicity. As a matter of fact, smaller NPs induced adverse effects on cells. Kim and colleagues [76] exposed New Zealand white rabbits (according to OECD test guidelines) to AgNPs in order to understand the possible eye irritation effect. Oedema and conjunctival redness have been observed after $1 \mathrm{~h}$ of AgNPs incubation, but the cornea, iris, or conjunctiva resulted unaffected after 24,48 , and $72 \mathrm{~h}$ of NPs exposure. In similar experiments, the instillation of AgNPs (5000 ppm) were tested on another in vivo model, the guinea pigs, observing a transient conjunctivae irritation during $24 \mathrm{~h}$ of early observation [77].

\section{Noble-Metal NPs as Therapy and Diagnostic for Retinal Disease}

AuNPs scatter or absorb light at specific wavelengths as a function of their physico-chemical properties. This characteristic is particularly suitable for bioimaging and to treat some diseases like cancer by NIR-triggered photothermal therapy (PTT), in which light is converted to heat in the tumor 
site where NPs accumulate [78]. In the NIR (650-900 nm), the absorption coefficients of haemoglobin and water are very low, and for this reason, the penetration of NIR wavelength in tissues is very high, allowing NPs stimulation without causing damage [79]. Song et al. [80] reported application of gold nanodisks (GNDs) on human retinal microvascular endothelial cells (HRMEC) and mouse oxygen-induced retinopathy model. The GNDs were optically suitable for nanomedicine applications due to their ability to produce detectable signals regardless of polarization or direction of light source and to scatter in NIR region $(830 \mathrm{~nm})$. In addition, GNDs were internalized less efficiently in cells with respect to AuNPs or Au rods (AuNRs), thus minimizing the ROS production and other several adverse effects. The authors used differently charged anisotropic NPs (nanorods or nanodisks) and compared them with AuNPs. After simulating the role of different sizes to select the best diameter $(160 \mathrm{~nm})$, they showed that GNDs were able to bind vascular endothelial growth factor (VEGF) inhibiting in vitro angiogenesis with high performance and abolished the VEGF-induced migration of endothelial cells. When these nanostructures were applied to mice eyes, a strong signal in optical coherence tomography (OCT) was detected with a consequent reduction of retinal degeneration (Figure 3). The OCT system involves the use of light energy that, once absorbed by tissues, causes a thermos-elastic expansion generating ultrasonic waves detected by a transducer.

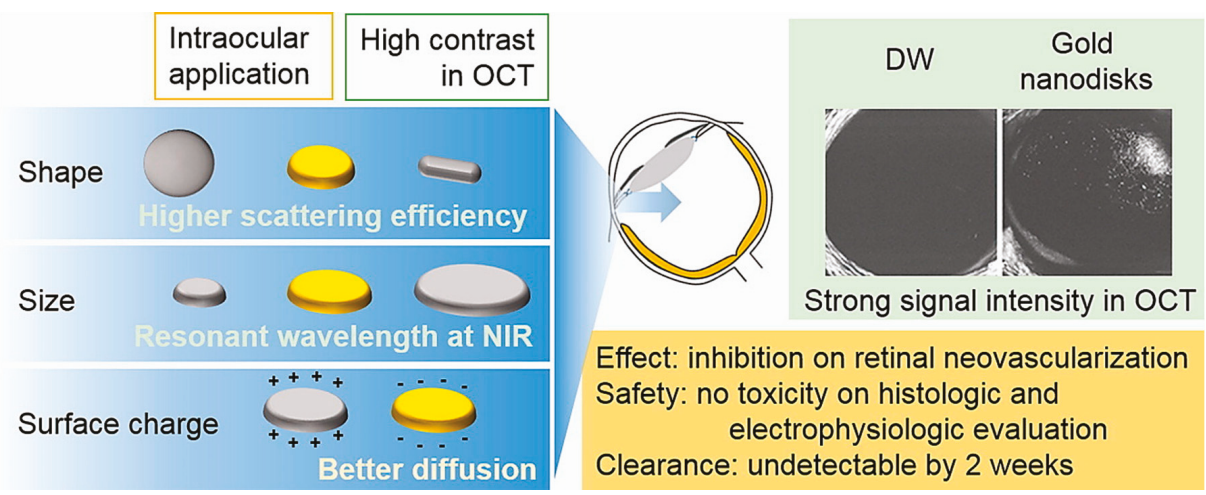

Figure 3. Schematic representation of gold nanodisks (GNDs) effects in retinal degeneration and Optical Coherence Tomography (OCT) imaging. The GNDs were particularly suitable for their optical properties connected to the shape: GNDs absorbed in the Near Infrared Region (NIR). The size and surface charge also influenced the eye diffusion. In OCT images, GNDs exhibited a strong signal compared to the dual window (DW) processing method, which is used to detect modulation of OCT signals due to scattering or absorption. Reprinted from [80] Copyright (2017), with permission from Elsevier.

Recent therapies to treat some diseases strictly connected to retinal degeneration consider the transplantation of photoreceptor precursors (PRPs) as efficient support improving visual acuity [81]. The analysis of cell survivor in the host retina is a key factor in this kind of treatment. Chemla et al. [82] used AuNPs in order to improve the tracking of transplanted PRPs. Cells were labelled with photothermic AuNPs and transplanted in the vitreous and sub-retinal space of Long-Evans pigmented rats (4-8 weeks old) by gauge needle.

By using coherence tomography, computed tomography and fluorescence fundus imaging, NPs have been tracked for 1 month without recording adverse effects. Real-time tracking is essential for evaluating the efficiency of retinal cell replacement therapies. These studies confirmed the possibility to use AuNPs to improve retinal cell therapy in humans. AuNPs-based photothermal therapy has been also used to treat eye tumors in vitro and in vivo. Some eye cancers provoke retinal detachment leading to a decreased visual eye activity. 
In this framework, Kim et al. [83] tested anticancer properties of Doxorubicin (Dox)-conjugated Fucoidan (Fu)-encapsulated AuNPs (Dox-Fu@AuNPs) for the treatment of VX2 squamous carcinoma cells and xenograft tumors in New Zealand White rabbits. After intra-tumoral injection of Dox-Fu@AuNPs in the eye, a complete suppression of the tumor has been observed by dual-chemotherapy and PTT treatment. No damage to retina has been also detected. These composite nanostructures enabled the improvement of the OCT imaging technique (thanks to the selective light absorption of AuNPs) [84].

The quality of photoacoustic-based imaging was also improved thanks to selective light absorption of AuNPs. In this way, a double effect in terms of therapy and diagnostic was achieved.

As reported above, OCT is largely used for imaging in ocular clinical research due to the possibility to take structural information based on the reflectivity of tissue. In this respect, Gordon and colleagues [85] confirmed that Au nanorods (with a peak absorption of $750 \mathrm{~nm}$ and diameter of 10-35 nm) injected in the retina of Wild-type C57BL/6 mice are able to enhance the contrast, thus significantly improving the quality of a scanned lesion. Additionally, intravitreal injection of $\mathrm{Au}$ nanorods functionalized by anti-VEGF antibody triggered a reduction in the extent of anatomic laser damage and lesion-associated photothermal signal density in mice treated in laser induced choroidal neovascularization (LCNV) model and injected with ICAM2-targeted GNR. AuNPs (3-5 nm) were also used as inhibitor of VEGF on choroid-retina endothelial cells (RF/6A) [86]. A similar effect was showed using AgNPs, which have been found to reduce the VEGF activation and induced cell proliferation and migration in bovine retinal endothelial cells [87]. In addition, AgNPs were shown to inhibit the angiogenesis, typical of metastasising cells and of retinal neovascularization development. Ag is widely used because it has the great advantage of being a cost-effective material. For this reason, it is a good candidate for the replacement of the current therapies used for the treatment of various retinal angiogenic disorders. In this respect, Sheikpranbabu and co-workers [88] investigated the effect of green AgNPs, obtained from Bacillus licheniformis, on VEGF-and Interleukin $1 \beta$ (IL-1 $\beta$ ) expression-that induced retinal endothelial cell permeability. AgNPs were able to stop VEGF-and IL-1 $\beta$-induced permeability in retinal endothelial cells from porcine retina.

\section{Bio-Inspired NPs for Ocular Drug Delivery}

The use of bio-inspired materials mimicking natural components for applications in living organisms has widely spread recently [89]. In fact, inorganic NPs have the serious drawback of not being degradable and are also difficult to get excreted from the body [90]. Bio-inspired nanomaterials, including liposomes, niosomes, and chitosan/alginate NPs have been then explored as valid alternatives to metal NPs for treatment of several diseases [91]. In addition, these 'soft' NPs have been applied for drug delivery in the posterior segment of eye, including retinal photoreceptors.

\subsection{Liposomes as Drug Delivery Carrier}

Liposomes consist of a lipid bilayer, resembling a cellular membrane, that surrounds an aqueous core. These structures are widely used in pre-clinical studies to improve drug delivery, for example through Blood Brain Barrier (BBB) [92]. The BBB and BRB have the same functional properties [93,94]: so liposomes improve drug delivery to the retina and its photoreceptors due to their high half-life, permitting the long term drug absorption [95]. Liposomes also offer the ability to significantly improve pharmacokinetics and pharmacodynamics of any loaded drug compared to its free-circulating counterpart. They are also considered as highly biocompatible and biodegradable as well up to some extent both in vitro and in vivo. For this reason, several anticancer drugs such as doxorubicin, daunorubicin, and epirubicin were encapsulated in liposomes core with results demonstrating a significant decrease of many severe adverse effects (nausea, weakness, vomiting) with respect to the same concentration of free (non-encapsulated) drug. In this context, Zhang et al. [96] subcutaneously injected tacrolimus-encapsulated liposomes in Lewis rats with autoimmune uveoretinitis (EAU) by subcutaneous injection. Confocal analysis over rhodamine-conjugated liposomes (containing also the 
tacrolimus) revealed that such vesicles remained in ocular fluids even after 14 days. They found a reduced intraocular inflammation and decrease of EAU development without observing significant adverse effects.

Liposomes encapsulated with the anti-inflammatory and anti-angiogenic drugs berberine hydrochloride and chrysophanol were applied in myoblasts and in ischemic animal models. These drugs have the ability to contrast oxidative stress by enhancing the activities of antioxidant enzymes such as superoxide dismutase (SOD) and glutathione peroxidase (GSH-Px) [97,98]. Berberine hydrochloride and chrysophanol have been used in ocular liposome-mediated delivery to overcome their low stability. In this respect, a recent study [99] showed a liposome system that can entrap the two drugs at same times using the third polyamidoamine dendrimer (PAMAM G3.0) as a delivery carrier for age-related macular degeneration (AMD) therapy in the ocular posterior chamber.

PAMAM G3.0-coated compound liposomes were used to evaluated HCECs (Human Corneal Epithelial Cells) internalization, trans-corneal permeability in New Zealand white rabbits (weighing $2.0-2.5 \mathrm{~kg}$ ) and male Sprague-Dawley rats (weighing 210-250 g). Pharmacokinetics were evaluated together with in vitro assessments of anti-reactive oxygen species (ROS) efficacy. The PAMAM G3.0-coated compound liposomes enhanced bio-adhesion on rabbit corneal epithelium after topical administration and exhibited great permeability in HCECs cells. The possibility to deliver a protective agent against photo-oxidative retinal damage in a light-damaged animal model was also investigated through the use of dendrimers (Figure 4A). Results showed that dendrimers encapsulated with chrysophanol-berberine hydrochloride induced the greatest protection of retinal function in light-exposed rats compared to unloaded dendrimers and free drug. In addition, no inflammation and damage of cornea, iris, and conjunctiva was observed after drug-loaded dendrimers instillation for 14 days. Upon exploring the fundus of the eye (interior surface of eye opposite the lens including the retina, optic disc, macula, fovea, and posterior pole) [100], modifications in the vessel structure and distribution have not been observed after different treatments. (Figure 4B).

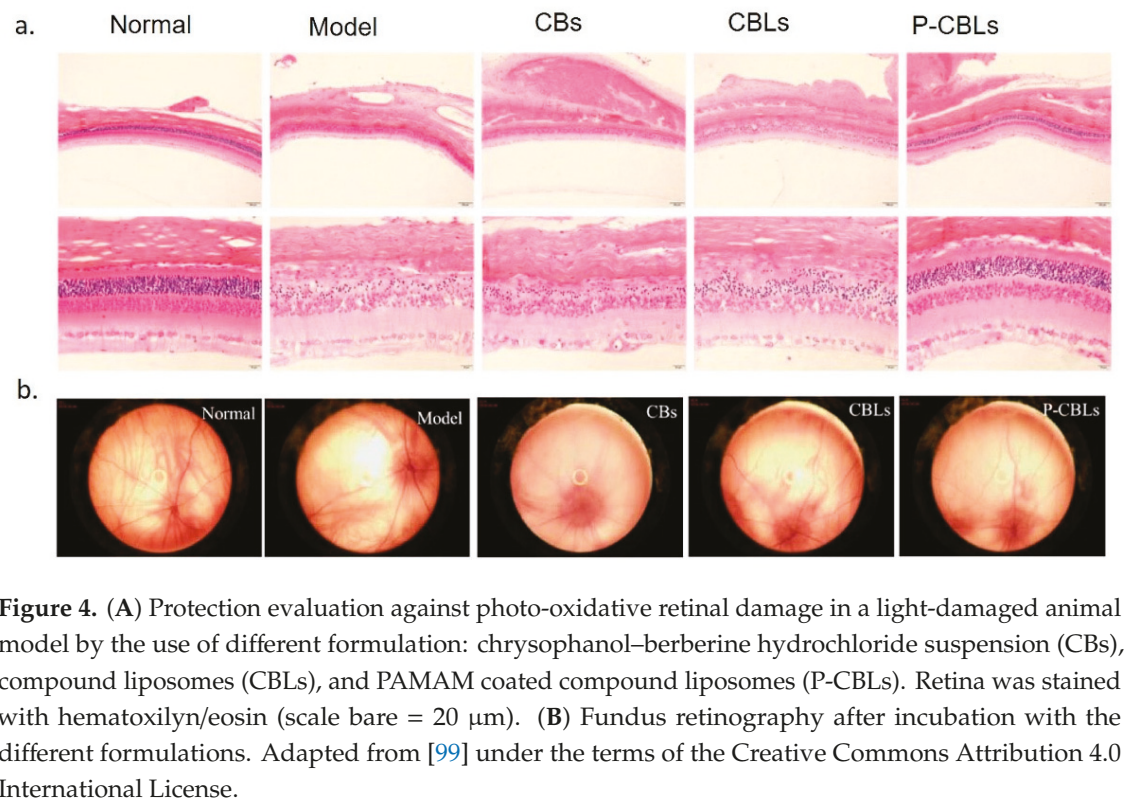


Liposomes offer also the great ability of an easy surface functionalization with a plethora of active macromolecules and targeting moieties that bind in a selective manner some specific receptors constitutive of different cell lines [101,102].

The surface modification of liposomes has also been widely studied to improve their specificity of targeting and to deliver them in different eye compartments. It has been reported [103] that the cationic PEGylated liposomes having an average zeta potentials below $+20 \mathrm{mV}$ were able to efficiently penetrate the murine retina. In contrast, liposome having surface zeta potential values above $+20 \mathrm{mV}$ were retained on vitreous section. This did not change whether PEGylated or non-PEGylated liposomes were used. Another critical factor for a liposome-based therapy is the phospholipids composition characterizing them. For example, edaravone-loaded egg L-phosphatidylcholine liposomes prevent retinal ganglion cells (RGCs) from undesired apoptotic processes [104]. This drug is a free radical scavenger used for acute brain infarction and amyotrophic lateral sclerosis treatment [105]. Surprisingly, minor RGC protection was observed with edaravone-loaded distearoyl phosphatidylcholine (DSPC) liposomes. This evidence suggest a link between the viscoelastic properties of the lipid bilayer and the final biological effect. In this specific case, the higher fluidity was a key factor to improve the ocular therapy. Another study [106] also tested the use of liposomes for the treatment of different kinds of hereditary degeneration of the retina. The degeneration of retina affects rod photoreceptors important for capturing light under low illumination conditions. Because of the pathology development, the patients affected by retina degeneration become aware of defects only when lesions are in an advanced stage. A key factor for this disease seems to be rods-specific Pde6b gene mutation in the transduction pathway that triggers an excessive accumulation of cGMP (that have two effectors: cyclic nucleotide gated ion channels (CNGCs) and cGMP-dependent protein kinase (PKG) [107]. Consequently, rod cell death was induced and in some cases the secondary loss of cones was observed [108]. The authors identified a liposomal cGMP analogue that was able to overcome the blood-retinal barrier (BRB) while strongly preserving retinal function and reducing rod photoreceptor loss in three different in vivo models for retina degeneration $[106,109]$. The vesicle structure of liposomes was suitable to confine proteins and peptides into aqueous core overcoming potential agglomeration and protein misfolding events. However, the surface functionalization with peptides is the most common method used for retina therapy. For example, liposomes functionalized with tanticoagulant annexin A5 have been demonstrated to have enhanced uptake levels through corneal epithelial barriers [110].

This vector has been injected topically to deliver $127 \mathrm{ng} / \mathrm{g}$ of bevacizumab to posterior segment of both rats and rabbit eye. Annexin A5-functionalized liposomes loaded with bevacizumab were also rapidly delivered to retina of the two animal models. Annexin A5 unilamellar liposomes made by different polymers (2-dipalmitoylsn-glycero-3-phosphocholine,1,2-dioleoyl-sn-glycero-3-phospho-L-serine and cholesterol) have been also used to target transforming growth factor (TGF)-B1 into the vitreous of rabbits [111]. It has been found that a higher concentration of molecule with respect to the experimental group treated with the unloaded drug only. The choice for human recombinant TGF- $\beta 1$ relied on the drug's ability to prevent retinal damage elicited by $A \beta$ oligomers.

\subsection{Niosomes for Drug and Gene Delivery}

Gene therapies is an approach where diseases are cured by modifying the expression of specific genes regulating pathology. This happens through the administration of nucleic acids aiming to replace abnormal gene sequence [112]. Liposomes and lipoplexes have been taken into consideration as suitable non-viral vectors platform for gene editing. Non-viral gene delivery approaches were widely applied in clinical trials since 2004 to attempt replacing the use of viral vectors that possess high risk of inducing oncogenesis, immunogenicity, mutagenicity phenomena [113].

Among non-viral vectors, niosomes have been recently tested as a potential candidate for gene delivery. Niosomes are vesicular systems comprising of bilayer made up of nonionic surfactants and they can internalize several types of bio-macromolecules such as nucleic acids and drugs. The most 
commonly used surfactant are Sorbitan fatty acid esters, Spans (Span 20, 40, 60, 65, 80, and 85), Tweens $(20,40,60$, and 80$)$ [114] and brij $(30,35,52,58,72$, and 76) [115].

In a recent study [116], niosomes made by cationic lipid 1,2-di-octadecenyl-3-trimethylammonium propane, Squalene, and Tween 20 have been combined with GFP-encoding genetic materials consisting on minicircle (MC-GFP, $2.3 \mathrm{~kb}$ ), its parental plasmid (pGFP, $3.5 \mathrm{~kb}$ ), and a larger plasmid (pEGFP, $5.5 \mathrm{~kb}$ ). Their efficacy in retinal disease treatment has been investigated. These noisome-DNA complexes, named as nioplexes, demonstrated good transfection ability in human ARPE19 retinal pigment epithelium cells (in vitro) and in embrionary rat retinal primary cells (in vivo). Fluorescence showed GFP positive signal emission after transfection with the nioplexes carrying plasmidic DNA. In addition, such transfected cells showed physiological glial cell morphology confirming good cell viability in all tested cases. (Figure 5). The successive injections of nioplexes in rat retinas confirmed the higher capacity of cationic niosomes vectoring minicircle DNA to deliver genetic material into retina cells. Therefore, nioplexes based on cationic niosomes vectoring minicircle DNA are demonstrated as powerful agents in retinal diseases therapies. Cationic formulations made by a combination of Tween-60:DOTMA:lycopene have been used to transfect ARPE-19 cells with pCMS-EGFP plasmid with a transfection efficiency close to 35\% [117]. Also in case, in vivo experiments in rat eye showed the efficiency of nioplexes to transfect outer segments of the retina, Niosomes constituted by Span 40 or Span 60 and cholesterol were produced to encapsulate and topically deliver in vivo acetazolamide. The intraocular pressure (IOP) after drug delivery system application was reduced in rabbits. In particular, multilamellar acetazolamide niosomes developed with Span 60 and cholesterol (7:4 molar ratio) were more efficient in reducing IOP, and at same time, no irritation and retinal damage were observed [118]. Gugleva et al. [119] prepared niosomes by different surfactants to deliver doxycycline hyclate in rats eye. Doxycycline hyclate is an antibiotic used for the treatment of eye infections. It was demonstrated to inhibit effect on matrix metalloprotease 9 (MMP-9) activity and mitogen activated protein kinase in experimental murine dry eye [120]. Niosomes were synthesized using several surfactants (Span 20, Span 60, Span 80, Tween 60) and cholesterol in different molar ratios and tested on male albino rabbits. The experiments clearly showed that the prepared niosomal formulations were high tolerated and did not induce any irritations.
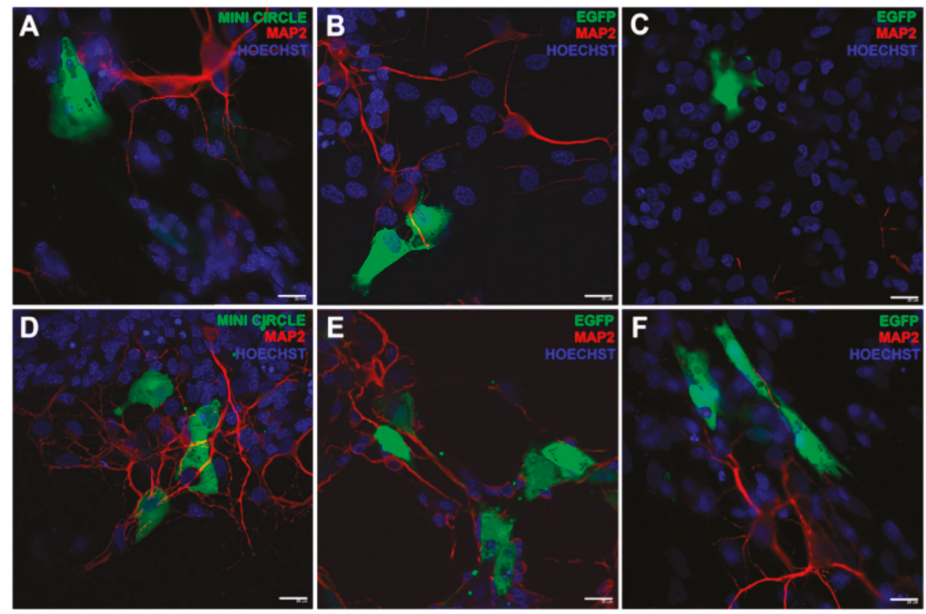

Figure 5. Fluorescence immonostaining by GFP expression in embrionary rat retinal primary cells. The green signal was referred to the transfection event of DST20 nioplexes with MC-GFP, (B) pGFP $3.5 \mathrm{~kb}$, or (C) pEGFP $5.5 \mathrm{~kb}$. (D-F) Positive controls incubated with Lipofectamine ${ }^{\mathrm{TM}} 2000$. Nuclei were stained with Hoechst 33,342 (blue) and neuronal dendrites with MAP2 (red). Scale bars: $20 \mu \mathrm{m}$. Reprinted from [116] Copyright (2019), with permission from Elsevier. 


\subsection{Hydrogels-Based Nanocarriers}

Hydrogels are three-dimensional, cross-linked networks of natural or synthetic water-soluble polymers that are suitable for applications like tissue engineering and controlled/sustained drugs delivery $[121,122]$. The aqueous environment is particularly advantageous to confine soluble active molecules, oligonucleotides, and proteins. For this reason, they are powerful tools in retinal treatment disease [123]. In addition, smart or environment-responsive hydrogels were synthetized to improve drug delivery efficacy [124].

\subsubsection{Natural Hydrogels: Chitosan NPs (CHNPs)}

Chitosan is a natural hydrogel cationic polysaccharide of co-polymers glucosamine and $\mathrm{N}$-acetyl glucosamine [125] obtained by alkaline deacetylation of chitin that is constitutive of crustacean shells of shrimps, lobster, and crab. It presents a positive charge that can be exploited to form polyelectrolyte vesicular complexes able to act as delivery systems. The positive charged chitosan is also ideal to bind a negatively charged corneal surface, to improve precorneal retention time and to decreases clearance [126]. In addition to chitosan, also the biocompatible alginate is extensively employed in biomedical applications. Alginate can also be a useful biopolymers for designing responsive hydrogels [127]. Because of its characteristics, alginate is also used for ophthalmic applications often in combination with chitosan [128]. For example, chitosan coated sodium alginate NPs [129] loaded with Fluorouracil (5-FU) have been successfully tested for eye delivery demonstrating a high 5-FU release in aqueous humor compared to free drug in rabbit eye. In another approach, chitosan NPs were encapsulated with levofloxacin for the treatment of ocular infection [130]. The formulation of NPs were optimized to enhance corneal retention without inducing eye irritation. These nanovectors demonstrated strong antibacterial agents against Pseudomonas aeruginosa and Stafiloccocus aureus.

Andrei and co-workers [131] designed biocompatible chitosan and alginate-based micro/NPs with a specific morphology aiming to deliver cefuroxim, a second-generation cephalosporin antibiotic. The authors performed an in vivo assessment to evaluate the biodistribution following an intravitreal administration of fluorescein-chitosan NPs on wistar rats. They observed an efficient NPs distribution in lens and retina. Similarly, chitosan-dextran NPs have been employed to deliver the antibiotic moxifloxacin that has been retained for a long time in vivo thanks to the mucoadhesive properties of NPs [132].

In recent work, humanin, a neuronal peptide that mediated inflammatory responses, was used in the treatment of age-related macular degeneration (AMD) [133]. This peptide was a suppressor of the IL-6 cytokine receptors and protects neuronal cells from necrosis [134]. The authors studied the effect on the inflammatory response reduction using chitosan NPs as vector vesicle (with a size of $346 \pm 52$ $\mathrm{nm}$ ) in which humanin (a micropeptide encoded in the mitochondrial genome) derivative AGA-(CR8) HNG17 (AGA-HNG) were encapsulated. The effects of NPs were assessed in retinal pigment epithelial cells. The percentage release profile of free protein after five days was $60 \%$, after which it enriched a plateau whereas the nanostructured exhibited a slow and substantial release with $80 \%$ of protein released after fifteen days. Cheng et al. [135] developed a thermosensitive hydrogel containing latanoprost and curcumin-loaded NPs (CUR-NPs). Curcumin was selected because of its antioxidative and antiinflammatory properties that made it suitable also for treatment of eye pathologies such as chronic anterior uveitis and light-induced retinal degeneration [136]. Latanoprost was chosen because it acted on IOP restore due to uveoscleral outflow increase [137]. In glaucoma disease, IOP is generated by loss of aqueous humor drainage through the uveoscleral or trabecular outflow pathway that can be damaged by oxidative stress at trabecular meshwork level [138]. In this condition, Latanoprost and CUR-NPs were demonstrated to decrease the oxidative stress in trabecular meshwork (TM) cells that resulted in decreased levels of apoptosis, inflammation-related gene expression, and mitochondrial damage [135]. In another work [139], neuroprotective and neuro regenerative agent Erythropoietin (EPO) was encapsulated in chitosan/hyaluronic acid (HA) nanoparticulate (size $\leq 300 \mathrm{~nm}$ ). Different 
formulations have been tested and delivered to porcine corneas, scleras, and conjunctivas by ex vivo permeation, finding out a good combination between targeting efficiency and biocompatibility.

Pandit et al. [140] encapsulated high levels of bevacizumab (an anti-angiogenesis drug) within chitosan-coated PLGA NPs (size 222.28 \pm 7.45 ) and demonstrated its efficient deliver to the retina followed by efficient release. As matter of fact, CS-coated NPs showed better permeability of the bevacizumab across sclera as compared to the free drug solution. Another category of carrier is made by glycol chitosan (GCS) NPs [141]. These biocompatible NPs, demonstrated to load high amount of plasmid DNA, have been successfully delivered to retinal pigment epithelium of adult wild-type albino mice. More importantly, the encapsulated pDNA was intact and maintained a proper conformation.

\subsubsection{Synthetic Hydrogel Based Nanocarriers}

The synthetic hydrogels are easy to customize and indeed are a close model of biological matrices. Hydrogels exhibit high hydrophilicity and high hydration [142]. The most commonly used are poly(hydroxyethyl methacrylate) (PHEMA), poly(ethylene oxide) (PEO, also called poly(ethyleneglycol) (PEG)), polyvinylpyrrolidone (PVP), poly(acrylic acid)(PAA), polyacrylamide (PAM), and poly(vinyl alcohol) (PVA) [143]. These polymers are all used in several ophthalmic applications with good results [144-147].

Anti-VEGF antibodies (anibizumab or aflibercept) were loaded within poly(lactic-co-glycolic acid) microspheres, and successively suspended within poly( $\mathrm{N}$-isopropylacrylamide)-based hydrogel and injected into a laser-induced rat model of choroidal neovascularization (CNV). Upon ocular administration, the rats showed smaller CNV lesions (less than 60\%) with respect to non-treated animals [148].

Agrahari et al. [149] obtained novel pentablock (PB) copolymer (PB-1: PCL-PLA-PEG-PLA-PCL) based nanoformulations suspended in a thermosensitive gelling copolymer (PB-2: mPEG-PCLPLA-PCL-PEGm) with a size of $150 \mathrm{~nm}$. This composite nanoformulation was used to deliver macromolecules to slowly release drugs without toxic effects on the posterior segment of the eye. In vitro tests performed on ocular and mouse macrophage (RAW 264.7) demonstrated their biocompatibility and potential to be clinically translated.

Yang et al. [150] reported the production of non-toxic polyamidoamine (PAMAM) dendrimer hydrogel/poly(lactic-co-glycolic acid) (PLGA) NP platform (HDNP) to treat glaucoma. The dendrimers were loaded with brimonidine and timolol maleat, which underwent an enhanced uptake together with a slow release in vitro up to 28-35 days. The topical administration of the same drug-loaded denndrimers in normotensive adult Dutch-belted male rabbits strongly reduced the introcular pressure (IOP) of about $18 \%$ maintaining a glaucoma reduction for four days. Conversely, Individual Dendrimer Hydrogel Formulations (DH) and NP Formulations (NP) decreased the disease symptoms only for $48 \mathrm{~h}$ (Figure 6). 


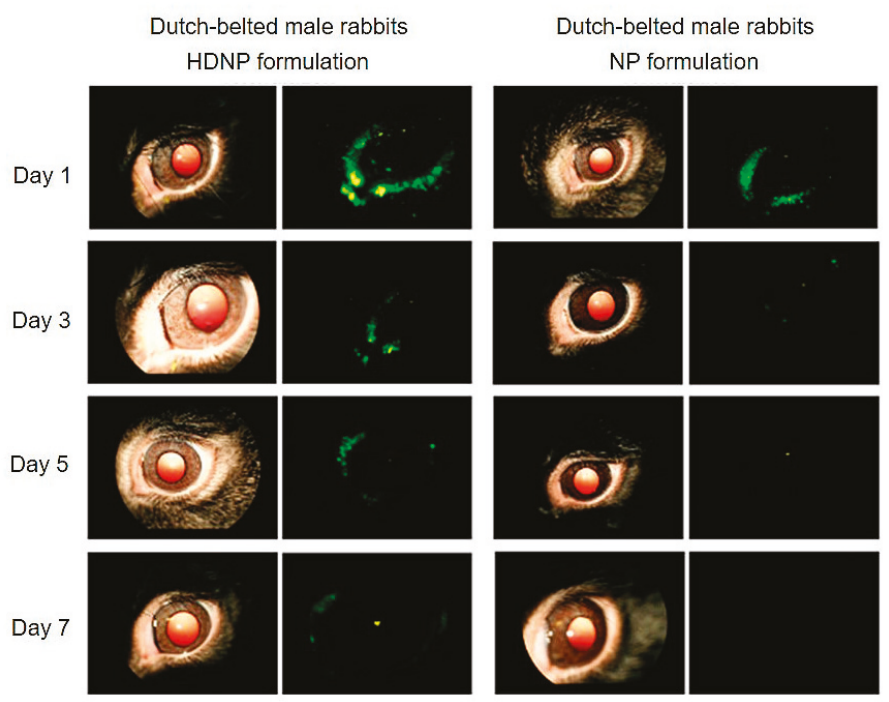

Figure 6. Fundus camera acquisitions of rabbits' eyes after hybrid polyamidoamine (PAMAM) dendrimer hydrogel/poly(lacticco-glycolic acid) (PLGA) nanoparticle platform (HDNP) and NP Formulations (NP) topical administration FluoSpheres were confined in nanosistems to follow the distribution and retention in the eye. The analysis was performed at the end of 1,3,5, and 7 days of formulations instillation. Left: regular fundus camera images of the eyes; right: fluorescent fundus camera images. Adapted with permission from [150], copyright (2012), American Chemical Society.

\subsection{Active and Self-Propelling Nanoparticles: New Generation of Nanobots in Retinal Disease}

The customization of NPs formulation at a molecular level has interesting outcomes for reproduction of fundamental phenomena such as macro- and microorganisms' survival mechanisms [151]. For example, bacteria, uni- or multicellular organisms, and sperm cells are able to sense and actively respond to external stimuli by the generation of movement. In particular, they convert an external energy sources (temperature, magnetic fields, adhesion forces, chemical gradients) into mechanical work that induce migration. This is typical in processes such as thermotaxis, magnetotaxis, haptotaxis, chemotaxis [152]. By taking inspiration from nature, we have also now a panel of NPs able to self-propel towards a gradient of nutrients and to mimic biological taxis [153]. However, the development of such NPs still has many challenges because at nanoscale level, the physical principles of propulsion are different due to the presence of the Brownian effect that can interfere with nanomptors by collisions with solvent molecules. One of these challenges is the generation of particles having asymmetrical geometries at the nanoscale, as this provides guidance for the movement directionality. Bacteria like Escherichia coli, in fact, achieve propulsion by non-time-reversible motion of long flagella, and this asymmetric shape of the body is critical for motion generation in a specific direction [154].

It is clear that self-propelling NPs represent a new powerful tool in drug delivery, which is usually dominated by NPs passive diffusion. It should not come as a surprise that such strategies have been employed also for delivery in ocular medicine. For example, Wu et al. [155] designed micro-vehicles that can actively propel through the vitreous humor to enrich retina. The movement can be activated by helical magnetic micropropellers ( $120 \mathrm{~nm}$ in diameter and $\sim 400 \mathrm{~nm}$ in length) with liquid layer coating to reduce the adhesion of biopolymeric network. These nanovectors propelled in nanoporous hyaluronan solution, a fluid mimicking the vitreous. The inspiration for nanotool development is due to the liquid layer that characterizes carnivorous Nepenthes pitcher plant, which presents a slippery surface on the peristome to catch insects $[156,157]$. Porcine eyes were used as a model of human eyes: 
applying the external magnetic field, micropropellers exhibited controllable propulsion from eyeball to retina within 30 minutes. Instead, passive silica microparticles did not penetrate the vitreus (Figure 7). Nanorobots were also used to perform microsurgery on the retina and surrounding membranes thanks to the possibility of injecting them elsewhere in the body and bringing the drug to the target eye region [158]. Some intravitreal implants have recently developed to perform more clinical-inspired experiments. These nanotools, constituted by polymeric containers (silicon, poly(vinyl alcohol), or ethylene vinyl acetate), were placed in the posterior segment of the eye and they are permeable to lipophilic drugs. They did not display any toxicity and had a long retention time. These nanosystems have been extremely useful to treat proliferative diabetic retinopathy, retinal vascular occlusion, and uveitis [159].

a.
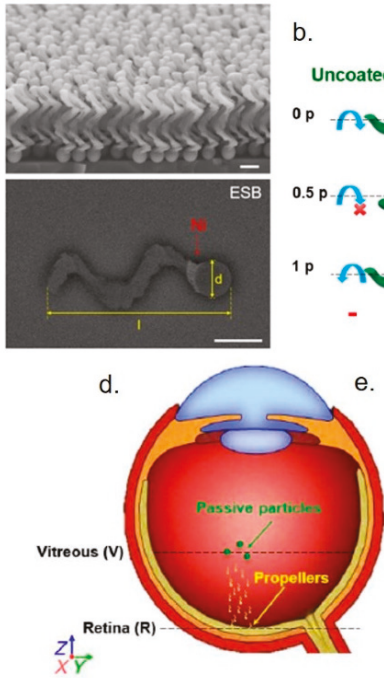

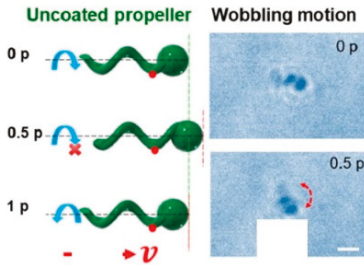

Propellers on retina $(\mathbf{R})$

c.

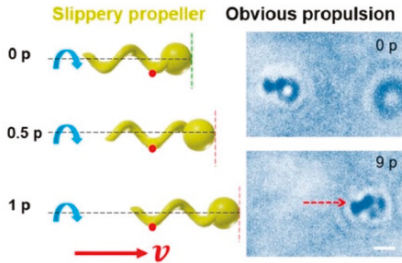

Passive particles in vitreous (V)
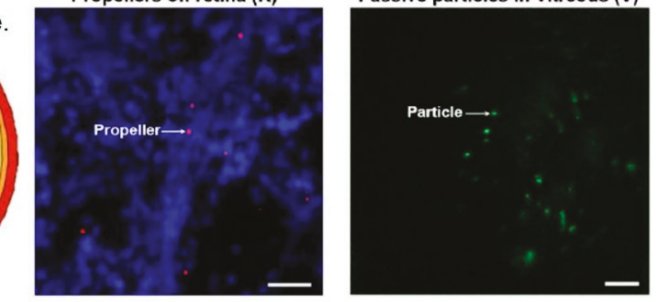

Figure 7. (a) SEM and ESB-SEM images of the micropropellers. (b,c) incomplete rotation of an uncoated micropropeller for one period (p) in the vitreous was showed in schematic and time-lapse microscopy, whereas the coated propeller exhibited propulsion under magnetic stimuli in vitreous for $1.5 \mathrm{~s}$ (nine periods). Red arrows showed the propulsion direction of propeller. The original position of nanotools and after the rotating magnetic field application were showed with green and red dashed lines. Scale bars, $1 \mu \mathrm{m}$. (d) Schematic illustration of micropropeller movement in the vitreous. (e) Left: fluorescent acquisitions showed the micropropellers labelled red on retina. Right: passive fluorescent particles were accumulated near center of vitreous. Nuclei of cells were stained with DAPI. Scale bar, $20 \mu \mathrm{m}$. Adapted from reference [155] under the terms of the Creative Commons Attribution-Non Commercial license.

\section{Conclusions and Perspectives}

It is established that eye damage is a leading cause of irreversible vision loss resulting from ocular diseases. The current standard treatments helped in both preventing and curing several pathological conditions, especially when related to the deep sections of the eye. However, many of these methods are rather invasive in terms of procedures, with a consequent possibility of promoting infection, inflammation, or even retinal detachment with potential risk of vision loss. These limitations impose considerable costs on patient quality of life and have an enormous economic impact on the health care system. The progress in the nano-engineering field can help boosting the discovery of a new approach for ocular diagnosis and therapy. Noble metals NPs and bio-inspired NPs represent a powerful tool to overcome all the limitations coming with standard treatments due to their physico-chemical properties. NPs can in fact penetrate deeper inside the ocular segments and also locally deliver high dosages of 
drugs, enriching local drug concentration when applied topically. In addition, the plasmonic properties of $\mathrm{Au}$ and $\mathrm{Ag}$ can have an added value to contrast eye diseases. However, numerous challenges still remain to be addressed as there are still limited numbers of in vivo studies. In addition, a deeper toxicology assessment is required for the development of new systems for ocular treatments.

Author Contributions: Conceptualization, V.D.M.; writing-original draft preparation, V.D.M.; review and editing, L.R. All authors have read and agreed to the published version of the manuscript.

Funding: This research received no external funding.

Acknowledgments: VDM kindly acknowledges Programma Operativo Nazionale (PON) Ricerca e Innovazione 2014-2020 Asse I "Capitale Umano", Azione I.2, Avviso "A.I.M: Attraction and International Mobility" CUP F88D18000070001.

Conflicts of Interest: The authors declare no conflict of interest.

\section{References}

1. Davson, H. The Physiology of The Eye; Elsevier: Amsterdam, The Netherlands, 2012.

2. Stephen, R.; Wilkinson, C.; Schachat, A.; Hinton, D.; Wilkinson, C. Retina; Elsevier: Amsterdam, The Netherlands, 2012.

3. Günhan, E.; van der List, D.; Chalupa, L.M. Ectopic Photoreceptors and Cone Bipolar Cells in the Developing and Mature Retina. J. Neurosci. 2003, 15, 1383-1389. [CrossRef]

4. Reichenbach, A.; Schnitzer, J.; Friedrich, A.; Brückner, G.; Schober, W. Development of the rabbit retina. Anat. Embryol. 1991, 183, 287-297. [CrossRef] [PubMed]

5. Dilnawaz, F.S.S.K. Nanotechnology-Based Ophthalmic Drug Delivery System. In Focal Controlled Drug Delivery; Domb, A., Khan, W., Eds.; Advances in Delivery Science and Technology; Springer: Boston, MA, USA, 2014.

6. Rakhi Dandonaa, L.D. Socioeconomic status and blindness. Br. J. Ophthalmol. 2001, 85, 1484-1488. [CrossRef] [PubMed]

7. Yorston, D. The Retina Retinal Diseases and VISION 2020. Comm Eye Health 2003, 16, 19-20.

8. Veleri, S.; Lazar, C.H.; Chang, B.; Sieving, P.A.; Banin, E.; Swaroop, A. Biology and therapy of inherited retinal degenerative disease: insights from mouse models. Dis. Models Mech. 2015, 8, 109-129. [CrossRef] [PubMed]

9. Jiang, S.; Franco, Y.L.; Zhou, Y.; Chen, J. Nanotechnology in retinal drug delivery. Int. J. Ophthalmol. $2018,11$. [CrossRef]

10. Gilbert, C.; Foster, A. Childhood blindness in the context of VISION 2020-The Right to Sight. Bull. World Health Organ. 2001, 79, 227-232.

11. Jones, B.W.; Marc, R.E.; Pfeiffer, R.L. Retinal Degeneration, Remodeling and Plasticity. In Webvision: The Organization of the Retina and Visual System; Salt Lake City (UT): University of Utah Health Sciences Center, UT, USA, 2016.

12. Rajappa, M.; Saxena, P.K.J. Ocular angiogenesis: Mechanisms and recent advances in therapy. Adv. Clin. Chem. 2010, 50, 103-121.

13. Aiello, L.P.; Avery, R.L.; Arrigg, P.G.; Keyt, B.A.; Jampel, H.D.; Shah, S.T.; Pasquale, L.R.; Thieme, H.; Iwamoto, M.A.; Park, J.E.; et al. Vascular endothelial growth factor in ocular fluid of patients with diabetic retinopa-thy and other retinal disorders. N. Engl. J. Med. 1994, 331, 480-1487. [CrossRef]

14. Weis, S.M.; Cheresh, D.A. Pathophysiological consequences of VEGF-induced vascular permeability. Nature 2005, 437, 497-504. [CrossRef]

15. Kim, S.J. Novel Approaches for Retinal Drug and Gene Delivery. Transl. Vis. Sci. Technol. 2014, 3, 7. [CrossRef] [PubMed]

16. Bennett, J.; Chung, D.C.; Maguire, A. Gene delivery to the retina: From mouse to man. Methods Enzym. 2012, $507,255-257$

17. $\mathrm{Xu}, \mathrm{H}$;; Chen, M. Targeting the complement system for the management of retinal in $\mathrm{fl}$ ammatory and degenerative diseases. Eur. J. Pharmacol. 2016, 787, 94-104. [CrossRef] [PubMed]

18. Díaz-Coránguez, M.; Ramos, C.; Antonetti, D.A. The inner Blood-Retinal Barrier: Cellular Basis and Development. Vis. Res. 2017, 139, 123-137. [CrossRef] 
19. Gaudana, R.; Jwala, J.; Boddu, S.H.S.; Mitra, A.K. Recent Perspectives in Ocular Drug Delivery. Pharm. Res. 2009, 26, 1197-1216. [CrossRef]

20. Kati-Sisko, V.; Hellinen, L. Expression, activity and pharmacokinetic impact of ocular transporters. Adv. Drug Deliv. Rev. 2018, 126, 3-22.

21. Souto, E.B.; Dias-ferreira, J.; Ana, L.; Ettcheto, M.; Elena, S. Advanced Formulation Approaches for Ocular Drug Delivery: State-Of-The-Art and Recent Patents. Pharmaceutics 2019, 11, 460. [CrossRef]

22. Le Bourlais, C.; Acar, L.; Zia, H.; Sado, P.A.; Needham, T.; Leverge, R. Ophthalmic drug delivery systems-Recent advances. Prog. Retin. Eye Res. 1998, 17, 33-58. [CrossRef]

23. Sugrue, M.F. The pharmacology of antiglaucoma drugs. Pharmacol. Ther. 1989, 43, 91-1989. [CrossRef]

24. Himawan, E.; Ekström, P.; Buzgo, M.; Gaillard, P.; Stefánsson, E.; Marigo, V.; Loftsson, T.; Paquet-Durand, F. Drug delivery to retinal photoreceptors. Drug Discov. Today 2019, 24, 1637-1643. [CrossRef]

25. Marmor, M.F.; Akira Negi, D.M.M. Kinetics of macromolecules injected into the subretinal space. Exp. Eye Res. 1985, 40, 687-696. [CrossRef]

26. Meyer, C.H.; Krohne, T.U.; Issa, P.C.; Liu, Z. Routes for Drug Delivery to the Eye and Retina: Intravitreal Injections. Retin. Pharmacother. Dev. Ophthalmol. 2016, 55, 63-70.

27. Falavarjani, K.G.; Nguyen, Q.D. Adverse events and complications associated with intravitreal injection of anti-VEGF agents: A review of literature. Eye 2013, 27, 787-794. [CrossRef] [PubMed]

28. Raghava, S.; Hammond, M.K.U. Periocular routes for retinal drug delivery. Expert Opin. Drug Deliv. 2004, 1, 99-144. [CrossRef]

29. Ghate, D.; Brooks, W.; McCarey, B.E.; Edelhauser, H.F. Pharmacokinetics of Intraocular Drug Delivery by Periocular Injections Using Ocular Fluorophotometry. Invest. Ophthalmol Vis. Sci. 2007, 48, 2230-2237. [CrossRef] [PubMed]

30. Castellarinand, A.; Pieramici, D.J. Anterior segment complications following periocular and intraocular injections. Ophthalmol. Clin. N. Am. 2004, 17, 583-590. [CrossRef]

31. Tsai, H.-H.; Wang, P.-Y.; Lin, I.-C.; Huang, H.; Liu, G.; Tseng, C.-L. Ocular Drug Delivery: Role of Degradable Polymeric Nanocarriers for Ophthalmic Application. Int. J. Mol. Sci. 2018, 19, 2830. [CrossRef]

32. ISO, Int. Organ Stand Nanotechnologies Vocab Part 1 Core Terms ISO/TS 80004-12010. 2010. Available online: https://www.iso.org/standard/51240.html (accessed on 1 March 2020).

33. ISO International Organization for Standardization. Technical Specification: Nanotechnologies Terminology and Definitions for Nanoobjects Nanoparticle, Nanofibre and Nanoplate. ISO/TS 80004-2:2008. 2008. Available online: https://www.iso.org/standard/44278.html (accessed on 1 March 2020).

34. De Matteis, V.; Rinaldi, R. Toxicity Assessment in the Nanoparticle Era. In Cellular and Molecular Toxicology of Nanoparticles; Springer: Cham, Switzerland, 2018; pp. 1-19.

35. Mu, Q.; Jiang, G.; Chen, L.; Zhou, H.; Fourches, D.; Tropsha, A.; Yan, B. Chemical Basis of Interactions Between Engineered Nanoparticles and Biological Systems. Chem. Rev. 2016, 7740-7781. [CrossRef]

36. Bhatia, S. Nanoparticles Types, Classification, Characterization, Fabrication Methods and Drug Delivery Applications. In Natural Polymer Drug Delivery Systems; Springer: Cham, Switzerland, 2016.

37. Ruth Duncan, R.G. Nanomedicine(s) under the Microscope. Mol. Pharm. 2011, 8, 2101-2214. [CrossRef]

38. Diebold, Y.; Calonge, M. Applications of nanoparticles in ophthalmology. Prog. Retin. Eye Res. 2010, 29, 596-609. [CrossRef]

39. Farjo, K.M.; Ma, J. The potential of nanomedicine therapies to treat neovascular disease in the retina. J. Angiogenes Res. 2010, 2, 21. [CrossRef] [PubMed]

40. JeffreyAdijanto, M.I.N. Nanoparticle-based technologies for retinal gene therapy. Eur. J. Pharm. Biopharm. 2015, 95, 353-367.

41. Soto, F.; Chrostowski, R.; Soto, F. Frontiers of Medical Micro/Nanorobotics: In vivo Applications and Commercialization Perspectives Toward Clinical Uses. Front. Bioeng. Biotechnol. 2018, 6, 1-12. [CrossRef]

42. Khlebtsovab, N.G.; Dykman, L.A. Optical properties and biomedical applications of plasmonic nanoparticles. J. Quant. Spectrosc. Radiat. Transf. 2010, 111, 1-35. [CrossRef]

43. Anker, J.N.; Hall, W.P.; Lyandres, O.; Shah, N.C.; Zhao, J.V. Biosensing with plasmonic nanosensors. Nat. Mater. 2008, 7, 442-453. [CrossRef] [PubMed]

44. Daniel, M.; Astruc, D. Gold Nanoparticles: Assembly, Supramolecular Chemistry, Quantum-Size-Related Properties, and Applications toward Biology, Catalysis, and Nanotechnology. Chem. Rev. 2004, 104, 293-346. [CrossRef] 
45. Asahi, T.; Uwada, T.; Masuhara, H. Single particle spectroscopic study on surface plasmon resonance probing local environmental conditions. Handai Nanophotonics 2006, 2, 219-228.

46. Boken, J.; Khurana, P.; Thatai, S. Plasmonic nanoparticles and their analytical applications: A review. Appl. Spectrosc. Rev. 2017, 52, 774-820. [CrossRef]

47. Loiseau, A.; Asila, V.; Boitel-aullen, G.; Lam, M. Silver-Based Plasmonic Nanoparticles for and Their Use in Biosensing. Biosens 2019, 2019, 9. [CrossRef]

48. Rizzello, L.; Pompa, P.P. Nanosilver-based antibacterial drugs and devices: Mechanisms, methodological drawbacks, and guidelines. Chem. Soc. Rev. 2014, 43, 1501-1518. [CrossRef]

49. Draslera, B.; Sayreb, P.; Günter, K.; Steinhäuserc, A.-R. In vitro approaches to assess the hazard of nanomaterials. NanoImpact 2018, 9, 51. [CrossRef]

50. De Matteis, V. Exposure to Inorganic Nanoparticles: Routes of Entry, Immune Response, Biodistribution and In Vitro/In Vivo Toxicity Evaluation. Toxics 2017, 5, 29. [CrossRef] [PubMed]

51. Naahidi, S.; Jafari, M.; Edalat, F.; Raymond, K.; Khademhosseini, A.C.P. Biocompatibility of engineered nanoparticles for drug delivery. J. Control. Release 2013, 10, 182-194. [CrossRef] [PubMed]

52. Turkevich, J.; Stevenson, P.C.; Hillier, J. A study of the nucleation and growth processes in the synthesis of colloidal gold. Faraday Soc. 1951, 11, 55-75. [CrossRef]

53. Brust, M.; Walker, M.; Bethell, D.; Schiffrin, D.J. Synthesis of thiol-derivatised gold nanoparticles in a two-phaseliquid-liquid system. J. Chem. Soc. Chem. Commun. 1994, 7, 801-802. [CrossRef]

54. Kumar, P.V.; Kala, S.M.J.; Prakash, K.S. Green synthesis of gold nanoparticles using Croton Caudatus Geisel leaf extract and their biological studies. Mater. Lett. 2019, 236, 19-22. [CrossRef]

55. Molnár, Z.; Bódai, V.; Szakacs, G.; Erdélyi, B.; Fogarassy, Z. Green synthesis of gold nanoparticles by thermophilic filamentous fungi. Sci. Rep. 2018, 8, 1-12. [CrossRef]

56. Giljohann, D.A.; Seferos, D.S.; Daniel, W.L.; Massich, M.D.; Patel, P.C. Gold Nanoparticles for Biology and Medicine. Angew. Chem. Int. Ed. Engl. 2010, 26, 3280-3294. [CrossRef]

57. Orlando, A.; Colombo, M.; Prosperi, D.; Corsi, F.; Panariti, A.; Rivolta, I.; Masserini, M.; Cazzinga, E. Evaluation of gold nanoparticles biocompatibility: A multiparametric study on cultured endothelial cells and macrophages. J. Nanopart. Res. 2018, 18, 58. [CrossRef]

58. Aghaie, T.; Jazayeri, M.H.; Manian, M.; Khani, l.; Erfani, M.; Rezayi, M.; Ferns, G.A.; Avan, A. Gold nanoparticle and polyethylene glycol in neural regeneration in the treatment of neurodegenerative diseases. J. Cell. Biochem. 2019, 120, 2749-2755. [CrossRef]

59. Bailly, A.-L.; Correard, F.; Popov, A.; Gleb Tselikov, F.; Appay, R.; Al-Kattan, A.; Andrei, V.; Kabashin, D.B.; Esteve, M.-A. In vivo evaluation of safety, biodistribution and pharmacokinetics of lasersynthesized gold nanoparticles. Sci. Rep. 2019, 9, 12890. [CrossRef] [PubMed]

60. Cassano, D.; Summa, M.; Pocoví-Martínez, S.; Mapanao, A.-K.; Catelani, T.; Bertorelli, R.; Voliani, V. Biodegradable Ultrasmall-in-Nano Gold Architectures: Mid-Period In Vivo Distribution and Excretion Assessment. Part. Part. Syst. Charact. 2019, 36, 1800464. [CrossRef]

61. Khan, H.A.; Abdelhalim, M.A.K.; Alhomida, A.S.; Al-Ayed, M.S. Effects of Naked Gold Nanoparticles on Proinflammatory Cytokines mRNA Expression in Rat Liver and Kidney. Biomed. Res. Int. 2013, 2013, 590730. [CrossRef] [PubMed]

62. Khan, H.A.; Alamery, S.; Ibrahim, K.E.; El-Nagar, D.M.; Al-Harbi, N.; Rusop, M.; Alrokayan, S.H. Size and time-dependent induction of proinflammatory cytokines expression in brains of mice treated with gold nanoparticle. Saudi J. Biol. Sci. 2019, 26. [CrossRef]

63. Karakoçak, B.B.; Raliya, R.; Davis, J.T.; Chavalmane, S.; Wang, W.N.; Ravi, N. Biocompatibility of gold nanoparticles in retinal pigment epithelial cell line. Toxicol. Vitr. 2016, 37, 61-69. [CrossRef]

64. Kim, J.H.; Kim, J.H.; Kim, K.-W.; Kim, M.H. Intravenously administered gold nanoparticles pass through the blood-retinal barrier depending on the particle size, and induce no retinal toxicity. Nanotechnology 2009, 20, 505101. [CrossRef]

65. Bakri, S.J.; Pulido, J.S.; Mukherjee, P.; Marler, R.J. Absence of histologic retinal toxicity of intravitreal nanogold in a rabbit model. Retin 2008, 2008, 147-149. [CrossRef]

66. Söderstjerna, E.; Bauer, P.; Cedervall, T.; Abdshill, H.; Johansson, F.; Johansson, U.E. Silver and Gold Nanoparticles Exposure to In Vitro Cultured Retina-Studies on Nanoparticle Internalization, Apoptosis, Oxidative Stress, Glial- and Microglial Activity. PLoS ONE 2014, 9, e0105359. [CrossRef]

67. Nakhlband, A. Impacts of nanomedicines in ocular pharmacotherapy. Bioimpacts 2011, 1, 7-22. 
68. Kittler, S.; Greulich Diendorf, M.; Köller, M.E. Toxicity of Silver Nanoparticles Increases during Storage Because of Slow Dissolution under Release of Silver Ions. Chem. Mater. 2010, 22, 4548-4554. [CrossRef]

69. Yang, X.; Gondikas, A.P.; Marinakos, S.M.; Auffan, M.; Li, J.; Hsu-Kim, H. Mechanism of Silver Nanoparticle Toxicity Is Dependent on Dissolved Silver and Surface Coating in Caenorhabditis elegans. Environ. Sci. Technol. 2012, 46, 1119-1127. [CrossRef] [PubMed]

70. De Matteis, V.; Malvindi, M.A.; Galeone, A.; Brunetti, V.; De Luca, E.; Kote, S.; Kshirsagar, P.; Sabella, S.; Bardi, G.; Pompa, P.P. Negligible particle-specific toxicity mechanism of silver nanoparticles: The role of $\mathrm{Ag}+$ ion release in the cytosol. Nanomedicine Nanotechnology. Biol. Med. 2015, 11, 731-739. [CrossRef] [PubMed]

71. Abbasi, E.; Milani, M.; Fekri Aval, S.; Kouhi, M.; Akbarzadeh, A.; Tayefi Nasrabadi, H.; Nikasa, P.; Joo, S.W.; Hanifehpour, Y.; Nejati-Koshki, K.; et al. Silver nanoparticles: Synthesis methods, bio- applications and properties. Crit. Rev. Microbiol. 2016, 42, 173-180. [CrossRef]

72. Zhang, T.; Song, Y.J.; Zhang, X.Y.; Wu, J.Y. Synthesis of silver nanostructures by multistep methods. Sensors (Switzerland) 2014, 14, 5860-5889. [CrossRef] [PubMed]

73. Singh, C.; Kumar, J.; Kumar, P.; Singh Chauhan, B.; Tiwari, K.N.; Mishra, S.K.; Srikrishna, S.; Saini, R.; Nath, G.; Singth, J. Green synthesis of silver nanoparticles using aqueous leaf extract of Premna integrifolia (L.) rich in polyphenols and evaluation of their antioxidant, antibacterial and cytotoxic activity. Biotechnol. Biotechnol. Equip. 2019, 359-371. [CrossRef]

74. Jun, Y.; Zi, Z.; Wanga, Y.; Zhaoa, G.; Liu, J.-X. Silver nanoparticles affect lens rather than retina development in zebrafish embryos. Ecotoxicol. Environ. Saf. 2018, 163, 279-288.

75. Sriram, M.I.; Kalishwaralal, K.; Barathmanikanth, S. Size-based cytotoxicity of silver nanoparticles in bovine retinal endothelial cells. Nanosci. Methods 2012, 1, 56-77. [CrossRef]

76. Kim, J.S.; Song, K.S.; Sung, J.H.; Ryu, H.R.; Choi, B.G.; Cho, H.S.; Lee, J.K. Genotoxicity, acute oral and dermal toxicity, eye and dermal irritation and corrosion and skin sensitisation evaluation of silver nanoparticles. Nanotoxicology 2013, 7, 953. [CrossRef]

77. Maneewattanapinyo, P.; Banlunara, W.; Thammacharoen, C.; Ekgasit, S.; Kaewamatawong, T. An evaluation of acute toxicity of colloidal silver nanoparticles. J. Vet. Med. Sci. 2011, 73, 1417-1423. [CrossRef]

78. De Matteis, V.; Cascione, M.; Cristina, C.; Rinaldi, R. Engineered Gold Nanoshells Killing Tumor Cells: New Perspectives. Curr. Pharm. Des. 2019, 25, 1477-1489. [CrossRef]

79. Weissleder, R. A clearer vision for in vivo imaging. Nat. Biotechnol. 2001, 19, 316-317. [CrossRef] [PubMed]

80. Song, H.B.; Wi, J.S.; Jo, D.H.; Kim, J.H.; Lee, S.W. Intraocular application of gold nanodisks optically tuned for optical coherence tomography: inhibitory effect on retinal neovascularization without unbearable toxicity. Nanomedicine 2017, 13, 1901-1911. [CrossRef] [PubMed]

81. Gasparini, S.J.; Llonch, S.; Borsch, O. Transplantation of photoreceptors into the degenerative retina: Current state and future perspectives. Prog. Retin. Eye Res. 2019, 69, 1-37. [CrossRef]

82. Chemla, Y.; Betzer, O.; Markus, A.; Farah, N.; Motiei, M. Gold nanoparticles for multimodal high-resolution imaging of transplanted cells for retinal replacement therapy. Nanomedicine 2019, 14, 1857-1871. [CrossRef]

83. Kim, H.; Nguyen, V.; Manivasagan, P.; Kim, S.W.; Oh, J. Doxorubicin-fucoidan-gold nanoparticles composite for dualchemo-photothermal treatment on eye tumors. Oncotarget 2017, 8, 113719-113733. [CrossRef] [PubMed]

84. Valluru, K.S.; Chinni, B.K.; Rao, N.A.; Bhatt, S.; Dogra, V.S. Basics and Clinical Applications of Photoacoustic Imaging. Ultrasound Clin. 2009, 4, 403-429. [CrossRef]

85. Gordon, A.Y.; Lapierre-Landry, M.; Skala, M.C.; Penn, J.S. Photothermal Optical Coherence Tomography of AntiAngiogenic Treatment in the Mouse Retina Using Gold Nanorods as Contrast Agents. Transl. Vis. Sci. Technol. 2019, 8, 18. [CrossRef] [PubMed]

86. Chan, C.-M.; Hsiao, C.-Y.; Li, H.-J.; Fang, J.-Y.; Chang, D.-C.; Hung, C.-F. The Inhibitory Effects of Gold Nanoparticles on VEGF-A-Induced Cell Migration in Choroid-Retina Endothelial Cells. Int. J. Mol. Sci. 2020, 21, 109. [CrossRef]

87. Gurunathanab, S.; Leeb, K.; Kalishwaralala, K.; Sheikpranbabua, S.; Vaidyanathana, R.; Eom, S.H. Antiangiogenic properties of silver nanoparticles. Biomaterials 2009, 30, 6341-6350. [CrossRef]

88. Sheikpranbabu, S.; Kalishwaralal, K.; Venkataraman, D.; Eom, S.H.; Park, J.; Gurunathan, S. Silver nanoparticles inhibit VEGF-and IL-1 $\beta$-induced vascular permeability via Src dependent pathway in porcine retinal endothelial cells. Nanobiotechnol. 2009, 7, 8. [CrossRef] 
89. Ngandeu Neubi, G.M.; Opoku-Damoah, Y.; Gu, X.; Han, Y.; Zhou, J.; Ding, Y. Bio-inspired drug delivery systems: an emerging platform for targeted cancer therapy. Biomater. Sci. 2018, 6, 958-973. [CrossRef] [PubMed]

90. Yang, L.; Kuang, H.; Zhang, W.; Aguilar, Z.P.; Wei, H.; Xu, H. Comparisons of the biodistribution and toxicological examinations after repeated intravenous administration of silver and gold nanoparticles in mice. Sci. Rep. 2017, 7, 3303. [CrossRef] [PubMed]

91. Madamsetty, V.S.; Mukherjee, A.; Mukherjee, S. Recent Trends of the Bio-Inspired Nanoparticles in Cancer Theranostics. Front. Pharmacol. 2019, 10, 1264. [CrossRef] [PubMed]

92. Birngruber, T.; Raml, R.; Gladdines, W.; Gatschelhofer, C.; Gander, E.; Ghosh, A.; Kroath, T.; Gaillard, P.J.; Pieber, T.R. Enhanced Doxorubicin Delivery to the Brain Administered Through Glutathione PEGylated Liposomal Doxorubicin (2B3-101) as Compared with Generic Caelyx, ${ }^{\circledR} /$ Doxil ${ }^{\circledR}$ _A Cerebral Open Flow Microperfusion Pilot Study. J. Pharm. Sci. 2014, 203, 1945-1948. [CrossRef]

93. Trost, A.; Lange, S.; Schroedl, F.; Bruckner, D.; Motloch, K.A.; Bogner, B.; Kaser-Eichberger, A.; Strohmaier, C.; Runge, C.; Aigner, L.; et al. Brain and Retinal Pericytes: Origin, Function and Role. Front. Cell Neurosci. 2016, 4, 20. [CrossRef] [PubMed]

94. Haque, S.; Shadab, M.D.; Alam, I.; Sahni, J.K.; Ali, J. Nanostructure-based drug delivery systems for brain targeting. Drug Dev. Ind. Pharm. 2012, 38, 387-411. [CrossRef] [PubMed]

95. Diebold, Y.; Jarrín, M.; Sáez, V.; Carvalho, E.L.; Orea, M.; Calonge, M.; Seijo, B. Ocular drug delivery by liposome-chitosan nanoparticle complexes (LCS-NP). Biomaterials 2007, 28, 1553-1564. [CrossRef] [PubMed]

96. Zhang, R.; He, R.; Qian, J.; Guo, J.; Xue, K.; Yuan, Y. Immunology and Microbiology Treatment of Experimental Autoimmune Uveoretinitis with Intravitreal Injection of Tacrolimus (FK506) Encapsulated in Liposomes. Investig. Ophthalmol. Vis. Sci. 2010, 51, 3575-3582. [CrossRef] [PubMed]

97. Yan, J.; Zheng, M. Chrysophanol liposome preconditioning protects against cerebral ischemia-reperfusion injury by inhibiting oxidative stress and apoptosis in mice. Int. J. Pharmacol. 2014, 10, 55-68. [CrossRef]

98. Choi, Y.H. Berberine hydrochloride protects $C_{2} C_{12}$ myoblast cells against oxidative stress-induced damage via induction of Nrf-2-mediated HO-1 expression. Drug Dev. Res. 2016, 77, 8. [CrossRef]

99. Lai, S.; Wei, Y.; Wu, Q.; Zhou, K.; Liu, T.; Zhang, Y.; Jiang, N.; Xiao, W.; Chen, J.; Liu, Q.; et al. Liposomes for efFective drug delivery to the ocular posterior chamber. J. Nanobiotechnol. 2019, 17, 64. [CrossRef] [PubMed]

100. Wolin, L.R.; Massopust, L.C. Characteristics of the ocular fundus in primates. J. Anat. 1967, 101, $693-699$. [PubMed]

101. Wang, J.-L.; Liu, Y.-L.; Li, Y.; Dai, W.-B.; Guo, Z.-M.; Wang, Z.-H.; Zhang, Q. EphA2 targeted doxorubicin stealth liposomes as a therapy system for choroidal neovascularization in rats. Ophthalmol. Vis. Sci. 2012, 53, 7348. [CrossRef] [PubMed]

102. Ravar, F.; Saadat, E.; Gholami, M.; Dehghankelishadi, P.; Mahdavi, M.; Azami, S. Hyaluronic acid-coated liposomes for targeted delivery of paclitaxel, in-vitro characterization and in-vivo evaluation. J. Control. Release 2016, 10, 10-22. [CrossRef]

103. Lee, J.; Goh, U.; Lee, H.J.; Kim, J.; Jeong, M. Effective retinal penetration of lipophilic and lipid-conjugated hydrophilic agents delivered by engineered liposomes. Mol. Pharm. 2017, 14, 423-430. [CrossRef]

104. Hironaka, K.; Inokuchi, Y.; Fujisawa, T.; Shimazaki, H.; Akane, M.; Tozuka, Y.; Tsuruma, K.; Shimazawa, M.; Hara, H. Edaravone-loaded liposomes for retinal protection against oxidative stress-induced retinal damage. Eur. J. Pharm. Biopharm. 2011, 79, 119-125. [CrossRef]

105. Yamamoto, Y.; Kuwahara, T.; Watanabe, K. Antioxidant activity of 3-methyl-1-phenyl-2-pyrazolin-5-one. Redox Rep. 1996, 2, 333-338. [CrossRef]

106. Vighi, E.; Trifunović, D.; Veiga-Crespo, P.; Rentsch, A.; Hoffmann, D.; Sahaboglu, A.; Strasser, T.; Kulkarni, M.; Bertolotti, E.; van den Heuvel, A.; et al. Combination of cGMP analogue and drug delivery system provides functional protection in hereditary retinal degeneration. Proc. Natl. Acad. Sci. USA 2018, 27, E2997-E3006. [CrossRef]

107. Lucas, K.A.; Pitari, G.M.; Kazerounian, S.; Ruiz-Stewart, I.; Park, J.; Schulz, S.; Chepenik, K.P.; Waldman, S.A. Guanylyl cyclases and signaling by cyclic GMP. Pharmacol. Rev. 2000, 52, 375-414.

108. Farber, D.B.; Lolley, R.N. Cyclic guanosine monophosphate: Elevation in degenerating photoreceptor cells of the C3H mouse retina. Science 1974, 186, 449-451. [CrossRef] 
109. Campbell, M.; Humphries, M.M.; Kiang, A.-S.; Nguyen, A.T.H.; Gobbo, O.L.; Tam, L.C.S.; Suzuki, M.; Hanrahan, F.; Ozaki, E.; Farrar, G.-J.; et al. Systemic low-molecular weight drug delivery to pre-selected neuronal regions. EMBO Mol. Med. 2011, 3, 235-245. [CrossRef]

110. Davis, B.M.; Normando, E.M.; Guo, L.; Turner, L.A.; Nizari, S. Topical Delivery of Avastin to the Posterior Segment of the Eye In Vivo Using Annexin A5-associated Liposomes. Small 2014, 24, 1575-1584. [CrossRef] [PubMed]

111. Platania, C.B.M.; Fisichella, V.; Fidilio, A.; Geraci, F.; Lazzara, F.; Leggio, G.M.; Salomone, S.; Drago, F.; Pignatello, R.; Caraci, F.; et al. Topical Ocular Delivery of TGF- $\beta 1$ to the Back of the Eye: Implications in Age-Related Neurodegenerative Diseases. Int. J. Mol. Sci. 2017, 18, 2076. [CrossRef] [PubMed]

112. Boulyjenkov, V.; Berg, K. Gene Therapy: Promises, Problems and Prospects; Genes Resist. to Dis.; Springer: Berlin/Heidelberg, Germany, 2000.

113. Ramamoorth, M.; Narvekar, A. Non viral vectors in gene therapy-An overview. J. Clin. Diagn. Res. 2015, 9 , GE01. [CrossRef]

114. Das, K.G.; Ram, A.R. Niosome ad a novel drug delivery system: A review. Appl. Pharm. 2013, 5, 1-7.

115. Seleci, D.A.; Seleci, M.; Walter, J.G. Niosomes as Nanoparticular Drug Carriers: Fundamentals and Recent Applications. J. Nanomat. 2016, 2016, 13. [CrossRef]

116. Gallego, I.; Villate-Beitia, I.; Martínez-Navarrete, G.; Menéndez, M.; López-Méndez, T.; Soto-Sánchez, C.; Zárate, J.; Puras, G.; Fernández, E.; Pedraz, J.L. Non-viral vectors based on cationic niosomes and minicircle DNA technology enhance gene delivery efficiency for biomedical applications in retinal disorders. Nanomedicine. 2019, 17, 308-318. [CrossRef] [PubMed]

117. Mashal, M.; Attia, N.; Puras, G.; Martinez-Navarrete, G.; Fernandez, E.P. Retinal gene delivery enhancement by lycopene incorporation into cationic niosomes based on DOTMA and polysorbate 60. J. Control. Release 2017, 254, 55-64. [CrossRef]

118. Guinedi, A.S.; Mortada, N.D.; Mansour, S. Preparation and evaluation of reverse-phase evaporation and multilamellar niosomes as ophthalmic carriers of acetazolamide. Int. J. Pharm. 2005, 306, 71-82. [CrossRef]

119. Guglevaa, V.; Titevaa, S.; Rangelovb, S.; Momekova, D. Design and in vitro evaluation of doxycycline hyclate niosomes as a potential ocular delivery system. Int. J. Pharm. 2019, 5, 567. [CrossRef]

120. Cintia, S.; De Paivaa Rosa, M.; Corralesa Arturo, L.; Villarreala William, J.; De-Quan, F.; Lia Michael, E.; Sternb Stephen, C. Corticosteroid and doxycycline suppress MMP-9 and inflammatory cytokine expression, MAPK activation in the corneal epithelium in experimental dry eye. Exp. Eye Res. 2006, 83, 526-535.

121. Sapino, S.; Chirio, D.; Peira, E.; Abellán Rubio, E.; Brunella, V.; Jadhav, S.A.; Chindamo, G.; Gallarate, M. Ocular Drug Delivery: A Special Focus on the Thermosensitive Approach. Nanomaterials (Basel) 2019, 9, 884. [CrossRef] [PubMed]

122. Hoare, T.R.; Kohane, D.S. Hydrogels in drug delivery: Progress and challenges. Polymer (Guildf) 2008, 49, 1993-2007. [CrossRef]

123. Wang, K.; Han, Z. Injectable hydrogels for ophthalmic applications. J. Control. Release 2017, 268, $212-224$. [CrossRef]

124. Ferreira, N.N.; Ferreira, L.M.B.; Cardoso, V.M.O.; Boni, F.I.; Souza, A.L.R.; Gremião, M.P.D. Recent advances in smart hydrogels for biomedical applications: From self-assembly to functional approaches. Eur. Polym. J. 2018, 99, 117-133. [CrossRef]

125. Elieh-Ali-Komi, D.; Hamblin, M.R. Chitin and Chitosan: Production and Application of Versatile Biomedical Nanomaterials. Int. J. Adv. Res. (Indore) 2016, 4, 411-427.

126. ValérieDodane, V.D. Pharmaceutical applications of chitosan. Pharm. Sci. Technol. Today 1998, 1, $246-253$.

127. Lee, K.Y.; Mooney, D.J. Alginate: Properties and biomedical applications. Prog. Polym. Sci. 2012, 37, $106-126$. [CrossRef]

128. Paliwal, R.; Paliwal, S.R.; Sulakhiya, K.; Kurmi, B.D.; Kenwat, R.; Mamgain, A. Chitosan-Based Nanocarriers for Ophthalmic Applications; Elsevier: Amsterdam, The Netherlands, 2019.

129. Nagarwal, R.C.; Kumar, R. Chitosan coated sodium alginate-chitosan nanoparticles loaded with 5-FU for ocular delivery: in vitro characterization and in vivo study in rabbit eye. Eur. J. Pharm. Sci. 2012, 20, 678-685. [CrossRef]

130. Ameeduzzafar Imam, S.S.; Abbas Bukhari, S.N.; Ahmad, J. Formulation and optimization of levofloxacin loaded chitosan nanoparticle for ocular delivery: In-vitro characterization, ocular tolerance and antibacterial activity. Int. J. Biol. Macromol. 2018, 108, 650-659. [CrossRef] 
131. Andrei, G.; Peptu, C.A.; Popa, M.; Desbrieres, J.; Peptu, C.; Gardikiotis, F.; Costuleanu, M.; Costin, D.; Dupin, J.C.; Uhart, A. Formulation and evaluation of cefuroxim loaded submicron particles for ophthalmic delivery. Int. J. Pharm. 2015, 30, 16-29. [CrossRef]

132. Kaskoos, R. Investigation of moxifloxacin loaded chitosan-dextran nanoparticles for topical instillation into eye: In-vitro and ex-vivo evaluation. Int. J. Pharm. Investig. 2014, 4, 164-173. [CrossRef] [PubMed]

133. Solanki, A.; Smalling, R.; Parola, A.H.; Nathan, I.; Kasher, R.; Pathak, Y.; Sutariya, V. Humanin Nanoparticles for Reducing Pathological Factors Characteristic of Age-related Macular Degeneration. Curr. Drug Deliv. 2019, 16, 226-232. [CrossRef]

134. Cohen, A.; Lerner-Yardeni, J.; Meridor, D.; Kasher, R.; Nathan, I.; Parola, A. Humanin derivatives inhibit necrotic cell death in neurons. Mol. Med. 2015, 21, 505. [CrossRef] [PubMed]

135. Cheng, Y.; Ko, Y.; Chang, Y.; Huang, S. Thermosensitive chitosan-gelatin-based hydrogel containing curcuminloaded nanoparticles and latanoprost as a dual-drug delivery system for glaucoma treatment. Exp. Eye Res. 2019, 179, 179-187. [CrossRef] [PubMed]

136. Radomska-leśniewska, D.M.; Osiecka-iwan, A.; Hyc, A.; Góźdź, A.; Dąbrowska, A.M.; Skopiński, P. Therapeutic potential of curcumin in eye diseases. Cent. Eur. J. Immunol. 2019, 44, 2-4. [CrossRef] [PubMed]

137. Nathan Gooch, S.A.M.; Condie, R.; Michael, B.R.; Bonnie Archer, B.K.A.; Ocular, B. Drug Delivery for Glaucoma Management. Pharmaceutics 2012, 4, 197-211. [CrossRef]

138. Michael, P.; Fautsch, D.H.J. Aqueous Humor Outflow: What Do We Know? Where Will It Lead Us? Investig. Ophthalmol. Vis. Sci. 2006, 47, 4181-4187.

139. Silva, B.; Marto, J.; Braz, B.S.; Delgado, E.; Almeida, A.J.; Gonçalves, L. New nanoparticles for topical ocular delivery of erythropoietin. Int. J. Pharm. 2020, 119020. [CrossRef]

140. Pandit, J.; Sultana, Y.; Aqil, M. Chitosan-coated PLGA nanoparticles of bevacizumab as novel drug delivery to target retina: optimization, characterization, and in vitro toxicity evaluation. Artif. Cells Nanomed. Biotechnol. 2016, 45, 1397-1407. [CrossRef]

141. Mitra, R.N.; Han, Z.; Merwin, M.; Taai, M.; Al Conley, S.M. Synthesis and Characterization of Glycol Chitosan DNA Nanoparticles for Retinal Gene Delivery. ChemMedChem 2014, 9, 189-196. [CrossRef]

142. Lee, J.-H.; Kim, H.-W. Emerging properties of hydrogels in tissue engineering. J. Tissue Eng. 2018, 9, 2041731418768285. [CrossRef] [PubMed]

143. Chang, D.; Kinam Park, A. Hydrogels for sustained delivery ofbiologics to the back of the eye. Drug Discov. Today 2019, 24, 1470-1482. [CrossRef] [PubMed]

144. Van Hovea, A.H.; Beltejar, M.G.; Benoit, D.S.W. Development and in vitro assessment of enzymatically-responsive poly(ethylene glycol) hydrogels for the delivery of therapeutic peptides. Biomaterials 2014, 35, 9719-9730. [CrossRef] [PubMed]

145. Drapala, P.W.; Jiang, B.; Chiu, Y.-C.; Mieler, W.F.; Brey, E.M. The Effect of Glutathione as Chain Transfer Agent in PNIPAAm-Based Thermo-responsive Hydrogels for Controlled Release of Proteins. Pharm. Res. 2014, 31, 742-753. [CrossRef] [PubMed]

146. Schultz, C.; Breaux, J.; Pharmd, J.S.; Douglas Morck, D.V.M. Drug delivery to the posterior segment of the eye through hydrogel contact lenses. Clin. Exp. Optom. 2011, 94, 212-218. [CrossRef]

147. Kabiri, M.; Kamal, S.H.; Pawar, S.V.; Roy, P.R.; Derakhshandeh, M.; Kumar, U.; Hatzikiriakos, S.G.; Hossain, S.; Yadav, V.G. A stimulus-responsive, in situ-forming, nanoparticle-laden hydrogel for ocular drug delivery. Drug Deliv. Transl. Res. 2018, 8, 484-495. [CrossRef]

148. Osswald, C.R.; Kang-Mieler, J.J. Controlled and Extended In Vitro Release of Bioactive Anti-Vascular Endothelial Growth Factors from a Microsphere-Hydrogel Drug Delivery System. Curr. Eye Res. 2016, 41, 1216-1222. [CrossRef]

149. Agrahari, V.; Agrahari, V.; Hung, W.-T.; Christenson, L.K. Composite Nanoformulation Therapeutics for Long-Term Ocular Delivery of Macromolecules. Mol. Pharm. 2016, 13, 2912-2922. [CrossRef]

150. Yang, H.; Tyagi, P.; Kadam, R.S.; Holden, C.A. Hybrid dendrimer hydrogel/PLGA nanoparticle platform sustains drug delivery for one week and antiglaucoma effects for four days following one-time topical administration. ACS Nano 2012, 25, 7595-7606. [CrossRef]

151. Wadhams, G.H.; Armitage, J.P. Making sense of it all: Bacterial chemotaxis. Nat. Rev. Mol. Cell. Biol. 2004, 5 , 1024-1037. [CrossRef] [PubMed]

152. Micali, G.; Endres, R.G. Bacterial chemotaxis: information processing, thermodynamics, and behavior. Curr. Opin. Microbiol. 2016, 30, 8-15. [CrossRef] [PubMed] 
153. Mei, Y.; Alexander, A. Solovev SS and OGS. Rolled-up nanotech on polymers: from basic perception to self-propelled catalytic microengines. Chem. Soc. Rev. 2011, 40, 2109-2119. [CrossRef] [PubMed]

154. Kumar, M.S.; Philominathan, P. The physics of flagellar motion of E. coli during chemotaxis. Biophys. Rev. 2010, 2, 13-20. [CrossRef] [PubMed]

155. Wu, Z.; Troll, J.; Jeong, H.-H.; Wei, Q.; Stang, M.; Ziemssen, F.; Wang, Z.; Dong, M.; Schnichels, S.; Qui, T.; et al. A swarm of slippery micropropellers penetrates the vitreous body of the eye. Sci. Adv. 2018, 4, eaat4388. [CrossRef] [PubMed]

156. Bohn, H.F.; Federle, W. Insect aquaplaning: Nepenthes pitcher plants capture prey with the peristome, a fully wettable water-lubricated anisotropic surface. Proc. Natl. Acad. Sci. USA 2004, 101, 14138-14143. [CrossRef]

157. Wong, T.-S.; Kang, S.H.; Tang, S.K.Y.; Smythe, E.J.; Hatton, B.D.; Grinthal, A. Bioinspired self-repairing slippery surfaces with pressure-stable omniphobicity. Nature 2011, 477, 443-447. [CrossRef]

158. Nistor, M.T.; Rusu, A.G. Nanorobots with Applications in Medicine; Elsevier: Amsterdam, The Netherlands, 2019.

159. Alhalafi, A.M. Applications of polymers in intraocular drug delivery systems. Oman J. Ophtalmol. 2017, 10, 3-8. [CrossRef]

(C) 2020 by the authors. Licensee MDPI, Basel, Switzerland. This article is an open access article distributed under the terms and conditions of the Creative Commons Attribution (CC BY) license (http://creativecommons.org/licenses/by/4.0/). 



\title{
The Intersection of Serine Metabolism and Cellular Dysfunction in Retinal Degeneration
}

\author{
Tirthankar Sinha ${ }^{\dagger}$, Larissa Ikelle ${ }^{\dagger}$, Muna I. Naash * and Muayyad R. Al-Ubaidi * \\ Department of Biomedical Engineering, University of Houston, Houston, TX 77204, USA; \\ tsinha2@Central.UH.EDU (T.S.); likelle@Central.UH.EDU (L.I.) \\ * Correspondence: mnaash@central.uh.edu (M.I.N.); malubaid@central.uh.edu (M.R.A.-U.); \\ Tel.: +1-713-743-1651 (M.I.N.); Fax: +1-713-743-0226 (M.I.N.) \\ + These authors contributed equally to this work.
}

Received: 4 February 2020; Accepted: 6 March 2020; Published: 10 March 2020

\begin{abstract}
In the past, the importance of serine to pathologic or physiologic anomalies was inadequately addressed. Omics research has significantly advanced in the last two decades, and metabolomic data of various tissues has finally brought serine metabolism to the forefront of metabolic research, primarily for its varied role throughout the central nervous system. The retina is one of the most complex neuronal tissues with a multitude of functions. Although recent studies have highlighted the importance of free serine and its derivatives to retinal homeostasis, currently few reviews exist that comprehensively analyze the topic. Here, we address this gap by emphasizing how and why the de novo production and demand for serine is exceptionally elevated in the retina. Many basic physiological functions of the retina require serine. Serine-derived sphingolipids and phosphatidylserine for phagocytosis by the retinal pigment epithelium (RPE) and neuronal crosstalk of the inner retina via D-serine require proper serine metabolism. Moreover, serine is involved in sphingolipid-ceramide balance for both the outer retina and the RPE and the reductive currency generation for the RPE via serine biosynthesis. Finally and perhaps the most vital part of serine metabolism is free radical scavenging in the entire retina via serine-derived scavengers like glycine and GSH. It is hard to imagine that a single tissue could have such a broad and extensive dependency on serine homeostasis. Any dysregulation in serine mechanisms can result in a wide spectrum of retinopathies. Therefore, most critically, this review provides a strong argument for the exploration of serine-based clinical interventions for retinal pathologies.
\end{abstract}

Keywords: serine; retinal degeneration; diabetic retinopathy; macular degeneration; macular telangiectasia; oxidative stress; sphingolipids; retina; RPE; Müller cells

\section{Why Is Serine Important to the Entire Retina?}

Serine is a non-essential amino acid directly involved in cellular homeostasis, proliferation, and differentiation $[1,2]$. The cells of the neural retina are no exception, and, in fact, exhibit a great dependence on serine and its exhaustive variety of metabolic intermediates [3]. Serine uptake occurs either by delivery from the bloodstream or it can be synthesized by the anabolism of the glycolytic intermediate, 3-phosphoglycerate (3-PG) [4] in the retina (neural retina-RPE, Figure 1). After uptake or synthesis, serine becomes available and serves as a central node in many cellular processes [5]. 


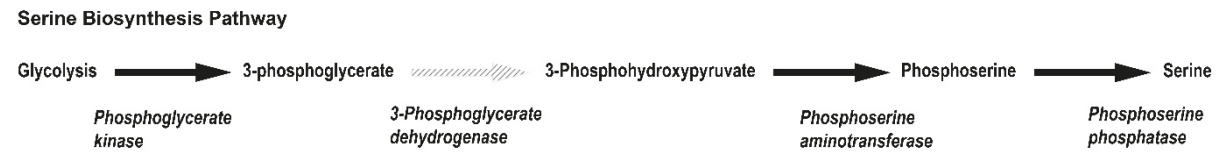

Figure 1. Pathway depicting serine biosynthesis from glycolysis. Metabolic intermediates involved in the enzymatic synthesis of L-serine from glycolysis is shown here with the rate limiting step marked with a dashed grey arrow. The enzymes involved in respective steps are shown in bold italics below the arrow for the individual reaction.

Other than being an integral amino acid in multiple essential proteins, free serine is essential for generating cysteine, glycine, methionine, and sphingolipids [5]. Glycine and cysteine are essential intermediates. Glycine is a precursor molecule for porphyrins and purine nucleotides [6]. Cysteine on the other hand is important in the protection of neuronal cells and for the production of taurine [7]. Together they form glutathione (GSH), a critical anti-oxidant in the retina [8]. Sphingolipids are elemental components of the phospholipid bilayer and are indispensable to cellular viability, homeostasis and function [2,3,5]. Furthermore, serine derived-metabolites have proven essential to methylation [2], apoptosis, and synaptic receptor activation [6].

The integrality of serine to cellular function has been appreciated by dysregulatory events appearing in many retinopathies. Reduced serine levels have been implicated in the etiology of macular related diseases such as macular telangiectasia type 2 (Mac Tel), age related macular degeneration, and diabetic retinopathy (DR) $[9,10]$. The retina is a complex stratified tissue consisting of the retinal pigment epithelium (RPE), a critical layer of cells for retinal homeostasis, and the neural retina containing the two types of photoreceptor cells. The neural retina also harbors the second order neurons and the retinal ganglion cells (RGC) that form the optic nerve. Serine proves to be a vital intermediate in many of these processes. Consequently, our goal is to provide a focused review of the role of serine homeostasis in maintaining optimum retinal function and proper oxidative balance.

\section{Why Does the Retina Need to Synthesize Serine?}

In most tissues, serine uptake from either blood or proteolysis sufficiently meets cellular metabolic requirements. However, there are tissues that mandate an elevated level of serine and, accordingly, upregulate enzymes for serine biosynthesis. As previously highlighted, this biosynthetic pathway (Figure 1) helps in maintaining the redox potential of the cell [11]. The primary source of serine biosynthesis is glucose [12], which in most cells is utilized via glycolysis. Serine biosynthesis branches from one of the glycolytic intermediates, 3-phosphoglycerate. The rate limiting step for serine biosynthesis is the reaction involving phosphoglycerate dehydrogenase (PHGDH), which converts 3-phosphoglycerate into phosphoserine. This is followed by the removal of the phosphate to generate l-serine.

It has been previously shown that the retina expresses high levels of all enzymes involved in serine biosynthesis. However, the RPE appears to express even higher levels than the neural retina [13] as has been shown by flux studies, whereby the RPE readily converts glucose into serine [10,14]. Further experiments verified that the neural retina possess an efficient system for serine uptake. [15].

\section{De Novo Serine Synthesis in Müller Cells}

Transport of serine, a neutral amino acid, across the tight blood-retinal barrier into the RPE or across the endothelial cells and to the neural retina is supposedly inadequate [16]. So the retina increases the levels of intracellular serine through de novo synthesis [12]. Further supply of serine to photoreceptors and the inner retina is provided by the RPE and retinal Müller cells [15]. The latter was demonstrated by co-immunofluorescence with anti-PHGDH and anti-cellular retinaldehyde-binding protein (CRALBP) antibodies showing that the serine de novo synthesis pathway is indeed present in 
the Müller cells [17]. Since serine metabolism is central to maintaining redox and oxidative balance, ion flux, glutamate levels, and many other support functions [18], Müller cells through their de novo synthesis of serine play major roles in these functions [17].

Many retinopathies are associated with loss of Müller glia [17]. Mac Tel, is a pathology of the retina recently characterized by significant reductions in serine synthesis and loss of central vision [16]. Although the macula (anatomic) is a small cone-dense region of the retina occupying only $1.4 \%$ of the total area of the retina, it harbors approximately $8 \%$ of the total cone population and $60 \%$ of all RGCs [19]. The macula is incredibly metabolically active and relies heavily on Müller glia [18]. Not only has localization of serine synthesis in the neural retina been demonstrated in Müller cells, but relative to peripheral Müller cells, those of the macula seem to show increased expression of PHGDH [10]. Moreover, the macular Müller cells show increased GSH and glycine production and are more susceptible to induced stress [10].

\section{Serine Homeostasis Plays an Important Role in the Maintenance of the Retina}

\subsection{Serine and Sphingolipids}

One of the many fates of biosynthesized L-serine is to combine with palmitoyl-Co-A to form sphingolipids (Figure 2) catalyzed by the enzyme serine palmitoyl-Co-A transferase (SPT) [20]. Even though there are multiple interconnected pathways, which can control the formation of various sphingolipids, the most common pathway is serine incorporation [21]. Neural retinal sphingolipids have been well characterized addressing their beneficial and toxic capacities [3,22-25]. It is very well known that the most vital role of sphingolipids is aiding in sphingomyelin formation, which enables efficient synaptic transmission [3]. Perhaps, that is one reason why the sphingolipid content in the inner retina is quite high [3]. Moreover, sphingolipids in the form of sphingosine-1-phosphate are thought to have anti-apoptotic role, further rationalizing the abundance of sphingolipids in the outer retina [3]. It is important to note that the role of sphingolipid levels and their derivatives have not yet been assessed in the RPE. Given that the RPE may be a vital source of serine for the neural retina, it is imperative to determine how its transport might be facilitated.

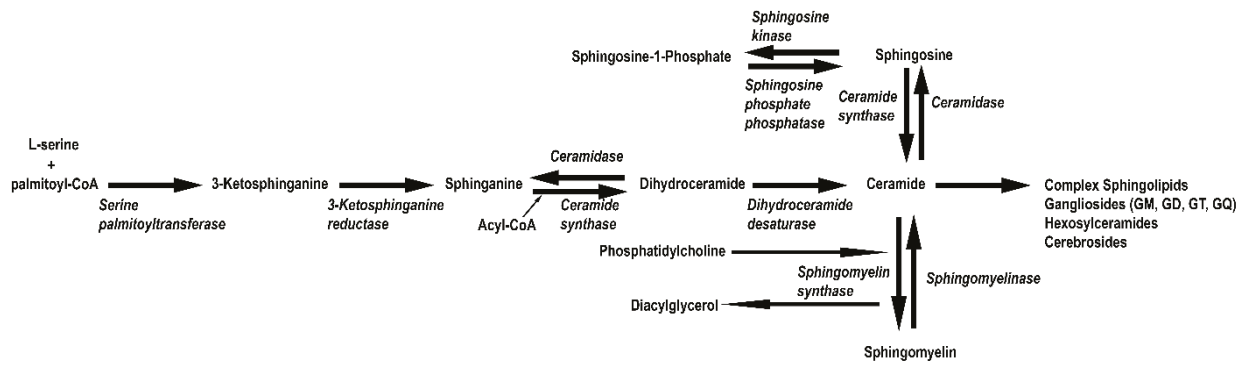

Figure 2. Pathway depicting sphingolipid biosynthesis from L-serine. Metabolic intermediates involved in the enzymatic synthesis of sphingolipids from L-serine are shown here. The enzymes involved in respective steps are shown in bold italics below the arrow for the individual reaction.

\subsection{Serine and RPE Phagocytosis}

The efficient phagocytosis of photoreceptor outer segments by the RPE is elemental to retinal health. From extensive examination of the process, it has been determined that any delay or inefficiency in the phagocytosis can lead to gross abnormalities for both the neural retina and the RPE [26]. The metabolism of the RPE is largely dependent on recycling the outer segment phospholipids, as they provide an important source of fuel [27-29]. The neural retina eliminates older disks in order to maintain optimal function. So how is serine vital for maintaining such an important step? In order for 
the RPE to recognize and phagocytose the correct portion of the outer segments, phosphatidylserine will localize to the extracellular surfaces of those digestible regions [30]. Since almost $10 \%$ of the entire outer segment is daily phagocytosed from each photoreceptor, it helps us appreciate the enormous amount of serine that needs to be available. Furthermore, it has been suggested that sphingolipids may also play a regulatory role in order to ensure efficient phagocytosis, since disruption of sphingolipid metabolism via SPT and ceramide synthase inhibition impaired phagocytosis [31,32]. In addition, glycosphingolipids like lactosylceramides and gangliosides are major lipid raft components and assist in cell adhesion, membrane polarity and phagocytosis initiation [33-36]. This raises the possibility that the sphingolipid pool in RPE may assist in facilitating outer segment phagocytosis as well as maintaining the tight junction barrier and cellular polarity. Since serine is an integral component of sphingolipids, it goes without saying that this presents another facet of serine availability contributing to efficient neural retina:RPE interdependence.

\subsection{The Role of D-Serine}

D-serine, an enantiomer of L-serine, is primarily released by glial cells, Müller cells and astrocytes [13]. Initially, it was discovered in the brain but has more recently been studied in the neural retina [13]. D-serine, formed by the racemization of L-serine by serine racemase (SRR) [13], functions as a neurotrophic factor and as a co-agonist for $N$-methyl-D-aspartate receptors (NMDARs) [37]. In the brain, NMDARs normally bind glutamate and glycine, but data suggests preferential or higher affinity binding to D-serine in the retina $[13,38]$. NMDARs locate at the synaptic terminals of RGCs and sparingly to photoreceptor terminals [39]. The role of NMDARs in the synaptic terminals has not been entirely substantiated, however, studies have indicated that they function in an excitatory role [13]. In the presence of D-serine, NMDA-mediated currents and light-evoked response showed increased amplitude over the D-serine absent control [13]. Irrespective of NMDAR functions, the importance of D-serine is evidenced by pathologies that are directly linked to D-serine irregularities. D-serine insufficiency has been associated with psychiatric and neurodevelopmental disorders [37]. Supplementation with D-serine has proven to mitigate some of the symptoms of psychosis [37]. Patients with DR, by contrast, suffer from increased SRR activity and over production of D-serine, which elicits an excitotoxic effect on RGCs and ultimately contributes to cell death [40]. This will be addressed in more detail in the following sections.

\subsection{Serine and Epigenetic Regulation}

One carbon metabolism is a fundamental process for purine, thymidine, and amino acid biosynthesis that involves the transfer of a one carbon unit to generate critical metabolites [8]. Serine is essential in this group of metabolic reactions [41] and, in this context, is a precursor for the synthesis of methionine. Briefly, serine donates a methyl group which reacts with homocysteine, originally derived from aspartate $[42,43]$. This reaction is catalyzed by serine transhydroxymethylase to ultimately form methionine $[42,43]$.

An adenosylation reaction of adenosine triphosphate (ATP) and methionine will yield $S$-adenosyl-L-methionine, which is the critical methyl donor for global methylation of DNA [41,44,45]. DNA methylation, an absolutely essential process to maintain cellular homeostasis [46], occurs on CpG islands of DNA and suppresses gene expression [47]. Therefore, irregularities in serine levels cause downstream hypo- or hyper-methylation of DNA [48], effecting stress responses, proliferation, metabolism, and even responses to extrinsic stimuli [46].

The link between epigenetic modulations and retinal disease is currently at a nascent phase [49], so correlations between aberrant serine metabolism, DNA methylation, and retinal disease remain unclear. However, recent studies performed on three pairs of monozygotic twins with different presentations of AMD indicated significant changes to DNA methylation patterns of genes that may be implicated in disease pathogenesis [50]. More critically, the diet of the studied twins also highlighted nutritional significance in epigenetic regulation. Subjects with reduced dietary methionine, vitamin D, 
and betaine had worse disease prognosis [50], implicating the importance of nutrient bioavailability and epigenetics. As serine is involved in the synthesis of methionine, further studies should explore serine levels in retinal disease in relation with epigenetic changes that may contribute to disease onset and/or progression.

\section{Serine Is an Anti-Oxidant and Mediator of Inflammation}

The role that serine plays as a central junction for critical intermediates also extends to anti-oxidative and inflammatory mechanisms. Experimental evidence has indicated that the retina shares some metabolic patterns with neoplastic cells [16]. In these systems, the Warburg effect predominates [51], and energy is generated primarily through glycolysis to ensure accelerated ATP production [16] As explained earlier, the retina is one of the highest energy consuming tissues and thus a source of excessive reactive oxygen species (ROS). Emerging data has shed light on extra-mitochondrial sources of ROS specifically in the outer retina and even in the outer segment of photoreceptors [52,53]. It was previously shown that oxygen is mainly absorbed at the level of the photoreceptors [54] and thus this site is most prone to oxidative stress, both in physiology and pathology [55]. To build on that, it seems that blue light exposure on outer segment discs as well as ectopic oxidative phosphorylation in the outer segment both pose a greater need for effective free radical scavengers in the outer segment-RPE interface [56-58]. Glycine and GSH and thus serine supply are essential for this ROS mitigation system of the rods and cones [59].

Fundamentally, intracellular redox homeostasis represents the equilibrium between oxidative and anti-oxidative species, creating a balance in which the environment is not cytotoxic and is sensitive enough to redox changes that may mandate an intracellular or extracellular response. Clearly, oxidative stress is caused by aberrations to this balance. Serine metabolism contributes to redox homeostasis through synthesis of glycine and its essential downstream products GSH and nicotinamide adenine dinucleotide phosphate (NADPH), as well as nicotinamide adenine dinucleotide (NADH) generation during serine biosynthesis [60]. In addition, GSH and NADPH deactivate ROS and other oxidative molecules [60]. GSH is the direct result of combining cysteine and glycine, both of which, as previously explored, are synthesized from serine [61]. NADPH is generated in many pathways, but recent studies suggest that metabolism of serine is a significant contributor of NADPH to the mitochondria, especially during hypoxic conditions [11]. During serine synthesis from 3-phosphoglycerate, serine donates a single carbon to folate forming glycine as well as tetrahydrofolate (THF). THF reductase forms NADP ${ }^{+}$. Then, GSH mediated-reduction of electrophilic molecules helps to maintain the balance of NADPH to $\mathrm{NADP}^{+}$, which is principle to redox homeostasis.

GSH anti-oxidative activity is vital for proper retinal function [62]. So in malignancies where serine is deficient, there is an obvious reduction in GSH level and activity, and, as a consequence, the supply of the precursor molecules have been significantly affected. However, it is not as simple as reduced supply. 5' adenosine monophosphate-activated protein kinase (AMP-K) can also influence the availability of GSH. AMP-K, like nuclear factor erythroid-derived 2-like 2 (NRF-2), is a "cellular sensor", and responds to metabolic and redox irregularities [63]. AMP-K supports cell survival by upregulating anti-oxidant molecules such as GSH. Elevating serum serine levels in mice fed high fat diet showed increased levels of phosphorylated AMP-K, which resulted in reduced oxidative side-effects and increased GSH expression [63]. AMP-K and NRF-2 are, by no means, the only transcription factors involved in moderating the anti-oxidative response, but they represent the profound integration of serine metabolism and retinal oxidative homeostasis.

NRF-2 is an important redox-activated transcription factor under conditions of stress and imbalance of damaging oxidative species [64]. NRF-2 targets critical anti-oxidant genes such as superoxide dismutase, catalase, and GSH by upregulating transcription. In culture of non-small cell lung cancer cells (NSCLCs), gene enrichment analysis has demonstrated a correlation between PHGDH, the enzyme necessary for shunting 3-phosphoglycerate to serine synthesis, and genes that target the activation of NRF-2 [64]. The correlation suggests that biosynthesis of serine is involved 
in the expression and regulation of essential anti-oxidant proteins [64]. In addition to the cohort of anti-oxidant proteins, NRF-2 also has some regulatory involvement in the bioavailability of nitric oxide (NO) [65], which has the ability to mitigate the effects of $\mathrm{H}_{2} \mathrm{O}_{2}$ and superoxide [66]. Treatment with serine increases NO levels in culture which directly links NO production to serine [65], whether endogenously or exogenously presented.

Oxidative stress can also elicit tremendous damage to membranes, DNA, and mitochondria. However, in many pathologies, oxidative stress and inflammation are intimately interlinked [67]. In particular, AMD and DR have been characterized by the slow infiltration of pro-inflammatory constituents of the innate immune system [68]. Serine is currently being explored as a possible therapy for addressing inflammation in these pathologies. However, the involvement of serine metabolism in inflammation and innate immunity remains unclear [69].

Interesting observations have demonstrated a contradictory picture. Exogenously administered serine has been shown to reduce levels of inflammatory cytokines such as interleukin- $1 \beta$ and interleukin-6 [63]. Serine has also been shown to weaken the pro-inflammatory response necessary for macrophage recruitment after bacterial infections in mice [70]. The conflict emanates from the upregulation of inflammatory elements by way of increased glycolytic flux. In DR and AMD, dietary mismanagement and genetic mutations lead to metabolic dysregulation [71-74], which may increase glycolysis in cells. This may lead to increased synthesis of serine since it is produced from the PHGDH shunt. The increase in serine glycolytic synthesis is linked to the amplified activation of toll-like receptor 4 (TLR-4) after activation by lipopolysaccharide (LPS) [69] or hydrogen peroxide [75]. TLR-4 then induces a cytokine response, namely interleukin-1 $\beta$ [69]. Furthermore, GSH, whose activity and expression is upregulated by stress and higher levels of serine, further contributes to cytokine production and maintenance of redox balance in the cellular environment [69].

Serine deprivation was shown to be effective in reducing the activation of TLR-4, and reducing cytokine levels [69]. Activation of TLR-4 has also been implicated in the etiology of AMD [76]. Contrarily, serine supplementation is being explored as a potential therapy. Exogenous supplementation has proven to be effective in many cases reducing oxidative stress and reducing cytokine levels, but endogenous synthesis (as discussed above) augments the inflammatory response [77]. Therefore, balancing the anti-oxidant effects of serine with the pro-inflammatory nature of its endogenous synthesis is something to consider when developing a serine-based therapy for retinopathies in which inflammation contributes to the pathogenesis.

\section{Consequences of Aberrations in Serine Metabolism}

Our exploration has thus far presented the extensive involvement of serine metabolism in retinal homeostasis. Therefore, aberrations to this important keg in the metabolic machinery can negatively influence retinal pathologies. Glycolytic serine synthesis provides the largest contribution to intracellular serine stores, so deficiencies generally result from abnormalities in that synthetic process. For instance, deleterious mutations in PHGDH have been associated with microcephaly, reduced cognition, and psychomotor abnormalities [78]. Neu-Laxova is a fatal congenital disease marked by serious systemic abnormalities and is attributed to homozygous mutations in enzymes involved in serine biosynthesis [1]. This is recapitulated in the $\mathrm{PHGDH}^{-/-}$mouse model, where mice suffer from embryonic lethality [1]. These irregularities can affect every part of the cell, from mitochondrial biosynthesis to oxidative imbalance.

An extensive comparative metabolomics study was performed by Gao et al., analyzing the metabolic and mitochondrial changes of colon cancer cell lines that occur as a result of serine deprivation [79]. Primarily, glycine, serine, threonine, pyrimidine, and sphingolipid pathways were the most significantly affected. Cells exhibited reduced fatty acid synthesis, and reduced TCA intermediates, consequently cells had a $45 \%$ reduction in ATP. Serine deprivation also caused major changes in mitochondrial membrane potential and increased fragmentation. However, phospholipid and phosphatidylserine levels remained comparably similar to standard culture conditions; sphingosine and 
ceramide levels on the other hand were significantly lowered [79]. It was concluded that mitochondrial fragmentation was exacerbated by reduced production of ceramides that serve as major constituents of the mitochondrial membrane. Ultimately, Gao et al. was able to isolate effected pathways that attenuated proper mitochondrial functions critical for cellular viability [79]. Considering the metabolic demands in the retina, such deficits would be extremely detrimental and could play a significant role in the etiology of many retinal diseases associated with metabolic dysregulation.

Cytotoxic aggregation of deoxysphingolipids is another important feature of serine deficiency [78]. As plasma serine levels decline, alanine flux increases and promotes the production of deoxysphingolipids [78]. As previously indicated, serine sphingolipids are synthesized through the condensation of serine and palmitoyl-CoA mediated by SPT. In cases of serine deficiency, SPT may incorporate alanine or glycine into the formation of the lipid, forming a cytotoxic analogue [78]. These lipids have been shown to aggregate and induce apoptosis in in vitro and in vivo models [78]. Retinal organoids treated with deoxysphingolipids exhibited dose-dependent apoptosis [9].

\section{Retinal Degeneration and Dysregulated Serine Metabolism}

\subsection{Inherited Retinal Degeneration}

Inherited retinal degeneration (IRD) can arise from mutations in various genes linked to photoreceptor development and structure or genes involved in phototransduction. To add to the complexity of IRDs, patients with similar mutations may present with very different phenotypes [80]. This is indicative of other underlying factors that can contribute to disease manifestation. It has been shown that metabolic dysregulation is a common element of various IRD models [81]. One example that has recently emerged is the dysregulation of sphingolipid metabolism [3]. Many investigators have shown that ceramide toxicity is elevated in various models of IRD [22]. In contrast, levels of the protective sphingolipid, sphingosine-1-phosphate, are significantly reduced [22]. What is yet to be determined is why and how the toxic ceramides are increased while sphingosine-1-phosphate levels diminish. One mechanism that has been postulated suggests that serine deficiency forces the sphingolipid metabolism to switch to the incorporation of alanine for sphingolipid synthesis [22]. However, in doing so, the reaction is skewed towards the formation of ceramides. Further investigation is required to determine why there is reduced serine availability. Since the glycolytic precursor, phosphoglycerate, is the primary source for serine biosynthesis, it is possible that dysregulation in glucose metabolism is the cause for the reduced serine availability.

\subsection{Diabetic Retinopathy}

Studies have demonstrated a correlation between serine deficiency and systemic diabetes [77]. Inflammation potentially increases the expression and activity of SRR, and consequently increases the availability of D-serine for receptor binding [40]. The elevated levels of D-serine contribute to glutamate toxicity and induce RGC apoptosis [40]. More precisely, as more D-serine binds to NMDARs, an excitotoxic response is induced [40]. As part of glutamate signal propagation in the retina, NMDAR excitation may be involved in decreasing neurotransmitter sensitivity [40]. Nevertheless, when D-serine levels are substantially elevated, the NMDAR-mediated excitation has a toxic effect in the retinal environment and induces cell death in RGCs [40]. In SRR null models with streptozocin (STZ) induced DR, degeneration of RGCs appeared attenuated, there was a reduction in neovascularization and the retina appeared significantly healthier than the control [40]. Most interestingly, multiple studies have found that if diabetic patients are treated with a serine supplement, blood glucose levels were reduced $[77,82]$. Currently, no mechanism is known to explain how this happens. But it is known that upon onset of diabetes, similar to serine deficiency, toxic sphingolipid accumulation starts occurring [83]. In fact, deoxysphingolipids have been shown to be potent biomarkers for diabetes mellitus [84]. As in IRD, DR results in similar toxic build-up of these aberrant sphingolipids, but this occurs far before any retinopathic complications start to develop [25]. These ceramides can 
actually be used as an early indicator of disease. It appears as if serine deprivation and toxic ceramide accumulation occur concomitantly. Perhaps, as the retina relies most heavily on serine, any deficiencies may cause the tissue to exhibit the first signs of disease in DR.

\section{Novel Insights: Macular Telangiectasia}

It has been elucidated recently that a dysregulated lipid metabolism can lead to increased and leaky vasculature [16]. Given the fact that serine homeostasis closely regulates lipid metabolism, it becomes a prime candidate for such monitoring. Interestingly, it was found in patients suffering from Mac Tel that their PHGDH activity is also compromised [85]. This led to severe serine deficiency, which contributed to vascular abnormalities [9]. As explained earlier, serine deficiency further leads to toxic deoxysphingolipid accumulation, which indeed plays a role in the etiology of Mac Tel type 2 [86]. However, administering exogenous serine to patients as a promising therapeutic approach has been marginally successful $[87,88]$. Importantly, how serine deficiency may affect the RPE and whether that has a role in Mac Tel is not yet known. Nevertheless, it is to be determined whether the restoration of physiological levels of serine in the retina can prevent further neovascularization in Mac Tel patients.

\section{Concluding Remarks}

Serine metabolism has vast interconnectivity with many of the homeostatic mechanisms that work in concert to maintain retinal health and function, as depicted in Figure 3. As reviewed above, considerable evidence has indicated that both the RPE and Müller cells of the inner retina have the requisite enzymes for serine biosynthesis. Besides the significant contribution of these two tissues to the serine pool of the entire retina, we also established the role de novo serine synthesis plays in redox currency generation and in mitigating free radical stress for both the neural retina and RPE. Given the retina is a metabolically high functioning tissue with high-energy demands and recent advancements indicating extra-mitochondrial contribution to the elevated presence of free radicals compared to other tissues, it calls for such extensive measures. Thus we show that while the pentose phosphate pathway might be sufficient to maintain the redox balance for other tissues, the retina depends upon additional tools, which it obtains primarily from serine metabolism: like NADPH from de novo biosynthesis, glycine, GSH and NADH. In addition, a review of the literature on serine-based lipid derivatives like phosphatidylserine, sphingolipids, and their toxic form, i.e., ceramides, helped us conclude the essential role these play in both RPE phagocytosis and membrane integrity in retinal homeostasis, while their imbalance is a critical factor in inherited retinal dystrophies. Coupled with the above observations and the recent advance on using D-serine as therapeutic candidate for diabetic retinopathy, we further postulate that there are a multitude of potential therapies targeting serine metabolism that hold tremendous promise against retinal diseases. We highlighted the prospect of using PHGDH replacement gene therapy or serine supplement therapy for Mac Tel patients and serine racemase and ceramide synthase inhibition for DR patients. Careful evaluation of recent literature also helped us align with the growing consensus that metabolic vulnerabilities add an extra layer of susceptibility for IRD patients. Thus, boosting serine biosynthesis and serine metabolism by pharmacological activators or complimentary gene therapy in these patients may reduce this risk factor and help postpone their onset of degeneration. Consequently, and in this review, our goal was to delineate some of the important roles of serine and demonstrate how they are imperative to the health of the retina. By providing a comprehensive view of the relationship between serine and retinal health, we hope to bring more awareness to the importance of serine to the retina so it can be further assessed for treatment options, and proteins that mediate its metabolic processes can be considered as viable targets for gene therapy. 


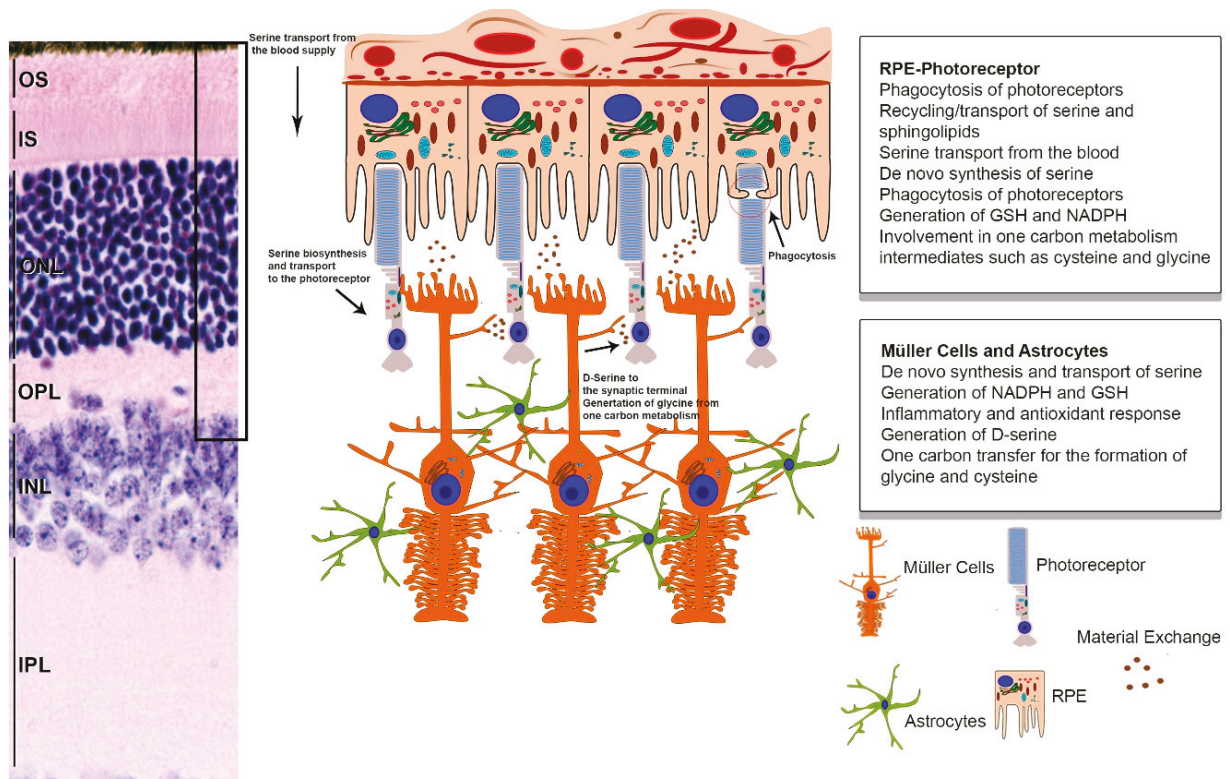

Figure 3. Graphical summary of serine metabolism in the retina. Serine homeostasis is primarily maintained by the RPE and retinal glia. The RPE transports and generates serine, which is ultimately transported to the photoreceptors. Additionally, important serine metabolic products such as glycine and cysteine are transported or catabolized to be used as fuel, as the energetic requirements of the RPE are very high. Photoreceptors also receive serine from the Müller glia and astrocytes. Glial cells are vital to the macula and generate serine from glycolysis, which is crucial in maintaining the redox balance in the photoreceptors, controlling neurotransmission, and mediating inflammation response elements. (IS, inner segment; OS, outer segment; ONL, outer nuclear layer; OPL, outer plexiform layer; INL, inner nuclear layer; IPL, inner plexiform layer).

Funding: This study was supported by a grant from the National Eye Institute (EY026499) to MIN and MRA.

Conflicts of Interest: The authors declare no conflict of interest.

\section{References}

1. El-Hattab, A.W. Serine biosynthesis and transport defects. Mol. Genet. Metab. 2016, 118, 153-159. [CrossRef] [PubMed]

2. Kalhan, S.C.; Hanson, R.W. Resurgence of serine: An often neglected but indispensable amino Acid. J. Biol. Chem. 2012, 287, 19786-19791. [CrossRef] [PubMed]

3. Simon, M.V.; Spalm, F.H.P.; Vera, M.S.; Rotstein, N.P. Sphingolipids as Emerging Mediators in Retina Degeneration. Front. Cell Neurosci. 2019, 13. [CrossRef] [PubMed]

4. Metcalf, J.S.; Dunlop, R.A.; Powell, J.T.; Banack, S.A.; Cox, P.A. L-Serine: A Naturally-Occurring Amino Acid with Therapeutic Potential. Neurotox. Res. 2018, 33, 213-221. [CrossRef]

5. Mattaini, K.R.; Sullivan, M.R.; Vander Heiden, M.G. The importance of serine metabolism in cancer. J. Cell Biol. 2016, 214, 249-257. [CrossRef]

6. Amelio, I.; Cutruzzolá, F.; Antonov, A.; Agostini, M.; Melino, G. Serine and glycine metabolism in cancer. Trends Biochem. Sci. 2014, 39, 191-198. [CrossRef]

7. Ripps, H.; Shen, W. Review: Taurine: A "very essential" amino acid. Mol. Vis. 2012, 18, 2673-2686.

8. Locasale, J.W. Serine, glycine and one-carbon units: Cancer metabolism in full circle. Nat. Rev. Cancer 2013, 13, 572-583. [CrossRef] 
9. Gantner, M.L.; Eade, K.; Wallace, M.; Handzlik, M.K.; Fallon, R.; Trombley, J.; Bonelli, R.; Giles, S.; Harkins-Perry, S.; Heeren, T.F.C.; et al. Serine and Lipid Metabolism in Macular Disease and Peripheral Neuropathy. N. Engl. J. Med. 2019, 381, 1422-1433. [CrossRef]

10. Zhang, T.; Zhu, L.; Madigan, M.C.; Liu, W.; Shen, W.; Cherepanoff, S.; Zhou, F.; Zeng, S.; Du, J.; Gillies, M.C. Human macular Müller cells rely more on serine biosynthesis to combat oxidative stress than those from the periphery. eLife 2019, 8, e43598. [CrossRef]

11. Ye, J.; Fan, J.; Venneti, S.; Wan, Y.-W.; Pawel, B.R.; Zhang, J.; Finley, L.W.S.; Lu, C.; Lindsten, T.; Cross, J.R.; et al. Serine Catabolism Regulates Mitochondrial Redox Control during Hypoxia. Cancer Discovery 2014, 4 , 1406-1417. [CrossRef]

12. de Koning, T.J.; Snell, K.; Duran, M.; Berger, R.; Poll-The, B.-T.; Surtees, R. L-serine in disease and development. Biochem. J. 2003, 371, 653-661. [CrossRef]

13. Stevens, E.R.; Esguerra, M.; Kim, P.M.; Newman, E.A.; Snyder, S.H.; Zahs, K.R.; Miller, R.F. D-serine and serine racemase are present in the vertebrate retina and contribute to the physiological activation of NMDA receptors. Proc. Natl. Acad. Sci. USA 2003, 100, 6789-6794. [CrossRef]

14. Chao, J.R.; Knight, K.; Engel, A.L.; Jankowski, C.; Wang, Y.; Manson, M.A.; Gu, H.; Djukovic, D.; Raftery, D.; Hurley, J.B.; et al. Human retinal pigment epithelial cells prefer proline as a nutrient and transport metabolic intermediates to the retinal side. J. Biol. Chem. 2017, 292, 12895-12905. [CrossRef]

15. Yam, M.; Engel, A.L.; Wang, Y.; Zhu, S.; Hauer, A.; Zhang, R.; Lohner, D.; Huang, J.; Dinterman, M.; Zhao, C.; et al. Proline mediates metabolic communication between retinal pigment epithelial cells and the retina. J. Biol. Chem. 2019, 294, 10278-10289. [CrossRef]

16. Joyal, J.-S.; Gantner, M.L.; Smith, L.E.H. Retinal energy demands control vascular supply of the retina in development and disease: The role of neuronal lipid and glucose metabolism. Prog. Retin Eye Res. 2018, 64, 131-156. [CrossRef]

17. Zhang, T.; Gillies, M.C.; Madigan, M.C.; Shen, W.; Du, J.; Grünert, U.; Zhou, F.; Yam, M.; Zhu, L. Disruption of De Novo Serine Synthesis in Müller Cells Induced Mitochondrial Dysfunction and Aggravated Oxidative Damage. Mol. Neurobiol. 2018, 55, 7025-7037. [CrossRef]

18. Poitry, S.; Poitry-Yamate, C.; Ueberfeld, J.; MacLeish, P.R.; Tsacopoulos, M. Mechanisms of Glutamate Metabolic Signaling in Retinal Glial (Müller) Cells. J. Neurosci. 2000, 20, 1809-1821. [CrossRef]

19. Curcio, C.A.; Allen, K.A. Topography of ganglion cells in human retina. J. Comp. Neurol. 1990, 300, 5-25. [CrossRef]

20. Gault, C.R.; Obeid, L.M.; Hannun, Y.A. An overview of sphingolipid metabolism: From synthesis to breakdown. Adv. Exp. Med. Biol. 2010, 688, 1-23. [CrossRef]

21. Merrill, A.H., Jr.; Schmelz, E.M.; Dillehay, D.L.; Spiegel, S.; Shayman, J.A.; Schroeder, J.J.; Riley, R.T.; Voss, K.A.; Wang, E. Sphingolipids-the enigmatic lipid class: Biochemistry, physiology, and pathophysiology. Toxicol. Appl. Pharmacol. 1997, 142, 208-225. [CrossRef]

22. Rotstein, N.P.; Miranda, G.E.; Abrahan, C.E.; German, O.L. Regulating survival and development in the retina: Key roles for simple sphingolipids. J. Lipid Res. 2010, 51, 1247-1262. [CrossRef]

23. Brush, R.S.; Tran, J.T.A.; Henry, K.R.; McClellan, M.E.; Elliott, M.H.; Mandal, M.N.A. Retinal Sphingolipids and Their Very-Long-Chain Fatty Acid-Containing Species. Invest. Ophth. Vis. Sci. 2010, 51, 4422-4431. [CrossRef]

24. Stiles, M.; Qi, H.; Sun, E.; Tan, J.; Porter, H.; Allegood, J.; Chalfant, C.E.; Yasumura, D.; Matthes, M.T.; LaVail, M.M.; et al. Sphingolipid profile alters in retinal dystrophic P23H-1 rats and systemic FTY720 can delay retinal degeneration. J. Lipid Res. 2016, 57, 818-831. [CrossRef]

25. Fox, T.E.; Han, X.; Kelly, S.; Merrill, A.H., 2nd; Martin, R.E.; Anderson, R.E.; Gardner, T.W.; Kester, M. Diabetes alters sphingolipid metabolism in the retina: A potential mechanism of cell death in diabetic retinopathy. Diabetes 2006, 55, 3573-3580. [CrossRef]

26. Kevany, B.M.; Palczewski, K. Phagocytosis of retinal rod and cone photoreceptors. Physiology 2010, 25, 8-15. [CrossRef]

27. Deigner, P.S.; Law, W.C.; Canada, F.J.; Rando, R.R. Membranes as the energy source in the endergonic transformation of vitamin A to 11-cis-retinol. Science 1989, 244, 968-971. [CrossRef]

28. Rando, R.R. Membrane phospholipids as an energy source in the operation of the visual cycle. Biochemistry 1991, 30, 595-602. [CrossRef] 
29. Rando, R.R. Membrane phospholipids and the dark side of vision. J. Bioenerg. Biomembr. 1991, 23, $133-146$. [CrossRef]

30. Sparrow, J.R.; Wu, Y.; Kim, C.Y.; Zhou, J. Phospholipid meets all-trans-retinal: The making of RPE bisretinoids. J. Lipid Res. 2010, 51, 247-261. [CrossRef]

31. Fadok, V.A.; Bratton, D.L.; Frasch, S.C.; Warner, M.L.; Henson, P.M. The role of phosphatidylserine in recognition of apoptotic cells by phagocytes. Cell Death Differ. 1998, 5, 551-562. [CrossRef]

32. Tafesse, F.G.; Rashidfarrokhi, A.; Schmidt, F.I.; Freinkman, E.; Dougan, S.; Dougan, M.; Esteban, A.; Maruyama, T.; Strijbis, K.; Ploegh, H.L. Disruption of Sphingolipid Biosynthesis Blocks Phagocytosis of Candida albicans. PLoS Pathog. 2015, 11, e1005188. [CrossRef]

33. Bryan, A.M.; Del Poeta, M.; Luberto, C. Sphingolipids as Regulators of the Phagocytic Response to Fungal Infections. Mediat. Inflamm. 2015. [CrossRef]

34. Sarantis, H.; Grinstein, S. Monitoring phospholipid dynamics during phagocytosis: Application of genetically-encoded fluorescent probes. Methods Cell Biol. 2012, 108, 429-444. [CrossRef]

35. Regina Todeschini, A.; Hakomori, S.I. Functional role of glycosphingolipids and gangliosides in control of cell adhesion, motility, and growth, through glycosynaptic microdomains. Biochim. Biophys. Acta 2008, 1780, 421-433. [CrossRef]

36. Hoekstra, D.; Maier, O.; van der Wouden, J.M.; Slimane, T.A.; van IJzendoorn, S.C. Membrane dynamics and cell polarity: The role of sphingolipids. J. Lipid Res. 2003, 44, 869-877. [CrossRef]

37. MacKay, M.-A.B.; Kravtsenyuk, M.; Thomas, R.; Mitchell, N.D.; Dursun, S.M.; Baker, G.B. D-Serine: Potential Therapeutic Agent and/or Biomarker in Schizophrenia and Depression? Front. Psychiatry 2019, 10. [CrossRef]

38. Kleckner, N.W.; Dingledine, R. Requirement for glycine in activation of NMDA-receptors expressed in Xenopus oocytes. Science 1988, 241, 835-837. [CrossRef]

39. Fletcher, E.L.; Hack, I.; Brandstätter, J.H.; Wässle, H. Synaptic localization of NMDA receptor subunits in the rat retina. J. Comp. Neurol. 2000, 420, 98-112. [CrossRef]

40. Ozaki, H.; Inoue, R.; Matsushima, T.; Sasahara, M.; Hayashi, A.; Mori, H. Serine racemase deletion attenuates neurodegeneration and microvascular damage in diabetic retinopathy. PLoS ONE 2018, 13, e0190864. [CrossRef]

41. Maddocks, O.D.K.; Labuschagne, C.F.; Adams, P.D.; Vousden, K.H. Serine Metabolism Supports the Methionine Cycle and DNA/RNA Methylation through De Novo ATP Synthesis in Cancer Cells. Mol. Cell 2016, 61, 210-221. [CrossRef]

42. Botsford, J.; Parks, L. Serine transhydroxymethylase in methionine biosynthesis in Saccharomyces cerevisiae. J. Bacterial. 1969, 97, 1176-1183. [CrossRef]

43. Stauffer, G.V.; Brenchley, J.E. Influence of methionine biosynthesis on serine transhydroxymethylase regulation in Salmonella typhimurium LT2. J. Bacterial. 1977, 129, 740-749. [CrossRef]

44. Lombardini, J.B.; Talalay, P. Formation, functions and regulatory importance of S-adenosyl-l-methionine. Adv. Enzyme Regul. 1971, 9, 349-384. [CrossRef]

45. Grundy, F.J.; Henkin, T.M. Synthesis of Serine, Glycine, Cysteine, and Methionine. In Bacillus subtilis and Its Closest Relatives; Sonenshein, A., Losick, R., Hoch, J., Eds.; ASM Press: Washington, DC, USA, 2002; pp. 245-254. [CrossRef]

46. Jobe, E.M.; Zhao, X. DNA Methylation and Adult Neurogenesis. Brain Plast. 2017, 3, 5-26. [CrossRef] [PubMed]

47. Kowluru, R.A.; Shan, Y.; Mishra, M. Dynamic DNA methylation of matrix metalloproteinase-9 in the development of diabetic retinopathy. Lab. Invest. 2016, 96, 1040-1049. [CrossRef]

48. Zeng, J.-D.; Wu, W.K.K.; Wang, H.-Y.; Li, X.-X. Serine and one-carbon metabolism, a bridge that links mTOR signaling and DNA methylation in cancer. Pharmacol. Res. 2019, 149, 104352. [CrossRef]

49. Li, W.; Liu, J.; Galvin, J.A. Epigenetics and Common Ophthalmic Diseases. Yale J. Biol. Med. 2016, 89, 597-600.

50. Liu, M.M.; Chan, C.C.; Tuo, J. Genetic mechanisms and age-related macular degeneration: Common variants, rare variants, copy number variations, epigenetics, and mitochondrial genetics. Hum. Genomics 2012, 6, 13. [CrossRef]

51. Ng, S.K.; Wood, J.P.M.; Chidlow, G.; Han, G.; Kittipassorn, T.; Peet, D.J.; Casson, R.J. Cancer-like metabolism of the mammalian retina. Clin. Exp. Ophthalmol. 2015, 43, 367-376. [CrossRef] 
52. Panfoli, I.; Calzia, D.; Ravera, S.; Bruschi, M.; Tacchetti, C.; Candiani, S.; Morelli, A.; Candiano, G. Extramitochondrial tricarboxylic acid cycle in retinal rod outer segments. Biochimie 2011, 93, 1565-1575. [CrossRef] [PubMed]

53. Calzia, D.; Panfoli, I.; Heinig, N.; Schumann, U.; Ader, M.; Traverso, C.E.; Funk, R.H.W.; Roehlecke, C. Impairment of extramitochondrial oxidative phosphorylation in mouse rod outer segments by blue light irradiation. Biochimie 2016, 125, 171-178. [CrossRef] [PubMed]

54. Alder, V.A.; Ben-Nun, J.; Cringle, S.J. PO2 profiles and oxygen consumption in cat retina with an occluded retinal circulation. Invest. Ophthalmol. Vis. Sci. 1990, 31, 1029-1034. [PubMed]

55. McHugh, K.J.; Li, D.A.; Wang, J.C.; Kwark, L.; Loo, J.; Macha, V.; Farsiu, S.; Kim, L.A.; Saint-Geniez, M. Computational modeling of retinal hypoxia and photoreceptor degeneration in patients with age-related macular degeneration. PloS ONE 2019, 14, e0216215. [CrossRef]

56. Roehlecke, C.; Schumann, U.; Ader, M.; Brunssen, C.; Bramke, S.; Morawietz, H.; Funk, R.H.W. Stress Reaction in Outer Segments of Photoreceptors after Blue Light Irradiation. PloS ONE 2013, 8, e71570. [CrossRef]

57. Roehlecke, C.; Schumann, U.; Ader, M.; Knels, L.; Funk, R.H.W. Influence of blue light on photoreceptors in a live retinal explant system. Mol. Vis. 2011, 17, 876-884.

58. Calzia, D.; Oneto, M.; Caicci, F.; Bianchini, P.; Ravera, S.; Bartolucci, M.; Diaspro, A.; Degan, P.; Manni, L.; Traverso, C.E.; et al. Effect of polyphenolic phytochemicals on ectopic oxidative phosphorylation in rod outer segments of bovine retina. Brit. J. Pharmacol. 2015, 172, 3890-3903. [CrossRef]

59. Panieri, E.; Santoro, M.M. ROS homeostasis and metabolism: A dangerous liason in cancer cells. Cell Death Dis. 2016, 7, e2253. [CrossRef]

60. Vučetić, M.; Cormerais, Y.; Parks, S.K.; Pouysségur, J. The Central Role of Amino Acids in Cancer Redox Homeostasis: Vulnerability Points of the Cancer Redox Code. Front. Oncol. 2017, 7, 319. [CrossRef]

61. Dringen, R.; Pfeiffer, B.; Hamprecht, B. Synthesis of the antioxidant glutathione in neurons: Supply by astrocytes of CysGly as precursor for neuronal glutathione. J. Neurosci. 1999, 19, 562-569. [CrossRef]

62. Winkler, B.S.; Giblin, F.J. Glutathione oxidation in retina: Effects on biochemical and electrical activities. Exp. Eye Res. 1983, 36, 287-297. [CrossRef]

63. Zhou, X.; He, L.; Zuo, S.; Zhang, Y.; Wan, D.; Long, C.; Huang, P.; Wu, X.; Wu, C.; Liu, G.; et al. Serine prevented high-fat diet-induced oxidative stress by activating AMPK and epigenetically modulating the expression of glutathione synthesis-related genes. Biochim. Biophys. Acta, Mol. Basis Dis. 2018, 1864, 488-498. [CrossRef] [PubMed]

64. DeNicola, G.M.; Chen, P.-H.; Mullarky, E.; Sudderth, J.A.; Hu, Z.; Wu, D.; Tang, H.; Xie, Y.; Asara, J.M.; Huffman, K.E.; et al. NRF2 regulates serine biosynthesis in non-small cell lung cancer. Nature Genet. 2015, 47, 1475-1481. [CrossRef] [PubMed]

65. Maralani, M.N.; Movahedian, A.; Javanmard, S.H. Antioxidant and cytoprotective effects of L-Serine on human endothelial cells. Res. Pharm. Sci. 2012, 7, 209-215.

66. Wink, D.A.; Miranda, K.M.; Espey, M.G. Cytotoxicity related to oxidative and nitrosative stress by nitric oxide. Exp. Biol. Med. 2001, 226, 621-623. [CrossRef]

67. Khansari, N.; Shakiba, Y.; Mahmoudi, M. Chronic Inflammation and Oxidative Stress as a Major Cause of Age- Related Diseases and Cancer. Recent Pat. Inflamm. Allergy Drug Discov. 2009, 3, 73-80. [CrossRef]

68. Perez, V.L.; Caspi, R.R. Immune mechanisms in inflammatory and degenerative eye disease. Trends Immunol. 2015, 36, 354-363. [CrossRef]

69. Rodriguez, A.E.; Ducker, G.S.; Billingham, L.K.; Martinez, C.A.; Mainolfi, N.; Suri, V.; Friedman, A.; Manfredi, M.G.; Weinberg, S.E.; Rabinowitz, J.D.; et al. Serine Metabolism Supports Macrophage IL-1 $\beta$ Production. Cell Metab. 2019, 29, 1003-1011. [CrossRef]

70. He, F.; Yin, Z.; Wu, C.; Xia, Y.; Wu, M.; Li, P.; Zhang, H.; Yin, Y.; Li, N.; Zhu, G.; et al. l-Serine Lowers the Inflammatory Responses during Pasteurella multocida Infection. Infect. Immun. 2019, 87. [CrossRef]

71. Dornan, T.L.; Ting, A.; McPherson, C.K.; Peckar, C.O.; Mann, J.I.; Turner, R.C.; Morris, P.J. Genetic susceptibility to the development of retinopathy in insulin-dependent diabetics. Diabetes 1982, 31, 226-231. [CrossRef]

72. Zarbin, M.A. Current concepts in the pathogenesis of age-related macular degeneration. Arch. Ophthalmol. 2004, 122, 598-614. [CrossRef] [PubMed]

73. Lambert, N.G.; ElShelmani, H.; Singh, M.K.; Mansergh, F.C.; Wride, M.A.; Padilla, M.; Keegan, D.; Hogg, R.E.; Ambati, B.K. Risk factors and biomarkers of age-related macular degeneration. Prog. Retin Eye Res. 2016, 54, 64-102. [CrossRef] [PubMed] 
74. Wong, M.Y.Z.; Man, R.E.K.; Fenwick, E.K.; Gupta, P.; Li, L.J.; van Dam, R.M.; Chong, M.F.; Lamoureux, E.L. Dietary intake and diabetic retinopathy: A systematic review. PLoS ONE 2018, 13, e0186582. [CrossRef] [PubMed]

75. Yin, Q.; Jiang, D.; Li, L.; Yang, Y.; Wu, P.; Luo, Y.; Yang, R.; Li, D. LPS Promotes Vascular Smooth Muscle Cells Proliferation Through the TLR4/Rac1/Akt Signalling Pathway. Cell Physiol. Biochem. 2017, 44, 2189-2200. [CrossRef]

76. Yi, H.; Patel, A.K.; Sodhi, C.P.; Hackam, D.J.; Hackam, A.S. Novel role for the innate immune receptor Toll-like receptor 4 (TLR4) in the regulation of the Wnt signaling pathway and photoreceptor apoptosis. PLoS ONE 2012, 7, e36560. [CrossRef]

77. Holm, L.J.; Buschard, K. L-serine: A neglected amino acid with a potential therapeutic role in diabetes. APMIS 2019, 127, 655-659. [CrossRef]

78. Esaki, K.; Sayano, T.; Sonoda, C.; Akagi, T.; Suzuki, T.; Ogawa, T.; Okamoto, M.; Yoshikawa, T.; Hirabayashi, Y.; Furuya, S. L-Serine Deficiency Elicits Intracellular Accumulation of Cytotoxic Deoxysphingolipids and Lipid Body Formation. J. Biol. Chem. 2015, 290, 14595-14609. [CrossRef]

79. Gao, X.; Lee, K.; Reid, M.A.; Sanderson, S.M.; Qiu, C.; Li, S.; Liu, J.; Locasale, J.W. Serine Availability Influences Mitochondrial Dynamics and Function through Lipid Metabolism. Cell Rep. 2018, 22, 3507-3520. [CrossRef]

80. Duncan, J.L.; Pierce, E.A.; Laster, A.M.; Daiger, S.P.; Birch, D.G.; Ash, J.D.; Iannaccone, A.; Flannery, J.G.; Sahel, J.A.; Zack, D.J.; et al. Inherited Retinal Degenerations: Current Landscape and Knowledge Gaps. Transl. Vis. Sci. Techn. 2018, 7. [CrossRef]

81. Punzo, C.; Xiong, W.; Cepko, C.L. Loss of daylight vision in retinal degeneration: Are oxidative stress and metabolic dysregulation to blame? J. Biol. Chem. 2012, 287, 1642-1648. [CrossRef]

82. Holm, L.J.; Haupt-Jorgensen, M.; Larsen, J.; Giacobini, J.D.; Bilgin, M.; Buschard, K. L-serine supplementation lowers diabetes incidence and improves blood glucose homeostasis in NOD mice. PLoS ONE 2018, 13, e0194414. [CrossRef]

83. Ross, J.S.; Russo, S.B.; Chavis, G.C.; Cowart, L.A. Sphingolipid regulators of cellular dysfunction in Type 2 diabetes mellitus: A systems overview. Clin. Lipidol. 2014, 9, 553-569. [CrossRef] [PubMed]

84. Bertea, M.; Rutti, M.F.; Othman, A.; Marti-Jaun, J.; Hersberger, M.; von Eckardstein, A.; Hornemann, T. Deoxysphingoid bases as plasma markers in diabetes mellitus. Lipids Health Dis. 2010, 9, 84. [CrossRef] [PubMed]

85. Scerri, T.S.; Quaglieri, A.; Cai, C.; Zernant, J.; Matsunami, N.; Baird, L.; Scheppke, L.; Bonelli, R.; Yannuzzi, L.A.; Friedlander, M.; et al. Genome-wide analyses identify common variants associated with macular telangiectasia type 2. Nat. Genet. 2017, 49, 559-567. [CrossRef] [PubMed]

86. Wang, L.; Miao, H.; Li, X. Tamoxifen retinopathy: A case report. Springerplus 2015, 4, 501. [CrossRef] [PubMed]

87. Othman, A.; Benghozi, R.; Alecu, I.; Wei, Y.; Niesor, E.; von Eckardstein, A.; Hornemann, T. Fenofibrate lowers atypical sphingolipids in plasma of dyslipidemic patients: A novel approach for treating diabetic neuropathy? J. Clin. Lipidol. 2015, 9, 568-575. [CrossRef]

88. Garofalo, K.; Penno, A.; Schmidt, B.P.; Lee, H.J.; Frosch, M.P.; von Eckardstein, A.; Brown, R.H.; Hornemann, T.; Eichler, F.S. Oral L-serine supplementation reduces production of neurotoxic deoxysphingolipids in mice and humans with hereditary sensory autonomic neuropathy type 1. J. Clin. Investig. 2011, 121, 4735-4745. [CrossRef] 



\title{
Review \\ Effects of Mitochondrial-Derived Peptides (MDPs) on Mitochondrial and Cellular Health in AMD
}

\author{
Sonali Nashine ${ }^{1}$ and M. Cristina Kenney ${ }^{1,2, *}$ \\ 1 Department of Ophthalmology, Gavin Herbert Eye Institute, University of California Irvine, Irvine, \\ CA 92697, USA; snashine@uci.edu \\ 2 Department of Pathology and Laboratory Medicine, University of California Irvine, Irvine, CA 92697, USA \\ * Correspondence: mkenney@uci.edu
}

Received: 2 February 2020; Accepted: 23 April 2020; Published: 29 April 2020

\begin{abstract}
Substantive evidence demonstrates the contribution of mitochondrial dysfunction in the etiology and pathogenesis of Age-related Macular Degeneration (AMD). Recently, extensive characterization of Mitochondrial-Derived Peptides (MDPs) has revealed their cytoprotective role in several diseases, including AMD. Here we summarize the varied effects of MDPs on cellular and mitochondrial health, which establish the merit of MDPs as therapeutic targets for AMD. We argue that further research to delve into the mechanisms of action and delivery of MDPs may advance the field of AMD therapy.
\end{abstract}

Keywords: MDPs; mitochondrial-derived peptides; Humanin; HNG; SHLPs; MOTS-c; Age-related Macular Degeneration (AMD); neuroprotection; RPE; mitochondria

\section{Introduction}

In the United States, geographic atrophy in dry AMD (Age-related Macular Degeneration) is a primary cause of vision loss in the elderly [1], and it has limited treatment options compared to those available for wet AMD $[2,3]$. Among the wide variety of factors that are instrumental in the etiology and pathogenesis of AMD, mitochondrial damage in the Retinal Pigment Epithelium (RPE), leading to RPE dysfunction, contributes significantly. AMD mitochondria are fragmented, have a higher number of lesions, altered ATP synthase activity, as well as compromised protein expression and nuclear-encoded protein import [4-6]. Furthermore, as confirmed by ATAC-sequencing, chromatin accessibility is decreased significantly in the RPE in AMD retinas [7].

The human mitochondrial genome is $~ 16.5$ kilobases, circular, double-stranded, and consists of 37 genes that code for 13 proteins of the respiratory chain complexes. These 13 proteins are a part of the electron transport chain and aid in oxidative metabolism and ATP production [8,9]. Some of the critical functions of mitochondria include, but are not limited to, ATP production via respiration, promoting thermogenesis via proton leak, regulation of cellular metabolism and calcium signaling, ROS generation, regulation of apoptosis, ion homeostasis, and heme synthesis [10]. The retina is a part of the central nervous system and is one of the highest energy-demanding tissues in the human body. Glycolysis in the cytosol and mitochondrial oxidative phosphorylation are the two primary sources of energy, i.e., ATP (adenosine triphosphate) generation. Retinal neurons derive their energy mostly from oxidative phosphorylation, which has a substantially higher ATP yield than glycolysis [11]. Unmet retinal energy demand puts the retinal neurons at a high risk of cell death that in turn causes impairment or loss of vision [12]. Mitochondrial function declines with age as a result of accumulated mtDNA damage/mutations [13]. The majority of proteins that support mitochondrial health and function are encoded by the nuclear genome. Therefore, coordinated regulation of mitochondrial and nuclear gene expression is essential to maintain cellular homeostasis [14]. Mammalian mitochondrial retrograde signaling, i.e., transmission of information from the mitochondria to the nucleus, is mediated 
by G-Protein Pathway Suppressor 2 (GPS2), which also functions as a transcriptional activator of nuclear-encoded mitochondrial genes. GPS2-regulated direct translocation from the mitochondria to the nucleus is essential for the transcriptional activation of a nuclear stress response to mitochondrial depolarization and for supporting basal mitochondrial biogenesis [15].

The mitochondrial genome encodes 22 tRNAs and two ribosomal RNAs, i.e., $12 \mathrm{~S}$ rRNA and $16 \mathrm{~S}$ rRNA, both of which are essential in synthesis of mitochondrial proteins. The 12S rRNA gene is 954 base pairs (bp) in length and spans 648-1601 bp of the mtDNA; it occupies 1/16 of the entire mtDNA and has 297 nucleotide substitutions, which account for $31 \%$ of the $12 \mathrm{~S}$ rRNA gene. Furthermore, the $16 \mathrm{~S}$ rRNA gene is $1559 \mathrm{bp}$ long, spanning 1671-3229 bp of the mtDNA; it occupies 1/10 of the entire mtDNA and has 413 nucleotide substitutions, which account for $25 \%$ of the $16 \mathrm{~S}$ rRNA gene [16]. Both $16 \mathrm{~S}$ rRNA and $12 \mathrm{~S}$ rRNA genes carry ORFs (Open Reading Frames) that encode mitochondrial-derived peptides.

\section{Mitochondrial-Derived Peptides (MDPs)}

Novel mitochondrial-derived peptides (MDPs), which are encoded within the mtDNA, serve as signals for organism cytoprotection and energy regulation. The MDPs encoded from the 16S rRNA region of the mtDNA include Humanin and SHLPs, which regulate cell survival and growth via distinct pathways. MOTS-c, encoded from the $12 \mathrm{~S}$ rRNA region of the mtDNA, plays a key role in regulation of muscle and fat metabolism and prevents hepatic steatosis. Numerous MDPs are well-characterized and are in preclinical development for aging-related diseases [17-20].

\section{Humanin}

Humanin was the first MDP discovered within the mammalian mitochondrial genome and is encoded from the $16 \mathrm{~S}$ rRNA coding region of the mtDNA. Humanin cDNA was first discovered in 2001 by functional expression screening of a cDNA library preparation from the occipital cortex of an Alzheimer's disease patient brain [21]. The study identified clones that protected against neuronal cell death induced by neurotoxic amyloid- $\beta$ peptides and by mutants of FAD (Familial Alzheimer's Disease) genes, namely APP (Amyloid Precursor Protein), PS1 (Presenilin 1), and PS2 (Presenilin 2). A $75 \mathrm{bp}$ open reading frame that codes for a 24-residue peptide was identified and its sequence was found to be similar to that of a human cDNA and $99 \%$ identical to the mtDNA sequence; it was therefore named Humanin. Humanin antagonized the cytotoxicity caused by APP (Amyloid Precursor Protein (APP), PS1 (Presenilin 1), and PS2 (Presenilin 2) mutants, by suppressing amyloid- $\beta_{1-42}$-triggered neurotoxicity and attenuating neuronal cell death.

\subsection{Structure}

Humanin is a secretory 24-amino acid peptide with the amino acid sequence H-Met-Ala-Pro-Arg-GlyPhe-Ser-Cys-Leu-Leu-Leu-Leu-Thr-Ser-Glu-Ile-Asp-Leu-Pro-Val-Lys-Arg-Arg-Ala-OH (H-MAPRGFSCL LLLTSEIDLPVKRRA-OH) and a molecular weight of 2687.26 Da (Figure 1). Following its discovery, the structure and biological functions of Humanin have been very well characterized. Using a combination of oligonucleotide synthesis, dimerization experiments, and bioinformatics, Yamagishi et al. conducted a comprehensive investigation that led to the identification of the amino acids essential for Humanin secretion and for its neuroprotective function [22]. This study revealed that Humanin is a signal peptide and the core domain of Humanin is formed by Leu9-Leu10-Leu11 residues with Leu10 being the key player. Leu9-Leu10-Leu11 and Pro19-Val20 are absolutely essential for the extracellular secretion of full-length Humanin. The neuroprotective action of Humanin requires Pro19, Ser14, Thr13, Leu12, Leu9, Cys8, Ser7, and Pro3. Ser7 and Leu9 residues are required for the self-dimerization of Humanin, which is necessary for its neuroprotective function. Providing critical insights into the amino acid requirements of Humanin would be useful in synthesis of peptides tailored to perform a specific biological function and for targeted improvement of particular cellular functions. 


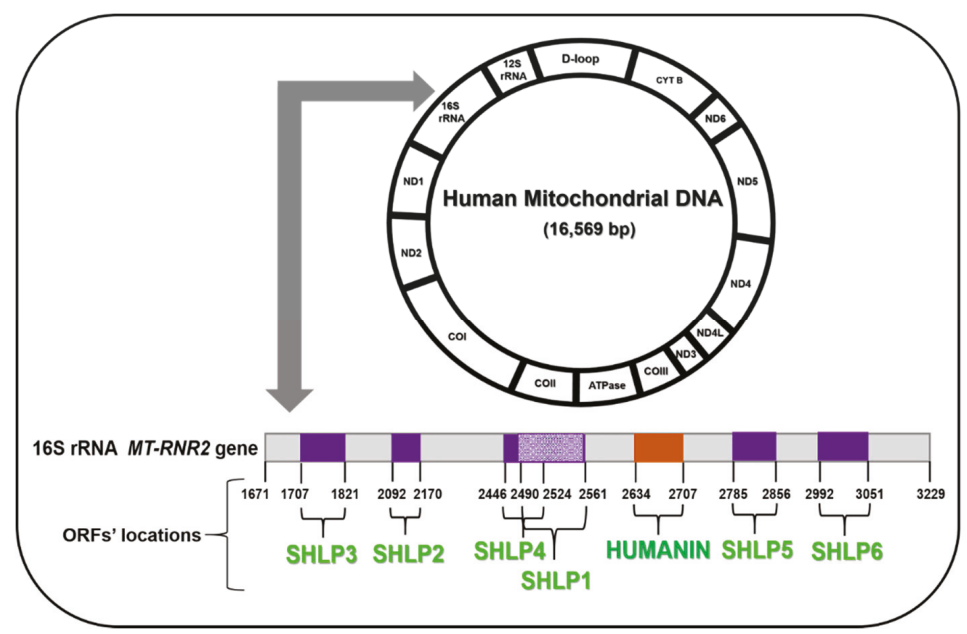

Figure 1. Humanin and Small Human-Like Peptides (SHLPs) Open Reading Frames (ORFs) in the human mitochondrial DNA.

\subsection{Tissue Distribution}

Measurable levels of Humanin are detectable in plasma, seminal fluid, and cerebrospinal fluid. A circulating Humanin pool has been demonstrated in various tissues of the human body, including hypothalamus, liver, heart, kidney, colon, testes, vasculature, and skeletal muscle [23-28]. Circulating plasma levels of Humanin decline with age in both humans and mice [29]. Demonstrating a proportional relationship between Humanin levels and human aging is of particular importance, as this could promote research that aims to boost Humanin levels and thereby delay aging in humans.

\subsection{Humanin Analogs}

Amino acid substitutions in Humanin have led to the development of synthetic Humanin analogs, which are more potent than the endogenous Humanin. Humanin G (HNG) is formed by a Ser to Gly amino acid substitution at position 14. HNGF6A is formed by the Phe to Ala amino acid substitution at position 6 [30].

\subsection{Humanin Receptors and Regulation}

Humanin exerts its cytoprotective effects by binding to receptors intracellularly/extracellularly, regulating the intrinsic mitochondrial pathway, and mediating downstream signaling. Humanin binds to the Formyl-Peptide Receptor-Like Receptor-1 (FPRL-1) and the IL-6 (Interleukin-6) receptor family trimeric receptor complex, comprised of CNTFR $\alpha$ (Ciliary Neurotrophic Factor Receptor $\alpha$ ), WSX-1, and GP130 (glycoprotein 130kDa), which are key contributors to the neuroprotective action of Humanin [31-33]. Substantial evidence suggests that mitochondrial retrograde signaling is involved in the endocrine regulation of aging and age-related pathologies. IGF-1 (Insulin Growth Factor-1) is a key player in the conserved endocrine pathway, which contributes to lifespan and healthspan. Humanin, a potent mediator of mitochondrial retrograde signaling, is directly regulated by IGF-1, and the levels of Humanin and IGF-1 simultaneously decline with age [34]. Humanin and IGF-1 are known to have distinct yet overlapping functions. By interacting with the C-terminal domain of IGFBP-3 (Insulin-like Growth Factor Binding Protein-3), Humanin interferes with the binding of Importin- $\beta$ to IGFBP-3, thereby suppressing IGFBP-3-mediated apoptosis [35]. Humanin regulates peripheral insulin action. In this study, peripherally administered HNGF6A in rats conferred insulin sensitivity via hypothalamic STAT3 (Signal transducer and activator of transcription 3) activation [29]. 


\section{Humanin Functions}

\subsection{Prevents Apoptosis}

Humanin binds to the pro-apoptotic protein BAX and prevents its translocation to the nucleus, thereby antagonizing the apoptotic activity of BAX and inhibiting the release of cytochrome c [36]. This finding reported by Guo et al. in the journal Nature was the first thorough study that delineated and reported the interactions of Humanin with the pro-apoptotic BAX protein. Since Humanin is encoded by the mitochondria, this report also suggested a possible retrograde signaling mechanism between the mitochondria and the nucleus that might be contributing to regulation of BAX-mediated apoptosis.

Humanin also binds and inactivates BAX-like proteins, such as Bid and BidEL [37]. Exogenously added HNG exerts substantive protective effects in transmitochondrial AMD RPE cells in vitro by (a) rescue of mitochondrial structure and function, (b) inhibiting the action of pro-apoptotic genes/proteins, and (c) intracellular and extracellular humanin receptor modulation (Figure 2) [38].

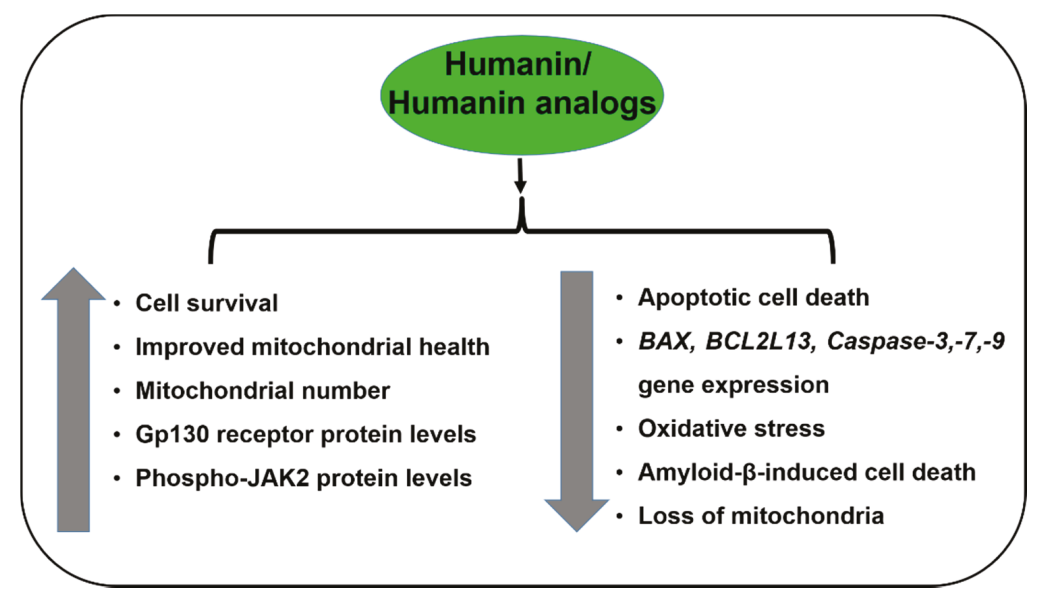

Figure 2. Effects of Humanin/Humanin analogs in RPE/AMD.

Humanin is cytoprotective against amyloid- $\beta$-mediated toxicity in neuronal cells, both in vitro and in vivo [17,18]; against cerebral ischemia and cardiac damage in mouse models [19,20]; and in numerous neurodegenerative disease models for Alzheimer's disease, Parkinson's disease, Huntington's disease, and Prion diseases $[18,19,39,40]$. Moreover, Hinton et al. in a comprehensive study published in the highly reputed IOVS eye journal demonstrated that Humanin rescues primary RPE cells from oxidative damage and subsequent death in vitro [41]. This showed that Humanin conferred RPE cell protection via two mechanisms: by enhancing mitochondrial biogenesis and function and by activation of STAT3. Further, Humanin mediated suppression of oxidative stress-induced cell senescence and maintained transepithelial resistance in human RPE monolayers. In summary, this study by Hinton et al. suggested the potential of Humanin as a therapeutic candidate for the treatment of geographic atrophy in dry AMD.

Each of the 24 amino acids in the Humanin peptide have a specific function. Serine at position 14 confers neuroprotection [22], but its substitution with glycine generates a variant called Humanin G/HNG that is 1000-fold more potent than its parent analog Humanin [16]. HNG is known to protect against cell death by preventing mitochondrial dysfunction [26].

Humanin potentially inhibits silver nanoparticles-induced cell death in human neuroblastoma cells by (a) protecting against redox imbalance and oxidative stress-induced DNA damage, (b) increasing mitochondrial membrane potential and enhancing the activity of mitochondrial succinate 
dehydrogenase, and (c) deactivation of the ER stress pathways that were upregulated by the silver nanoparticles [42].

\subsection{Prevents Amyloid- $\beta$-Induced Toxicity}

Amyloid- $\beta$ is a key component of drusen deposits that are formed in the AMD retinas. Administration of Humanin $G$ reduces amyloid- $\beta$ loads and inhibits amyloid- $\beta$-induced cell apoptosis by (a) restoring amyloid- $\beta$-mediated decline in calcium homeostasis, (b) suppressing amyloid- $\beta$-induced membrane fluidity changes, (c) restoring mitochondrial membrane potential, and (d) decreasing intracellular reactive oxygen species [43]. This finding is crucial as Humanin G's ability to mitigate amyloid- $\beta$-induced cytotoxicity might be used as a therapeutic approach to delay the progression of dry AMD.

\subsection{Stress Resistance Against ER Stress-Induced Apoptosis}

Humanin exerts its therapeutic benefits by antagonizing the action of a plethora of cellular insults, thereby protecting the RPE cells against cytotoxicity. Treatment with Humanin downregulates the expression of an ER stress marker CHOP (C/EBP Homologous Protein), inhibits ROS (Reactive Oxygen Species) production, and regulates intracellular calcium influx, thereby preventing RPE cell apoptosis [44,45]. Treatment of primary human RPE cells with three ER stress sensors, i.e., Tunicamycin, Brefeldin A, and Thapsigargin, induced mitochondrial damage and dose-dependent loss of RPE cells. However, pretreatment with Humanin provided significant cytoprotection against ER stress-induced cell death by restoring the depleted mitochondrial glutathione (GSH) levels to normal, reducing mitochondrial superoxide generation, and downregulating ER stress-induced apoptotic Caspase 4 and Caspase 3 [46].

\subsection{Activation of the ERK, AKT, and STAT3 Signaling Pathways}

As a secretory peptide, Humanin regulates both intracellular and extracellular signaling pathways. Exogenously administered Humanin both in vitro and in vivo rapidly increases AKT-1 phosphorylation and activates the PI3K (Phosphoinositide 3-Kinase)/AKT pathway; AKT-1 is the AKT Serine/Threonine Kinase-1 protein that plays a role in cell motility, metabolism, and proliferation [47]. Moreover, intraperitoneal injection of Humanin in vivo elevates endothelial NOS (Nitric Oxide Synthase) phosphorylation and also increases the phosphorylation of AMPK (5' Adenosine MonophosphateActivated Protein Kinase), a protein which contributes to cellular energy homeostasis [25]. The signaling pathway mediated by Humanin involves its interaction with various molecular entities, including protein kinases, integral membrane receptors, and transcription regulators. In neuronal cell lines, Humanin interacts with and activates the GP130 receptor and mediates its effects via its canonical AKT, ERK1/2 (Extracellular Signal-Regulated Kinase 1/2), and STAT3 signaling cascades. Humanin acts as a primary agonist for the GP130 receptor in neuronal cell lines in vitro, and Humanin treatment transiently activates GP130, thereby mediating cellular protective effects, such as anti-apoptosis, enhanced insulin sensitivity, and protection from hypoxic and ischemic stressors [48].

\subsection{Preserves Endothelial Function in Atherosclerosis}

Oh et al. demonstrated that sustained administration of Humanin G (a more potent analog of Humanin) to ApoE-knockout mice inhibited the progression of atherosclerotic plaques and preserved endothelial function [49].

\subsection{Prevents Vascular Remodeling and Inflammation}

Exogenously added Humanin attenuated angiogenesis, inflammation, apoptosis, and microvascular remodeling in an ApoE-deficient mouse model of atherosclerosis [23]. 


\subsection{Cytoprotective Against LDL-Induced Oxidative Stress}

Bachar et al. demonstrated that Humanin is expressed in the endothelial cell layer of human blood vessels. In human endothelial cells in vitro, exogenous supplementation of Humanin attenuated oxidized (Ox)-LDL-induced ROS generation and apoptotic cell death by $50 \%$ and reduced the levels of cellular ceramide, which is a lipid second messenger that initiates apoptotic signaling cascades. Therefore, Humanin renders protection against Ox-LDL-mediated oxidative stress and is cytoprotective in human vasculature [50].

\subsection{Protects Germ Cells/Leukocytes_Reduces Cancer Metastases}

A recent study by Jia et al. provided evidence of the role of Humanin in maintaining germ cell homeostasis. Substantive Humanin-mediated protection against chemotherapy-induced male germ cell apoptosis both in vivo and ex vivo was observed. The study also demonstrated that this anti-apoptotic effect is primarily mediated via STAT3 and BAX signaling [51].

\subsection{Germ Cell Apoptosis by Chemo Drugs}

Another similar study by Lue et al. demonstrated that Humanin G prevents cyclophosphamideinduced toxicity and death of male germ cells and leukocytes. In this study, HNG protected normal cells against stress and suppressed cancer metastases [52].

\subsection{Cytoprotection in Carotid Atherosclerotic Plaques}

In addition, it has been reported that Humanin is present in carotid atherosclerotic plaques and higher expression of Humanin in symptomatic patients compared to asymptomatic patients could be a part of a stress-response defense mechanism to delay the progression of the disease [53].

\section{SHLPs (Small Humanin-Like Peptides)}

The $16 \mathrm{~S}$ rRNA region of the mitochondrial DNA also codes for another category of MDPs called Small Humanin-Like Peptides (SHLPs), which include SHLP1, SHLP2, SHLP3, SHLP4, SHLP5, and SHLP6. SHLPs are 24-38 amino acids long and each SHLP may differentially regulate mitochondrial and cellular health and functions. Extensive characterization of SHLP2, SHLP3, and SHLP6 has been detailed in recent literature but the specific biological functions of the SHLPs are still being studied.

\subsection{SHLP2}

SHLP2 has a molecular weight of $3017.54 \mathrm{Da}$ and is 26 amino acids long with the sequence H-MGVKFFTLSTRFFPSVQRAVPLWTNS-OH (Figure 1). The plasma SHLP2 levels decline with age, suggesting its critical role in aging. SHLP2 stabilizes the AMD mitochondria by preserving the mitochondrial oxidative phosphorylation protein complex subunits (I-V) in AMD RPE transmitochondrial cells, and also promotes mitochondrial metabolism. This study also highlighted the potential of an exogenously added SHLP2 peptide to enhance mitochondria-specific mtGFP fluorescence staining, increase mtDNA copy numbers, and upregulate the PGC-1 $\alpha$ gene, which is a master regulator of mitochondrial biogenesis [54]. This in vitro study by Nashine et al. is the first study that demonstrates an association between SHLP2 and AMD transmitochondrial RPE cells, and therefore provides a basis for further explorative studies in the field of AMD. Pretreatment with SHLP2 inhibited apoptotic cell death as evidenced by higher live cell numbers and substantive downregulation of effector caspases, namely Caspase- 3 and Caspase-7, in AMD RPE cells. Moreover, SHLP2 rescued and protected AMD RPE cybrid cells against amyloid- $\beta$-induced toxicity in vitro (Figure 3 ). Therefore, SHLP2 could be considered a potential therapeutic candidate for macular degeneration. SHLP2 also mediates chaperone-like activity, prevents the misfolding of amyloid polypeptides, and prevents neuronal cell death [55]. 


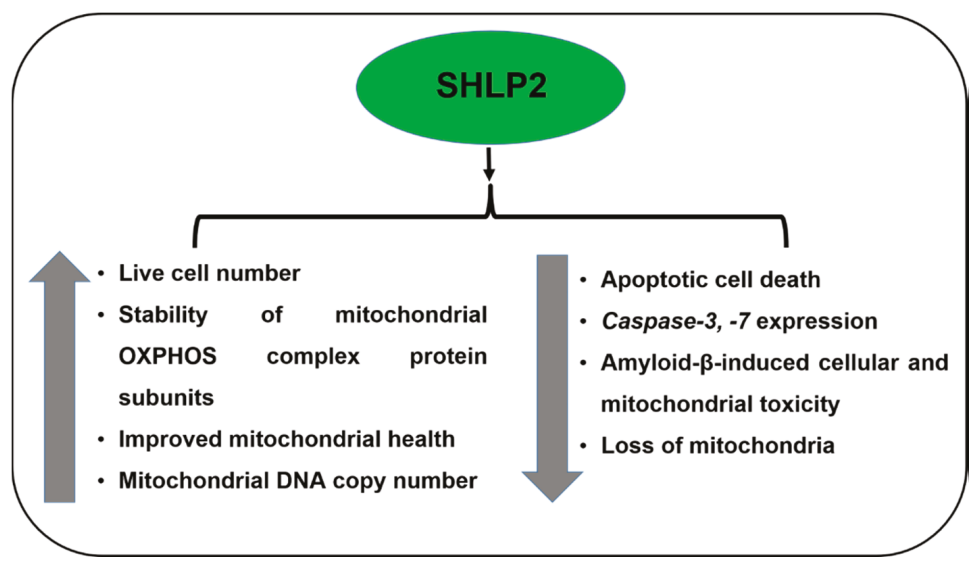

Figure 3. Effects of SHLP2 in AMD.

\subsection{SHLP3}

SHLP3 is a 38 amino acids-long peptide with the sequence H-MLGYNFSSFPCGTISIAPGFN FYRLYFIWVNGLAKVVW-OH and has a molecular weight of $4380.15 \mathrm{Da}$. SHLP3 increases cellular ATP levels and mitochondrial oxygen consumption rate (OCR) in vitro. SHLP3 suppresses ROS generation, mediates ERK signaling, promotes adipocyte cellular differentiation, and blocks staurosporine-induced apoptosis, thereby promoting mitochondrial and cellular survival and functions. SHLP2 and SHLP3 have insulin-sensitizing effects both in vivo and in vitro, and are known to increase leptin levels. SHLP2 functions as an insulin sensitizer both peripherally and centrally as it enhances peripheral glucose uptake and inhibits glucose production in the liver. SHLP3 also increases IL-6 and MCP-1 levels.

SHLP4 increases cell proliferation in the NIT-1 and 22RV-1 cell lines in vitro. SHLP6 drastically enhances apoptotic cell death in the NIT-1 and 22RV-1 cell lines, and exerts an effect opposite of SHLP2 and SHLP3, which are cytoprotective molecules [56]. Among all the SHLPs, SHLP2 is the only peptide whose cytoprotective function has been established in AMD (Figure 3).

\section{MOTS-c}

MOTS-c (Mitochondrial ORF (Open Reading Frame) within the Twelve S rRNA c) is encoded in the $12 \mathrm{~S}$ rRNA region of the mtDNA, and is a 16-amino acid peptide with the sequence H-MRWQEMG YIFYPRKLR-OH and a molecular weight 2174.7 Da (Figure 4). It is associated with insulin resistance and is found in plasma, brain, liver, and muscle tissues. MOTS-c enhances insulin sensitivity and regulates plasma metabolites in three metabolic pathways, namely sphingolipid metabolism, monoacylglycerol metabolism, and dicarboxylate metabolism. MOTS-c indirectly decreases cellular oxidative stress by reducing plasma oxidized glucose levels. MOTS-c also activates AKT phosphorylation and AMPK pathways. Moreover, MOTS-c reduces skeletal muscle fatigue and improves performance in vivo [57]. This is the first report by Cohen et al. that delineates the specific roles of MOTS-c and opens new avenues for further research with this MDP. MOTS-c enhances mitochondrial respiration in senescent cells by regulating fatty acid oxidation and increasing the senescence-related effectors [58]. Since MOTS-c levels also decline with age, it has been implicated in the regulation of lifespan and healthspan in organisms. This lifespan/healthspan prolonging ability of MOTS-c may be attributed to (1) MOTS-c-mediated increase in intracellular $\mathrm{NAD}^{+}$, a key redox metabolic coenzyme that activates sirtuins, which in turn regulate aging; and (2) MOTS-c-mediated reduction in methionine metabolism via inhibition of the folate/methionine cycle [59]. Although the function of MOTS-c as a crucial player in cell longevity, mitochondrial function, and metabolic homeostasis has been well-established, its specific protective function in the eye is yet to be reported. Since the AMD etiology involves a metabolic component as 
well as mitochondrial dysregulation, it would be interesting to investigate the cytoprotective effects of MOTS-c in AMD pathology (Figure 5).

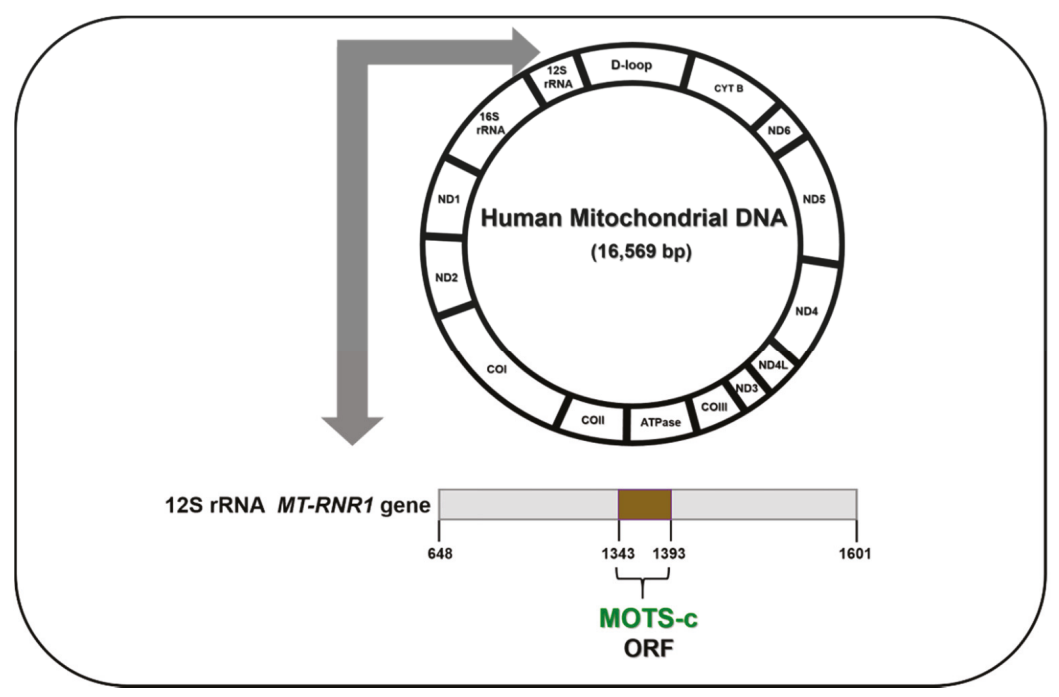

Figure 4. MOTS-c ORF in the human mitochondrial DNA.

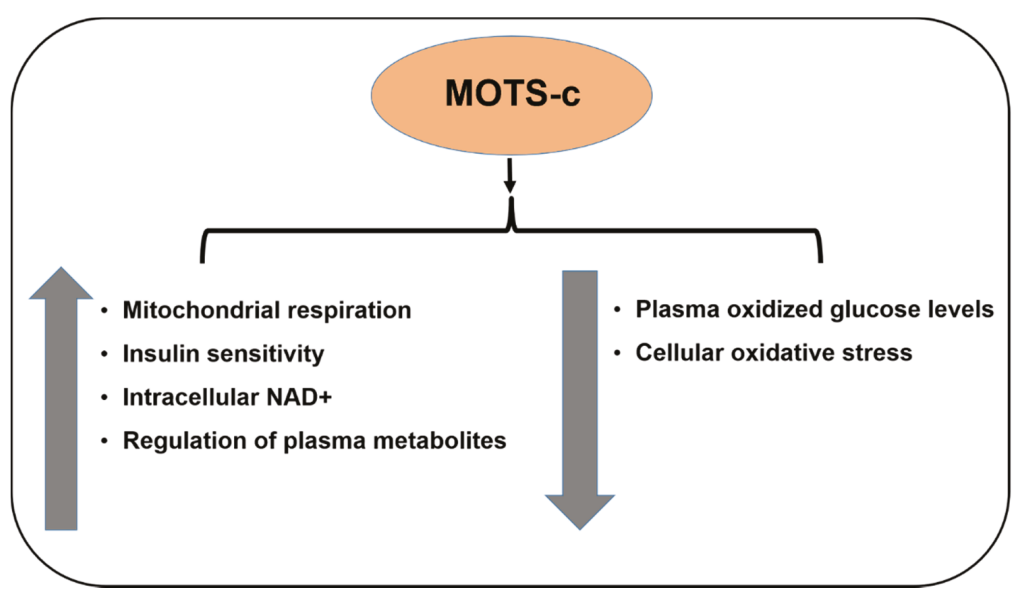

Figure 5. Effects of MOTS-c.

CohBar, a clinical-stage biotechnology company focused on the research and development of mitochondria-based therapeutics, has successfully completed a Phase 1a clinical study and initiated the Phase $1 \mathrm{~b}$ stage of a double-blind, placebo-controlled clinical trial of CB4211 as a potential treatment for nonalcoholic steatohepatitis (NASH) and obesity. CB4211 is the first therapeutic candidate based on a mitochondrial-derived peptide to enter clinical testing in humans. The completed Phase 1a stage of the CB4211 clinical study evaluated safety and tolerability, and the drug was safe and well tolerated after seven days of dosing. The Phase $1 \mathrm{~b}$ stage of the study will be an assessment of safety, tolerability, and activity in obese subjects with nonalcoholic fatty liver diseases (NAFLD). Assessments will include changes in liver fat assessed by MRI-PDFF, body weight, and biomarkers relevant to NASH and obesity [60]. 


\section{Conclusions and Future Directions}

The dry and wet forms of AMD have some common denominators in terms of AMD disease pathology, since the characteristic features of dry AMD, i.e., RPE cell apoptosis and accumulation of drusen deposits (in which amyloid- $\beta$ is a key component), may subsequently lead to choroidal neovascularization observed in wet AMD pathology. To our knowledge, differential effects of MDPs in dry AMD versus wet AMD have not been delineated and reported yet.

Since this manuscript presents the literature that establishes the role of MDPs in mitigating RPE cell apoptosis and reducing amyloid- $\beta$-induced toxicity, it suggests that MDPs are potential therapeutic candidates for treatment of dry AMD, and may delay its progression to the late form, i.e., wet AMD. However, the specific therapeutic effects of MDPs in reducing or preventing choroidal neovascularization in wet AMD need to be characterized. Experimentally, the effects of MDPs on the angiogenesis-promoting factors should be tested to evaluate if MDPs are able to downregulate the pro-angiogenic factors and related pathways, and thereby prevent or suppress neovascularization in wet AMD.

In conclusion, MDPs, especially Humanin (and its analogs) and SHLPs, provide cytoprotection in ocular diseases, including AMD, and may be considered as potential therapeutic targets for AMD. However, the use of MDPs as therapeutic agents for AMD will require development of appropriate delivery techniques and formulations. Currently, nanoparticles encapsulating Humanin are being tested in some labs to subsequently facilitate its efficient delivery and sustained action. In a recent study by Li et al., it was demonstrated that the Humanin peptide mediates elastin-like polypeptide nano-assembly and protects human RPE cells against oxidative stress-induced apoptotic cell death. This technology may facilitate cellular delivery of biodegradable nanoparticles with potential protection against AMD [61]. Furthermore, with the development of precision medicine, the use of MDPs can be customized for AMD therapy to match patient needs.

Author Contributions: S.N.: Wrote and edited the manuscript; M.C.K.: Corresponding author. All authors have read and agreed to the published version of the manuscript.

Funding: This work is supported by Arnold and Mabel Beckman foundation, NEI R01 EY0127363, Discovery Eye Foundation, Polly and Michael Smith, Edith and Roy Carver, Iris and B. Gerald Cantor Foundation, Unrestricted Departmental Grant from Research to Prevent Blindness, UCI School of Medicine, and support of the Institute for Clinical and Translational Science (ICTS) at University of California Irvine. S.N. is a recipient of the 2017 Genentech/ ARVO AMD Translational Research Fellowship and the 2016 RPB pilot research grant.

Conflicts of Interest: The authors declare no conflict of interest.

\section{References}

1. Klein, R.; Chou, C.F.; Klein, B.E.; Zhang, X.; Meuer, S.M.; Saaddine, J.B. Prevalence of age-related macular degeneration in the US population. Arch Ophthalmol. 2011, 129, 75-80. [CrossRef] [PubMed]

2. McCusker, M.M.; Durrani, K.; Payette, M.J.; Suchecki, J. An eye on nutrition: The role of vitamins, essential fatty acids, and antioxidants in age-related macular degeneration, dry eye syndrome, and cataract. Clin. Dermatol. 2016, 34, 276-285. [CrossRef] [PubMed]

3. Au, A.; Parikh, V.S.; Singh, R.P.; Ehlers, J.P.; Yuan, A.; Rachitskaya, A.V.; Sears, J.E.; Srivastava, S.K.; Kaiser, P.K.; Schachat, A.P.; et al. Comparison of anti-VEGF therapies on fibrovascular pigment epithelial detachments in age-related macular degeneration. Br. J. Ophthalmol. 2016, 101, 970-975. [CrossRef] [PubMed]

4. Karunadharma, P.P.; Nordgaard, C.L.; Olsen, T.W.; Ferrington, D.A. Mitochondrial DNA. Damage as a potential mechanism for age-related macular degeneration. Invest Ophthalmol Vis. Sci. 2010, 51, 5470-5479. [CrossRef]

5. Nordgaard, C.L.; Karunadharma, P.P.; Feng, X.; Olsen, T.W.; Ferrington, D.A. Mitochondrial proteomics of the retinal pigment epithelium at progressive stages of age-related macular degeneration. Invest Ophthalmol. Vis. Sci. 2008, 49, 2848-2855. [CrossRef]

6. Nordgaard, C.L.; Berg, K.M.; Kapphahn, R.J.; Reilly, C.; Feng, X.; Olsen, T.W.; Ferrington, D.A. Proteomics of the retinal pigment epithelium reveals altered protein expression at progressive stages of age-related macular degeneration. Invest Ophthalmol. Vis. Sci. 2006, 47, 815-822. [CrossRef] 
7. Wang, J.; Zibetti, C.; Shang, P.; Sripathi, S.R.; Zhang, P.; Cano, M.; Hoang, T.; Xia, S.; Ji, H.; Merbs, S.L.; et al. ATAC-Seq analysis reveals a widespread decrease of chromatin accessibility in age-related macular degeneration. Nat. Commun. 2018, 9, 1364. [CrossRef]

8. Clayton, D.A. Structure and function of the mitochondrial genome. J. Inherit. Metab. Dis. 1992, 15, 439-447. [CrossRef]

9. Holt, I.J.; Reyes, A. Human mitochondrial DNA replication. Cold Spring Harb. Perspect. Biol. 2012, 4, a012971. [CrossRef]

10. Brand, M.D.; Orr, A.L.; Perevoshchikova, I.V.; Quinlan, C.L. The role of mitochondrial function and cellular bioenergetics in ageing and disease. Br. J. Dermatol. 2013, 169, 1-8. [CrossRef]

11. Wong-Riley, M.T. Energy metabolism of the visual system. Eye Brain 2010, 2, 99-116. [CrossRef]

12. Eells, J.T. Mitochondrial Dysfunction in the Aging Retina. Biology 2019, 8, 31. [CrossRef]

13. Chistiakov, D.A.; Sobenin, I.A.; Revin, V.V.; Orekhov, A.N.; Bobryshev, Y.V. Mitochondrial aging and age-related dysfunction of mitochondria. Biomed Res. Int. 2014, 2014, 238463. [CrossRef]

14. Taanman, J.W. The mitochondrial genome: Structure, transcription, translation and replication. Biochim. Biophys. Acta 1999, 1410, 103-123. [CrossRef]

15. Cardamone, M.D.; Tanasa, B.; Cederquist, C.T.; Huang, J.; Mahdaviani, K.; Li, W.; Rosenfeld, M.G.; Liesa, M.; Perissi, V. Mitochondrial Retrograde Signaling in Mammals Is Mediated by the Transcriptional Cofactor GPS2 via Direct Mitochondria-to-Nucleus Translocation. Mol. Cell 2018, 69, 757-772.e7. [CrossRef]

16. Yang, L.; Tan, Z.; Wang, D.; Xue, L.; Guan, M.X.; Huang, T.; Li, R. Species identification through mitochondrial rRNA genetic analysis. Sci. Rep. 2014, 4, 4089. [CrossRef]

17. Yen, K.; Lee, C.; Mehta, H.; Cohen, P. The emerging role of the mitochondrial-derived peptide humanin in stress resistance. J. Mol. Endocrinol. 2013, 50, R11-R19. [CrossRef]

18. Fuku, N.; Pareja-Galeano, H.; Zempo, H.; Alis, R.; Arai, Y.; Lucia, A.; Hirose, N. The mitochondrial-derived peptide MOTS-c: A player in exceptional longevity? Aging Cell 2015, 14, 921-923. [CrossRef]

19. Kim, K.H.; Son, J.M.; Benayoun, B.A.; Lee, C. The Mitochondrial-Encoded Peptide MOTS-c Translocates to the Nucleus to Regulate Nuclear Gene Expression in Response to Metabolic Stress. Cell Metab. 2018, 28, 516-524.e7. [CrossRef]

20. Mehta, H.H.; Xiao, J.; Ramirez, R.; Miller, B.; Kim, S.J.; Cohen, P.; Yen, K. Metabolomic profile of diet-induced obesity mice in response to humanin and small humanin-like peptide 2 treatment. Metabolomics 2019, 15, 88. [CrossRef]

21. Hashimoto, Y.; Niikura, T.; Tajima, H.; Yasukawa, T.; Sudo, H.; Ito, Y.; Kita, Y.; Kawasumi, M.; Kouyama, K.; Doyu, M.; et al. A rescue factor abolishing neuronal cell death by a wide spectrum of familial Alzheimer's disease genes and Abeta. Proc. Natl. Acad. Sci. USA 2001, 98, 6336-6341. [CrossRef]

22. Yamagishi, Y.; Hashimoto, Y.; Niikura, T.; Nishimoto, I. Identification of essential amino acids in Humanin, a neuroprotective factor against Alzheimer's disease-relevant insults. Peptides 2003, 24, 585-595. [CrossRef]

23. Zhang, X.; Urbieta-Caceres, V.H.; Eirin, A.; Bell, C.C.; Crane, J.A.; Tang, H.; Jordan, K.L.; Oh, Y.K.; $\mathrm{Zhu}$, X.Y.; Korsmo, M.J.; et al. Humanin prevents intra-renal microvascular remodeling and inflammation in hypercholesterolemic ApoE deficient mice. Life Sci. 2012, 91, 199-206. [CrossRef]

24. Widmer, R.J.; Flammer, A.J.; Herrmann, J.; Rodriguez-Porcel, M.; Wan, J.; Cohen, P.; Lerman, L.O.; Lerman, A. Circulating humanin levels are associated with preserved coronary endothelial function. Am. J. Physiol. Heart Circ. Physiol. 2013, 304, H393-H397. [CrossRef]

25. Muzumdar, R.H.; Huffman, D.M.; Calvert, J.W.; Jha, S.; Weinberg, Y.; Cui, L.; Nemkal, A.; Atzmon, G.; Klein, L.; Gundewar, S.; et al. Acute humanin therapy attenuates myocardial ischemia and reperfusion injury in mice. Arterioscler. Thromb. Vasc. Biol. 2010, 30, 1940-1948. [CrossRef]

26. Moretti, E.; Giannerini, V.; Rossini, L.; Matsuoka, M.; Trabalzini, L.; Collodel, G. Immunolocalization of humanin in human sperm and testis. Fertil. Steril. 2010, 94, 2888-2890. [CrossRef]

27. Colón, E.; Strand, M.L.; Carlsson-Skwirut, C.; Wahlgren, A.; Svechnikov, K.V.; Cohen, P.; Söder, O. Anti-apoptotic factor humanin is expressed in the testis and prevents cell-death in leydig cells during the first wave of spermatogenesis. J. Cell Physiol. 2006, 208, 373-385. [CrossRef]

28. Tajima, H.; Niikura, T.; Hashimoto, Y.; Ito, Y.; Kita, Y.; Terashita, K.; Yamazaki, K.; Koto, A.; Aiso, S.; Nishimoto, I. Evidence for in vivo production of Humanin peptide, a neuroprotective factor against Alzheimer's disease-related insults. Neurosci. Lett. 2002, 324, 227-231. [CrossRef] 
29. Muzumdar, R.H.; Huffman, D.M.; Atzmon, G.; Buettner, C.; Cobb, L.J.; Fishman, S.; Budagov, T.; Cui, L.; Einstein, F.H.; Poduval, A.; et al. Humanin: A novel central regulator of peripheral insulin action. PLoS ONE 2009, 4, e6334. [CrossRef]

30. Chin, Y.P.; Keni, J.; Wan, J.; Mehta, H.; Anene, F.; Jia, Y.; Lue, Y.H.; Swerdloff, R.; Cobb, L.J.; Wang, C.; et al. Pharmacokinetics and tissue distribution of humanin and its analogues in male rodents. Endocrinology 2013, 154, 3739-3744. [CrossRef]

31. Hashimoto, Y.; Kurita, M.; Aiso, S.; Nishimoto, I.; Matsuoka, M. Humanin inhibits neuronal cell death by interacting with a cytokine receptor complex or complexes involving CNTF receptor alpha/WSX-1/gp130. Mol. Biol. Cell. 2009, 20, 2864-2873. [CrossRef] [PubMed]

32. Hashimoto, Y.; Suzuki, H.; Aiso, S.; Niikura, T.; Nishimoto, I.; Matsuoka, M. Involvement of tyrosine kinases and STAT3 in Humanin-mediated neuroprotection. Life Sci. 2005, 77, 3092-3104. [CrossRef]

33. Taga, T.; Kishimoto, T. Gp130 and the interleukin-6 family of cytokines. Annu. Rev. Immunol. 1997, 15, 797-819. [CrossRef]

34. Lee, C.; Wan, J.; Miyazaki, B.; Fang, Y.; Guevara-Aguirre, J.; Yen, K.; Longo, V.; Bartke, A.; Cohen, P. IGF-I regulates the age-dependent signaling peptide humanin. Aging Cell. 2014, 13, 958-961. [CrossRef]

35. Njomen, E.; Evans, H.G.; Gedara, S.H.; Heyl, D.L. Humanin Peptide Binds to Insulin-Like Growth Factor-Binding Protein 3 (IGFBP3) and Regulates Its Interaction with Importin- $\beta$. Protein Pept. Lett. 2015, 22, 869-876. [CrossRef]

36. Guo, B.; Zhai, D.; Cabezas, E.; Welsh, K.; Nouraini, S.; Satterthwait, A.C.; Reed, J.C. Humanin peptide suppresses apoptosis by interfering with Bax activation. Nature 2003, 423, 456-461. [CrossRef]

37. Luciano, F.; Zhai, D.; Zhu, X.; Bailly-Maitre, B.; Ricci, J.E.; Satterthwait, A.C.; Reed, J.C. Cytoprotective peptide humanin binds and inhibits proapoptotic Bcl-2/Bax family protein BimEL. J. Biol. Chem. 2005, 280, 15825-15835. [CrossRef]

38. Nashine, S.; Cohen, P.; Chwa, M.; Lu, S.; Nesburn, A.B.; Kuppermann, B.D.; Kenney, M.C. Humanin G (HNG) protects age-related macular degeneration (AMD) transmitochondrial ARPE-19 cybrids from mitochondrial and cellular damage. Cell Death Dis. 2017, 8, e2951. [CrossRef]

39. Ikonen, M.; Liu, B.; Hashimoto, Y.; Ma, L.; Lee, K.W.; Niikura, T.; Nishimoto, I.; Cohen, P. Interaction between the Alzheimer's survival peptide humanin and insulin-like growth factor-binding protein 3 regulates cell survival and apoptosis. Proc. Natl. Acad. Sci. USA 2003, 100, 13042-13047. [CrossRef]

40. Gong, Z.; Tasset, I. Humanin enhances the cellular response to stress by activation of chaperone-mediated autophagy. Oncotarget 2018, 9, 10832-10833. [CrossRef]

41. Sreekumar, P.G.; Ishikawa, K.; Spee, C.; Mehta, H.H.; Wan, J.; Yen, K.; Cohen, P.; Kannan, R.; Hinton, D.R. The Mitochondrial-Derived Peptide Humanin Protects RPE Cells from Oxidative Stress, Senescence, and Mitochondrial Dysfunction. Invest Ophthalmol. Vis. Sci. 2016, 57, 1238-1253. [CrossRef]

42. Gurunathan, S.; Jeyaraj, M.; Kang, M.H.; Kim, J.H. Mitochondrial Peptide Humanin Protects Silver Nanoparticles-Induced Neurotoxicity in Human Neuroblastoma Cancer Cells (SH-SY5Y). Int. J. Mol. Sci. 2019, 20, 4439. [CrossRef]

43. Li, X.; Zhao, W.; Yang, H.; Zhang, J.; Ma, J. S14G-humanin restored cellular homeostasis disturbed by amyloid-beta protein. Neural Regen. Res. 2013, 8, 2573-2580. [PubMed]

44. Minasyan, L.; Sreekumar, P.G.; Hinton, D.R.; Kannan, R. Protective Mechanisms of the Mitochondrial-Derived Peptide Humanin in Oxidative and Endoplasmic Reticulum Stress in RPE Cells. Oxid. Med. Cell Longev. 2017, 2017, 1675230. [CrossRef] [PubMed]

45. Knapp, A.; Czech, U.; Polus, A.; Chojnacka, M.; Śliwa, A.; Awsiuk, M.; Zapała, B.; Malińska, D.; Szewczyk, A.; Dembińska-Kieć, A. Humanin Peptides Regulate Calcium Flux in the Mammalian Neuronal, Glial and Endothelial Cells under Stress Conditions. J. Cell Sci. Ther. 2012, 3, 128. [CrossRef]

46. Matsunaga, D.; Sreekumar, P.G.; Ishikawa, K.; Terasaki, H.; Barron, E.; Cohen, P.; Kannan, R.; Hinton, D.R. Humanin Protects RPE Cells from Endoplasmic Reticulum Stress-Induced Apoptosis by Upregulation of Mitochondrial Glutathione. PLoS ONE 2016, 11, e0165150. [CrossRef]

47. Xu, X.; Chua, C.C.; Gao, J.; Chua, K.W.; Wang, H.; Hamdy, R.C.; Chua, B.H. Neuroprotective effect of humanin on cerebral ischemia/reperfusion injury is mediated by a PI3K/Akt pathway. Brain Res. 2008, 1227, 12-18. [CrossRef]

48. Kim, S.J.; Guerrero, N.; Wassef, G.; Xiao, J.; Mehta, H.H.; Cohen, P.; Yen, K. The mitochondrial-derived peptide humanin activates the ERK1/2, AKT, and STAT3 signaling pathways and has age-dependent signaling differences in the hippocampus. Oncotarget 2016, 7, 46899-46912. [CrossRef] 
49. Oh, Y.K.; Bachar, A.R.; Zacharias, D.G.; Kim, S.G.; Wan, J.; Cobb, L.J.; Lerman, L.O.; Cohen, P.; Lerman, A. Humanin preserves endothelial function and prevents atherosclerotic plaque progression in hypercholesterolemic ApoE deficient mice. Atherosclerosis 2011, 219, 65-73. [CrossRef]

50. Bachar, A.R.; Scheffer, L.; Schroeder, A.S.; Nakamura, H.K.; Cobb, L.J.; Oh, Y.K.; Lerman, L.O.; Pagano, R.E.; Cohen, P.; Lerman, A. Humanin is expressed in human vascular walls and has a cytoprotective effect against oxidized LDL-induced oxidative stress. Cardiovasc. Res. 2010, 88, 360-366. [CrossRef]

51. Jia, Y.; Ohanyan, A.; Lue, Y.H.; Swerdloff, R.S.; Liu, P.Y.; Cohen, P.; Wang, C. The effects of humanin and its analogues on male germ cell apoptosis induced by chemotherapeutic drugs. Apoptosis 2015, 20, 551-561. [CrossRef] [PubMed]

52. Lue, Y.; Swerdloff, R.; Wan, J.; Xiao, J.; French, S.; Atienza, V.; Canela, V.; Bruhn, K.W.; Stone, B.; Jia, Y.; et al. The Potent Humanin Analogue (HNG) Protects Germ Cells and Leucocytes While Enhancing Chemotherapy-Induced Suppression of Cancer Metastases in Male Mice. Endocrinology 2015, 156, 4511-4521. [CrossRef] [PubMed]

53. Zacharias, D.G.; Kim, S.G.; Massat, A.E.; Bachar, A.R.; Oh, Y.K.; Herrmann, J.; Rodriguez-Porcel, M.; Cohen, P.; Lerman, L.O.; Lerman, A. Humanin, a cytoprotective peptide, is expressed in carotid atherosclerotic [corrected] plaques in humans. PLoS ONE. 2012, 7, e31065. [CrossRef]

54. Nashine, S.; Cohen, P.; Nesburn, A.B.; Kuppermann, B.D.; Kenney, M.C. Characterizing the protective effects of SHLP2, a mitochondrial-derived peptide, in macular degeneration. Sci. Rep. 2018, 8, 15175. [CrossRef]

55. Okada, A.K.; Teranishi, K.; Lobo, F.; Isas, J.M.; Xiao, J.; Yen, K.; Cohen, P.; Langen, R. The Mitochondrial-Derived Peptides, HumaninS14G and Small Humanin-like Peptide 2, Exhibit Chaperone-like Activity. Sci. Rep. 2017, 7, 7802. [CrossRef]

56. Cobb, L.J.; Lee, C.; Xiao, J.; Yen, K.; Wong, R.G.; Nakamura, H.K.; Mehta, H.H.; Gao, Q.; Ashur, C.; Huffman, D.M.; et al. Naturally occurring mitochondrial-derived peptides are age-dependent regulators of apoptosis, insulin sensitivity, and inflammatory markers. Aging (Albany NY) 2016, 8, 796-809. [CrossRef]

57. Kim, S.J.; Miller, B.; Mehta, H.H.; Xiao, J.; Wan, J.; Arpawong, T.E.; Yen, K.; Cohen, P. The mitochondrial-derived peptide MOTS-c is a regulator of plasma metabolites and enhances insulin sensitivity. Physiol. Rep. 2019, 7, e14171. [CrossRef]

58. Kim, S.J.; Mehta, H.H.; Wan, J.; Kuehnemann, C.; Chen, J.; Hu, J.F.; Hoffman, A.R.; Cohen, P. Mitochondrial peptides modulate mitochondrial function during cellular senescence. Aging (Albany NY) 2018, 10, 1239-1256. [CrossRef]

59. Lee, C.; Kim, K.H.; Cohen, P. MOTS-c: A novel mitochondrial-derived peptide regulating muscle and fat metabolism. Free Radic. Biol. Med. 2016, 100, 182-187. [CrossRef]

60. CohBar to Resume its Phase 1a/1b Clinical Trial. Available online: https://www.cohbar.com/news-media/ press-releases/detail/81/cohbar-to-resume-its-phase-1a1b-clinical-trial (accessed on 3 May 2019).

61. Li, Z.; Sreekumar, P.G.; Peddi, S.; Hinton, D.R.; Kannan, R.; MacKay, J.A. The humanin peptide mediates ELP nanoassembly and protects human retinal pigment epithelial cells from oxidative stress. Nanomedicine 2019, 24, 102111. [CrossRef]

(C) 2020 by the authors. Licensee MDPI, Basel, Switzerland. This article is an open access article distributed under the terms and conditions of the Creative Commons Attribution (CC BY) license (http://creativecommons.org/licenses/by/4.0/). 
MDPI

St. Alban-Anlage 66

4052 Basel

Switzerland

Tel. +41616837734

Fax +41 613028918

www.mdpi.com

Cells Editorial Office

E-mail: cells@mdpi.com www.mdpi.com/journal/cells

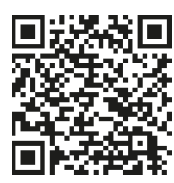



MDPI

St. Alban-Anlage 66

4052 Basel

Switzerland

Tel: +41 616837734

Fax: +41 613028918

www.mdpi.com 